\title{
Bevölkerungspolitik im Kontext ökologischer Generationengerechtigkeit
}

Von der Fakultät Wirtschafts- und Sozialwissenschaften der Universität Stuttgart zur Erlangung der Würde eines Doktors der Wirtschafts- und Sozialwissenschaften (Dr. rer. pol.) genehmigte Abhandlung.

Vorgelegt von

Jörg Tremmel

aus Frankfurt am Main.

Hauptberichter:

Prof. Dr. Ortwin Renn

Mitberichter:

Prof. Dr. Konrad Ott

Tag der mündlichen Prüfung: 14. Juli 2005

Institut für Sozialwissenschaften

2005 


\section{Für meine Laura}

Diese Promotion wurde von der Friedrich-Naumann-Stiftung mit Mitteln des Bundesministeriums für Bildung und Forschung gefördert. 


\section{Gliederungsübersicht}

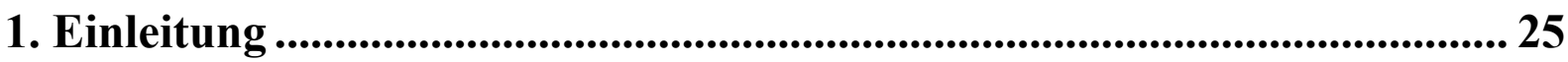

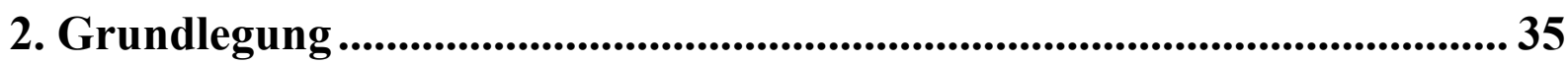

3. Ist Bevölkerung eine Ursache für Umweltbelastungen? ............................. 84

4. Ist intertemporale Generationengerechtigkeit ein sinnvolles Konzept? 112

5. Kann Bevölkerungspolitik ethisch vertretbar sein? ..................................... 125

6. Demografischer Wandel und Bevölkerungspolitik in Deutschland....... 213

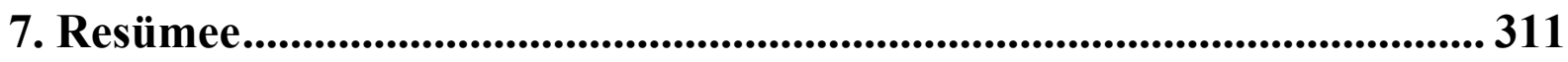




\section{Inhaltsverzeichnis}

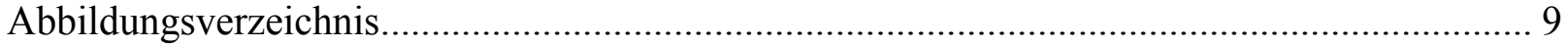

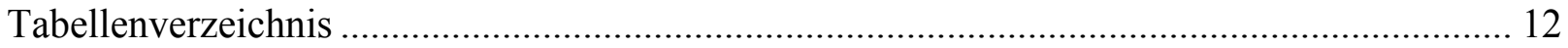

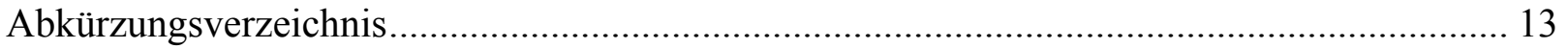

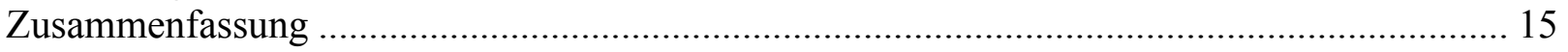

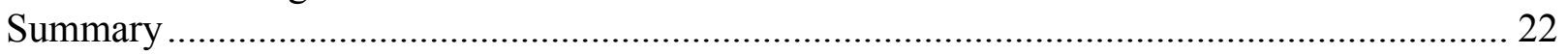

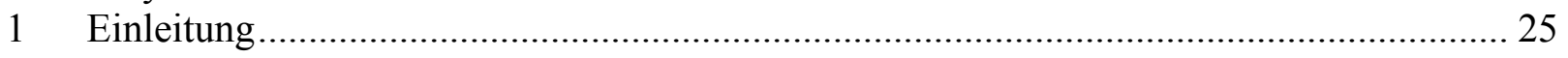

1.1 Problemstellung und Erkenntnisinteresse ......................................................... 25

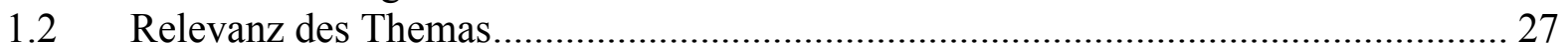

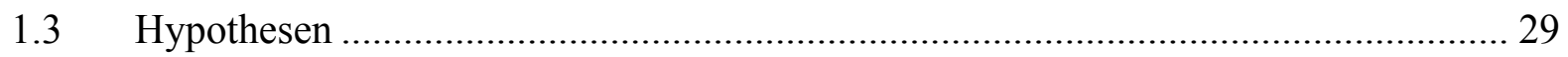

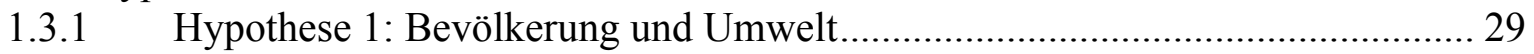

1.3.2 Hypothese 2: Verantwortung für spätere Generationen .................................. 30

1.3.3 Hypothese 3: Ethische Vertretbarkeit von Bevölkerungspolitik ........................ 30

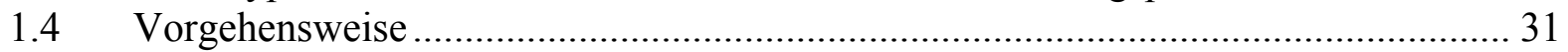

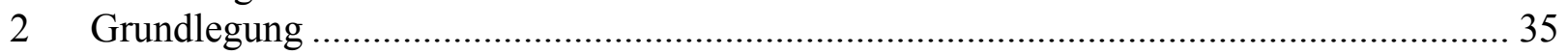

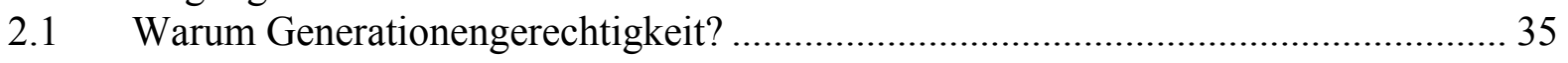

2.1.1 Beispiellose technologische Möglichkeiten................................................... 35

2.1.2 Beispielloses Wissen über die Zukunft......................................................... 39

2.1.3 Die Entdeckung des Themas Generationengerechtigkeit ................................ 39

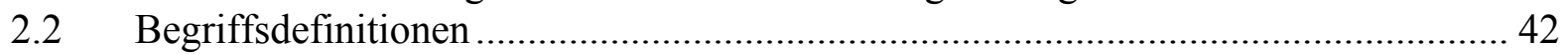

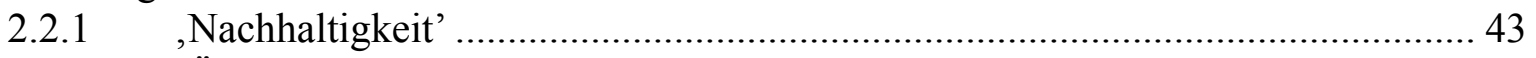

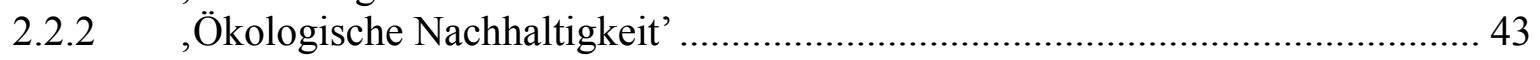

2.2.3 ,Dritte Welt', ,Entwicklungsländer' oder, Weniger Entwickelte Länder'? ....... 45

2.3 Relevanz demografischer Fragen für die Soziologie ............................................ 46

2.3.1 Demografie - eine verunsicherte Wissenschaft ................................................ 46

2.3.2 Erkenntnisobjekt und Teildisziplinen der Bevölkerungswissenschaft ............... 49

2.3.3 Der Unterschied zwischen, Bevölkerung' und, Gesellschaft' ............................ 50

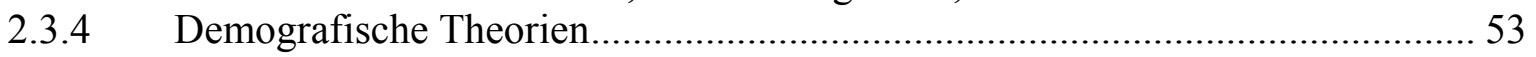

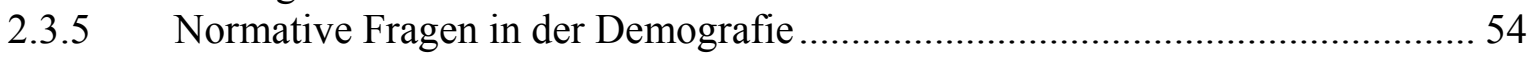

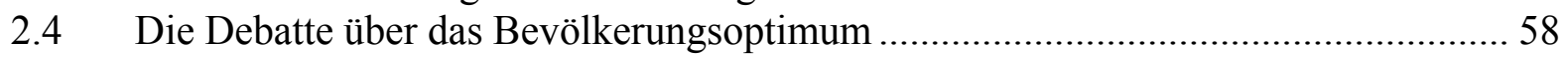

2.4.1 Das Bevölkerungsgesetz von Thomas Robert Malthus ..................................... 58

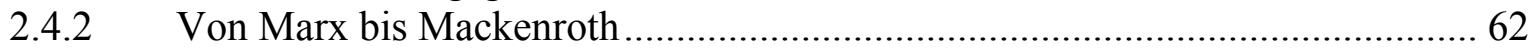

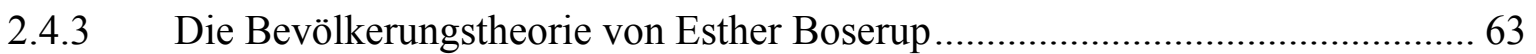

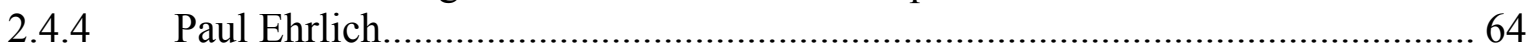

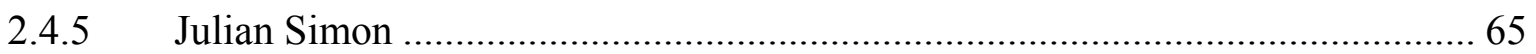

2.4.6 Das Bevölkerungsoptimum gemäß der Youth Bulge-Theorie ........................... 67

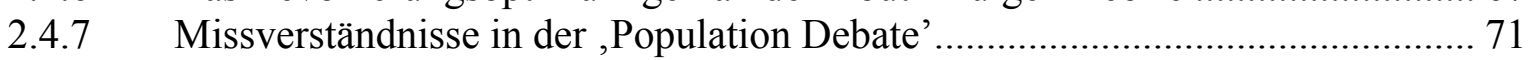

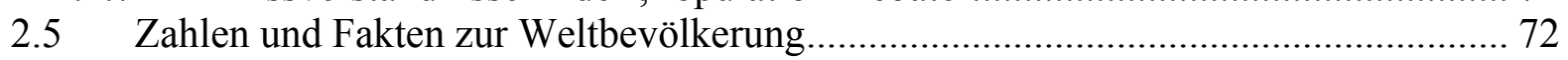

2.5.1 Bevölkerungsdynamik global und regional .................................................. 72

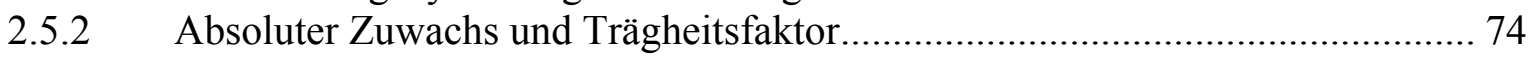

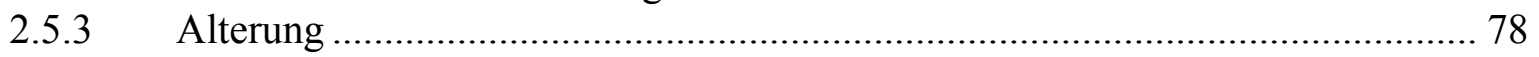

2.5.4 Regionale Verteilung der Bevölkerung in Nord und Süd............................... 79

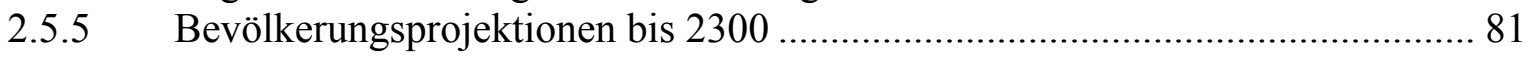

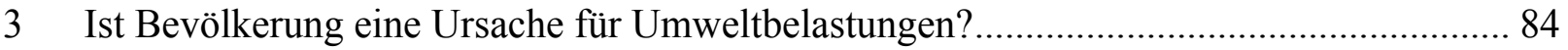

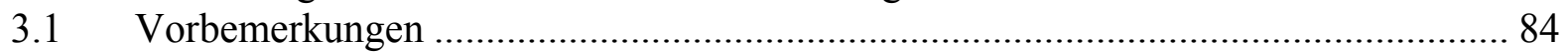

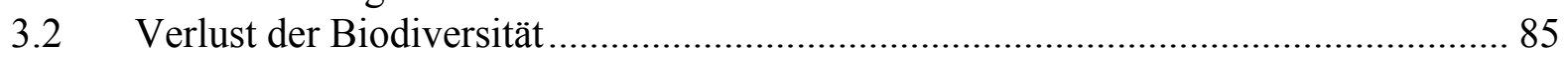

3.2.1 Drei Phasen anthropogen verursachten Artensterbens .................................... 85 


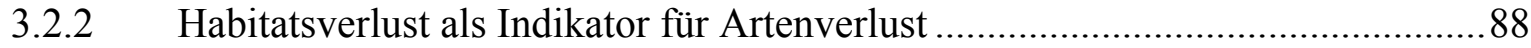

3.2.3 Fallstudie: Ranomafana-Wald in Madagaskar ................................................ 92

3.2.4 Populationsverlust als Indikator für Artenverlust..........................................93

3.2.5 Bevölkerungsgröße, -dichte oder wachstum als Ursache für Artenverlust? .......95

3.2.6 Zwischenfazit zu Bevölkerungsdichte und Artenverlust..................................96

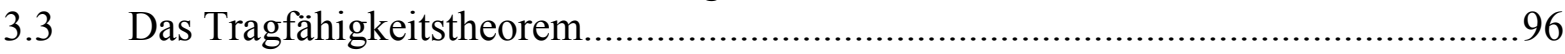

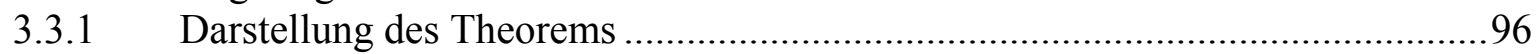

3.3.2 Inhärente Schwächen des Tragfähigkeits-Theorems ....................................... 97

3.3.2.1 Systematische Veränderung der Ressourcenbasis ........................................97

3.3.2.2 Abhängigkeit von sozialen und kulturellen Faktoren..................................101

3.3.3 Kritik am Tragfähigkeitstheorem aus ökologischer Sicht ................................. 102

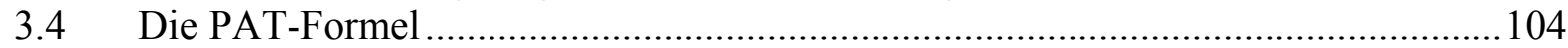

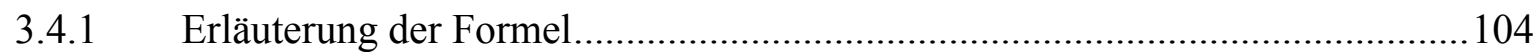

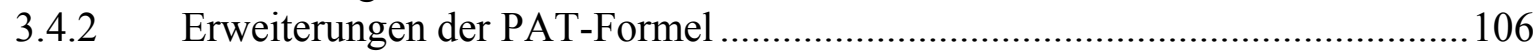

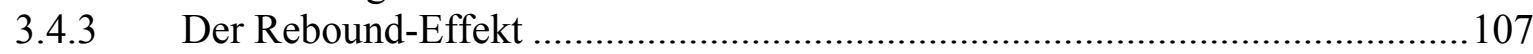

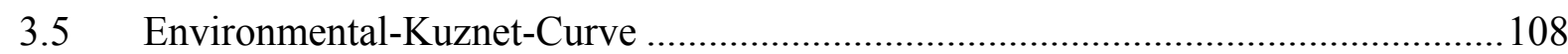

3.6 Anwendung der PAT-Formel auf verschiedene Umweltprobleme ........................... 108

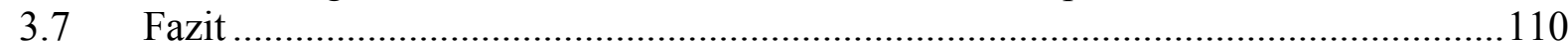

4 Ist intertemporale Generationengerechtigkeit ein sinnvolles Konzept? .........................112

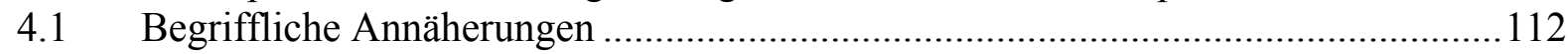

4.1.1 Die Mehrdeutigkeit des Begriffs , Generation' ................................................. 112

4.1.2 Soziologische und chronologische Definition von ,Generation' ........................ 114

4.1.3 Temporale und intertemporale Generationengerechtigkeit ..............................116

4.1.4 Eine Definition von intertemporaler Generationengerechtigkeit ....................... 118

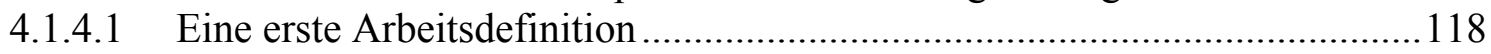

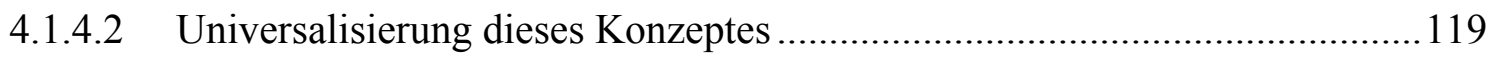

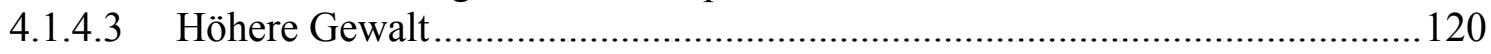

4.1.4.4 Fortschritt und Verbesserung - die zweite Arbeitsdefinition........................121

4.1.4.5 ,Künftige Generationen' vs. ,Angehörige künftiger Generationen'?.............122

4.1.4.6 Endergebnis: Eine Definition von Generationengerechtigkeit.......................123

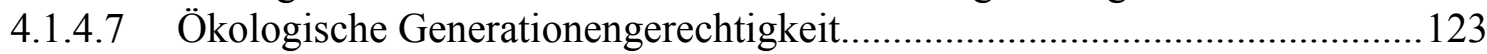

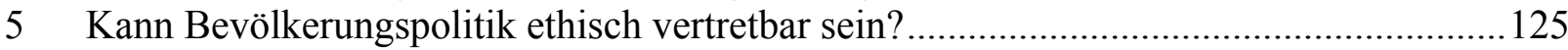

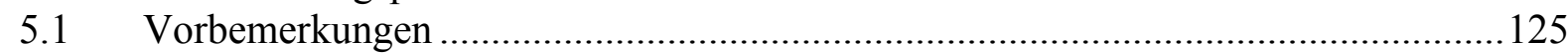

5.2 Ethische Bewertung von Bevölkerungspolitik in der abstrakt-philosophischen

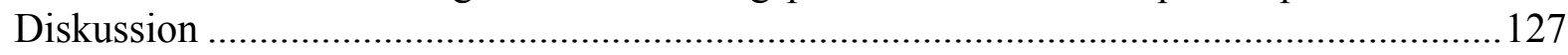

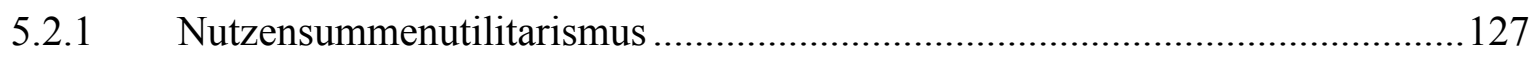

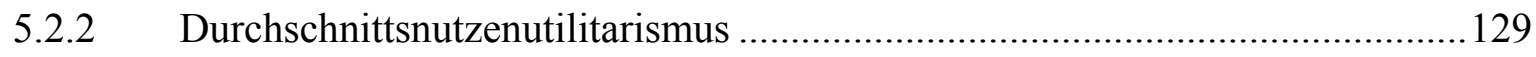

5.2.3 Argumente gegen einen reinen DNU bei bevölkerungsethischen Fragen..........130

5.2.4 Der Erhalt der Menschheit als Ausgangspunkt einer Generationenethik...........133

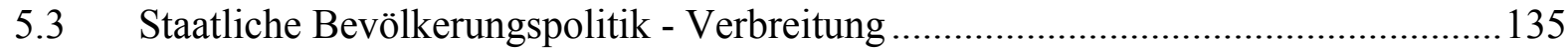

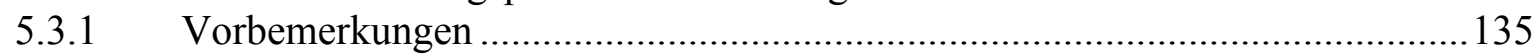

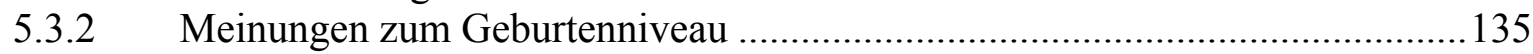

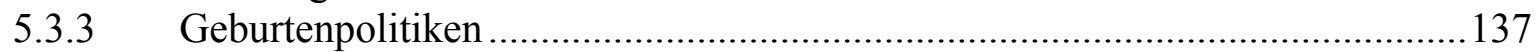

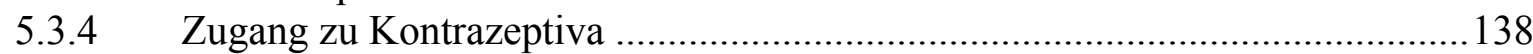

5.4 Die Bevölkerungspolitik Chinas........................................................................ 138

5.4.1 Instrumente und Reichweite der Ein-Kind-Politik ......................................... 140

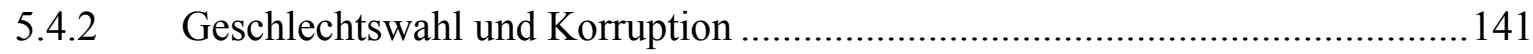

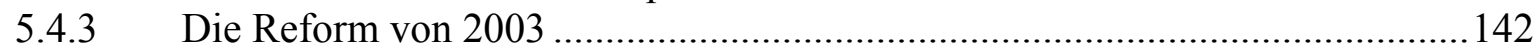

5.4.4 Der chinesische Blick auf die eigene Bevölkerungszahl .................................. 143 


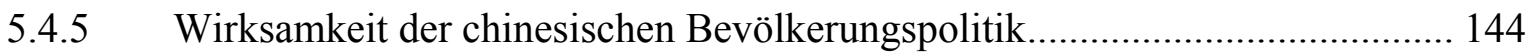

5.4.6 Die geringere Wirksamkeit der indischen Geburtenpolitik ............................. 147

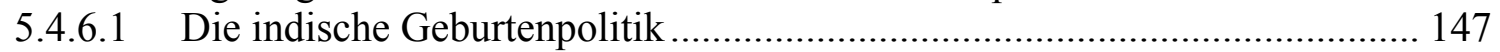

5.4.6.2 Vergleich der Geburtenpolitiken Chinas und Indiens.................................. 150

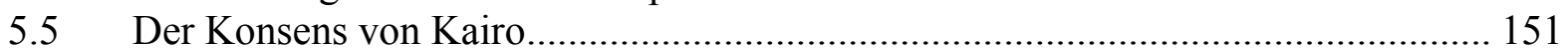

5.5.1 Reproduktive Gesundheit als neues Paradigma.......................................... 151

5.5.1.1 Stärkung von Frauen in allen Bereichen.................................................... 153

5.5.1.2 Der Bezug auf zukünftige Generationen ................................................ 154

5.5.1.3 Die Ausführungen zu Bevölkerungspolitik und Familienplanung ................ 156

5.5.1.4 Klares Bekenntnis zum Durchschnittsnutzenutilitarismus .......................... 157

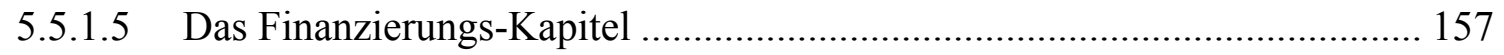

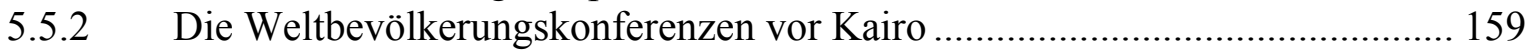

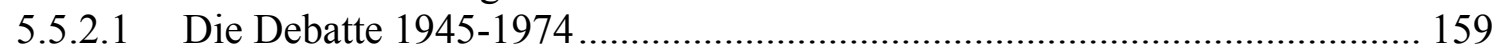

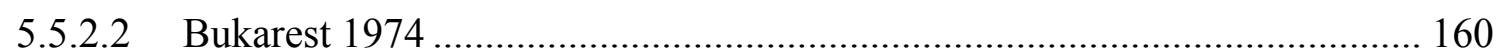

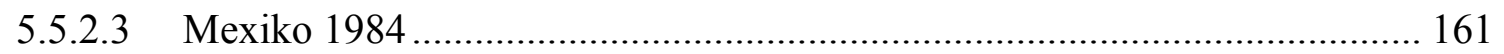

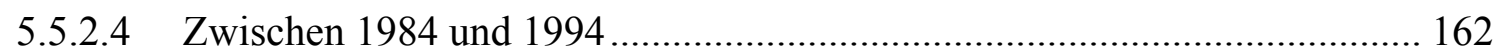

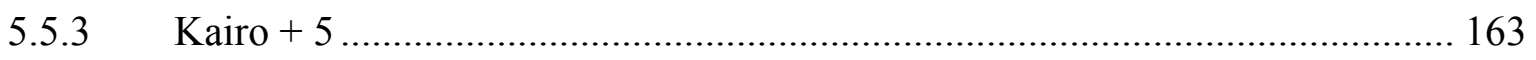

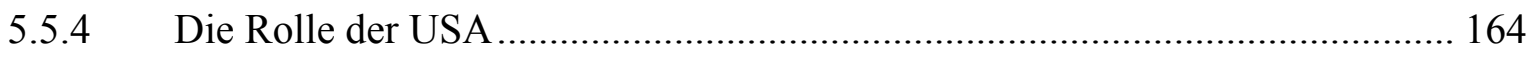

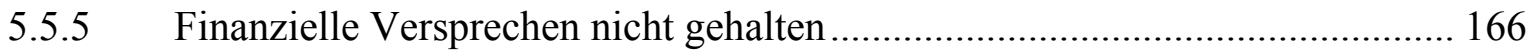

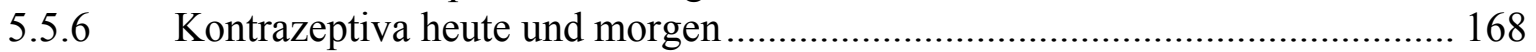

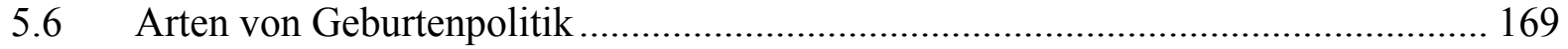

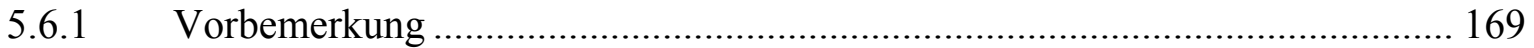

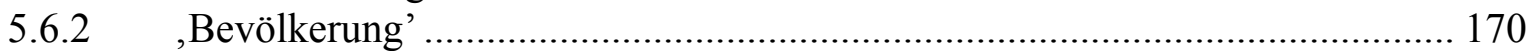

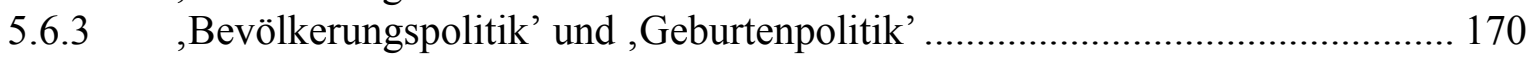

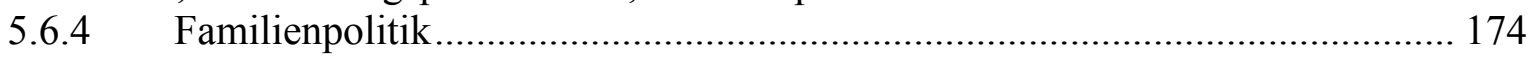

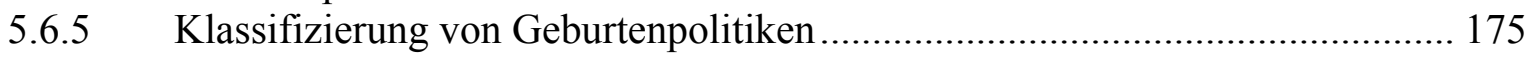

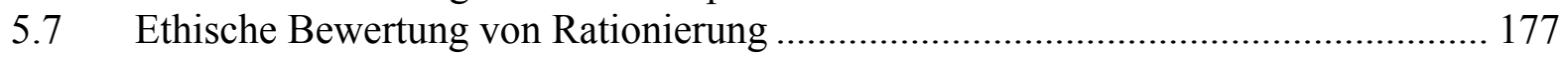

5.8 Ethische Bewertung des Konsenses von Kairo................................................... 178

5.8.1 „Menschen dürfen nie als Objekte betrachtet werden“ - Kritik am Ergebnis von

Kairo 178

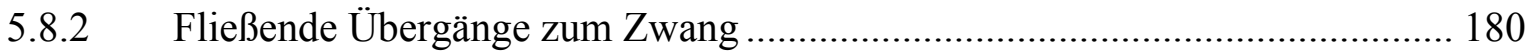

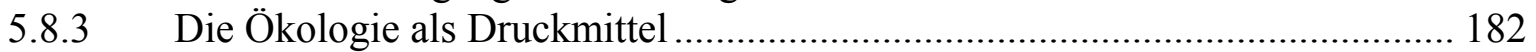

5.8.4 Differenzierte Beurteilung der Kritik notwendig........................................... 183

5.8.5 Bewertung der Kritik an quantitativer Familienplanung .................................. 186

5.8.6 Kann es eine selbstbestimmte, ,freie' Entscheidung geben? ............................. 190

5.8.7 Kinderwunsch und Kinderzahl ..................................................................... 192

5.8.8 Fortpflanzung - eine Entscheidung der Frau, des Mannes oder des Paares? ... 193

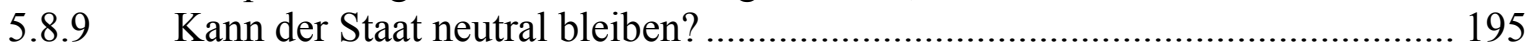

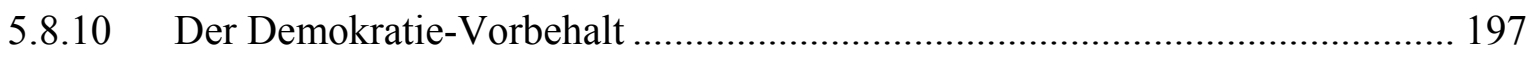

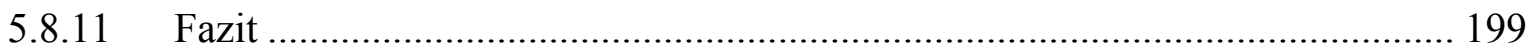

5.9 Ethische Bewertung von finanziellen Steuerungsinstrumenten............................... 200

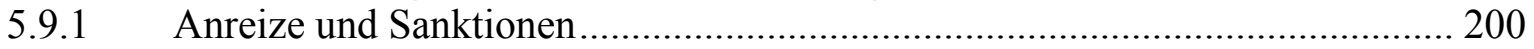

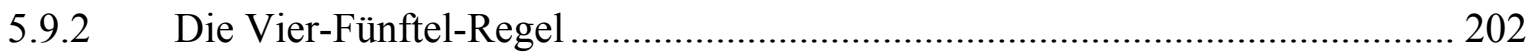

5.10 Ethische Bewertung ordnungspolitischer Ansätze .............................................. 206

5.10.1 Veränderung des Heiratsalters ........................................................................ 206

5.10.2 Generationengerechtigkeit und umlagefinanzierte Rentensysteme in

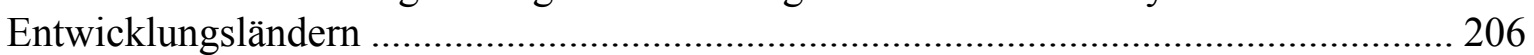

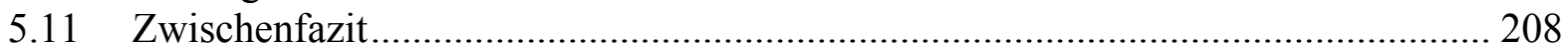

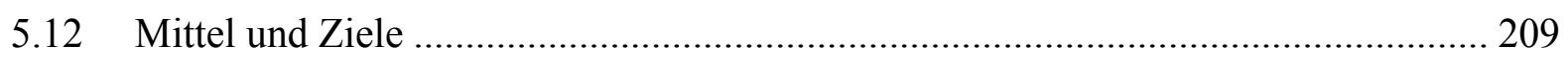


5.12.1 Die Rechte heutiger und künftiger Individuen ...........................................209

5.12.2 Ethische Dilemmata und ethisch doppelt gebotene Handlungsstrategien .........210

6 Demografischer Wandel und Bevölkerungspolitik in Deutschland .................................213

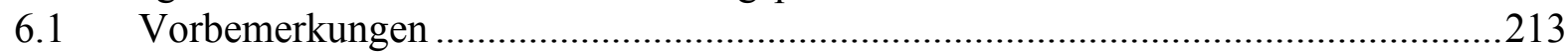

6.2 Die demografische Situation Deutschlands in Zahlen...........................................213

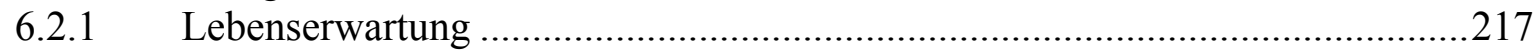

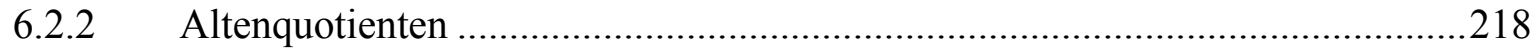

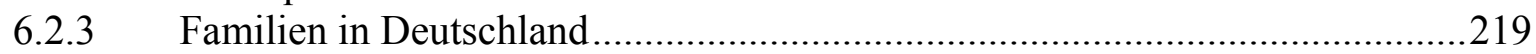

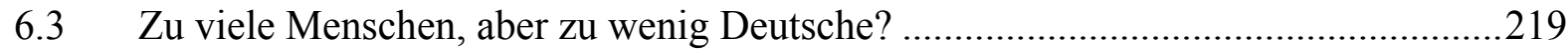

6.3.1 Die deutsche Debatte über den demografischen Wandel .................................219

6.3.2 Der Richtungswechsel der deutschen Politik zwischen 1994 und 2004 ...........222

6.3.3 Eine Welt im demografischen Gleichgewicht ...............................................224

6.3.4 Was sagt das Aktionsprogramm über die Geburtenentwicklung in den reichen

Ländern? 226

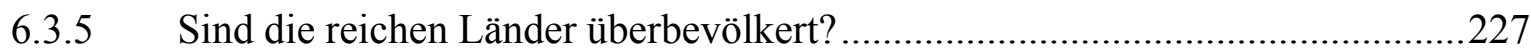

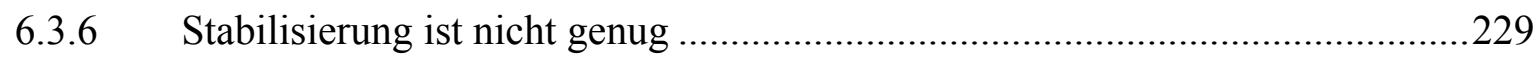

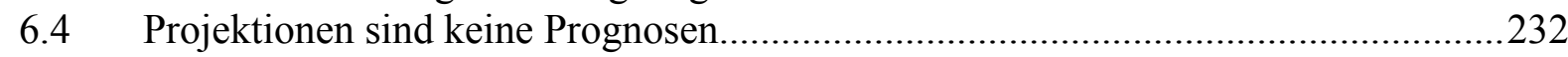

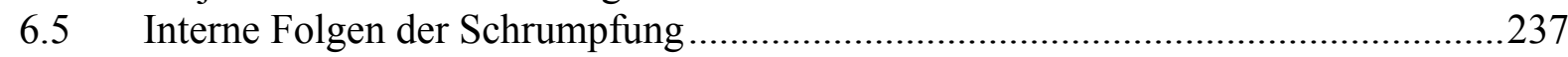

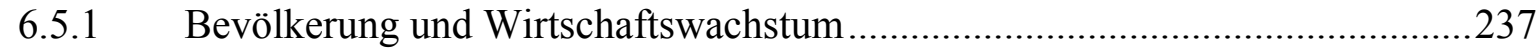

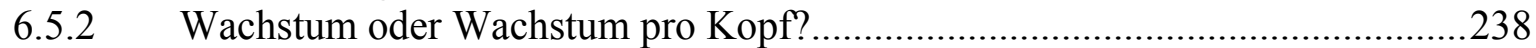

6.5.3 Auslastung der Infrastruktur- und Versorgungssysteme .................................241

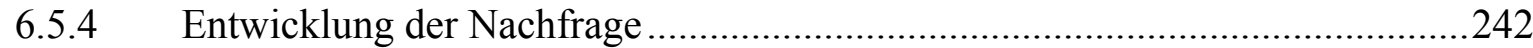

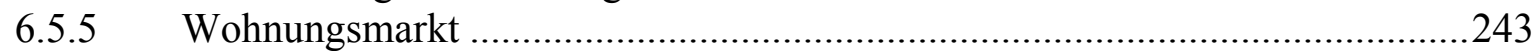

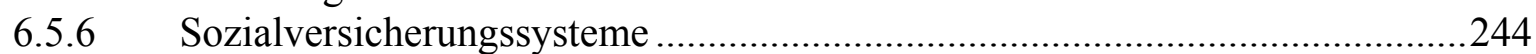

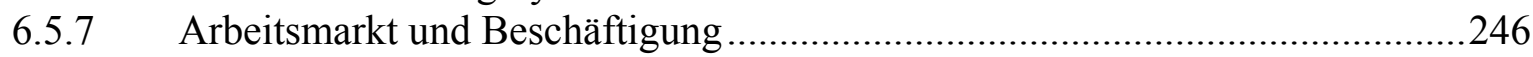

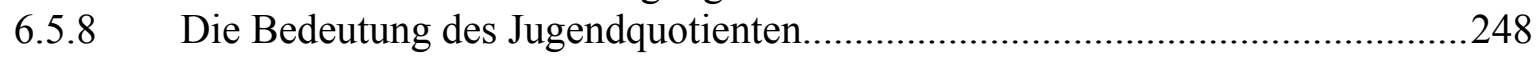

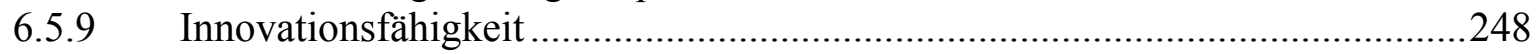

6.5.10 Die Macht der Generationen an der Wahlurne .................................................2.253

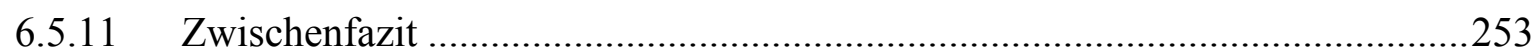

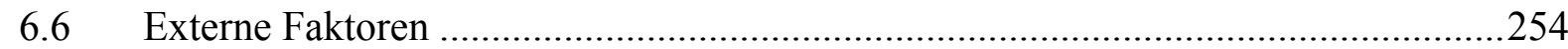

6.6.1 Machtpolitik oder Kooperation als Maxime von Außenpolitik?......................254

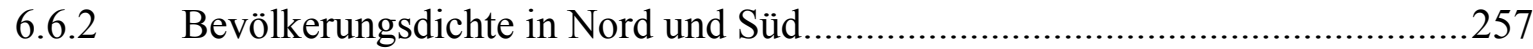

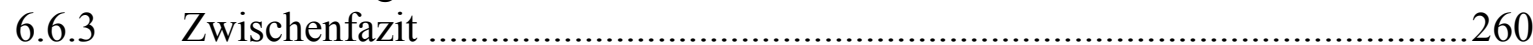

6.7 „Hilfe wir schrumpfen!“ - Rationalität und biologisches Erbe................................260

6.8 Eine Strategie für den Übergang in Deutschland .................................................261

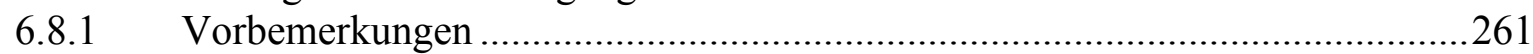

6.8.2 Die Chancen der Schrumpfung nutzen .......................................................262

6.8.2.1 Bevölkerungsentwicklung und Naturschutz in Deutschland.........................262

6.8.2.2 Fortdauernde Bodenversiegelung und Artensterben ...................................262

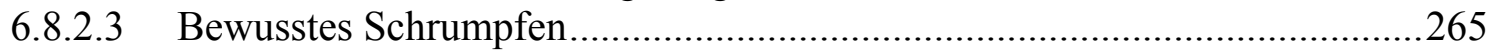

6.8.2.4 Herausforderungen für den Städtebau ...................................................266

6.8.2.5 Eine attraktive Vision für Planer und Architekten entwickeln......................267

6.8.2.6 Gezielterer Abriss ...........................................................................................268

6.8.2.7 Wohnen auf der, Grünen Wiese' unattraktiver, in den Städten attraktiver machen 269

6.8.2.8 Demografische Daten bei jeder Planung berücksichtigen

6.8.2.9 Keine öffentliche Förderung mehr für die Erschließung neuer Bauflächen in

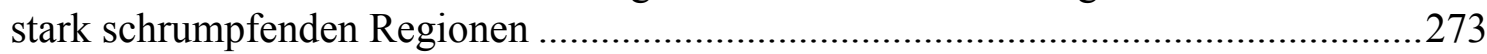

6.8.2.10 Fallstudie: Die Rückkehr der Wölfe in die Lausitz ................................275 


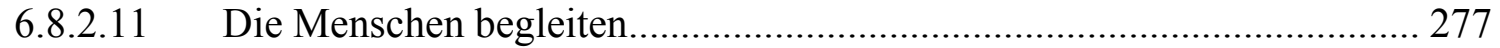

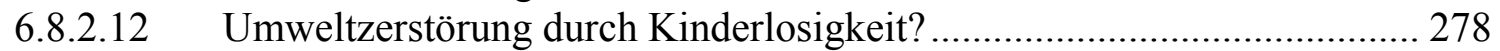

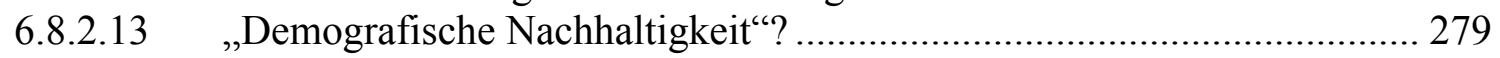

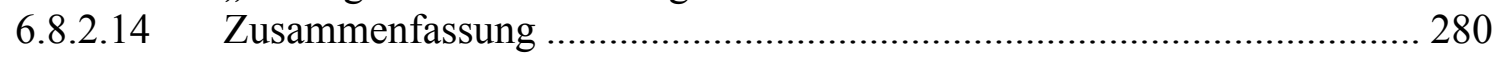

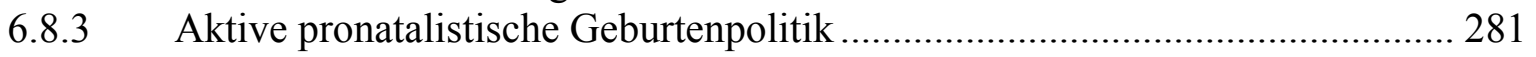

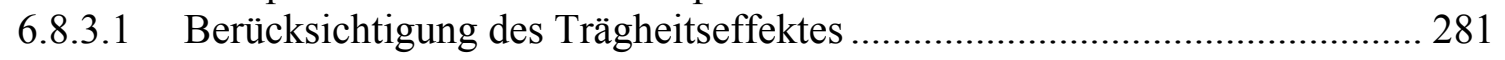

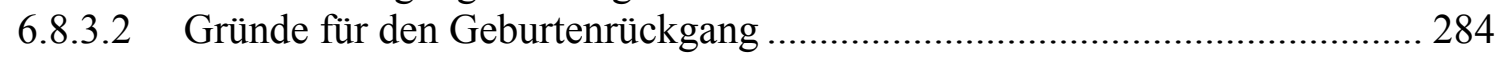

6.8.3.3 Die Debatte über eine ,Rente nach Kinderzahl' ........................................... 296

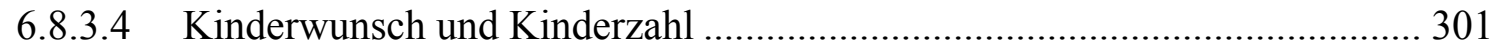

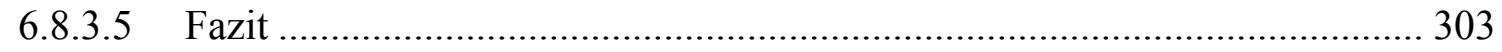

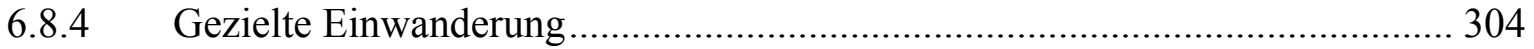

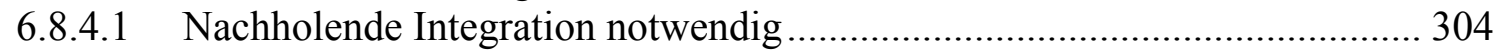

6.8.4.2 Deutschlands erstes Einwanderungsgesetz................................................ 308

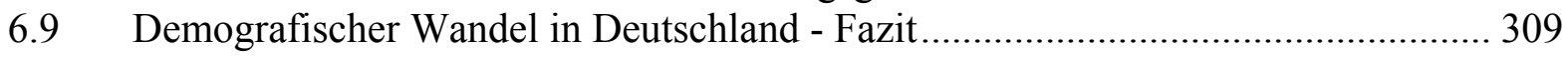

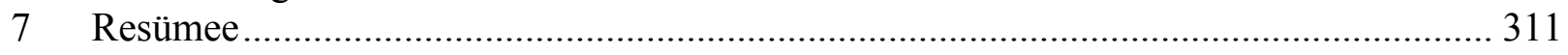

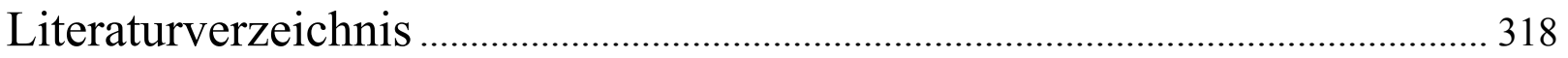




\section{Abbildungsverzeichnis}

Abb. 1: Relevante Zeitskalen für Mensch und Umwelt 38

Abb. 2: Teilbereiche der Demografie 53

Abb. 3: Fünf-Phasen-Schema des demografischen Übergangs 55

Abb. 4: Länder im Krieg und Bevölkerungswachstum 70

Abb. 5: Statistische Wahrscheinlichkeit von Krieg nach dem Anteil $\begin{array}{ll}\text { der Youth Bulges } & 71\end{array}$

Abb. 6: Historische Entwicklung der Weltbevölkerung 73

Abb. 7: Weltbevölkerungsprojektionen bis 2050

Abb. 8: Wachstumsrate und Zuwachs der Weltbevölkerung 77

Abb. 9: Einfluss des Trägheitsfaktors auf Bevölkerungwachstum in Entwicklungsländern $\quad 77$

Abb. 10: Regionale Verteilung der Weltbevölkerung, 1950-2050 80

Abb. 11: Bevölkerungsprojektionen Welt 2000-2300 82

Abb. 12: Artenzahl und Habitatsfläche 89

Abb. 13: Populationsindices landlebender Arten, Meeres-Arten und Süsswasser-Arten, 1970-2000 94

Abb. 14: Populationsindices landlebender Arten, 1970-2000 95

Abb. 15: Schätzungen der maximalen Tragfähigkeit der Erde 98

Abb. 16: Temporale und intertemporale Generationen 117

$\begin{array}{ll}\text { Abb. 17: Die abstoßende Schlussfolgerung } & 128\end{array}$

Abb. 18: Geburtenrate, Todesrate und natürliche Wachstumsrate in China 144

Abb. 19: Zusammengefasste Geburtenziffer in China, 1949-1999 145

Abb. 20: Die Entwicklung von Chinas Gesamtfruchtbarkeitsziffer im Vergleich zu anderen Ländern der Region 1975-1998 146

Abb. 21: Kontinuum geburtenpolitischer Maßnahmen 177 
Abb. 22: Relation zwischen Verwendung von Kontrazeptiva und Geburtenrate

Abb. 23: Kinderwunsch und Kinderzahl in Entwicklungsländern

Abb. 24: Entwicklung der Bevölkerung in Deutschland bis 2050

Abb. 25: Lebendgeborene und Gestorbene in Deutschland bis 2050

Abb. 26: Saldo der Wanderungen über die Grenzen Deutschlands

Abb. 27: Fernere Lebenserwartung im Alter von 60 Jahren

Abb. 28: Altenquotienten nach verschiedenen Altersabgrenzungen

Abb. 29: Ökologischer Fußabdruck nach Regionen

Abb. 30: Entwicklung der deutschen Bevölkerung und

Weltbevölkerungsentwicklung

Abb. 31: Zusammengefasste Geburtenziffern in Deutschland

Abb. 32: Vorausberechnete und tatsächliche Bevölkerungsentwicklung

Abb. 33: Demo-ökonomisches Paradoxon

Abb. 34: Vorausschätzung der Ein- und Mehrpersonenhaushalte bis 2050243

Abb. 35: Besiedelungsdichte der Welt 1700

Abb. 36: Besiedelungsdichte der Welt 1800

Abb. 37: Besiedelungsdichte der Welt 1900

Abb. 38: Besiedelungsdichte der Welt 1998

Abb. 39: Rote Listen im europäischen Vergleich (Säugetiere)

Abb. 40: Veränderung der Einwohnerzahlen ausgewählter ostdeutscher Städte 1988 bis 1999

Abb. 41: Deutschland zieht um

Abb. 42: Bevölkerungsentwicklung und kommunale Infrastrukturbereiche 272

Abb. 43: Deutschland renaturiert 
Abb. 45: Geburtenraten in der EU

Abb. 48: Zuwanderungen in Staaten der Europäischen Union und der Schweiz, 1990-1996 (kumuliert) 


\section{Tabellenverzeichnis}

Tab. 1: Auftreten des Begriffs, Generationengerechtigkeit' in deutschen Qualitätszeitungen

Tab. 2: Tragekapazität für den Menschen in unterschiedlichen Stadien seiner Entwicklung

Tab. 3: Kinderzahl und Glückswert pro Kind, Fall 1

Tab. 4: Kinderzahl und Glückswert pro Kind, Fall 2

Tab. 5: Regierungsmeinungen zum Geburtenniveau in Entwicklungsländern, 1976-2001

Tab. 6: Regierungsmeinungen zum Geburtenniveau in den am wenigste entwickelten Ländern, 1976-2001

Tab. 7: Regierungsmeinungen zum Geburtenniveau in den

Stärker Entwickelten Ländern, 1976-2001

Tab. 8: Geburtenpolitik nach Regionen

Tab. 9: Official Development Assistance für Gesundheit

Tab. 10: Methoden der Kontrazeption

Tab. 11: Divergierende Kinderwünsche von Männern und Frauen

Tab. 12: Eine antinatalistische Geburtenpolitik im Rahmen der Vier-Fünftel-Regel (Malusmodell)

Tab. 13: Eine antinatalistische Geburtenpolitik im Rahmen der Vier-Fünftel-Regel (Bonusmodell)

Tab. 14: Langzeitprojektionen der UNO

Tab. 15: Wirtschaftswachstum pro Kopf in Ostdeutschland

Tab. 16: Wohnfläche pro Person in Leipzig 1849-2001

Tab.17: Gründe von Frauen in Deutschland gegen ein (weiteres) Kind 


\section{Abkürzungsverzeichnis}

AAAS

$\mathrm{BiB}$

BIP

BMU

$\mathrm{BMZ}$

BSP

BUND

c.p.

DNU

EKC

FAO

FFH

i.O.

IPPF

ISEW

IUCN

LDC (WEL)

LPI

MDC (SEL)

Mio.

Mrd.
American Association for the Advancement of Sciences

Bundesinstitut für Bevölkerungsforschung

Bruttoinlandsprodukt

Bundesministerium für Umwelt, Naturschutz und Reaktorsicherheit

Bundesministerium für wirtschaftliche Zusammenarbeit und Entwicklung

Bruttosozialprodukt

Bund für Umwelt und Naturschutz Deutschland

ceteris paribus (alle anderen Variablen bleiben konstant)

Durchschnittsnutzenutilitarismus

Environmental-Kuznets-Curve

Food and Agriculture Organization (Welternährungsorganisation)

Flora-Fauna-Habitat

im Original

International Planned Parenthood Federation

Index of Sustainable Economic Welfare

International Union for the Conservation of Nature

Less Developed Countries (Weniger entwickelte

Länder/Entwicklungsländer)

Living Planet Index

More Developed Countries (Stärker entwickelte Länder)

Million

Milliarde 
NGO/NRO

Nabu

NPP

NSU

ODA

OECD

PoA

o.V.

RH

SEL

SRU

$\mathrm{SuV}$

SWR

UBA

UNFPA

UNEP

UNO

WBGU

WEL

WHO

WPPA

WWF
Non Governmental Organization

(Nichtregierungsorganisation)

Naturschutzbund Deutschland

Nettoprimärproduktion

Nutzensummenutilitarismus

Official Development Assistance

Organization for Economic Cooperation and Development

Programme of Action (Aktionsprogramm der

Weltbevölkerungskonferenz 1994)

ohne Verfasser

Reproduktive Gesundheit/Reproductive Health

siehe MDC

Rat von Sachverständigen für Umweltfragen

Siedlungs- und Verkehrsfläche

Sachverständigenrat zur Begutachtung der gesamtwirtschaftlichen Entwicklung

Umweltbundesamt

United Nations Fund for Population Activities

United Nations Environment Programme

United Nations Organization

Wissenschaftlicher Beirat der Bundesregierung für Globale Umweltveränderungen

siehe LDC

World Health Organisation (Weltgesundheitsorganisation)

World Population Plan of Action (Weltbevölkerungsaktionsplan der Weltbevölkerungskonferenz 1974)

World Wide Fund for Nature (früher: World Wildlife Fund) 


\section{Zusammenfassung}

Ziel der Arbeit ist, die folgende Hypothese zu prüfen:

Bevölkerungspolitik ist ethisch vertretbar, wenn sie durchgeführt wird, um ökologische Generationengerechtigkeit zu erreichen.

Diese Hypothese ist nur sinnvoll, wenn sich zwei vorgelagerte Hypothesen bestätigen, nämlich

Es besteht eine Kausalbeziehung zwischen Bevölkerung und Naturbelastung.

Und:

Die heute lebende Generation hat eine Verantwortung für zukünftige Generationen, u.a. in ökologischer Hinsicht. Intertemporale Generationengerechtigkeit ist ein sinnvolles Konzept.

Die erste vorgelagerte Hypothese ist empirischer Natur. Die Untersuchung zeigt, dass ein enger Zusammenhang zwischen dem Verlust an Biodiversität und Bevölkerungswachstum besteht. Allerdings lässt sich das Artensterben nicht monokausal auf Bevölkerungszunahme zurückführen. Daraus darf man aber auch nicht den falschen Umkehrschluss ziehen, dass die Bevölkerung für das globale Artensterben nur ein Faktor unter vielen sei. Selbst wenn Menschen theoretisch einfach ,zusammenrücken können', so ist dies in der Praxis keine Option. Daher bietet eine stabile bzw. rückläufige Weltbevölkerung große Chancen für den Erhalt von Artenreichtum und biologischer Vielfalt auf unserem Planeten. Für andere Umweltprobleme mag das Bevölkerungswachstum im Konzert der Kausalfaktoren eine weniger große Rolle spielen, aber selbst dann führt es oft zu größerer Umweltbelastung. Die erste Hypothese dieser Arbeit kann also als bestätigt angesehen werden.

Auch die zweite vorgelagerte Hypothese lässt sich bestätigen. Von den Grundsätzen intragenerationeller Gerechtigkeit ist vor allem die Formel, dass Gleiches gleich, Ungleiches seiner Eigenart entsprechend verschieden zu behandeln sei, etabliert. Generationen setzen sich aus Individuen zusammen, die alle die gleiche Menschenwürde besitzen. Prima facie sind ,Generationen' gleichwertig zueinander. Allerdings ist letztlich nicht die bloße Erhaltung des Status Quo das Ziel - sonst befänden wir uns alle noch auf dem Niveau der Neandertaler - sondern Fortschritt in der Lebensqualität ist erstrebenswert. Mit Hilfe dieser Überlegungen, wird , Generationengerechtigkeit' wie folgt definiert: 
Generationengerechtigkeit ist erreicht, wenn die Angehörigen der heutigen Generation A den Angehörigen der nächsten Generation B die Möglichkeit geben, sich ihre Bedürfnisse mindestens im gleichen Ausmaß wie A zu erfüllen.

Da antinatalistische Bevölkerungspolitiken häufig mit dem Wohlergehen und den Interessen kommender Generationen begründet werden, wird dann untersucht, ob und wenn ja, wie man die Bedürfnisse und Interessen zukünftiger Generationen ermitteln kann. Die Grundbedürfnisse aller Menschen - heutiger und künftiger sind ähnlich. Angesichts der in allen Teilen der Welt steigenden Lebenserwartung bei gleichzeitigem Bevölkerungswachstum ergeben sich wenig Anhaltspunkte, dass die Menschheit derzeit durch eine Zerstörung der lebenserhaltenden ökologischen Systeme die Basis für die Erfüllung der Grundbedürfnisse zukünftiger Generationen untergräbt. Die Lebenserwartung dürfte auch in Zukunft weiter ansteigen. Allerdings ist das ungebremste Artensterben eine klare Verletzung unserer Pflichten gegenüber kommenden Generationen. Dieses einzuschränken wird wiederum nicht ohne eine möglichst rasche Stabilisierung der Weltbevölkerung möglich sein.

Die Antwort auf die dritte und wichtigste Hypothese der Studie (Legitimität von Bevölkerungspolitik) lautet: Es kommt darauf an. Zunächst wird geprüft, ob überhaupt irgendeine Art von Bevölkerungspolitik ethisch vertretbar sein kann. Staaten haben das Wohl ihrer Bürger zu fördern. Dazu kann auch gehören, dass demokratische Staaten demografische Zielgrößen haben bzw. Stellungnahmen (z.B. in dem Sinne, dass die Geburtenrate des eigenen Landes zu hoch oder zu niedrig ist) abgeben. Bevölkerungspolitische Ziele sind also nicht generell unethisch. Das Spektrum möglicher geburtenpolitischer Maßnahmen reicht von einer Vergrößerung der Optionen bis hin zum Zwang. Um diese Einteilung greifbarer zu machen, wurde die wohl umstrittenste und zugleich am besten untersuchte Bevölkerungspolitik der Welt - die chinesische - im Detail dargestellt. Dieses Beispiel zeigt, dass in der Praxis keine Idealtypen, sondern Mischformen vorherrschen.

Ein Staat darf in jedem Fall durch indirekte Maßnahmen (Verbesserung der Gesundheitsvorsorge, der Bildungschancen von Frauen und der verbesserten Aufklärung) seine demografischen Ziele anstreben. Es ist ihm erlaubt, dabei die quantitative Deckung des , ungedeckten Bedarfs' an Kontrazeptiva in einen integrierten Ansatz einzubinden. Diese Erkenntnis bestimmt seit der Weltbevölkerungskonferenz in Kairo 1994 die bevölkerungspolitische Debatte.

Im Rahmen direkter Maßnahmen sind finanzielle Steuerungsmechanismen dann legitim, wenn sie der Vier-Fünftel-Regel folgen. Das heißt: Der Staat muss sicherstellen, dass durch seine Geburtenpolitik keiner seiner Bürger, der eine vom Staat als ,unerwünscht' angesehene Kinderzahl wählt, dadurch mehr als ein Fünftel seines Einkommens verliert im Vergleich zu einer Wahl der vom Staat als 
,ideal' angesehenen und entsprechend ökonomisch geförderten Kinderzahl. Rationierungspolitik ist generell als unethisch einzustufen.

Bezüglich der Kernfrage der Studie sind vier mögliche ethisch relevante Konstellationen zu unterscheiden:

Fall 1: Staat wünscht Senkung der Geburtenrate, Individuen wünschen sich weniger Kinder als sie tatsächlich haben.

Fall 2: Staat wünscht Senkung der Geburtenrate, Individuen wünschen sich mehr Kinder als sie tatsächlich haben.

Fall 3: Staat wünscht Erhöhung der Geburtenrate, Individuen wünschen sich weniger Kindern als sie tatsächlich haben.

Fall 4: Staat wünscht Erhöhung der Geburtenrate, Individuen wünschen sich mehr Kindern als sie tatsächlich haben.

Davon ist nur Fall 1 ethisch unproblematisch. Fall 1 ist die Situation in vielen schnell wachsenden Entwicklungsländern, in denen Paare bzw. Individuen sich weniger Kinder wünschen als sie haben müssen und der Staat versucht, Kontrazeptiva bereit zu stellen. Hier liegt eine doppelt gebotene ethische Handlungsstrategie vor: Das Handeln im Interesse heutiger Menschen ist identisch mit dem Handeln im Interesse zukünftiger Generationen. Bei Fall 2 sind die Interessen kommender Generationen, v.a. auf Erhalt der biologischen Vielfalt, abzuwägen gegen die Interessen heutiger Menschen. Bei Fall 3 schadet eine Regierung angesichts des heutigen Bevölkerungsdrucks auf die Umwelt scheinbar heutigen Zeitgenossen ebenso wie zukünftigen Generationen. Auf den ersten Blick scheint sich ein solches Verhalten kaum rechtfertigen zu lassen. Bei Fall 4 handelt der Staat im Einklang mit den Interessen seiner Bürger. Auch hier sind - wie bei Fall 2 aber die Interessen heutiger Menschen gegen die künftiger Generationen abzuwägen.

Nach der Betrachtung der Situation in Deutschland im letzten Hauptteil der Studie können auch die Fälle 3 und 4 unter dem Kriterium der ökologischen Generationengerechtigkeit bewertet werden. Hierbei gerät das Urteil über die pronatalistisch eingestellten Regierungen in Fall 4-Szenarios ins Wanken. In den alternden reichen Ländern sprechen sich immer mehr Regierungen für eine pronatalistische Politik aus. Dies ist im Zusammenhang mit ökologischer Generationengerechtigkeit ein komplexes und ambivalentes Thema, dessen Bewertung zwiespältig ausfällt.

So wie in Bezug auf Gebiete in anderen Teilen der Welt die Kausalität zwischen zunehmender Bevölkerungsdichte und Artenverlust gilt, so gilt diese Beziehung natürlich auch für Deutschland. Gilt der Zusammenhang zwischen Bevölkerungswachstum und Artenverlust auch in umgekehrter Richtung? Was ist von der These zu halten, dass mit einem Bevölkerungsrückgang die Artenvielfalt zunimmt 
(These 1). Es gibt kaum empirische Hinweise, dass eine abnehmende Bevölkerungsdichte eine Zunahme an neuen endemischen Arten bewirken würde. Zunächst einmal erschafft die Natur neue Arten nur in Zeitskalen, die im Bereich von Hundertausenden bzw. Millionen von Jahren liegen. Wenn also im Zeitraum von wenigen Jahrzehnten die Bevölkerungsdichte in einem Gebiet sinkt, so führt dies nicht zu einem Feuerwerk von ganz neuen Lebensformen. These 1 kann also nicht bestätigt werden. Anders sieht es bei den ausgewanderten Arten aus. Es spricht viel dafür, dass Arten, die es zwar nicht mehr in Deutschland, aber in seinen weniger dicht besiedelten Nachbarländern gibt, bei einer abnehmenden Bevölkerungsdichte in Deutschland wieder heimisch werden würde (These 2). Ebenso ist es wahrscheinlich, dass die Bestände heimischer Arten zunehmen, wenn die Bevölkerungsdichte sinkt (These 3). Allerdings ist bei These 2 und 3 der Zusammenhang nicht monokausal. Eine quantitative Prognose - etwa dass bei einer Senkung der Bevölkerungsdichte um 10 Prozent die Bestände der Tierarten im gleichen Gebiet im Durchschnitt um 10 Prozent steigen - wäre unseriös.

Trotz dieser Differenzierungen gilt: Eine Schrumpfung der Bevölkerung Deutschlands bietet große Chancen, die Flächenversiegelung zu verlangsamen, zusammenhängende Schutzgebiete auszuweisen und die Roten Listen bedrohter heimischer Tier- und Pflanzenarten zu verkürzen. Zudem können Länder wie Deutschland viel für die Rückkehr ausgewanderter Arten tun, wenn sie schrumpfen. Viele Arten, die heute nur noch ex situ (d.h. nicht mehr in der freien Wildbahn, sondern in Zoos) überleben, könnten wir leicht rückbürgern, wenn die Landbevölkerung dem zustimmen würde. Das gilt z.B. für den in Deutschland im Freiland ausgerotteten Wisent, den Braunbär, den Luchs oder den Wolf. Voraussetzung ist, dass große zusammenhängende Nationalparks ausgewiesen werden. Die bisher ausgewiesenen Nationalparks sind zu klein, um die Ansiedelung solcher ausgewanderter Arten $\mathrm{zu}$ ermöglichen. Erfolge bei der Reduzierung der menschlichen Flächeninanspruchnahme vergrößern also die Chancen für die Erhaltung der Artenvielfalt für zukünftige Generationen. Zudem würde der ökologische Fußabdruck Deutschlands in der Welt voraussichtlich kleiner. Dies alles ist im Sinne der ökologischen Generationengerechtigkeit positiv zu bewerten. Andererseits kann es nicht darum gehen, die Zahl der Menschen unbegrenzt schrumpfen zu lassen. Aus dem Plädoyer für eine Stabilisierung der Bevölkerung auf ökologisch nachhaltigem Niveau folgt die Notwendigkeit, entsprechende Stabilisierungsmaßnahmen auch tatsächlich einzuleiten. Es besteht zumindest theoretisch die Gefahr des Überschießens nach unten, wenn jede Generation ihre Vorgängergeneration nur zu zwei Dritteln ersetzt. Insofern kann eine ethische Bewertung nicht pauschal für die Fälle 3 und 4 abgegeben werden. In beiden Fällen kann das pronatalistische staatliche Handeln ausnahmsweise gerechtfertigt sein, wenn ohne staatliche Interventionen die Bevölkerung sehr stark abnähme. In jedem Fall ist Fall 4 aber unproblematischer als Fall 3, da der Staat bei Fall 4 im Einklang mit den Wünschen der heutigen Bürger 
handelt, wenn er die Geburtenrate zu erhöhen versucht. Die Grenze zwischen einem Fall 3-Szenario und einem Fall 4-Szenario liegt in Deutschland bei einer Geburtenrate von 1,7 Kindern pro Frau, da dies die gewünschte Kinderzahl ist. Dementsprechend ist die Steigerung der Geburtenrate von 1,4 auf 1,7 im Rahmen einer intratemporalen Betrachtung ethisch unproblematisch. Berücksichtigt man nun zusätzlich die intergenerationelle Perspektive, so lässt sich zwar nicht bestreiten, dass dadurch die Schrumpfung abgebremst und dadurch die Entlastungseffekte für die Umwelt geringer würden, aber die Schrumpfung ginge ja dennoch weiter. Da es auch im Sinne der ökologischen Generationengerechtigkeit nicht das Ziel sein kann, Deutschland unverhältnismäßig stark zu entvölkern, erscheinen pronatalistische Maßnahmen mit dem Ziel, eine Geburtenrate von 1,7 zu erreichen, bei Einhaltung der Vier-Fünftel-Regel in jedem Fall ethisch gerechtfertigt. Fraglich ist, ob eine Politik zur Erzielung einer Geburtenrate von 2,1 ethisch gerechtfertigt wäre. Dazu wäre zu dem Zeitpunkt, zu dem die Bevölkerung auf ein niedrigeres Niveau wie z.B. 70 Mio. gesunken ist, zu prüfen, ob die Prinzipien ökologischer Nachhaltigkeit nun eingehalten werden. Ist dies so, so spricht aus Sicht der ökologischen Generationengerechtigkeit nichts gegen pronatalistische Maßnahmen, welche auf eine Geburtenrate von 2,1 zielen. Ist dies nicht so, dann muss erneut abgewogen werden, ob sich die ökologischen Vorteile einer weiteren Schrumpfung vor dem Hintergrund der Nachteile in anderen Bereichen vertreten lassen.

In jedem Fall sollte aber im mit 83 Mio. bevölkerten Deutschland eine tatsächliche Schrumpfung eingeleitet und nicht etwa durch eine überzogene Einwanderungspolitik oder eine zu massive Geburtenpolitik von Anfang an verhindert werden. Diese Studie zeigt, dass ein Rückgang der Bevölkerung nicht per se generationenungerecht ist. Dies folgt bereits theoretisch aus den Überlegungen zu DNU und NSU und lässt sich auch praktisch durch viele Beispiele einer wachsenden Lebensqualität bei gleichzeitiger Verbesserung der ökologischen Rahmendaten zeigen. Deshalb ist der Begriff ,demografische Nachhaltigkeit“ auch irreführend. In dieser Studie wird eine dreiteilige Strategie vorgeschlagen, um den demografischen Übergang bis zur Erlangung eines neuen, niedrigen Bevölkerungsgleichgewichts in Deutschland zu bewältigen. Der erste Teil lässt sich mit ,Die Chancen der Schrumpfung nutzen' zusammenfassen. Damit sind Ansätze gemeint, die den Rückgang der Bevölkerung nicht umzukehren versuchen, sondern unsere Gesellschaft darauf einstellen und sogar Chancen dieser Entwicklung zu identifizieren versuchen. Aus ökologischer Sicht sind diese Chancen bereits beschrieben worden. Im größeren Maßstab können v.a. in Ostdeutschland gezielt Pläne entwickelt werden, welche Dörfer aufgegeben werden sollten, um zusammenhängende Naturschutzgebiete einzurichten. Aber auch im Westen könnten durch weitsichtige städtebauliche Planung im Zuge der nächsten 50 Jahre die schlimmsten architektonischen Sünden der Vergangenheit beseitigt werden. Anstatt abzureißen, was sich von selbst entleert hat, müssten in jeder Gemeinde diejenigen 
Liegenschaften identifiziert werden, die zuerst entleert werden sollen, um sie dann abzureißen.

Eine wichtige Herausforderung ist die Dämpfung negativer Auswirkungen des demografischen Wandels auf die Wirtschaft. Hier ist allerdings eher die Alterung das Problem, die generell gedanklich häufig nicht hinreichend von der Schrumpfung getrennt wird. Viele Probleme, z.B. der Verlust an Innovationsfähigkeit, lassen sich nicht gänzlich vermeiden, aber sie lassen sich zumindest dämpfen.

Der zweite Teil der Strategie umfasst Maßnahmen, um die Geburtenrate wieder auf Bestandhaltungsniveau zu steigern. Dieses Plädoyer für eine aktive Bevölkerungspolitik steht nicht im Widerspruch zu den bisherigen Politikempfehlungen. Wenn die derzeitige Fertilitätsrate von 1,4 anhielte, wird nach den Langzeitprojektionen der UN Population Division Deutschland im Jahr 2300 nur noch rund 3 Mio. Einwohner haben, so viele wie heute Berlin. Dies ist keine attraktive Vorstellung. Das heißt für Deutschland, dass die Faktoren, die dafür verantwortlich sind, dass sich Paare gegen Kinder entscheiden, abgebaut werden müssen. Dazu sind u.a. ein gesellschaftlicher Wertewandel, stärkere finanzielle Anreize und ein besseres Betreuungsangebot notwendig.

Der dritte Teil der Strategie beschäftigt sich mit Einwanderung. Angesichts der verfehlten Integrationspolitik der letzten Jahrzehnte wird verstärkte Migration als Königsweg zur Bewältigung des demografischen Wandels abgelehnt, auch wenn Einwanderung in bestimmtem Maße notwendig ist.

Auch wenn die Geburtenraten im weltweiten Maßstab zurückgegangen sind, ist die Stabilisierung der Weltbevölkerung frühestens in der zweiten Hälfte des 21. Jahrhunderts zu erwarten. Die zunächst in den reichen Ländern und den Schwellenländern einsetzende Schrumpfung, die sich nach und nach auch auf die Entwicklungsländer ausweitet, eröffnet Potenziale für die Erreichung der ökologischen Nachhaltigkeit im globalen Maßstab. Die Weltbevölkerung könnte nach UN-Projektionen ab 2075 (bei 9,2 Mrd.) stagnieren und dann relativ konstant bleiben bzw. bis 2300 leicht abnehmen. Wenn die reichen Länder tatsächlich in das Stadium der Schrumpfung einträten, so könnten sie auf internationalen Bevölkerungskonferenzen ihre Vorleistungen betonen und damit allen Verfechtern von Neokolonialismus- und Rassismusthesen den Wind aus den Segeln nehmen. „Keines der auf dem Erdgipfel 1992 diskutierten (...) Probleme wäre wirklich schwerwiegend, wenn auf der Welt nur 500 Millionen Menschen mit Nahrung, Kleidung und einem Zuhause versorgt werden müssen“, schreibt Ernst-Ulrich von Weizsäcker.'

Es besteht weiterer Forschungsbedarf. Umweltwissenschaftler sollten stärker die positiven Effekte des Schrumpfens der Bevölkerungen der Nordhalbkugel untersuchen - die direkten Effekte für die Ökologie in diesen Ländern selbst, sowie die indirekten durch die Reduzierung ihres ökologischen Fußabdrucks und

\footnotetext{
${ }^{1}$ Weizsäcker (1995), 291
} 
auf der internationalen Bühne, wenn globale demografische Zielgrößen verhandelt werden. In den Umweltgutachten des SRU und des WBGU fehlt bisher eine detaillierte Untersuchung dieser Beziehungen. 


\section{Summary}

Are population growth and the death of species really connected to each other? Can politics and programs influence population growth? And can they do it without infringing the fundamental right of individuals and couples for independent reproductive decisions? These are the questions of this study, with a main emphasis to the latter question.

Hypothesis 3: A population policy based on 'ecological generational justice' is ethically legitimised.

In order to answer this crucial normative question, two other hypothesis need to be discussed first.

The first hypothesis is: There is a causal relation between population and environmental degradation.

The second hypothesis is: The generation living today has a responsibility for future generations, among others in terms of ecology. Intertemporal generational justice is a possible and ingenious concept.

In the first part of this study, the first hypothesis about the interactions between nature and population will be examined. The loss of biodiversity is selected as an example. By using this environmental problem, the connection between population growth, population density and destruction of nature can clearly be shown. The complex relation between changes in population and changes in nature is further explained by a case study. It follows a description of the theory behind this relation: carrying capacity theory, Environmental-Kuznets-curve und PATformula.

The outcome: The first hypothesis is confirmed. There is a strong connection between population growth and loss of biodiversity. Even if there is no monocausal relation between the two, population growth is a key driver for this ecological problem, probably more than for other ecological problems.

The development of a theory of intertemporal generational justice is the theme of the second main part. First the different possible definitions and concepts of "generation" are pointed out. The word has at least four different meanings. In the following part a definition of intergenerational justice is drafted. Concerning the principles discussed in the context of intragenerational justice one of them has explicitly shown to be true: What is equal is to be treated equally, what is unequal is to be treated according to its character. Generations are composed by individuals each one owning the same human dignity. Prime facie all generations are equal to each another. In the face of this considerations generational justice is defined as following: 
Generational justice is accomplished if the individuals of the present generation A allow the individuals of the future generation B to have the possibilities to fulfil their needs at least in the same amount as the individuals of the generation $\mathrm{A}$ have today.

The answer to the third and most important hypothesis of the work about the ethical legitimisation of population policies is: It depends. First of all it must be checked if any kind of population policy can be legitimised. Only if yes, it must be examined to which methods that applies. The government has to improve the well-being of its people. In this context a democratic state can have demographic targets and certain attitudes concerning the right number of birth rate. Therefore population policy itself is not generally unethical. The spectrum of birth policy methods reaches out from an enlargement of options to a restriction. The example of the Chinese population policy shows that in practice there are no pure 'one type' population policies but only hybrid forms.

A government is absolutely allowed to accomplish their goals by indirect means. The quantitative coverage of the 'unmet need' of contraceptives in a comprehensive approach to improve health prevention needs to be connected with an improvement of educational opportunities (especially for women) and of their reproductive health. This insight dominates the debate about population policies since the International Conference on Population and Development in Cairo 1994.

Financial steering mechanisms can be legitimised, if they follow the four-fifthrule. That is: The government should assure that through its birth policy people who have a number of children not favoured by the state, do not have a financial disadvantage of more than a fifth in their income compared to their situation if they chose having an 'optimal' and most supported number of children. The policy of rationing in general is considered as unethical.

With regard to the central question of the study four different possible and ethically relevant constellations are distinguished:

Case 1: The Government wishes to decrease the rate of birth, the individuals want to have less children than they actually have at present.

Case 2: The Government wishes to decrease the rate of birth, the individuals want to have more children than they actually have at present.

Case 3: The Government wishes to increase the rate of birth, the individuals want to have less children than they actually have at present. 
Case 4: The Government wishes to increase the rate of birth, the individuals want to have more children than they actually have at present.

Among these, only case 1 is ethically unproblematic. Case 1 corresponds to the situation in many fast growing developing countries, in which couples wish to have less children than they do and the government tries to provide contraceptives. In this case the necessary means are ethically legitimised for even two reasons. In case 2 the interests of future generations, especially of conserving the biological variety, needs to be balanced with the interests of the present people. In case 3, the attitude of the government seems to do damage to present as well as to future generations. In case 4, the aims of the government and its present citizens coincide. Still, they have to be weighted against the interests of future generations like in case 2.

Regarding cases 3 and 4, in the last main section of this study the situation in Germany is discussed. In the aging rich countries more and more governments argue for a pronatalistic policy. The projected decline in population until the year 2050 is cause for concern to the elite. Lately, the demographic change is not only considered as fatal for the wealth and for foreign politics but also as not generational just. Here - in contrast to the ecological debate - a decline in population is considered as a disadvantage for future generations not for ecological but for economic reasons. The demographic shrinking of the rich countries is not easy to call either, generational just' or ,generational unjust'. It is ambivalent. The process of shrinking offers great potential for fewer ground sealings, enlarged nature protection areas and therefore a reduced 'red list' of endangered local animals and plants. This can be judged positively in terms of ecological generational justice. On the other hand, obviously the ultimate goal is not to keep on shrinking forever. If we want to stabilize the German population on a ecological sustainable level, we must actually also think about raising birth rates. Otherwise there will be an over-shooting to a level which is too low. For this reason, there can be no general ethical judgement about cases 3 and 4 . In both cases, a pronatalistic policy can be ethically legitimate, if otherwise the population would decrease dramatically. It is sure that case 4 is less problematic than case 3 because in case 4, the government acts in accordance with the choices of the citizens. For Germany, the threshold when a case 4-scenario becomes a case 3 -scenario is probably 1,7 children per woman. 


\section{Einleitung}

\subsection{Problemstellung und Erkenntnisinteresse}

Führende internationale Umweltforschungsinstitute, -einrichtungen und -expertinnen $^{2}$ betonen, dass es kaum gelingen kann, eine ökologisch nachhaltige Welt zu schaffen, wenn nicht das weltweite Bevölkerungswachstum (weiter) gedrosselt wird. $^{3} 1993$ veröffentlichten mehr als 600 der renommiertesten Wissenschaftler der Welt, darunter die Mehrheit der lebenden Nobelpreisträger, eine Warnung der Wissenschaftler an die Menschheit:

„Die Erde ist begrenzt. Ihre Fähigkeit, eine steigende Zahl von Menschen zu erhalten, ist begrenzt. Und wir nähern uns mit großer Geschwindigkeit diesen Grenzen. Gegenwärtige Wirtschaftsstile zum Schaden der Umwelt können weder in den entwickelten Ländern noch in den Entwicklungsländern aufrecht erhalten werden, ohne das Risiko einzugehen, dass lebenserhaltende globale Systeme irreversibel geschädigt werden. (...) Belastungen aus dem unbeschränkten Bevölkerungswachstum stellen Anforderungen an die Natur, die alle Anstrengungen für eine nachhaltige Zukunft zunichte machen können. Wenn wir die Zerstörung unserer Umwelt stoppen wollen, müssen wir Grenzen für dieses Wachstum akzeptieren. ${ }^{\text {"4 }}$

Die Frage, ob der Staat aber aus ökologischen Gründen dem Einzelnen Anreize für wenige Kinder setzen darf, ist ethisch äußerst komplex. Ist dies nicht ein unzulässiger Eingriff in die Menschenrechte? Erreichen denn nicht auch Strategien zur effizienteren Ressourcennutzung das gewünschte Ziel? Ist nicht ein Wertewandel in Richtung eines freiwilligen Konsumverzichtes des Nordens die eigentliche Aufgabe des nächsten Jahrhunderts?

Die Kernfrage der Studie lautet: Sind ökologisch begründete bevölkerungspolitische Maßnahmen ethisch legitimierbar? Diese normative Frage wird unter dem Aspekt der Generationengerechtigkeit als ethisches Leitbild für die Verantwortung gegenüber künftigen Generationen und ihren Interessen diskutiert.

Die Fragen von Ökologie, Generationengerechtigkeit und Bevölkerungs- bzw. Geburtenpolitik sind in dieser Studie nicht willkürlich miteinander verbunden worden - sie sind sachlich eng miteinander verknüpft. In dem Sammelband von

\footnotetext{
2 Aus Gründen der Geschlechtergerechtigkeit wird abwechselnd die männliche und die weibliche Schreibweise benutzt.

${ }^{3}$ Z.B. AAAS (2001); Center for International Earth Science Information Network (2003); IUCN (1990); Meadows/Meadows/Randers (1992); UN Environment Programme (2000); Worldwatch Institute (1993); World Resources Institute (2000); Word Wide Fund für Nature (2004).

${ }^{4}$ Zitiert nach Ehrlich/Ehrlich/Daily (1995), 2
} 
Ernest Partridge, der 1980 auf dem Höhepunkt der frühen angloamerikanischen Debatte über Generationengerechtigkeit erschien, wird dies im Vorwort so formuliert:
"Seminal thinkers realize already that the vaulting question, »What do we owe posterity? « will haunt humankind as long as men and nations are forced to grapple with the intertwined problems of overpopulation, resource depletion, and ecologi- cal exploitation." 5

Vierzehn Jahre später trafen sich die Vertreter von 179 Staaten zur Internationalen Konferenz über Bevölkerung und Entwicklung in Kairo. Auch dort wurden die drei Themen Bevölkerungswachstum, Umwelt und Verantwortung für zukünftige Generationen gemeinsam diskutiert und im Aktionsprogramm der Konferenz eine integrierte Antwort darauf gegeben. ${ }^{6}$

Unsere Verantwortung für die Nachwelt trat erst in den Vordergrund der philosophischen, später auch der politischen Debatte, als das Potenzial der Menschheit, die Umwelt irreversibel zu beeinflussen, Ausmaße erreicht hatte, die früher undenkbar gewesen wären. Das weltweite Bevölkerungswachstum aber ist einer der maßgeblichen Treibsätze für die Umweltkrise, und Bevölkerungspolitik ist potenziell eine wichtige Stellschraube, um diese Krise zu lösen.?

„Warum gehört Bevölkerungspolitik überhaupt in den Kontext intergenerationeller Gerechtigkeit?“ fragt der Philosoph Anton Leist. ${ }^{8}$ Und gibt sogleich die Antwort: „Deshalb, weil mit der Zahl der Nachkommen auch über das Wohlstandniveau der nächsten Generation entschieden wird." ${ }^{\text {"9 }}$ Weder eine umweltwissenschaftliche Studie, die die Frage, wie wir eine ökologisch nachhaltige Welt erreichen können, erörtert, noch eine philosophische Studie zur Frage, was ,Generationengerechtigkeit' eigentlich bedeutet, könnte es sich leisten, bevölkerungspolitische Fragen zu ignorieren. Diese interdisziplinäre Studie muss versuchen, mehrere Gebiete abzudecken, die schon einzeln sehr umfangreich sind. Dies birgt besondere Anforderungen, ist aber ohne Alternative, da der Erkenntnisverlust noch höher wäre, wenn man ineinander vernetzte Fragestellungen künstlich trennen wollte. ${ }^{10}$

Die Wahl der gewünschten Kinderzahl ist ein elementares, mit dem eigenen Lebensglück eng verknüpftes Interesse jeder Einzelnen. Wenn sich allerdings nachweisen ließe, daß Bevölkerungspolitik ein erfolgversprechendes Mittel ist, um einen Zusammenbruch zentraler ökologischer Systeme abzuwenden, so ist das

\footnotetext{
${ }^{5}$ Udall (1980), xi

${ }^{6}$ Vgl. Abschnitt 5.5. Der Konsens von Kairo.

${ }^{7}$ Dies würde freilich nur gelten, wenn sie ethisch vertretbar ist, was erst noch zu untersuchen ist.

${ }^{8}$ Leist (1991), 330

${ }^{9}$ A.a.O.

10 Partridge $(1980,6)$ nennt die Trennung der Themenbereiche ,Verantwortung für zukünftige Generationen' und ,Bevölkerungspolitik' „artificial““.
} 
individuelle Interesse abzuwägen gegenüber dem Gemeinwohl und den Interessen künftiger Generationen. Ohne einen unaufhörlichen Nachschub an Menschen hätte sich unsere Spezies nicht erhalten, geschweige denn weiterentwickeln können. Deshalb wird der Fruchtbarkeit seit biblischen Zeiten ein hoher Wert beigemessen. ${ }^{11}$ Doch zu hohes Bevölkerungswachstum gefährdet möglicherweise das Wohlergehen der betroffenen Gesellschaften. Wenn die Zukunft der Spezies Mensch gefährdet ist, dann ist es auch der Anspruch auf individuelle Freiheit, den jeder Mensch legitimerweise hat. Diese Gefahren sind ernst genug, um den traditionellen Wert uneingeschränkter Fortpflanzung und einer wachsenden Bevölkerung zu hinterfragen. Wie ist vorzugehen, wenn die Wahrung der Menschenwürde und die volle Entfaltung der Persönlichkeit heutiger Generationen mit der Wahrung der menschlichen Würde künftiger Generationen und deren voller Persönlichkeitsentfaltung kollidiert? Hier gibt es ethische Dilemmata. Möglicherweise müssen in jedem Fall Freiheitsrechte eingeschränkt werden - entweder die heutiger oder die künftiger Generationen.

\subsection{Relevanz des Themas}

Im globalen Rahmen wurde das Ziel der ,nachhaltigen Entwicklung' 1992 auf dem Erdgipfel in Rio de Janeiro von 180 Entwicklungsländern (im Folgenden: Weniger entwickelte Länder, WEL) und Industrieländern (im Folgenden: Stärker entwickelte Länder, SEL) verabschiedet. Es ist seitdem zu einem der Schlüsselbegriffe des neuen Milleniums geworden. Fragen der Bevölkerungspolitik werden als wichtiges Element von Nachhaltigkeitsstrategien diskutiert. Die Mitgliedsstaaten der Vereinten Nationen melden der Population Division der UN zudem regelmäßig, ob sie eine aktive Bevölkerungspolitik betreiben: dies traf zuletzt auf 131 von 193 Staaten zu. ${ }^{12}$ Zwischen 2005 und 2500 wird sich die Bevölkerung nach den Projektionen der UN Population Division in folgenden Ländern mindestens verdreifachen: Afghanistan, Burkina Faso, Burundi, Tschad, Kongo, Demokratische Republik Kongo, Ost-Timor, Guinea-Bissau, Liberia, Mali, Niger und Uganda. ${ }^{13}$ Auch China und Indien, die zusammen für mehr als ein Drittel der Weltbevölkerung verantwortlich sind, empfinden ihre Bevölkerungszunahme als zu hoch. Indien trägt mit 16,5 Mio. einen Anteil von rund 22 Prozent am jährlichen Wachstum der Weltbevölkerung um ca. 76 Mio. ${ }^{14}$

$\mathrm{Ob}$ mit dem Argument der Generationengerechtigkeit Bevölkerungszielgrößen oder bevölkerungspolitische Maßnahmen begründet und legitimiert werden können, ist für die Politik zahlreicher Staaten von erheblicher Relevanz. Dies gilt auch für die Menschheit als ganze: Geht man wie die mittlere Variante der UNProjektionen von einer Weltbevölkerungsgröße von 9,1 Mrd. Menschen im Jahr

\footnotetext{
${ }^{11}$ Vgl. Bischöfe in Deutschland (2004), Genesis 1 (28) u. Genesis 17 (1 ff.)

${ }^{12}$ UN Population Division (2003a), 4

${ }^{13}$ UN Population Division (2005), 7

${ }^{14}$ UN Population Division (2005), 52; Government of India (2005)
} 
2050 aus, ${ }^{15}$ so wird der Bevölkerungsdruck auf erneuerbares Süßwasser, Ackerland, Wälder, Fischereizonen, Artenvielfalt und die Atmosphäre so groß wie nie zuvor in der Geschichte der Menschheit. Die Vereinten Nationen gehen in ihrer neuesten Revision der Bevölkerungsprojektionen von 200 Mio. Menschen mehr im Jahr 2050 aus - so viele wie insgesamt in Deutschland, Frankreich und Italien leben - als noch bei ihrer letzten Revision vor drei Jahren. Der Zuwachs der Weltbevölkerung um weitere 40 Prozent oder 2,6 Milliarden Menschen stellt auch die Frage nach antinatalistischen Geburtenpolitiken mit neuer Dringlichkeit, nachdem an dieser Front in den letzten Jahren schon fast Entwarnung gegeben worden war.

Fragen der Bevölkerungspolitik stehen in Deutschland weniger selbstverständlich auf der politischen Tagesordnung als bei vielen anderen Staaten, da Bevölkerungspolitik hierzulande ein historisch belasteter Begriff ist. Erst im Sommer 2003 verkündete die zuständige Familienministerin Renate Schmidt, dass auch Deutschland fortan eine ,aktive Bevölkerungspolitik“16 betreiben werde. Diese Politik soll die Geburtenraten ${ }^{17}$ erhöhen, da die prognostizierte Alterung und Schrumpfung der deutschen Bevölkerung in hohem Maße von Politik, Wirtschaft und Wissenschaft negativ gesehen wird. Einer der renommiertesten deutschen Demografen, Herwig Birg, zieht explizit das Konzept der ,Generationengerechtigkeit' als Begründung für seine Forderung nach Ankurbelung der Geburtenraten heran. ${ }^{18}$

Im nationalen Rahmen besteht aber auch ein gesellschaftlicher Konsens für einen weiteren ökologischen Umbau der Industriegesellschaft; die Umwelt nimmt einen hohen Platz in der Werteskala der Deutschen ein. Obwohl die Zahl der nationalen Umwelt-Forschungsinstitute hoch ist, werden Fragen des Faktors Bevölkerung im Zusammenhang mit ökologischen Nachhaltigkeitsstrategien meist nur dann thematisiert, wenn es um die Entwicklungsländer geht. In den meisten Kon-

\footnotetext{
${ }^{15}$ UN Population Division (2005), 6. Die Wissenschaftler der Population Division trafen die Annahme, dass die zusammengefasste Geburtenziffer bis zum Jahr 2050 auf 2,05 Kinder pro Frau fällt. Läge die zusammengefasste Geburtenziffer bei 2,53, so müsste für 2050 mit 10,6 Mrd. Menschen gerechnet werden (hohe Variante), bei einer zusammengefassten Geburtenziffer von 1,56 mit 7,7 Mrd. Menschen (niedrige Variante). Bei konstantem Wachstum lebten zur Jahrhundertmitte 11,7 Mrd. Menschen auf der Erde.

${ }^{16}$ o.V. $(10.07 .2003)$

${ }^{17}$ Mit ,Geburtenrate' ist im Folgenden stets die sog. zusammengefasste Geburtenziffer (auch als ,Gesamtfruchtbarkeitsrate' bezeichnet, englisch: Total Fertility Rate, TFR) gemeint. Die TFR bezeichnet die Zahl an Kindern, die eine Frau im Laufe ihres Lebens hätte, wenn die altersspezifischen Fruchtbarkeitsziffern des Bezugsjahres von ihrem 15. bis zu ihrem 49. Lebensjahr gelten würden. Die TFR ist unabhängig von der jeweiligen Altersstruktur der Bevölkerung. Sie ist aber anfällig für Periodeneffekte (Vorziehen oder Nachholen von Geburten). Die TFR betrug in Deutschland 2003 genau 1,34 Geburten je Frau. Unter der Voraussetzung, dass die altersspezifischen Fruchtbarkeitsziffern des Jahres 2003 konstant bleiben, würden die Frauen in Deutschland im Laufe ihres Lebens durchschnittlich 1,34 Kinder gebären.

${ }^{18} \operatorname{Birg}(2001), 12-14$ u. 213-218
} 
zepten für Deutschland und andere Industrienationen, z.B. dem UmweltraumKonzept, wird die Bevölkerungsgröße als exogene Variable betrachtet, die die anderen Faktoren (wie z.B das Konsumniveau) bestimmt. Während die Zusammenhänge zwischen Umweltbelastung und rückständiger Technologie (Effizienzrevolution) und zwischen Umweltbelastung und Konsum (Suffizienzansatz) in Deutschland breit diskutiert werden, wird die wichtige Rolle des Bevölkerungsfaktors bei der Suche nach einer umfassenden nationalen Strategie für ökologische Nachhaltigkeit bisher kaum thematisiert. Es stellt sich also die Frage, ob Strategien für ökologische Nachhaltigkeit auch Deutschland (und anderen SEL) das Bevölkerungsthema einschließen sollten. Es sollte vor diesem Hintergrund diskutiert werden, ob sich eine mögliche geburtensteigernde Politik in Deutschland mit dem Argument der Generationengerechtigkeit rechtfertigen lässt. Bei der ethischen Bewertung des demografischen Wandels in Deutschland hat die vorliegende Studie teilweise Pioniercharakter.

\subsection{Hypothesen}

Der Report über die Entwicklung der Weltbevölkerung und die Zukunft der Artenvielfalt beginnt seinen Überblick so: „Hängen Bevölkerungswachstum und Artensterben tatsächlich zusammen? Können Politik und Programme die Trends des Bevölkerungswachstums beeinflussen? Und können sie das, ohne gegen das Grundrecht von Paaren und Individuen auf freie reproduktive Entscheidungen zu verstoßen?" ${ }^{\text {19 }}$ Dies sind auch die Fragen dieser Studie. Um die letzte, die Kernfrage der Studie beantworten zu können, sind zunächst zwei vorgelagerte Hypothesen zu prüfen.

\subsubsection{Hypothese 1: Bevölkerung und Umwelt}

Die erste Hypothese lautet:

Hypothese 1: Es besteht eine Kausalbeziehung zwischen Bevölkerung und Naturbelastung.

Nach der umfassenden PAT-Formel (Ecological Impact $=\mathrm{f}$ (Population, Affluence, Technology)) ist menschliche Bevölkerung ceteris paribus immer auch eine Ursache für Naturbelastung. Je schneller wachsend, größer und dichter die menschliche Bevölkerung auf einem bestimmten Raum ist, desto größer ist tendenziell die Naturbelastung. Zu klären bleibt, ob die PAT-Formel auch unabhängig von c.p.-Analysen Erklärungswert beanspruchen kann. Zu klären bleibt zweitens, was genau unter ,Bevölkerung' verstanden werden soll (z.B. Bevölkerungswachstum, absolute Bevölkerungsgröße, Bevölkerungsdichte, Bevölkerungsstruktur) und wie sich Veränderungen dieser Größen auf die Natur auswirken. Beides wird untersucht im Hinblick auf die Verminderung der Artenvielfalt.

\footnotetext{
${ }^{19}$ Cincotta/Engelmann (2001), 16
} 


\subsubsection{Hypothese 2: Verantwortung für spätere Generationen}

Hypothese 2: Die heute lebende Generation hat eine Verantwortung für zukünftige Generationen, u.a. in ökologischer Hinsicht. Intertemporale Generationengerechtigkeit ist ein sinnvolles Konzept.

„Why should I do anything for posterity? What has posterity done for me?" Diese Frage von Narveson macht deutlich, dass bis heute in der Ethik nicht unumstritten ist, ob wir überhaupt Verantwortung für das Wohl der Nachwelt übernehmen müssen. ${ }^{21}$ Es ist offensichtlich, dass die Kernfrage dieser Studie Sind ökologisch begründete bevölkerungspolitische Maßnahmen mit dem Konzept der Generationengerechtigkeit ethisch legitimierbar? keine sinnvolle Frage wäre, wenn es nicht gelänge, plausibel zu zeigen, dass wir heute Lebenden überhaupt eine Verantwortung gegenüber zukünftigen Generationen haben.

Zweitens muss der Umfang unserer Verpflichtungen bestimmt werden. Hier reichen die Antworten von Minimalverplichtungen, die keinerlei Änderung unseres Lebenstils zur Folge hätten, bis zu einer völligen Aufopferung für kommende Generationen. Letzteres ergibt sich, wenn man der Überlegung folgt, dass der heutigen Generation eine gigantische, möglicherweise unendlich große Zahl zukünftiger Generationen gegenübersteht. Im Sinne einer bestimmten Interpretation der utilitaristischen Maxime, die Handlung zu wählen, die das größtmögliche Glück der größtmöglichen Zahl von Menschen zur Folge hat, wäre die Einbuße der heutigen Generation dem ersten Anschein nach gering im Vergleich zum Zuwachs an Glück für kommende Generationen. ${ }^{22}$

Ich versuche zu begründen, dass das richtige Maß zwischen diesen beiden Extrempositionen ,Keine Verpflichtungen' und ,Völlige Aufopferung' liegt.

\subsubsection{Hypothese 3: Ethische Vertretbarkeit von Bevölkerungspolitik}

Hypothese 3: Bevölkerungspolitik ist ethisch vertretbar, wenn sie durchgeführt wird, um ökologische Generationengerechtigkeit zu erreichen.

Zunächst muss untersucht werden, ob überhaupt irgendeine Art von Bevölkerungspolitik zulässig sein kann. Falls ja, so sind verschiedene konkrete bevölkerungspolitische Maßnahmen auf ihre ethische Vertretbarkeit zu überprüfen. Dazu werden unterschiedliche Arten von Bevölkerungspolitik auf einer Skala angeordnet, an deren beiden Enden ,Erhöhung der Optionen/Freiwilligkeit' und ,Vermin-

\footnotetext{
${ }^{20}$ Narveson (1978), 38

${ }^{21}$ Dies behauptet z.B. Beckerman (2004), 2. Dazu Tremmel (2004c).

${ }^{22}$ Narveson (1978), 39
} 
derung der Optionen/Zwang' stehen. Jede Art der Bevölkerungspolitik stellt zunächst eine Abwägungsentscheidung zwischen Regierungen und Bürgern in der Gegenwart dar. Das Ziel (ökologische Generationengerechtigkeit) rechtfertigt nicht die Mittel, insofern müssen erst die Mittel (bevölkerungspolitische Maßnahmen) ohne Bezug auf Generationengerechtigkeit auf ihre ethische Vertretbarkeit untersucht werden. In einem zweiten Schritt wird danach geprüft, ob sich das Urteil ändert, wenn die (vermuteten) ökologischen Interessen kommender Generationen ins Kalkül miteinbezogen werden.

\subsection{Vorgehensweise}

Um die zwei Hypothesen prüfen zu können, müssen zunächst einige Grundlagen gelegt werden. $\mathrm{Zu}$ Beginn wird begründet, warum gerade das Konzept der Generationengerechtigkeit ausgewählt wurde, um Bevölkerungspolitik ethisch zu beurteilen. Darauf folgend werden einige Kernbegriffe definiert. Im nächsten Abschnitt wird auf das Erkenntnisobjekt und die inneren Spannungen des Faches ,Bevölkerungswissenschaft' eingegangen und hervorgehoben, dass die Soziologie die Leitwissenschaft für bevölkerungswissenschaftliche Fragestellungen ist. Zur Vertiefung wird eine zentrale demografische Debatte, namentlich jene über das sog. Populationsoptimum, dargestellt. Um das gesamte Zahlenmaterial, auf das in den kommenden Abschnitten immer wieder zurückgegriffen werden muss, kompakt greifbar zu haben, wird ein Abschnitt über Zahlen und Fakten zur Weltbevölkerung eingefügt.

Der Rest der Studie ist in vier große Abschnitte unterteilt:

1.) Bevölkerung und Umwelt (Abschnitt 3)

2.) Entwicklung eines Konzeptes von intertemporaler Generationengerechtigkeit und seine Anwendung auf ökologische Fragestellungen (Abschnitt 4)

3.) Ethische Zulässigkeit von antinatalistischer Bevölkerungspolitik (Abschnitt 5)

4.) Bewertung des vorausberechneten Bevölkerungsrückgangs in Deutschland (Abschnitt 6)

Im ersten dieser Hauptteile (Kapitel 3) wird die erste Hypothese geprüft, d.h. es werden die Wechselwirkungen zwischen Bevölkerung und Umwelt untersucht. Dabei wird der Verlust der Biodiversität als Beispiel herausgegriffen. An diesem Problem lässt sich der Zusammenhang zwischen Bevölkerungswachstum bzw. dichte und Naturzerstörung am deutlichsten zeigen. Durch eine Fallstudie wird das Verständnis der komplexen Beziehungen zwischen Bevölkerungsveränderungen und Umweltveränderungen vertieft. Anschließend wird die Theorie dieser Beziehungen dargestellt: Tragfähigkeitstheoreme, Environmental-Kuznets-Curve und PAT-Formel. Diese Konzepte werden diskutiert und kritisch beurteilt. 
Die Entwicklung einer Definition von intertemporaler Generationengerechtigkeit $^{23}$ ist Thema des relativ kurzen zweiten Hauptteils (Kap. 4).

Im dritten Hauptteil der Studie (Kap. 5) werden verschiedene Bevölkerungspolitiken kategorisiert und auf einem Kontinuum eingetragen, welches von absolutem Zwang bis zu einer Ausweitung der individuellen Freiheitsgrade bzw. Handlungsoptionen reicht. Um diese Einteilung greifbarer zu machen, wird die wohl umstrittenste und zugleich am besten untersuchte Bevölkerungspolitik der Welt die chinesische - im Detail dargestellt.

$\mathrm{Zu}$ Beginn der ethischen Bewertung wird auf die komplexen theoretischen $\mathrm{Zu}-$ sammenhänge von Generationengerechtigkeit und Bevölkerungspolitik eingegangen. Nutzensummenutilitarismus und Durchschnittsnutzenutilitarismus führen im Hinblick auf die Wahl zwischen zwei Alternativen stets zum gleichen Ergebnis, wenn zwei Generationen gleich groß sind. Da in der wirklichen Welt aber die Zahl der Individuen je Generation variiert, kommen beide Ethiken im Hinblick auf Bevölkerungspolitik zu radikal unterschiedlichen Schlussfolgerungen.

Anschließend wird die praxisorientierte ethische Diskussion begonnen und dazu zunächst der aktuelle internationale Konsens über Bevölkerungspolitik, also das Ergebnis der Weltkonferenz zu Bevölkerung und Entwicklung in Kairo 1994 rezipiert. Der Konsens von Kairo ist von verschiedener Seite kritisiert worden, u.a. von feministischer. Es wird geprüft, wie berechtigt diese Kritik ist. Es wird sodann hinterfragt, was ,,selbstbestimmte Entscheidung über die Zahl der eigenen Kinder" eigentlich heißen kann. Finanzielle Anreiz- und Sanktionsinstrumente werden miteinander verglichen und eine allgemeine Regel aufgestellt, mit der überprüft werden kann, ob finanzielle Anreize oder Sanktionen legitim sind.

Antinatalistische Programme und Maßnahmenkataloge in den Entwicklungsländern lassen sich nicht abschließend beurteilen, ohne den Blick auch auf die Länder in der Nordhalbkugel zu richten. Schließlich wirken Umweltprobleme grenzüberschreitend. Formeln wie „Es gibt zu viele Menschen auf der Welt, aber zu wenig Deutsche" müssen kritisch hinterfragt werden. Deshalb wird das im dritten Hauptteil gefundene Ergebnis im vierten Hauptteil (Kap. 6) am Beispiel Deutschland konkret auf seine Tauglichkeit überprüft. Die Zahlen und Fakten des demografischen Wandels in Deutschland werden zu Beginn dieses Teils ausführlich dargestellt. In Deutschland gibt es keine antinatalistische Politik, dennoch bekommen die Deutschen weit weniger Kinder, als zur Bestanderhaltung notwendig wäre. Der vorausberechnete Bevölkerungsrückgang bis zum Jahr 2050 wird von den Eliten mit Sorge gesehen. Neuerdings wird der demografische Wandel nicht nur als wohlstandsvernichtend und außenpolitisch fatal, sondern auch als

\footnotetext{
${ }^{23}$ Temporale Generationengerechtigkeit ist die Gerechtigkeit zwischen jungen, mittelalten und älteren heute lebenden Menschen. Intertemporale Generationengerechtigkeit wird definiert als die Gerechtigkeit zwischen Menschen, die früher lebten, die heute leben und die zukünftig leben werden. Die Gesamtheit der zu einer bestimmten Zeit lebenden Menschen wird also als Generation im intertemporalen Sinne bezeichnet, vgl. Abschnitt 4.1.3. Temporale und intertemporale Generationengerechtigkeit.
} 
nicht generationengerecht bezeichnet. Hier wird also - genau umgekehrt zur ökologischen Debatte - eine sinkende Bevölkerung als Benachteiligung für die nächste Generation gesehen - nicht wegen ökologischer, sondern wegen wirtschaftlicher Zusammenhänge. Auch wenn ökonomische bzw. finanzielle Generationengerechtigkeit nicht im Mittelpunkt dieser Studie steht, so wäre es unbefriedigend, die Wechselwirkungen zwischen (dem ökologischen Aspekt von) Generationengerechtigkeit und Bevölkerungsentwicklung im weltweiten Maßstab darzustellen, ohne die gegenläufige Debatte zwischen (dem wirtschaftlichen Aspekt von) Generationengerechtigkeit und Bevölkerungsschrumpfung zu thematisieren. Auf letztere Debatte wird daher im vorletzten Abschnitt in gebotener Kürze eingegangen. Dabei wird diskutiert, wie Güter- und Wohnungsnachfrage, Sozialversicherungsbeiträge, Innovationskraft, Arbeitslosigkeit und weitere wirtschaftliche Größen vom demografischen Wandel beeinflusst werden. Schließlich wird eine dreitteilige Strategie für die demografische Zukunftsfähigkeit Deutschlands entwickelt. Zuletzt fasst ein Resumee die Ergebnisse der Studie zusammen.

Wie bei jeder Studie muss das Themenfeld abgegrenzt werden. Nicht behandelt wird erstens die Frage nach der temporalen Generationengerechtigkeit, d.h. - vereinfacht gesagt - die Diskussion, was Eltern ihren Kindern schulden und was diese umgekehrt ihren Eltern schuldig sind. In Bezug auf Gerechtigkeitsfragen unterscheiden sich temporale von intertemporalen Generationenkonflikten v.a. in einer fundamentalen Hinsicht: Fehlentscheidungen im Hinblick auf das Vorsorgeverhalten früherer Generationen könnten bei temporalen Vergleichen von der unmittelbar nachfolgenden Generation direkt sanktioniert werden.

Wie im ökologischen Kontext üblich, behandle ich die Frage nach der intertemporalen Generationengerechtigkeit, d.h. die Frage, was die Gesamtheit der heute lebenden Menschen zukünftigen intertemporalen Generationen schuldig ist. Mit dieser Beschränkung vermeide ich theoretische Probleme, die sich zwangsläufig stellen würden, wenn ich den Anspruch erheben würde, eine umfassendere Theorie der Generationengerechtigkeit zu entwickeln, die auch überlappende Generationen einschließt. Zahlreiche Kriterien, die für eine Theorie intertemporaler Generationengerechtigkeit zentral sind, z.B. dass keine Kommunikation, keine Verhandlungen oder Verträge zwischen Generationen möglich sind, oder dass es statt direkter nur indirekte Reziprozität geben kann, gelten im Fall überlappender Generationen nicht. In dieser Studie wird also keine allgemeine, alle Bereiche umfassende Theorie der Generationengerechtigkeit entwickelt.

Zweitens werden Fragen einer generationengerechten Wirtschafts-, Finanz-, Bildungs-, Arbeitsmarkt-, Gesundheits- oder Jugendpolitik nicht vertieft. Eine komplette Theorie der Generationengerechtigkeit müßte auf ökologische Zerstörungen, ausufernde Staatsverschuldung, ungleiche Renditen in der Rentenversicherung, Jugendarbeitslosigkeit, Bildungsmisere, fehlende Stimmrechte von Unterachtzehnjährigen und all das andere, was - ob zu Recht oder Unrecht - als un- 
gerecht beklagt wird, ${ }^{24}$ Bezug nehmen. Der Schwerpunkt dieser Studie liegt aber klar auf dem ökologischen Kontext. Die Überschreitung dieses Rahmens wird allerdings notwendig bei der Diskussion über das gemischt strukturierte Kapital, welches die heutige Generation der nächsten hinterläßt. Auf die Diskussion von schwacher vs. starker Nachhaltigkeit soll nicht verzichtet werden. Dennoch wird aus Gründen des Gesamtumfangs dieser Studie weder ausführlich auf „Generationenbilanzierung" (besser: finanzielle Generationenbilanzierung) noch auf Humankapital- oder Sozialkapitalbilanzierung eingegangen. Die methodischen Probleme, die sich bei einer Bilanzierung des von der heutigen an zukünftige Generationen zu vererbenden Gesamtkapitals stellen, können nur benannt werden.

Des weiteren beanspruche ich nicht, eine umfassende Darstellung von Theorien der intragenerationellen Gerechtigkeit zu liefern. Vielmehr wird auf herkömmliche Gerechtigkeitstheorien nur so weit eingegangen, wie dies zum Verständnis der Unterschiede zwischen den Konzepten von intergenerationeller Gerechtigkeit und intragenerationeller Gerechtigkeit notwendig ist.

\footnotetext{
${ }^{24}$ Gronemeyer (1989); Schüller (1995); Ederer/Ederer (1995) Tremmel (1996); Gesellschaft für die Rechte zukünftiger Generationen (1997); Schüller (1997); Tremmel (1998a); SPIEGEL Spezial (1999); Podszun (2000); Klöckner (2003); Gronemeyer (2004); Bülow (2004). Vgl. auch www.srzg.de; www.yois-europe.org, www.futur-x.de,
} 


\section{Grundlegung}

\subsection{Warum Generationengerechtigkeit?}

\subsubsection{Beispiellose technologische Möglichkeiten}

Ein erheblicher Teil des Textes beschäftigt sich mit der normativen Idee der Generationengerechtigkeit. Warum diese Schwerpunktsetzung auf ein bestimmtes ethisches Konzept?

Jede Sozialphilosophie, die die gegenwärtigen Zustände kritisiert oder auch nur bewertet, bezieht sich auf mögliche Alternativen, d.h. auf nicht realisierte gesellschaftliche Zustände. Würde man sie denn realisieren wollen, so könnte dies zwangsläufig nur in der Zukunft geschehen. Insofern hatten alle Gesellschaftsentwürfe, egal ob die Hauptströmungen der Philosophie der Aufklärung (Kant), des Marxismus, der ,neoklassischen' ökonomischen Theorie oder der liberalen politischen Philosophie einschließlich Rawls' Theorie der Gerechtigkeit die zukünftigen Generationen mit im Blick. Sie erhofften sich im Falle der Verwirklichung ihrer Grundsätze für die Zukunft eine Verbesserung, z.B. verstanden als ein Prozess der zunehmenden Vervollkommnung (Hegel, Condorcet), des zunehmenden Wohlstands (Rawls, Neoklassik) oder der zunehmenden Entlastung von Arbeitsleid (Marx) ${ }^{25}$

Explizit in den Blick sind die zukünftigen Generationen (bzw. die Frage nach der Generationengerechtigkeit) aber erst seit dem Aufkommen der ökologischen Frage und später des Nachhaltigkeitsthemas gerückt. Dem Club of Rome verbleibt das historische Verdienst, durch seine Studien die ethische Einsicht befördert zu haben, dass der Nutzung der Natur Grenzen gesetzt werden müssen, ${ }^{26}$ ohne allerdings eine konkrete Generationenethik daraus zu entwickeln. Während noch Rawls $^{27}$ wie Kant Jahrhunderte zuvor von einer quasi naturgesetzlichen ständigen Verbesserung der Lebensbedingungen kommender Generationen ausgeht, weist Hans Jonas in seinem epochemachenden ${ }^{28}$ Buch Das Prinzip Verantwortung ${ }^{29}$ auf das mit der modernen Technik gewachsene Potenzial hin, das zukünftige Schicksal von Menschheit und Natur durch Handeln und Unterlassen irreversibel negativ $\mathrm{zu}$ beeinflussen. Jonas arbeitet überzeugend heraus, was in allen Zeitaltern bis zum 20. Jahrhundert galt: Ungeachtet seiner schrankenlosen Erfindsamkeit war der Mensch, gemessen an den Elementen, immer noch klein. ${ }^{30}$ Alle Wagnisse, die er unternimmt, alle Erdumrundungen und Entdeckungsreisen, sind eben deshalb Wagnisse, weil der Mensch die ihn umgreifende Natur und ihre Kräfte nicht wirklich beeinflussen kann. „Ihnen tut er nicht wirklich weh, wenn er sein kleines Königreich aus ihrem großen herausschneidet. Sie [die Kräfte der Natur, J.T.]

\footnotetext{
${ }^{25}$ Birnbacher (2003), 81 u. 87

${ }^{26}$ Meadows/Meadows/Zahn/Milling (1972)

${ }^{27}$ Rawls (1971/1979), 319-335

${ }^{28}$ Jonas Thesen werden von mir nicht uneingeschränkt unterstützt, vgl. Abschnitt 5.2.4.

${ }^{29}$ Jonas (1979)

${ }^{30}$ A.a.O., 19. Siehe auch Jonas (1980), 25.
} 
dauern, während seine Unternehmen ihren kurzlebigen Lauf nehmen. So sehr er auch die Erde Jahr um Jahr mit seinem Pfluge plagt - sie ist alterslos und unermüdbar; ihrer ausdauernden Geduld kann und muß er trauen und ihrem Zyklus muß er sich anpassen. Und ebenso alterslos ist das Meer. Kein Raub an seiner Brut kann seine Fruchtbarkeit erschöpfen, kein Durchkreuzen mit Schiffen ihm Schaden tun, kein Abwurf in seine Tiefen es beflecken. ${ }^{\text {" }}{ }^{31}$

Der Mensch konnte sich noch so anstrengen, sein Einfluss auf das ihn umgebende Ökosystem blieb begrenzt und brachte es nicht aus dem Gleichgewicht. Wie ein kleines Kind konnte er seine ganze Macht austesten, die Natur vermochte er nicht zu ändern. Sie war die große Konstante, während er spielerisch seine Aktionen ihr gegenüber variierte. ${ }^{32}$ Unter diesen Umständen war natürlich eine Ethik der Verantwortung gegenüber der Natur ${ }^{33}$ mehr als überflüssig. Der Mensch war lediglich gut beraten, der Natur mit möglichst großer Klugheit und Effizienz zu begegnen, um ausreichend an ihren Schätzen teilzuhaben.

Man mag Jonas vorwerfen, dass sein Naturbild zu sehr von einer stabilen, unverwüstlichen Natur ausgeht. Diese Vorstellung ist sicher einseitig und wird in der Ökologie in dieser Allgemeinheit nicht mehr vertreten. Denkt man an die fünf geologischen Phasen globalen Artensterbens, ${ }^{34}$ sowie an den Wechsel von Eisund Warmzeiten, so muss man die Natur als deutlich dynamischer, ja katastrophenanfälliger einstufen.

Jonas entscheidender, unstrittiger Punkt ist jedoch, dass der Mensch früher relativ wenig Einfluss auf die globale, überregionale Natur hatte. Solange dies galt, konnte der Mensch als Ethiker sich darauf beschränken, eine Ethik für den intragenerationellen Kontext zu entwickeln.

Dies erklärt, warum selbst die bedeutendsten Ethiker früherer Epochen intergenerationellen ethischen Problemen kaum Interesse widmeten. Kant etwa schrieb dazu nur folgendes: „Befremdend bleibt es immer hierbei: dass die älteren Generationen nur scheinen um der späteren willen ihr mühseliges Geschäft zu treiben, um nämlich diesen eine Stufe zu bereiten, von der diese das Bauwerk, welches die Natur zur Absicht hat, höher bringen könnten; und das nur noch die spätesten das Glück haben sollen, in dem Gebäude zu wohnen, woran eine lange Reihe ihrer Vorfahren (zwar freilich ohne Absichten) gearbeitet hatten, ohne doch selbst an dem Glück, das sie vorbereiteten, Anteil nehmen zu können. ${ }^{\text {“35 }}$

In der Staatstheorie und der politischen Philosophie wandte sich Thomas Jefferson als einer der ersten den Verpflichtungen der heutigen Generationen ge-

\footnotetext{
${ }^{31}$ Jonas (1979), 19

${ }^{32}$ A.a.O.

${ }^{33}$ Rolston (1974) spricht von einer ,ökologischen Ethik'.

${ }^{34}$ Cincotta/Engelmann (2001), 31

${ }^{35}$ Kant (1785/1968), 53
} 
genüber ihren Nachkommen zu, und zwar im Hinblick auf die Staatsverschuldung. ${ }^{36}$

Was früher schicksalhaft hinzunehmen war, rückte in der zweiten Hälfte des 20. Jahrhunderts mehr und mehr in den Umkreis menschlicher Steuerung. Dies gilt inzwischen sogar für die biophysikalischen Rahmenbedingungen der Erde selbst, wie z.B. das Klima. Wer die Worldwatch-Grafiken über den menschengemachten Klimawandel, über die Entfischung der Meere, die Veränderung der Wildnis zu Ackerland, den Verlust von Artenvielfalt und die menschengemachte Veränderung des Ozongehalts der Atmosphäre anschaut, der erkennt, dass die scheinbar unerschöpfliche Erneuerungskraft der Natur doch nicht so unerschöpflich ist. ${ }^{37}$ Die in dem herkömmlichen Verantwortungsbegriff mehr oder weniger selbstverständlich mitgemeinte Verantwortung für zukünftige Generationen erscheint unter den Umständen der Gegenwart in einem völlig neuen Licht..$^{38}$ Leider hat in den letzten Jahrzehnten die Weiterentwicklung der Moral und Weitsicht der Entscheidungsträger nicht mit dem enormen Zuwachs der technischen Möglichkeiten Schritt gehalten. Laut Jonas besteht das Universum der herkömmlichen Ethik aus Zeitgenossen, d.h. sein Zukunftshorizont ist beschränkt auf deren voraussichtliche Lebensspanne. Das Neuland, das die Menschheit mit der Hochtechnologie betreten habe, sei für die ethische Theorie noch ein Niemandsland. ${ }^{39}$

Dieses überzeugend vorgetragene Plädoyer für eine grundsätzliche und radikale Erweiterung des Geltungsbereiches der Ethik steht in krassem Gegensatz zu der Ansicht vieler Ethiker, dass alle wichtigen moralischen Prinzipien in der langen Geschichte der Ethik eigentlich schon ausgesprochen und diskutiert wurden, so dass es grundsätzliche Neuerungen nicht geben könne. ${ }^{40}$ Wie weit soll die von Jonas postulierte neue Ethik reichen? Jonas geht so weit zu sagen:

"The presence of man in the world had been a first and unquestionable given, from which all idea of obligation in human conduct started out. Now it has itself become an object of obligation - the obligation namely to ensure the very premise of all obligation, i.e. the foothold for a moral universe in the physical world - the existence of mere candidates for a moral order..." ${ }^{41}$

\footnotetext{
${ }^{36}$ „Funding I consider as limited, rightfully, to a redemption of the debt within the lives of a majority of the generation contracting it“", zitiert nach Ehmke (1953), 129. Vgl. auch Bühler (1949), 29-33.

${ }^{37}$ Siehe die Serien Vital Signs und State of the World, erhältlich im pdf-Format unter www.worldwatch.org.

${ }^{38}$ Birnbacher (2003), 81

${ }^{39}$ Jonas (1979), 7

40 "Wo es um Fragen des richtigen Lebens geht, kann nur Falsches wirklich neu sein." (Spaemann 1990, 9)

${ }^{41}$ Jonas (1980), 31. Hervorhebungen im Original.
} 
Auch wenn es sicher vernünftig ist, den schlimmstmöglichen Fall zu erwägen, so ist doch eine ökologische oder sonstige Katastrophe, die zum völligen Aussterben der Menschheit führen könnte, im Moment so unwahrscheinlich, dass dieser Fall nicht der Ausgangspunkt einer Zukunftsethik bzw. Generationenethik sein sollte. ${ }^{42}$ Bedroht sind weder die Menschheit noch die Natur als ganzes, sondern Teile der Menschheit und bestimmte Funktionen und Elemente der Natur. ${ }^{43}$ Sogar die Auffassung, dass die Natur überhaupt durch den Menschen Schaden nimmt oder genommen hat, ist jüngst bestritten worden. ${ }^{44}$ Eine nüchterne Bestandsaufnahme der anthropogenen Einwirkungen auf die Ökosysteme zeigt jedoch deutlich, wie stark die Menschheit die Natur schon geschädigt hat. ${ }^{45}$

Dass wir aufgrund gewachsener technologischer Möglichkeiten kritisch hinterfragen müssen, ob die geltenden ethischen Normen die neuen Probleme abdecken, ist jedoch nicht zu bestreiten. Wie die Gegenüberstellung der Maßstäbe von Mensch und Natur in Abb. 1 illustriert, haben wir durch unsere Eingriffe in den Naturhaushalt die Chance, die Zukunft zu prägen, wie noch keine Generation vor uns.

\section{Abb. 1: Relevante Zeitskalen für Mensch und Umwelt}

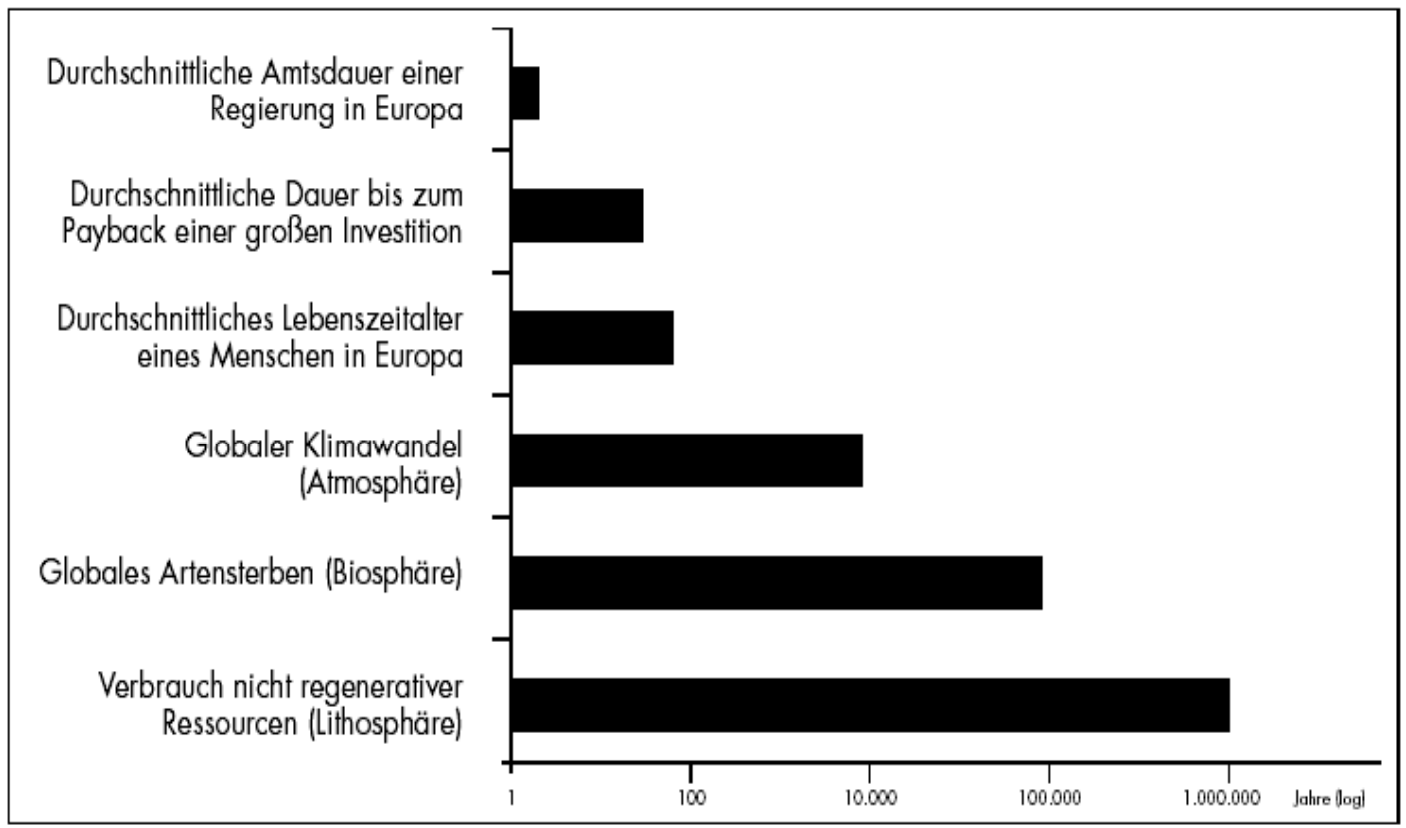

\section{Quelle: Tremmel (2003c), 350}

\footnotetext{
${ }^{42}$ Vgl. Abschnitt 5.2.4. Der Erhalt der Menschheit als Ausgangspunkt einer Generationenethik. Viele v.a. US-amerikanische Ethiker wählen diesen anderen Ausgangspunkt für die Entwicklung einer Zukunftsethik, auch Jonas (1979), 87 und wohl auch Birnbacher (1988), 67-70.

${ }^{43}$ Knaus/Renn (1998), 18

${ }^{44}$ Lomborg (2002); Maxeiner/Miersch (2002); Maxeiner/Miersch (1999)

${ }^{45} \mathrm{Vgl}$. Abschnitt 3. Ist Bevölkerung eine Ursache für Umweltbelastungen?
} 
Nur ein Beispiel: Bis 1999 wurden in deutschen Atomkraftwerken 7196 Tonnen Plutonium (PU-239) als Abfallstoff produziert. Plutonium hat eine Halbwertszeit von 24.110 Jahren. Nach heutigem Kenntnisstand wird also noch in 789.471 Jahren ein Gramm aus heutiger Hinterlassenschaft übrig sein - und bereits ein Gramm kann einen Menschen tödlich erkranken lassen. Wenn man bedenkt, dass erst seit 10.000 Jahren Menschen ihre Geschichte aufschreiben, wird deutlich, für welch lange Zeit die heute lebenden Generationen kommenden Generationen ihre Hinterlassenschaften aufbürden. ${ }^{46}$

\subsubsection{Beispielloses Wissen über die Zukunft}

Dennoch folgt - wenn auch zeitverzögert - die ,Merkwelt' der ,Wirkwelt' ${ }^{47}$ Dies ist der zweite Umstand, der für eine ausführliche Beschäftigung mit dem Konzept der Generationengerechtigkeit spricht. „Es wird für die Akteure immer schwerer, sich zu ihrer Entlastung auf schlichtes Unwissen zu berufen. (...) Das Wissen um die (wahrscheinlichen) Fernfolgen gegenwärtigen Handelns und Unterlassens drängt uns - wie sehr wir uns auch dagegen sträuben mögen - eine entsprechende Fernverantwortung, diese wiederum eine entsprechende Fernethik auf. Mit zunehmendem Wissen wächst die Macht, gleichzeitig aber auch die Verantwortung." schreibt der Zukunftsethiker Dieter Birnbacher. ${ }^{48}$ Und der Philosoph Vittorio Hösle bringt es auf die knappe Formel: „Wer mehr Macht hat, hat auch mehr Pflichten. “49

Beides - die zunehmende Macht und das zunehmende Wissen darum - sind neue Trends. ,Generationengerechtigkeit' scheint also ein erfolgversprechender Ansatz zu sein, um sich den neuen Problemen zu nähern. Ob sich aber mit Generationengerechtigkeit der Schutz der Natur ethisch begründen lässt, ist damit noch nicht gesagt.

\subsubsection{Die Entdeckung des Themas Generationengerechtigkeit}

Wo gibt es Anknüpfungspunkte? Generell wird das Thema der Verantwortung für künftige Generationen nach Rawls (also nach 1971) in der angloamerikanischen Philosophie ausführlich behandelt, ${ }^{50}$ mit zehnjähriger Verspätung auch im deut-

\footnotetext{
${ }^{46}$ Tremmel (2003c), 349

${ }^{47}$ Birnbacher (2003), 81

${ }^{48}$ A.a.O. Hervorhebungen im Original

${ }^{49}$ Hösle (1991), 126

50 Delattre (1972), 254-258; Bayles (1976); Barry (1977), 204-248; English (1977), 91-104; Barry/Sikora (1978); Partridge (1980); Bayles (1980); Parfit (1981), 113-172; Bandman (1982), 95-102; Ahrens (1983); Daniels (1988); Barry (1989); Weiss (1989); Partridge (1990), 40-66; De-Shalit (1992); Laslett/Fishkin (1992); Walzer (1992); Auerbach (1995); Clayton (1996), 195-212
} 
schen Sprachraum, ${ }^{51}$ u.a. unter dem Stichwort der ,Langzeitverantwortung, ${ }^{52}$ Parallel fällt hierzulande der Begriff, Generationengerechtigkeit' in der wirtschaftswissenschaftlichen Debatte über die Krise der Sozialversicherungssysteme. ${ }^{53}$ Auch die Finanzwissenschaft hat die Situation der zukünftigen Generationen als Forschungsobjekt entdeckt. ${ }^{54}$ Schließlich sind noch die Gerontologie und die Familienforschung, die sich immer mehr als Generationenforschung versteht, als Diskussionsstränge zu nennen..$^{55}$

Auch auf politischer Bühne ist der Begriff „Generationengerechtigkeit“ dabei, die Agenda zu erobern. Im April 2002 veröffentlichte z.B. die Deutsche Bundesregierung eine ,Nationale Nachhaltigkeitsstrategie', in der ,Generationengerechtigkeit' als eine Koordinate von ,Nachhaltigkeit' genannt wird. $^{56} \mathrm{Im}$ Grundsatzprogramm der Grünen, verabschiedet am 17. März 2002 in Berlin, ist ,Generationengerechtigkeit' ein eigener Unterabschnitt gewidmet, ${ }^{57}$ auch in der Diskussion um ein neues Grundsatzprogramm der SPD spielt der Begriff eine zentrale Rolle. In den Wiesbadener Grundsätzen der F.D.P. wird er bereits häufiger verwandt als, soziale Gerechtigkeit'. ${ }^{58}$

Die noch relativ junge intergenerationelle Ethik ist im Kommen, sie ist - im doppelten Sinne - eine Ethik der Zukunft. Trendforscher prognostizieren, dass "Generationengerechtigkeit" in den nächsten Jahren das Schlüsselwort unserer Gesellschaft wird. ${ }^{59}$ Dafür spricht auch, dass der Bezug auf kommende Generationen in zahlreiche nach 1990 verabschiedete Verfassungen aufgenommen wurde, v.a. in Mittelosteuropa und auch in die Länderverfassungen der fünf neuen Länder.

\footnotetext{
${ }^{51}$ Kleger (1986); Birnbacher (1988); Saladin/Zenger (1988); Posner (1990); Leist (1991); Kettner (1992); Brumlik (1992); Ketelhodt (1993); Brumlik/Brunkhorst (1993); Brumlik (1995); Unnerstall (1999); Acker-Widmaier (1999); Birnbacher/Brudermüller (2001); SRzG (2003). Seit 2001 existiert mit der Zeitschrift ,Generationengerechtigkeit!' auch eine interdisziplinäre Fachzeitschrift zu diesem Thema.

${ }^{52}$ Siehe z.B. die Aufsatzsammlung von Gethmann/Kloepfer/Nutzinger (1993), später: Irrgang (2002) aus technikhermeneutischer Sicht.

${ }^{53}$ Nell-Breuning/Fetsch (1981); Fogt (1982); Hanns-Martin-Schleyer-Stiftung (1984); Stiftung für Kommunikationsforschung (1988); Leisering (1990); Kaufmann (1990); Hondrich/Koch-Arzberger (1992); Mohl (1993); Becker (1994); Kronberger Kreis (1994); Tremmel (1997); Tremmel/Viehöver (2000); Alfred-Herrhausen-Gesellschaft (2000); Butterwegge/Klundt (2001); Deutscher Bundestag (2002); Pfeiffer/Domitra (2003)

${ }^{54}$ Unter anderem Deutsche Bundesbank (2001), 29-44; Raffelhüschen/Walliser (1997), 65-89; Grütz (1999); Wissenschaftlicher Beirat beim Bundesministerium der Finanzen (2001); Börstinghaus (2002); Holstein (2003); Lorz (2003)

55 Baltes/Mittelstrass (1992); Lüscher/Schultheis (1993); Fux (1994); Klose (1994); Kohli/Szydlik (2000)

${ }^{56}$ Bundesregierung Deutschland (2002)

${ }^{57}$ Bündnis 90/Die Grünen (2002), $74 \mathrm{ff}$.

58 ,Soziale Gerechtigkeit' wird einmal genannt, ,Generationengerechtigkeit' zweimal (FDP 1997).

${ }^{59}$ Opaschowski (2000), 6
} 
Trotz des Aufstiegs des Konzeptes der ,Generationengerechtigkeit' besitzt der Konflikt alt-jung in der soziologischen, philosophischen oder ökonomischen Debatte immer noch eine weit geringere Bedeutung als z.B. die Konfliktlinie armreich. ${ }^{60}$ Gerade in den Sozialwissenschaften hat ,Generation' als soziologische Kategorie (abgesehen von seiner familialen Bedeutung) ${ }^{61}$ bisher weit weniger Aufmerksamkeit erfahren als die Kategorien ,Klasse' (bzw. deren Abwandlungen ,Schicht' oder ,Milieu') und ,Geschlecht'. Immerhin nahm in den letzten Jahren die Zahl der Publikationen zu Fragen der Generationengerechtigkeit und Zukunftsethik (im weitesten Sinne) deutlich zu. ${ }^{62}$ Parallel dazu stieg die Verwendung des Begriffs ,Generationengerechtigkeit' in Qualitätszeitungen seit 2001 stark an, wie Tabelle 1 zeigt. $^{63}$

\section{Tab. 1: Auftreten des Begriffs ,Generationengerechtigkeit' in deut- schen Qualitätszeitungen (Anzahl der Artikel)}

\begin{tabular}{|l|c|c|c|c|c|c|c|c|c|c|}
\hline & $\begin{array}{l}\text { Gesamt- } \\
\text { zahl } \\
\text { Artikel }\end{array}$ & $\begin{array}{c}1995 / \\
1996\end{array}$ & 1997 & 1998 & 1999 & 2000 & 2001 & 2002 & 2003 & $\begin{array}{c}2004 \\
\text { (bis } \\
\text { 03.03.) }\end{array}$ \\
\hline $\begin{array}{l}\text { Süd- } \\
\text { deutsche } \\
\text { Zeitung }\end{array}$ & 86 & 0 & 5 & 0 & 6 & 13 & 8 & 24 & 30 & 0 \\
\hline FAZ & 80 & $\begin{array}{c}- \\
\text { (verfügbar } \\
\text { ab 1997) }\end{array}$ & 0 & 0 & 1 & 1 & 1 & 24 & 51 & 2 \\
\hline $\begin{array}{l}\text { taz - die } \\
\text { tages- } \\
\text { zeitung }\end{array}$ & 96 & $\begin{array}{c}- \\
\text { (verfügbar } \\
\text { ab 1997) }\end{array}$ & 0 & 0 & 16 & 7 & 9 & 20 & 41 & 3 \\
\hline $\begin{array}{l}\text { Der } \\
\text { Spiegel }\end{array}$ & 29 & $\begin{array}{c}- \\
\text { (verfügbar } \\
\text { ab 1996) }\end{array}$ & 0 & 0 & 8 & 5 & 1 & 6 & 7 & 2 \\
\hline
\end{tabular}

\section{Quelle: Nullmeier (2004a)}

\footnotetext{
${ }^{60}$ Nullmeier (2004b), 65

${ }^{61}$ Siehe dazu den Abschnitt 4.1.1. Die Mehrdeutigkeit des Begriffs, Generation'.

${ }^{62}$ Partridge bemängelte 1980: „The lack of manifest philosophical interest in the topic is further indicated by the fact that of the almost 700.000 doctoral dissertations on file at University Microfilms in Ann Arbor, Michigan, only one has in its title either the words 'posterity', 'future generations' or 'unborn generations'." (Partridge 1980, 10). Für Deutschland gilt dies inzwischen nicht mehr. Eine Anfrage am 5.6.2004 an die Datenbank der Deutschen Bibliothek ergab: Immerhin 6 Publikationen tragen ,zukünftige Generationen' im Titel, 12 Publikationen 'intergenerationelle Gerechtigkeit', 6 ,Generationengerechtigkeit' und 9 ,Zukunftsverantwortung'. Ein beträchtlicher Teil der Veröffentlichungen ist erst nach 2000 erschienen. Die Deutsche Bibliothek hat zwei Schlagwörter gebildet: Unter ,Zukunftsethik' finden sich 13 Einträge, unter dem wirtschaftlichen Begriff ,Intergenerationentransfer' sind es 16 Einträge.

${ }^{63}$ Nullmeier (2004b), 66
} 
Grund dafür mag die Wahrnehmung vieler Menschen sein, dass die Jüngeren nicht nur in ökologischer Hinsicht, sondern neuerdings auch in wirtschaftlicher Hinsicht schlechter gestellt sein werden als die Älteren, wenn man Gesamtlebensverläufe betrachtet. In einer repräsentativen Studie des Zukunftsforschers Opaschowski gab eine deutliche Mehrheit der Deutschen an, dass nach ihrer Einschätzung die Jugend nicht mehr auf dem gleichen Niveau leben wird wie die heutige Elterngeneration. ${ }^{64}$

Im Sommerloch 2003 erreichte die öffentliche Debatte über das Thema erstmals die Massenmedien (BILD-Zeitung, Haupt-Abendnachrichten), als der damals 23jährige Vorsitzende der CDU-Jugendorganisation, Philipp Mißfelder, forderte, Über85jährigen keine künstlichen Hüftgelenke auf Kosten der Solidargemeinschaft mehr zu verschreiben.

Zusammenfassend kann man sagen, dass es viele Anknüpfungspunkte für Konzepte von Generationengerechtigkeit gibt. Trotz alledem steht eine interdisziplinäre, wissenschaftliche Generationengerechtigkeitsforschung meiner Meinung nach noch ganz Anfang, da nur wenige Publikationen konsequent interdisziplinär aufgezogen sind. Die Frage nach den Generationenbeziehungen greift sehr tief. Sie spricht faktisch alle Lebensbereiche einer Gesellschaft an. Sie fragt nach den grundlegenden Beziehungen in einer Gesellschaft, die - zumindest im Alltag - als selbstverständlich hingenommen und kaum reflektiert werden. Und sie berührt grundsätzlichste ethische Fragen, z.B. solche nach den Verpflichtungen jedes Einzelnen oder auch der Menschheit als ganzer.

\subsection{Begriffsdefinitionen}

Wissenschaft ist nur möglich, wenn die verwendeten Wörter interpersonal nicht auf Dauer missverstanden werden. Wie Begriffe, gerade in den Sozial- und Geisteswissenschaften, definiert werden können, ist allerdings ein schwieriges und umstrittenes Thema. Die Kernbegriffe in dieser Studie werden nicht anhand mehrerer Kritierien ${ }^{65}$ definiert, sondern nur grob bestimmt. Die Begriffe ,Bevölkerungspolitik' und ,Generationengerechtigkeit' werden später im Hauptteil definiert, da ihren Definitionen zu ausführliche Überlegungen vorausgehen, um diese noch im Grundlegungsteil zu platzieren.

\footnotetext{
${ }^{64}$ Die Frage lautete exakt: „Für die junge Generation wird es in Zukunft viel schwieriger, ebenso abgesichert und im Wohlstand zu leben wie die heutige Elterngeneration." Dieser Aussage stimmten 61 Prozent der 18-49jährigen, 58 Prozent der 50-64jährigen, 49 Prozent der 65-79jährigen und 43 Prozent der Über79jährigen zu (Opaschowski 2004, 199).

${ }^{65}$ Siehe für ein solches kriteriengebundenes Definitionsverfahren Tremmel (2003a), 32-85. Dazu kritisch Ott (2004) und Brand (2004).
} 


\subsection{1, Nachhaltigkeit'}

Der Begriff ,Nachhaltigkeit' wurde von mir anhand des kriteriengebundenen Definitionsverfahrens in einer früheren Studie definiert. ${ }^{66}$ Demnach ist Nachhaltigkeit definiert als ein Konzept, das intergenerationelle und intragenerationelle Gerechtigkeit auf der normativen Ebene gleichrangig behandelt. Aus der Forderung nach intergenerationeller Gerechtigkeit ergeben sich die beiden Aktivitätsfelder Ökologie und Finanzen (,ökologische' und ,finanzielle' Nachhaltigkeit). Im Rahmen von intragenerationeller Gerechtigkeit soll v.a. internationale Gerechtigkeit (Angleichung der Lebensverhältnisse zwischen Nord und Süd), Gerechtigkeit zwischen Arm und Reich innerhalb eines Landes und Gerechtigkeit zwischen Männern und Frauen hergestellt werden.

Inzwischen existieren mehr als 300 Definitionen von Nachhaltigkeit - und fast täglich kommen neue hinzu. ${ }^{67}$ Die Verwendung durch die wissenschaftliche Gemeinde zeigt klar, dass ,Nachhaltigkeit' zwischen 1998 und Mitte 2004 (Gegenwart) nicht an Bedeutung verloren, aber eine andere Bedeutung bekommen hat eine, die im Fall von Zielkonflikten nicht mehr notwendigerweise zugunsten ökologischer Belange sein muss. Daraus folgt, dass ,Nachhaltigkeit' als umweltwissenschaftlicher Leitbegriff problematisch geworden ist. ${ }^{68}$ Diese Definition hat ihrerseits Kritik erfahren. ${ }^{69}$ Wegen seiner Umstrittenheit wird in dieser Studie der Begriff der ,Nachhaltigkeit' nicht als ökologische Zielbeschreibung verwendet, stattdessen benutze ich den aus meiner Sicht schärferen Begriff, ökologische Nachhaltigkeit'.

\subsection{2, Ökologische Nachhaltigkeit'}

Wie bereits hervorgehoben, ist der Mensch „zu einem aktiven Systemfaktor von planetarischer Bedeutung" geworden. ${ }^{70}$ Von der Ökologie werden Antworten auf folgende Fragen erwartet: Wo liegen die Belastungsgrenzen natürlicher Systeme? Wie kann der langfristige Bestand der Biosphäre gewährleistet werden? Wie muss sich der Mensch verhalten, um langfristig im Einklang mit den Rahmenbedingungen, die die Natur ihm setzt, zu leben $?^{71}$ Diese rein physikalischen Fragen nach der Belastbarkeit der ökologischen Systeme werden durch das Konzept der ,ökologischen Nachhaltigkeit' beantwortet. ${ }^{72}$ Ökologische Nachhaltigkeit lässt sich

\footnotetext{
${ }^{66}$ Tremmel (2003a), 85-137

${ }^{67}$ Dobson (2000), 62

${ }^{68}$ Tremmel (2004a); Andere Autoren hielten den Begriff von Anfang für ungeeignet für die Wissenschaft, da er ein politischer Begriff sei, dessen wesentliche Funktion es sei, Interessenkonflikte zuzudecken, siehe z.B. Jüdes (1997), 1; Lélés (1991), 613.

${ }^{69} \mathrm{Ott}(2004)$

${ }^{70}$ WBGU (1996), 3

${ }^{71}$ Kopfmüller u.a. (2001), 52

${ }^{72}$ In meiner Definition des Konzeptes der ökologischen Nachhaltigkeit ist also keine normative Begründung desselben enthalten. Ob anthropogene Einwirkungen die Belastungsgrenzen ökologischer Systeme überschreiten, ist ein im Prinzip naturwissenschaftlich feststellbarer Sachverhalt. Ob dies ,gut' oder
} 
definieren als ein Zustand, in dem der Mensch im Umgang mit seiner Umwelt bestimmte Regeln einhält. Diese Handlungsleitlinien wurden Anfang der 90er Jahre von Pearce und Turner (1990) ${ }^{73}$ und von Daly $(1991)^{74}$ entwickelt und im deutschen Sprachraum u.a. vom Rat von Sachverständigen für Umweltfragen (SRU) weiterentwickelt. ${ }^{75}$ Wenn in dieser Studie von ,ökologischer Nachhaltigkeit' gesprochen wird, dann ist ein Zustand gemeint, in dem Folgendes gilt:

1.) Schädlich wirkende Stoffe ${ }^{76}$ werden in die Umweltmedien (v.a. Luft, Wasser, Boden und Atmosphäre) nur in dem Ausmaß emittiert, wie die Umweltmedien sie aufgrund ihrer natürlichen Regenerationsfähigkeit im entsprechenden Zeitraum wieder abbauen können. Emissionen nicht abbaubarer Schadstoffe werden unabhängig von dem Ausmaß, in dem noch freie Tragekapazitäten verfügbar sind, minimiert.

2.) Erneuerbare Ressourcen werden nicht stärker genutzt, als sie sich erneuern. Nichterneuerbare Rohstoffe und Energieressourcen werden so sparsam wie mit vertretbarem Aufwand möglich genutzt. ${ }^{77}$

3.) Es werden keine Gefahrenquellen aufgebaut, die zu Schäden führen können, die nicht mehr oder nur mit unverhältnismäßig großem Aufwand beseitigt werden können. ${ }^{78}$

4.) Die bestehende Vielfalt sowohl von Tier- und Pflanzenarten als auch von Ökosystemen wird nicht durch menschliches Handeln verringert.

Obwohl der Kern dieser Regeln unstrittig ist, finden sie sich in vielen Studien in modifizierter Form. So fehlt bei mir z.B. die von der Enquete-Kommission des

,schlecht' ist, ist eine Bewertungsfrage, die das Konzept der ökologischen Nachhaltigkeit selbst nicht geben kann (Kopfmüller u.a. 2001, 56). Dazu sind Überlegungen über gesellschaftliche Ziele notwendig, die ich im Zuge der Entwicklung eines Konzeptes von, Generationengerechtigkeit' anstelle. Einen Zustand als ,ökologisch nachhaltig' (oder auch nicht) zu bezeichnen, ist nach dieser Definition eine deskriptive, keine normative Aussage.

${ }^{73}$ Pearce/Turner (1990)

${ }^{74}$ Daly (1991), 32-46

${ }^{75} \mathrm{SRU}(2002), 67 \mathrm{f}$.

${ }^{76}$ In den Regeln des SRU $(2002,67)$ ist von ,Schadstoffen' die Rede. Kohlendioxid ist aber bspw. kein Schadstoff, sondern nur ein in der Atmosphäre schädlich wirkender Stoff.

${ }^{77}$ Abweichend SRU (2002), 67. Die Forderung, für jede verbrauchte Einheit an erschöpfbaren Rohstoffen einen physisch und funktionell gleichwertigen Ersatz an erneuerbaren Ressourcen zu schaffen, ist zu weitgehend (siehe auch Abschnitt 4.4.2. Substituierbarkeit zwischen natürlichem und künstlichem Kapital).

${ }^{78}$ Vgl. z.B. UBA (1997), 12. Der Wissenschaftliche Beirat Globale Umweltveränderungen (WBGU) formuliert: „Verbot für alle Eingriffe des Menschen (...), bei denen globale Regelkreise nachweislich gefährdet sind“, vgl. WBGU (1999). 
12. Bundestages aufgestellte Regel, dass menschliche Eingriffe in die Umwelt in einem ausgewogenen Verhältnis zu dem Reaktionsvermögen der Umwelt stehen müssen. ${ }^{79}$ Wie Abb.1 zeigte, fallen die relevanten Zeitmaße von Mensch und bestimmten natürlichen Prozessen so weit auseinander, dass diese Forderung praktisch nicht umzusetzen ist.

\subsection{3 ,Dritte Welt', ,Entwicklungsländer' oder , Weniger Entwickelte Länder'?}

Die Einteilung der Länder der Erde in eine ,Erste Welt', ,Zweite Welt' und ,Dritte Welt' stammt aus einer Epoche, als zahlreiche Nationen noch einen kommunistischen Block bildeten und insofern begrifflich als ,Zweite Welt' zusammengefasst wurden. Diese Einteilung ist mit dem Systemwechsel dieser Länder und mit der Ausdifferenzierung der ehemals armen Länder (v.a. auf der Südhalbkugel) in Schwellenländer mit gehobenem Lebensstandard auf der einen Seite und den ,least developed countries' auf der anderen Seite obsolet geworden. Diese Dreiteilung der Welt war ohnehin seit jeher problematisch, weil man aus ihr auch eine Wertung herauslesen konnte.

Der Begriff ,Unterentwickelte Länder' ist, auch wenn er dem Begriff ,Dritte Welt' vorzuziehen ist, ebenfalls nicht unproblematisch. Der Begriff ,Entwicklung' entstand philosophiegeschichtlich mit dem Auftreten und der Verbreitung von Darwins Evolutionslehre. ${ }^{80}$ Der politische Begriff , unterentwickelte Gebiete' wurde erstmalig vom US-amerikanischen Präsident Harry S. Truman in seiner Inaugurationsrede am 20.1.1949 benutzt. ${ }^{81}$,Entwicklung' bedeutet nach diesem Denkschema das Voranschreiten auf einer einheitlichen Route. Die Konzeption von ganz verschiedenen, aber gleichberechtigten Wegen für unterschiedliche Gesellschaften ist mit dem Begriff kaum kompatibel. Auch impliziert die Gegenüberstellung von ,Unterentwickelten Ländern' und ,Entwickelten Ländern' bzw. ,Industrienationen', dass die Länder Europas und Nordamerikas Modell für den Rest der Welt sein könnten, was ökologisch nicht möglich ist. Der Begriff ,Industrieländer' ist zudem wegen des Übergangs von der Industrie- zur Dienstleistungs- bzw. Wissensgesellschaft problematisch.

Andererseits ist eine Unterscheidung der Länder dieser Welt weiterhin konzeptionell notwendig. Im Text wird daher die UNO-Terminologie benutzt, die von ,Developing Countries' bzw. ,Less Developed Countries' (LDC) und ,More Developed Countries' (MDC) (auf deutsch ,Entwicklungsländern' bzw. ,Weniger entwickelten Ländern' (WEL) und ,Stärker entwickelten Ländern' (SEL)) spricht.

\footnotetext{
${ }^{79}$ Deutscher Bundestag (1994), 44

${ }^{80}$ Störig (1998), $483 \mathrm{f}$.

${ }^{81}$ Tremmel (2003a), 140
} 


\subsection{Relevanz demografischer Fragen für die Soziologie}

\subsubsection{Demografie - eine verunsicherte Wissenschaft}

Diese Arbeit ist interdisziplinär angelegt und berührt Fragen der Soziologie, Ethik, Demografie und Ökonomie. Im Kern ist das Thema dieser Arbeit jedoch zwischen Bevölkerungssoziologie und Bevölkerungsethik angesiedelt. Um dies zu begründen, sollen in diesem Abschnitt die Erkenntnisobjekte und Kernfragen der Demografie als wissenschaftlicher Disziplin untersucht werden.

Die Bevölkerungswissenschaft ${ }^{82}$ kann auf eine bis in die Antike zurückreichende Tradition zurückblicken. Volkszählungen wurden schon von den Römischen Kaisern durchgeführt - die Geschichte der Geburt Jesu ${ }^{83}$ erinnert uns daran. Das Zählen der Bevölkerung, seiner Geburten und Sterbefälle, ist bis heute die Grundlage aller weiterführenden Berechnungen der Bevölkerungswissenschaft. In Deutschland wurde die Bevölkerungswissenschaft zuerst durch Leibniz gefördert, der die Arbeiten der englischen ,politischen Arithmetiker' aufgriff. Der Londoner Captain der Stadtwache John Graunt und der Medizinprofessor William Petty hatten systematisch wöchentliche ,Totenzettel' und Aufzeichnungen von Heiraten und Taufen ausgewertet und versucht, die Bevölkerungsgröße von Städten, die Auswirkungen der damals grassierenden Pest und das Geschlechterverhältnis bei der Geburt zu bestimmen. Die von ihnen entwickelte ,Sterbetafel' gehört noch heute zu den wichtigen Analyseinstrumenten der Bevölkerungsmathematik und findet v.a. in der Versicherungsbranche breite Anwendung. Als Vater der deutschen Bevölkerungswissenschaft gilt Johann Peter Süßmilch. Sein Hauptwerk Die göttliche Ordnung in den Veränderungen des menschlichen Geschlechts ${ }^{84}$ fasst das gesamte bevölkerungsstatistische Wissen seiner Zeit zusammen. Es enthält neben Berechnungen der Sterblichkeit und Geburtenhäufigkeit auch bevölkerungspolitische Vorschläge wie Abschaffung von Heiratsverboten und Förderung der Einwanderung. Dass Süßmilch die Bevölkerungsgesetze als Ausdruck göttlicher Ordnung verstand, war zu dieser Zeit für den überwiegenden Teil aller wissenschaftlichen Argumentationen üblich und schützte vor dem Bannstrahl der Kirche. Interessant für die spätere Diskussion ist, dass in der Epoche des Merkantilismus Wirtschafts- und Bevölkerungswachstum generell gleichgesetzt wurden und man insofern zu pronatalistischen Politiken neigte. Dagegen formulierte Thomas Malthus in seinem Essay on the principle of population as it Affects the Future Improvement of Society $(1798)^{85}$ sein berühmtes Bevölkerungsgesetz. Es besagt, dass das Wachstum der Nahrungsmittelproduktion nur in arithmetischer Progression zunehme $(1,2,3,4,5 \ldots)$, während die Bevölkerung, wenn sie nicht ge-

\footnotetext{
${ }^{82}$ Die Begriffe ,Bevölkerungswissenschaft' und ,Demografie' werden synonym benutzt.

${ }^{83}$ Bischöfe in Deutschland (2004), Lukas 2 (1 f.)

${ }^{84}$ Süssmilch (1741)

${ }^{85}$ Malthus (1798/1977)
} 
bremst würde, in geometrischer Progression $(1,2,4,8,16 \ldots)$ wachse ${ }^{86}$ Hungersnöte seien die unausweichliche Folge.

In der zweiten Hälfte des 19. Jahrhunderts begann in Deutschland eine kritische Auseinandersetzung über die richtigen Messverfahren, die in verschiedene Methodenstreitigkeiten mündete. ${ }^{87}$ Der Siegeszug quantitativer Verfahren in der Demografie wurde auch dadurch begünstigt, dass zu dieser Zeit Volkszählungen im heutigen Sinne eingeführt wurden und die amtliche Registrierung von Wohnortswechseln, Lebend- und Totgeburten, Eheschließungen, Sterbefällen nach dem Alter und der Todesursache, grenzüberschreitenden Migrationen, etc. immer genauer und vollständiger wurde.

In den 1920er-Jahren entwickelte sich, nicht nur in Deutschland, neben der soziologisch orientierten Bevölkerungswissenschaft auch die biologistische Richtung. Schon mit der Darwinschen Revolution war die biologische Kategorie der ,Population' stärker ins Zentrum der Bevölkerungswissenschaft gerückt. Als Population können Gruppen von Lebewesen einer bestimmten Art gelten, die auf einem bestimmten Territorium leben. Alle Populationen, die prinzipiell miteinander fortpflanzungsfähig sind, machen wiederum die Art aus ${ }^{88}$ Wenn es sich bei einer Population um Menschen handelt, spricht man von ,Bevölkerung'.

Die bahnbrechenden Entdeckungen auf den Gebieten der Vererbungswissenschaft und der Genetik führten zu einer veränderten Perspektive auf die Bevölkerung. Die ,Qualität' der Bevölkerung und die ,Degenerationsgefahr', die fixen Ideen der Sozialdarwinisten, wurden zu Themen der Bevölkerungswissenschaft. Die propagierte Lösung - die ,Verbesserung des Erbguts' - führte zu den Gräueltaten der Nationalsozialisten mit der langfristigen Folge, dass die Bevölkerungswissenschaft bis heute in Deutschland einen schweren Stand hat. Noch heute wird Bevölkerungspolitik gelegentlich mit Rassenhygiene und Eugenik gleichgesetzt und mit den unter Adolf Hitler eingeführten Mutterkreuzen in Verbindung gebracht. Vor diesem Hintergrund ist es nicht überraschend, dass speziell die deutschen Demografen bis heute Debatten über Bevölkerungspolitik und -ethik scheuen.

Nach 1945 war ein Neuanfang für die deutsche Bevölkerungswissenschaft, anders als für andere ,belastete' Wissenschaften, schwer. Viele vorher hier wirkende Wissenschaftler waren in die USA emigriert und lehrten dort nun ,Population studies'. Auch die Finanzierung von Bevölkerungswissenschaft wurde vernachlässigt. In Frankreich und England war es v.a. die Verantwortung für die Menschen in den Kolonien, die die Beschäftigung mit demografischen Fragen zum

\footnotetext{
${ }^{86}$ Siehe Abschnitt 2.4. Die Debatte über das Bevölkerungsoptimum.

${ }^{87}$ Zunächst in den ,Methodenstreit' in der deutschsprachigen Ökonomik seit 1870, der sich fortsetzte im Wiener Werturteilsstreit 1909 und in der Auseinandersetzung über Max Webers Werturteilsfreiheitspostulat.

${ }^{88}$ Der Begriff der ,Art' wird in der Biologie weit präziser definiert. Für die Fragestellungen dieses Textes reicht die grobe Definition aber aus.
} 
nationalen Interesse machte. In den USA wurde das Population Office in Princeton zu einem intellektuellen Zentrum ausgebaut. In Westdeutschland wurde Demografie bis in die 1970er Jahre nur als spezielle Soziologie oder als Bevölkerungstatistik gepflegt. In der ehemaligen DDR sammelte sich zwar ein größerer Wissensbestand an als im Westen, aber die sozialistische Demografie musste stets den Koordinaten marxistischer Wissenschaft angepasst werden, wodurch sie unter ihren Möglichkeiten blieb. In der Gründungsphase neuer Universitäten in den 70er Jahren entstanden an den Universitäten Bielefeld ${ }^{89}$ und Bamberg ${ }^{90}$ eigene Lehrstühle, zu denen sich nach der Vereinigung auch die Humboldt-Universität in Berlin gesellte. ${ }^{91} 1996$ wurde in Rostock das Max-Planck-Institut für Demografische Forschung eingerichtet. ${ }^{92}$ In diesem Zusammenhang wurden auch an der Universität Rostock zwei Lehrstühle für Demografie geschaffen. ${ }^{93}$ Inzwischen kann an der Universität Rostock - einmalig in Deutschland - der Diplom-Studiengang Demografie studiert werden. In Deutschland gibt es also (Stand Juli 2004) nur an vier Universitäten Lehrstühle für Bevölkerungswissenschaft. Weitere universitäre Lehr- und Forschungseinrichtungen existieren in Kiel ${ }^{94}$, Karlsruhe, ${ }^{95}$ Bayreuth, ${ }^{96}$ München ${ }^{97}$ und an der FU Berlin. ${ }^{98}$ In den meisten Fällen sind sie angesiedelt bei den soziologischen Instituten. Auch einige ökonomische Institute untersuchen die mikro- und makroökonomischen Aspekte des demografischen Wandels; das bekannteste ist das Mannheim Research Institute for the Economics of Ageing. ${ }^{99}$ In Wiesbaden existiert als eine der wichtigsten nichtuniversitären Forschungseinrichtungen das Bundesinstitut für Bevölkerungsforschung, ${ }^{100}$ das inzwischen an das Statistische Bundesamt angegliedert ist. Auch die Deutsche Stiftung Weltbevölkerung ${ }^{101}$ und das Berlin Institut für Weltbevölkerung und globale Entwicklung ${ }^{102}$ sind zu nennen, da es sich bei beiden Einrichtungen nicht nur um advokatorische Nichtregierungsorganisationen, sondern durchaus auch um Forschungsinstitute handelt. In Frankreich gibt es

\footnotetext{
${ }^{89}$ www.ibs.uni-bielefeld.de

${ }^{90}$ www.uni-bamberg.de/sowi/bevoelkerung

${ }^{91}$ www.demographie.de

${ }^{92} \mathrm{http}: / / \mathrm{www}$.demogr.mpg.de

${ }^{93}$ www.soziologie.uni-rostock.de

${ }^{94}$ www.uni-kiel.de/anthrop/index.html

${ }^{95}$ www.uni-karlsruhe.de/ soziologie

${ }^{96}$ www.uni-bayreuth.de/departments/sozialgeographie

${ }^{97}$ www.selapo.vwl.uni-muenchen.de und www.geo.wiso.tu-muenchen.de/index.html

${ }^{98}$ www.fu-berlin.de/aeimhof

${ }^{99} \mathrm{http} / / /$ mea.uni-mannheim.de. Eine Liste, welche Ökonomen in Deutschland bevölkerungsökonomisch forschen, liefert der Ausschuss für Bevölkerungsökonomie des Vereins für Socialpolitik (www.socialpolitik.org).

${ }^{100}$ www.bib-demographie.de

${ }^{101}$ www.dsw-online.de

${ }^{102}$ www.berlin-institut.org
} 
dagegen 50, in Italien 20, in Belgien und den Niederlanden jeweils 10 und in Spanien 5 Lehrstühle für Bevölkerungswissenschaft. ${ }^{103}$

Die Bevölkerungswissenschaft hat kein einheitliches Theoriegebäude. Der Bevölkerungswissenschaftler arbeitet an einzelnen Fragestellungen, die statistisch, soziologisch, geographisch, biologisch, ökonomisch, historisch oder ethisch sein können. Die Paradigmenvielfalt ist dafür verantwortlich, dass sich die Ergebnisse bevölkerungswissenschaftlicher Forschung häufig nur begrenzt aufeinander beziehen lassen und sich die Demografie schwer tut in ihrer Selbstdefinition in Abgrenzung zu anderen Disziplinen. Manche Demografen sind sogar der Meinung, die Bevölkerungswissenschaft befinde sich erst auf dem Weg zu einer eigenständigen wissenschaftlichen Disziplin. ${ }^{104}$

\subsubsection{Erkenntnisobjekt und Teildisziplinen der Bevölkerungswissen- schaft}

Unstrittig ist, dass die Bevölkerungsstatistik das formale ,Rückgrat' der Demografie ist. Alle Teildisziplinen (Bevölkerungssoziologie, -ökonomik, -ethik, -geografie) greifen auf ihre Zahlen zurück. Die Grundgleichung der Bevölkerungswissenschaft ist einfach:

Bevölkerungsanfangsbestand + Geburten - Sterbefälle $+/$ - Wanderungssaldo $=$ Bevölkerungsendbestand. ${ }^{105}$

Daraus lassen sich differenzierte Kennzahlen und Maßzahlen wie z.B. die Geburtenrate, die allgemeine Fruchtbarkeitsrate, die Gesamtfruchtbarkeitsrate, die Sterberate, die Nettowanderungsrate oder die Wachstumsrate berechnen. Die Mehrheit der Bevölkerungsforscher ist der Ansicht, dass diese Kennzahlen für sich genommen wenig aussagekräftig sind, und dass sozialwissenschaftliche Theorien benötigt werden, um Bevölkerungsentwicklungen zu erklären. ${ }^{106}$ Die Geburten und Sterbevorgänge sind zwar Teil der biologischen Natur des Menschen, aber längst „sozial überformt“, wie Mackenroth es auszudrücken pflegte. ${ }^{107}$

Nach Pulte kann die Bevölkerungswissenschaft in vier große Komplexe eingeteilt werden: die Bevölkerungsstatistik, die Bevölkerungstheorien, die Bevölkerungspolitik und die historische Demografie: ${ }^{108}$

1.) Die Bevölkerungsstatistik befasse sich mit der Ermittlung der Zahl, Entwicklung und des Aufbaus einer Bevölkerung und ihrer Gliederung nach Merkmalen wie Alter, Geschlecht und Familienstand.

\footnotetext{
${ }^{103}$ Mackensen (1998), 38

${ }^{104}$ Mackensen (1989), 19; Schimany (2003), 49

${ }^{105}$ Im weltweiten Maßstab fällt Migration als Einflussgröße weg.

${ }^{106}$ Höpflinger (1997), 12

${ }^{107}$ Mackenroth (1953)

${ }^{108}$ Pulte (1972), $15 \mathrm{f}$.
} 
2.) Die Bevölkerungstheorien bauten auf der Statistik auf, gäben aber auch Hinweise für statistische Erhebungen. Um zu Erklärungen für bestimmte Erscheinungen der Bevölkerungsentwicklung zu kommen, werde dabei auch der Versuch unternommen, allgemeine, d.h. auch für die Zukunft gültige Gesetze aufzustellen.

3.) Bevölkerungspolitik sei die Wissenschaft von den Maßnahmen, derer sich ein Staat bedienen kann, um Größe, Wachstum und räumliche Verteilung einer Bevölkerung zu beeinflussen.

4.) Die historische Demografie befasse sich mit dem historischen Ablauf einer Bevölkerungsentwicklung.

Offensichtlich ist diese Einteilung, so hilfreich sie auch auf den ersten Blick scheinen mag, mit Kategorienfehlern behaftet. Bevölkerungstheorie ist eine andere Kategorie als Bevölkerungsstatistik oder die historische Demografie. Wissenschaftliche Theorien können von der gesamten Bevölkerungswissenschaft aufgestellt und falsifiziert werden - von der Bevölkerungssoziologie genauso wie von der Bevölkerungsökonomik oder der historischen Demografie.

Die ,Bevölkerungspolitik' als Wissenschaft zu bezeichnen, ist ebenfalls unglücklich. Wenn schon, dann müsste man die Wissenschaft von den Maßnahmen, denen sich ein Staat in bevölkerungspolitischer Hinsicht bedienen kann, als ,Bevölkerungspolitologie' bezeichnen. Wir müssen also Pultes Einteilung verwerfen und tiefer forschen.

\subsubsection{Der Unterschied zwischen ,Bevölkerung' und ,Gesellschaft'}

Der Grund, warum es eine immer noch diskutierte Frage ist, ob die Demografie lediglich ein Teilgebiet der Soziologie, eine eigenständige Sozialwissenschaft ${ }^{109}$ oder aber gar keine Sozialwissenschaft darstellt, ist die schwierige Abgrenzung zwischen ,Bevölkerung' und ,Gesellschaft', die Mackensen anschaulich hervorhebt:
„Bevölkerungen sind, das ist offenkundig, nichts als Men- schen. Diese Menschen stehen miteinander in Beziehung. Sie gehen miteinander um, sie verständigen sich miteinander. Ihr Handeln ist aufeinander bezogen, aufeinander angewiesen: Menschen können ihre Absichten nicht ohne einander errei-

\footnotetext{
${ }^{109}$ Ich benutze folgende Definitionen von ,Soziologie' und ,Sozialwissenschaften': ,Soziologie' ist die Wissenschaft, deren Gegenstandsbereich das soziale Handeln des Menschen umfasst, darin eingeschlossen die Beschäftigung mit sozialen Normen, Rollen und Handlungsmustern, Einstellungen, Wertorientierungen, mit regelgemäßem und abweichendem Verhalten, sowie die Untersuchung sozialer Klassen, Schichten, Generationen und Geschlechter, sozialer Prozesse und Institutionen.

Der Begriff ,Sozialwissenschaften' wird im weiteren Sinne verstanden als Sammel-Bezeichnung für diejenigen Wissenschaften und Forschungszweige, in denen die gesellschaftlichen Aspekte menschlichen Verhaltens und Zusammenlebens behandelt werden (Soziologie, aber auch Kulturanthropologie, Politologie, Ethnologie, Pädagogik, Geschichts-, Rechts- und Sprachwissenschaft sowie die Sozialpsychologie und die Ökonomie).
} 
chen. Sie haben sich schon immer darauf eingerichtet, einander zu unterstützen, zu helfen: sie konkurrieren miteinander und verfolgen einander widersprechende Interessen. (...) Die Akteure sind immer an irgendeinem Ort. (...) Über nichts anderes hat Bevölkerungswissenschaft also zu berichten. ${ }^{\text {"110 }}$

Bei dieser Beschreibung sind ,Bevölkerung' und ,Gesellschaft' quasi deckungsgleich. Eine interessante alternative Antwort gibt der Bamberger Bevölkerungswissenschaftler Josef Schmid. Bei ihm ist ,Bevölkerung' das ,reproduktive Substrat' der Gesellschaft. ${ }^{111}$ Die Gesellschaft mit ihren Schichten, Geschlechtern und Generationen ist also nach Schmids Meinung nicht deckungsgleich mit der Bevölkerung. Letztere sei eine besondere Kategorie mit der bemerkenswerten Eigenschaft, sich und damit die Gesellschaft über die Zeit hinweg zu reproduzieren. Anders als die sprachlich-sozialen Konstrukte, mit denen es die Sozialwissenschaften bei der Beschreibung gesellschaftlicher Zusammenhänge oft zu tun haben (z.B. ,soziale Rolle', ,Demokratie', ,Totalitarismus'), ${ }^{112}$ besitzt eine Bevölkerung eine spezifische Materialität, weswegen Hummel behauptet, dass die Demografie den Naturwissenschaften näher stünde als den Sozialwissenschaften. ${ }^{113}$ Dem freilich widerspricht Schmid: „Bevölkerungssoziologie ist das Studium der Bewegungen und Veränderungen einer Bevölkerung, soweit sie durch gesellschaftliche Prozesse verursacht oder zumindest beeinflusst werden. Bevölkerungssoziologie ist somit das Geschichte und Theorie umfassende ,Herzstück' der Bevölkerungswissenschaft. "“14

Bei Schmids Definition von Bevölkerung als das reproduktive Substrat einer Gesellschaft ist eine konzeptionelle Nähe zwischen Bevölkerungsprozessen und den Generationen, also zeitlich hintereinander lebenden Bevölkerungen, unverkennbar. Tatsächlich beschäftigt sich die Demografie offensichtlich - wenn man den Großteil der Artikel in Fachzeitschriften berücksichtigt - weniger mit ,Bevölkerung', sondern eher mit ,Bevölkerungsentwicklungen'. Gemessen werden zwar Bevölkerungsbestände und auf sie bezogene Ereignisdaten; Folgerungen für die Geburten-, Sozial-, Gesundheits- und Wohnungspolitik lassen sich aber nur aus den Veränderungen dieser Daten über die Zeit ableiten. ${ }^{115}$ „Wie und warum vollziehen sich Entwicklungen der Bevölkerung bzw. Entwicklungen ihrer Komponenten?“ „In welchem Ausmaß und warum verändern sich das Geburtenverhalten, die Sterberate, der Wanderungssaldo, die Lebenserwartung?" Das sind die Kernfragen der Demografie. Der erste Teil der Fragen, das ,Wie', wird von der Bevöl-

\footnotetext{
${ }^{110}$ Mackensen (1997), 196

${ }^{111}$ Schmid (1984), 17

${ }^{112}$ Tremmel (2003a), 49

${ }^{113}$ Hummel (2000), 229

${ }^{114}$ Schmid (2003), 1

${ }^{115}$ Mackensen (2003)
} 
kerungsstatistik beantwortet, die Frage nach dem ,Warum' aber nicht. Hinter einer Variablen wie der Geburtenziffer stehen eine Vielzahl weiterer Variablen wie Ehe- und Familienformen, Erwerbstätigkeit, Beruf und Ausbildungsgrad, Einkommen und Vermögen, Sozialstruktur und Größe des Wohnorts, religiöse, politische, kulturelle und wirtschaftliche Einflussfaktoren. Theoriebildung mit diesen Variablen ist nicht mehr Teil der Bevölkerungsmathematik, sondern der Sozialwissenschaften. Bevölkerungswissenschaft steht also den Sozialwissenschaften näher als den Naturwissenschaften oder den Formalwissenschaften. Dem steht nicht entgegen, dass sie statistische bzw. mathematische Methoden benutzt, so wie es auch die empirische Sozialforschung macht.

Schwieriger ist die Frage, ob die Demografie eine Bindestrich-Soziologie ist. Die Übergänge zwischen einer als eigenständige Sozialwissenschaft verstandenen Demografie und der Bevölkerungssoziologie sind sicher fließend. Bevölkerungswissenschaft wird aber heute von verschiedenen Disziplinen betrieben, von denen jede die aus der Statistik gewonnenen Kennziffern auf ihre fachspezifischen Fragestellungen bezieht und Bevölkerungsvorgänge vor dem Hintergrund ihres Wissenschaftsverständnis analysiert. ${ }^{116}$ Die Demografie ist eine Disziplin mit einer breiten interdisziplinären Ausstrahlung, an der verschiedene Disziplinen partizipieren. Zusammenfassend kann man sagen, dass die Demografie eine eigenständige Wissenschaft im Rahmen der Sozialwissenschaften ist, ebenso wie Wirtschaftswissenschaften oder die Anthropologie. Wie diese nutzt sie die Statistik als Hilfswissenschaft, allerdings in stärkerem Maße als die meisten anderen Sozialwissenschaften. Es wäre nicht richtig, der Demografie eine Mittelstellung zwischen den Naturwissenschaften (Biologie) und den Sozialwissenschaften zuzuschreiben - da sie biologische Faktoren wie Alter oder Geschlecht immer als soziale Faktoren behandelt, gehört sie zu den Sozialwissenschaften. Sie hat verschiedene Unterdisziplinen (z.B. Bevölkerungssoziologie, Bevölkerungsökonomie, Bevölkerungsgeografie, historische Demografie), von denen die Bevölkerungssoziologie nach Ansicht der meisten Demografen die wichtigste ist. ${ }^{117}$

Die Überlegungen zum Erkenntnisobjekt der Demografie werden von Abbildung 2 zusammengefasst:

\footnotetext{
${ }^{116}$ Schimany (2004), 51

${ }^{117}$ So auch bei Mackenroth (1953), 111; Höpflinger (1997), 11; Schimany (2004), 52-57. In Bezug auf familienbezogene Fragestellungen gibt es eine große Nähe und z.T. überlappende Erkenntnisinteressen mit der „Familiensoziologie“.
} 


\section{Abb 2.: Teilbereiche der Demografie}

\begin{tabular}{|c|c|c|c|c|c|}
\hline & I & & & & \\
\hline $\begin{array}{l}\text { Bevölkerungs- } \\
\text { statistik }\end{array}$ & $\begin{array}{l}\text { Bevölkerungs- } \\
\text { soziologie }\end{array}$ & $\begin{array}{l}\text { Bevölkerungs- } \\
\text { ökonomie }\end{array}$ & $\begin{array}{l}\text { Bevölkerungs- } \\
\text { geografie }\end{array}$ & $\begin{array}{l}\text { Historische } \\
\text { Demografie }\end{array}$ & $\begin{array}{l}\text { Bevölkerungs- } \\
\text { ethik }\end{array}$ \\
\hline $\begin{array}{l}\text { befasst sich } \\
\text { mit der } \\
\text { Ermittlung und } \\
\text { Aufbereitung } \\
\text { der } \\
\text { Kennzahlen } \\
\text { einer } \\
\text { Bevölkerung } \\
\end{array}$ & $\begin{array}{l}\text { stellt Theorien } \\
\text { auf, z.B. zur } \\
\text { Erläuterung } \\
\text { des demografi- } \\
\text { schen } \\
\text { Übergangs }\end{array}$ & $\begin{array}{l}\text { stellt Theorien } \\
\text { auf, z.B. } \\
\text { "Value of } \\
\text { children"- } \\
\text { Ansatz, } \\
\text { Opportunitäts- } \\
\text { kostenansatz }\end{array}$ & $\begin{array}{l}\text { stellt Theorien } \\
\text { auf, z.B. zum } \\
\text { Bevölkerungs- } \\
\text { maximum }\end{array}$ & $\begin{array}{l}\text { stellt Theorien } \\
\text { auf, z.B. zur } \\
\text { historischen } \\
\text { Entwicklung } \\
\text { einer } \\
\text { bestimmten } \\
\text { Bevölkerungs- } \\
\text { struktur } \\
\end{array}$ & $\begin{array}{l}\text { stellt } \\
\text { normative } \\
\text { Fragen: Darf } \\
\text { der Staat } \\
\text { Geburten- } \\
\text { politik (z.B. } \\
\text { Ein-Kind-Pol.) } \\
\text { betreiben? }\end{array}$ \\
\hline beschreibend & $\begin{array}{l}\text { beschreibend, } \\
\text { erklärend }\end{array}$ & $\begin{array}{l}\text { beschreibend, } \\
\text { erklärend }\end{array}$ & $\begin{array}{l}\text { beschreibend, } \\
\text { erklärend }\end{array}$ & $\begin{array}{l}\text { beschreibend, } \\
\text { erklärend }\end{array}$ & wertend \\
\hline
\end{tabular}

\subsubsection{Demografische Theorien}

Als Beispiel für die Theoriebildung in der Demografie soll kurz ihre wohl bekannteste Theorie besprochen werden, die des demografischen Übergangs. Sie behandelt die Entwicklung der Geburten- und Sterbeziffer während der Phase des Übergangs eines Landes von der Agrar- zur Industriegesellschaft. In traditionellen Gesellschaften ist sowohl die Geburten- als auch die Sterberate hoch, sodass die Wachstumsrate der Bevölkerung sehr gering ist (unter ein Prozent). Durch medizinischen Fortschritt beginnt zunächst die Sterberate, mit zunehmender allgemeiner Bildung und einsetzender Industrialisierung auch die Geburtenrate zu sinken. In dieser Übergangsphase kommt es zu einem starken Bevölkerungswachstum. Erst wenn sich Geburten- und Sterberate auf niedrigem Niveau stabilisieren, stellt sich Nullwachstum der Bevölkerung ein. ${ }^{118}$

Der Theoriestatus des demografischen Übergangs ist aber umstritten, sofern zu konkreten Bevölkerungen keine hinreichend erklärenden Aussagen oder gar Vorhersagen gemacht werden. Nur wenn der demografische Übergang auch versuchsweise erklärt (und nicht nur beschrieben) wird, kann man von einer Theorie sprechen. Welche die entscheidenden und auslösenden Faktoren des demografischen Übergangs sind, hängt von historischen und regionalen Besonderheiten ab, die aus dem bloßen Schema nicht hervorgehen. Der Wunsch nach weniger Kinder kann z.B. soziologisch durch Hypothesen über einen Wertewandel in Richtung

${ }^{118}$ Vgl. Schimany (2003), 81-93; Chesnais (1992); Schmid (1984) 
Individualisierung, Materialismus und Verwestlichung erklärt werden. Umstritten ist bei dem Schema auch, ob diese aus der europäischen Bevölkerungsgeschichte abgeleitete Theorie überhaupt auf andere Gesellschaften bzw. Kulturkreise übertragbar ist. Ist ein Zustand von niedriger Geburten- und Sterberate wirklich die letzte Phase des Modells?

\section{Abb. 3: Fünf-Phasen-Schema des demografischen Übergangs}

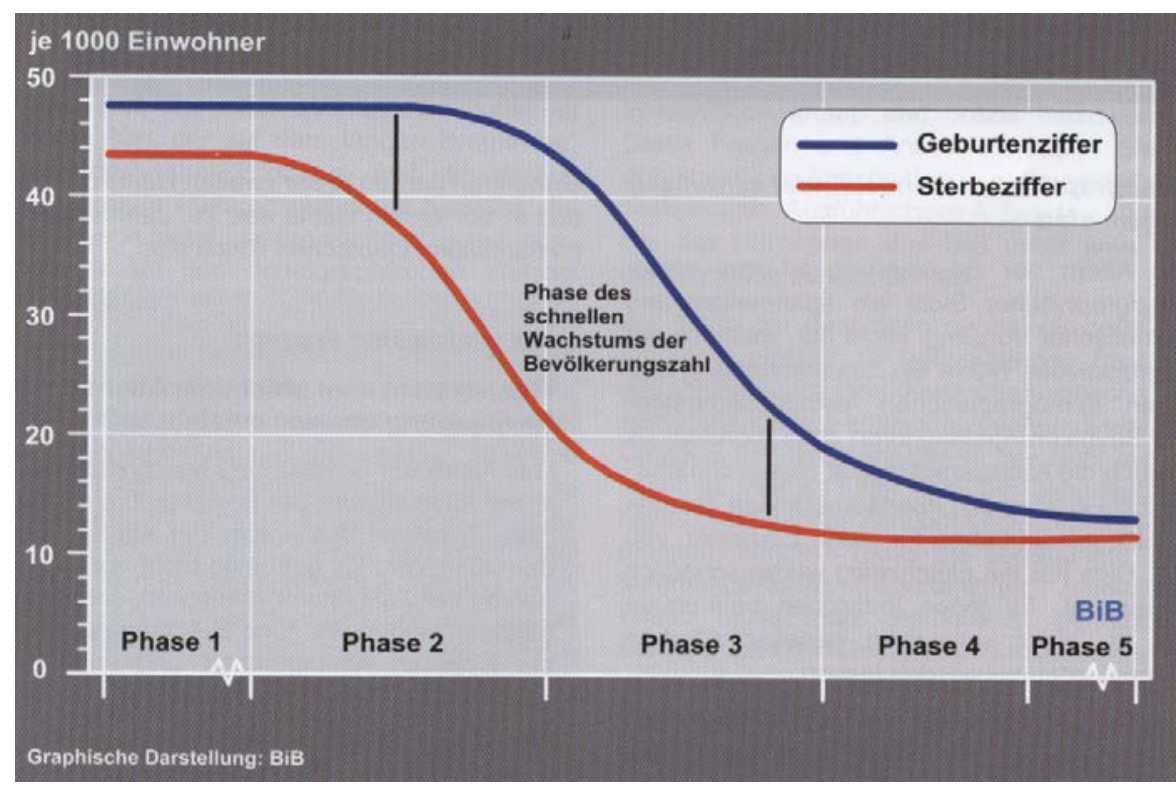

Quelle: Bundesinstitut für Bevölkerungsforschung/BiB (2004), 10

In vielen SEL sind die Geburtenraten unter die Sterberaten gesunken - ohne dass ein neuer Anstieg ersterer in Sicht ist. ${ }^{119}$ In vielen Entwicklungsländern dagegen sind wegen AIDS die Sterberaten wieder neu angestiegen, nachdem diese Länder schon die dritte Phase erreicht hatten.

\subsubsection{Normative Fragen in der Demografie}

Der letzte Ast von Abbildung 2, die Bevölkerungsethik, wirft weitere Fragen auf. Unbestreitbar ist die Demografie, verstanden als Sozialwissenschaft, eine beschreibende und eine erklärende Wissenschaft, d.h. sie arbeitet sowohl beschreibend als auch erklärend im Sinne der Bildung von Theorien über Ursache-Wirkungsbeziehungen. Stellen sich ihr darüber hinaus auch normative Fragen?

Wir können die Frage genereller formulieren: „Gehören normative Fragen zum Erkenntnisobjekt der Gesellschaftswissenschaften ${ }^{120}$, oder sind diese allein der

\footnotetext{
119 Dies wird oft - terminologisch unglücklich - als „2. Demografischer Übergang“ bezeichnet. Treffender wäre es, dies als 6. Phase im bestehenden Schema des Demografischen Übergangs einzubauen.

${ }^{120}$ Die Begriffe „Gesellschaftswissenschaften“ und „Sozialwissenschaften“ werden synonym benutzt.
} 
,Ethik' und ,Philosophie' vorbehalten?“ Für das Erkenntnisobjekt bzw. ,die Kernfrage' der Sozialwissenschaften kommen grundsätzlich zwei Alternativen in Frage:

(1) Wie funktioniert Gesellschaft? Wie organisieren Menschen ihr Zusammenleben?

(2) Wie sollte Gesellschaft funktionieren? Wie sollten Menschen ihr Zusammenleben organisieren? Welche Gesellschaftsordnung ist die richtige?

Besonders die sog. Kritische Theorie verlangte mit Vehemenz, dass (Sozial)-Wissenschaft nicht nur die bestehenden gesellschaftlichen Verhältnisse zu beschreiben und zu erklären habe, sondern auch versuchen müsse, die Gesellschaft emanzipatorisch zu verändern. Übertragen auf die Demografie lautet die Frage dann

(3) Wie sollte sich die Bevölkerung bzw. wie sollten sich ihre Komponenten entwickeln?

Zweifellos ist die Unterscheidung von Werturteilen und Tatsachen, von Sollen und Sein, nicht ein spezifisches Gedankenprodukt des ausgehenden 19. Jahrhunderts, als die Sozialwissenschaften entstanden, sondern so alt wie die ,Wissenschaft' selbst. ${ }^{121}$ Aber die Sozialwissenschaften scheinen von der Frage besonders betroffen zu sein. Das Verhältnis der Sozialwissenschaft zu präskriptiven Fragen stand schließlich im 20. Jahrhundert im Mittelpunkt zweier bekannter sozialwissenschaftlicher Kontroversen: des Werturteilsstreits und des Positivismusstreits. Dies zeigt, dass zumindest ein Teil der Sozialwissenschaftler normative Fragestellungen für einen wichtigen Teil der Sozialwissenschaften hält.

Die wesentliche Kritik der sog. Frankfurter Schule gegen eine rein ,positivistisch' ausgerichtete Sozialforschung war, dass sie die Soziologie wehrlos mache gegen die Gefahren des Faschismus. ${ }^{122}$ Wer - wie die Nonkognitivisten - moralische und normative Urteile grundsätzlich für nicht begründbar hält, für den endet der Bereich der Wissenschaft bei den empirischen Fragestellungen. Die Frage, ob der Faschismus, der Kommunismus oder der Liberalismus besser ist, lässt sich dann nicht mehr wissenschaftlich beantworten. Andererseits grenzte sich die Soziologie auf ihrem Weg zu einer eigenständigen Wissenschaft aus guten Gründen auch ab gegen die Ethik, auf deren Erkenntnisse sie nichtsdestotrotz zurückgreifen kann.

\footnotetext{
${ }^{121}$ Comte prägte den Begriff ,Soziologie' in den 20er Jahren des 18. Jahrhunderts (Comte 1822/1914), also zu einer Zeit, als Physik, Chemie, Astronomie, Geowissenschaften, Biologie und andere Naturwissenschaften bereits etabliert waren. Die Philosophie bestand schon viel früher, umfasste aber als ,Naturphilosophie' die gesamte Wissenschaft und war somit keine reine Geisteswissenschaft.

${ }^{122}$ Dahms (1994), 50 u. 401
} 
An dieser Stelle bietet es sich an, kurz in die Geschichte der Soziologie zurückzuschauen und zu fragen, wie Klassiker wie Montesquieu, Comte, Marx, Tocqueville, Durkheim, Pareto und Weber das Verhältnis von deskriptiver und normativer Betrachtung bestimmten. Darin ist nach Vittorio Hösle eine Entwicklungslogik zu entdecken. ${ }^{123}$ „Ist für die Antike und das Hochmittelalter das Sein im wesentlichen ein normativ aufgeladener Begriff - weswegen es mit dem Guten zusammenfällt -, so bildet sich in einem Jahrhunderte währenden Prozess ein neuer Begriff von Sein als Faktizität: Die Welt wird - wie im Logischen Positivismus - das, was der Fall ist; die Frage nach dem Guten gehört bestenfalls einer anderen Ordnung an, wenn sie nicht sogar gegenstandslos wird. “124 Montesquieu, Tocqueville oder auch Vico haben noch ein Verständnis von Sozialwissenschaft, das zwar einerseits aufklärerisch beeinflusst, aber auch noch stark im christlichen Seinsverständnis verwurzelt ist. Hier ist die normative Frage also religiös begründet und wird damit beantwortet, dass die christliche Gesellschaft die beste sei. Comte und Marx brechen radikal mit dem christlichen Weltbild. Comte will die Metaphysik überwinden, die unbeweisbaren Aussagen von Hegel sind ihm ein Gräuel. ${ }^{125}$ Der unhaltbare Begriff Gottes und der vage metaphysische Begriff der Natur müssten durch empirisch belegbare Begriffe abgelöst werden. Marx deckt mit seiner Ideologiekritik Pseudomoral auf, ohne eine eigene Moral begründen zu können.

Durkheim, Weber und Pareto, der Mathematik und Naturwissenschaften studiert hatte, gelten als Herolde der wertfreien Soziologie. Durkheim kann als ihr eigentlicher Begründer gelten. Seine ,sozialen Fakten' ${ }^{126}$ zeichnen sich durch ihre Äußerlichkeit gegenüber dem individuellen Bewusstsein aus.

Mit großer Vehemenz forderte im Werturteilsstreit 1904-1917 Max Weber von Wissenschaftlern den Verzicht auf wertende Stellungnahmen im Rahmen ihrer wissenschaftlichen Arbeit. ${ }^{127}$ Für ihn stellt sich die Soziologie als ,Wissenschaft vom sozialen Handeln’128 die Frage: „Wie handeln Menschen und warum?“ Die Frage: „Wie sollten Menschen handeln?“ gehört für Weber nicht mehr zum Erkenntnisbereich der Soziologie.

Ausgehend von der Prämisse, dass auch normative Fragen wissenschaftlich entscheidbar sind, neige ich der Auffassung zu, dass die Soziologie zwar nicht Handlungen von Individuen normativ bewerten sollte, durchaus aber Gesellschaftsordnungen. Wenn sich eine totalitäre Gesellschaftsordnung durchzusetzen beginnt, dann dürfen gerade Soziologen, die sich ja schließlich wissenschaftlich mit der Erforschung von Gesellschaftsordnungen beschäftigen, nicht schweigen.

\footnotetext{
${ }^{123}$ Hösle (1999), 125-165

${ }^{124}$ Hösle (1999), 129

${ }^{125}$ A.a.O.

${ }^{126}$ Durkheim (1895/1965)

${ }^{127}$ Weber (1904), 146-214; Weber (1913), 147-186; Weber (1917), 489-540

${ }^{128}$ Vgl.Weber (1980), 1
} 
Meiner Meinung nach gibt es fließende Übergänge zwischen Soziologie und Ethik. Die interessantesten Studien, z.B. über Gerechtigkeit, kombinieren empirische Ansätze mit normativen Überlegungen. Beides sollte aber natürlich dennoch analytisch stets klar getrennt werden.

Ein etwas anders gelagertes Argument lautet, dass es eine ,wertfreie' oder politisch neutrale Bevölkerungswissenschaft nicht geben könne, weil der Staat ihre Ergebnisse nutze, um damit seine Interessen bzw. die politischen Interessen einzelner Schichten durchzusetzen. ${ }^{129}$ Dieses Argument kennt man eher in Bezug auf die Naturwissenschaften. Es spricht meines Erachtens nicht gegen die Demografie als Wissenschaft, sondern dafür, bevölkerungsethischen Fragen nicht zu geringen Raum einzuräumen. Ob dies ethisch vorgebildete Demografen oder demografisch gebildete Ethiker tun, ist für die Beantwortung dieser Fragen letztlich irrelevant.

${ }^{129}$ Butterwegge (2002), 177 


\subsection{Die Debatte über das Bevölkerungsoptimum}

\subsubsection{Das Bevölkerungsgesetz von Thomas Robert Malthus}

Die Frage nach dem Bevölkerungsoptimum hat eine lange Tradition in der Bevölkerungswissenschaft. Eine Einführung in diese Debatte vermittelt ein Gefühl für das Denken berühmter Demografen (bzw. demografisch interessierter Biologen und Ökonomen) und erleichtert das Verständnis der folgenden Kapitel.

Eine der bekanntesten Theorien zur Überbevölkerung war der Maßstab die Fähigkeit eines Landes, seine Bevölkerung zu ernähren. Die Debatte eröffnete der englische Pfarrer Thomas Robert Malthus mit seiner Behauptung, dass die Bevölkerung sich ohne Hemmnisse stärker vermehren werde als die Nahrungsmittelproduktion. ${ }^{130}$ Hungersnöte und Elend seien ,eine absolut unausweichliche“ Folge dieses Ungleichgewichts. ${ }^{131}$ Malthus schreibt: „Ich habe behauptet, dass die Bevölkerung bei ungehindertem Wachstum in einer geometrischen Reihe zugenommen hat, der Unterhalt für den Menschen aber in einer arithmetischen Reihe. Wir wollen überprüfen, ob diese Behauptung richtig ist." ${ }^{\text {“132 }}$ Wer nun eine empirische oder auch nur theoretisch stringente Beweisführung erwartet, wird allerdings enttäuscht. Malthus fährt fort: „In den Vereinigten Staaten von Amerika, wo die Unterhaltsmittel bis heute in weit größerer Menge zur Verfügung stehen, die Sitten des Volkes reiner und demzufolge die Hindernisse für frühe Heiraten geringer sind als in einem der modernen Staaten Europas, hat es sich erwiesen, dass sich die Bevölkerungszahl in 25 Jahren verdoppelt. Diese Wachstumsquote wollen wir (...) als unsere Richtschnur annehmen und demnach behaupten, dass die Bevölkerung, wenn keine Hindernisse auftreten, jeweils im Zeitraum von 25 Jahren auf die doppelte Zahl von Köpfen anwächst, somit also in einer geometrischen Reihe zunimmt. “'133 Das singuläre Beispiel der Vereinigten Staaten reicht Malthus also als Begründung für eine allgemeine Gesetzmäßigkeit. Dabei war sogar dieses eine Beispiel umstritten: Zeitgenössische Denker sprachen von einer Verdoppelung zwischen 15 und 25 Jahren, der berühmteste Demograf vor Malthus, Johann Süßmilch, gab die Zeitspanne, in dem sich eine Bevölkerungszahl verdoppeln könne, mit 42 bis 96 Jahren an. ${ }^{134}$

Noch einfacher machte Malthus es sich mit dem zweiten Teil seines Gesetzes, der Zunahme der Nahrungsmittel in arithmetischer Reihe: „Wenn ich voraussetze, dass durch die bestmögliche Politik, durch die Bebauung von mehr Boden und durch erhebliche Förderung der Landwirtschaft die Erträge unseres Inselreiches in den ersten 25 Jahren auf das Doppelte gesteigert würden, so ist damit gewiß soviel zugestanden, wie man eben noch mit Fug und Recht erwarten kann. Unmög-

\footnotetext{
${ }^{130}$ Malthus (1798/1977), 18

${ }^{131}$ A.a.O., 19

${ }^{132}$ A.a.O., 20

${ }^{133}$ A.a.O., 21

${ }^{134}$ Malthus (1798/1977), Kommentar des Herausgebers Barth, 188. Malthus selbst (53 f.) gesteht für einzelne Staaten unterschiedliche Zeiträume für die Verdoppelung zu.
} 
lich aber wäre es, wollte man hoffen, dass in den darauffolgenden Jahren der Ertrag vervierfacht werden könnte. Das würde allen unseren Kenntnissen von den Eigenschaften des Bodens widersprechen. Das Höchste, was wir uns vorstellen können, liefe darauf hinaus, dass die Zunahme im zweiten Zeitraum von 25 Jahren dem gegenwärtigen Ertrag entsprechen würde. Wir wollen diese Annahme, obschon sie gewiß weit von der Wahrheit entfernt ist, als unsere Regelquote aufstellen und behaupten, dass aufgrund gewaltiger Anstrengungen der Gesamtertrag der Insel in jeweils 25 Jahren um eine ebensogroße Menge an Unterhaltsmitteln wachse, wie sie gegenwärtig hervorgebracht wird. ${ }^{135}$

Die Aufstellung einer Hypothese ist hier schon zugleich ihr Beweis. Zudem enthält dieses sog. Bevölkerungsgesetz offensichtlich den Denkfehler, dass die Verdoppelungsrate entweder dauerhaft gilt - dann muss die Rate der Nahrungsproduktion falsch angesetzt worden sein. Oder, wenn die Rate der Nahrungsproduktion tatsächlich das Wachstum begrenzt und Hungersnöte als natürliches Regulativ auftreten, so muss langfristig auch in den USA die Vermehrungsrate der Bevölkerung falsch eingeschätzt worden sein. Dass in den USA Hungersnöte ausbrechen, bezeichnet Malthus aber selbst als „fast unmöglich“"136. Offensichtlich sieht er sich genötigt, in einer Fußnote auf diesen Widerspruch einzugehen, indem er schreibt: „An Beispielen dieser Art [der raschen Verdoppelung der US-amerikanischen Bevölkerung, J.T.] scheint sich zu zeigen, dass die Möglichkeiten der Erde voll ausreichen, um jegliches Bedürfnis nach Nahrung, das der Mensch hegt, zu befriedigen. Wir würden uns aber eines Irrtums schuldig machen, wenn wir daraus die Annahme ableiteten, dass Bevölkerung und Nahrungsmittel stets im gleichen Verhältnis zunehmen. ${ }^{\text {"137 }}$ Dass die letztgenannte Annahme nicht aus dem vorherigen Satz folgt, heißt aber ja nicht, dass im Umkehrschluss richtig sein muss, dass Nahrung in arithmetischer und Bevölkerung in geometrischer Reihe zunehmen. Weist man Malthus Anspruch, quantitative Zusammenhänge erkannt und belegt zu haben, zurück, so reduziert sich sein Bevölkerungsgesetz auf die qualitative Aussage, ,dass die Vermehrungskraft der Bevölkerung unbegrenzt größer ist als die Kraft der Erde, Unterhaltsmittel für den Menschen hervorzu-

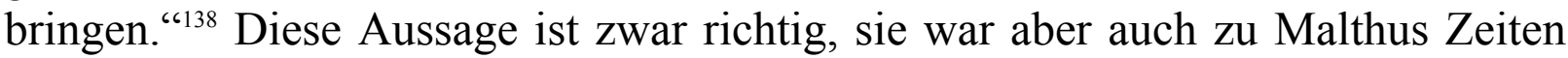
nicht neu. Der Übersetzer seines Werkes, Christian Barth, stellt eine Vielzahl von Quellen zusammen, die schon vor Malthus diesen Zusammenhang erwähnten. ${ }^{139}$

Malthus freilich vertieft diesen Gedanken. Er unterscheidet vorbeugende Hemmnisse (preventive checks) und nachwirkende Hemmnisse (positive checks). Beide seien dafür verantwortlich, dass die Bevölkerung langfristig nicht stärker

\footnotetext{
${ }^{135}$ A.a.O., 21

${ }^{136}$ A.a.O., 63

137 A.a.O., 54. Fußnote.

138 A.a.O., 18

${ }^{139}$ A.a.O., 180-183. Eine Aufzählung findet sich auch bei Cohen (1995), 5-8
} 
wachse als die Nahrungsmittelproduktion. ${ }^{140}$ Als vorbeugendes Hemmnis nennt Malthus die Hinausschiebung der Eheschließung. Je früher die Menschen heirateten, desto tugendhafter sei eine Gesellschaft, aber desto mehr Kinder würden auch geboren, so der von ihm konstruierte Zusammenhang. ${ }^{141}$ Angesichts der heutigen Debatte über die Gründe für die Kinderlosigkeit in SEL ist es interessant zu sehen, dass schon zu Malthus Zeiten Kinder mit gesellschaftlichem Abstieg in Verbindung gebracht wurden: „Ein Mann mit höherer Bildung, aber mit einem Einkommen, das gerade ausreicht, um ihm einen Platz in der guten Gesellschaft zu ermöglichen, dürfte ganz gewiß einsehen, dass er im Fall einer Heirat und einer Familiengründung genötigt ist, sich den wohlhabenderen Pächtern und der Klasse der kleineren Händler anzuschließen, insofern er überhaupt gesellschaftlichen Umgang pflegt. "'142 Auch eine Frau müsse nach Malthus Ansicht Abstriche beim Lebensstandard hinnehmen und ihr gesellschaftliches Leben aufgeben, sobald sie sich für Heirat und Kinder entscheide. Dies habe eine geburtenaufschiebende Wirkung. Versagt dieses vorbeugende Hemmnis und die Bevölkerung wächst zu schnell an, so bewirkten die nachwirkenden Hemmnisse eine Rückkehr ins Gleichgewicht: „Die Angst vor der Eheschließung, die sich daraus ergebenden lasterhaften Gewohnheiten, Krieg, Luxus, die unmerklich, aber dennoch mit Gewissheit vor sich gehende Entvölkerung der großen Städte, die beengten Behausungen und die unzureichende Nahrung für viele Arme: all dies hält die Bevölkerung davon ab, sich über die Unterhaltsmittel hinaus zu vermehren; dadurch wird - wenn ich eine aufs erste sicherlich befremdlich klingende Formulierung gebrauchen darf - die Notwendigkeit großer, verheerender Seuchen, die den Überschuss beseitigen müssten, entbehrlich. “143 Und an anderer Stelle: „Sollten sie aber versagen in diesem Vernichtungskrieg, dann dringen Krankheitsperioden, Seuchen und Pest in schrecklichem Aufgebot vor und raffen Tausende und Abertausende hinweg. Sollte der Erfolg immer noch nicht vollständig sein, gehen gewaltige, unvermeidbare Hungersnöte als Nachhut um und bringen mit einem mächtigen Schlag die Bevölkerungszahl und die Nahrungsmenge der Welt auf den gleichen Stand. "'144 Dieses nachwirkende Hemmnis sei hauptsächlich, wenn auch nicht ausschließlich auf die niederste Gesellschaftsschicht beschränkt. ${ }^{145}$

Soweit Malthus recht magere bevölkerungstheoretische Kernthesen, die heute von zahlreichen Demografen negativ rezipiert werden. ${ }^{146}$ Aber war es überhaupt sein Hauptanliegen, eine bevölkerungswissenschaftliche Analyse durchzuführen?

\footnotetext{
${ }^{140}$ Kurzfristig sei dies natürlich schon möglich, aber wie bei einem Pendel würden Ausschläge, d.h. Abweichungen von der Wachstumsrate der Nahrungsmittel, in die eine oder andere Richtung früher oder später stets zurückgeführt.

${ }^{141}$ Malthus (1798/1977), 24 und 35-38

${ }^{142}$ A.a.O., 38

${ }^{143}$ A.a.O., 63

${ }^{144}$ A.a.O., 68

${ }^{145}$ A.a.O., 41

${ }^{146}$ Deutliche Kritik auch bei Appleman (1976), Khalatbari/Otto (1999)
} 
Malthus Argumentation ist nur unter Berücksichtigung des geschichtlichen Hintergrunds zu verstehen, vor dem sein Werk entstand. Er schrieb seinen Text unter dem Eindruck der amerikanischen Unabhängigkeitserklärung von 1776 und der Französischen Revolution von 1789, deren gemeinsame revolutionäre Idee die "Gleichheit" aller Menschen war. Eines von Malthus Anliegen war zweifellos, mit seinem Essay argumentative Munition zu liefern gegen die egalitaristischen Theorien, die damals in ganz Europa diskutiert wurden. Er wollte die Unmöglichkeit aller Bestrebungen beweisen, die das Los der Arbeiter verbessern und gesellschaftlichen Reichtum umverteilen wollten. ${ }^{147}$ Malthus war Sohn eines Landbesitzers und sah wohl, dass im Zuge der geschichtlichen Zeitläufe auch in Großbritannien der Ruf nach Reformen lauter und die Vorrechte der Grundbesitzer in Frage gestellt werden würden. In der gesamten zweiten Hälfte seines Buches wendet Malthus dann sein ,Bevölkerungsgesetz' als Argument gegen die Gleichheitstheorien an, wie sie insbesondere von Condorcet ${ }^{148}$ und Godwin ${ }^{149}$ formuliert worden waren. Mit fragwürdigen Argumentationsketten folgert Malthus aus dem ,Bevölkerungsgesetz', dass die von diesen beiden Denkern angegriffenen Institutionen Ehe und Eigentum erhalten werden müssten. ${ }^{150}$ Die Idee der Gleichheit aller Bürger lehnt Malthus grundsätzlich ab: „Leid und Not des Lebens stellen eine andere Art von Anreiz dar, der notwendig zu sein scheint, um mittels einer besonderen Abfolge von Eindrücken das Herz empfindungsfähig und menschlicher zu machen, das soziale Mitgefühl zu wecken, all die christlichen Tugenden zu entfalten und Spielraum für die umfassenden Bemühungen der Nächstenliebe zu geben." ${ }^{151}$ Der ganze ,Essay' weist eine konservative, anti-revolutionäre Grundtendenz auf, wobei die Verknüpfung der „Systemfrage“ (Eigentumsordnung, politische Ordnung, etc.) mit der bevölkerungstheoretischen Frage sachlich kaum zu rechtfertigen ist.

Dass Malthus als Bevölkerungstheoretiker im Gedächtnis der Menschheit bleiben würde, hätte sicherlich kaum ein Zeitgenosse zu prophezeien gewagt. Malthus selbst hat seine Schrift nur als schnell dahingeschriebene Gelegenheitsarbeit ver-

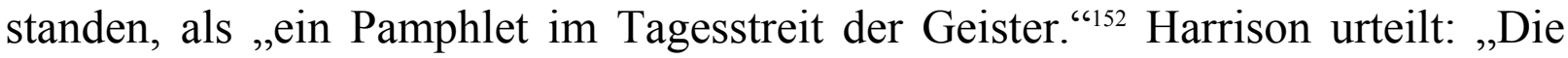
erste Fassung seines Essay war flüchtig zusammengestellt, schlecht dokumentiert, argumentierte unzusammenhängend und war vor allem polemisch. ${ }^{\text {"153 }}$

\footnotetext{
${ }^{147}$ Harrison (1994), 31

${ }^{148}$ Condorcet (1795/1976)

${ }^{149}$ Godwin (1793)

${ }^{150}$ Malthus (1798/1977), $92 \mathrm{f}$.

${ }^{151}$ Malthus (1798/1977), 161. Siehe auch 49-50. Malthus wurde von der Nachwelt häufig des Zynismus geziehen (vgl. Nachwort von Malthus 1798/1977), er selbst beteuert allerdings in seiner Schrift, dass ihm nichts besser gefiele, als wenn sein ,Gesetz' nicht gälte (Malthus 1798/1977, 15).

${ }^{152}$ Malthus (1798/1977), Kommentar des Herausgebers Barth, 195. Siehe auch Malthus eigene Aussage, dass er darauf verzichtete, eine ,ansehnlichere Zahl von Belegen“ zur Erläuterung der Kernthesen zu sammeln (Malthus 1798/1977, 11).

${ }^{153}$ Harrison (1994), 31
} 
Erst als Malthus merkt, dass seine Thesen sofort nach ihrem Erscheinen ein lebhaftes Echo auslösen, bringt er 1803, fünf Jahre nach der ersten Fassung seines Essay, eine zweite, erweiterte Auflage heraus, ${ }^{154}$ die stark verbessert war. Sein Fatalismus wird darin zumindest etwas abgeschwächt. Während in der ersten Auflage viel von der unerbittlichen Logik der ,Gesetze' und kaum von Lösungsansätzen die Rede ist - nur eine Förderung der Landwirtschaft empfiehlt er mehrmals ${ }^{155}$-, setzt Malthus nun auf Geburtenbeschränkung durch Junggesellentum, Spätheirat und freiwillige Enthaltsamkeit, um die natürlichen ,checks' zu ersetzen. Man kann diese zweite Auflage als historischen Beginn der wissenschaftlichen Bevölkerungssoziologie betrachten, ${ }^{156}$ da die Untersuchung menschlicher Verhaltensweisen die rein biologischen Prozesse des Kinderkriegens und Sterbens in den Hintergrund drängt.

Was der unmittelbaren Nachwelt von Malthus in Erinnerung blieb, war aber in erster Linie sein ,ehernes Bevölkerungsgesetz', nach dem die Bevölkerung in geometrischer Progression, die Nahrungsmittelproduktion aber nur in arithmetischer Progression zunehme. Davon beeinflusst formulierte Ricardo 15 Jahre später sein ,ehernes Lohngesetz', nach dem sich Löhne der Arbeiter niemals weit vom Mindestlohn, der zu ihrem Überleben und dem ihrer Kinder notwendig ist, wegbewegen würden. ${ }^{157}$

\subsubsection{Von Marx bis Mackenroth}

Die erste Fassung von Malthus Essay war ein Angriff auf die Ideale des Sozialismus, daher verwundert es nicht, dass Karl Marx ihn als „Sensationalpamphlet“ und als „Pasquill auf das Menschengeschlecht“ verurteilte. ${ }^{158}$ Marx stellte erstmals einen Zusammenhang zwischen Wirtschaftsordnung und Bevölkerungsentwicklung her: Das Populationsgesetz des Kapitalismus bewirkt nach Marx das Entstehen der industriellen Reservearmee, also von ,überflüssigen' Arbeitern, die keine Beschäftigung finden könnten. Es handle sich um Überbevölkerung im Hinblick auf ein Wirtschaftssystem, nicht um , wirkliche Überbevölkerung' im Hinblick auf Nahrungsmittel. ${ }^{159}$ Friedrich Engels schrieb dazu: „Die Bevölkerung drückt auf die Mittel - nicht der Subsistenz, sondern der Beschäftigung. Die Menschheit könnte sich rascher vermehren, als die moderne bürgerliche Gesellschaft vertragen kann." ${ }^{\text {160 }}$

Auf die Abhängigkeit von Bevölkerungsgesetzen von den wirtschaftlichen Rahmenbedingungen wies 1953 auch Mackenroth hin. ${ }^{161}$ Er vertrat die These einer

\footnotetext{
${ }^{154}$ Malthus (1803)

${ }^{155}$ Malthus (1798/1977), 50 u. 60

${ }^{156}$ Schmid (2003), 1

${ }^{157}$ Ricardo (1817/1994)

${ }^{158}$ Zitiert nach Harrison (1994), 33. ,Pasquill’ = anonyme Schmähschrift

${ }^{159}$ Harrison (1994), 33

${ }^{160}$ Zitiert nach Harrison (1994), 33

${ }^{161}$ Mackenroth (1953)
} 
jeweils historisch und gesellschaftlich mit der Wirtschaftsweise abgestimmten „Bevölkerungsweise'. Damit konnte er das Phänomen des säkularen Geburtenrückgangs theoretisch erfassen, was mit den älteren ,Gesetzen' nicht möglich war. Der Zusammenhang zwischen sozialem Wandel und Reproduktionsprozess spielt in Mackenroths strukturtheoretischem Ansatz eine zentrale Rolle. Damit wurden die klassischen Theorien, denen eine eindimensionale biologische oder ökonomische Betrachtung zu Grunde lag, von einer Sichtweise abgelöst, die den vielfältigen, komplexen Wechselbeziehungen zwischen demografischen und gesellschaftlichen Bedingungen Rechnung trug. ${ }^{162}$

\subsubsection{Die Bevölkerungstheorie von Esther Boserup}

1965 stellte die dänische Ökonomin Ester Boserup eine Bevölkerungstheorie auf, welche bis heute großen Einfluss in der Debatte über das Bevölkerungsoptimum genießt. Ihre sog. Boserup-These bezog sich zunächst in ihrem grundlegenden Werk The Conditions of Agricultural Growth ${ }^{163}$ nur auf die Landwirtschaft, wurde aber später auch auf die Industrialisierung angewendet. ${ }^{164}$ Boserup führte Änderungen in der Landwirtschaft auf den Faktor Bevölkerungswachstum zurück. Immer wenn ein bestimmter Schwellenwert erreicht sei, würden kulturelle und technologische Neuerungen eingeleitet, die dazu führten, dass die größere Bevölkerung im Sinne der Tragfähigkeit ernährt werden könne.

Solange die Bevölkerungen klein waren, betrieben die Bauern laut Boserup Wanderfeldbau, d.h. sie holzten Wälder ab, bebauten die gerodeten Flächen für ein bis zwei Jahre und zogen dann weiter. ${ }^{165}$ Nach zwanzig Jahren hatten sich die Böden so weit regeneriert, dass die Wanderbauern erneut dort aussäen konnten. Mit der zunehmenden Besiedlungsdichte eines Territoriums entstanden Probleme. Die Bevölkerung musste in immer geringeren Abständen auf dieselben Parzellen zurückgreifen. Infolgedessen verkrusteten die Böden und die Ernteerträge sanken. Um ihre größere Bevölkerung zu ernähren, mussten sich die Gesellschaften etwas einfallen lassen: Einfache Grabstöcke wurden durch Hacken ersetzt, später durch Pflüge, die von Ochsen gezogen wurden. In mehreren Revolutionen in der Agrartechnik führten die Bauern Bewässerung, Kompost, Fruchtfolgen, Dünger, Pestizide und schließlich die grüne Gentechnik ein. Das Bevölkerungswachstum war sozusagen die ,Peitsche', die technologischen Fortschritt erzwang. Boserup sieht diese Entwicklung als großen Fortschritt an: „Ein kleines Volk, dessen Größe stagniert, wird nur mit sehr geringer Wahrscheinlichkeit über das Stadium der primitiven Landwirtschaft hinauskommen und ein höheres Niveau der Technik und der kulturellen Entwicklung erreichen. ${ }^{\text {"166 }}$

\footnotetext{
${ }^{162}$ Schimany (2003), 43

${ }^{163}$ Boserup (1965)

${ }^{164}$ Boserup (1981)

${ }^{165}$ Harrison (1994), 35

${ }^{166}$ Boserup (1965), 118
} 
Ohne Boserups Thesen ausführlich diskutieren zu können, ist kritisch zu fragen, ob Fortschritte in der Agrarwirtschaft wirklich monokausal oder auch nur maßgeblich auf den Faktor ,Bevölkerungszunahme' zurückzuführen sind. Die Landwirtschaft war in Europa bis ins 19. Jahrhundert der vorherrschende Wirtschaftszweig, in dem 90 Prozent der Menschen arbeiteten. Anfang des 21. Jahrhunderts sind in Deutschland nur noch 2 Prozent der Menschen darin tätig. Im 19. Jahrhundert konnte ein Beschäftigter in der Landwirtschaft kaum mehr als sich selbst mit den erzeugten Nahrungsmitteln versorgen. Im Jahr 1950 war dagegen eine Arbeitskraft in der Landwirtschaft in Westdeutschland in der Lage, 14 weitere Personen mit zu versorgen. ${ }^{167}$ Bis zum Beginn des 21. Jahrhunderts hatte sich das Verhältnis auf 88 Personen mehr als versechsfacht. Die Bevölkerung stieg in den letzten 50 Jahren in Westdeutschland aber nur um rund 20 Prozent. Offensichtlich hängt der Produktivitätsfortschritt in der Landwirtschaft also noch von anderen Faktoren ab als der Bevölkerungsentwicklung.

\subsubsection{Paul Ehrlich}

In der zweiten Hälfte des 20. Jahrhunderts war es vor allem Paul Ehrlich, Biologieprofessor an der Stanford Universität, der zu den einflussreichsten Warnern vor weiterer Bevölkerungszunahme zählte. „Der Kampf um die Welternährung ist entschieden. In den siebziger Jahren werden gewaltige Hungersnöte die Menschheit heimsuchen. Hunderte von Millionen Menschen werden trotz aller sofort eingeleiteter Hilfsprogramme verhungern. Es ist zu spät, eine ernste Zunahme der Weltsterberate zu verhindern. Viele Menschenleben können allerdings gerettet werden durch umfangreiche Sofortprogramme zur Erhöhung der Nahrungsmittelproduktion. Werden sie jedoch nicht von entschiedenen und erfolgreichen Anstrengungen der Geburtenbeschränkung begleitet, so können solche Programme das Massensterben freilich nur hinauszögern", so beginnt Ehrlichs Buch Die Bevölkerungsbombe. ${ }^{168}$

Ehrlich thematisiert auch die negativen Folgen von Bevölkerungsdichte auf die Umwelt, letztlich ist seine Argumentation aber auf die Ernährungssicherheit bezogen. Dabei berücksichtigt er - das unterscheidet ihn von Malthus - dass die Zerstörung der Umwelt Auswirkungen auf die Ernährungssicherheit hat. Dass aber die neomalthusianische Frage, und nicht etwa die ökologische Frage nach dem Erhalt der Biodiversität letztlich im Mittelpunkt von Ehrlichs umfassendem Werk steht, verdeutlicht die folgende Stelle:

\footnotetext{
${ }^{167}$ Verdi (Oktober 2003), 13

${ }^{168}$ Ehrlich (1971). Man kann Ehrlich leicht angreifen, z.B. wenn er das (von ihm so genannte) Gefühl von Überbevölkerung beschreibt, das ihn in Dehli überwältigte (Ehrlich 1971, 15). Zu Gute muss man ihm jedoch halten, dass er sich stets auch für eine antinatalistische Geburtenpolitik in den SEL aussprach und insofern keine doppelten Moralstandards hatte. Ganz im Gegenteil betont er, dass jedes US-amerikanische Baby durch sein höheres Konsumniveau den gleichen negativen Effekt auf die globale Umweltsituation hat wie 25 indische Babies (Ehrlich/Ehrlich 1970, 322-324).
} 
„No human activity causes as much direct environmental damage as agriculture, yet no other activity is more dependent for its success on environmental integrity. No lack of the material ingredients of well-being causes as much human suffering as lack of food. And no index so plainly measures failure of a population to remain within its carrying capacity as the extent of hunger-related disease and death." 169

\subsubsection{Julian Simon}

Ehrlichs Thesen blieben nicht lange unwidersprochen. Vor allem der Ökonom Julian Simon besetzte seit 1977 die intellektuelle Gegenposition. ${ }^{170}$ Er nennt Ehrlich und den angeblichen Mainstream ,Doomsters' (Schwarzseher) ${ }^{171}$ und betonte die stimulierende Wirkung von Bevölkerungswachstum für technischen Fortschritt, Innovationen und damit Produktivität und Wirtschaftswachstum. ${ }^{172}$ Simon behauptet, dass nichts dafür spricht, dass wir unseren Kindern eine schlechtere Welt hinterlassen: „We are confident that the nature of the physical world permits continued improvement in humankind's economic lot in the long run, indefinitely. Of course, there are always newly arising local problems, shortages, and pollutions... and the solutions usually leave us better off than if the problem had never arisen." 173

Wie nach ihm Lomborg oder Maxeiner/Miersch versucht Simon mit Statistiken zu belegen, dass jeder Umweltindikator sich verbessert habe und alles Gerede von einer Krise auf einer falschen Wahrnehmung beruhe. Der Titel von Simons Hauptwerk The Ultimate Resource gibt aber einen Hinweis darauf, dass Simon fernab aller Polemik gegen Ökologen ${ }^{174}$ im wesentlichen eine ganz einfache, schlüssige These vertritt, nämlich dass die Innovationskraft einer Gesellschaft ceteris paribus mit wachsender Bevölkerung steige, da die Ressource ,menschliche Intelligenz' dadurch zunehme. Sein Schlüsselerlebnis beschreibt Simon so:

„One spring day about 1969, I visited the US AID office on the outskirts of Washington, D.C., to discuss a project intended to lower fertility in less-developed countries. I arrived early for my appointement, so I strolled outside in the warm sunshine. Below the building's plaza I noticed a road sign that said Iwo Jima Memorial. There came to me the memory

\footnotetext{
${ }^{169}$ Ehrlich/Ehrlich/Daily (1995), 6

${ }^{170}$ Simons erstes bevölkerungsökonomisches Buch The Economics of Population Growth erschien 1977.

${ }^{171}$ Simon und Ehrlich behaupten beide stets, der Mainstream stehe auf der anderen Seite.

${ }^{172}$ Simon (1977); Simon (1981); Simon (1992); Simon (1998); Vgl. auch Steinmann (1988), 19-35

${ }^{173}$ Simon (1992), $29 \mathrm{f}$.

${ }^{174}$ Kostprobe: „The Limits to Growth simulation of 1972, in which people breed to the exhaustion of natural resources, is so devoid of meaning that it is not worth detailed discussion or criticism. Yet it is taken seriously by many people to this day, and it is therefore a fascinating example of how scientific work can be outrageously bad and yet very influential." (Simon 1988, 508)
} 
of reading a eulogy delivered by a Jewish chaplain over the dead on the battlefield at Iwo Jima, saying something like: »How many who would have been a Mozart or Michelangelo or an Einstein have been buried here? « And I thought: Have I gone crazy? What business do I have trying to help arrange it that fewer beings will be born, each one of whom might be a Mozart or a Michelangelo or an Einstein - or simply a joy to his or her family and community, and a person who will enjoy life?"175

Simon nennt in der Danksagung der letzten Auflage von The Ultimate Resource als seine geistigen Väter die ,Bevölkerungsoptimisten' William Petty, Adam Smith, Friedrich Engels, Jules Verne, H.G. Wells (letztere beide wegen ihres Lobs der menschlichen Visionskraft), Simon Kuznets, Harold J. Barnett, Chandler Morse, A.V. Chayanov und Ester Boserup. Sie hätten ihm zu der Überzeugung verholfen, dass „Bevölkerungswachstum, zusammen mit der Verlängerung der Lebenserwartung, ein moralischer und materieller Triumph" ${ }^{\text {“176 }}$ sei. Simon expliziert diesen Punkt so: „Adding more people to any community causes problems, but people are also the means to solve these problems. The main fuel to speed the world's progress is our stock of knowledge, an the brake is our lack of imagination. The ultimate resource is people - skilled, spirited, hopeful people who will exert their wills and imaginations for their own benefit as well as in a spirit of faith and social concern. Inevitably they will benefit not only themselves but the poor and the rest of us as well." ${ }^{177}$

Simon beschreibt eine c.p.-Beziehung. Insofern stellt sich die Frage nach der Richtigkeit seiner Prämissen. In der wirklichen Welt hat aber das Wachstum einer Bevölkerung Auswirkungen auf das Bildungsniveau und die Innovationskraft eben dieser Bevölkerung. ${ }^{178}$ Simons Kernaussage, im Modell zweifellos richtig, scheitert also an der Empirie. Damit soll nicht gesagt werden, dass seine umfangreichen Ausführungen zu den positiven Wirkungen von Bevölkerungswachstum auf Wirtschaftswachstum, Produktivität, Lebensstandard und psychologisches Wohlbefinden, sowie seine Kritik an den Thesen von Ehrlich oder dem Club of Rome gänzlich falsch sind. Es ist hier nicht der Platz, auf allen Feldern eine Debatte mit Simon zu führen. Beim Thema Artenvielfalt aber sind seine Äußerungen sehr angreifbar.

Simon vertritt hier einen rein instrumentellen Anthropozentrismus, der Tiere als Sachen ansieht und ihren Wert nach dem Nutzen für den Menschen bemisst: „Society properly is concerned about possible dangers to species. Individual species, and perhaps all species taken together, constitute a valuable endowment, and we

\footnotetext{
${ }^{175}$ Simon (1998), xxxi

${ }^{176}$ A.a.O., xxxvii

${ }^{177}$ A.a.O., xxxviii

${ }^{178}$ Siehe ausführlich den Abschnitt 6.5.9. Innovationsfähigkeit.
} 
should guard their survival just as we guard our other physical and social assets." ${ }^{179}$ Simon sagt zwar, dass wir den zukünftigen Nutzen der Arten für den Menschen abschätzen müssen, gibt aber im nächsten Satz zu, dass er keine Antwort darauf hat, wie ein Bewertungsverfahren aussehen könnte. ${ }^{180}$ Hinsichtlich der Berechnung der Kosten einer Artenschutzpolitik macht er aber konkrete Vorschläge: „Lastly, any policy analysis concerning species loss must explicitly evaluate the total cost of protective actions - for example the cost of not logging or not building roads in an area. And such a total cost estimate must include the long-run indirect costs of reduction in economic growth to a community`s education and general advancement." 181

Den überwiegenden Rest seiner Ausführungen kritisiert Simon die Schätzungen der Biologen. ${ }^{182}$ Er weist darauf hin, dass es große Bandbreiten und Unsicherheiten in den Schätzungen gibt und hebt die nachgewiesenen Ausrottungsraten hervor. Aber auch die meisten Biologen betonen, dass die nachgewiesenen Ausrottungsraten sehr gering sind. ${ }^{183}$ Und würde man Simons nicht-konstruktiver Kritik genügen wollen, so dürften überhaupt keine wissenschaftlichen Schätzungen über die Aussterberate noch unentdeckter Arten gemacht werden.

Auf der einen Seite verlangt Simon (zu Recht) eine Verbesserung der wissenschaftlichen Datenbasis, damit die Schätzungen verfeinert werden können, auf der anderen Seite lehnt er die (von Biologen erhobene) Forderung nach einem umfassenden Forschungsprogramm aus Kostengründen strikt ab. „[It] would absorb the careers of 25.000 biologists according to Wilson and cost billions of dollars that could be used for other scientific and social purposes, or even just for poor and not-poor individuals'personal benefits." 184

Die bleibende Leistung in Simons Lebenswerk ist der Hinweis auf den Mensch als unerschöpfliche Quelle von Innovationen. Wie viele Personen, die einen guten Gedanken hatten, versucht Simon alles, um Gegenargumente zu entkräften manchmal auch um den Preis eigener Glaubwürdigkeit.

\subsubsection{Das Bevölkerungsoptimum gemäß der Youth Bulge-Theorie}

In die Debatte über das Bevölkerungsoptimum lässt sich auch die These von der ,Youth Bulge' (,Jugendbeule') einordnen. Damit wird ein demografischer Erklä-

\footnotetext{
${ }^{179}$ Simon (1998), 440

${ }^{180}$ A.a.O., 448

${ }^{181}$ A.a.O., 449

${ }^{182}$ In der 734 Seiten dicken letzten Auflage von The Ultimate Resource von 1998 sind 19 Seiten dem Problem des Artensterbens gewidmet (439-458). Davon werden fünf Seiten verwandt, um die Annahmen in Norman Myers Buch The Sinking Ark (1979) zu kritisieren. Simon ignoriert den gesamten Fortschritt, den die Taxonomie zwischen 1979 und 1998 gemacht hat. Selbst wenn es sich hier um eine Schlampigkeit handelte, so viele Seiten der ersten Auflage von The Ultimate Resource von 1981 in die Auflage von 1998 zu übernehmen, so wirft es doch ein grelles Licht auf die Defizite dieses Kapitels.

${ }^{183}$ Heywood/Stuart (1992), 93. Siehe dazu auch ausführlich Abschnitt Verlust der Biodiversität.

${ }^{184}$ Simon (1998), 455
} 
rungsversuch für die Neigung von Ländern zu Angriffskriegen, Revolutionen, Terrorismus und sonstigen Unruhen bezeichnet, bei dem zwar keine Bevölkerungsgröße oder -dichte, aber eine bestimmte Bevölkerungsstruktur als Erklärungsansatz herangezogen wird.

Manche Kriegsursachenforscher sehen einen Zusammenhang zwischen einem hohen Anteil von Heranwachsenden an der Gesamtbevölkerung und der Neigung eines Landes zu Umstürzen und kriegerischen Konflikten. Die seit 1945 fast parallel verlaufende Entwicklung von Bevölkerungswachstum und dem Anstieg bewaffneter Konflikte wird als Beleg herangezogen. In den 1970er Jahren sah der französische Soziologe und Kriegsforscher Gaston Bouthoul in einer ,Youth bulge', sofern sie mit einer hohen Bevölkerungsdichte zusammenfällt, einen Nährboden für Kriege. Er führt aus, dass in den zurückliegenden zweihundert Jahren die Kampfhandlungen in der Welt im selben Maße zugenommen hätten, wie die Kindersterblichkeit gesunken sei. Starkes Bevölkerungswachstum, die von ihm so genannte ,demografische Inflation“, führe zu einer Entwertung menschlichen Lebens und sei ein grundlegendes Element kollektiver Aggressivität. ${ }^{185}$

Heute lebt von den 1,2 Milliarden Jugendlichen zwischen 10 und 19 Jahren rund ein Viertel in extremer Armut. ${ }^{186}$ Drohen uns also verstärkt Kriege aus Unruhen und Kriege aus demografischen Gründen, wie Dießenbacher befürchtet? ${ }^{187}$ Dießenbacher untersucht den Genozid in Ruanda, der 1994 in nur hundert Tagen 800.000 Opfer forderte. Die ruandische Bevölkerung hatte sich von 3,6 Millionen im Jahr 1952 auf 7,5 Millionen 1993 mehr als verdoppelt. Mit durchschnittlich 8,3 Lebendgeburten je Frau gehörte Ruanda damals zu den Ländern mit den weltweit höchsten Fertilitätsraten. Trotz ausschließlich agrarischer Wirtschaftsweise lag die Bevölkerungsdichte in Ruanda höher als in einer Industrienation wie Deutschland. Diese „Übervölkerungsstruktur“ habe laut Dießenbacher maßgeblich das Morden ausgelöst und sie sei keineswegs eine Besonderheit Ruandas.

Der Bremer Völkermordforscher Gunnar Heinsohn schreibt dazu, nicht Bevölkerungswachstum an sich oder absolute Zahlen von Bevölkerungen oder Bevölkerungsgruppen lösten Kriege aus, sondern der Anteil der Jugendlichen und jungen Erwachsenen innerhalb einer Gesellschaft. ${ }^{188}$ Besonders gefährlich wird es laut Heinsohn, wenn die Altersgruppe der 15 bis 24jährigen einen Anteil von 20 Prozent an der Gesamtbevölkerung übersteigt - eine solche Altersverteilung führe fast zwangsläufig zu Krieg und Gewalt. Heinsohn stellt eine Verbindung mit bestimmten gesellschaftlichen Arrangements her, die gegeben sein müsse, damit der Überhang der Jugend ihre kriegerische Wirkung entfalte. In vielen Teilen der Welt können gemeinhin nur die erstgeborenen Söhne etwas erben. Alle Nachgeborenen, aber auch jene, für die nach einer Realteilung zu wenig zum Überleben

\footnotetext{
${ }^{185}$ Bouthoul (1972)

${ }^{186}$ Kröhnert (2004), 1

${ }^{187}$ Dießenbacher (1998)

${ }^{188}$ Heinsohn (2003)
} 
übrig bleibe, fänden keinen angemessenen Platz in der Gesellschaft. Die überzähligen Söhne seien auf der Suche nach Status zu allem bereit - auch zu Gewalt. Dieses Heer an potenziellen Kriegern, die in Heroismus und Märtyrertum ihre einzige Chance sähen, würde durch die demografische Entwicklung in den kommenden 15 Jahren immer kopfstärker. Es sei kein Zufall, dass diese Zeitspanne unter amerikanischen Strategen als die gefährlichste im Kampf gegen den internationalen Terrorismus gelte. So glaubt der CIA-Direktor George Tennet, dass die Wahrscheinlichkeit für einen jungen Menschen, in den nächsten Jahren Terrorist zu werden, im Mittleren Osten und in Afrika südlich der Sahara am größten ist. ${ }^{189}$ Besonders groß sei die Gefahr, wenn die Faktoren ,youth bulge', politische Unzufriedenheit und sinkende Realeinkommen zusammentreffen. Ein Musterbeispiel sei Saudi-Arabien. Zwischen 1984 und 2002 hat sich die saudische Bevölkerung mehr als verdoppelt. Die Wirtschaft hätte einen außergewöhnlichen Wachstumsschub erleben müssen, um die Einkommen in diesem Zeitraum stabil zu halten. Tatsächlich sank aber das Pro-Kopf-Einkommen um mehr als die Hälfte, weil sich die Bevölkerung im Arbeitsalter stärker mit der Versorgung der Kinder beschäftigen musste. ${ }^{190}$

Das Problem besteht nach Ansicht von Schmid nicht so sehr in den inneren Probleme der Entwicklungsgesellschaften, sondern in der Wechselwirkung zwischen schrumpfenden und wachsenden Gesellschaften: „Das Problem besteht im Aneinanderrücken von schrumpfenden und alternden Bevölkerungen, die heute noch 80 Prozent der Weltproduktion erwirtschaften und die Welthandelsströme beherrschen, und von jungen und wachsenden Bevölkerungen der „Dritten Welt", die bereits 80 Prozent der Weltbevölkerung stellen, aber aus weltwirtschaftlicher Perspektive überwiegend Teil der Peripherie sind. Die Probleme der modernisierten Welt und die diametral entgegengesetzten der Entwicklungsregionen lassen ein ,demografisches Jahrhundert' erwarten: die Frustration, das westliche Lebensmodell für sich nicht übernehmen und einrichten zu können, wird große Entwicklungsländer auf ihr Bevölkerungsgewicht und eigenkulturelles Selbstbewusstsein verweisen." ${ }^{191}$

Zwar wird sich das Durchschnittsalter in allen Gesellschaften bis 2050 erhöhen, aber ein vielleicht noch bedeutsamerer Effekt ist, dass die Spanne zwischen den jüngsten und den ältesten Gesellschaften größer wird. Unter der Überschrift Die Geopolitik des globalen Alterungsprozesses schreibt der Direktor der Initiative Global Aging am Centre for Strategic and International Studies (CSIS), Paul S. Hewitt: „Heute liegt der Unterschied des Durchschnittsalters zwischen der jüngsten und der ältesten Gesellschaft, Jemen und Japan, bei 27 Jahren - im Jahr 1950 waren es nur 3,4 Jahre. Aber im Jahr 2015 wird der durchschnittliche Jemenit 32 Jahre jünger sein als der durchschnittliche Europäer und 34 Jahre jünger als der

\footnotetext{
${ }^{189}$ Zitiert nach Kröhnert (2004), 3

${ }^{190}$ Hewitt (23.3.2004), 39

${ }^{191}$ Schmid (2004); Schmid (2003), 3 f.
} 
durchschnittliche Japaner. Es ist bedeutsam, dass Länder auf beiden Seiten dieser Kluft Herde wirtschaftlicher und politischer Unruhe sein werden. ${ }^{\text {" } 192}$

Die These vom ,youth bulge' klingt plausibel. Der zweifellos vorhandenen Testosteronüberschuss in diesen Gesellschaften veranlasste schon Cicero zu dem Ausspruch: „Es sind die jungen Männer, die die Staaten erschüttern!“ Allerdings konnte empirisch ein solcher Zusammenhang bisher nicht nachgewiesen werden (siehe auch Abb. 2).

\section{Abb. 4: Länder im Krieg und Bevölkerungswachstum}

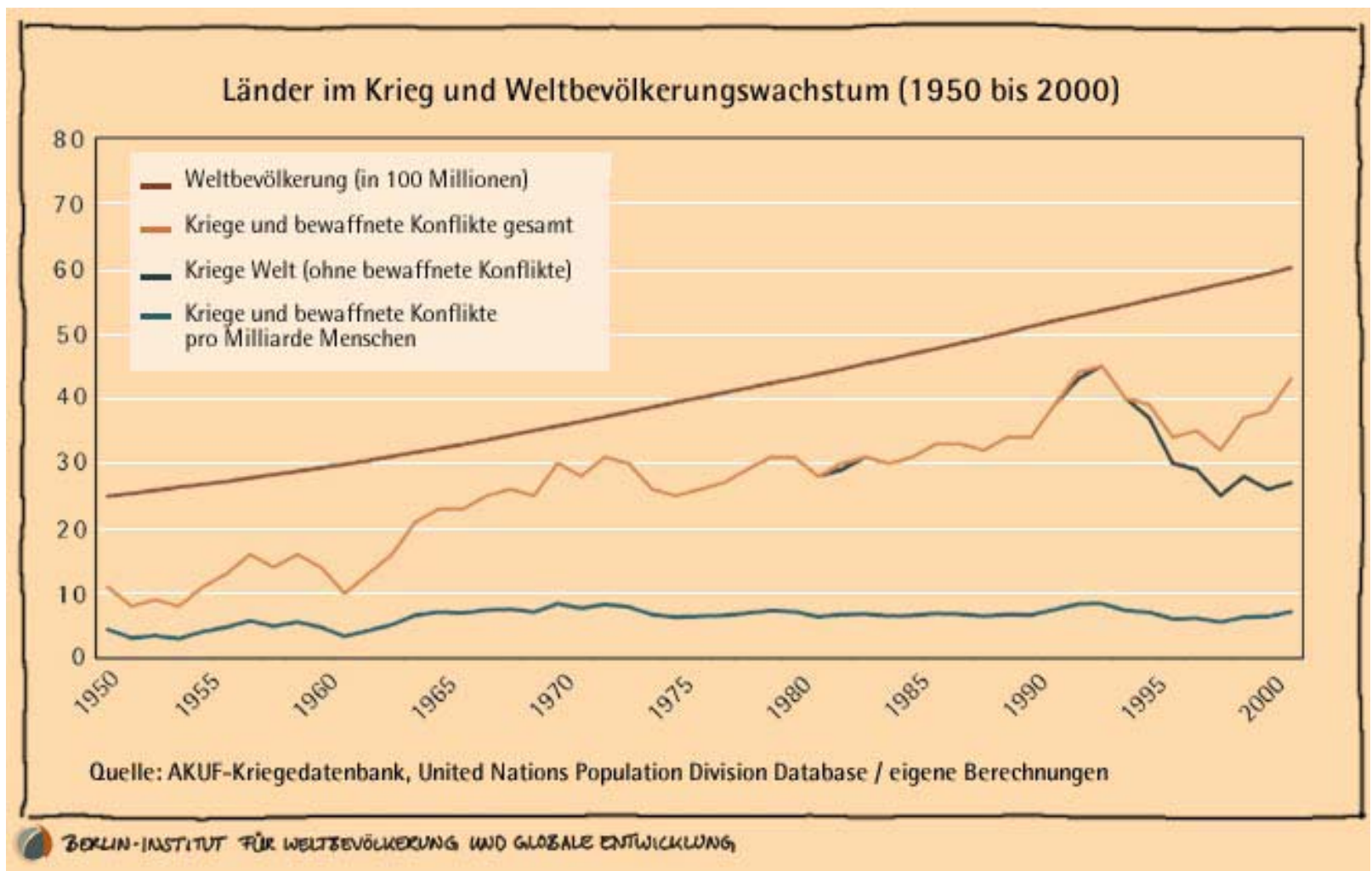

\section{Quelle: Kröhnert (2004)}

Eine Untersuchung des Berlin-Instituts für Weltbevölkerung und globale Entwicklung von 2004 überprüfte die These vom ,youth bulge' anhand demografischer Daten aus 159 Ländern und aller registrierten Kriege zwischen 1950 und 2000. Sie kam zu dem Ergebnis, dass ,youth bulges' keine monokausale Erklärung für die Entstehung von Kriegen liefern, sondern lediglich einen Stressfaktor unter vielen darstellen. ${ }^{193}$ Länder mit einem sehr hohen Youth Bulge haben sogar eine niedrigere „Kriegswahrscheinlichkeit“" als solche mit einem mittleren.

\footnotetext{
${ }^{192}$ Hewitt (23.4.2003), 39

${ }^{193}$ Kröhnert (2004), 1
} 


\section{Abb. 5: Statistische Wahrscheinlichkeit von Krieg nach dem Anteil der Youth Bulges}

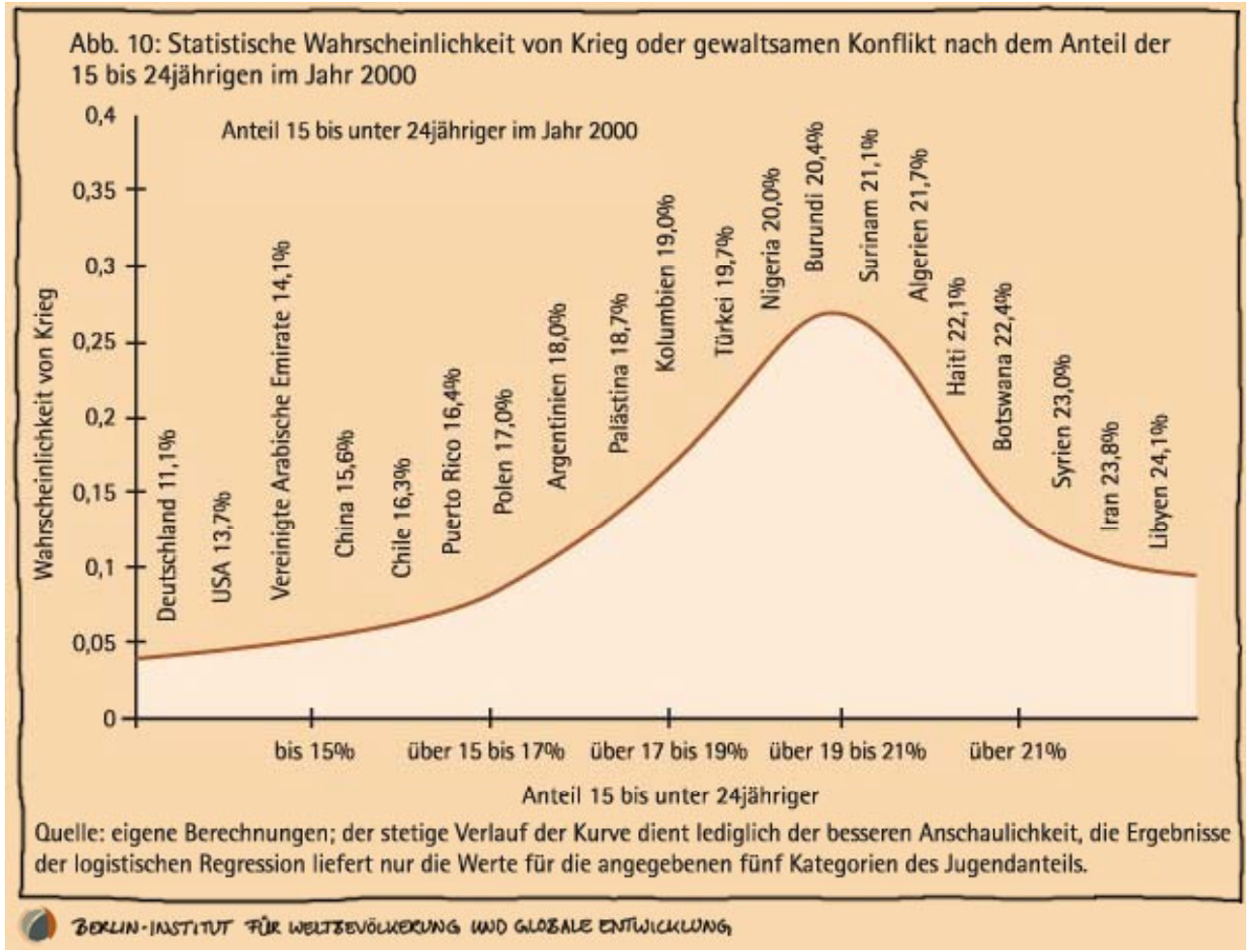

\section{Quelle: Kröhnert (2004)}

Es besteht weiterer Forschungsbedarf, um abschließend zu klären, ob und wenn ja, unter welchen Umständen der intuitiv einleuchtende Youth-Bulge-Ansatz einen echten Erklärungswert hat.

\subsubsection{Missverständnisse in der ,Population Debate'}

Wer hat nun das Bevölkerungsoptimum richtig bestimmt? In den letzten Jahrzehnten wurden von den Bevölkerungsoptimisten wie Simon und -pessimisten wie Ehrlich zahlreiche Studien und Statistiken erstellt, die den einen oder anderen Standpunkt belegen sollten. Das Ergebnis ist mager. Eine überzeugende Beweisführung, welche Bevölkerungsgröße für ein Land oder für die Erde als ganzes optimal ist, gelang keiner Seite.

Es ist nicht Ziel dieses Abschnitts, die Debatte über das Bevölkerungsoptimum erschöpfend darzustellen. Vielmehr sollte die kurze Übersicht über diese Debatte zeigen, dass die Standpunkte nicht direkt aufeinander beziehbar sind. Die Protagonisten der ,Population Debate' haben verschiedene Kontexte im Kopf und müs- 
sen zwangsläufig zu verschiedenen Bevölkerungsoptima gelangen. Sie reden zumindest zum Teil aneinander vorbei. Wenn man von Unterbevölkerung, Bevölkerungsoptimum und Überbevölkerung spricht, so muss, so meine These, genau gesagt werden, im Hinblick auf welchen Maßstab die Betrachtung angestellt wird. Es lässt sich kein für alle Zeiten und alle Bereiche gültiges Optimum aufstellen, ${ }^{194}$ sondern nur Optima für bestimmte Teilbereiche. Wenn z.B. Friedfertigkeit das Kriterium ist, welches das Optimum bestimmt, so wäre eine ältere Bevölkerungsstruktur einer jüngeren vorzuziehen. Autoren wie z.B. Julian Simon dagegen fragten, welche Bevölkerungsgröße die richtige sei, um eine hohe Innovationsrate zu erzielen. Hierbei ist c.p. eine jüngere, größere Bevölkerung erfolgreicher als eine ältere und kleinere. In der vorliegenden Studie geht es darum, welche Bevölkerungsgröße für ein Land bzw. die Welt optimal im Sinne der ökologischen Nachhaltigkeit (inklusive Erhalt der Artenvielfalt) ist. Dies ist eine andere Fragestellung als bei Malthus, Boserup oder Simon.

Es ist unmöglich, ein Bevölkerungsoptimum zu bestimmen, welches universell gültig ist und den Kriterien ,Ökologische Nachhaltigkeit', ,Innovationsfähigkeit', ,Friedfertigkeit' und ,Wirtschaftswachstum' genügt. „If the ethical problems of population limitation could be reduced to one overriding issue, matters would be simplified. They cannot", so fasst der Ethiker Daniel Callahan die Debatte über das Optimum zusammen. ${ }^{195}$ Wohl aber lassen sich Aussagen über das Bevölkerungsoptimum hinsichtlich eines der genannten Bereiche machen. Wir werden daher später darauf eingehen, wie das Bevölkerungsoptimum in ökologischer Hinsicht aussähe.

Nun wenden wir uns aber zunächst einer Bestandsaufnahme der Bevölkerungsdynamik zu. Es erscheint angemessen, diese den Überlegungen Bevölkerung-Umwelt-Zusammenhängen voranzustellen, da bei jenen auf dieses Zahlenmaterial zurückgegriffen werden muss.

\subsection{Zahlen und Fakten zur Weltbevölkerung}

\subsubsection{Bevölkerungsdynamik global und regional}

Die Weltbevölkerung entwickelte sich in den Jahrhunderten vor Christi Geburt zahlenmäßig nur sehr langsam. Bei einer Wachstumsrate von nahezu null bedurfte es mehrerer Jahrtausende, bis sich die Weltbevölkerung verdoppelt hatte. Vor der neolithischen Revolution zählte die Weltbevölkerung nur ca. fünf bis zehn Millionen Menschen. Die Bevölkerungsdichte - heute in Deutschland etwa 231 Menschen pro Quadratkilometer - lag bei weniger als 0,1 Menschen/qkm. Um 300 v.C. wird die Weltbevölkerung auf 100 Millionen Menschen geschätzt. Um 1650 war die Erde mit einer halben Milliarde Menschen bevölkert. 1804 war die erste Milliarde erreicht. Bis 1900 stieg die Weltbevölkerung auf 1,65 Milliarden Jahre.

\footnotetext{
${ }^{194}$ Vgl. die Debatte über das Populationsmaximum im Abschnitt 3.3. Das Tragfähigkeitstheorem.

${ }^{195}$ Callahan (1976), 20
} 
Im 20. Jahrhundert wuchs sie von 1,6 auf 6,1 Milliarden, d.h. sie vervierfachte sich dann fast, wovon der größte Teil dieser Zunahme - 80 Prozent - in der zweiten Jahrhunderthälfte erfolgte. Anders ausgedrückt: Die Bevölkerung hat seit 1960 so stark zugenommen wie in den vier Millionen Jahren zuvor, seit dem ersten aufrechten Gang unserer Urahnen (vgl. Abb. 3). ${ }^{196}$

\section{Abb. 6: Historische Entwicklung der Weltbevölkerung}

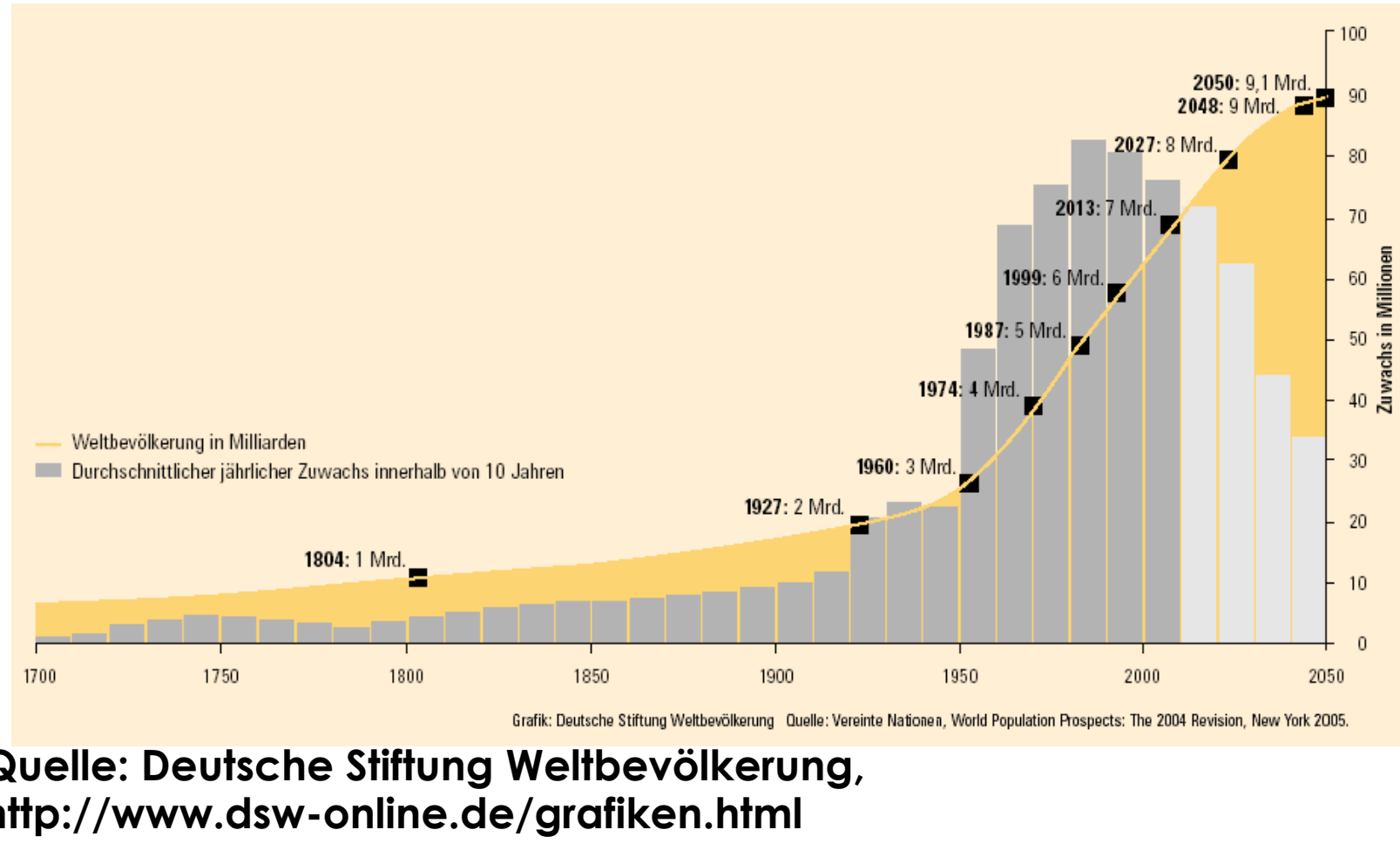

Der Direktor der Population Division, Joseph Chamie, bezeichnet das 20. Jahrhundert aus demografischer Sicht als das Jahrhundert der absoluten Superlative: ${ }^{197}$

- Nie zuvor wurde eine zusätzliche Milliarde in so kurzer Zeit erreicht. Von der fünften zur sechsten Milliarde brauchte die Welt nur 12 Jahre, von 1987 bis 1999.

\footnotetext{
${ }^{196}$ Worldwatch Institute (2000), 11. Der Politologe Bernd Guggenberger zeichnet folgendes Bild: „Nehmen wir an, die Kette menschlichen Lebens seit den Uranfängen vor zwei bis drei Millionen Jahren umfasse 100.000 einzelne Glieder, für jede Generation eines, welches jeweils in seiner Größe und seinem Gewicht die Anzahl der in dieser Generation lebenden Individuen darstelle. Dann ist das letzte Kettenglied, welches die jetzt lebende Generation symbolisiert, nicht einfach nur das größte und gewichtigste aller Kettenglieder; nein, es ist größer und schwerer als alle hunderttausend Glieder der gesamten Menschenkette davor zusammengenommen.“ (Guggenberger 27.1.1990). Dieses Beispiel ist allerdings nicht richtig. Nach Berechnungen von Birg $(1996,14)$ beläuft sich die Gesamtzahl aller bis heute jemals gelebten Menschen auf rund $80 \mathrm{Mrd}$. Die heute Lebenden machen also 8 Prozent all derjenigen aus, die jemals gelebt haben. Aber auch dieses Zahlenbeispiel zeigt, dass wir aus demografischer Sicht in außergewöhnlichen Zeiten leben. Mit der prognostizierten Stabilisierung der Weltbevölkerung wird dieser Prozentsatz in Zukunft stark zurückgehen und wohl niemals mehr den heutigen Wert erreichen.

${ }^{197}$ Vgl. Chamie (2004), $12 \mathrm{f}$.
} 
- Nie zuvor gab es so viele zusätzliche Menschen in einem Jahr: 87 Millionen Mitte der 1980er Jahre.

- In den Jahren 1962 und 1963 gab es eine jährliche weltweite Wachstumsrate von 2,2 Prozent, soviel wie nie zuvor.

Diese Zahlen machen deutlich, dass die Menschheit sich nicht noch mal ein Jahrhundert wie das 20. leisten kann.

\subsubsection{Absoluter Zuwachs und Trägheitsfaktor}

Nach der mittleren Variante der Langzeitprojektionen zur Bevölkerungsentwicklung wird die Weltbevölkerung von derzeit 6,3 Milliarden auf 9,1 Milliarden steigen. ${ }^{198}$ Die Hälfte dieses Zuwachses von 2,6 Milliarden Menschen wird von nur acht Ländern beigesteuert: Indien, Pakistan, Nigeria, den USA, China, Bangladesch, Äthiopien und der Demokratischen Republik Kongo. ${ }^{199}$

\section{Abb. 7: Weltbevölkerungsprojektionen für 2050}

\section{Weltbevölkerungsprojektionen für 2050}

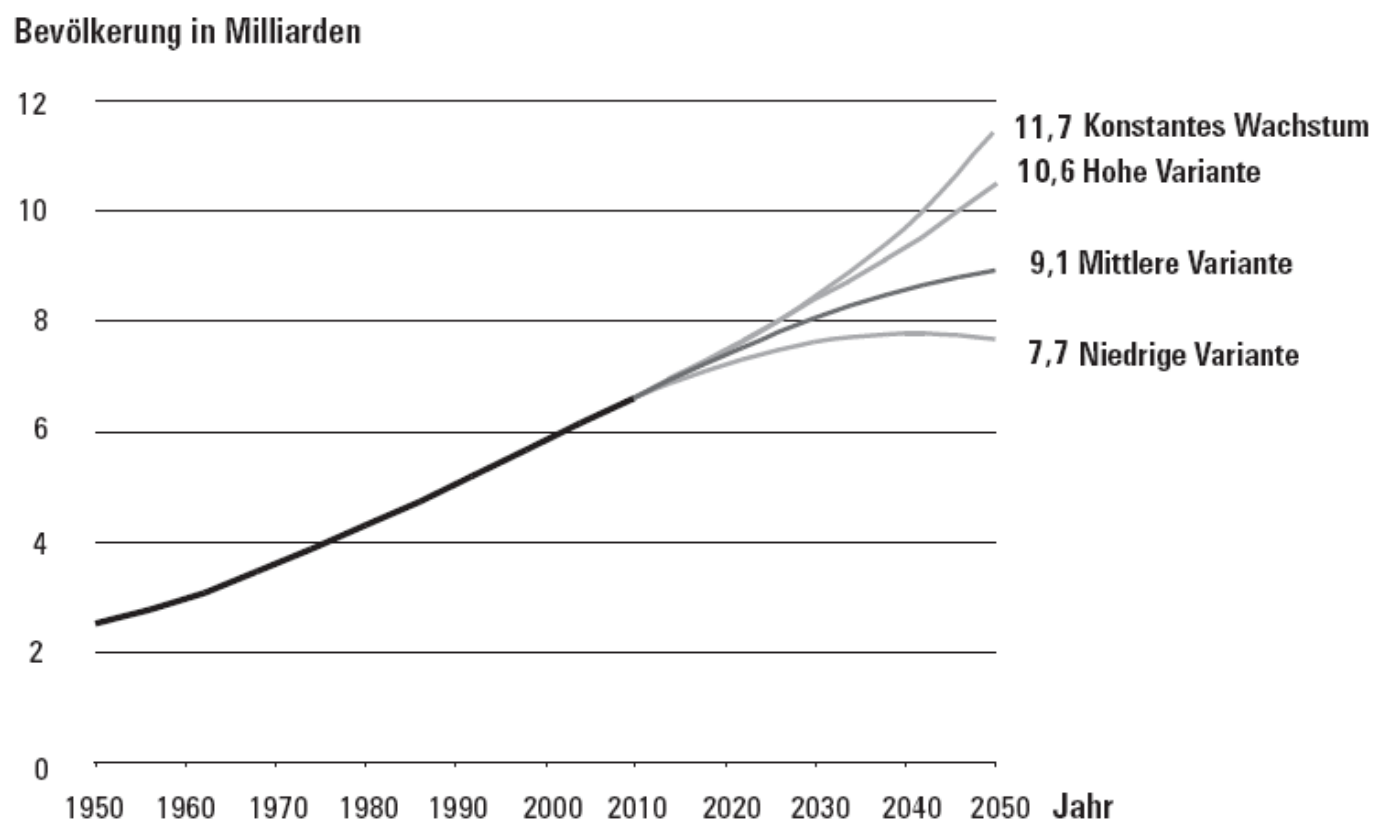

Quelle: Deutsche Stiftung Weltbevölkerung, http://www.dsw-online.de/grafiken.html

\footnotetext{
${ }^{198}$ UN Population Division (2005)

${ }^{199}$ UN Population Division (2003c), 8 (Die Aufzählung der Länder erfolgte in der Reihenfolge ihrer Beteiligung am Zuwachs).
} 
Die drei Wachstumsszenarien stehen nicht im konventionellen statistischen Sinn für Fehlertoleranzen. Die UN-Demografen treffen keine Aussage darüber, wie wahrscheinlich das untere und das obere Szenario im Vergleich zum mittleren Szenario ist. Vielmehr wurden unterschiedliche Prämissen zu Grunde gelegt. Die Wissenschaftler der Population Division trafen bei ihrer mittleren Variante die Annahme, dass die zusammengefasste Geburtenziffer bis zum Jahr 2050 auf 2,05 Kinder pro Frau fällt. Wenn Frauen im Durchschnitt 2,53 Kinder hätten, so würde die Weltbevölkerung bis 2050 auf 10,6 Milliarden steigen (hohe Variante), hätten sie 1,56 Kinder, so ergäbe sich eine Weltbevölkerung von 7,7 Milliarden (niedrige Variante). Würde die Wachstumsrate dagegen entgegen den Annahmen unverändert bleiben, so würde die Weltbevölkerung bis 2050 11,7 Milliarden erreichen (vgl. Abb. 7).

\section{Abb. 8: Wachstumsrate und Zuwachs der Weltbevölkerung}

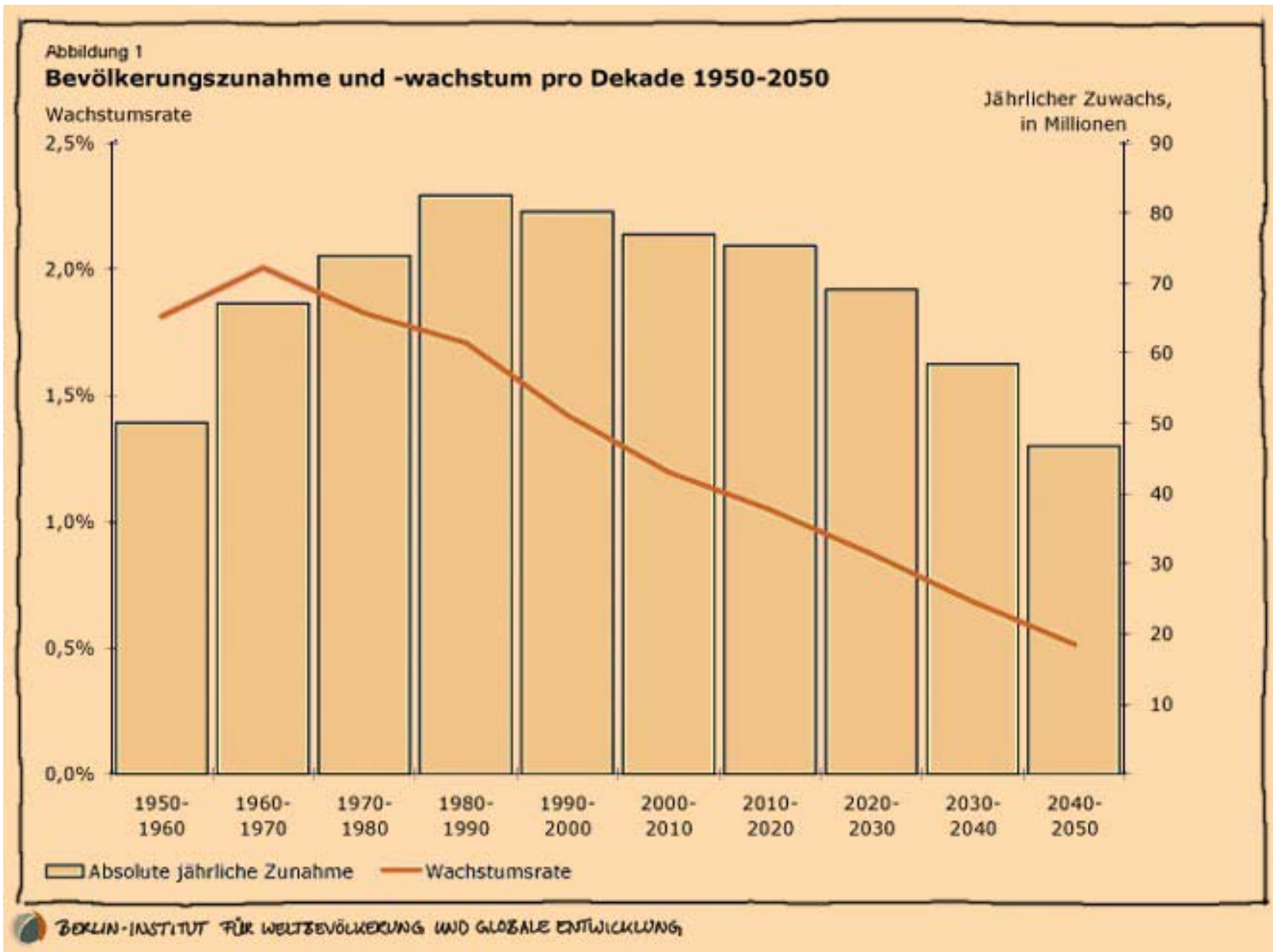

Quelle: Berlin-Institut für Weltbevölkerung und globale Entwicklung, http://www.berlin-institut.org/pages/fs/fs_grafiken.html

Die mittlere Schätzung in der 2004 Revision geht von 200 Millionen Erdenbürgern mehr im Jahr 2050 aus als die 2002 Revision, diese wiederum von 400 Milli- 
onen weniger als die 2000 Revision. Insgesamt schätzt man heute die weitere $\mathrm{Zu}$ nahme der Weltbevölkerung geringer ein als noch in den 1980er und 1990er Jahren. Dies ist zum einen auf eine Zunahme der prognostizierten Todesfälle, in erster Linie wegen HIV/AIDS, zurückzuführen, zum anderen ist der prognostizierte Rückgang an Geburten dafür verantwortlich. Die Bevölkerungsabteilung der UNO prognostizierte 2003 erstmals, dass in den meisten Entwicklungsländern die Gesamtfruchtbarkeitsrate, die bereits zwischen 1950 und 2000 von 6 auf 2,8 gesunken war, in der ersten Hälfte des 21. Jahrhunderts (2030-2035) unter das Bestandhaltungsniveau von 2,1 Kindern pro Frau sinken wird. Insgesamt hatten im Jahr 2000 rund 60 Länder und Territorien, die 44 Prozent der Weltbevölkerung ausmachen, Geburtenraten unter dem Bestandhaltungsniveau. ${ }^{200}$ Viele Demografen erwarten, dass auf dieser Liste bald auch Brasilien, Mexiko, Iran und sogar Indien stehen. ${ }^{201}$

Die Wachstumsrate der Weltbevölkerung beträgt derzeit 1,2 Prozent pro Jahr. Der jährliche Zuwachs an Menschen liegt heute bei 76 Millionen und wird auch in den nächsten Jahrzehnten zunächst nur langsam sinken (siehe Abb. 8).

Auf den ersten Blick erscheint es widersprüchlich, dass der jährliche Zuwachs an Menschen trotz gesunkener Geburtenraten heute höher ist als etwa 1960. Dies ist aber lediglich eine einfache Folge der Tatsache, dass demografische Änderungen stets mit erheblicher Zeitverzögerung sichtbar werden. Das rasante Wachstum der zweiten Hälfte des letzten Jahrhundert wirkt fort, da sich in Ländern mit einer jungen Altersstruktur (d.h. einer breiten Basis der Bevölkerungspyramide) die Zahl derer, die in das Alter der Fortpflanzungsfähigkeit eintreten, in Zukunft automatisch erhöhen wird. Wegen der dadurch wachsenden Zahl an potenziellen Müttern und Vätern erhöhen sich die absolute Geburtenzahl und die absolute Bevölkerungszahl in diesen Ländern auch dann, wenn die Kinderzahl je Frau in der Zukunft weiter sinkt. Im Jahr 1960 lebten drei Milliarden Menschen auf der Erde. Bei der damaligen Wachstumsrate der Weltbevölkerung von 2,2 Prozent kamen jährlich 66 Millionen Erdenbürger dazu. Heute vermehrt sich die Erdbevölkerung von knapp 6,4 Milliarden mit einer Wachstumsrate von 1,2 Prozent - also wächst die Weltbevölkerung trotz der fast halbierten Wachstumsrate heute mit 77 Mio. Menschen jährlich. Zum Bevölkerungswachstum tragen zwei Familien mit je drei Kindern nun einmal mehr bei als eine Familie mit fünf Kindern. Dieser Effekt ist der wichtigste Faktor beim weiteren Bevölkerungswachstum (vgl. Abb. 9).

${ }^{200}$ A.a.O., 5

${ }^{201}$ Hewitt (23.3.2004), 39 


\section{Abb. 9: Einfluss des Trägheitsfaktors auf das Bevölkerungwachstum in Entwicklungsländern}

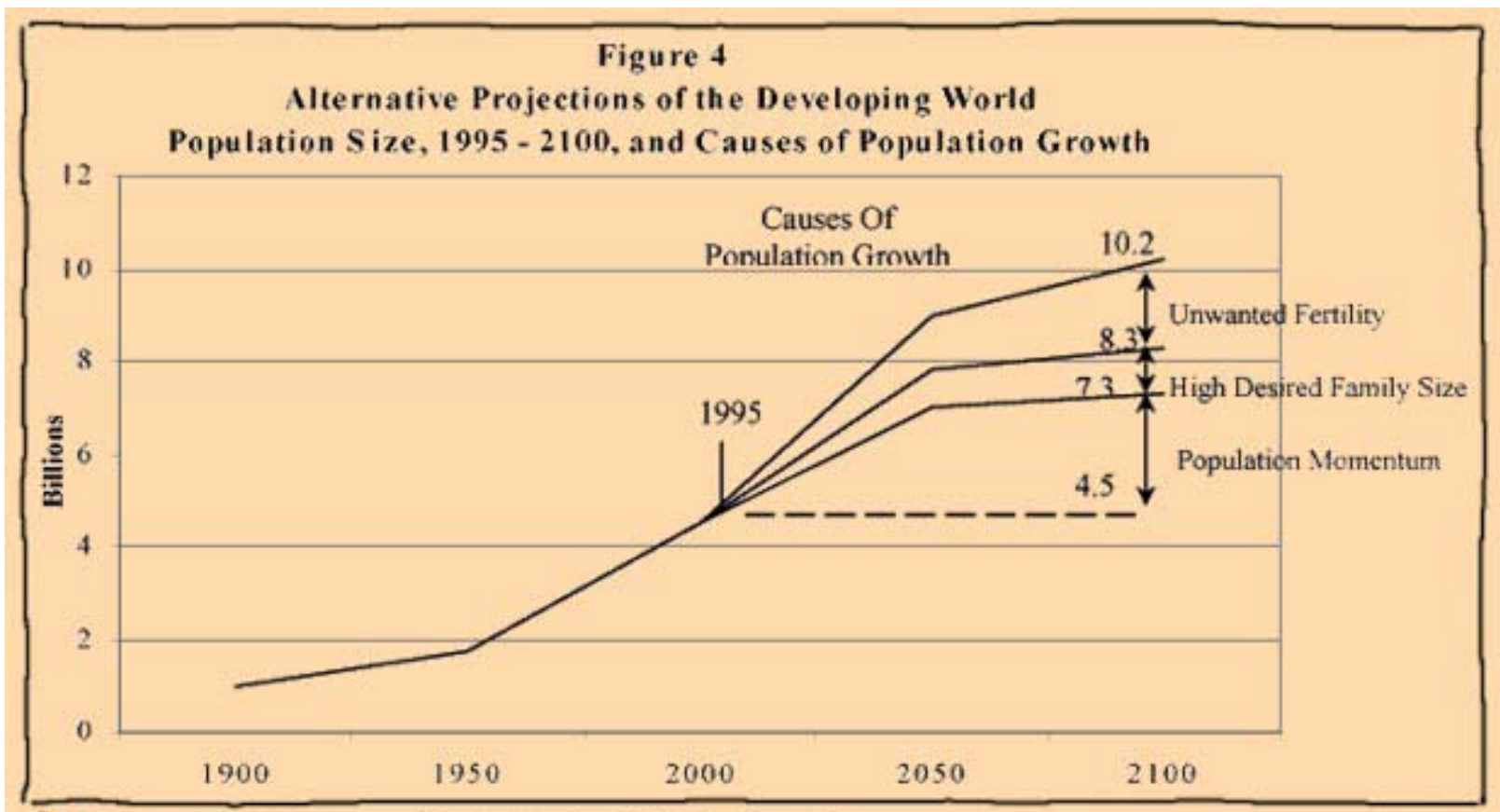

BOXUN-INSTITTT FÜR WEUTBEVÖKERUNG WNO GLOBALE ENTWICKLWNG

\section{Quelle: Berlin-Institut für Weltbevölkerung und globale Entwicklung, http://www.berlin-institut.org/pages/fs/fs_grafiken.html}

Unter der Annahme, dass die Kinderzahl je Frau innerhalb eines einzigen Jahres auf die für die langfristige Konstanz der Bevölkerung ausreichende Kinderzahl von 2,1 je Frau sinkt, lässt sich mit Simulationsmodellen die daraus resultierende Bevölkerungszahl in der Zukunft errechnen. Das Verhältnis dieser hypothetischen zur gegenwärtigen Bevölkerungszahl wird als Trägheitsfaktor oder ,PopulationMomentum' bezeichnet. ${ }^{202}$ Es wurde in seiner Wirkung von dem Bevölkerungswissenschaftler Herwig Birg mit einem Tanker verglichen, bei dem

\footnotetext{
202 Auch wenn das Ersatzniveau (Gesamtfruchtbarkeitsrate von 2,1) erreicht ist, setzt sich der vorangegangene Trend eine Zeitlang fort, d.h. die Bevölkerung wächst weiter, oder sie nimmt weiter ab. Das Ersatzniveau wird erst wirksam, wenn die vorangegangenen geburtenstarken bzw. -schwachen Jahrgänge das reproduktive Alter überschritten haben. Bis dahin ist jede Jahreskohorte, die in ein bestimmtes Alter eintritt, größer bzw. kleiner als die vorhergehende. Erst nach zwei bis drei Generationen, wenn sich die Zahl der Geburten und Sterbefälle dauerhaft ausgleicht, kommt die Bevölkerungsentwicklung ins Gleichgewicht (Deutsche Stiftung Weltbevölkerung 1999, 34).
} 
in voller Fahrt der Motor abgestellt wird. ${ }^{203}$ Auf hoher See wird er noch eine weite Strecke zurücklegen, bis er zum Stillstand kommt. ${ }^{204}$

\subsubsection{Alterung}

Durch die Abnahme der Fertilität und die Zunahme der Lebenserwartung kommt es zu einer erheblichen Alterung der Weltbevölkerung. Die Lebenserwartung wird nach den Prognosen der Population Division von 65 Jahren (2003) auf 74 Jahre (2045-2050) zunehmen, wobei aber große regionale Unterschiede bestehen bleiben. Während sie in den entwickelten Ländern von 76 auf 82 Jahre steigen wird, wird jemand in den Entwicklungsländern Mitte dieses Jahrhunderts im Durchschnitt 73 Jahre alt werden (2003: 63 Jahre). Die Zahl der Übersechzigjährigen wird sich nahezu verdreifachen und 2050 fast 2 Milliarden Menschen betragen. In den weiter entwickelten Ländern wird dann rund ein Drittel der Bevölkerung über 60 Jahre alt sein (2003: 19 Prozent). Diese Entwicklung schlägt sich auch im Medianalter nieder. ${ }^{205}$ Es stieg global zwischen 1950 und 2002 gerade mal um dreieinhalb Jahre, von 23,6 auf 27 Jahre. Der Grund ist das Übergewicht der jungen Gesellschaften der Entwicklungsländer. Während in jungen Gesellschaften wie z.B. Jordanien 2003 das Medianalter bei 18 Jahren liegt, liegt es in Europa heute bei 38 Jahren. Auch 2050 werden die Europäer (zusammen mit den Japanern) noch mit einem Medianalter von etwa 50 Jahren die Statistik anführen. Lateinamerikaner werden nach UNO-Berechnungen Mitte des Jahrhunderts 38 Jahre erreichen, soviel wie die Europäer heute. Afrikaner werden ihr Medianalter von 19 auf 31 Jahre steigern können. Weltweit wird bis 2050 das Medianalter von heute 27 Jahren auf 37 Jahre steigen. Der Alterungsprozess, den die SEL heute schon durchmachen, wird also die ganze Welt erfassen.

Anfangs wurde die durchschnittliche Lebenserwartung dadurch erhöht, dass die Säuglingssterblichkeit gesenkt wurde. Die große Überraschung ist, dass sie auch nach Abschluss dieser Entwicklung weiter steigt, weil die Menschen am Ende der Lebensspanne kontinuierlich Jahre hinzugewinnen. In den entwickelten Ländern nimmt die Lebenserwartung Jahr für Jahr um etwa 2,5 Monate zu. ${ }^{206}$ In den WEL vollzieht sich die Zunahme der Lebenserwartung sehr viel rascher. Die Lebenserwartung wuchs dort in der zweiten Hälfte des 20. Jahrhunderts jährlich um 5,3

\footnotetext{
${ }^{203} \operatorname{Birg}(1994), 13$

${ }^{204}$ Das ,Population Momentum' gilt natürlich auch für schrumpfende Gesellschaften. Nicht vollständig ersetzte Generationen geben eine geringere Basis ab, die überhaupt die Chance hat, Nachkommen in die Welt zu setzen.

${ }^{205}$ Das Medianalter ist dasjenige Alter, das von je genau der Hälfte der Bevölkerung unter- und überschritten wird.

${ }^{206}$ Hewitt (23.3.2004), 39
} 
Monate. In China schoß sie zwischen 1950 und 1970 um erstaunliche 20 Jahre nach oben.

Diese Zunahme der durchschnittlichen Lebenserwartung ist evolutionsgeschichtlich mehr als außergewöhnlich. Anhand archäologischer Skelettfunde ermittelte man, dass sie bis vor 10.000 Jahren bei ca. 20-25 Jahren lag. ${ }^{207}$ Bis ins 18. Jahrhundert betrug sie im Durchschnitt relativ gleichbleibend 30-35 Jahre - und hat sich somit überraschenderweise kaum gegenüber der Frühgeschichte erhöht. Erst mit Beginn des 20. Jahrhunderts nahm die Lebenserwartung vergleichsweise rasch zu.

Es wird kontrovers diskutiert, ob es überhaupt biologische Grenzen für eine weitere Zunahme der Lebenserwartung gibt. Der Leiter des Max-Planck-Instituts für demografische Forschung in Rostock, James Vaupel, wirft dem Statistischen Bundesamt vor, es gehe in seinen Bevölkerungsvorausberechnungen von einem zu niedrigen Zuwachs der Lebenserwartung aus. ${ }^{208}$ Vaupel argumentiert, dass seit 1840 die höchste Lebenserwartung, die in einem Land gemessen wurde, kontinuierlich um 2,5 Jahre pro Jahrzehnt (also 3 Monate pro Jahr) gestiegen ist. Legt man diesen Trend zu Grunde, so würde die mittlere Lebenserwartung im Jahr 2050 in Deutschland bei 90 Jahren liegen. Die zehnte Bevölkerungsvorausberechnung des Statistischen Bundesamtes geht aber davon aus, dass die Lebenserwartung (kombiniert für Frauen und Männer) nur auf 84 Jahre steigen wird.

\subsubsection{Regionale Verteilung der Bevölkerung in Nord und Süd}

Das rasante Bevölkerungswachstum ist ungleich verteilt (vgl. Abb. 10). Die Bevölkerung der SEL wächst laut der 2002 Revision mit einer Wachstumsrate von 0,25 Prozent pro Jahr, das Wachstum der Entwicklungsländer beträgt 1,46 Prozent, wobei die am wenigsten entwickelten Länder sogar noch schneller wachsen $(2,4$ Prozent pro Jahr). Afrika, der Kontinent mit der höchsten Wachstumsrate, wird seine Bevölkerung bis 2050 mehr als verdoppeln - von 800 Millionen auf 2,3 Milliarden. 98 Prozent des jährlichen Zuwachses an Menschen entfällt schon heute auf die WEL, ${ }^{209}$ daran wird sich auch in den nächsten Jahrzehnten nichts ändern. In Europa wurde 1998 ein historischer Wendepunkt erreicht: Die Bevölkerung hörte bei 728 Millionen Menschen auf zu wachsen zum ersten Mal seit Jahrhunderten.

\footnotetext{
${ }^{207}$ Schimany (2003), 104

${ }^{208}$ Vaupel (8.4.2004); siehe ausführlich Oeppen/Vaupel (2002)

${ }^{209}$ Schimany (2003), 14
} 


\section{Abb. 10: Regionale Verteilung der Bevölkerung 1950-2050}

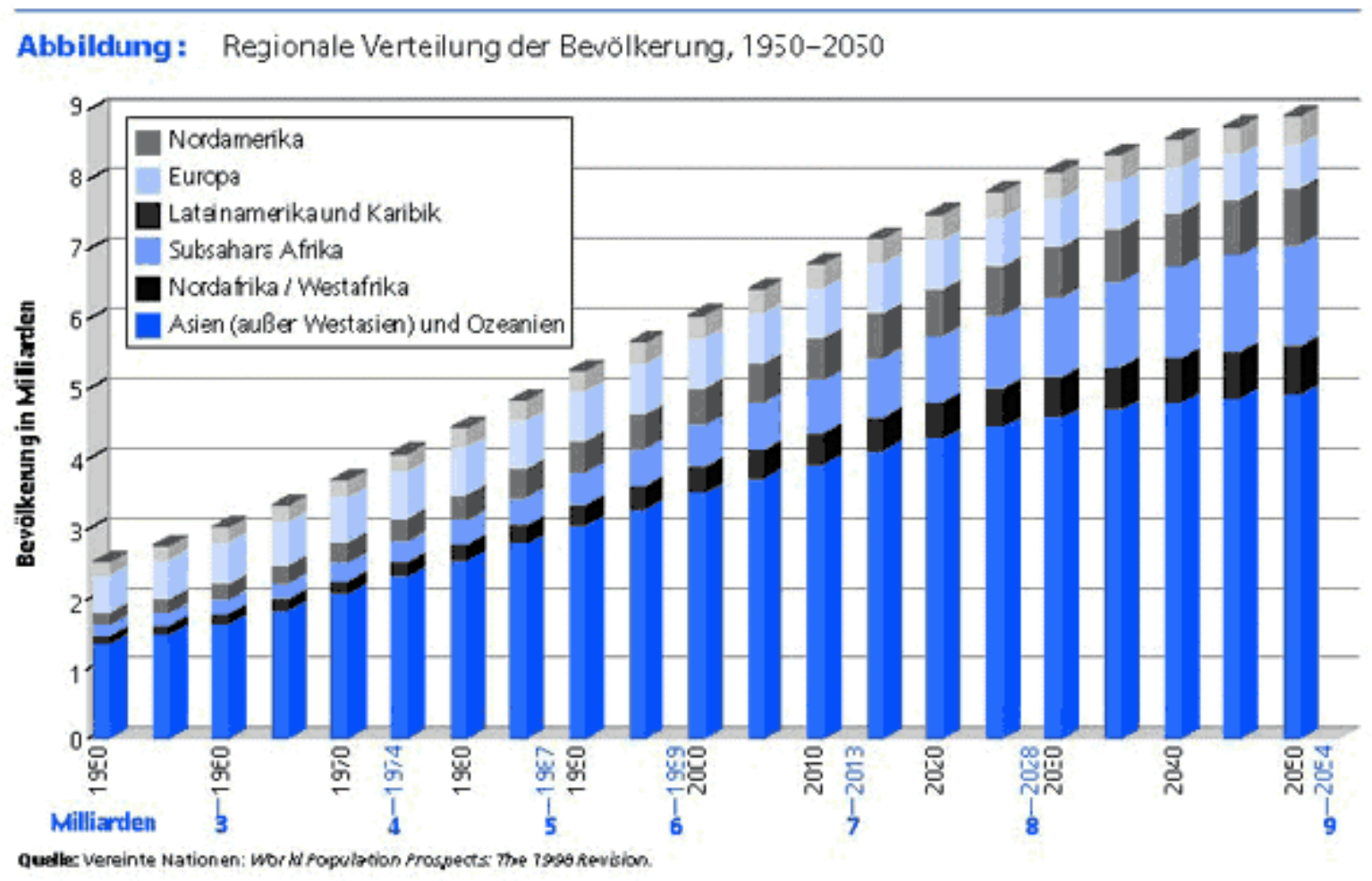

Quelle: UN Population Division, http://www.un.org/esa/population/publications.htm

Es gibt einen Trend zur Verringerung des demografischen Gewichts der heute entwickelten Staaten bei zunehmendem Anteil Afrikas und anderer Regionen der heutigen Entwicklungsländer. Die Ursachen für diese regionale Verteilung des Weltbevölkerungswachstums sind sicher komplex und multikausal. Dennoch deutet die Tatsache, dass dieses zuvor ungekannte Wachstum in fast allen Regionen der WEL zeitgleich einsetzte, auf die Dominanz eines ursächlichen Faktors hin. ${ }^{210}$ Die Schlüsselrolle scheint dabei dem von der modernen Medizin hervorgebrachten Rückgang der Sterblichkeit zuzukommen. Gemäß der Grundannahme des Modells des demografischen Übergangs bleibt die Bevölkerung auf einem niedrigen Niveau, wenn Geburtenrate und Sterberate hoch sind. Wenn dann die Säuglingssterblichkeit sinkt (bzw. die Lebenserwartung ansteigt), wächst die Bevölkerung zunächst stark an, bis sich die geöffnete Schere von hoher Geburtenrate und niedriger Sterberate wieder schließt, indem sich das Geburtenniveau der gesunkenen Sterblichkeit angleicht. ${ }^{211}$ In den letzten Jahren hat sich in den Entwick-

\footnotetext{
${ }^{210}$ Schockenhoff (1996), 13

${ }^{211}$ A.a.O. Zur klassischen Theorie des demografischen Wandels siehe schon Mackenroth (1972), 29-33
} 
lungsländern der Rückgang der Geburtenraten beschleunigt und mehr und mehr Entwicklungsländer scheinen den Abschluss der demografischen Transformation erreicht zu haben. Es sind heute nicht mehr die Entwicklungsländer insgesamt, deren Bevölkerungswachstum ,explodiert', sondern vielmehr wachsen nur noch bestimmte Regionen wie Schwarzafrika, der indische Subkontinent und die meisten islamischen Länder exponentiell.

\subsubsection{Bevölkerungsprojektionen bis 2300}

Anfang Dezember 2003 veröffentlichte die Bevölkerungsabteilung der Vereinten Nationen (UN Population Division) eine neue Langzeit-Bevölkerungsprojektion. ${ }^{212}$ Diese Publikation setzt eine Serie von sieben vergleichbaren Studien seit Mitte der 1970er Jahre fort. Sie erweitert den bisherigen Zeithorizont um 150 Jahre bis 2300. Es sollen die langfristigen Implikationen einer Fortsetzung von demografischen Tendenzen der letzten Jahrzehnte illustriert werden. In dieser Langzeitprojektion wurden erstmals auch die Werte für einzelne Nationalstaaten ausgewiesen. Der Ersatz einer Elterngeneration durch eine gleich große Kindergeneration und damit eine langfristige demografische Stabilität wird wie erwähnt mit einer durchschnittlichen Zahl von ca. 2,1 Kindern je Frau erreicht. Dieses Ersatzniveau der Fertilität übte über Jahrzehnte eine fast normative Kraft auf die Fertilitätsannahmen der UN-Bevölkerungsabteilung aus. Für die Entwicklungsländer brachte der sich immer weiter ausbreitende Fertilitätsrückgang scheinbar einen empirischen Beleg für das Sinken auf das Ersatzniveau der Fertilität. In den 1990er Jahren wurde jedoch deutlich, dass der Fertilitätsrückgang in China, Thailand, Südkorea und anderen Entwicklungsländern keineswegs bei 2,1 Kindern je Frau enden würde. Seit Jahrzehnten liegt in zahlreichen westeuropäischen Staaten die Gesamtfruchtbarkeitsrate unter diesem Wert. Die UN-Bevölkerungsprojektionen der 1970er, 1980er und frühen 1990er Jahre unterstellten beharrlich, dass die Kinderzahlen bereits wenige Jahre nach der Veröffentlichung der jeweiligen Prognosen wieder auf das Ersatzniveau steigen würden.

Das mittlere Szenario der aktuellen langfristigen Projektionen geht immer noch von einer Annahme der zukünftigen Annäherung aller Nationen an das Ersatzniveau der Fertilität als tragendes Element aus. Dieses Szenario unterstellt, dass die Fertilität auch in Entwicklungsländern unter das Ersatzniveau sinken werde. Die Fertilität soll jedoch in keinem Land länger als 100 Jahre unter dem Ersatzniveau verbleiben, sondern dann wieder auf 2,1 Kinder je Frau ansteigen. In diesem Fall würde die Weltbevölkerung Mitte des 21. Jahrhunderts 8,9 Mrd. Menschen erreichen und danach nur noch geringfügig auf ein Maximum von 9,2 Mrd. im Jahr 2075 wachsen. Danach käme es zu einem geringfügigen Rückgang auf 8,4 Mrd. im Jahr 2200 und zu einem erneuten leichten Anstieg auf knapp 9 Mrd. in 2300.

${ }^{212}$ Quelle dieses Abschnitts ist UN Population Division (2003b). 
Die Autoren unterstreichen mit der Auswahl der anderen Szenarien die Tatsache, dass bereits geringfügige Abweichungen im angenommenen langfristigen Niveau der Fertilität gravierende Wirkungen auf die zukünftige Größe der Weltbevölkerung hätten. Eine Anpassung der Fertilität von 0,25 Kindern über dem Ersatzniveau wurde im hohen Szenario angenommen. Eine Weltbevölkerung von 36,4 Mrd. wäre im Jahr 2300 die Folge. Läge die langfristige Fertilität um 0,25 Kindern unter dem Ersatzniveau (niedriges Szenario) würde die Weltbevölkerung bis 2300 auf nur 2,3 Mrd. schrumpfen, den Stand von 1940. Wie das Szenario ,konstantes Wachstum' in Abb. 8 zeigt, sind die heutigen Geburtenraten nicht bis 2300 in die Zukunft extrapolierbar - die Ergebnisse werden zu unrealistisch. ${ }^{213}$

\section{Abb. 11: Bevölkerungsprojektionen Welt 2000-2300}

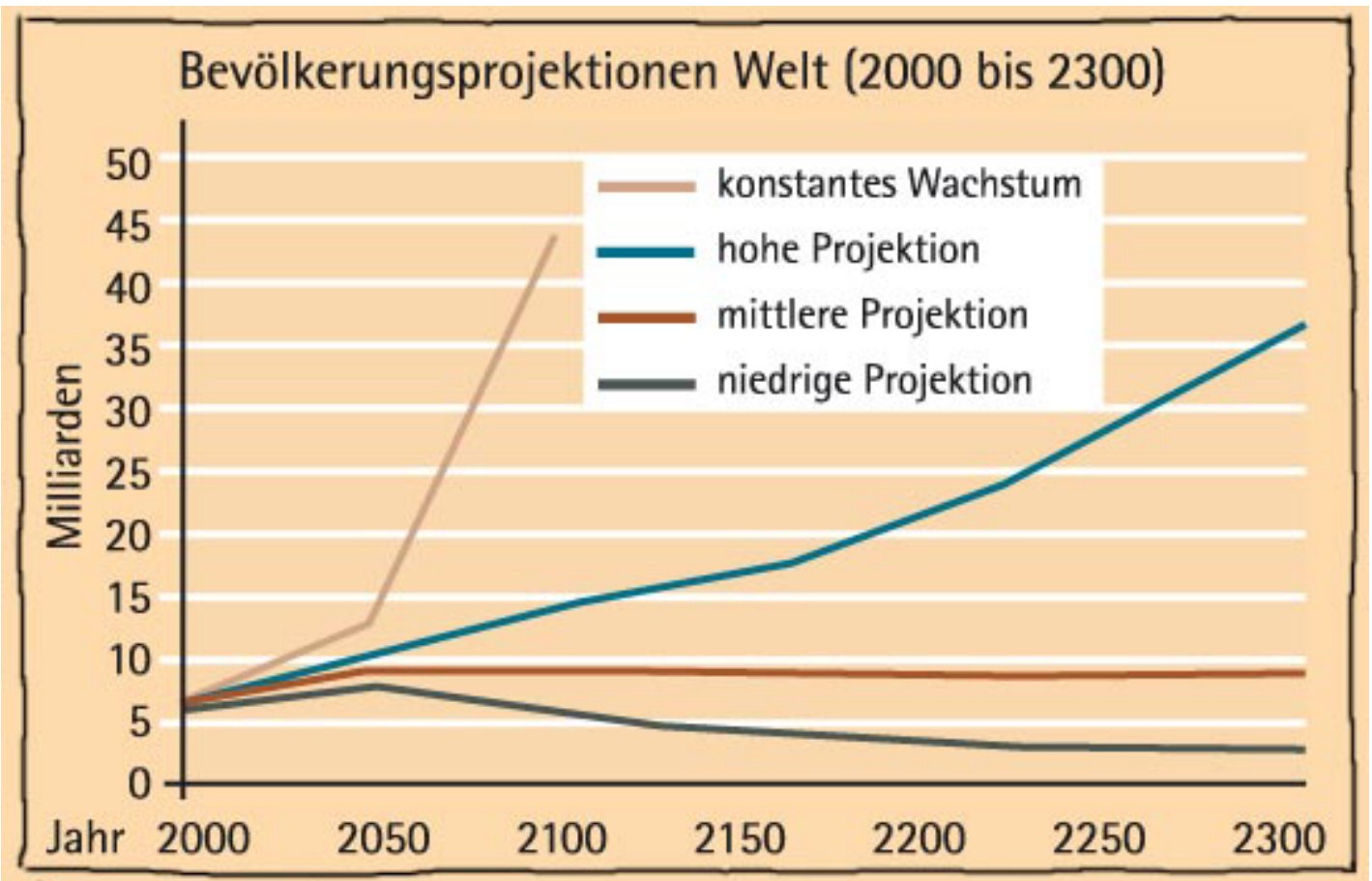

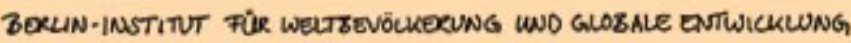

\section{Quelle: Berlin-Institut für Weltbevölkerung und globale Entwicklung, http://www.berlin-institut.org/pages/fs/fs_grafiken.html}

Die regionalen Unterschiede bei der Bevölkerungsentwicklung werden sich bei dieser Langzeitprognose noch weiter vertiefen. Die Bevölkerung der europäischen Länder wird, wenn die Prämissen der Population Division zutreffen, bis 2300 von heute 728 Millionen auf etwa 611 Millionen Menschen sinken. Die Bevölkerungszahlen in Deutschland (heute: 82 Millionen) werden nach einem Rückgang in den nächsten 100 Jahren (73 Millionen in 2100) im Jahre 2300 auf etwa das

${ }^{213}$ Siehe Abschnitt 6.4. Projektionen sind keine Prognosen. 
heutige Niveau zurückkehren. Die Bevölkerungszahlen in Afrika werden sich bis 2300 fast verdreifachen: von heute 796 Millionen auf 2,1 Milliarden Menschen. Die Bevölkerung Ugandas wird sich im selben Zeitraum sogar von 23 Millionen auf 155 Millionen Menschen mehr als versechsfachen. Afrikas Anteil an der Weltbevölkerung wird sich nach der mittleren Variante von heute 13 Prozent auf 24 Prozent im Jahre 2300 fast verdoppeln. Der Anteil Europas schrumpft hingegen um etwa die Hälfte - von zwölf auf sieben Prozent. 


\section{Ist Bevölkerung eine Ursache für Umweltbelastungen?}

\subsection{Vorbemerkungen}

Um die Auswirkungen menschlichen Handelns auf seine Umwelt ins rechte Verhältnis zu setzen, kann man die 4,55 Milliarden Jahre Erdgeschichte auf die Länge eines Jahres projizieren. Nimmt man an, dass am 1. Januar die Erde entstanden sei, so würden am 27. Februar die ersten Lebewesen auftauchen, aber erst am 1. September hätten sich die ersten Einzeller mit echtem Zellkern (Eukaryonten) entwickelt. Fische, die ersten Wirbeltiere, gäbe es seit dem 21. November, Säugetiere ab dem 12. Dezember. Um 19.30 des 31.12. erschien der älteste bekannte Ahne der Menschheitsfamilie, der erste ,anthropos'. Erst um 23.57 zeigt sich der Homo Sapiens und besiedelt in weniger als einer Minute alle Kontinente. Zwei Sekunden vor Mitternacht endet das Mittelalter, vor eineinhalb Sekunden ereignet sich die Industrielle Revolution. Die Moderne dauert noch nicht mal eine einzige Sekunde an. ${ }^{214}$

Die heutige Umweltkrise ist ohne Beispiel in der Menschheitsgeschichte. Während alle Umweltkrisen der Vergangenheit Regionalkrisen waren, ist sie global. Luft- und Wasserschadstoffe sind bis in die letzten Winkel der Erde nachzuweisen, die Reduzierung der tropischen Regenwälder erfolgt weltweit in einem atemberaubenden Tempo und die Belastung der Atmosphäre mit Treibhausgasen verändert das Klima auf der ganzen Welt. ${ }^{215}$

Der Wettlauf zwischen Bevölkerungsvermehrung und Steigerungen der Lebensmittelproduktion zwingt zu immer mehr durchgreifenden und zügigen Veränderungen in der Umwelt: erforderlich sind der umfassende und effizienzsteigernde Einsatz von neuen Techniken und ein intensiver Eingriff in verbliebene Naturflächen. Dies geschieht nicht nur im globalen Rahmen, es vollzieht sich auch alles mit wesentlich höherer Geschwindigkeit als früher.

Kennzeichen der heutigen Lage sind also das anhaltende Bevölkerungswachstum, Globalität der Auswirkungen unserer Aktivitäten sowie die Geschwindigkeit der Veränderungen. ${ }^{216}$

Dennis Meadows zieht anlässlich der im Frühjahr 2004 erschienenen Neuauflage seines epochemachenden Buches Die Grenzen des Wachstums Bilanz: „Die meisten Naturwissenschaftler sind davon überzeugt, dass die Menschheit das Klima ändert. Viele Fischbestände sind bereits verschwunden. Die Wälder, die Binnengewässer, der fruchtbare Boden - auf sämtlichen Kontinenten der Erde wird all das heruntergewirtschaftet. (...) Wir haben 30 Jahre verloren.“'”217

\footnotetext{
${ }^{214}$ Nach Gorke (2000), 103; siehe auch Zahrnt (1993), 115.

${ }^{215}$ Kreibich/Weizsäcker/Renn/Radermacher/Borchert/Thunert (2003), 12. Siehe vertiefend auch Knaus/Renn (1998), 37-43

${ }^{216}$ A.a.O., 13

${ }^{217}$ Meadows (2004), 20
} 
Im folgenden Abschnitt wird der Zusammenhang zwischen Bevölkerung und Umweltproblemen untersucht. Dabei konzentriere ich mich auf das weltweite Problem des Verlustes an Biodiversität, da sich an ihm der Zusammenhang am deutlichsten manifestiert. ${ }^{218}$ Anschließend wird die Theorie dieser Beziehungen dargestellt: Tragfähigkeitstheoreme, PAT-Formel und Environmental-KuznetsCurve werden diskutiert und beurteilt.

\subsection{Verlust der Biodiversität}

\subsubsection{Drei Phasen anthropogen verursachten Artensterbens}

Seit es Menschen gibt, haben sie mit anderen Arten um Lebensraum konkurriert, haben sie Tiere und Pflanzen ausgerottet und verdrängt. Man kann mehrere anthropogene Ausrottungsphasen unterscheiden. Als die Menschen von Afrika aus die anderen Kontinente besiedelten, vor 50.000 bis 10.000 Jahren, führten ihre Aktivitäten nach Ansicht einiger Paläontologen zum Aussterben von 200 großen Tierarten. ${ }^{219}$ Ihre Fossilien datieren auf die Epochen, als Menschen die entsprechenden Kontinente besiedelten (Australien und Europa: vor ca. 50.000 Jahren, Sibirien vor ca. 20.000 Jahren, Nordamerika vor ca. 11.000 Jahren). Durch ihre Bejagung und den Eingriff in ihre Ökosysteme beraubten diese frühen Menschen die ihnen nachfolgenden Generationen um Arten wie die Glyptodonten (Riesen-Gürteltiere) und das Mammut.

In einer zweiten Phase, vor 1.500 bis 500 Jahren, besiedelten die Menschen auch die entlegensten Inseln in den Ozeanen. Dort trafen sie auf einzigartige endemische Artenwelten, die sich über Jahrmillionen herangebildet hatten, und bejagten sie so gnadenlos, dass sie ausstarben. Lange bevor westliche Entdecker wie James Cook 1769 Neuseeland entdeckten, hatten z.B. die Maori zahlreiche Großvogelarten dort ausgerottet. ${ }^{220}$ Insgesamt starben in dieser zweiten Phase des Artensterbens allein in Polynesien wohl über 1.000 Inselvogelarten. ${ }^{221}$

Diese beiden ersten Phasen anthropogen verursachten Artensterbens unterscheiden sich grundsätzlich von der dritten, der heutigen: Niemals zuvor hat der Homo Sapiens so viele und so unterschiedliche Arten in so vielen Gegenden der Welt ausgerottet oder an den Rand des Aussterbens getrieben. Heute gehen Arten aller evolutionären Formen und Größen verloren, von den größten Bäumen bis hin zu den winzigsten Bodenmikroben. Dazu kommt noch, dass die meisten Pflanzenund Tierarten, die heute vom Aussterben bedroht sind, Tausende von Jahren vom Menschen bejagt bzw. abgeerntet wurden, ohne jemals in ihrem Bestand gefähr-

\footnotetext{
218 Andere Facetten der so genannten Umweltkrise, die in Wirklichkeit ein Konglomerat sehr unterschiedlicher Problemfelder ist, sind u.a. der Klimawandel, die Abfallproblematik, die Übernutzung von Ressourcen, Wasser und Land und der Aufbau von Gefahrenquellen.

${ }^{219}$ Martin (1984). Diese These ist aber nicht unumstritten. Andere Forscher machen Klimaveränderungen für dieses frühe Artensterben verantwortlich.

${ }^{220} \mathrm{Cincotta} /$ Engelman (2001), 47

${ }^{221} \mathrm{Pimm} /$ Russell/Gittleman/Brooks (1995)
} 
det zu sein. Erst in der Gegenwart wurden aus solchen ,ko-existierenden Arten' gefährdete Arten. ${ }^{222}$ Zudem hat sich die Geschwindigkeit erhöht, mit der sich das vom Menschen verursachte Artensterben abspielt: Während die Mammuts im Pleistozän mehrere Jahrtausende und die Inselvogelarten im Holozän immerhin Jahrhunderte der Jagd und sonstiger menschlicher Eingriffe überstanden, werden heute stabile Arten innerhalb von wenigen Jahrzehnten ausgerottet oder an den Rand der Ausrottung getrieben. ${ }^{223}$

Wie bei den meisten Umweltfragen variieren die Schätzungen, wie groß der vom Menschen verursachte Schaden tatsächlich ist. ${ }^{224}$ Gegenwärtig sind etwa 1,5 Millionen Arten wissenschaftlich beschrieben. ${ }^{225}$ Monatlich werden neue Arten entdeckt, insbesondere in den Tropen und der Tiefsee. Doch inzwischen sind die Zuwächse an Taxa zumindest bei den Wirbeltieren eher modernen biochemischen und genetischen Erkenntnissen zu verdanken als klassischen taxonomischen Methoden. $\mathrm{Zu}$ den angewandten Methoden sollen beispielhaft Vögel, die zu den besterforschtesten Arten überhaupt gehören, betrachtet werden. Das internationale Artenregister verzeichnet ungefähr 10.000 gesicherte Vogelarten und große $\mathrm{Zu}-$ wächse waren eigentlich nicht mehr zu erwarten. ${ }^{226}$ Seit kurzem gibt es jedoch eine Diskussion unter Ornithologen, ob nicht in Wirklichkeit viel mehr Arten existieren. Es gibt Vogelpopulationen, die sich zwar hinsichtlich der traditionell zur taxonomischen Klassifizierung verwendeten anatomischen Eigenschaften wie Größe, Gefieder und Schnabelform nicht wesentlich unterscheiden, im Hinblick auf andere wie bevorzugter Lebensraum oder Paarungsruf aber äußerst verschieden sind. Geht man davon aus, dass Populationen verschiedenen Arten angehören, wenn sie sich unter natürlichen Lebensbedingungen nicht miteinander paaren, so gibt es weit mehr Arten, als mit dem klassisch-anatomischen Methodenspektrum feststellbar.

Je nach verwendeter Methode schätzt man, dass es zwischen 3,6 und 100 Millionen Arten gibt, ${ }^{227}$ wobei die meisten Schätzungen von zwischen 7 und 15 Millionen Arten ausgehen. ${ }^{228}$ Der renommierte Biologe Edward O. Wilson warnt aber: „Der Mittelwert dieser Schätzungen liegt bei etwas über 10 Millionen, doch nur wenige Experten würden ihren wissenschaftlichen Ruf aufs spielen setzen und

\footnotetext{
${ }^{222}$ Allerdings gibt es auch Arten, die im Zuge der rasanten Eroberung des Planeten durch den Menschen zu Gewinnern wurden. Dazu zählen die domestizierten Pflanzen und Tiere. Das Wachstum des Nutztierbestandes hält mit dem Wachstum der Weltbevölkerung Schritt. Die weltweite Zunahme des frei verfügbaren Einkommens wird die Populationen dieser kleinen Minderheit von Arten (und ihr Abfallaufkommen) voraussichtlich weiter ansteigen lassen - auf Kosten von wildlebenden Arten.

${ }^{223} \mathrm{Cincotta} /$ Engelman (2001), 49

${ }^{224}$ Für einen Überblick siehe Gibbs (2001).

${ }^{225}$ Müller (2003), 197. Wilson (2004), 36

${ }^{226}$ Vertiefend zu diesem Beispiel (2004), 42

${ }^{227}$ Wilson (2004), 36

${ }^{228}$ Cincotta/Engelmann (2001), 6. Der Botaniker Peter H. Raven von der Universität Missouri schätzt 810 Millionen (Raven 1994), der Biologe Edward O. Wilson aus Harvard schätzt 10 Millionen allein in den Regenwäldern (Wilson 1992, 280).
} 
sich auf diese oder eine andere Zahl festlegen. Die Wahrheit ist, dass wir mit der Erforschung des Lebens auf der Erde eben erst begonnen haben. ${ }^{\text {" } 229}$ Basierend auf einer Annahme von 10 Millionen Arten hat der britische Ökologe Stuart Pimm anhand der Verlustraten der am besten untersuchten Tierfamilien berechnet, dass die gegenwärtige Rate der Artenausrottungen durch den Menschen auf das 100bis 1.000fache der ,natürlichen Hintergrundrate', also der Rate, mit der Arten auch ohne Existenz des Menschen aussterben und neu entstehen würden, gestiegen ist. ${ }^{230}$ Wilson schätzt, dass pro Jahr zwischen 0,1 und einem Prozent aller Arten aussterben, mindestens 27.000 pro Jahr. ${ }^{231}$ Fast alle Wissenschaftler sind sich einig, dass Entwarnung nicht in Sicht ist. Ganz im Gegenteil zeigen Computermodelle, dass sich in den nächsten Jahrzehnten das Artensterben noch einmal beschleunigen dürfte. Was die Evolution in Jahrmillionen erschaffen hat, wird in wenigen Bruchteilen davon zerstört. „Die Vernichtung der Artenvielfalt könnte dasjenige Vergehen sein, welches uns künftige Generationen am wenigsten vergeben werden", fürchtet Wilson. ${ }^{232}$ Anzeichen für eine Stabilisierung der Weltbevölkerung zitierend, schreibt er an anderer Stelle zum Artensterben: „Ich nenne das einen Flaschenhals, denn wir müssen durch die Trümmer der verbleibenden Ressourcen hindurch zu einer neuen Ära gelangen, in der die Bevölkerung wieder sinkt, vielleicht im 22. Jahrhundert. Unser Ziel muss sein, so viel wie möglich von der Artenvielfalt da durch zu bringen." ${ }^{233}$

Die große Einigkeit der Biologen über das Bevölkerungswachstum als treibende Kraft für das Artensterben ist bezeichnend. Dennoch ist klar, dass angesichts der großen Unsicherheiten über die Zahl der überhaupt existierenden Arten auch die Schätzungen über die Zahl der ausgestorbenen Arten relativ spekulativ sein müssen. Das Problem ist ein grundsätzliches: Wie soll man das Verschwinden einer Art wissenschaftlich zweifelsfrei belegen? Selbst wenn kein Vertreter der Art seit Jahrhunderten mehr gesichtet wurde, ist es nicht verifizierbar, dass wirklich nicht irgendwo noch ein paar Exemplare überlebt haben. Naturschutzpolitiker bzw. gesellschaftspolitisch aktive Biologen und Ökologen werden von ihren Gegnern gelegentlich harsch kritisiert oder gar lächerlich gemacht, wenn eine ,ausgestorbene' Art sich plötzlich wieder blicken lässt. ${ }^{234}$ Die Taxonomie behilft sich deswegen zunehmend mit Kategorien wie, verschollen', selbst wenn die Wahrscheinlichkeit sehr hoch ist, dass diese Tiere, ausgestorben' sind.

\footnotetext{
${ }^{229}$ Wilson (2004), 37

${ }^{230} \mathrm{Pimm} /$ Russell/Gittleman/Brooks (1995). Die ,natürliche Hintergrundrate' liegt bei ein bis zehn Arten pro Jahr.

${ }^{231}$ Wilson (1992), 280. Basierend auf unterschiedlichen Annahmen über die Zahl existierender Arten konstatiert Cohen, dass die Ausrottungsrate zwischen 0,5 Prozent und 40 Prozent pro Jahrzehnt liegen muss (Cohen 1995, 337).

${ }^{232}$ Wilson (1984), 121

${ }^{233}$ Gibbs (2001), 49

${ }^{234}$ Tendenzen in diese Richtung weist auch der Artikel von Müller (2003), 199 auf.
} 
Um die grundsätzlichen Probleme bei der Ermittlung der Zahl aussterbender Tierund Pflanzenarten in den Griff zu bekommen, behilft man sich mit der empirisch einfacheren Messung der Zerstörung von Habitaten. Dem liegt eine überzeugende Hypothese zu Grunde: Wenn ihre Lebensräume zerstört werden, dann verschwinden auch die Arten, die in ihnen zu Hause sind.

\subsubsection{Habitatsverlust als Indikator für Artenverlust}

Das Artensterben ist nicht monokausal erklärbar. $\mathrm{Zu}$ den wichtigsten Gründen gehören 1.) Lebensraumverlust, 2.) direkte Verfolgung, 3.) Übernutzung der Bestände, 4.) Einschleppung von Exoten, 5.) chemische Belastung, 6.) Massentourismus, 7.) Treibhauseffekt und 8.) intensive Landwirtschaft. ${ }^{235}$ Diese Gründe sind aber nicht gleichgewichtig für den Artentod verantwortlich. Viel seltener als früher sterben heute Arten wegen zu intensiver Bejagung aus, verantwortlich ist vielmehr nach übereinstimmender Ansicht der Biologen in der Mehrzahl der Fälle die Zerstörung ihres Lebensraumes. ${ }^{236}$ Das englische Akronym HIPO listet die schädlichen Faktoren in der Reihenfolge ihrer Wichtigkeit auf: ${ }^{237}$

- Habitatzerstörung (Habitat destruction). Die Wälder Hawaiis bsp. Wurden zu 75 Prozent abgeholzt, was unvermeidlich zur Dezimierung und Ausrottung vieler Arten führte.

- Invasive Arten (Invasive species). Ameisen, Schweine und andere fremde Arten verdrängten die natürliche Tier- und Pflanzenwelt Hawaiis.

- Umweltverschmutzung (Pollution). Durch die Verschmutzung des Süsswassers, der Böden und der flachen Küstengewässer werden weitere Arten geschwächt und dezimiert.

- Übernutzung (Overharvesting). Manche Arten, v.a. Vögel, wurden schon während der polynesischen Besiedelung durch übermäßige Jagd stark dezimiert oder gar ausgerottet.

Durch das Bevölkerungswachstum verbrauchen mehr Menschen mehr Land. Da die tropischen Wälder einen Großteil der noch erhaltenen Arten der Erde beheimaten, fällt ihr Verlust besonders stark ins Gewicht. ${ }^{238}$ Der Zusammenhang zwischen dem Verlust von Arten und Ökogebieten wird durch die schon in den 1960er Jahren entwickelte und vielfach empirisch belegte Arten/Areal-Beziehung modelliert. ${ }^{239}$ Wird ein Habitat, z.B. eine Insel von Regenwald, zerstört, so sterben

\footnotetext{
${ }^{235}$ Gorke (1999), 13

${ }^{236}$ Wilson (2004), 74; Pimm/Raven (2000), 843.

${ }^{237}$ Normalerweise werden die Faktoren, die zu Artensterben führen können, als HIPPO-Formel bezeichnet, wobei das weitere P für Population steht (vgl. Wilson 2004, 74). Bevölkerung ist aber nicht bloss ein weiterer Faktor, sondern - in den Worten Wilsons - „die Triebfeder für alle anderen schädlichen Faktoren“ (a.a.O.). Nur um gängige Akronyme zu erhalten (Hippo = griech.: Pferd), sollten keine inhaltlichen Kompromisse eingegangen werden.

${ }^{238}$ UNFPA (2001), 7. Wilson (1992)

${ }^{239}$ Gibbs (2001), 46; Cincotta/Engelmann (2001), 32
} 
zwar die darin heimischen Arten aus, aber nicht linear. Eine 50prozentige Verkleinerung des Lebensraums bedeutet nur für 10 bis 20 Prozent der Arten das Aus. Selbst wenn 90 Prozent des Ursprungshabitats zerstört sind, bleiben noch 55 bis 30 Prozent der Arten erhalten.

\section{Abb. 12: Artenzahl und Habitatfläche}

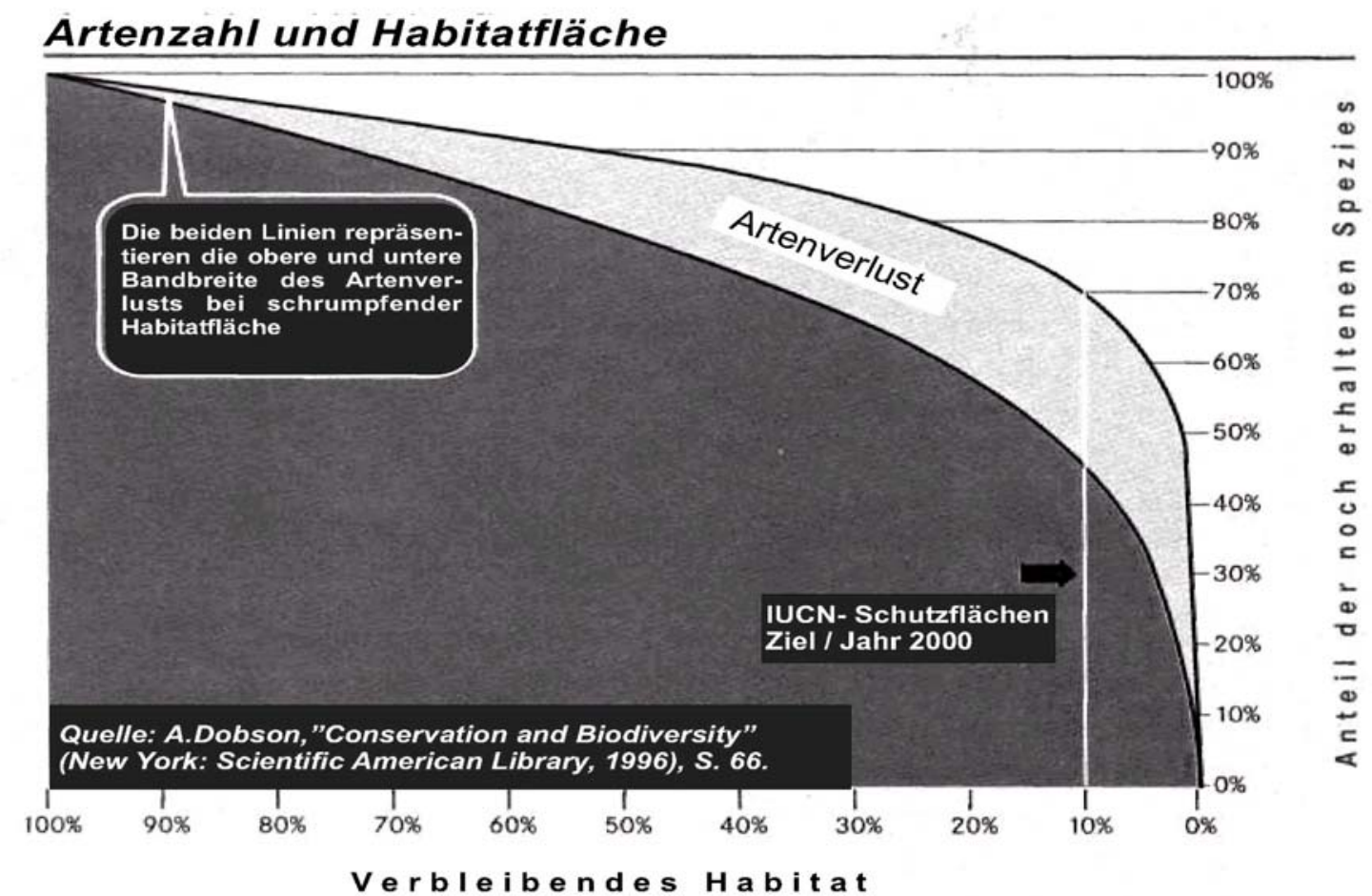

\section{Quelle: Dobson (1996), 66}

Die Arten/Areal-Beziehung wurde von MacArthur und Wilson in Bezug auf endemische Arten auf Inseln nachgewiesen ${ }^{240}$. So richtet sich etwa auf den Westindischen Inseln die Zahl der Reptilien- und Amphibienarten nach der Inselgröße: Kuba (114384 qkm, ungefähr 100 Arten), Puerto Rico (8896 qkm, 40 Arten), Montserrat (85 qkm, 25 Arten), Saba (13 qkm, 10 Arten) und Redonda (2,6 qkm, 5 Arten). ${ }^{241}$ Wird also die aus der Inselbiogeografie gewonnene Arten-ArealBeziehung auf den artenreichen Regenwald angewandt und unterstellt, dass alle dort lebenden Arten ökologisch an ihn gebunden sind, so lässt sich berechnen, dass bei der Vernichtung von jährlich einem Prozent der Regenwaldfläche ein

\footnotetext{
240 MacArthur/Wilson (1967). Die Arten/Areal-Kurve wird erzeugt durch eine modifizierte Exponentialfunktion $\mathrm{S}=\mathrm{cA}^{-\mathrm{z}}$. Dabei steht $\mathrm{S}$ für die Zahl der verbleibenden einheimischen Arten, $\mathrm{c}$ ist eine Konstante und $\mathrm{z}$ beschreibt die Form der Kurve. Die meisten wissenschaftlichen Untersuchungen ermittelten einen z-Wert von 0,15 bis 0,35 (Cincotta/Engelmann 2001, 142).

${ }^{241}$ Wilson (2004), 83
} 
globaler Artenrückgang auf 0,2 bis 0,3 Prozent pro Jahr auftreten müsse. ${ }^{242}$ Geht man nun von 10 Millionen Arten weltweit aus, so entspricht das 20.000 bis 30.000 Arten im Jahr.

Zwar verhalten sich Artenverlust und Habitatsverlust also nicht proportional, aber auch das vergleichsweise günstige Verhältnis nützt nichts, wenn der Mensch sich immer weiter ausbreitet. Die Ausdehnung der Siedlungs- und landwirtschaftlichen Anbauflächen verschlingt jedes Jahr riesige Wald- und Feuchtgebiete. ${ }^{243}$ Die Befriedigung der Grundbedürfnisse der wachsenden Weltbevölkerung führt zu einem hohen Druck auf die weltweit verbliebene Wildnis. Die Ausweisung von großflächigen Naturschutzgebieten durch Staaten trifft fast immer auf mehr Widerstand bei einer Bevölkerung, wenn diese wächst. Der Aufkauf solcher Flächen durch private Initiativen wie die Stiftung Conservation International wird teurer. Wo schon Nationalparks bestehen, wird bei einer wachsenden Bevölkerung die Gefahr größer, dass die Tiere in der Nähe der Parkgrenzen Menschen verletzen oder Eigentum beschädigen.

Alle Regenwälder der Erde bedeckten, je nach Definition, ${ }^{244}$ ursprünglich zwischen 14 und 18 Millionen Quadratkilometer. In den wenigen Jahrzehnten seit dem Ende des Zweiten Weltkriegs - einer evolutionsgeschichtlich extrem kurzen Zeit - wurde die Hälfte davon gerodet. ${ }^{245}$ Je nach Schätzung verschwindet alle 510 Jahre eine weitere Million Quadratkilometer. ${ }^{246}$ Nimmt man den mittleren Wert an, ${ }^{247}$ so werden rechnerisch in spätestens 60 Jahren die letzten großflächigen Tropenwälder abgeholzt sein. ${ }^{248}$ Wegen des Bevölkerungswachstums ist in den letzten 45 Jahren die Waldfläche pro Kopf von 1,2 Hektar auf etwa 0,5 Hektar pro Erdbewohner gesunken. ${ }^{249}$ Der proportional größte Waldverlust ${ }^{250}$ hat in Asien stattgefunden, gefolgt von Afrika und Lateinamerika. Die am stärksten von der Entwaldung betroffenen Einzelstaaten sind Brasilien, China, Indonesien, Sudan, Sambia, Mexiko, die Demokratische Republik Kongo und Myanmar. ${ }^{251}$ In den gemäßigten Klimazonen nahm die Waldfläche zwischen 1990 und 2000 hingegen um zehn Prozent $z u{ }^{252}$

\footnotetext{
${ }^{242}$ Wilson (1992)

${ }^{243}$ Cincotta/Engelmann (2001), 18

${ }^{244}$ Nicht nur die Definition von ,Regenwald', auch die von ,Entwaldung' ist umstritten. Die FAO definiert Entwaldung als dauerhafte Umwandlung von Waldgebieten in genutzte Flächen (z.B. für Landwirtschaft) mit einer langfristigen (mehr als 10 Jahre) Reduzierung der Baumkronenfläche auf weniger als 10 Prozent (FAO 2001, 31). Nach dieser Definition werden also auch Waldflächen, die seit neun Jahren stark beansprucht wurden, noch als Wälder gezählt.

${ }^{245}$ Pimm/Raven (2000), 844, UNFPA (2001), 26

${ }^{246}$ Schätzungen über jährliche Verlustraten schwanken zwischen 0,3 und 2 Prozent (Wilson 2004, 85).

${ }^{247}$ Die Rechnung basiert auf: Entwaldung von 150.000 qkm/Jahr, 7-9 Mio. qkm Restfläche.

${ }^{248}$ Terborgh (1999) kommt in komplexeren Berechnungen auf einen Zeitraum von 50 Jahren.

${ }^{249}$ UNFPA (2001), Angaben bis 2005 extrapoliert.

${ }^{250}$ Das Verhältnis von verlorener zu noch erhaltener Waldfläche.

${ }^{251}$ Worldwatch Institute (2002), 104

${ }^{252}$ Word Wide Fund für Nature (2004), 4
} 
Eine beträchtliche Zahl der Spezies konzentriert sich in ca. 25 sog. Biodiversitäts-Hotspots, so nennen Biologen die am stärksten gefährdeten artenreichen Regionen, und in den noch relativ unberührten drei großen tropischen Waldregionen (dem oberen Amazonas und dem Bergland von Guayana, dem Kongobecken sowie der aus Neuguinea und Melanesien bestehenden Inselgruppe). ${ }^{253}$ Obwohl diese Wälder nur 2,3 Prozent der Erdoberfläche bedecken, kommen dort schätzungsweise über die Hälfte aller Arten vor ${ }^{254}$ In diesen letzten großen, geschlossenen Waldgebieten ist der Bevölkerungsdruck aber mit 3 Prozent Wachstum jährlich mehr als doppelt so groß wie im weltweiten Durchschnitt. ${ }^{255}$ Falls sich an der Rate, mit der die Entwaldung fortschreitet, nichts ändert, werden diese tropischen Wälder in den nächsten Jahrzehnten auf ein paar isolierte Wälder zusammenschmelzen..$^{256}$

Die Beziehung zwischen Bevölkerungswachstum und Umwelt vollzieht sich also - vereinfacht gesagt - über folgenden Wirkungsmechanismus: Bevölkerungswachstum bedeutet Ausweitung der Aktivitäten zur Befriedigung menschlicher Grundbedürfnisse, v.a. Erschließung neuer Anbau-, Weide- und Siedlungsflächen, dies führt zu Zerstörung von Habitaten (derzeit v.a. in Entwicklungsländern) und damit zum Verlust von Biodiversität.

Laut UNFPA deuten bisherige Erkenntnisse darauf hin, „dass weitgehend intakte, zusammenhängende Waldflächen nur dort erfolgreich geschützt werden können, wo die Bevölkerungsdichte auf einem sehr niedrigen Niveau (ein bis zwei Menschen pro Quadratkilometer) gehalten werden kann. ${ }^{\text {“257 }}$ Diese Aussage gilt wohl für eine traditionelle Lebensweise, die einfache Methoden des Ackerbaus betreibt. Darunter ist in erster Linie die Brandrodung zu verstehen, die v.a. in Afrika noch weit verbreitet ist. Afrika ist geologisch stabiler als andere Kontinente - vulkanische Aktivität hat dort weniger oft fruchtbares Material für Böden produziert als anderswo. ${ }^{258}$ Um diese Böden zu bewirtschaften, hat sich das Prinzip der Brandrodung über Jahrtausende bewährt. Abgegrenzte Waldgebiete werden niedergebrannt, wodurch sich die Bodenbeschaffenheit verbessert, da die Asche Nährstoffe hinzuführt. ${ }^{259}$ Die dort lebenden Arten fliehen einfach in den intakten umliegenden Wald. Die Felder werden dann ein bis drei Perioden bewirtschaftet, bevor verlassen werden. Gräser, Büsche und schließlich Bäume erobern das Gebiet zurück und führen im wieder Stickstoff zu. Nach etwa 20 Jahren beginnt der Kreislauf von neuem. Ehrlich und Mitautoren fassen zusammen: „Shifting cultivation of this sort has persisted for millenia in the tropics of South-

\footnotetext{
${ }^{253}$ Myers/Mittermeier/Mittermeier/Fonseca/Kent (2000).

${ }^{254}$ A.a.O., 35

${ }^{255}$ A.a.O., 21

${ }^{256}$ Cincotta/Engelmann (2001), 109

${ }^{257}$ UNFPA (2001), 27

${ }^{258}$ Ehrlich/Ehrlich/Daily (1995), 153

${ }^{259}$ A.a.O.
} 
east Asia, Africa and South America, relatively undisturbed until the late 20th century when population growth began to upset the balance between people and the land they depended on for sustenance. “260

\subsubsection{Fallstudie: Ranomafana-Wald in Madagaskar}

Zur Verdeutlichung der empirischen Zusammenhänge zwischen Bevölkerungsentwicklung und Artensterben soll als Fallstudie der Ranomafana-Wald in Madagaskar betrachtet werden. ${ }^{261}$

Die Insel Madagaskar ist in biologischer Hinsicht einzigartig. Vor 200 Millionen Jahren verband sie Afrika mit Indien innerhalb des südlichen Riesenkontinents Gondwanaland. Als die beiden Kontinentalplatten auseinanderdrifteten, entfernte sich Madagaskar vom afrikanischen Kontinent. Durch die Isolation konnten neue Arten entstehen und Spezies, die anderswo ausstarben, blieben am Leben. Madagaskar besitzt mehr Orchideen- und Schmetterlingsarten als das ganze restliche Afrika zusammen. Auf der Insel leben auch alle bis auf zwei Lemurenarten der Welt, acht Arten des Baobab-Baums (in Rest-Afrika dagegen nur eine Art) und zwei Drittel der Chamäleonarten der Erde.

Im Durchschnitt hatten Frauen zwischen 1995-2000 in Madagaskar 6,1 Kinder. Nach der mittleren Bevölkerungsschätzung der Vereinten Nationen wird die Bevölkerung von heute 15,9 Mio. bis 2050 auf 46,3 Mio. ansteigen. ${ }^{262}$

In Madagaskar werden zur Zeit jedes Jahr 111.000 Hektar Wald gerodet. Dadurch ist der Lebensraum der Lemuren, der vielleicht anmutigsten Halbaffen der Welt, vernichtet. Von drei Arten - dem Goldenen Bambuslemuren, dem Breitschnauzenhalbmaki und dem Fingertier - gibt es nur noch wenige hundert Tiere, die meisten im Ranomafana-Wald. Dieser Wald war 1973 noch $60 \mathrm{~km}$ breit, aber Ende der 1990er Jahre bereits auf einen Streifen von sieben bis $15 \mathrm{~km}$ Breite zurückgedrängt worden. Die Dorfbewohner, zum größten Teil bitterarme Menschen, roden den Wald und bauen Reis an. In den trockenen Monaten des südlichen Frühlings brennt der Wald jeden Tag. Das Dorf Amobiaviavy z.B. ist in den letzten 50 Jahren von 32 Personen auf über 320 Menschen gewachsen. Zunächst hatten die Menschen nur ein Tal bewässert, mit der steigenden Bevölkerung wurden immer mehr Felder angelegt. Als sie nicht mehr ausreichten, um die Dorfbewohner zu ernähren, begannen die Menschen, die Hänge zu roden, die das Tal begrenzten. Trotz allem werden die von jeder Familie bewirtschafteten Nassreisfelder immer kleiner. Die Bereitschaft, unter diesen Umständen den Lebensraum von ein paar Affen zu schonen, die man sowieso kaum zu Gesicht bekommt, ist verständlicherweise gering. Der Wald ist da und wartet scheinbar nur auf den ersten Mann, der mit einer Axt hineingeht.

\footnotetext{
${ }^{260}$ A.a.O., 145

${ }^{261}$ Das folgende Beispiel ist Harrison (1994), 109-128 entnommen.

${ }^{262}$ UN Population Division (2003b), 5
} 
Der Ranomafana-Wald wurde wider Erwarten gerettet. Um die letzten Halbmakis zu schützen, begann der WWF Druck auszuüben, und das Gebiet wurde 1990 als Nationalpark ausgewiesen. Um illegale Fortsetzungen der Rodungen zu verhindern und das Los der Menschen zu erleichtern, wurde ein mit 4,3 Millionen Dollar ausgestattetes Entwicklungsprojekt ins Leben gerufen. Harrison schließt seine Fallstudie mit den Worten: „Im Ranomafana-Wald wurde die ökologische Tragödie gestoppt. Doch in 999 von 1000 Fällen wird sie bis zu ihrem alles zerstörenden Ende fortgesetzt - auf Madagaskar und in allen anderen tropischen Regenwaldgebieten. Der Wald wird gerodet, um das Überleben der Menschen zu sichern. Die Natur zieht sich zurück oder stirbt. Doch der Aufschub für die Menschen ist nur vorübergehend. Schließlich sind die Grenzen des Waldes erreicht, und die Abrechnung folgt. (...) Die Erträge sinken, und die Bauern können dem Land nicht länger ihren Lebensunterhalt abringen. Für vielleicht 20 oder 30 Jahre armseligen Überlebens gehen der Wald und die Natur in ihm verloren." ${ }^{2633}$

Das Beispiel des Ranomafana-Waldes legt es nahe, die These von Esther Boserup kritisch zu bewerten. Bevölkerungswachstum verursacht ErnährungsProbleme, ist aber zugleich auch der Schlüssel zur Lösung dieser Probleme, so lautete verkürzt gesagt diese These. Doch die von Boserup erwarteten Technologie-Sprünge in der Landwirtschaft stellen sich eben nicht immer ein. Wenn sie in Madagaskar, aus welchen Gründen auch immer, ausbleiben, so droht der unkontrolliert wachsenden Population eine Hungersnot.

\subsubsection{Populationsverlust als Indikator für Artenverlust}

Die Populationen der meisten Arten durchlaufen Expansions- und Reduktionsphasen - dies ist durchaus ein Normalzustand in der Evolution. Dennoch ist der Populationsverlust ähnlich dem Habitatsverlust ${ }^{264}$ ein Indikator für drohenden Artenverlust. Wenn die Bestände fast aller Arten massiv zurückgehen, dann ist dies ein untrüglicher Hinweis für präzendenzlose menschliche Eingriffe in die Biosphäre. Laut dem „Living Planet Index“ des World Wildlife Funds (WWF) sind 40 Prozent der Wildtiere zwischen 1970 und 2000 verschwunden. Der Living Planet Index (LPI) misst die Entwicklung der Bestände von über 1100 Vogel-, Säugetier-, Reptilien-, Amphibien- und Fischarten. Der LPI berechnet sich aus drei Indizes der Ökosysteme Land, Süsswasser und Meere. In den letzten 30 Jahren sank der Populationsindex von 555 an Land lebenden Arten (Säugetiere, Vögel und Reptilien) um etwa $30 \%$, der aus 267 Meeresarten zusammengesetzte Populationsindex um rund $30 \%$ und der aus 323 Arten bestehende Süsswasser-Populationsindex um $50 \%$.

\footnotetext{
${ }^{263}$ Harrison (1994), 121

${ }^{264}$ Beide Variablen sind natürlich nicht unabhängig voneinander.
} 


\section{Abb. 13: Populationsindices landlebender Arten, Meeres-Arten und Süsswasser-Arten, 1970-2000}
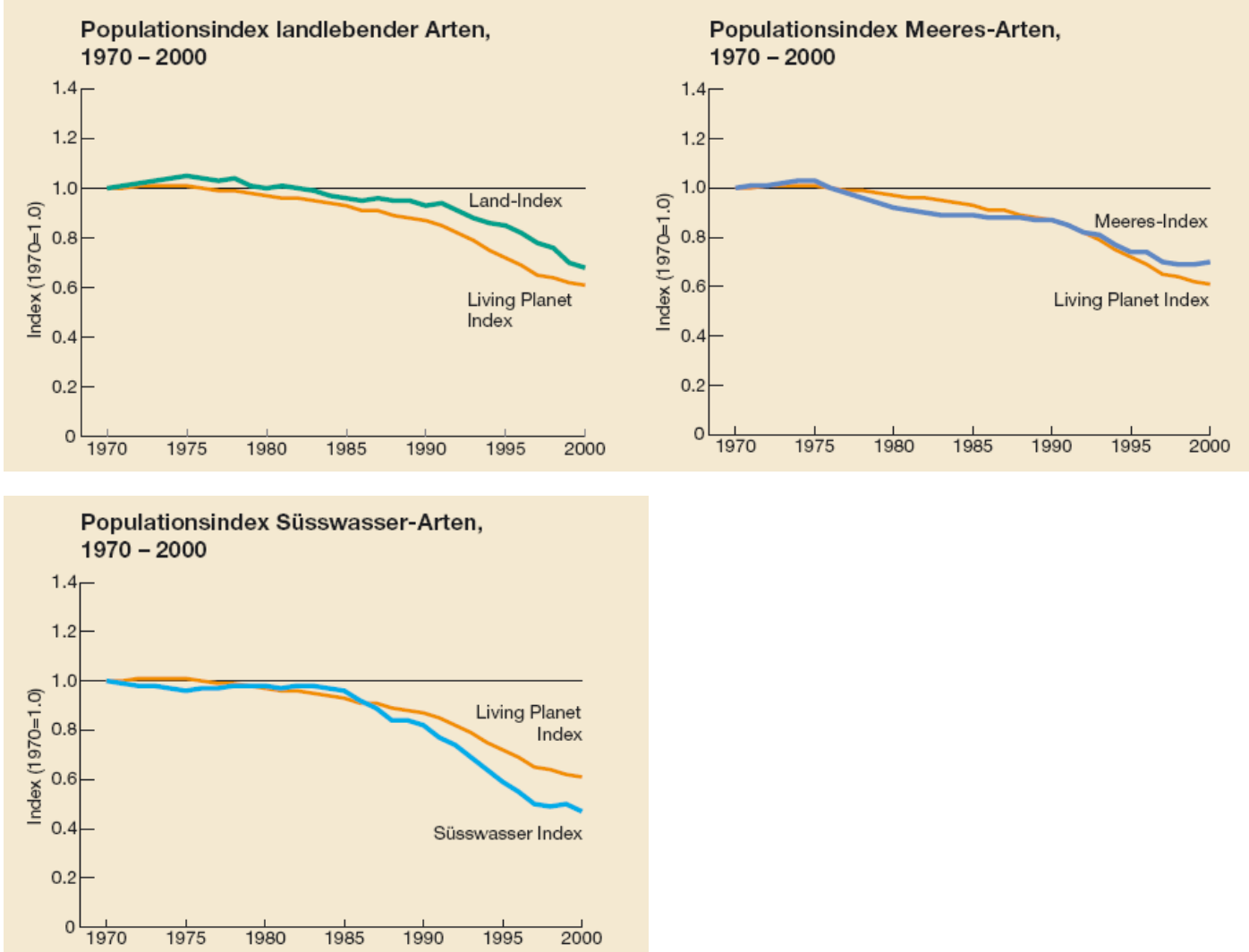

\section{Quelle: World Wide Fund for Nature (2004b): Living Planet Report 2004. Deutsche Zusammenfassung. http://www.wwf.de/service/publikationen/.}

Die Trends bei den Populationen von Wildtieren sind also - ebenso wie der Trend bei den Habitaten - dem Trend des weltweiten Bevölkerungswachstums, das zwischen 1970 und 2000 um 65 Prozent zunahm, entgegengesetzt. Ein kausaler Zusammenhang liegt - ungeachtet der Schwierigkeit, ihn genau zu quantifizieren auf der Hand. Diese empirischen Hinweise verstärken sich, wenn man die Populationsindices für landlebende Arten und Süsswasser-Arten weiter nach Regionen untergliedert. ${ }^{265}$ Die Tierpopulationen, die in tropischen Ländern mit hohem Bevölkerungswachstum beheimatet sind, ${ }^{266}$ haben weit stärker abgenommen als die Tierpopulationen in den moderaten Klimazonen. Der Index für letztere fiel um 65 Prozent, während ersterer nur 10 Prozent abnahm. ${ }^{267}$

\footnotetext{
${ }^{265}$ Beim dritten Populationsindex, dem für Meeresarten, ist eine solche Untergliederung nicht möglich, man kann nur die einzelnen Ozeane gesondert betrachten.

${ }^{266}$ Die 555 Arten in diesem Index setzen sich zusammen aus 124 tropischen und 431 nicht-tropischen.

${ }^{267}$ World Wide Fund for Nature (2004b), 4. Ähnlich sieht es beim Populationsindex der Süsswasserarten aus.
} 


\section{Abb. 14: Populationsindices landlebender Arten, 1970-2000}

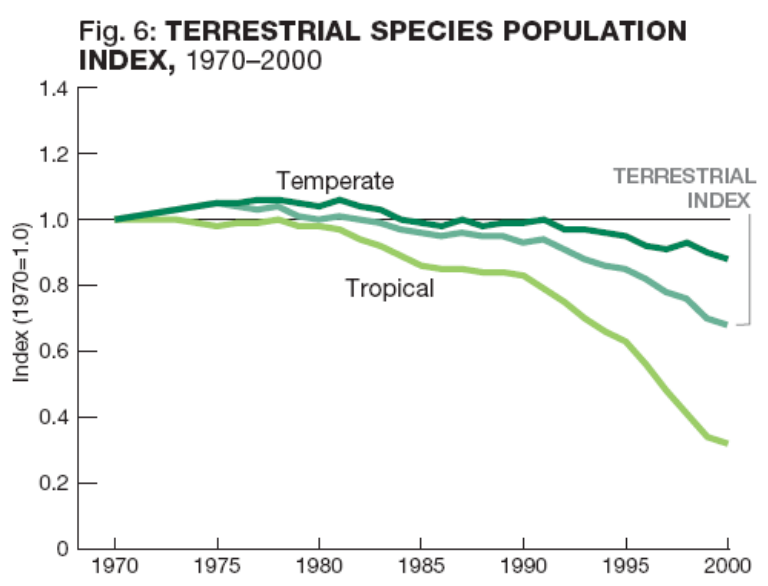

\section{Quelle: World Wide Fund for Nature (2004a): Living Planet Report 2004. http://www.panda.org/news_facts/publications/index.cfm}

\subsubsection{Bevölkerungsgröße, -dichte oder wachstum als Ursache für Arten- verlust?}

Wie gezeigt gibt es eine Fülle empirischer Hinweise dafür, dass Bevölkerung eine Ursache für Artensterben (vermittelt über Habitats- und Populationsverluste) ist. Es stellt sich die Frage, ob eigentlich Bevölkerungsgröße, -dichte oder -veränderungsrate die entscheidende Determinante für den Artenverlust ist. Weit verbreitet ist die Ansicht, dass es v.a. auf den Entwicklungspfad ankomme. ${ }^{268}$ Ausgehend von einer gegebenen Bevölkerung gelten geringe Veränderungsraten in ökologischer Hinsicht als vorteilhafter als höhere, da dann Mensch und Tier mehr Zeit für Anpassung bleibt. ${ }^{269}$ Auch wenn dies unmittelbar überzeugt, soll darauf hingewiesen werden, dass eine hohe Wachstumsrate in einem dünn besiedelten Gebiet nicht per se problematisch sein muss. Wie am Beispiel des Ranomafana-Wald gezeigt, ist letztlich die Bevölkerungsdichte der wichtigste Faktor. Die Bevölkerungsgröße, z.B. 10 Mio. Menschen, ist hingegen relativ wenig aussagekräftig. Wenn sich diese 10 Mio. Menschen auf einer großen Fläche verteilen, so ist die demografische Belastung für die Umwelt gering.

\footnotetext{
268 „Sofern man sich überhaupt auf eine bevölkerungspolitische Optimierung einlassen will (...), so kann sich das Optimum nicht auf die Bevölkerungsdichte, sondern nur auf den Entwicklungspfad beziehen“, schreibt der Bevölkerungssoziologie Franz-Xaver Kaufmann (2004b, 23).

${ }^{269}$ Bayles (1976a), xxi
} 
Die Dichte und die absolute Bevölkerungsgröße nehmen natürlich dort zu, wo die Bevölkerung wächst, insofern sind die drei Größen Bevölkerungswachstum, Bevölkerungsdichte und absolute Bevölkerungsgröße miteinander verknüpft. Wer - wie auch immer - eine optimale Veränderungsrate einer gegebenen Bevölkerung berechnet zu haben glaubt, könnte mit einfachen Rechenoperationen auch die optimale Dichte berechnen.

Das Ergebnis, dass die Dichte letztlich ein wichtigerer Faktor als die Größe ist, wird in in späteren Kapiteln noch beschäftigen. Deutschland bspw. ist ein Land mit niedrigem Bevölkerungswachstum aber hoher Bevölkerungsdichte.

\subsubsection{Zwischenfazit zu Bevölkerungsdichte und Artenverlust}

Es gibt zahlreiche empirische Hinweise für einen engen kausalen Zusammenhang zwischen zunehmender Bevölkerungsdichte und Artenverlust. Die Beziehungen sind komplex. Zwischen Bevölkerungsdichte und Artenverlust gibt es zahlreiche intermediäre Variablen. So führt Bevölkerungszuwachs zu Habitatsverlust - und dieser entsprechend der HIPO-Formel zu Artenverlust. Ein anderer Zusammenhang lautet: Bevölkerungszuwachs bzw. zunehmende Bevölkerungsdichte bewirkt verstärkte Emission der sog. Treibhausgase $\left(\mathrm{CO}_{2}\right.$, Methan etc.). Dies bewirkt eine Erhöhung der durchschnittlichen Temperatur und eine Verschiebung der Klimazonen. Da die Arten sich nicht schnell genug umstellen können, kommt es zu einem Massensterben. ${ }^{270}$

Aus der empirischen Bestätigung des Zusmmenhangs zwischen zunehmender Bevölkerungsdichte und Artenverlust kann noch nicht abgeleitet werden, dass eine solcher Zusammenhang auch für alle anderen Umweltprobleme besteht. Klimawandel, die Entfischung der Meere, Wüstenbildung, Erosion, die anthropogene Veränderung des Ozongehalts der Atmosphäre, Abfallaufkommen, Luft-, Wasserund Bodenverschmutzung, um nur einige weitere Umweltprobleme zu nennen, hier ähnlich detailliert zu untersuchen, würde einen Großteil des Raums dieser Studie verbrauchen. Stattdessen wenden wir uns den Theorien über die Beziehungen zwischen Umwelt und Bevölkerung zu, die all diese isolierten Umweltprobleme abzubilden versuchen.

\subsection{Das Tragfähigkeitstheorem}

\subsubsection{Darstellung des Theorems}

Eine wichtige Rolle in der Bevölkerungswissenschaft spielt das aus der Biologie stammende Konzept der Tragfähigkeit (engl: ,Carrying Capacity'). Es ist vor allem diese Fragestellung, in der sich die Diskussion über Bevölkerungsentwicklung und Überbevölkerung mit dem Diskurs um Umwelt und Entwicklung verschränkt. ${ }^{271}$ Führende Demografen wie Josef Schmid bezeichnen das Konzept als

\footnotetext{
${ }^{270}$ Wilson (2004), 94

${ }^{271}$ Hummel (2000), 79
} 
den „empirischen Schlüssel zum Verständnis des Zusammenhangs von Bevölkerung und Ressourcen". ${ }^{272}$

In der Biologie bezeichnet ,Tragfähigkeit' einen Zustand, bei dem eine maximale Anzahl einer bestimmten Spezies in einem begrenzten Territorium langfristig leben und ernährt werden kann. Paul Ehrlich definiert ,Carrying Capacity' so: „the maximum population size of any organism that an area can support, without reducing its ability to support the same species in the future."273

Es geht um eine Lebensweise, die nicht nur wenigen Exemplaren, sondern einer größtmöglichen Anzahl das Überleben sichert. Wenn die maximale Bevölkerungsgrenze überschritten wird, dann vernichtet die Spezies ihren eigenen Lebensraum. Mit einiger Verzögerung beginnt dann die eigene Population dieser Art zu sinken. Nachfolgend untersuchen wir also kein bedingtes Tragfähigkeitskonzept (,Wenn die Menschen diesen und jenen Level an Konsum und Technologie verwirklichen würden, dann könnten so und so viele auf der Erde leben"), sondern ein unbedingtes im Sinne der maximalen Tragekapazität (,Wie viele Menschen können maximal auf Erden leben?“).

\subsubsection{Inhärente Schwächen des Tragfähigkeits-Theorems}

\subsubsection{Systematische Veränderung der Ressourcenbasis}

Grundsätzlich ist zu fragen, ob das der Populationsbiologie entstammende Konzept jenseits kultureller, ökonomischer und historischer Zusammenhänge in den humanen Kontext übertragen werden kann. ${ }^{274}$ Fast alle Tiere sind ausschließlich auf die Nahrung angewiesen, die sie in ihrer Umgebung finden. ${ }^{275}$ Dagegen hat der Mensch im Laufe seiner Geschichte immer neue Wege gefunden, die Erde an seine Nahrungsmittelbedürfnisse anzupassen. Etwa die Hälfte der Landfläche wurde dadurch transformiert - rund 11 Prozent für Ackerland wie auch für Forstwirtschaft, rund 26 Prozent als Weideland und rund 2,5 Prozent für Wohnfläche, Industrie, Dienstleistungen und Transport. ${ }^{276} \mathrm{Im}$ Hinblick auf die menschliche Spezies hat sich die Grenze der maximalen Tragekapazität im Verlauf der Geschichte stetig nach oben verlagert. Entsprechend Tabelle 2 konnte die Erde in der Jäger- und Sammlerzeit des Paläolithikums zwischen 91.000 und 78 Millionen Menschen ernähren, wenn diese jeden Winkel der Erde (also rund 130 Millionen Quadratkilometer) besiedelt hätten. Dies ist aber natürlich nicht möglich, da ein Großteil der Erdoberfläche aus Wüsten und Eis besteht. Kratochwil verengt diese

\footnotetext{
${ }^{272}$ Schmid (1994b), 33

${ }^{273}$ Ehrlich/Ehrlich/Daily (1995), 3 f. Eine Liste von 26 Definitionen von 'Carrying capacity' liefert Cohen (1995), 419-425

${ }^{274}$ Vgl. Cohen (1995), 261 ff.; Heilig (1996), 244; Allenby (1999), 34

${ }^{275}$ Ehrlich/Ehrlich/Daily (1995), 139. Nurwenige Arten erhöhen systematisch ihre Nahrungsbasis, in dem sie z.B. wie Ameisen Blattläuse „melken“ oder Pilze „anbauen“.

${ }^{276}$ AAAS (2001), 3
} 
Schätzspanne auf fünf bis zehn Millionen Menschen, die die Erde damals zu ernähren bzw. ,tragen' vermochte. ${ }^{277}$

Tab. 2: Tragekapazität für den Menschen in unterschiedlichen Stadien seiner Entwicklung

\begin{tabular}{|l|l|}
\hline Produktionsbedingungen & Tragekapazität pro $\mathbf{~ k m}^{2}$ (Menschen) \\
\hline Jäger und Sammler & 0,0007 bis 0,6 \\
\hline Hirtenvölker & 0,9 bis 1,6 \\
\hline Frühe Agrikultur & 2 bis 100 \\
\hline Technisch verbesserte Agrikultur & 8 bis 120 \\
\hline Frühindustrialisierung & 90 bis 145 \\
\hline Moderne Industriegesellschaft & 140 bis 300 \\
\hline Postindustrielle Gesellschaft & $?$ \\
\hline
\end{tabular}

\section{Quelle: Knaus/Renn (1997), 41}

Wie aber sieht die Zukunft aus? In der Geschichte hat es viele Versuche gegeben, die für alle Zeiten maximale Tragekapazität ${ }^{278}$ der Erde zu bestimmen. Wie Abb. 15 zeigt, schwanken die Schätzungen stark, wobei mehr als die Hälfte aller Schätzungen zwischen 4 und 16 Milliarden rangieren. ${ }^{279}$

\section{Abb. 15: Schätzungen der maximalen Tragfähigkeit der Erde (in Mrd.)}

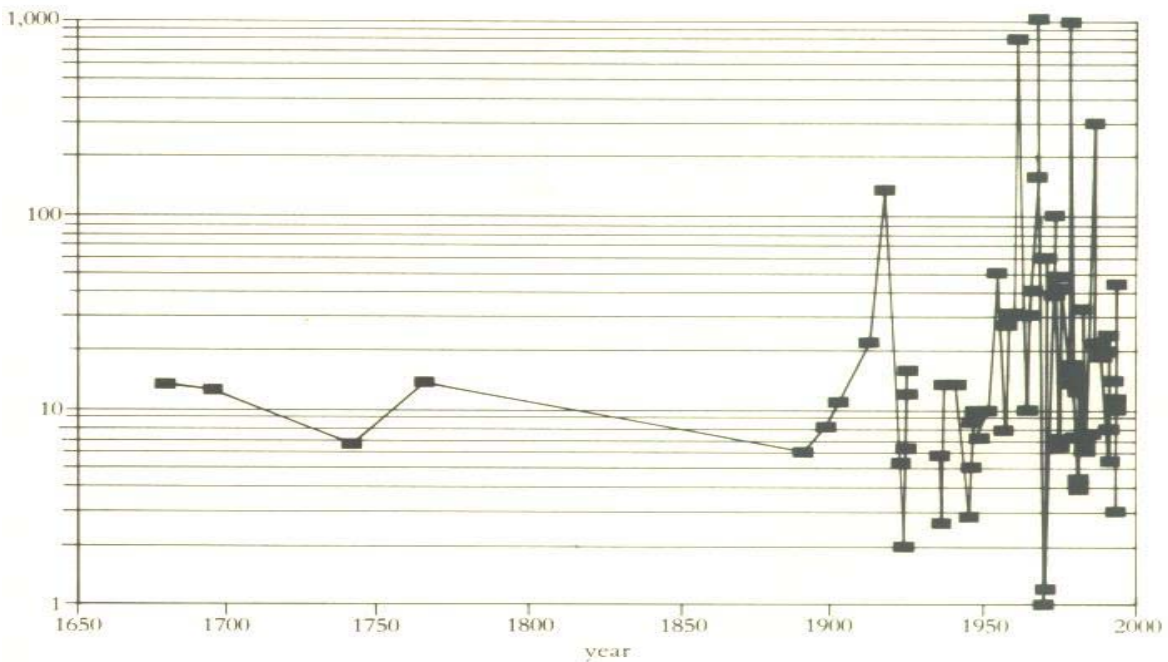

\section{Quelle: Cohen (1995), 213}

\footnotetext{
${ }^{277}$ Kratochwil (1996), 14

${ }^{278}$ Hiermit ist tatsächlich die maximale gemeint, nicht etwa die optimale.

${ }^{279}$ Cohen (1995), 10
} 
Die erste wissenschaftliche Berechnung stammt von dem Klassiker der deutschen Bevölkerungswissenschaft, Johann Peter Süßmilch. Er schätzte, dass die Erde, auf der damals etwa 750 Mio. lebten, gut 14 Mrd. Menschen tragen kann. ${ }^{280}$ In den 1960er Jahren, damals lebten schon 3 Mrd. Menschen, veröffentlichte H.J. Fremlin in der Zeitschrift New Scientist einen Artikel, in dem er argumentierte, dass $10^{16}$ bis $10^{18}$ Menschen, also bis zu eine Milliarde Milliarden Menschen, die Erde bevölkern können. ${ }^{281}$ Dabei ging er davon aus, dass die gesamte Erde mit Wolkenkratzern bedeckt werden könnte, auf deren Dächern Lebensmittel angebaut werden. Alle Produkte außer den Nahrungsmitteln würden künstlich hergestellt werden. In Fremlins Szenario ist die Hitzeabfuhr der limitierende Faktor für das Wachstum der Weltbevölkerung. ${ }^{282}$

Die meisten modernen Schätzungen sehen die Nahrungsmittelproduktion als den limitierenden Faktor an. ${ }^{283}$ Die Welternährungsorganisation FAO erstellt seit 1983 regelmäßig Tragfähigkeitsanalysen. ${ }^{284}$ Üblicherweise werden die Potenziale ackerbaulicher Flächen bewertet, wobei die jeweils angewandten Bodennutzungsund Fruchtfolgesysteme berücksichtigt werden. In diese Berechnungen gehen agrarische Beschränkungsfaktoren wie Hanglagen, Kulturpflanzenwahl und Brachanforderungen ein. Aus diesen Berechnungen wird die höchstmögliche potenzielle Nahrungsmittelproduktion abgeleitet. Alle anbaubaren Produkte, z.B. Gemüse, Kartoffeln, Hirse oder Fleisch, werden dafür in Getreideeinheiten umgerechnet. Anhand des Kalorien- und Proteinbedarfs und des Verbrauchs von Viehfutter für die Weidewirtschaft wird die maximal versorgbare Menschenzahl ermittelt. ${ }^{285}$ Die benötigten Nahrungsmittel richten sich dabei nach der Mindestkalorienmenge pro Kopf, die nach Angaben der WHO empfehlenswert ist.

Im Grundsatz folgen die Berechnungen der bereits 1925 benutzten Formel: ${ }^{286}$

$$
B=\frac{F \cdot E}{N},
$$

wobei B die Bevölkerungsgröße, F die für den Ackerbau geeignete Fläche, E der Ertrag pro Flächeneinheit und $\mathrm{N}$ der Nahrungsbedarf eines Menschen ist. Da alle Größen endlich sind und der Nahrungsbedarf größer Null sein muss, ist die Zahl

\footnotetext{
${ }^{280}$ Süßmilch (1765), 177

281 Fremlin (1964), 287. Diese Schätzung ist in Cohens Grafik nicht enthalten, da sie nicht maßstabsgetreu hätte dargestellt werden können.

${ }^{282}$ A.a.O. Fremlin erläutert den Sinn dieser Rechnung so: "The point of the article - written with a very dry humor - is not that such a heat limit is likely ever to be encountered but that, if people are not comfortable with the prospect of such population density, then they must be willing to choose (Hervorhebung i.O.) a lower population density at which they would feel comfortable."

${ }^{283}$ Cohen (1995), 218. Alternativ sind aber auch Wasser, Energie, Ressourcen, Landfläche, Bodenqualität, Krankheiten, Senken, Biodiversität, einzelne Mineralien oder Hitzeabfuhr als limitierende Faktoren vorgeschlagen worden (Cohen 1995, 329). Viele der Faktoren stehen in Wechselwirkung miteinander.

${ }^{284}$ FAO (1983)

${ }^{285}$ Geist (1993), 199

${ }^{286}$ Penck (1925), zitiert nach Cohen (1995), 166
} 
der Menschen auf der Erde begrenzt. Diese Formel scheint die Frage, wie viele Menschen können maximal auf der Erde leben, in eindeutiger Weise beantworten zu können. Der Mathematiker und Demograf Joel Cohen, der einst selbst versuchte, eine eindeutige Antwort darauf zu geben, stieß jedoch auf so viele kulturelle und soziale Variablen, dass er - in seinen Worten - „lernte, die Frage zu hinterfragen. " ${ }^{287}$ Zunächst mal ist jede physikalische Größe umstritten. Cohen untersuchte acht wissenschaftliche Untersuchungen über die maximale Tragfähigkeit der Erde. ${ }^{288}$ Die Angaben, wie viel Hektar der Erdoberfläche fruchtbares Land sind, schwankten beträchtlich. Große Unterschiede gab es auch in den Prämissen, wie sich die Nahrung zusammensetzen soll (nur vegetarische Kost oder auch Fleisch), wie viel Kalorien ein Mensch überhaupt braucht und welche Fruchtsorten unter welchen Boden- und klimatischen Bedingungen als die ertragreichsten angesehen werden können. Albrecht Penck nahm 1925 als erster die Köppenschen Klimazonen als Grundlage für eine räumlich differenzierte Tragfähigkeitstabelle der Erde. Die biophysischen Bedingungen eines Territoriums können sich aber verändern. So kann beispielsweise aufgrund eines Temperaturwandels der Anbau neuer Getreidesorten in bestimmten Gegenden möglich werden. Dies wurde meist nicht berücksichtigt. Zu Pencks Zeiten galten die Tropen als Räume mit höchster Tragfähigkeit - heute geht die Bevölkerungsgeografie davon aus, dass sie ökologisch benachteiligt sind. ${ }^{289}$ Eine Untersuchung über die maximale Tragfähigkeit der Erde stößt auf immer neue grundsätzliche und komplexe Schwierigkeiten. Manche Untersuchungen, die hohe Bevölkerungszahlen als Ergebnis hatten, berücksichtigten nicht einmal, dass jede Getreidesorte unterschiedlich lange Fruchtzeiten hat, und dass sich Boden regenerieren muss. Weiter blieb oft unberücksichtigt, dass es Missernten gibt oder dass Nahrungsmittel auf dem Transportweg verderben. Der Transport braucht Energie, so dass möglicherweise gar nicht Nahrungsmittel (oder Trinkwasser), sondern Energie der limitierende Faktor für das weitere Wachstum der Menschheit ist. Möglicherweise wichtiger als die physikalischen sind auch die ökonomischen Gegebenheiten. Keine der acht Studien über die maximale Tragekapazität der Erde berücksichtigte, dass den ärmsten zehn Prozent der Menschheit schlicht die Kaufkraft fehlt, um produzierte Nahrungsmittel zu Marktpreisen zu erwerben. Zudem kann eine menschliche Gesellschaft aus ästhetischen, religiösen, kulturellen oder biozentrischen Gründen Teile des Territoriums ungenutzt oder sogar unbetreten lassen. Das Tragfähigkeitskonzept unterstellt dagegen, dass alles verfügbare Land produktiv genutzt wird.

Auch wenn das Tragfähigkeitskonzept durchaus auf die Erde angewandt werden kann und wird, so geht es zumindest in seiner biologischen Ursprungsbedeutung von einem regionalen Territorium aus. Bezogen auf menschliche Populationen ist

\footnotetext{
${ }^{287}$ Cohen (1995), 359

${ }^{288}$ A.a.O., 162-211. Die FAO-Untersuchung war eine von diesen acht.

${ }^{289}$ Bähr (1997), 263
} 
die Verbindung zwischen Territorium und Ernährungspotenzial bzw. natürlichen Ressourcen allenfalls für Agrargesellschaften zutreffend. Die Energiebasis von Agrargesellschaften besteht fast nur aus der in Biomasse gespeicherten Sonnenenergie. Deren energetische Transformation erfolgt im wesentlichen über die menschliche Arbeitskraft und den Stoffwechsel des menschlichen, zuweilen auch des tierischen Organismus. Die Energieversorgung von Agrargesellschaften ist daher in hohem Masse flächenabhängig und der Transport größerer Mengen von Materialien ist nur über kurze Strecken wirtschaftlich sinnvoll. In Industriegesellschaften wird dagegen die menschliche Arbeitskraft durch Maschinen substituiert, deren Betrieb nicht Fläche, sondern bloß Zufuhr fossiler Brennstoffe benötigt. Plakativ gesagt: „Die Kartoffeln, die der Mensch heute isst, sind nicht mehr durch Solarenergie hergestellt worden, sondern durch Öl.“" ${ }^{290}$ Transportkosten machen in der industriellen Gesellschaft nur mehr einen Bruchteil der Kosten von Gütern aus. Damit wird aber die metabolische Abhängigkeit einer Bevölkerung von dem Territorium, über das sie verfügt, außerordentlich relativiert. ${ }^{291}$ Handel ist eine Methode, material- und energieintensive Prozesse nach außen zu verlagern. Während in den letzten beiden Jahrzehnten Materialintensität und Energieintensität in den hochentwickelten Industrienationen stagnierten oder sanken, ${ }^{292}$ scheinen sie in vielen Entwicklungsländern zu steigen. ${ }^{293}$ Entwicklungsländer fördern also mit vielen negativen Umweltfolgen die Rohmaterialien, die dann in SEL weiterverarbeitet und konsumiert werden. Durch solche Externalisierungen ist die globale Ökonomie heute gekennzeichnet.

\subsubsection{Abhängigkeit von sozialen und kulturellen Faktoren}

Während Tiere von ihren Instinkten getrieben werden, kann der Mensch seinen Lebensstil relativ leicht ändern und damit seine noch ,tragfähige' Bevölkerungsgröße variieren. Um eine einzelne numerische Zahl als Antwort auf die Frage nach der Tragfähigkeit der Erde als Antwort zu geben, müsste man die Bedürfnisse und tatsächlichen Entscheidungen zukünftiger Generationen vollständig kennen. Dies ist nicht möglich. Welchen Wert haben Berechnungen maximaler Tragfähigkeit, wenn sie von den Annahmen ausgehen, dass alle Menschen Vegetarier werden, dass kein Quadratmeter nutzbares Land für Erholung oder für die Natur reserviert bleibt, dass die ärmsten zehn Prozent der Menschheit die Kaufkraft haben, sich die produzierten Lebensmittel leisten zu können und dass die zukünftigen Menschen als einzigen Wunsch denjenigen hegen, genug zu essen zu

\footnotetext{
${ }^{290}$ Zitiert nach Ehrlich/Ehrlich/Daily (1995), 147

${ }^{291}$ Fischer-Kowalski (2003), Editorial (ohne Seitenzahl)

${ }^{292}$ Eurostat (23-1-2003). Zitiert nach Fischer-Kowalski (2003)

${ }^{293}$ Fischer-Kowalski (2001)
} 
haben ${ }^{294}$ Falls es eine numerische Zahl anzugeben vermag, wie viele Menschen die Erde zu tragen vermag, dann liegt diese Zahl mit Sicherheit über dem, was Menschen für erträglich halten. ${ }^{295}$

Gegen das Konzept maximaler Tragekapazität werden noch weitere Einwände vorgebracht. ${ }^{296}$

\subsubsection{Kritik am Tragfähigkeitstheorem aus ökologischer Sicht}

Ein wichtiger weiterer Vorwurf gegen das Tragfähigkeitskonzept ist, dass es auch aus ökologischer Sicht unzureichend ist. Zunächst ist festzuhalten, dass das Tragfähigkeitstheorem gerade in der Umweltdebatte, speziell in der Humanökologie, ${ }^{297}$ eine große Rolle spielt. Bereits 1972 warnte der Club of Rome vor der Überfrachtung der Tragfähigkeit der Erde durch das rapide Bevölkerungswachstum, welches die nicht-erneuerbaren Ressourcen erschöpfe. ${ }^{298}$ Diese Studie des USamerikanischen Ökonomen Dennis Meadows und seiner Mitarbeiter benutzte ein Computermodell, um Vorhersagen über Bevölkerungsentwicklung, Verfügbarkeit von Ressourcen, industrieller Produktion und Nahrungsmittelproduktion zu machen. ${ }^{299}$ Ohne eine Änderung der Verhaltensmuster prognostizierte die Studie etwa im Jahr 2025 einen katastrophalen Zusammenbruch der Menschheit, verursacht durch eine dramatische Abnahme der Ressourcen (v.a. Mineralien und Land). Wie man die Variablen auch veränderte, Bevölkerungswachstum und Produktion überschritten stets die langfristige Tragfähigkeit der Erde. Das einzige Szenario, in dem ein Zusammenbruch verhindert werden könne, sah eine massive Reduzierung der Ressourcennutzung und der Umweltverschmutzung, die Nutzung der Sonnenenergie, die Verringerung der industriellen Produktion zugunsten des tertiären Sektors, Recycling und als Kern eine Stabilisierung der Bevölkerungsgröße auf dem Niveau von 1970 vor. Sollte sich aber eines der anderen Szenarien

\footnotetext{
${ }^{294}$ Wenn alle Mensch sich vegetarisch ernährten und kein Getreide mehr für die Viehzucht verwendeten, so würde die derzeit für landwirtschaftliche Zwecke genutzte Fläche von 1,4 Mrd. Hektar rund 10 Milliarden Menschen ernähren (Wilson 2004, 57).

${ }^{295}$ Cohen (1995), 359

${ }^{296}$ Ein eher zweifelhafter Einwand ist, dass das Konzept Bevölkerungspolitik salonfähig mache. „Vor allem der demografische Tragfähigkeitsansatz kann zur Ausbildung von Denkstilen, Mentalitätsfiguren und Planungsvorstellungen führen, die mit autoritären Ordnungsvorstellungen korrespondieren“, schreibt die Soziologin Diana Hummel (Hummel 1995, 87). Und der Soziologe Helmut Geist ergänzt: „Die geläufige Bestimmung wird dahin zielen, den Tragfähigkeitsdiskurs um das bio-politische Bedürfnis einer regulierenden Kontrolle der Bevölkerung zu zentrieren“ (Geist 1993, 200). Ob diese Kritik berechtigt ist, wird im Abschnitt über die ethische Legitimierbarkeit von Bevölkerungspolitiken beantwortet werden.

${ }^{297}$ Die Humanökologie sieht Menschen nicht nur als stoffwechselnde Organismen, die in ökologische Systeme eingebettet sind. Sie berücksichtigt auch, dass sie Wesen mit Kultur sind, die Wirtschaftsordnungen und soziale Systeme entwickelt haben, vgl. Diekmann/Jäger (1996), 15; Renn (1996a), 37

${ }^{298}$ Meadows/Meadows/Zahn/Milling (1972)

${ }^{299}$ Die Tatsache, dass solche Computermodelle damals noch etwas Neuartiges waren und man ihnen schon deshalb großen Glauben schenkte, trug maßgeblich bei zu der Schockwirkung, die das Buch weltweit auslöste.
} 
bewahrheiten, so zeichnet Meadows die Zukunft in düsteren Farben: „Mit Sicherheit besäßen die überlebenden Menschen, wie groß ihre Zahl auch wäre, nicht mehr genug, um eine neue Form der Gesellschaft aufzubauen, die wir uns heute vorstellen können. ${ }^{\text {“300 }}$ Diese vermutlich falsche Voraussage - tatsächlich deutet heute nichts darauf hin, dass die menschliche Zivilisation in rund 20 Jahren zusammenbricht - wurde Meadows bzw. dem ganzen Club of Rome oft zum Vorwurf gemacht, und die Studie wurde als Vorhersage des Jüngsten Gerichts karikiert.

Eine verbesserte Computersimulation von Meadows und Mitarbeitern 1992 griff den Hauptkritikpunkt auf, dass Die Grenzen des Wachstums zu sehr auf die Begrenztheit der Quellen abstellt, anstatt die Aufnahmefähigkeit der Senken als maßgeblichen limitierenden Faktor zu erkennen. Im Grundsatz kam aber auch die Studie von 1992 zu der Schlussfolgerung, dass die fortgesetzte Wachstumsdynamik von Bevölkerung, Industrieproduktion und Rohstoffverbrauch zu einer steigenden Umweltverschmutzung und dadurch zu einer Überschreitung der Tragfähigkeit der Erde führen werde. ${ }^{301}$

Das Tragfähigkeitskonzept spielt also für die Ökologie-Bewegung eine wichtige, prägende Rolle. Nicht nur in seiner biologischen Ursprungsbedeutung, sondern auch in seinen Anwendungen auf menschliche Populationen ist das Konzept der Tragfähigkeit aber weit stärker mit Ernährungssicherheit verknüpft als mit ökologischer Nachhaltigkeit. Bei dem Tragfähigkeitstheorem handelt es sich letztlich um eine Neuauflage der malthusianischen Fragestellung. Das Wort ,tragen' bezeichnet die Nähe zur Ernährungssicherheit. Eine Lebensweise im Sinne der ökologischen Nachhaltigkeit ist damit noch lange nicht erreicht. So wäre es durchaus ,tragfähig' im Sinne der maximalen Tragekapazität, alle Urwälder in Weideflächen umzuwandeln, um mehr Menschen ernähren zu können. Zur ökologischen Nachhaltigkeit gehört aber auch, dass keine Tier- und Pflanzenarten und Biotope durch anthropogene Eingriffe zerstört werden. Vereinfacht kann man es so ausdrücken: Ökologische Tragfähigkeit ist ökologische Nachhaltigkeit minus die vierte Regel, dass Biodiversität und Ökosysteme nicht durch anthropogene Eingriffe zerstört werden dürfen. ${ }^{302}$ Es wurde von den Ökologen, die das Tragfähigkeitstheorem benutzten, leider oft ignoriert, dass dieser Bereich mit dem Tragfähigkeitstheorem nicht zu fassen ist. Damit soll nicht gesagt werden, dass keine Wechselbeziehungen zwischen Ernährungssicherheit und Biodiversität (und somit mit ökologischer Nachhaltigkeit) bestehen: Ökologisches Fehlverhalten kann zur Ausbreitung von Wüsten, zu Waldrodung und zur Überfischung der Meere führen, wodurch sich die Ernährungssicherheit der Menschen und die Artenvielfalt verschlechtert bzw. verringert. Trotzdem kann nicht verkannt werden, dass die Erhaltung der ästhetischen Funktionen der Natur und die Erhaltung

\footnotetext{
${ }^{300}$ Meadows/Meadows/Zahn/Milling (1972), 153

${ }^{301}$ Meadows/Meadows/Randers (1992)

${ }^{302} \mathrm{Vgl}$. Abschnitt Begriffsdefinitionen.
} 
der Mitwelt andere Überlegungen und Berechnungen erfordern als sie im Rahmen des Tragfähigkeits-Diskurses angestellt werden. Das Bevölkerungsoptimum im Sinne der ökologischen Nachhaltigkeit zu ermitteln ist eine andere Fragestellung als die Frage nach der Ernährungssicherheit, die nach wie vor im Mittelpunkt von Tragfähigkeitskonzeptionen steht.

Das an den Schiffsbau erinnernde Tragfähigkeitskonzept impliziert, dass das Schiff mehr Menschen tragen kann, wenn es keine Arche Noah ist, sondern wenn alle Plätze von Menschen besetzt werden. Die Frage nach der Tragfähigkeit der Erde ist also, so wichtig sie unbestreitbar ist, nicht die Frage dieser Studie.

Abschließend kann man sagen, dass die Frage nach der maximalen Tragfähigkeit der Erde nicht sinnvoll ist. Die gesamte heutige Menschheit ließe sich auf der Fläche der Insel Mallorca zusammenpferchen, ${ }^{303}$ die Sonne liefert mehr Energie als wir jemals verbrauchen können, die Böden geben so viel her, dass niemand hungern braucht. Wer so rechnet, für den ist alles im Überfluss vorhanden. Die bloße Möglichkeit, den Hunger in den Entwicklungsländern aus der Welt zu schaffen, hat aber noch niemanden satt gemacht. Joel Cohen stellt einen Katalog von Aspekten auf, die bei der Frage „How many people can the Earth support?“ zu berücksichtigen sind, z.B. die Art der Technologie, die physikalische, biologische und chemische Umwelt, das Durchschnittsniveau und die Verteilung materiellen Wohlstands, politische Institutionen, ökonomische Arrangements, Normen und Werte. ${ }^{304}$ Das Tragfähigkeitstheorem ist aufgrund seiner Herkunft aus der Biologie nicht geeignet, mehrere Variablen zu berücksichtigen. ${ }^{305}$ Eine Veränderung von Technologie und Konsumgewohnheiten - der beiden wichtigsten Variablen wird hingegen in der PAT-Formel berücksichtigt, der wir uns nun zuwenden.

\subsection{Die PAT-Formel}

\subsubsection{Erläuterung der Formel}

Nachdem sich das Tragfähigkeits-Theorem (im Sinne maximaler Tragekapazität) als wenig brauchbar herausgestellt hat, werden in diesem Abschnitt die theoreti-

\footnotetext{
${ }^{303}$ Dies hat einer der Studenten von Herwig Birg ausgerechnet (Birg 2001, 10). Rechnet man nach, wie viel Platz dabei jedem Einzelnen auf der $3640 \mathrm{qkm}^{2}$ großen Insel zugestanden wird, so kommt man auf gerade $0,57 \mathrm{qm}^{2}$. Falls Birgs Student verdeutlichen wollte, dass die Erde heute noch nicht so dicht bevölkert sei wie man gemeinhin annimmt, dann dürfte er eher das Gegenteil erreicht haben.

${ }^{304}$ Cohen (1995), 16 u. 262

305 Tiere und Pflanzen variieren weder bewusst ihr Konsumverhalten noch ihre Technologie. Versucht man die Optionen, die nur der Mensch hat, in Definitionen von Tragfähigkeit zu integrieren, so läuft man Gefahr, diese zu überladen, vgl. z.B. die Definition von Bähr: „Die Tragfähigkeit eines Raums gibt diejenige Menschenmenge an, die in diesem Raum unter Berücksichtigung des hier/heute erreichten Kultur- und Zivilisationsstandes auf agrarischer/natürlicher/gesamtwirtschaftlicher Basis mit/ohne Handel mit anderen Räumen unter Wahrung eines bestimmten Lebensstandards/des Existenzminimums auf längere Sicht leben kann.“ (Bähr 1997, 265)
} 
schen Beziehungen zwischen Umwelt und Bevölkerung anhand der PAT-Formel (manchmal auch IPAT-Formel) ${ }^{306}$ vertieft. Diese Formel findet als fruchtbares gedankliches Modell heute breite Anerkennung unter Wissenschaftlern, die sich mit den menschlichen Einwirkungen auf die Natur beschäftigen. ${ }^{307}$ Die Formel „Ecological Impact $=$ Population $\cdot A$ ffluence $\cdot T$ echnological Impact" geht davon aus, dass jede Form von Naturbelastung letztlich eine Funktion von Bevölkerung, Konsum und Technologie ist. Die (umstrittene) multiplikative Verknüpfung der Formel außerachtlassend, kann man allgemein schreiben:

$$
\mathrm{N}=\mathrm{f}(\mathrm{B}, \mathrm{K}, \mathrm{T})
$$

Während mit B üblicherweise die absolute Bevölkerungsgröße gemeint wird, meint K den Konsum pro Person. T schließlich bezeichnet den jeweiligen Stand von Technik und Technologie, der eingesetzt wird, um eine Einheit von $\mathrm{K}$ zu erzeugen. ${ }^{308}$ Eine Verbesserung von $\mathrm{T}$ ist gleichbedeutend mit der Senkung bestimmter Intensitäten, v.a. der Rohstoffintensität und der Energieintensität. Die Einwirkung auf die Natur $(\mathrm{N})$ wird üblicherweise verstanden entweder als Verbrauch einer natürlichen Ressource oder als Emission eines Schadstoffs.

Die Bedeutung der drei Faktoren kann gut am Beispiel des Schadstoffaustoßes deutscher Pkw erläutert werden. Gegenwärtig besitzt laut Statistischem Bundesamt in Deutschland eine Bevölkerung von rund 83 Millionen Menschen eine Pkw-Flotte von rund 42,3 Millionen Pkw mit einem durchschnittlichen Kraftstoffverbrauch je Pkw von ca. 8,8 Liter Benzin auf $100 \mathrm{~km}$.

Man kann nun ceteribus paribus jeden der drei Faktoren variieren:

$$
N=f(\bar{B}, \bar{K}, T) \Rightarrow N=f(T)
$$

Wenn es z.B. gelänge, den Durchschnittsverbrauch um die Hälfte auf 4,4 Liter Benzin auf $100 \mathrm{~km} \mathrm{zu}$ senken, so wäre rein mathematisch der Schadstoffausstoß nur etwa halb so hoch (technische Details werden bei diesem groben Beispiel vernachlässigt).

$$
N=f(\bar{B}, K, \bar{T}) \Rightarrow N=f(K)
$$

Wenn die Menschen ihre Pkw-Flotte nur halb so viel benutzen, d.h. wenn sie also

\footnotetext{
${ }^{306}$ Dabei steht I schlicht für Impact, also für die Auswirkungen der drei Variablen auf die Natur.

${ }^{307}$ AAAS (2001), 7-10; UN Population Division (2001b), 35-40. Entwickelt wurde die Formel von Ehrlich und Holdren in den 1970er Jahren (Ehrlich/Holdren 1971; Holdren/Ehrlich 1974).

${ }^{308}$ UN Population Division (2001b), 35
} 
nur noch halb so viel Auto führen, so wäre ebenfalls c.p. der Schadstoffausstoß nur rund halb so hoch.

$$
N=f(B, \bar{K}, \bar{T}) \Rightarrow N=f(B)
$$

Und wenn die Bevölkerung geringer wäre bzw. in Zukunft sinken würde, so nähme ebenfalls die Naturbelastung ab, selbst wenn die Pkw-Zahl pro Person, das Fahrverhalten und die Technologie gleich blieben.

\subsubsection{Erweiterungen der PAT-Formel}

Von zahlreichen Autoren wird gefordert, die PAT-Formel um den Faktor ,Organisationsgrad' (O) zu erweitern. ${ }^{309}$ Wenn etwa ein Stamm von Jägern und Sammlerinnen seine Lebensweise umstellt und sesshaft wird, so wird argumentiert, sei dies nicht unbedingt eine Veränderung von Konsum oder Technologie, sondern der Organisationsstruktur. Auch das Rechts- und Wirtschaftssystem, obwohl von enormem Einfluss, ist nicht explizit in dem Grundmodell der PAT-Formel vertreten. Mitte der 1960er Jahre schlug der Humanökologe Otis Duncan eine neue Formel mit dem schönen Namen POET vor (Ecological Impact $=\mathrm{f}$ (Human Population, Organisation, Environmental Conditions, and Technology)). ${ }^{310}$ Eine anderer Ansatz zur Erweiterung der Formel bezieht verschiedene Formen der Naturbelastung mit ein. „The impact measured in the IPAT formula (...) takes the amount of resources used or pollution produced as a proxy for environmental damage. In many situations an extra factor has to be added to arrive at the true damage: the sensitivity of the environment. So a fuller formula would read: $\mathrm{I}=\mathrm{P}$. $\mathrm{C} \cdot \mathrm{Tr} \cdot \mathrm{Tw} \cdot \mathrm{S}$. Tr refers to the technology of resource use, Tw to the technology of waste management, and $\mathrm{S}$ to the amount by which the environment changes in response to a given amount of resource extraction or pollution" schreibt eine Gruppe amerikanischer Wissenschaftler im AAAS Atlas on Population and Environment. ${ }^{311}$

Die Forderung nach mehr Variablen ist im Grundsatz berechtigt. Es ist sicher richtig, wenn die Wissenschaftlergruppe weiter schreibt: „Reality is still more complex. There are many other factors which affect each element of the „pressure" side of the equation. Population change, for example, is determined by fertility, mortality and migration. Each of these, in turn, is affected by a host of other factors, from patterns of breastfeeding and the status and education of women, to child health, availability of contraception, the distribution of land and income, and the opportunities for migration. ${ }^{\text {"312 }}$ Andererseits ist die PAT bzw. POET-Formel ein Modell. Ein Modell ist ein vereinfachtes Abbild der Realität, nicht die Realität

\footnotetext{
${ }^{309}$ Renn (1996a), 37 f.

${ }^{310}$ Duncan (1964), Dunlap (1994), zitiert nach Renn (1996), 38

${ }^{311}$ AAAS (2001), 7

${ }^{312}$ AAAS (2001), 8
} 
selbst. Eine Formel mit fünf Variablen auf der linken und 30 Variablen auf der rechten Seite verlöre ihren Charme. ${ }^{313}$ Für unsere Betrachtungen reicht es aus, wenn die Vielzahl der tatsächlichen Einflussfaktoren zu einigen wenigen Größen aggregiert werden. Im Folgenden wird daher aus pragmatischen Gründen weiter von der PAT-Formel gesprochen.

\subsubsection{Der Rebound-Effekt}

Anders als im Modell gibt es in der wirklichen Welt keine c.p.-Beziehungen zwischen Bevölkerung, Konsum, Technologie und Naturbelastung. Vielmehr ist stets der Rebound-Effekt (Bumerang-Effekt) zu beachten, d.h. eine Veränderung einer Variablen wirkt auf mindestens eine andere Variable zurück. ${ }^{314}$ So wird der vom Statistischen Bundesamt vorausberechnete Rückgang der Bevölkerung in Deutschland aller Voraussicht tendenziell nicht zu einem entsprechenden Rückgang des Faktors ,Konsum' führen, da die bestehende Infrastruktur nicht proportional verringert werden wird. Als Beispiel dafür mag die prognostizierte Bevölkerungsentwicklung und der Wohnflächenbedarf in Thüringen dienen. Die Bevölkerung Thüringens wird, wenn die vom Statistischen Bundesamt gesetzten Annahmen eintreffen, von 2,431 Mio. Personen Ende 2000 auf 1,730 Mio. Ende 2050 zurückgehen. Das ist eine Verringerung um 701000 Personen bzw. 28,8 Prozent und entspricht einem durchschnittlichen jährlichen Rückgang um 14100 Personen. Um abzuschätzen, wie sich das auf die bebaute Fläche auswirkt, sollte die Entwicklung der letzten Jahrzehnte betrachtet werden. Die Wohnfläche je Einwohner hat sich von 25,5 Quadratmeter je Einwohner im Jahr 1981 auf 36,0 Quadratmeter je Einwohner im Jahr 2000 erhöht, bei einem gleichzeitigen Sinken der Bevölkerung von 2,714 Mio. auf 2,431 Mio. Offensichtlich entwickelt sich die Wohnflächengröße je Einwohner nicht parallel zur Einwohnerzahl. Eine Verkleinerung der Wohnungen proportional zur Bevölkerungsgröße um 28,8 Prozent ist auch deshalb unwahrscheinlich, weil Wohnungen nicht beliebig teilbar sind. Dieselben Wohnungen werden also von weniger Menschen bewohnt. Dies zeigt, dass der Rückgang der Naturbelastung durch Bevölkerungsrückgang (über)kompensiert werden kann durch organisatorische Faktoren oder durch die steigenden Ansprüche der verbliebenen kleineren Bevölkerung.

Auch Rebound-Effekte zwischen B und T werden vermutet: Für die Entwicklungsländer behauptete Boserup wie bereits erwähnt, dass eine zunehmende Bevölkerungsgröße bzw. - dichte technologischen Fortschritt induziert oder zumindest beschleunigt. Umgekehrt gilt für die WEL, dass ein Rückgang ihrer Bevölke-

\footnotetext{
${ }^{313}$ Allenfalls eine Erweiterung von PAT zu POET-Formel ist sinnvoll, sofern die Variablen trennscharf definiert und dann klar voneinander abgegrenzt werden. Bei der POET-Formel wird z.B. der Einflussfaktor ,Effizienz der Ressourcennutzung' den Produktionsverhältnissen (und somit ,O') zugerechnet, während er bei der PAT-Formel ,T’ zugerechnet wird.

${ }^{314}$ AAAS (2001), 8
} 
rung zu einem Nachlassen der Innovationskraft im Hinblick auf umweltfreundliche Innovationen führen könnte. ${ }^{315}$

\subsection{Environmental-Kuznet-Curve}

Politisch ist v.a. umstritten, ob eine positive Kausalbeziehung zwischen ,Konsum' und ,Naturbelastung' besteht. ${ }^{316}$ Mit dem Schlagwort „Poverty is the biggest polluter" vertrat Indira Gandhi die Auffassung, dass ganz im Gegenteil die Armutsbekämpfung auch ein Beitrag zum Umweltschutz sei. Ökologen weisen allerdings darauf hin, dass eine Bekämpfung der Armut nicht immer und automatisch umweltentlastend wirke. ${ }^{317}$

Die Verbindung zwischen Wohlstandsentwicklung und Naturbelastung wird derzeit vor allem in der Debatte über die „Environmental-Kuznets-Curve“ (EKC) thematisiert. ${ }^{318}$ Gemäß diesem Modell wachsen Umweltbelastungen mit steigendem BSP zunächst an, bei weiterer Steigerung des Bruttosozialprodukts tritt jedoch eine Trendwende ein und gewisse Umweltbelastungen gehen trotz weiteren wirtschaftlichen Wachstums zurück. Das Musterbeispiel hierfür sind die $\mathrm{SO}_{2}$ Emissionen der Industriestaaten. Lässt sich daraus folgern, dass ein beschleunigtes Wirtschaftswachstum der beste Weg für die Entwicklungsländer sei, ihre Umweltprobleme zu lösen? Der SRU nennt diese Argumente „höchst fragwürdig“, da die Verlaufsbeobachtungen, die der Kurve zugrunde liegen, „eben nicht verallgemeinerbar" seien. ${ }^{319}$ So trifft die EKC zwar auf die Qualität der Fließgewässer und auf die Luftqualität zu, aber nicht auf den Flächenverbrauch, die Abfallmenge, die $\mathrm{CO}_{2}$-Emissionen und die Biodiversität. Zumal dieser Verlauf selbst bei den Luftschadstoffen kein Automatismus ist, sondern durch das steigende Umweltbewusstsein der prosperierenden Gesellschaft verursacht wird. Dieses kann sich nur in Staaten mit Demokratie, Meinungsfreiheit, freier Presse und entwickelter Zivilgesellschaft so entfalten, dass sich der am Beispiel westlicher Länder beobachtete Kurvenverlauf wiederholen dürfte.

\subsection{Anwendung der PAT-Formel auf verschiedene Umweltprobleme}

Es spricht vieles dafür, dass bestimmte Umweltprobleme zu unterschiedlichen Anteilen von den Faktoren Bevölkerung, Konsum und Technologie verursacht werden. Artensterben und Landschaftsverbrauch sind eher eine Funktion von ,Bevölkerung' als von ,Konsum'. Das Ozonloch ist stärker eine Funktion von ,Technologie' als von der ,Bevölkerung'. Klimawandel, Energieverbrauch,

\footnotetext{
${ }^{315}$ Vgl.dazu 6.5.9. Innovationsfähigkeit im Abschnitt 6.5. Interne Folgen der Schrumpfung

${ }^{316}$ Vgl. Tremmel (2003a), 143

${ }^{317}$ Knaus/Renn (1998), 69

${ }^{318}$ SRU (2002), $62 \mathrm{ff}$.

${ }^{319}$ SRU (2002), 63
} 
Verbrauch nicht-erneuerbarer Ressourcen und Umweltverschmutzung hängen vermutlich etwas stärker von ,B' und ,T' ab als von ,K', da das Konsumverhalten der Menschen relativ konstant ist.

Über solche qualitativen Aussagen hinaus ist es aber sehr schwierig bis unmöglich, wissenschaftlich abgesicherte Aussagen zu machen. Auf globaler Ebene wird man nie sagen können, dass das Artensterben in einer bestimmten Dekade zu 60 Prozent durch B, zu 25 Prozent durch T und zu 15 Prozent durch K verursacht wurde.

Nach Angaben des UNFPA ist das Bevölkerungswachstum in Entwicklungsländern die Ursache für 79 Prozent der Entwaldung, für 72 Prozent der Ausweitung des Ackerlandes und für 69 Prozent des Wachstums des Nutztierbestandes. ${ }^{320}$ Ich halte dies für eine Scheingenauigkeit, die mehr Schaden als Nutzen stiftet. Wenn in einer Fallstudie über einen bestimmten Zeitraum der Stand der Technologie und des Konsums konstant bliebe, könnte theoretisch, wenn die Bevölkerung im gleichen Zeitraum variiert, der Zusammenhang zwischen Bevölkerungswachstum (-rückgang) und Umweltbelastung (-entlastung) quantifiziert werden. Solche c.p.-Beziehungen gibt es aber wie gesagt so gut wie nicht außerhalb von Modellen, d.h. in der wirklichen Welt. Eine neuere Studie der UN Population Division kommt zu dem gleichen Schluss: „It is generally not possible to partition ,blame' for environmental problems among the various contributing factors, except in a very rough manner." ${ }^{\text {"321 }}$

Es muss also zwischen c.p.-Analysen und beobachtbaren Zusammenhängen unterschieden werden. Ceteris paribus lässt sich das Problem des Artensterbens auch über die Variablen Konsum und Technologie lösen. Wenn 6,3 Milliarden Menschen ihre Ernährung auf vegetarische Nahrungsmittel umstellen würden, so würde das Artensterben verlangsamt werden können. Das gleiche gilt für den Platzbedarf: Würden die bald neun Milliarden Menschen alle in Hochhäusern wohnen, so wäre das Wachstum der Weltbevölkerung kein Problem für die Biodiversität. Die Beispiele machen deutlich, dass theoretisch selbst ein Problem wie der Verlust von Artenvielfalt allein auf den Faktor ,Konsum' zurückgeführt werden kann, dass dies aber praktisch - eben weil aus einer Vielzahl von Gründen die meisten Menschen ihren heutigen Lebensstil nicht radikal umstellen wollen und können - kein sinnvoller Ansatz zur Lösung des Problems ist.

Dies heißt nicht, dass Umweltschutzprogramme und Programme, die das Konsumverhalten des Menschen ändern wollen, sinnlos sind. Für einen nicht zu vernachlässigenden Prozentsatz von Arten waren sicher die Folgen des Konsumverhaltens der maßgebliche Grund des Aussterbens. Einige Schildkrötenarten sind

\footnotetext{
${ }^{320}$ UNFPA (1992), ii

${ }^{321}$ UN Population Division (2001b), 38
} 
mit hoher Wahrscheinlichkeit v.a. deshalb verschwunden, weil sie als Delikatesse galten. ${ }^{322}$

\subsection{Fazit}

Die erste Hypothese dieser Studie kann als bestätigt angesehen werden. Es besteht ein enger Zusammenhang zwischen dem Verlust an Biodiversität und Bevölkerungswachstum bzw. dadurch zunehmender Bevölkerungsdichte. Allerdings lässt sich das Artensterben nicht monokausal auf Bevölkerungszunahme zurückführen. Daraus darf man aber auch nicht den falschen Umkehrschluss ziehen, dass die Bevölkerung für das globale Artensterben nur ,ein Faktor unter vielen“ sei. Selbst wenn Menschen theoretisch einfach ,zusammenrücken können“, so ist dies in der Praxis keine Option. Daher bietet eine stabile bzw. rückläufige Weltbevölkerung große Chancen für Artenreichtum und biologische Vielfalt auf unserem Planeten. ${ }^{323}$ Für andere Umweltprobleme mag das Bevölkerungswachstum im Konzert der Kausalfaktoren eine weniger große Rolle spielen, aber selbst dann führt es oft zu größerer Umweltbelastung. Über die tatsächlichen Anteile verschiedener Einflussfaktoren auf verschiedene Umweltprobleme lassen sich kaum quantitative Angaben machen.

Es wurde bereits in Abschnitt 2.4. darauf hingewiesen, dass ein Bevölkerungsoptimum nur für Teilbereiche bestimmt werden kann. Wie also sähe das Bevölkerungsoptimum in ökologischer Hinsicht aus? Ausgehend von der PAT-Formel muss man zwischen ,absoluter' und ,relativer' Überbevölkerung unterscheiden. ,Absolute Überbevölkerung' besteht, wenn ein Land trotz eines grundbedürfnisorientierten Lebensstils und einer höchstmöglichen Rate umweltfreundlicher Innovationen die Gebote ökologischer Nachhaltigkeit nicht einhalten kann. Dabei ist die Bestimmung der höchstmöglichen Innovationsrate ein größeres theoretisches Problem als die Bestimmung des grundbedürfnisorientierten Konsums. ,Relativ überbevölkert' ist ein Land, wenn es bei seinem gegenwärtigen (über einem grundbedürfnisorientierten liegenden) Lebensstil und der gegenwärtigen (steigerbaren) Rate der umweltfreundlichen Innovationen die ökologischen Nachhaltigkeitsgebote nicht einhalten kann.

Diese Ergebnisse sind keine Definitionen, sondern Ableitungen aus der PATFormel und den ökologischen Nachhaltigkeitsgeboten. Zahlreiche WEL haben zumindest im Durchschnitt der Bevölkerung ein sehr niedriges Konsumniveau.

\footnotetext{
${ }^{322}$ Andere Faktoren lassen sich nicht eindeutig in das PAT-Schema einordnen. So hat die außerordentliche Mobilität des Menschen, wenn auch meistens unbeabsichtigt, zur Einfuhr von fremden Arten in bisher isolierte Ökosysteme geführt. Die Besiedelung der hawaiianischen Inseln durch die Polynesier führte dazu, dass sich dort Ratten und Schweine ausbreiteten und rund die Hälfte der endemischen Vogelarten ausstarb (Cincotta/Engelmann 2001, 51). Es ist schwer zu sagen, ob man das Bevölkerungswachstum oder eher einen anderen Faktor dafür verantwortlich machen muss. Die PAT-Formel ist nicht komplex genug, um solche Sachverhalte zu erfassen.

${ }^{323}$ Cincotta/Engelmann (2001), 24
} 
Dieses ist nicht weiter absenkbar, zumal es sich ohnehin nicht um einen selbstgewählten, grundbedürfnisorientierten Lebensstil handelt, sondern um ein Entwicklungsdefizit, welches aus verschiedenen Gründen sehr problematisch ist. Das Konsumniveau etwa der Menschen am Rande des Ranomafana-Waldes kann sicher nicht mehr weiter gesenkt werden. Aus rein ökologischer Perspektive bedeutet dies, dass nur zwei Möglichkeiten bleiben: Entweder muß über Familienplanung die Geburtenrate reduziert werden oder es muß die Innovationsrate gesteigert werden (Stichwort: Technologietransfer), um ökologische Nachhaltigkeit zu erreichen.

Ein relativ überbevölkertes Land kann dagegen an jeder der drei Stellschrauben drehen, um ökologische Nachhaltigkeit zu erreichen. In zahlreichen reichen Ländern ist der Lebensstandard relativ hoch. Erfolge bei der Verringerung der Naturbelastung sind bei allen drei Faktoren zu erwarten. Bei dieser theoretischen Betrachtung darf aber nicht vergessen werden, dass ein radikaler Konsumverzicht von der breiten Mehrheit der Bevölkerung in den SEL abgelehnt wird und daher praktisch nicht durchführbar ist. Diese Länder sind (zum Glück) Demokratien, keine Öko-Diktaturen, wo das Notwendige einfach dekretiert werden könnte. Eine freiwillige Schrumpfung der Bevölkerung bietet daher für diese Länder große ökologische Chancen. ${ }^{324}$

\footnotetext{
${ }^{324}$ Wir kommen in Abschnitt 6 darauf zurück, welche Chancen sich aus der vorausberechneten Bevölke-
} rungsschrumpfung in Deutschland ergeben könnten. 


\section{Ist intertemporale Generationengerechtigkeit ein sinnvolles Konzept?}

\subsection{Begriffliche Annäherungen}

\subsubsection{Die Mehrdeutigkeit des Begriffs, Generation'}

Generationengerechtigkeit setzt sich aus den Einzelwörtern ,Generationen' und ,Gerechtigkeit' zusammen. Von diesen beiden Bestandteilen ist ,Gerechtigkeit' mit Sicherheit schwieriger zu definieren, aber auch der Begriff, Generation' wird, wie sich bei näherem Hinsehen zeigt, in unterschiedlichen Zusammenhängen gebraucht und ist mehrdeutig. Durch diese Mehrdeutigkeit mag sich zwar die politische Schlagkraft des Generationenbegriffs noch erhöhen, ${ }^{325}$ für die wissenschaftliche Betrachtung sollte aber stets verdeutlicht werden, welche Bedeutungen von ,Generation' gerade gemeint wird. Vier Bedeutungen gilt es zu unterscheiden, in denen das Wort in der englischen, der deutschen und zahlreichen weiteren Sprachen gebraucht wird:

1.) Chronologische (temporale) Generation. Erstens werden unter ,Generationen' Altersgruppen verstanden, wobei man z.B. von der jungen, mittleren und älteren Generation spricht. Grundlage der Zuordnung ist das aktuelle Alter und damit ein bestimmter Geburtsjahrgang. In Deutschland gebären Frauen heute durchschnittlich das erste Kind mit etwa 29 Jahren. Daraus abgeleitet werden die Jahrgänge, die zu einem bestimmten Zeitpunkt die Unterdreißigjährigen stellen, als die junge, die 30-60jährigen als die mittlere und die Übersechzigjährigen als die alte bzw. ältere Generation bezeichnet. In der Bevölkerungswissenschaft werden auch kleinere Abschnitte unterschieden (Jahre, Jahrfünfte, Jahrzehnte und andere Kohorten). In diesem Sinne leben stets mehrere Generationen gleichzeitig. ${ }^{326}$

2.) Chronologische (intertemporale) Generation. Zweitens wird das Wort ,Generation' verwendet, um die Gesamtheit der heute lebenden Menschen zu bezeichnen. In diesem Sinn lebt jeweils nur eine Generation zur gleichen Zeit. ${ }^{327}$

3.) Soziale Generation. In der Soziologie bezeichnet nach Karl Mannheims klassischer Definition ${ }^{328}$,Generation' eine Gruppe von Menschen, deren Einstellungen, Orientierungen und Verhaltensweisen weitgehend homogen sind. Sie sind häufig durch ähnliche Schlüsselerlebnisse sozialisiert worden und drücken einer zeitlichen Epoche den Stempel auf (z.B. die

\footnotetext{
${ }^{325}$ Nullmeier (2004b), 67

${ }^{326}$ Häufig wird weiter differenziert, z.B. in ,junge Alte' und , alte Alte'. Wenn im Folgenden meist von drei Generationen (der jungen, mittleren und alten) gesprochen wird, so dient dies lediglich der Vereinfachung.

${ }^{327}$ Birnbacher (1988), 23

${ }^{328}$ Mannheim (1928)
} 
,68er-Generation', die ,89er-Generation'). Was benachbarte Geburtsjahrgänge zu einer Generation macht, ist das Gefühl der gleichartigen Betroffenheit durch eine geschichtliche oder gesellschaftliche Situation, durch die sich ,eine eigenartige Nähe von sich ansonsten fremden Personen ergibt“ “. ${ }^{329}$ Diese kollektive Identität als , Generation' kann sich trotz unterschiedlicher Herkunft, Religion und ethnischer Zugehörigkeit herausbilden. Auch im Bereich der Kunst (z.B. die ,Romantiker') und Literatur (Generation von $1898^{330}$, Generation von $1927^{331}$, Lost Generation ${ }^{332}$ ) spielt der Begriff in ähnlicher Hinsicht eine Rolle. Hier bezieht er sich auf die Gemeinsamkeit der Stile und Themen. ${ }^{333}$ Schon bei der ,Erfahrungsgeneration ${ }^{334}$ ist annähernde Altersgleichheit nicht zwingend, wenn auch die vertretenen Jahrgänge selten mehr als eine Dekade auseinanderliegen. ${ }^{335}$ In der Literatur oder Kunst können 20jährige und 50jährige gleichermaßen zu einer, Generation' gehören.

4.) Familiale Generation. Schließlich gibt es auf der Mikroebene die familiäre oder ,familiale' Bedeutung des Generationenbegriffs. Familiäre Generationen bezeichnen die Glieder der Abstammungslinie (lineage). ${ }^{336} \mathrm{Im}$ Rahmen der Verwandtschaftsbeziehungen gehören die Väter einer anderen Generation an als ihre Söhne. Sogar gleichaltrige Verwandte können durch eine ,Generation' voneinander getrennt sein, z.B. wenn eine Frau erst mit $36 \mathrm{ihr}$ erstes Kind bekam, ihre Schwester aber bereits mit 18 und deren Tochter auch bereits wieder mit 18. Bedeutung 4 ist vor allem in der Familiensoziologie anzutreffen. Man spricht vom familiären Genera-

\footnotetext{
${ }^{329}$ Bude (2000), 187

${ }^{330}$ Von Martínez Ruiz 1913 geprägte Bezeichnung für eine Gruppe spanischer Schriftsteller, die (besonders seit dem Verlust der letzten überseeischen Kolonien im Kubakrieg 1898) eine geistige Erneuerung Spaniens u.a. durch den Anschluss an die Entwicklung im übrigen Europa anstrebte.

${ }^{331}$ Gruppe von spanischen Autoren, die sich im Zusammenhang mit dem 300. Todestag von L. de Góngora y Argote zu dessen Feier und Würdigung konstituierte. Aus ihrer ursprünglichen Zielsetzung zeitadäquate Lektüre und Aktualisierung der Klassiker - entstand die bedeutendste Dichtergruppe seit dem spanischen goldenen Zeitalter.

${ }^{332}$ Angeblich von Gertrude Stein geprägte Bezeichnung für eine Gruppe amerikanischer Schriftsteller (u.a. Ernest Hemingway, F.S. Fitzgerald) in den 1920er-Jahren, für die die Erfahrung des Ersten Weltkrieges zu persönlicher Desillusionierung und zur Entfremdung von tradierten Wertvorstellungen geführt hatte.

${ }^{333}$ Im technischen Bereich spricht man ebenfalls von Generationen (,die neueste Generation nuklearer Vergeltungswaffen“; „ein Computer mit einem Prozessor der dritten Generation“). Diese Arbeit beschäftigt sich aber nur mit Sprachgebräuchen, die auf Gruppen von Menschen bezogen sind.

${ }^{334}$ Synonym: , gesellschaftlicher Generationenbegriff'. Teilweise werden soziale bzw. gesellschaftliche Generationen noch unterschieden in ,politische', ,kulturelle' und ,ökonomische' Generationen (Kohli/Szydlik 2000, 8-10).

${ }^{335}$ Tremmel (1998b), 215

336 Kohli/Szydlik (2000), 11. Daher spricht man hier auch von ,geneologischen' Generationen (Lüscher/Liegle 2003, 243 f.).
} 
tionenkonflikt (im Gegensatz zum gesellschaftlichen), wenn es z.B. um die Probleme der Ablösung der Kinder von ihren Eltern geht.

Der Substantiv ,Generation' wurde im 17. Jahrhundert aus lateinisch ,generatio' entlehnt, was ,Zeugung', ,Zeugungsfähigkeit ${ }^{337}$ bedeutet. ,Generativ' ist stärker auf das Fortpflanzungsverhalten bezogen, während, generationell' gesellschaftliche Zusammenhänge beschreibt. Ich spreche im Folgenden daher von intergenerationeller Gerechtigkeit (Generationengerechtigkeit), nicht von intergenerativer Gerechtigkeit.

\subsubsection{Soziologische und chronologische Definition von, Generation'}

Die Problematik des Generationenbegriffs ist für die Sozialwissenschaften nicht neu. Der Begriff gehört sozusagen in die Oberliga soziologischer Topoi. Häufig benutzt man in der Soziologie den Mannheim'schen Generationenbegriff. Karl Mannheim unterschied dabei zwischen ,Generationslagerung', ,Generationszusammenhang' und ,Generationseinheit'. Gleiche Geburtsjahrgänge bilden nach seiner Ansicht noch keine Generation: „Es wird niemand behaupten wollen, die chinesische und die deutsche Jugend um 1800 hätten sich in einer verwandten Lagerung befunden. ${ }^{\text {"'}}{ }^{338}$ Eine ,Lagerung' ist das zeitliche Zusammentreffen der Jugendphase mit geschichtlichen Ereignissen, die das Potenzial haben, erstens zu sozialisieren und zweitens eine gemeinsame Werthaltung der jungen Menschen zu schaffen. Als ein solches geschichtliches Ereignis nennt Mannheim die Befreiungskriege gegen Napoleon; ${ }^{339}$ heutige Gesellschaftswissenschaftler würden möglicherweise an den 2. Weltkrieg, die 68er-Zeit, den Fall der Berliner Mauer 1989/1990 oder den Terroranschlag vom 11.9.2001 denken. Aber dass sich geschichtliche Ereignisse vollziehen, heißt noch nicht, dass sie wirklich Menschen sozialisieren. Mannheim nennt das Beispiel der entlegen lebenden bäuerlichen Jugend, die von den sozialen und geistigen Umwälzungen, die nach 1800 die städtische Jugend bewegten, gar nicht berührt wurden. ${ }^{340}$ Selbst wenn die Sozialisation gegeben ist, d.h. wenn Individuen tatsächlich an der Geschichte partizipieren, also einen ,Generationszusammenhang' bilden, so folgt daraus für Mannheim noch nicht, dass sie auch eine ,Generationseinheit' (d.h. eine soziale Generation) bilden. „Ab 1800 steht z.B. (beiläufig gesprochen) immer klarer abhebbar eine romantische, im Laufe der Zeit immer konservativere Gruppe einer rationalistisch-liberal werdenden Jugend gegenüber. Man kann bei weitem nicht

\footnotetext{
${ }^{337}$ Brockhaus - Die Enzyklopädie in 24 Bänden. Internetausgabe. Rev. 2004-03-05. Für eine ausführliche Begriffsgeschichte siehe Lüscher/Liegle (2003), 35-38. Lüscher/Liegle leiten aus der Etymologie eine doppelte Bedeutung (Schöpfertum und Mitgliedschaft) ab. Dies erscheint mir als eine Überdehnung der etymologischen Bedeutung.

${ }^{338}$ Mannheim (1928), 180

${ }^{339}$ A.a.O., 310

${ }^{340}$ A.a.O.
} 
sagen, dass diese Gruppen durch dieselben modernen Gehalte verbunden sind", so Mannheim. ${ }^{341}$ Dennoch gehörten sie einem einheitlichen ,Generationenzusammenhang' an, da sie beide von den äußeren geschichtlichen Ereignissen geprägt wurden und dann nur in der geistigen Verarbeitung zwei diametral unterschiedliche Antworten auf ihre historische Situation gaben. ${ }^{342}$ Die jungen Individuen, die in einem ,Generationenzusammenhang' stehen, bilden also nur dann eine ,Generationseinheit', wenn sie dieselben Überzeugungen und Werte herausbilden, oder anders gesagt: wenn sie die gleiche Antwort auf eine geschichtliche Frage geben. Ein Beispiel aus der Gegenwart für einen ,Generationenzusammenhang' ohne Bildung einer sozialen Generation ist die Prägung durch die Globalisierung. Während ein Teil der Jugend sich an diesen Megatrend anpasst und ihn als neue Chance begrüßt, steht ihm ein anderer Teil kritisch gegenüber und versucht, die Geschwindigkeit der Globalisierung zu bremsen.

Es ist umstritten, welche soziale Generationen es in Deutschland gibt. Der Politologe Leggewie favorisiert die Generationsbezeichnungen ,Flakhelfer-Generation', ,68er-Generation' und ,89er-Generation'. ${ }^{343}$ Die Unter30jährigen werden aber auch als 98er, Generation X, Generation @ und Generation Berlin, sowie als Cyber-, Techno-, Golf- und Ally-Generation tituliert. ${ }^{344}$ Es ist offensichtlich, dass es ein wenig sinnvoller Ansatz ist, Generationengerechtigkeit als Gerechtigkeit zwischen sozialen Generationen, z.B. der 68er und der 89er-Generation, zu definieren. $\mathrm{Zu}$ unbestimmt und umstritten sind die soziologischen Generationenbezeichnungen. Für Vergleiche zwischen Generationen im Rahmen von Gerechtigkeitsuntersuchungen braucht man aber einen Generationenbegriff, der nicht überlappend ist und auf einem zeitunabhängigen Merkmal basiert. Dies leistet nur der chronologische ${ }^{345}$ Generationenbegriff. Geburtsjahrgänge sind als konstant bleibende Merkmale geeignet, Prägungen nicht. „Verantwortung für kommende Generationen" ist kein sinnvolles Konzept, wenn man soziale Generationen im Sinn hat. Schließlich weiß man überhaupt nicht, ob eine zukünftige soziale Generation als ,2011er' oder ,2020er' tituliert werden wird.

Auch der familiale Generationenbegriff ist für Untersuchungen über Generationengerechtigkeit kaum relevant. Wer in der Auseinandersetzung mit den eigenen Eltern einen belastenden familiären Generationenkonflikt erlebt hat, muss deshalb das Verhalten seiner Vorgängergeneration in gesamtgesellschaftlicher

\footnotetext{
${ }^{341}$ A.a.O., 311. Hervorhebung im Original.

${ }^{342}$ Mannheim scheint davon auszugehen, dass es stets die äußeren geschichtlichen Tatsachen sind, die die Menschen prägen. Es ist eine alte Debatte, ob nicht eher „Menschen Geschichte machen“. Darauf einzugehen, ist hier jedoch nicht der Platz.

${ }^{343}$ Leggewie (1998); SRzG (1998); Leggewie (1995).

${ }^{344}$ Vgl. Kohli/Szydlik 2000, 7; Bude 2001. Frühere Bezeichnungen für angebliche Generationen (z.B. ,Wandervögel', ,Skeptische Generation') sind auch nicht allgemein akzeptiert.

${ }^{345}$ synonym: ,demografisch'
} 
Hinsicht keineswegs als problematisch empfinden; umgekehrt gilt das gleiche. ${ }^{346}$ Wenn etwa ein 28jähriger beklagt, dass es ungerecht gegenüber seiner Generation sei, dass die amtierende Politikergeneration es unterlässt, Umwelt und Natur zu schützen, so ist irrelevant, ob dieser selbst schon Vater ist oder noch nicht. ${ }^{347} \mathrm{Ge}-$ rechtigkeitsfragen in familiären Generationenkonflikten, also auf der Mikroebene, untersuchen zu wollen, ist nicht der Ansatz dieses Textes. Hier geht es um die Makroebene, also um die Ebene, auf der in der Vergangenheit schon andere große Gerechtigkeitsthemen, z.B. zwischen Klassen oder Geschlechtern, untersucht wurden.

\subsubsection{Temporale und intertemporale Generationengerechtigkeit}

In der Debatte um Generationengerechtigkeit, Ökologie und Bevölkerungsentwicklung sind also v.a. die chronologischen Bedeutungen 1.) und 2.) relevant. Den Unterschied zwischen beiden gilt es nun näher zu untersuchen. So kann die Aussage eines 28jährigen: „Meine Generation wird benachteiligt, aber die nach uns kommende Generation wird noch mehr benachteiligt sein!" in doppelter Hinsicht verstanden werden. In der erstgenannten, engen Definition wäre der sprachliche Ausdruck „meine Generation“, bezogen auf Deutschland, gleichbedeutet mit 26,7 Mio. Menschen. Die Designate dieser Definition wären die Jahrgänge der 0 bis 30 jährigen. Verwendete er dagegen bei seiner sprachlichen Äußerung die weite Definition, so würden 82,4 Mio. Menschen darunter fallen.

Folgende Terminologie halte ich für fruchtbar: Wird ,Generation' im Sinne der ,engen' Bedeutung gebraucht, so sprechen wir von ,temporaler Generation' bzw. ,temporaler Generationengerechtigkeit', sonst von ,intertemporaler Generation' bzw. ,intertemporaler Generationengerechtigkeit' ${ }^{348}$ Temporale Generationengerechtigkeit ist also die Gerechtigkeit zwischen jungen, mittelalten und älteren heute lebenden Menschen. Intertemporale Generationengerechtigkeit wird definiert als die Gerechtigkeit zwischen Menschen, die früher lebten, die heute leben und die zukünftig leben werden. Die klare Unterscheidung zwischen beiden Bedeutungen ist fundamental. So ist etwa der Satz „Die gegenwärtige Generation

\footnotetext{
${ }^{346}$ Wie oben erwähnt heizte im Sommerloch 2003 der Vorsitzende der Jungen Union, Philipp Mißfelder, den gesellschaftlichen Generationskonflikt an. Es ist damit aber keineswegs gesagt, dass Mißfelder auf familiärer Ebene ein schlechtes Verhältnis zu seinen Eltern oder Großeltern hat.

${ }^{347}$ Man könnte einwenden, dass man einfach den statistischen Durchschnitt nimmt, zu dem aus Kindern Eltern, aus Eltern Großeltern und aus Großeltern Urgroßeltern werden. Dann ist man aber wieder auf der Makroebene und bei dem ersten der beiden chronologischen Generationenbegriffe. Man betrachtet dann die Gesamtbevölkerung, die anhand von drei oder vier Daten in Kohorten eingeteilt wird.

${ }^{348}$ Diese Terminologie hat den Vorteil, dass beide Begriffe Assoziationen mit ,zeitlich' wecken, wobei intertemporal für größere Zeiträume steht. In ähnlichem Sinne spricht Laslett $(1971,172)$ vom ,temporal term of generation“. Auch Viehöver $(2002,21)$ benutzt diese Begrifflichkeiten, wenn auch mit anderem Inhalt.

Alternativ könnte man auch von ,Altersgruppen' sprechen, wenn man ,Generationen' in der engen Wortbedeutung meint; dies aber läuft dem eingebürgerten Sprachgebrauch entgegen, da dann die Verwendung des Ausdrucks, die junge Generation’ nicht mehr zulässig wäre.
} 
kann mit jetzigen Entscheidungen die Nachwelt erheblich treffen, hingegen ist dies umgekehrt nicht möglich“ richtig, wenn man einen intertemporalen Generationenbegriff benutzt und falsch, wenn man einen temporalen Generationenbegriff benutzt. Leider wurde diese Unterscheidung in der wissenschaftlichen Debatte über Generationengerechtigkeit lange komplett vernachlässigt. ${ }^{349}$

In Abbildung 10 sind die temporalen Generationen grau und die intertemporalen doppelt umrandet dargestellt.

\section{Abb. 16: Temporale und intertemporale Generationen}

\begin{tabular}{|c|c|c|c|c|c|}
\hline & Periode 1 & Periode 2 & Periode 3 & Periode 4 & $\ldots$ \\
\hline 1. Generation & $\begin{array}{l}\text { Kind } \\
(0-30)\end{array}$ & $\begin{array}{l}\text { Eltern } \\
(31-60)\end{array}$ & $\begin{array}{c}\text { Großeltern } \\
(61-)\end{array}$ & & \\
\hline 2. Generation & & $\begin{array}{l}\text { Kind } \\
(0-30)\end{array}$ & $\begin{array}{c}\text { Eltern } \\
(31-60)\end{array}$ & $\begin{array}{c}\text { Großeltern } \\
(61-)\end{array}$ & \\
\hline 3. Generation & & & $\begin{array}{l}\text { Kind } \\
(0-30)\end{array}$ & $\begin{array}{l}\text { Eltern } \\
(31-60)\end{array}$ & $\begin{array}{c}\text { Großeltern } \\
(61-)\end{array}$ \\
\hline 4. Generation & & & & $\begin{array}{l}\text { Kind } \\
(0-30)\end{array}$ & $\begin{array}{c}\text { Eltern } \\
(31-60)\end{array}$ \\
\hline
\end{tabular}

Quelle: eigene Darstellung in Anlehnung an Birnbacher (1988), 24

Zur Vereinfachung wird Generation 1 als die allererste in der Menschheitsgeschichte angenommen, also als eine Generation ohne Vorgänger. Ebenfalls zur Vereinfachung wird angenommen, dass die betrachteten Populationen im Durchschnitt genau zu dem Zeitpunkt sterben, zu dem ihre Urenkel geboren werden. ${ }^{350}$

Im Beispiel überlappen sich die intertemporalen Generationen, da zum Zwecke der Veranschaulichung eine Periodenlänge von 30 Jahren gewählt wurde. Würden wir jede Periode mit 90 Jahren ansetzen, wie es dem Konzept der intertemporalen Generationen angemessen ist, so käme es nicht zu einer Überlappung.

\footnotetext{
${ }^{349}$ Eine der seltenen Ausnahmen ist der Text von Delattre (1972).

${ }^{350}$ Gibt man diese restriktiven Prämissen auf und betrachtet ,the real world', so besteht zu jedem Zeitpunkt, also zu jedem Querschnitt der Zeitachse, die intertemporale Generation aus allen Menschen, deren Lebenszeit sich überlappt. Da die älteste lebende Deutsche, eine 110jährige aus Stuttgart, 1893 geboren wurde (www.stuttgarter-zeitung.de/stz/page/detail.php/485705. Rev. 2003-12-23), bilden also zum Zeitpunkt 2005 die Vertreter der Jahrgänge 1893 bis 2005 eine intertemporale Generation.
} 


\subsubsection{Eine Definition von intertemporaler Generationengerechtigkeit}

\subsubsection{Eine erste Arbeitsdefinition}

Als Ausgangspunkt für die Entwicklung einer Definition für Generationengerechtigkeit nehmen wir der Einfachheit halber die populäre Brundtland-Definition für Sustainability, die in der direkten Übersetzung folgendermaßen lautet:

1.1 Dauerhafte Entwicklung ist Entwicklung, die die Bedürfnisse der Gegenwart befriedigt, ohne zu riskieren, dass künftige Generationen ihre eigenen Bedürfnisse nicht befriedigen können. ${ }^{351}$

Die Brundtland-Kommission, die den Auftrag hatte, eine für Nord und Süd akzeptable politische Kompromissformel auszuhandeln, spricht von 'Entwicklung'. Dieser Begriff wird für eine Definition von Generationengerechtigkeit nicht gebraucht. ${ }^{352}$ Lässt man ihn weg und ergänzt den Begriff , Generationengerechtigkeit', so ergibt sich sinngemäß

1.2 Generationengerechtigkeit ist erreicht, wenn die heutige Generation ihre Bedürfnisse erfüllt, ohne zu riskieren, dass künftige Generationen ihre eigenen Bedürfnisse nicht erfüllen können. ${ }^{353}$

Definition 1.2 stellt die heutige Generation sprachlich ins Zentrum: ihre Bedürfnisse sollen erfüllt werden, wenn auch unter einer Nebenbedingung. Eine Definition für Generationengerechtigkeit sollte die Gleichberechtigung heutiger und zukünftiger Generationen auch sprachlich klar zum Ausdruck bringen. Wenn wir diese Punkte berücksichtigen und auch die Erwähnung des ,Risikos' eliminieren, ${ }^{354}$ so können wir formulieren:

\subsubsection{Generationengerechtigkeit ist erreicht, wenn zukünftige}

Generationen ihre Bedürfnisse im selben Ausmaß erfüllen

können wie die heutige Generation. ${ }^{355}$

\footnotetext{
${ }^{351}$ Hauff (1987), 46

352 Tremmel (2003c), 54

${ }^{353}$ Das Wort ,eigenen' vor Bedürfnissen halte ich für redundant. Zwar ist es völlig richtig, dass es um die subjektiven Bedürfnisse zukünftiger Individuen geht und nicht um die von den Heutigen festgelegten, aus unserer Sicht ,richtigen' Bedürfnisse, aber dies steckt bereits im Ausdruck ,ihre Bedürfnisse“.

Die Verben ,erfüllen' und ,befriedigen' sind für mich fast synonym, allerdings ist ,befriedigen' gelegentlich mit der Befriedigung sexueller Bedürfnisse konnotiert, so dass ich ,erfüllen` bevorzuge.

${ }^{354} \mathrm{Im}$ englischen Original lautet die Brundtland-Definition: "Sustainable development is development that meets the needs of the present without compromising the ability of future generations to meet their own needs.“ (WCED 1987, 43). In der deutschen Übersetzung ist „compromising“ unglücklich mit ,riskieren“ übersetzt worden, richtiger wäre z.B. „,in Kauf nehmen“ gewesen. Eine spezielle Erwähnung der Risiko-Dimension ist der Brundtland-Definition nicht zu entnehmen.

${ }^{355}$ Tremmel (2004b), 58
} 
Dies ist eine Definition für intertemporale Generationengerechtigkeit, da ihr der weite Generationenbegriff zu Grunde liegt. ${ }^{356}$

\subsubsection{Universalisierung dieses Konzeptes}

Nun wird in dieser Definition eine einzige Generation mit Tausenden anderen verglichen. In der Terminologie des Utilitarismus lässt sich dieses Argument so ausdrücken: Wenn das größte Glück für eine größtmögliche Zahl erreicht werden soll, wobei ,größtmögliche Zahl' sich offensichtlich auf die zukünftigen Generationen bezieht, dann werden die Zukünftigen als Aggregat immer die Heutigen um ein Vielfaches (bzw. wenn man eine unendliche Reihe von Generationen annimmt, um ein Unendliches) übertreffen. ${ }^{357}$ Der Nutzen einer Generation über z.B. 115 Jahre (z.B. 115 Millionen Nutzen-Einheiten) kann offensichtlich nicht sinnvoll mit dem potenziellen Nutzen von vielen Generationen über z.B. 1.150 Jahre (z.B. 1,15 Mrd. Einheiten) verglichen werden. Betrachten wir diesen Vergleich zwischen A (einer Generation) und B (vielen Generationen) etwas ausführlicher. Wenn man in dieser Weise vergleicht, so muss man $\mathrm{zu}$ der falschen Schlussfolgerung gelangen, dass entweder überhaupt nichts gespart werden sollte, weil sonst die Schlechtgestellten A für die Bessergestellten B sparen würden. Dies wäre der Fall, wenn man sozusagen die 1,15 Mrd. Nutzeneinheiten von B schon als gegeben hinnehmen würde, quasi im Sinne einer autonomen Sparrate, so dass man nach dem Prinzip der Verteilungsgerechtigkeit diesen vorhandenen Gewinn lediglich noch gerecht aufteilen muss.

Oder man würde (im Sinne der Chancengerechtigkeit) zu der falschen Schlussfolgerung gelangen, dass die heutige Generation A sehr viel sparen muss, da sie durch ihren Verzicht auf nur 115 Mio. Nutzeneinheiten dem Vergleichsobjekt B die Chance auf 1,15 Mrd. Einheiten erhält. Deutlicher wird dieser zweite Fall, wenn wir keinen Nutzen, sondern einen Schaden annehmen, den A anrichten kann, z.B. die Zerstörung der Biodiversität der Erde. Unter diesem Schaden leidet dann nicht nur die direkt auf A folgende Generation, sondern alle kommenden Generationen hätten darunter zu leiden. Auf A liegt dann die riesige Bürde, nicht durch einen falschen Schritt unzähligen zukünftigen Generationen ihre Zukunft zu verderben.

Im Sinne der indirekten Reziprozität ist es sinnvoller, die Wohlbefindens- bzw. Nutzenwerte von nur zwei aufeinanderfolgenden Generationen zu vergleichen. Wenn jede ,nächste Generation' im Sinne der indirekten Reziprozität das Ererbte erhält und weitergibt, dann wirkt sich dies auf die gesamte Reihe von Generatio-

\footnotetext{
${ }^{356}$ Bei einer Entsprechung für temporale Generationengerechtigkeit müsste man ,zukünftige Generationen“ durch „nachrückende Generationen“ und "heutige Generation” durch "heute mittlere Generation” oder „,vorangegangene Generation“ oder „Vorgänger-Generation“ ersetzen. Nachfolgend wird aber lediglich eine Definition für intertemporale Generationengerechtigkeit entwickelt.

${ }^{357}$ Derr (1980), 43. Narveson (1978), 38
} 
nen, auf alle zukünftigen Generationen, aus. Wir können also 1.3.1 abwandeln und formulieren:

1.4 Generationengerechtigkeit ist erreicht, wenn sich die nächste (d.h. die erste zukünftige) Generation ihre Bedürfnisse im gleichen Ausmaß erfüllen kann wie die heutige Generation.

Betrachtet man drei intertemporale Generationen (G1, G2, G3), so führt $1.4 \mathrm{zu}$ der problematischen Konsequenz, dass G1 seine Nachfolgergeneration G2 gleich gut oder besser stellen könnte, um den Preis, dass es G3 schlechter als beiden geht. Aber hat G1 seine Verpflichtungen bei so einem Verhalten wirklich erfüllt? Die Generation G2 müßte bei diesem Beispiel, wenn sie selbst an der Reihe wäre, ihre Verpflichtungen gegenüber G3 zu erfüllen, extra Anstrengungen machen und hätte damit im Endeffekt c.p. möglicherweise doch ein schlechteres Leben als G1. Damit hätte G1 aber auch bereits gegen ihre Verpflichtungen gegenüber G2 verstoßen.

Die erste Nebenbedingung zu $1.4^{358}$ ist also, dass jede zukünftige Generation die Gelegenheit haben muss, sich ihre Bedürfnisse im gleichen Ausmaß wie ihre Vorgängergeneration zu erfüllen.

\subsubsection{Höhere Gewalt}

Es gibt eine weitere Nebenbedingung. Eine Frage, die in einer Debatte über Generationengerechtigkeit so wichtig ist wie in einer über intragenerationelle Gerechtigkeit, lautet: „Wo verläuft die Grenze zwischen Unglück und Ungerechtigkeit?“"359 Manchmal sind die Lebensumstände von Generationen schlicht das Ergebnis höherer Gewalt, etwa wenn eine neue, unbekannte Krankheit ausbricht oder einige Jahrzehnte später geheilt wird. Dagegen ist z.B. bei der Belastung der Erde mit Atommüll sehr wohl berechenbar, welche Generation besser gestellt ist, und es ist auch möglich, eine Gleichbehandlung herzustellen. Allerdings ist dies nicht immer politisch gewollt oder durchsetzbar. Wenn eine kommende Generation es also schlechter hat, so ist ethisch ganz entscheidend, ob - juristisch gesprochen - Fahrlässigkeit bzw. sogar Vorsatz ihrer Vorgängergeneration der Grund dafür ist - oder ob „niemand etwas dafür kann.“

Der Hinweis auf höhere Gewalt ist also eine weitere Nebenbedingung. Er ist kein Argument gegen Zukunftsethiken an sich. Denn natürlich ist es wahr, dass wir nicht die Macht haben, das Schicksal zukünftiger Generationen allein zu gestalten, weder zum Guten noch zum Schlechten. Die Zukunft ist gerade deshalb vieldeutig, weil sie nicht nur von unserer menschlichen Einflussnahme abhängt. ${ }^{360}$

\footnotetext{
${ }^{358}$ Und späteren Verbesserungen dieser Arbeitsdefinition.

${ }^{359}$ Horn/Scarano (2002), 9

${ }^{360}$ Renn (1996b), 24
} 
Nichtsdestotrotz haben wir einen immensen Einfluss, und das gibt uns Verantwortung, alles uns Mögliche zu tun.

„Anders als Naturgewalten oder auch menschliche Schwächen lässt sich Technikeinsatz nicht als unglücklicher Zufall, nicht als unvermeidbares Schicksal oder als Manifestation externer Kräfte deuten. Mag auch das einzelne Individuum wenig Einflussmöglichkeiten über den Einsatz einer Technik haben, irgend jemand in der Gesellschaft muss den Einsatz wollen, ansonsten würde es nicht dazu kommen. »Irgend jemand « kann auch eine Institution sein oder sich als Resultat des Zusammenwirkens vieler Kräfte einstellen, entscheidend bleibt, dass Technikeinsatz eine Entscheidung für die Verwirklichung dieser Technik voraussetzt. (...) Je mehr sich Technik als Gestaltungselement in der Lebenswelt durchsetzt und zudem Schutz vor Naturkräften bietet, um so mehr erweitert sich der Verantwortungsspielraum des Menschen“", schreibt Ortwin Renn. ${ }^{361}$

Unsere Verantwortung gegenüber zukünftigen Generationen bezieht sich auch nicht nur auf die zukünftigen Folgen unserer eigenen Handlungen. Wenn wir zukünftige Unglücke (also per definitionem keine durch menschliche Aktivitäten hervorgerufenen Effekte) vorhersehen könnten, wären wir, die Heutigen, sofort zu einer höheren Sparrate verpflichtet. Wenn wir exakt berechnen könnten, dass in 100 Jahren durch einen knapp vorbeifliegenden Meteoriten alle Ernten dieses Jahres vernichtet würden, wäre es ethisch geboten, ab sofort gewaltige Ressourcen in die Vorsorge für dieses Ereignis, also z.B. den Bau extrem großer Lager, zu investieren. ${ }^{362}$

\subsubsection{Fortschritt und Verbesserung - die zweite Arbeitsdefinition}

Von den Grundsätzen intragenerationeller Gerechtigkeit ist vor allem die Formel, dass Gleiches gleich, Ungleiches seiner Eigenart entsprechend verschieden zu behandeln sei, etabliert. Generationen setzen sich aus Individuen zusammen, die alle die gleiche Menschenwürde besitzen. Prima facie sind ,Generationen' gleichwertig zueinander. Unsere bisherige Arbeitsdefinition für Generationengerechtigkeit lautet:

1.4 Generationengerechtigkeit ist erreicht, wenn die nächste Generation sich ihre Bedürfnisse im gleichen Ausmaß erfüllen kann wie die heutige Generation.

unter den beiden Nebenbedingungen

$\mathrm{N}$ 1: Dabei ist erstens zu beachten, dass die Chancen jeder zukünftigen Generation so groß wie die ihrer Vorgängergeneration sein sollten.

\footnotetext{
${ }^{361}$ A.a.O., 27

${ }^{362} \mathrm{Vgl}$. Gosseries (2002), 470
} 
N 2: Dabei ist zweitens zu beachten, dass sich die ethischen Verpflichtungen der heutigen Generation nur auf ihren Macht- bzw. Verantwortungsbereich erstrecken.

Nun berücksichtigt diese Arbeitsdefinition aber offensichtlich immer noch zu wenig die Besonderheiten des intergenerationellen Kontextes. Dem Dichter Heinrich Heine wird der Satz zugeschrieben: „Jede Zeit hat ihre Aufgabe, und durch die Lösung derselben rückt die Menschheit weiter." Die bisherigen Generationen haben Wachstum und Wohlstandszunahme erfahren, sie sollten im Sinne der Gerechtigkeit dafür sorgen, dass dies auch für zukünftige Generationen möglich bleibt. Es war das Motiv früherer Eltern, dass es ihren Kindern einmal besser gehen soll. Eine positive intergenerationelle Sparrate sollte somit auch das Ziel der heute Lebenden im Umgang mit zukünftigen Generationen sein. ${ }^{363}$ Fortschritt in der Lebensqualität ist sicherlich wünschenswerter als ein Stillstand auf dem Status Quo, letzterer wiederum ist einer Verschlechterung vorzuziehen.

Für eine Erweiterung lässt sich auch ins Felde führen, dass es niemals gelingen wird, rechnerisch exakt einen Zustand der Gleichbehandlung herbeizuführen. Um nicht das Risiko einer versehentlichen Schlechterstellung der Zukünftigen einzugehen, sollten die Heutigen im Sinne des Vorsorgeprinzips eine Besserstellung anstreben. ${ }^{364}$ Akzeptiert man diese Gedankengänge, so ist in die zweite, verbesserte Arbeitsdefinition noch ein „mindestens“ einzufügen.

1.5 Generationengerechtigkeit ist erreicht, wenn die heutige Generation A der nächsten Generation B die Möglichkeit gibt, sich ihre Bedürfnisse mindestens im gleichen Ausmaß wie A zu erfüllen.

\subsubsection{5 ,Künftige Generationen' vs. ,Angehörige künftiger Generationen'?}

Eine wichtige Frage ist, ob sich diese Definition auf die zukünftigen bzw. nachrückenden Generationen als Entitäten bezieht oder auf die Angehörigen zukünftiger bzw. nachrückender Generationen? Die Implikationen sind weitreichend. Stellen wir uns in einem vereinfachten Beispiel vor, die heutige Generation A, die sich aus 20 Individuen zusammensetzt, wollte eine nicht-erneuerbare Ressource, von der insgesamt 100 Einheiten vorhanden sind, mit einer zukünftigen Generation B gerecht teilen. Es erscheint generationengerecht, wenn sie 50 Einheiten verbraucht und 50 Einheiten für B spart. Wegen des Bevölkerungswachstums wird B aber aus 30 Individuen bestehen (was A aufgrund ihrer Prognosen bereits bekannt ist). Die heutige Generation A müsste dann - wenn die Definition von Generationengerechtigkeit auf die Angehörigen zukünftiger Generationen abstellt - nicht nur die Hälfte, sondern 60 Einheiten der Ressource sparen und könnte

\footnotetext{
${ }^{363}$ Tremmel (2003b), 34

${ }^{364}$ Vgl. Birnbacher/Schicha (1996), 151
} 
dementsprechend weniger selbst verbrauchen, um den zukünftigen Individuen pro Kopf ein gleiches Nutzungsniveau zu ermöglichen. ${ }^{365}$

Im Kontext finanzieller Generationengerechtigkeit wird es dagegen von Nachteil für die nächste Generation sein, zahlenmäßig kleiner als ihre Vorgänger-Generation zu sein, da diese dann die Rentenansprüche und sonstigen Zahlungsversprechen des Sozialstaats begleichen muss.

Allerdings wurde auch die These aufgestellt, dass eine geringere Jahrgangstärke die wirtschaftlichen Lebenschancen einer Generation positiv beeinflusst, da sie beim Übergang vom Bildungs- ins Beschäftigungssystem mehr Möglichkeiten vorfindet. ${ }^{366}$

Es ist also in jedem Fall eine relevante Frage, ob eine Definition von Generationengerechtigkeit auf Generationen als Entititäten oder auf Individuen abstellen muss. Für ersteres könnte argumentiert werden, dass es bei Generationengerechtigkeit nicht um einzelne Individuen, sondern um Generationen gehe und dass die diese Generationen bevölkernden Individuen erst sekundär Gegenstand einer Zukunftsethik seien. Ich würde aber mit Unnerstall konform gehen, dass jede Definition von Generationengerechtigkeit sinnvollerweise nur auf die Angehörigen zukünftiger Generationen, auf die ,zukünftigen Individuen' abstellen kann. ${ }^{367}$ Generationengerechtigkeit ist ein Ansatz, der mit seiner Bedürfnisorientierung auf das Individuum abzielt. Wenn ein zukünftiges Individuum ohne eigenes Verschulden deutlich schlechter gestellt ist als ein Angehöriger der heutigen Generation, so kann dies nicht als generationengerecht bezeichnet werden.

\subsubsection{Endergebnis: Eine Definition von Generationengerechtigkeit}

Wir kommen damit zur unserer endgültigen Definition von Generationengerechtigkeit:

1.6.1 Generationengerechtigkeit ist erreicht, wenn die Angehörigen der heutigen Generation A den Angehörigen der nächsten Generation B die Möglichkeit geben, sich ihre Bedürfnisse mindestens im gleichen Ausmaß wie A zu erfüllen.

\subsubsection{7 Ökologische Generationengerechtigkeit}

Daraus lässt sich für den Teilbereich Ökologie folgende Definition ableiten:

\footnotetext{
365 Entsprechend der ersten Nebenbedingung muss die heutige Generation das voraussichtliche Wachstum nicht nur der nächsten, sondern auch weiterer Generationen berücksichtigen.

${ }^{366}$ Hillmert/Mayer (2004), Vgl. auch schon Easterlin (1980). Die Grundthese von Easterlin, dass die Entscheidung von Eltern über die Zahl ihrer Kinder sich maßgeblich danach richtet, ob die Kinder denselben Lebensstandard genießen werden wie ihre Eltern früher, lässt sich aber nicht empirisch belegen (vgl. Vorholt 1984).

${ }^{367}$ Dies wurde v.a. von Unnerstall (1999) herausgearbeitet.
} 
Ökologische Generationengerechtigkeit ist erreicht, wenn Generation A ein Naturkapital in der gleichen Höhe an Generation B weitergibt, wie A es selbst empfangen hat.

Eine themen- bzw. sektorenbezogene Anwendung zusätzlich zu einer allumfassenden Anwendung der Theorie der Generationengerechtigkeit ist für den ökologischen Bereich schon deshalb gerechtfertigt, weil sie auch in zahlreichen anderen Feldern, wie z.B. Rente, gemacht wird. ${ }^{368}$

Nun ist bei dem Ziel „Ökologische Generationengerechtigkeit“" prima facie nur das Ziel einer Erhaltung, nicht einer Steigerung sinnvoll. Das Naturkapital ist nicht vom Menschen gemacht worden, er steht daher auch nicht in der Pflicht, es zu vermehren. Lediglich wenn die ökologischen Nachhaltigkeits-Regeln nicht eingehalten werden, kann eine Steigerung des Naturkapitals geboten sein. Diese Pflicht zur Steigerung endet aber, sobald sie erreicht worden sind.

Letztlich ist es das normative Ziel, Generationengerechtigkeit sektorenübergreifend entsprechend der Definition 1.6.1 einzuhalten, nicht bloß in Teilbereichen davon wie "Generationengerechtigkeit in der Ökologie“ oder "Generationengerechtigkeit in der Rentenversicherung“. Das Ziel „Ökologische Generationengerechtigkeit" kann in Konflikt mit anderen Ziele wie z.B. „Finanzielle Generationengerechtigkeit“" geraten. Dieser Zielkonflikt wird im sechsten Abschnitt in Bezug auf die demografische Situation in Deutschland vertieft. Vorerst beschäftigen wir uns aber weiter mit der Situation in Entwicklungsländern, die durch wachsende Bevölkerungen und stark abnehmendes Naturkapital gekennzeichnet ist.

\footnotetext{
${ }^{368}$ Vgl. Tremmel (2003d); Tremmel (1997). Eine gute Zusammenfassung über Generationengerechtigkeit in der gesetzlichen Rentenversicherung bietet auch der Dokumentationsband der Tagung „Generationengerechtigkeit in der Rentenversicherung" vom 3.12.2003 in Erfurt (VDR 2004).
} 


\section{Kann Bevölkerungspolitik ethisch vertretbar sein?}

\subsection{Vorbemerkungen}

Nachdem die ersten beiden Hypothesen dieser Studie bestätigt wurden, stellt sich die Frage, ob daraus die Konsequenz gezogen werden kann, bevölkerungspolitische Ziele und Maßnahmen in Strategien für ökologische Nachhaltigkeit zu integrieren, wann immer solche Strategien konzipiert werden. Die dritte Hypothese dieser Studie soll dafür nun überprüft werden.

Generell wirft Bevölkerungssteuerung als staatlicher Eingriff in die Entscheidungsfreiheit von Individuen oder Familien tiefgreifende ethische Fragen auf. Nachwuchs zu bekommen ist eine äußerst intime und private Angelegenheit und gleichzeitig ein gesellschaftlich höchst relevanter Prozess. ${ }^{369}$ Diese Doppelseitigkeit ist mit verantwortlich für die Brisanz des Themas Bevölkerungspolitik und die Emotionalität, mit der es diskutiert wird. Die ethischen Fragen sind schwerwiegend und schwer zu beantworten: Darf ein Staat die Kinderzahl seiner Bürger beeinflussen, indem er Anreize für ein bestimmtes gewünschtes Reproduktionsverhalten setzt? Oder ist die Entscheidungsfreiheit des Einzelnen in jedem Fall ein höherwertiges Gut als die von Geburtenpolitik erhofften positiven Effekte für zukünftige Generationen, so dass schon aus diesem Grund Geburtenpolitik niemals ethisch legitimiert sein kann? Ist die Entscheidung über die Fortpflanzung ein Individualrecht, so dass das Individuum völlig selbstbestimmt entscheiden kann? Oder in welchem Maße können die Familie, der Clan, der Staat oder gar die Weltgemeinschaft für sich beanspruchen, in dieser Angelegenheit zu intervenieren? Ist selbstbestimmte Entscheidung überhaupt möglich? Ist es praktisch möglich, dass ein Staat neutral bleibt und keine bevölkerungswirksame Politik macht?

Diese komplexen Beziehungen zu untersuchen, ist für Ethiker unverzichtbar, da wir mit der Entscheidung über die Zahl unserer Nachkommen auch zugleich über ihr Wohlstandsniveau entscheiden. „Intergenerational ethics is therefore inevitably connected with population and procreation ethics (...)", schreibt Gosseries. ${ }^{370}$

In den nächsten Abschnitten wird eine ethische Bewertung von Bevölkerungspolitiken vorgenommen. Solche ethischen Argumente lassen sich auf zwei Ebenen identifizieren - auf einer abstrakt-analytischen und auf einer praktisch-politischen. Um den Gegensatz deutlich zu machen: Als die Gegner bei der internationalen Weltbevölkerungskonferenz in Kairo 1994 das beratene Aktionsprogramm als ,unethisch' geißelten, kam Parfits ,Mere Addition Paradox' in ihrer Argumentation nicht vor. Umgekehrt spielen die Texte, auf die sich die Weltgemeinschaft verständigte, und die somit auf politischer Ebene regeln, was für ethisch akzeptabel gehalten wird, keine Rolle in der einschlägigen ,reinen' philosophischen Debatte. Man könnte auch von einer abstrakten und einer angewand-

\footnotetext{
${ }^{369}$ Wichterich (1994), 9

${ }^{370}$ Gosseries (2002), 471
} 
ten ethischen Herangehensweise an das Problem sprechen, die relativ unverknüpft nebeneinander stehen.$^{371}$ Abstrakte ethische Prinzipien können Hintergrundwissen vermitteln und den Blick schärfen. Zunächst wird daher auf die Debatte über Nutzensummen- und Durchschnittsnutzenutilitarismus eingegangen. Dann wird gefragt, ob der Erhalt der Menschheit der Ausgangspunkt einer Generationenethik sein sollte.

Der Schwerpunkt in dieser Studie liegt aber auf dem induktiven Ansatz einer angewandten Ethik. ,Auch auf dem Gebiet der Bevölkerungspolitik und Familienplanung kann die ethische Beurteilung demografischer Handlungsziele oder konkreter Familienplanungsprogramme nicht einlinig aus allgemeinen sittlichen Grundsätzen oder dem obersten Moralprinzip abgeleitet werden“, schreibt der Ethiker Schockenhoff. In Situationen hoher Bevölkerungswachstumsraten (und gleichzeitiger großer Naturzerstörung) könnten tiefergehende Eingriffe in die Autonomie gerechtfertigt sein als bei geringerem Problemdruck. Meine erste Prämisse ist also, dass bevölkerungsethische Prinzipien ,situationsbezogen' sein müssen. Daneben ist meine zweite Prämisse, dass die Ziele und Mittel jeweils in sich gerechtfertigt sein müssen, bevor sie instrumentell aufeinander bezogen werden können. Das Ziel der, Generationengerechtigkeit' (und im Rahmen dessen des Erhaltes der Artenvielfalt) wurde bereits ausführlich gerechtfertigt. Dieser gute Zweck heiligt aber nicht alle Mittel, v.a. wenn dadurch das Wohl der heutigen Generation verletzt würde. Eine bevölkerungspolitische Einzelmaßnahme erweist sich dann als legitim, wenn sie erstens in sich ethisch legitimierbar ist, d.h. nicht schon wie Zwangsabtreibung oder -sterilisierung in sich ein Unrecht darstellt, und zweitens als geeigneter oder gar notwendiger Teilschritt zu einem ethisch legitimen Gesamtziel erscheint. ${ }^{372}$ Der enge Zusammenhang von Artensterben und Bevölkerungswachstum spricht für antinatalistische Politiken. Diese Zielvorgabe muss aber in Einklang gebracht werden mit dem Recht heute lebender Menschen, die Zahl ihrer Kinder eigenverantwortlich und frei zu bestimmen. Zunächst sind also die Mittel in sich zu prüfen, d.h. wir müssen untersuchen, ob geburtenpolitische Maßnahmen als solche überhaupt ethisch sein können.

\footnotetext{
${ }^{371}$ Diese Dichotomie wird auch von von Leist $(1991,355)$ und Singer $(1976,83)$ konstatiert. Leist billigt den rein philosophischen Ausführungen Klarheit auf Kosten der Praxisnähe zu, Singer weist darauf hin, dass es letztlich aber darum gehe, Prinzipien für die Praxis zu entwickeln.

${ }^{372}$ Schockenhoff (1996), 23
} 


\subsection{Ethische Bewertung von Bevölkerungspolitik in der abstrakt- philosophischen Diskussion}

\subsubsection{Nutzensummenutilitarismus}

In diesem Abschnitt wird zunächst überprüft, ob Nutzensummenutilitarismus (NSU) oder Durchschnittsnutzenutilitarismus (DNU) besser geeignet ist, um moralische Urteile über Bevölkerungspolitiken zu fällen. ${ }^{373}$ Die Unterscheidung zwischen NSU und DNU ist grundlegend für die Frage der Generationengerechtigkeit. Dabei muss es nicht zwangsläufig ,Nutzen' sein, der aufaddiert wird, es kann auch ,Glück', ,Zufriedenheit' ,Fehlen von Leid' oder etwas Ähnliches sein. Jedenfalls muss es das sein, ,was das Leben lebenswert macht'. Sprechen wir der Einfachheit halber von ,Glück'. Der Nutzensummenutilitarist addiert die Glückswerte aller Menschen in einer Gesellschaft auf.. ${ }^{374}$ Der Durchschnittsnutzenutilitarist teilt die gefundene Glückssumme durch die Zahl der Personen einer Gesellschaft. Für ihn ist diejenige Gesellschaft vorzuziehen, in der dann die Glückswerte am höchsten sind.

NSU und DNU führen im Hinblick auf die Wahl zwischen zwei Alternativen stets zum gleichen Ergebnis, wenn zwei hintereinanderfolgende Generationen gleich groß sind. ${ }^{375}$ In der wirklichen Welt variiert aber die Zahl der Individuen je Generation. In Bezug auf mögliche Bevölkerungspolitiken kommen beide Ethiken zu radikal unterschiedlichen Schlussfolgerungen. Für den NSU ist die ethisch gebotene Strategie relativ simpel: Solange jeder zusätzliche Mensch zum Gesamtnutzen beiträgt, muss die Welt weiter bevölkert werden. Selbst wenn die Erde schon von 500 Milliarden Menschen bevölkert wäre, würde ein Nutzensummenutilitarist für weiteres Wachstum plädieren, solange jeder zusätzliche Mensch sein Leben selbst als lebenswert empfindet. Es wäre allerdings nach dieser Methode unmoralisch, die Welt mit zusätzlichen Menschen zu bevölkern, die negative Glückswerte aufweisen, also ihr Leben nicht als lebenswert empfinden. ${ }^{376}$ Formal ist unter vielen Weltzuständen derjenige vorzuziehen, für den gilt:

\footnotetext{
${ }^{373}$ Vgl. zu dieser Frage auch die Sammelbände Bayles (1976) und von Barry/Sikora (1978); außerdem Parfit (1987, Kapitel 17); Birnbacher (1998; 2003); Wolf (1995, 1996, 1997); Fehige/Wessels (1998); Arrhenius (2000); Gosseries (2002). Stets geht es auch um Bevölkerungspolitik, da im wesentlichen Weltzustände mit unterschiedlichen Populationen verglichen werden.

${ }^{374}$ Die Glückswerte jedes Einzelnen ergeben sich wiederum aus den Durchschnitten aus mehr oder weniger glücklichen Zeiten (Nettoglückswert), die dieser Mensch in seinem Leben bis zum Beobachtungszeitpunkt $t_{0}$ erlebt. Wenn ich in den letzten fünf Jahren meines Lebens Nettoglückswerte von 8, 4, 6, 2 und 10 hatte, dann ist mein Durchschnitts-Nettoglückswert 6. Wenn ein zehnjähriges Individuum $A$ also bei einer Weltbevölkerung $X$ in $t_{0}$ den Glückswert 10 erhält, und ein 70jähriges Individuum B den Glückwert 12, so sind dies die Durchschnitte ihrer bisherigen Lebensjahre. Von Ausnahmen abgesehen (vgl. Fußnote 737) können die Werte nicht negativ sein.

${ }^{375}$ Singer (1976), 81

${ }^{376}$ Bayles (1976a), xix
} 
$\max \sum_{i=1}^{n} U_{i}, \forall i=1, \ldots .$, ..$^{377}$

In Abb. 17 symbolisiert die Höhe der Rechtecke das Nutzenniveau eines Mitglieds der Menschheit, die Breite der Rechtecke symbolisiert die Zahl der auf der Erde lebenden Menschen in verschiedenen Szenarios. B hat die doppelte Breite wie A. C ist nochmals doppelt so breit wie B. Nach dem NSU würde Zustand B besser sein als $\mathrm{A}$. $\mathrm{C}$ würde besser sein als $\mathrm{B}$ und $\mathrm{Z}$ wäre der bestmögliche Zustand von allen. ${ }^{378}$

\section{Abb. 17: Die abstoßende Schlussfolgerung}

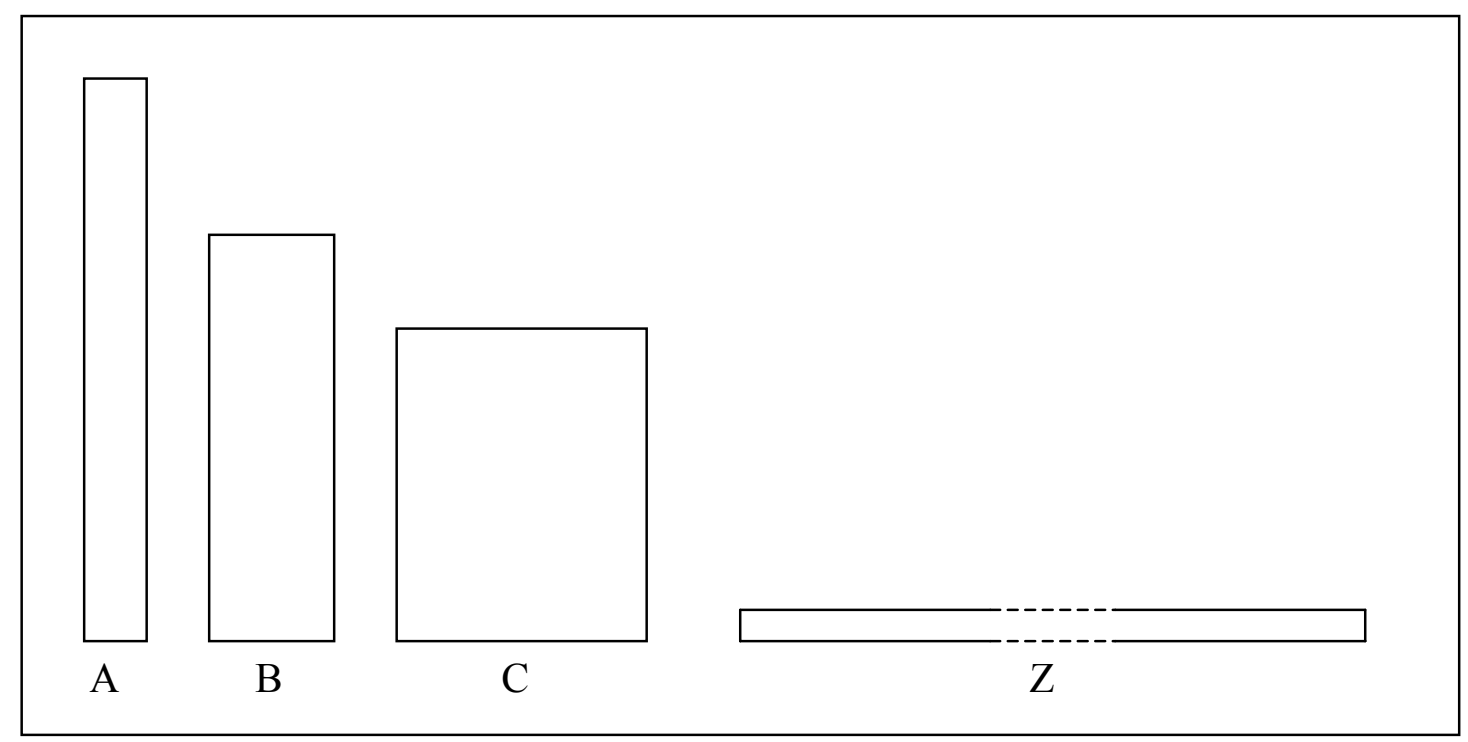

\section{Quelle: Parfit (1987), 388}

Dies bezeichnet Parfit als „repugnant conclusion“. ${ }^{379}$,Abstoßend' sei diese Folgerung, weil wir intuitiv der Meinung sind, dass es für eine Bevölkerung besser wäre, wenn weniger Menschen auf einem hohen Wohlstandsniveau existierten. ${ }^{380}$ Nach dieser Folgerung gilt aber das Gegenteil als besser: Dass eine riesige Bevölkerung auf einem Niveau dahinvegetiert, welches gerade noch ein bisschen mehr lebenswert als unlebenswert erscheint. Mathematisch gesehen geht die Zahl der Menschen gegen unendlich, das Glück bzw. die Lebensqualität jedes Einzelnen

\footnotetext{
${ }^{377}$ Es wird angenommen, dass für jedes Individuum i eine interpersonelle, kardinale Nutzenfunktion $\mathrm{U}_{\mathrm{i}}$ existiert.

378 "For any possible population of at least ten billion people, all with a very high quality of life, there must be some much larger imaginable population whose existence, if other things are equal, would be better, even though its members have lives that are barely worth living." (Parfit 1987, 388)

${ }^{379}$ Parfit (1987), 388. Narveson verwendet den Begriff, repugnant' für diesen Fall schon 1973 in einem Aufsatz für The Monist. Vol. 57. Nr.1, der inhaltsgleich mit Narveson (1976) ist.

${ }^{380}$ Leist (1991), 339
} 
asymptotisch gegen eine sehr kleine Zahl, die das absolute Überlebensminimum darstellt (nicht gegen Null). Es stellt sich die Frage, wie man feststellen kann, wann der Punkt erreicht ist, an dem die Menschen ihr Leben als unerträglich empfinden würden. Angesichts der für die Mehrheit der Menschen realistischen Möglichkeit, ihrem Leben durch Suizid jederzeit ein Ende zu setzen, kann man von einem positiven Nutzen ausgehen, solange Menschen Selbsttötung für sich selbst ablehnen. ${ }^{381}$ Da sich aber wohl auch in einer viel dichter als heute besiedelten Welt nur wenige Menschen selbst töten würde, läuft das Kriterium des NSU de facto auf eine Politik der Vergrößerung der Bevölkerung bzw. Beschleunigung des Bevölkerungswachstums hinaus.

\subsubsection{Durchschnittsnutzenutilitarismus}

Insbesondere Narveson wandte sich gegen alle utilitaristischen Konzepte, die von der Person des Menschen abstrahieren. ${ }^{382}$ Der NSU wolle nur vordergründig das ,Glück von Individuen' maximieren, in Wirklichkeit gehe es ihm um die Maximierung von ,menschlichem Glück'. Der Mensch als Wesen sei dabei lediglich der Container für das Maximandum ,Glück'. Narveson fordert, dass sich die Moralphilosophie, da sie sich mit Menschen befasst, immer am Glück des einzelnen Individuums orientieren sollte. Parfit fasst diesen Standpunkt so zusammen:

„On his view, it is not good that people exist because their lives contain happiness. Rather, happiness is good because it is good for people. Narveson also claims that, in causing someone to exist, we cannot be benefiting this person. $\mathrm{He}$ therefore claims that, of the two ways of increasing the sum of happiness - making people happy, and making happy people - only the first is good for people." ${ }^{\prime 383}$ Damit ist Narveson Durchschnittsnutzenutilitarist. Bei dem Durchschnittsnutzenkriterium würde unter vielen möglichen Weltzuständen derjenige gewählt werden, für den gilt:

$\max \frac{1}{n} \sum_{i=1}^{n} U_{i}$

,Das größtmögliche durchschnittliche Glück der in einer Gesellschaft lebenden Menschen' wird dann zur neuen Zielformel. In Bezug auf Bevölkerungspolitik

\footnotetext{
381 Ähnlich Birnbacher (1988), 85 [Fn. 23]. Wir betrachten wie gesagt Gesamtlebensverläufe. Jeder Selbsttöter hat bis zu seiner Selbsttötung einen positiven Nettoglückswert, insofern trägt er (zunächst) positiv zu Z bei. Dies gilt auch für einen Verhungernden bis zu dem Zeitpunkt seines Verhungerns oder für einen Teilnehmer eines durch zu hohe Bevölkerungdichte ausgelösten Krieges bis zu dem Zeitpunkt seines Todes.

Negative Nettoglückswerte könnten folglich nur Menschen haben, die ihr Leben nicht mehr als lebenswert empfinden und Suizid rational befürworten würden, aber durch z.B. religöse oder kulturellen Gebote an der Selbsttötung gehindert werden. Die Zahl dieser Menschen dürfte relativ gering sein.

${ }^{382}$ Narveson (1976), 66-68

${ }^{383}$ Parfit (1987), 394. Nur am Rande sei angemerkt, dass bei der personenunabhängigen Variante der Maximierung des ,Glückes auf Erden' auch das Glück von Tieren und Pflanzen in die Berechnung einbezogen werden kann.
} 
bedeutet das, dass eine pronatalistische Bevölkerungspolitik (nur) dann sinnvoll bzw. ethisch geboten ist, wenn durch Bevölkerungswachstum das durchschnittliche Glück der Generationsangehörigen erhöht wird. Nehmen wir an, dass in der Welt 6 Milliarden Menschen leben, von denen jeder 10 Einheiten Glück besitzt. Werte über 10 ergäben sich, wenn bspw. die Vertreter einer zusätzlichen Milliarde pro Person 15 Glückseinheiten aufwiesen $(10,714)$ oder wenn durch ihr Dazukommen jeder der $7 \mathrm{Mrd}$. Erdenbürger 11 Einheiten Glück aufwiese (11). Eine antinatalistische Bevölkerungspolitik wäre ethisch geboten, wenn sich bei einer Abnahme der Weltbevölkerung das durchschnittliche Glück der Generationenangehörigen vergrößern würde. Diese moralische Implikation ist derjenigen des Nutzensummenutilitarismus diametral entgegen gesetzt, der stets eine pronatalistische Bevölkerungspolitik fordert.

\subsubsection{Argumente gegen einen reinen DNU bei bevölkerungsethischen Fragen}

Es sind zahlreiche Einwände gegen den DNU vorgebracht worden, die auf kreativen Gedankenexperimenten beruhen. ${ }^{384}$ Allerdings sind diese Beispiele häufig konstruiert und wenig relevant für einen Entscheidungsträger, der heute über eine konkrete bevölkerungspolitische Maßnahme entscheiden soll. Es erscheint mir z.B. weit hergeholt, wenn gegen den DNU Weltzustände als Beispiele vorgebracht werden, in denen nur 10.000 oder gar nur zwei Menschen leben. ${ }^{385}$ Realistischerweise wird die Weltbevölkerung in den nächsten Jahrhunderten zwischen 1 Mrd. und 15 Mrd. Menschen liegen. In beiden Zuständen ist die Weiterexistenz der Menschheit gesichert.

Das ,Mere-Addition-Paradox' ist ein weiteres Argument, das gegen den DNU vorgebracht worden. ${ }^{386}$ Es lautet:

„Angenommen, man entdeckt eine Gruppe von bislang völlig isoliert lebenden Menschen (...), die auf einem relativ niedrigen Wohlstandsniveau leben. Nennen wir sie kurz „die Indianer“. Der Durchschnittsnutzen unter Einbezug der Indianer würde also sinken, und folglich wäre ein solcher Zustand zu bedauern. Eine Welt ohne diese Gruppe wäre also besser. Das scheint vor allem deshalb kaum akzeptabel, weil das Wohlstandsniveau der besser lebenden Menschen nicht ver-

\footnotetext{
${ }^{384}$ Z.B. der Hinweis, dass der DNU beim Vergleich von Leidenszuständen (negativen Nettoglückswerten) intuitiv unbefriedigende Ergebnisse bringt, wie etwa im ,Zwei-Höllen-Beispiel' bei Parfit (1987, 393) dargestellt.

${ }^{385}$ Parfit (1987), 420

${ }^{386}$ Nach Auffassung von Anton Leist ist es sogar das scharfsinnigste Argument, vgl. Leist (1991), 340.
} 
ändert würde und die Alternative für die schlechter gestellte Gruppe der Indianer Nichtexistenz wäre. “3877

Das Problem steckt hier in dem Wort ,Angenommen'. Bei diesem Beispiel geht es offensichtlich nicht mehr um die ethische Bewertung von Bevölkerungspolitiken. Ausgangsthese aller diesbezüglichen Überlegungen war, dass wir heute durch Handeln in unserer realen Welt neben den Lebensbedingungen auch die zahlenmäßige Größe zukünftiger Generationen beeinflussen können und dabei bedenken sollten, dass die Lebensbedingungen von der Größe der nächsten Generation abhängig sein können. Die Frage nach der ethischen Bewertung von Bevölkerungspolitik bezieht sich auf Zustandsänderungen, d.h. von Übergängen von einer gegebenen Bevölkerung zu einer kleineren oder größeren neuen Bevölkerung. Die Indianer sind aber schon da. Es handelt sich bei diesem Fall also gar nicht mehr um ein Zwei-Generationen-Modell, sondern um ein Ein-GenerationenModell. ${ }^{388}$ Die Basis der dritten Hypothese dieser Studie ist ein Zwei-Generationen-Modell, d.h. die heute lebende Generation beeinflusst durch ihre Handlungen das Los der nächsten Generation. Nehmen wir in folgendem Beispiel an, dass die Kinder, die ein Paar nach und nach bekommt, bestimmte Nettoglückswerte haben.

Tab. 3: Kinderzahl und Glückswert pro Kind, Fall 1

\begin{tabular}{|l|l|l|l|l|l|l|l|l|l|l|}
\hline Kinderzahl & 1 & 2 & 3 & 4 & 5 & 6 & 7 & 8 & 9 & 10 \\
\hline $\begin{array}{l}\text { Glückswert } \\
\text { je Kind }\end{array}$ & 8 & 10 & 9 & 7 & 5 & 4,5 & 4 & 3,5 & 3 & 2,5 \\
\hline
\end{tabular}

\section{Quelle: eigene Darstellung}

Eltern könnten in diesem Beispiel also moralisch handeln, wenn sie ihrem ersten Kind ein weiteres hinzufügen und sie würden moralisch handeln, wenn sie auf ein weiteres Kind verzichten, wenn sie schon zwei haben.

Wie aber sähe moralisches Handeln unter folgenden Rahmenbedingungen aus?

Tab. 4: Kinderzahl und Glückswert pro Kind, Fall 2

\begin{tabular}{|l|l|l|l|l|l|l|l|l|l|l|}
\hline Kinderzahl & 1 & 2 & 3 & 4 & 5 & 6 & 7 & 8 & 9 & 10 \\
\hline $\begin{array}{l}\text { Glückswert } \\
\text { je Kind }\end{array}$ & 7 & 7 & 7 & 7 & 7 & 7 & 7 & 7 & 7 & 7 \\
\hline
\end{tabular}

\section{Quelle: eigene Darstellung}

${ }^{387}$ Leist (1991), 340. Vgl. für ähnliche Formulierungen auch Narveson (1976), 74 und Parfit (1987), 419-441

${ }^{388}$ Singer (1976), 96-98 
Offensichtlich ist die Annahme, dass das Glück jedes Menschen eine Funktion der Größe seiner Familie (auf der Mikroebene) bzw. seiner Generation (auf der Makroebene) ist, hier außer Kraft gesetzt. Diese Annahme wird nachfolgend als Annahme 1 bezeichnet. Im Kern müssen wir also fragen, ob Annahme 1 realistisch ist. Ist sie es, dann brauchen wir uns im Rahmen der ethischen Bewertung von Bevölkerungspolitik bloß auf das durchschnittsnutzenutilitaristische Prinzip verlassen.

Annahme 1 kann meiner Meinung nach als belegt gelten, sowohl im kleinen familiären wie auch im großen, weltweiten Kontext. Natürlich besteht die Schwierigkeit, dass man Glück nicht einfach empirisch messen kann, dass Glücksgefühle kulturabhängig sind, etc. Diese Schwierigkeiten wurden bereits thematisiert und der HDI wurde als ein Indikator zur Operationalisierung von Bedürfniserfüllung vorgeschlagen. Nun nimmt das Bildungsniveau, aber auch letztlich die Gesundheit pro Kind ab, wenn man Durchschnittsverdiener, z.B. mangels Verfügbarkeit von Verhütungsmitteln, zwingt, statt zwei bis vier Kindern doppelt oder dreifach so viele Kinder zu haben. Wer die Erschöpfung von Frauen mit zehn und mehr Kindern in WEL persönlich erlebt hat, wird kaum die Ansicht vertreten, eine Mutter könne für jedes dieser Kinder ausreichend Zeit und Aufmerksamkeit aufwenden. Selbst wenn es in Entwicklungsländern für die Eltern günstiger sein mag, wenn viele Kinder ihre Rente erwirtschaften, so ist auch der Staat ist überfordert mit dem Bau von Schulen und der Versorgung mit Basisgesundheitsleistungen.

In SEL können sich die Fähigkeiten dieser Kinder sich nicht so gut entwickeln, einfach weil die Eltern i.d.R. keine finanziellen Mittel haben, um sportliche, musische und künstlerische Anlagen zu testen und zu fördern. Sie werden als Durchschnittsverdiener bei einer großen Kinderzahl nicht allen Geigenunterricht, Tennisverein und Sprachkurse im Ausland bieten können. Die individuelle Förderung je Kind ist nicht mehr so gut möglich wie bei einer überschaubaren Zahl von Kindern.

Gilt Annahme 1 im Hinblick auf die Weltbevölkerung? Solange die Erde mittelmäßig dicht bevölkert ist, mag unklar sein, ob durch ein zusätzliches Individuum der Durchschnittsnutzen aller steigt, sinkt oder unverändert bleibt. Mit immer größerer Bevölkerungsdichte schwindet diese Unklarheit: die Nettoglückswerte jedes einzelnen Individuums nehmen ab. Es ist schon aus logischen Gründen unabweisbar, dass das individuelle Glück letztlich keine von der Bevölkerungszahl unabhängige Größe sein kann.

Ich sehe daher auch keine Notwendigkeit, bei der Bewertung heutiger bevölkerungsethischer Fragen nach einem Mittelweg zwischen DNU und NSU zu suchen. Es mag kontraintuitiv sein, dass nach dem Prinzip des DNU auch eine aussterbende Menschheit bei gleichbleibendem Wohlstandsniveau nicht als negativ be- 
urteilt werden könnte, ${ }^{389}$ relevant für die Beurteilung von Bevölkerungspolitiken wie z.B. der chinesischen im Jahr 2004 ist es nicht. ${ }^{390}$

\subsubsection{Der Erhalt der Menschheit als Ausgangspunkt einer Generationenethik}

Philosophen haben seit dem Beginn der Philosophie unterschiedliche Ansichten darüber geäußert, was letztlich als intrinisch gut angesehen werden soll. Neben den bereits behandelten Maximanden ${ }^{391}$ kommt noch eine weitere Antwort in Betracht: ,menschliches Leben' ${ }^{392}$ Hans Jonas macht explizit diesen Fall zum Ausgangspunkt seiner ,Heuristik der Furcht' und dürfte damit, obwohl er dies selbst nicht klar ausspricht, DNU und NSU anders bewerten als ich dies im letzten Abschnitt getan habe.

„Handle so, dass die Wirkungen deiner Handlung verträglich sind mit der Permanenz echten menschlichen Lebens auf Erden. ${ }^{\text {“'393 }}$

Dies ist nach Jonas die Maxime, nach der wir leben sollten. Jonas erstem Imperativ folgt ein zweiter:

„Existenz des Menschen heißt einfach: dass Menschen leben.

Dass sie gut leben, ist das nächste Gebot. “394

Hier wird eine Reihenfolge aufgestellt, die den Implikationen des DNU entgegen zu laufen scheint. Wenn die reale Gefahr eines Aussterbens der Menschheit besteht, dann scheint eine pronatalistische Bevölkerungspolitik ein Gebot der Vorsicht zu sein. Damit soll Jonas nicht eine solche Schlussfolgerung unterstellt werden, aber er könnte so interpretiert werden, dass er die Gefährdungen der Existenz der Gattung Mensch und die der Lebensbedingungen der zukünftigen Generationen de facto zusammenwirft. In diese Richtung könnte man seine folgende Aussage verstehen: „Mindestens lässt sich sagen, dass die Gefahren, die das künftige Sosein bedrohen, im allgemeinen dieselben sind, die im größeren Maßstab das Dasein bedrohen, und die Vermeidung der einen a fortiori die Vermeidung der

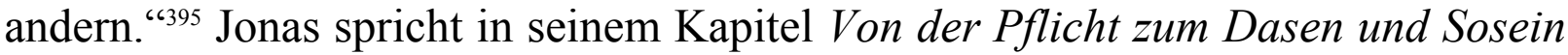
einer Nachkommenschaft überhaupt von einer Pflicht zur Fortpflanzung, auch wenn er betont, dass nicht notwendig jeder Einzelne diese Pflicht habe. ${ }^{396}$

\footnotetext{
${ }^{389}$ Leist (1991), 340; Birnbacher (1986), 37-39; Singer (1976), 97 f.

390 Überflüssig zu sagen, dass eine Übereinkunft aller 1,3 Milliarden Chinesen, ab sofort auf Familiengründungen zu verzichten, zwar denkmöglich, aber sonst in jeder Hinsicht unmöglich ist.

${ }^{391}$ Z.B. ,Wohl', ,Nutzen', ,Glück', ,Zufriedenheit' oder ,Lust'

${ }^{392}$ Callahan (1976), 23

${ }^{393}$ Jonas (1979), 186

${ }^{394}$ A.a.O.

${ }^{395}$ Siehe Jonas (1979), 87. Hervorhebung im Original.

${ }^{396}$ Jonas (1979), 86
} 
Die moralischen Konsequenzen, die sich aus der Jonas'schen ,Heuristik der Furcht' und einer ,Ethik für die Bedürfnisse der zukünftigen Menschen', ergeben, sind also nicht deckungsgleich, denn für die Generationenethik ist, wie gezeigt, der DNU der Angelpunkt. ${ }^{397}$ Der unterschiedliche Ausgangspunkt beider Konzeptionen $^{398}$ mag rein der Tatsache geschuldet sein, dass unterschiedliche Wahrscheinlichkeiten für den Fall des Aussterbens der Menschheit angenommen werden. Schon im Abschnitt 2.1. Warum Generationengerechtigkeit? wurde darauf hingewiesen, dass die Wahrscheinlichkeit, dass die Menschheit komplett ausstirbt, im Moment so gering ist, dass dieser Fall nicht zum Ausgangspunkt der Entwicklung einer Zukunftsethik gemacht werden sollte. Wie unverwüstlich das Wachstum geworden ist, illustriert ein makaberes Beispiel: Selbst beim Abwurf einer Hiroshima-Bombe pro Tag würde sich am rasanten Bevölkerungswachstum nicht viel ändern. ${ }^{399}$ Durch die Atombombe wäre zwar mit 90000 Todesfällen zu rechnen, gegenwärtig gibt es aber pro Tag rund 211.000 mehr Neugeborene als Todesfälle. Dieser makabere Vergleich soll deutlich machen, dass die Norm, das Aussterben der Menschheit zu vermeiden, zwar eminent wichtig, aber im Moment völlig unzeitgemäß ist. Höherrangige moralische Normen für Probleme, die sich nicht stellen, können aber durch eigentlich nachrangigere Normen in den Hintergrund gedrängt werden. Daher ist es im Moment angemessen, die Existenz von zukünftigen Individuen als Prämisse für jede Generationenethik zu nehmen. ${ }^{400}$

Auch wenn die heutigen Generationen die Pflicht haben, die Generationenkette nicht abreißen zu lassen und für ein Fortbestehen der Menschheit zu sorgen, sind sie nicht verpflichtet, die nächste Generation so zahlreich werden zu lassen, wie dies technisch, d.h. beim Verzicht auf jede Art von Verhütungsmitteln etc. möglich wäre. ${ }^{401}$ Ganz im Gegenteil würde dies der Pflicht heutiger Generationen, der nächsten Generation mindestens die gleichen Chancen auf Erfüllung ihrer Bedürfnisse zu erhalten, diametral zuwider laufen. Allen potenziellen zukünftigen

\footnotetext{
${ }^{397}$ Unnerstall (1999), 269. Zu einem ähnlichen Ergebnis wie Jonas kommt man, wenn man die Frage stellt: „Wie viele Generationen sollten insgesamt leben?“ bzw. „Was ist die optimale Lebensdauer der Gattung Mensch?“ Vgl. Birnbacher (1986), 34-39; Birnbacher (1988), 67-70

${ }^{398}$ Dass es diese möglichen beiden unterschiedlichen Ansatzpunkte für eine Generationenethik gibt, führte dazu, dass Sikora/Barry ihren Sammelband Obligations to Future Generations in zwei Teile ausspalteten: „Part I: Should intrinsic value be placed on the preservation of mankind“ und „Part II: Assuming that future generations will exist and that the future population size is taken as given, what obligations do we have to future generations?".

399 Wie bereits gesagt, ist eine Erhöhung der Sterberate nirgendwo auf der Welt Bestandteil einer staatlichen Bevölkerungspolitik.

${ }^{400}$ Auch Unnerstall setzt sich in dieser Weise mit Jonas auseinander (Unnerstall 1999, 268).

${ }^{401}$ Das Maximum menschlicher Fruchtbarkeit wird erreicht, wenn die reproduktive Periode voll ausgenützt würde und von der Geburt eines Kindes bis zur erneuten Empfängnis nur drei Monate vergingen. Unter diesen Bedingungen würde eine Frau, Mehrlingsgeburten ausgeschlossen, 35 Kinder gebären können. Das Guiness Buch der Rekorde gibt die größte Anzahl von Kindern, die eine Frau im Laufe ihres Lebens gebar, mit 55 an. Die Chilenin Leontida Albina bekam diese Kinder, darunter fünf Drillinge, in einem Zeitraum von 38 Jahren.
} 
Generationen ein Recht auf Gezeugt- bzw. Geborenwerden einzuräumen, ist schlicht absurd - binnen kürzester Zeit wäre die Erde von mehreren hundert Milliarden Menschen bevölkert. ${ }^{402}$

Der hinter uns liegende Abschnitt kann uns in begrenztem Umfang helfen, praktische Probleme in einen theoretischen Kontext einzuordnen. Die theoretische Debatte würde aber stärker dadurch befruchtet, dass sie praktische Probleme in ihren Kontext einbezieht als umgekehrt.

\subsection{Staatliche Bevölkerungspolitik - Verbreitung}

\subsubsection{Vorbemerkungen}

Bevor nun mit der praktisch-ethischen Bewertung von Bevölkerungspolitiken begonnen wird, werden diese zunächst beispielhaft dargestellt. Man kann nur bewerten, was man gut kennt. Nach einer Übersicht über die Verbreitung von Bevölkerungspolitiken weltweit wird dazu zunächst auf die Bevölkerungspolitik Chinas und anschließend auf den heute international gültigen, in Vertrags-Dokumenten der UN-Weltbevölkerungskonferenzen niedergelegten Konsens der internationalen Gemeinschaft zu Bevölkerungspolitik eingegangen. ${ }^{403}$ Die alternative Herangehensweise wäre gewesen, praktisch bei Null beginnend eigene Prinzipien zur Bewertung von Bevölkerungspolitiken abzuleiten. ${ }^{404}$ Mir erscheint es jedoch nicht sinnvoll, zu ignorieren, was die Weltgemeinschaft bereits beschlossen hat. Insbesondere ist das Programme of Action (PoA) der Weltbevölkerungskonferenz 1994 ein fruchtbarer Ausgangspunkt für eigene ethische Überlegungen.

\subsubsection{Meinungen zum Geburtenniveau}

Die Population Division der Vereinten Nationen befragt die Regierungen der Mitgliedsländer in regelmäßigen Abständen, wie sie ihre demografische Lage beurteilen. Die Befragung 2001 ergab, dass nur 38 Prozent aller Länder mit ihrem Geburtenniveau zufrieden sind. 58 Prozent der Entwicklungsländer und mehr als drei Viertel der Least Developed Countries (78 Prozent) bewerten ihr Geburtenniveau als zu hoch. ${ }^{405}$

\footnotetext{
402 Theoretisch könnte die Menschheit ihre Bevölkerung alle drei Jahre verdoppeln, wenn jede Frau so viele Kinder bekommen würde, wie sie biologisch gesehen austragen kann (Harrison 1994, 25).

${ }^{403}$ Die Darstellung dieses Konsenses ist selbst natürlich deskriptiv, nicht normativ.

${ }^{404}$ So wie etwa Callahan (1976) oder Schockenhoff (1996) es tun.

${ }^{405}$ Alle Zahlen dieses Abschnitts sind entnommen aus UN Population Division (2001a).
} 
Tab. 5: Regierungsmeinungen zum Geburtenniveau in Entwicklungsländern, 1976-2001

\begin{tabular}{|l|l|l|l|l|l|l|l|l|}
\hline \multicolumn{5}{c|}{ Zahl der Länder } & \multicolumn{3}{|l}{ Prozentsatz } \\
\hline 1976 & 9 & 52 & 55 & 116 & 8 & 45 & 47 & 100 \\
\hline 1986 & 13 & 50 & 67 & 130 & 10 & 38 & 52 & 100 \\
\hline 1996 & 9 & 50 & 86 & 145 & 6 & 34 & 60 & 100 \\
\hline 2001 & 10 & 51 & 84 & 145 & 7 & 35 & 58 & 100 \\
\hline
\end{tabular}

Quelle: UN Population Division (2003a), 2

Tab. 6: Regierungsmeinungen zum Geburtenniveau in den am wenigsten entwickelten Ländern, 1976-2001

\begin{tabular}{|l|l|l|l|l|l|l|l|l|}
\hline \multicolumn{4}{l}{ Zahl der Länder } & \multicolumn{3}{l}{ Prozentsatz } \\
\hline 1976 & 3 & 26 & 13 & 42 & 7 & 62 & 31 & 100 \\
\hline 1986 & 2 & 20 & 26 & 48 & 4 & 42 & 54 & 100 \\
\hline 1996 & 0 & 11 & 38 & 49 & 0 & 22 & 78 & 100 \\
\hline 2001 & 0 & 11 & 38 & 49 & 0 & 22 & 78 & 100 \\
\hline
\end{tabular}

Quelle: UN Population Division (2003a), 2

Dagegen schätzt die Hälfte der SEL (50 Prozent) die Zahl der Geburten in ihrer Bevölkerung als zu gering ein. Wie die nächste Tabelle zeigt, nahm der Anteil dieser Länder, die dieser Meinung sind, in den fünf Jahren zwischen 1996 und 2001 um zehn Prozentpunkte zu.

Tab. 7: Regierungsmeinungen zum Geburtenniveau in den Stärker entwickelten Ländern, 1976-2001

\begin{tabular}{|l|l|l|l|l|l|l|l|l|}
\hline \multicolumn{5}{c|}{ Zahl der Länder } & \multicolumn{3}{|l}{ Prozentsatz } \\
\hline 1976 & 7 & 27 & 0 & 34 & 21 & 79 & 0 & 100 \\
\hline 1986 & 9 & 25 & 0 & 34 & 26 & 74 & 0 & 100 \\
\hline 1996 & 19 & 28 & 1 & 48 & 40 & 58 & 2 & 100 \\
\hline 2001 & 24 & 23 & 1 & 48 & 50 & 48 & 2 & 100 \\
\hline
\end{tabular}

Quelle: UN Population Division (2003a), 2 


\subsubsection{Geburtenpolitiken}

Unzufriedenheit der Regierung über das Geburtenniveau muss nicht zwangsläufig zur Formulierung einer Geburtenpolitik führen. ${ }^{406}$ Tab. 8 zeigt die Verbreitung von Geburtenpolitiken.

\section{Tabelle 8: Geburtenpolitiken nach Regionen}

\begin{tabular}{|c|c|c|c|c|c|c|c|c|c|c|}
\hline & \multicolumn{10}{|c|}{ Afrika } \\
\hline & \multicolumn{5}{|c|}{$\begin{array}{l}\text { Zahl der Länder, die versuchen, ihr } \\
\text { Fertilitätsnivau... }\end{array}$} & \multicolumn{5}{|c|}{ Prozentsatz } \\
\hline & $\begin{array}{l}\mathrm{zu} \\
\text { erhöhen }\end{array}$ & $\begin{array}{l}\text { beizu- } \\
\text { behalten }\end{array}$ & $\begin{array}{l}\mathrm{zu} \\
\text { senken }\end{array}$ & $\begin{array}{l}\text { keine } \\
\text { Inter- } \\
\text { vention }\end{array}$ & Total & $\begin{array}{l}\text { zu } \\
\text { erhöhen }\end{array}$ & \begin{tabular}{|l|} 
beizu \\
behalten
\end{tabular} & $\begin{array}{l}\mathrm{zu} \\
\text { senken }\end{array}$ & $\begin{array}{l}\text { keine } \\
\text { Inter- } \\
\text { vention }\end{array}$ & Total \\
\hline 1976 & 2 & 2 & 12 & 32 & 48 & 4 & 4 & 25 & 67 & 100 \\
\hline 1986 & 3 & 3 & 21 & 24 & 51 & 6 & 6 & 41 & 47 & 100 \\
\hline 1996 & 2 & 3 & 36 & 12 & 53 & 4 & 6 & 68 & 23 & 100 \\
\hline 2001 & 1 & 3 & 38 & 11 & 53 & 2 & 6 & 72 & 21 & 100 \\
\hline \multicolumn{11}{|c|}{ Asien } \\
\hline 1976 & 2 & 9 & 14 & 12 & 38 & 5 & 24 & 38 & 32 & 100 \\
\hline 1986 & 8 & 6 & 13 & 11 & 38 & 21 & 16 & 34 & 29 & 100 \\
\hline 1996 & 7 & 6 & 19 & 11 & 46 & 15 & 20 & 41 & 24 & 100 \\
\hline 2001 & 8 & 7 & 20 & 11 & 46 & 17 & 15 & 43 & 24 & 100 \\
\hline \multicolumn{11}{|c|}{ Europa } \\
\hline 1976 & 7 & 7 & 0 & 15 & 29 & 24 & 24 & 0 & 52 & 100 \\
\hline 1986 & 8 & 6 & 0 & 15 & 29 & 28 & 21 & 0 & 52 & 100 \\
\hline 1996 & 16 & 4 & 1 & 22 & 43 & 37 & 9 & 2 & 51 & 100 \\
\hline 2001 & 15 & 5 & 1 & 22 & 43 & 35 & 12 & 2 & 51 & 100 \\
\hline \multicolumn{11}{|c|}{ Lateinamerika und Karibik } \\
\hline 1976 & 2 & 0 & 10 & 15 & 27 & 7 & 0 & 37 & 56 & 100 \\
\hline 1986 & 0 & 0 & 15 & 18 & 33 & 0 & 0 & 45 & 55 & 100 \\
\hline 1996 & 1 & 1 & 18 & 13 & 33 & 3 & 3 & 55 & 39 & 100 \\
\hline 2001 & 1 & 1 & 19 & 12 & 33 & 3 & 3 & 58 & 36 & 100 \\
\hline \multicolumn{11}{|c|}{ Nordamerika } \\
\hline 1976 & 0 & 0 & 0 & 2 & 2 & 0 & 0 & 0 & 100 & 100 \\
\hline 1986 & 0 & 0 & 0 & 2 & 2 & 0 & 0 & 0 & 100 & 100 \\
\hline 1996 & 0 & 0 & 0 & 2 & 2 & 0 & 0 & 0 & 100 & 100 \\
\hline 2001 & 0 & 0 & 0 & 2 & 2 & 0 & 0 & 0 & 100 & 100 \\
\hline
\end{tabular}

406 So trug z.B. auch Deutschland 2001 in der Rubrik ,View on fertility level' ein, dass das Geburtenniveau als zu niedrig betrachtet wird, bei der Frage nach der Bevölkerungspolitik wurde dagegen ,No intervention' angegeben. 


\begin{tabular}{|c|c|c|c|c|c|c|c|c|c|c|}
\hline \multicolumn{11}{|c|}{ Ozeanien } \\
\hline 1976 & 0 & 1 & 4 & 2 & 7 & 0 & 14 & 57 & 29 & 100 \\
\hline 1986 & 0 & 1 & 5 & 5 & 11 & 0 & 9 & 45 & 45 & 100 \\
\hline 1996 & 1 & 3 & 8 & 4 & 11 & 6 & 19 & 50 & 25 & 100 \\
\hline 2001 & 1 & 3 & 8 & 4 & 16 & 6 & 19 & 50 & 25 & 100 \\
\hline
\end{tabular}

\section{Quelle: UN Population Division (2003a), 4}

Insgesamt 131 von 193 Staaten (68 Prozent) haben explizit formulierte, auf eine Beeinflussung des Gebärverhaltens zielende Bevölkerungspolitiken. ${ }^{407} 77$ Prozent der Entwicklungsländer haben solche Politiken, 59 Prozent antinatalistische, 8 Prozent pronatalistische und 10 Prozent solche, die das gegenwärtige Fruchtbarkeitsniveau beibehalten wollen. Bei den SEL verfolgen 43 Prozent aller Länder Bevölkerungspolitiken, bei 31 Prozent der SEL sind das pronatalistische, 2 Prozent antinatalistische und 10 Prozent auf Beibehaltung des gegenwärtigen Niveaus abzielende. Die Tabelle zeigt die Aufteilung nach Regionen.

Geburtenpolitik - und somit Bevölkerungspolitik - ist also weltweit stark verbreitet. Daraus abzuleiten, dass sich die Frage nach ihrer ethischen Legitimierbarkeit gar nicht stellt, wäre verfehlt. Aber man muss sich vergegenwärtigen, dass eine solche Forderung wohl auf lange Sicht keine Chance auf Durchsetzung hätte.

\subsubsection{Zugang zu Kontrazeptiva}

Auch solche Länder, die keine explizite Bevölkerungspolitik verfolgen, werden von der UN befragt, ob sie Zugang zu Kontrazeptiva ermöglichen. Unterschieden werden dabei folgende Kategorien: ,Beschränkung des Zugangs', ,keine Unterstützung', ,indirekte Unterstützung', , direkte Unterstützung'. Mit ,direkter Unterstützung' ist gemeint, dass Regierungseinrichtungen direkt tätig werden; bei ,indirekter Unterstützung" unterstützt die Regierung lediglich die Aktivitäten von Nichtregierungsorganisationen und Familienberatungszentren. 2001 unterstützte ein Rekordwert von 92 Prozent aller Regierungen den Zugang zu Kontrazeptiva, entweder direkt (75 Prozent) oder indirekt (17 Prozent).

Unter den vielen Bevölkerungspolitiken, die weltweit zur Zeit von Regierungen umgesetzt werden, ist die chinesische besonders umstritten. Ihr wenden wir uns jetzt zu.

\subsection{Die Bevölkerungspolitik Chinas}

Als bevölkerungsstärkstes Land der Welt sieht sich China angesichts seines zum zwei Dritteln aus Gebirgen und Wüsten bestehenden Territoriums besonderen Problemen bei Ernährung und Versorgung, Ausbildung und Beschäftigung seiner

${ }^{407}$ UN Population Division (2003a), 5 
Menschen gegenüber. ${ }^{408}$ Es hat darauf mit einer besonders rigiden, weltweit einmaligen, aber auch mehrfach grundlegend revidierten Bevölkerungspolitik reagiert.

In der Zeit der Republik (1912-1949) galt die große Bevölkerung als wichtigste Ressource zur Sicherung der Zukunft einer erstarkenden Nation. Abtreibungen und Verhütungsmittel waren in China verboten. Geburtenkontrolle galt als imperialistisches Komplott zur Dezimierung des chinesischen Volkes.

Nach dem Ende der japanischen Besatzung und dem Sieg der Kommunisten im Bürgerkrieg 1945-1949 wurde am 1.10.1949 von Mao Zedong die Volksrepublik China gegründet. Erste Warnungen vor den ökologischen und ökonomischen Folgen eines ungehemmten Bevölkerungswachstums in den 50er Jahren führten zu einer ersten zögerlichen Geburtenplanungskampagne, in deren Verlauf Kontrazeptiva zugelassen und Ausnahmen vom strikten Abtreibungsverbot erlaubt wurden. Maos Diktum, dass jeder zusätzliche Mund auch zwei zusätzliche Hände bedeute, wurde aber erst durch die Hungerkatastrophe des Großen Sprungs vorwärts (1958-1961) ernsthaft in Frage gestellt. ${ }^{409}$ Durch die überstürzten Industrialisierungsversuche auf Kosten der Landwirtschaft kam es im ganzen Land zu Versorgungsengpässen. 20 Millionen Chinesen verhungerten. Nach diesem Fiasko setzte 1962 in den Städten eine zweite Geburtenplanungskampagne ein, die 1965 auch auf das Land ausgedehnt wurde. ${ }^{410}$ Ab 1963/64 wurden Verhütungsmittel kostenlos abgegeben und Abtreibungen legalisiert. In der Kulturrevolution 1966-1976, in der junge Rotgardisten das Land in der Absicht umkrempelten, alles Alte und Traditionelle auszumerzen, waren keine koordinierten bevölkerungspolitischen Maßnahmen durchsetzbar. Sie wurden erst 1971 mit der dritten Geburtenplanungskampagne (Erhöhung des Heiratsalters, Zwei-Kind-Beschränkung und Planziffern für den Bevölkerungszuwachs) wieder aufgenommen. Zwischen 1950 und 1970 war die Bevölkerungszahl von rund 550 auf 830 Millionen gestiegen, vor allem bedingt durch die seit Beginn der 1960er Jahre rapide gesunkene Sterblichkeit infolge verbesserter medizinischer Versorgung. Aus Sicht der Kader war China ,überbevölkert' und sein rasches Bevölkerungswachstum galt als Haupthindernis für die Modernisierung des Landes. Die Vorgängerin der Ein-Kind-Politik, die moderate antinatalistische Später-länger-weniger-Kampagne, funktionierte vorwiegend über Propaganda-Kampagnen, durch, Überzeugung' und sozialen Druck. Sie konnte (aus Sicht der Regierung) einige Erfolge aufweisen: Zwischen 1970 und 1980 sank das Bevölkerungswachstum von jährlich 2,6 Prozent auf ca. 1,2 Prozent. Die Gesamtfruchtbarkeitsziffer (durchschnittliche Zahl der

\footnotetext{
${ }^{408}$ Scharping (2003), 1. Viele Chinesen leben in den Einzugsgebieten des Jangtsekiang und des Gelben Flusses, die ungefähr die Fläche von der Größe der amerikanischen Oststaaten einnehmen. Die Bevölkerungsdichte dort ist eine der höchsten der Welt. Im Westen bilden Wüsten und Berge eine unüberwindliche Grenze, im Süden andere Völker (Wilson 2004, 59).

${ }^{409}$ Stoyke (2004), 16

${ }^{410}$ Scharping (2003), 1
} 
Geburten je Frau) sank im selben Zeitraum von 5,8 auf 2,7, näherte sich also dem zum Erhalt einer konstanten Bevölkerungszahl nötigen Wert von 2,1 an.

\subsubsection{Instrumente und Reichweite der Ein-Kind-Politik}

Aufgrund des Bevölkerungsmoments, d.h. der aufrückenden besonders starken Jahrgänge gebärfähiger Frauen, wuchs die Bevölkerung nominal jedoch mit fast unverminderter Geschwindigkeit weiter. 1981 überschritt die Bevölkerungszahl die Grenze von einer Milliarde.

„Eine weitere Reduzierung des Wachstums mit ,soften' Methoden und die letztendliche Stabilisierung hätte noch mehrere Generationen in Anspruch genommen und dabei die Früchte wirtschaftlicher Reformen verschlungen und die natürlichen Ressourcen des Landes durch Luft- und Wasserverschmutzung, Entwaldung und Bodenerosion und durch den enormen Verbrauch knappen Agrarlandes zum Siedlungsbau ruiniert", schreibt Stoyke. ${ }^{411}$ Deshalb entschied sich Deng Xiaoping 1979 zur Ein-Kind-Politik.

Die chinesische Ein-Kind-Politik wurde mehrfach modifiziert und insbesondere durch das Gesetz von 2003 stark gelockert, insofern kann man nur bedingt vom 25jährigen Jubiläum dieser Politik sprechen, wie es das Worldwatch-Institut im Herbst 2004 tat. ${ }^{412}$ In den ersten Jahren vertrat die kommunistische Führung aber den Anspruch, die Ein-Kind-Politik radikal in Stadt und Land durchzusetzen; die mangelnde Konsequenz früherer bevölkerungspolitischer Maßnahmen wurde als großer Fehler gesehen.

Nach der Geburt des ersten Kindes wurde der Gebrauch von Verhütungsmitteln vorgeschrieben, bei ungenehmigten Schwangerschaften war eine sofortige Abtreibung vorgesehen. Bei Verweigerung einer Abtreibung bei einer ungenehmigten zweiten Schwangerschaft drohten Sanktionen wie relativ hohe Lohnkürzungen, Wegfall von sozialen Diensten und Strafgelder.

Das gesetzlich vorgesehene Prinzip der Wahlfreiheit in Bezug auf Verhütungsmittel ist in der Praxis durch Vorschriften über Pessareinsetzungen und Sterilisationen wieder eingeschränkt worden. Nur letztgenannte Verhütungsmethoden erschienen der Bürokratie zuverlässig und kostengünstig genug. Auf dem Lande wurde für ,überzählige Kinder' in der Regel eine einmalige Strafzahlung in Höhe eines Großteils eines Jahreseinkommens gefordert. ${ }^{413}$ Von Anfang an war die EinKind-Politik ein System, das Anreize und Sanktionen einander gegenüber stellte. „Infolge notorisch knapper Mittel der Familienplanungsbehörden dominierte jedoch lange Zeit die Peitsche, nicht das Zuckerbrot", resümiert Stoyke. ${ }^{414}$ Dennoch zwangen die Widerstände gegen die Ein-Kind-Politik die Regierung mehrmals zu Kompromissen.

\footnotetext{
${ }^{411}$ Stoyke (2004), 16

${ }^{412}$ Meulenberg (2004), 31

${ }^{413}$ McElroy/Yang (2000), 390

${ }^{414}$ Stoyke (2004), 17
} 
1984 kam es zu einer regionalen Liberalisierung, die die Ein-Kind-Politik bis heute charakterisiert. ${ }^{415}$ In den meisten ländlichen Gebieten wird Ehepaaren, deren erstes Kind eine Tochter ist, eine zweite Schwangerschaft zugebilligt. Diese Regelung betrifft ca. 70 Prozent der Gesamtbevölkerung. Ein zweites Kind mit mindestens vier Jahren Abstand zum ersten ist in den Provinzen Guangdong, Hainan und Yunnan sowie in Teilen der Provinzen Hebeis und Hunans zulässig (zusammen ca. 10 Prozent der Bevölkerung). Ausnahmen von der Ein-Kind-Politik werden ebenfalls im Falle einer starken Behinderung des ersten Kindes sowie für die 56 ethnischen Minoritäten gemacht, deren zerstreute Siedlungen in unwegsamen Gebieten zu einer schlechteren medizinischen Versorgung und infolgedessen zu einem sehr niedrigen Wachstum geführt hatte. Sie machen jedoch nur ca. acht Prozent der Gesamtbevölkerung Chinas aus. ${ }^{416}$ Auf dem Lande sind aufgrund der zahlreichen Ausnahmen der Ein-Kind-Politik heute zwei Kinder die Regel. Die Familienplaner nehmen Rücksicht auf die Bauern, die nach alter Tradition (siehe unten), wegen ihrer Altersversorgung und aus praktischen Gründen noch häufig unbedingt einen männlichen Nachkommen haben möchten. Die Bauern leben vom Reisanbau, und für die harte körperliche Arbeit braucht man nach Ansicht vieler Bauern einen Sohn. Wenn das erste Kind ein Mädchen ist, darf ein zweites geboren werden. Doch wenn auch das zweite ein Mädchen ist, dann gibt es keine Erlaubnis mehr für ein drittes Kind.

Die Lohnkürzungen für Städter liegen seit 1984 bei zehn bis 20 Prozent der Löhne beider Eltern für eine Dauer von drei bis 14 Jahren. ,Überzählige' Kinder sind bis heute vom staatlichen Schulsystem ausgeschlossen und müssen auf teurere Privatschulen geschickt werden, was für die Eltern eine weitere ökonomische Belastung ist.

\subsubsection{Geschlechtswahl und Korruption}

Eines der größten Probleme der Ein-Kind-Politik ist die Verheimlichung von ungeplanten Schwangerschaften und Geburten, insbesondere bei erstgeborenen Mädchen, die dann als formal nicht existente Unterklasse von Frauen nur schwer ihren Anspruch auf Bildung und medizinische Versorgung geltend machen können. Die Geschlechtswahl des Kindes, obwohl strikt verboten, ist in Zeiten leicht verfügbarer Ultraschallgeräte schwer einzudämmen. Die Kinder, insbesondere die Söhne, sind v.a. auf dem Land wichtige Arbeitskräfte und in Abwesenheit eines staatlichen Rentensystems auch einzige Altervorsorge. Sie spielten darüber hinaus eine wichtige Rolle im Ahnenkult, für den der Stammhalter der Familie Sorge zu tragen hatte. Auf 100 geborene Mädchen kommen in China heute mehr als 117

\footnotetext{
${ }^{415}$ Xie (2000), 54

${ }^{416}$ Stoyke (2004), 17
} 
Jungen, während der globale Durchschnitt bei 105-106 Jungen liegt. In einigen Regionen Chinas werden Werte von mehr als 125:100 erreicht. ${ }^{417}$

Andere Probleme, denen sich die Regierung gegenübersieht, sind Bestechung von Kadern und Fälschung von Papieren. ${ }^{418}$ Das Ausmaß dieser Manipulationen ist schwer abzuschätzen, da einerseits der Anreiz für Datenfälschungen und Korruption hoch ist, andererseits die Zentralregierung solche Vorkommnisse auch drakonisch bestraft. ${ }^{419}$ Die Kader haben die Pflicht, überzählige Geburten anzuzeigen und Geldstrafen für ein drittes Kind zu verhängen. Das führt oft zu Konflikten und Streitigkeiten zwischen den für die Familienplanung zuständigen Funktionären und den Dorfbewohnern. ${ }^{420}$ Bestechungsversuche sind weit verbreitet. Die chinesische Regierung versucht durch drastische Strafen, darunter sogar die Todesstrafe, die Korruption zu bekämpfen. Lokale Kader, die ihre Quoten erfüllen wollen, erzwangen in der Vergangenheit in einigen Fällen Abtreibungen, auch in späten Schwangerschaftsmonaten. Solche Gewaltanwendungen übereifriger nachgeordneter Beamter sind jedoch niemals von der Führung gebilligt worden. Staatliche Angestellte und Parteikader sind in ihren eigenen Familien streng an die Ein-Kind-Politik gebunden. ${ }^{421}$

Das Angebot an kostenlosen Verhütungsmitteln und medizinischer Versorgung für Schwangere wurde im Zuge der Reformen der Ein-Kind-Politik mehrfach verbessert, auch wurden verschiedene Aufklärungskampagnen durchgeführt. Dies wurde vor allem in Zeiten von HIV/AIDS intensiviert, wodurch die Epidemie die Nation offiziellen Zahlen zufolge deutlich weniger als in Nachbarländern erfasst hat. Charlotte Höhn, die Direktorin des BiB, signalisiert dazu Zustimmung: „Auch das chinesische Großexperiment der rigorosen Ein-Kind-Politik mit für Europäern unverständlichen und abzulehnenden Anreiz- und Strafmaßnahmen ist nur deswegen von einem abstrakten Gesichtspunkt aus als einigermaßen akzeptabel einzustufen, weil die Chinesen ihre drastische Bevölkerungspolitik nicht isoliert betrieben, sondern mit einer systematischen Frauen-, Gesundheits-, Beschäftigungs- und allgemeinen Entwicklungspolitik verbanden. ${ }^{6{ }^{6422}}$

\subsubsection{Die Reform von 2003}

2003 wurde die Ein-Kind-Politik erneut grundlegend reformiert. Das neue Gesetz schreibt eine Verbindung der Geburtenkontrolle mit einer Verbesserung der Situation der Frau vor, führt Schutzbestimmungen und Arbeitsplatzsicherheit für Schwangere ein und überträgt die Verantwortung für die Verhütung Mann und

\footnotetext{
${ }^{417}$ Li/Peng (2000), 64-76. Damit liegt China allerdings noch hinter einigen arabischen Ländern. Bahrain, Oman; Katar, Saudi-Arabien und die Vereinigten Arabischen Emirate haben Werte zwischen 116:100 und 186:100 (vgl. Meulenberg 2004, 33).

${ }^{418}$ White (2000), 102-119

${ }^{419}$ Merli/Raftery (2000), 109-126

${ }^{420}$ Meulenberg (2004), 32

${ }^{421}$ Maass (16.6.2004)

${ }^{422}$ Höhn (1995), 5
} 
Frau. Im Moment wird in China unter dem Eindruck der demografischen Alterung des Landes diskutiert, die dekretierte Geburtenrationierung durch eine Besteuerung höherer Kinderzahlen abzulösen oder zu einer sozial verträglicheren ZweiKind-Politik zurückzukehren. ${ }^{423}$ Die Regionalisierung, die es den einzelnen Verwaltungsregierungen überlässt, wie sie die Geburtenpolitik umsetzen, ist weit vorangeschritten. In über der Hälfte der 31 Verwaltungseinheiten brauchen Paare, die ein zweites Kind bekommen dürfen - zum Beispiel weil das erste eine Tochter ist - nicht mehr eine vierjährige Frist zwischen erster und zweiter Entbindung einzuhalten. In der Yangtze-Metropole Shanghai dürfen nun auch Eltern in ihrer zweiten Ehe ein weiteres Kind bekommen. ${ }^{424}$ In Peking und vielen anderen Städten dürfen zwei Einzelkinder, die heiraten, heute zwei Kinder haben. Chinesen, die im Ausland studieren, dort heiraten und mit ihren ausländischen Ehepartnern nach China zurückkehren, sind von der Ein-Kind-Politik ausgenommen. ${ }^{425}$

Wo keine Ausnahmeregelungen gelten, wird seit einigen Jahren verstärkt auf Anreize statt auf Strafen gesetzt. Als Ansporn dienen Zuschüsse zu den Schulgebühren, billige Kredite und höhere Renten. Ehepaare, die sich schriftlich auf nur ein Kind verpflichten, erhalten neben einem Ein-Kind-Zertifikat von der Provinzregierung in Yunnan eine Prämie von 1.000 Yuan (rund 100 Euro). ${ }^{426}$ Dies entspricht für viele Bauern in der 43 Mio. Menschen zählenden Südprovinz einem Jahreslohn. Zudem erhalten die Paare dadurch einen Anspruch auf 750 Yuan Jahresrente im Alter. Neben Anreizen setzt die Regierung verstärkt auf Aufklärung. In einigen Dörfern und Städten der Nordostprovinz Heilongjiang können Frauen neuerdings über Telefonhotlines oder in sog. Flüsterräumen mit Ärzten sexuelle Probleme besprechen und sich über Kontrazeptiva informieren.

\subsubsection{Der chinesische Blick auf die eigene Bevölkerungszahl}

Es ist wenig überraschend, dass die antinatalistischen Maßnahmen der Staatskommission für Familienplanung von offizieller Seite als notwendig dargestellt werden. Den Tag des sechsmilliardensten Erdenbürgers im Oktober 1999 nahm China zum Anlass, eine Fortführung der strikten Geburtenkontrolle im Land zuzusichern. Auch wenn die demografische Explosion gebremst sei, bleibe die Lage sehr ernst, kommentierte die Volkszeitung, das offizielle Organ der Kommmunistischen Partei Chinas. Ohne die strikte Ein-Kind-Politik hätte die Weltbevölkerung bereits vor vier Jahren die Sechs-Milliarden-Marke überschritten, hieß es weiter. ${ }^{427}$

Wie aber wird die Ein-Kind-Politik der letzten Jahrzehnte von den Chinesen selbst bewertet? Aus westlicher Sicht würde man erwarten, dass angesichts der

\footnotetext{
${ }^{423}$ Scharping (2003), 2

${ }^{424}$ o.V. (29.05.2004), 108

${ }^{425}$ Meulenberg (2004), 32

${ }^{426}$ Maass (16.6.2004), 16

${ }^{427}$ o.V. (13.10.1999), 9
} 
massiven Eingriffe des Staates in die private Entscheidung über die Zahl der Kinder Volksaufstände losbrechen. Es ist aber keineswegs so, dass die Politik der Regierung auf den einhelligen, erbitterten Widerstand der Bevölkerung stößt. Das Ziel, die eigene Bevölkerung so bald wie möglich zu stabilisieren, wird von einer Mehrheit der chinesischen Wissenschaftler und der Normalbürger gutgeheißen. Natürlich stellt sich die Frage, inwieweit in einer Autokratie freie Meinungsäußerungen möglich sind. Aber viele ausländische Beobachter berichten über Äußerungen, die auf ein relativ hohes $\mathrm{Maß}$ an Zustimmung zu einer antinatalistischen Politik schließen lassen. In jeder Menschenschlange beschweren sich die Chinesen, dass es ihrer zu viele gebe. ${ }^{428}$

\subsubsection{Wirksamkeit der chinesischen Bevölkerungspolitik}

Wie Abb. 18 zeigt, sinkt seit der Einführung der Ein-Kind-Politik die Geburtenrate und (bei annähernd gleichbleibender Sterberate) auch die Wachstumsrate der Bevölkerung. Deutlich spiegelt der Wiederanstieg der Geburtenkurve zwischen 1980 und 1982 das Nachholen der Geburten wieder, die nach dem Beginn der Ein-Kind-Politik 1979 zunächst aufgeschoben wurden.

\section{Abb. 18: Geburtenrate, Todesrate und natürliche Wachstumsrate (je 10.000 Einwohner), 1950-2003}

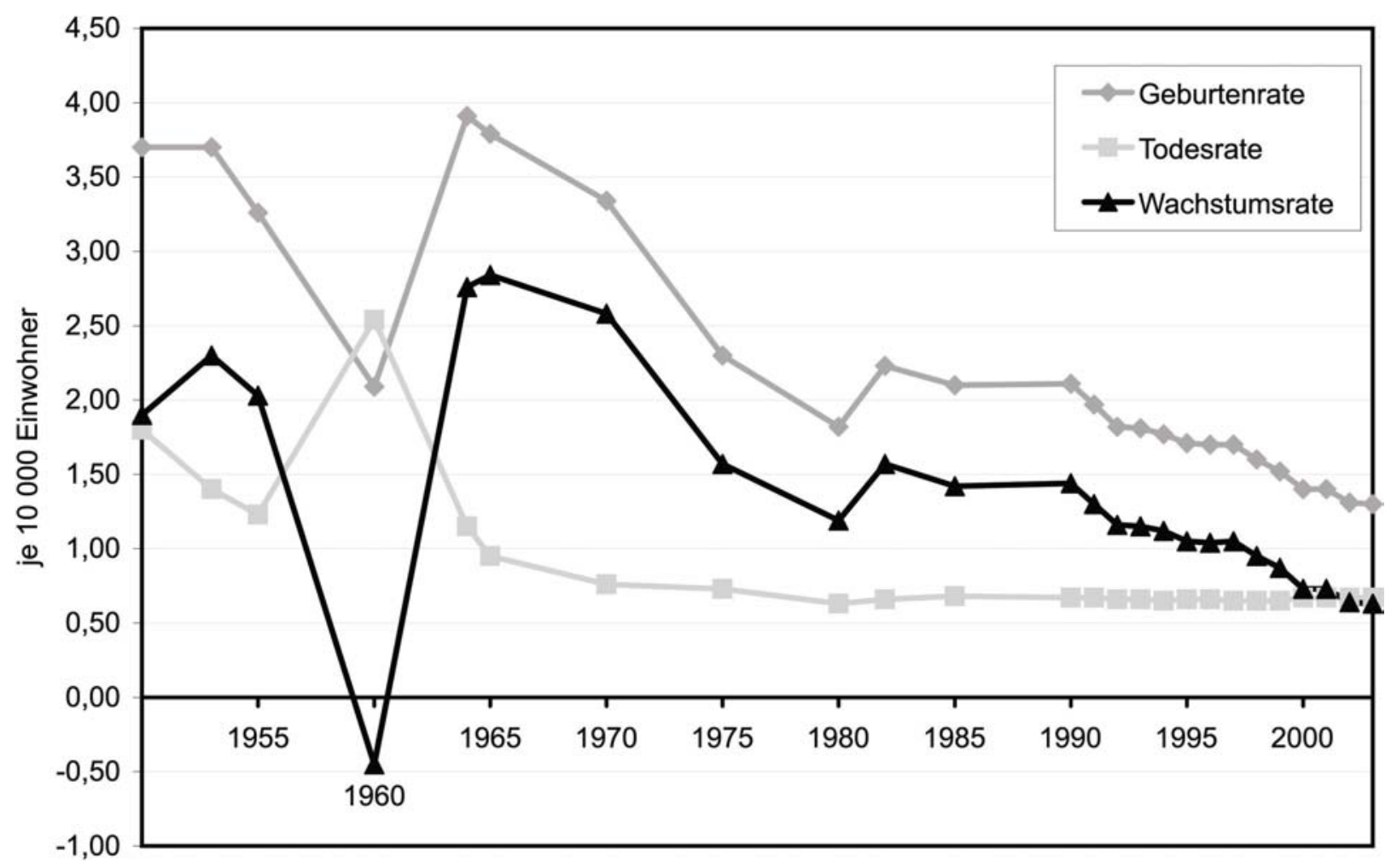

Quelle: Wong, John (2001), 70

\footnotetext{
${ }^{428}$ Blume (2003), 7
} 
Wie Abb. 19 zeigt, nahm in den Städten die Zahl der Geburten pro Frau weit stärker ab als auf dem Land. Dies spiegelt die beschriebenen Schwierigkeiten der Ein-Kind-Politik in ländlichen Regionen wieder.

\section{Abb. 19: Zusammengefasste Geburtenziffer (Kinder pro Frau), 1949- 1999}

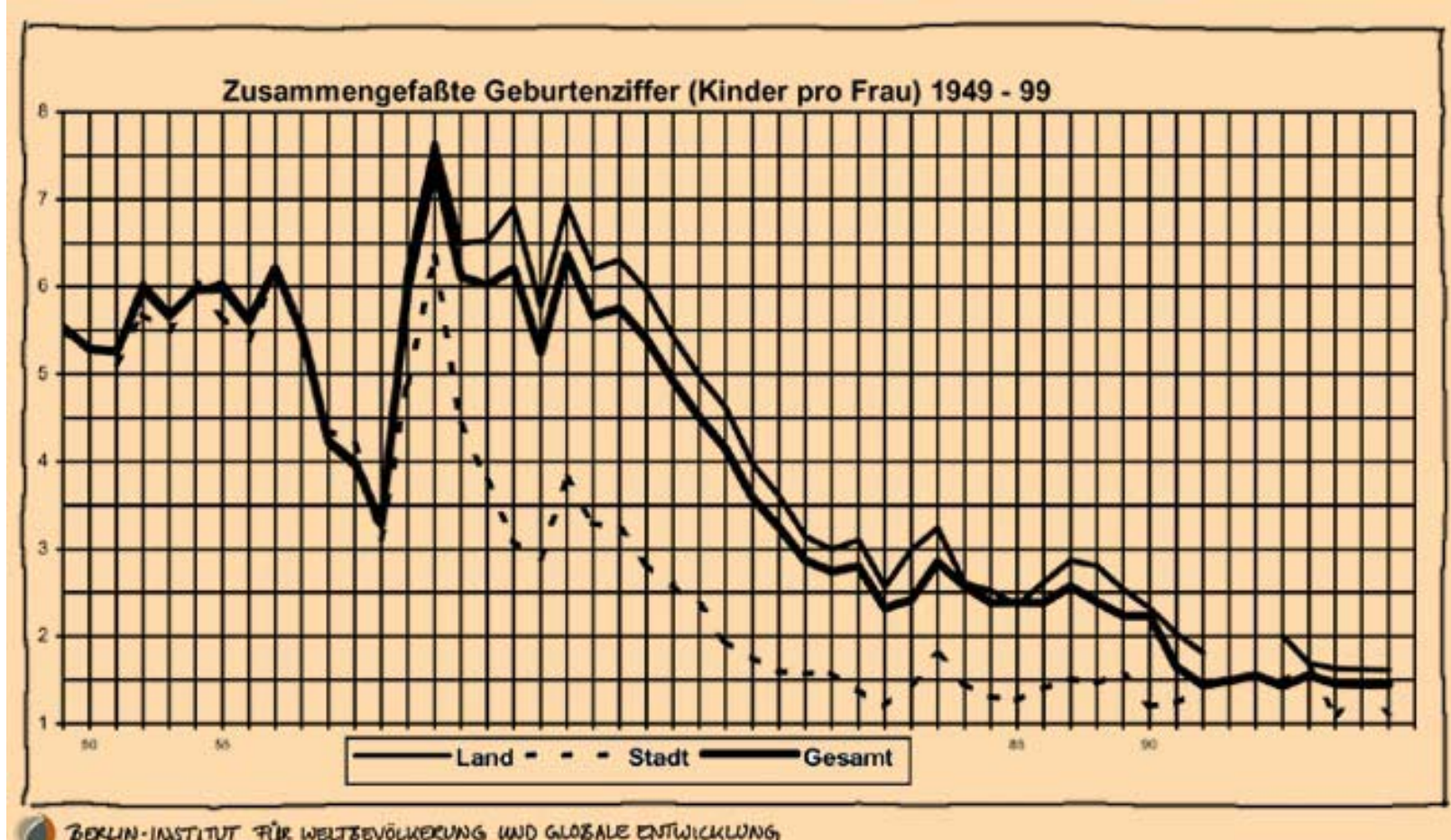

\section{Quelle: Berlin-Institut für Weltbevölkerung und globale Entwicklung, http://www.berlin-institut.org/pages/fs/fs_grafiken.html}

In den bisherigen 20 Jahren der Ein-Kind-Politik konnte das Bevölkerungswachstum auf unter ein Prozent gesenkt werden. ${ }^{429}$ Die Gesamtfruchtbarkeitsziffer liegt seit Anfang der 1990er Jahre unterhalb der Reproduktionsrate und beträgt heute ca. 1,7. Die Zahl der aufgrund der Ein-Kind-Politik unterbliebenen Geburten wird auf 340 Mio. geschätzt. ${ }^{430}$ Hält die gegenwärtige Entwicklung an, so wird sich Chinas Bevölkerungszahl in den 2050er Jahren bei etwa 1,6 Mrd. (Stand 2003: 1,3 Mrd.) stabilisieren und danach leicht rückläufig sein.

Wie Abb. 20 zeigt, war die chinesische Familienplanungspolitik wirkungsvoller als in den Nachbarländern, sofern diese ebenfalls WEL sind. Zwar haben auch andere Entwicklungsländer wie Thailand, Indonesien, Vietnam, die Philippinen und Laos in den letzten 25 Jahren Erfolge in der Reduzierung ihres Bevölkerungswachstums erzielt, aber doch deutlich geringere als China.

\footnotetext{
${ }^{429}$ Die Zahl stammt aus den offiziellen Statistiken Chinas, die nach Ansicht des Demografen Scharping mit Vorsicht zu genießen sind (vgl. Scharping 2003, 2).

${ }^{430}$ Scharping (2003), 2
} 


\section{Abb. 20: Entwicklung von Chinas Gesamffruchtbarkeitsziffer (TFR) im Vergleich zU anderen Ländern der Region 1975-1998}

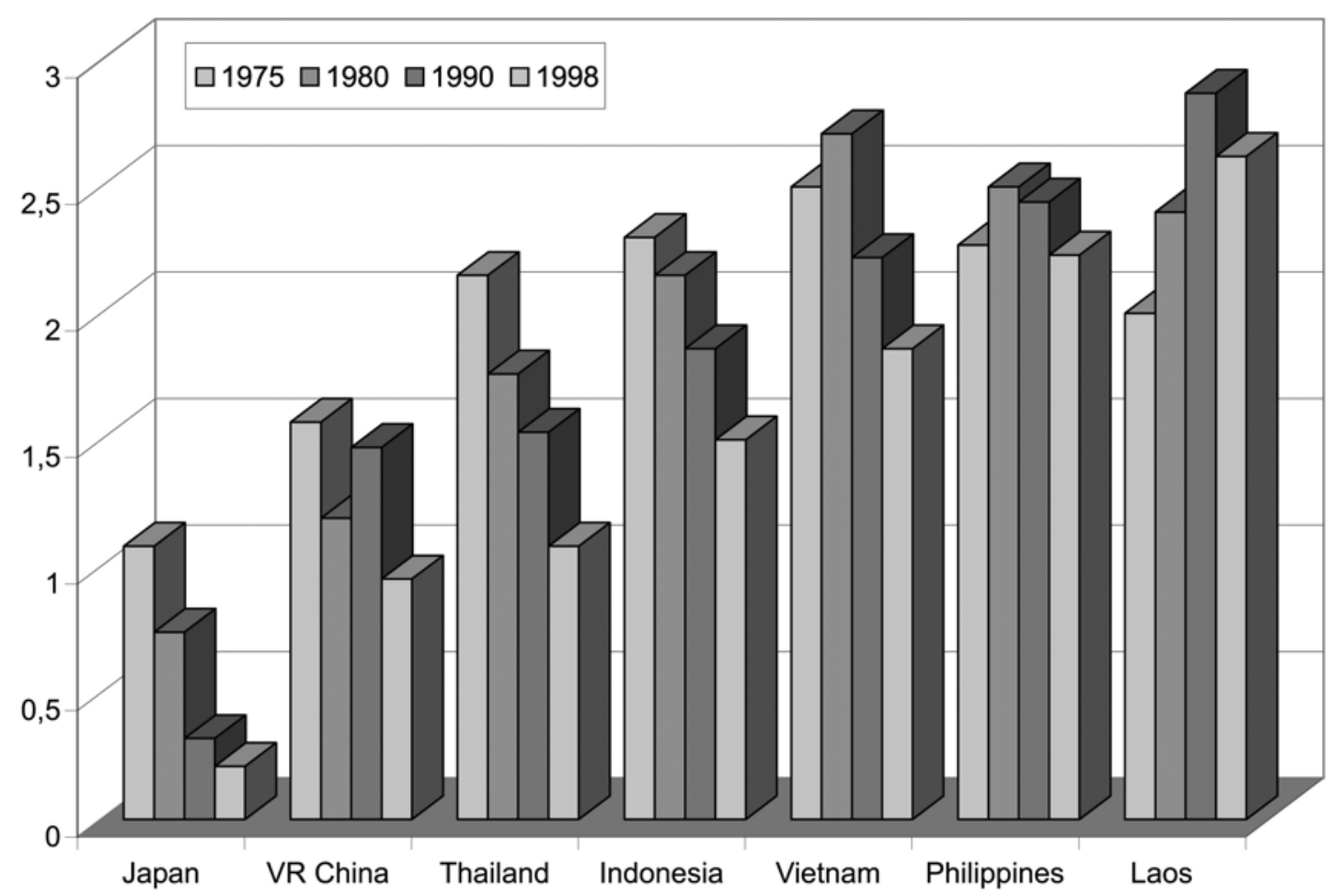

\section{Quelle: Wong (2001), 71}

Zwar stellen manche Ökonomen die Frage, inwieweit die staatliche Regulierung überhaupt den entscheidenden Faktor für die Bevölkerungsentwicklung der Volksrepublik darstellt. Ihrer Ansicht nach sind die sozioökonomischen Anreize infolge des rasanten chinesischen Wirtschaftswunders der eigentliche Motor der Geburtenreduzierung. ${ }^{431}$ Aber selbst diese relativierende Sicht bestreitet nicht die Tatsache, dass die staatliche chinesische Bevölkerungspolitik einen direkten Einfluss auf die Höhe der Geburtenrate hatte, sondern nur die Höhe der Auswirkung.

China zeigt, dass es grundsätzlich möglich ist, durch Geburtenpolitik das Reproduktionsverhalten $\mathrm{zu}$ beeinflussen. ${ }^{432}$ Eine ökologisch begründete Bevölkerungspolitik wäre also nicht schon mangels Unwirksamkeit zum Scheitern verurteilt. Chinas Geburtenpolitik wurde und wird in erster Linie aus Gründen der Ernährungssicherheit, nicht aus ökologischen Gründen durchgeführt. Nach FAO-Berechnungen hungern mit heute rund 135 Millionen (11 Prozent der Bevölkerung) auch tatsächlich 58 Millionen Chinesen weniger als noch vor einem Jahrzehnt. Dagegen hat sich der Hunger im weiter rasch wachsenden Indien mit

\footnotetext{
${ }^{431}$ So z.B. Zhang (1990), 105-123; Schultz/Yi (1995), 329-350

${ }^{432}$ Diese Aussage gilt zunächst für den Fall antinatalistischer Politiken, pronatalistische haben wir noch nicht untersucht.
} 
214 Millionen (21 Prozent der Bevölkerung) in der letzten Dekade verschärft. ${ }^{433}$ Auch nach Ansicht von westlichen Beobachtern ist die Bevölkerungspolitik maßgeblich mitverantwortlich für den wirtschaftlichen Aufschwung, der einigen hundert Millionen Chinesen einen bescheidenen Wohlstand gebracht hat. ${ }^{43}$ Auch wenn die Umweltpolitik nicht das Hauptmotiv der Bevölkerungsplaner war und ist, werden die positiven ökologischen Nebeneffekte durchaus anerkannt. ${ }^{435} \mathrm{Die}$ immensen Umweltprobleme Chinas - Luft- und Gewässerverschmutzung, Verwüstung, Rodung und Artensterben - werden dadurch gelindert. Im September 2003, schrieb das chinesische Magazin Qiushi: "Since population has always been at the core of sustainable development, it is precisely the growth of population and its demands that have led to the depletion of resources and the degradation of the environment. The reduction in birth rate, the changes in the population age structure, especially the improvement in the quality of the population, can effectively control and relieve the pressure on our nation's environment and resources and strengthen our nation's capability to sustain development." ${ }^{936}$

Das Beispiel China mit seinen verschiedenen Stadien von Bevölkerungspolitik zeigt aber auch: Ob staatliche Maßnahmen wirksam sind, hängt auch davon ab, wie konsequent sie sind bzw. wie weitreichend sie in die Entscheidungsfreiheit des Einzelnen eingreifen.

Je rigider die geburtenpolitischen Maßnahmen, desto effizienter wirken sie, aber desto ethisch problematischer sind sie auch. Dieser Zusammenhang ist so plausibel und logisch, dass wir uns nicht lange mit Beispielen aufhalten müssen. Ein empirisches Beispiel mag genügen - der Vergleich zwischen den Geburtenpolitiken Chinas und Indiens. ${ }^{437}$

\subsubsection{Die geringere Wirksamkeit der indischen Geburtenpolitik}

\subsubsection{Die indische Geburtenpolitik}

Die Geschichte der bevölkerungspolitischen Maßnahmen Indiens lässt sich unterteilen in das erste Familienplanungsprogramm 1952-1975, die Bevölkerungspolitik unter den Notstandsgesetzen 1975-1977, bevölkerungspolitische Maßnahmen bis 2000 und schließlich die aktuell verfolgte Bevölkerungspolitik seit der "National Population Policy-2000“. Indien formulierte als erster Staat weltweit, 1952 eine offizielle antinatalistische Geburtenpolitik. Jedoch wurde konkreten Maßnahmen der Familienplanung im ersten fünf Jahres Plan (19511956) nur ein geringer Stellenwert zugemessen. Man ging davon aus, dass allein

\footnotetext{
${ }^{433}$ FAO (2003)

${ }^{434}$ Maass (16.6.2004), 3

${ }^{435} \mathrm{Qu} / \mathrm{Li}(1994)$

${ }^{436}$ Zitiert nach Meulenberg (2004), $31 \mathrm{f}$.

${ }^{437}$ Zur Vertiefung der Bevölkerungspolitik Indiens, siehe: Haub (2005); Gans (2005); Bronger (1996); Lambrecht/Mertens (1989) sowie die Verlautbarungen der indischen Regierung (z.B. http://www.mohfw.nic.in/dofw\%20website/Health\%20\&\%20Poulation\%20indicators/hpi\%20frame.htm )
} 
aufgrund der zunehmenden Industrialisierung die Geburtenrate sinken würde. Aus Gründen der kulturellen Akzeptanz beschränkte sich der Staat auf die Propagierung der Rhythmusmethode - die Vermittlung anderer Methoden blieb privaten Organisationen vorbehalten. Bis 1956 wurde nur die Hälfte des zur Verfügung stehenden Etats ausgegeben. In der zweiten Fünfjahresphase (1956-1961) kam es zu einer Erhöhung des Etats und Verhütungsmaßnahmen wurden flächendeckend durch Krankenhäuser zur Verfügung gestellt. Des weiteren wurden in allen Bundesstaaten Familienplanungskomitees eingerichtet. Eine Neuerung war auch, dass Sterilisationen als zulässige Methode der Geburtenregelung anerkannt wurden. Im Jahre 1961 fand eine Volkszählung statt, die in der indischen Regierung eine Katastrophenstimmung auslöste. Die Bevölkerung war in der Dekade von 1951-1961 um ca. 80 Mio. gestiegen. Da mit diesen Zahlen niemand gerechnet hatte, begann man nun mit dem dritten Fünfjahresplan (1961-1966) das Familienplanungsprogramm um einiges ernster zu nehmen. Der klinische, angebotsorientierte Ansatz der Familienplanung wurde kritisiert und es kam nun stärker zu dem Versuch, die Bevölkerung für die Familienplanung zu motivieren. Um das Wissen über Familienplanung zu verbreiten, begann man Laien wie Lehrer, Gemeinderäte oder Hebammen auszubilden. Des weiteren kam es zum ersten Mal zu finanziellen Anreizen in Form von Prämien, die für Sterilisationen gezahlt wurden (zwischen zwei und zehn Rupien für Patienten, Ärzte und Motivatoren). Eine weitere konzeptionelle Veränderung war die Einführung der Spirale, die in Massenkampagnen propagiert wurde. Die Berichte über Nebenwirkungen verbreiteten sich jedoch recht schnell, so dass viele Frauen den Einsatz verweigerten. Generell wurden Frauen vermehrt in bevölkerungspolitische Programme einbezogen. Es entstanden Mutter-Kind Programme, die Kinderschutzimpfungen beinhalteten, um die Säuglingssterblichkeit zu senken und gleichzeitig die Mutter für Familienplanung zu sensibilisieren. Post Partum-Programme wurden in Entbindungsstationen ins Leben gerufen, da man der Ansicht war, dass sich Mütter nach Entbindungen besonders leicht für Verhütungsmethoden wie Spiralen oder Sterilisationen entscheiden würden. Ende der 60er Jahre entstanden auch „soziale Vermarktungsprogramme“ durch die, parallel zu den Krankenhäusern, Verhütungsmittel verbreitet wurden. Diese Verhütungsmittel waren einerseits staatlich subventioniert und somit erschwinglich und andererseits gelangten sie so auch in ländlichere Gebiete, in denen es keine umliegenden Krankenhäuser gab, da sie durch Lokalhändler verkauft wurden.

Mit dem vierten Fünfjahresplan (1969-1674) wurden das erste Mal Planziffern für die Senkung der Geburtenrate veröffentlicht. So sollte bis 1983/84 die Geburtenrate auf 25 Promille gesenkt werden. Eine weitere neue Maßnahme dieses Planes war die Errichtung von Sterilisationscamps einhergehend mit einer wesentlichen Erhöhung der Prämie. 1971 wurden in einem solchen Camp von 40 Ärzten ca. 60.000 Männer sterilisiert. Des weiteren trat 1972 ein Abtreibungsgesetz in Kraft, 
durch das Abtreibungen mit zu dem Familienplanungsservice gezählt werden durften, obwohl sie offiziell nicht als Verhütungsmethode anerkannt waren.

Im Jahre 1975 verhängte Indira Gandhi aufgrund großer innenpolitischer Unruhen den Aus-nahmezustand. Zu dieser Zeit kam es zu einer aggressiven Verfolgung des Sterilisations-programms, bei der das Planziel von 4,3 Mio. Sterilisationen für 1976/77 weit übertroffen wurde. Insgesamt wurden in diesen zwei Jahren 11 Mio. Sterilisationen durchgeführt. Auf allen administrativen Ebenen wurde Druck ausgeübt, um die Sterilisationszahlen in die Höhe zu treiben. Lizenzen wie z.B. Führerscheine, Geschäftslizenzen, Landregistrationen waren von Nachweisen einer Sterilisation anhängig. Besonders untere Klassen waren von Zwangssterilisationen betroffen. Aufgrund der diktatorischen Maßnahmen war die Familienplanung am Ende der Notstandregierung zu einem ,negativ besetzten Synonym für Regierungsgewalt geworden." Der heftige Widerstand in der Bevölkerung gegen dieses Programm führte 1977 zu dem Sturz Indira Gandhis.

Nach dem Sturz Indira Gandhis musste das Image der Familienplanung rehabilitiert werden. Aus diesem Grund wurde das Ministerium für Gesundheit und Familienplanung in Ministerium für Gesundheit und Familienwohlfahrt unbenannt. Es sollte nun die Deckung der Grundbedürfnisse im Vordergrund stehen - und nicht nur die bloße Reduzierung der Geburtenrate. 1996 wurden offiziell die Zielvorgaben für die Versorgung mit Verhütungsmitteln abgeschafft. Damit wendete man sich allmählich von dem zielorientierten Ansatz ab und indirekten, nachfrageorientierten Ansätzen zu. Es entstanden Programme zu Ernährung, Beschäftigung, Frauenwohlfahrt und Mutter-Kind Gesundheitsversorgung. Außerdem kam es zu weiteren Anreizen wie die der Ehrenbürgerschaft bei nur zwei Kindern. Diese beinhaltete eine spezielle Karte, die zu billigeren Versicherungen, Teilnahme an Lotterien, Pensionen und anderen Vergünstigungen berechtigte (ab 1984). Mit dieser Karte sollte man sehen, dass sich die Appelle bewahrheiten - nämlich dass Familien mit nur zwei Kindern automatisch reicher und materiell abgesicherter seien (so auch der Slogan „Small family - happy family"). Das Heiratsalter wurde von 15 auf 18 Jahre bei Frauen und bei Männern von 18 auf 21 Jahre heraufgesetzt.

Eltern mit zwei Töchtern, bei denen sich ein Elternteil sterilisieren ließ, wurden entweder ein monatliches Stipendium (50 R.) oder eine Prämie von $100.000 \mathrm{Ru}$ pien gewährt. Zudem gab es auch Anreize in Form von Sozialversicherungsgutscheinen (ab 1987), die immer Töchter und Sterilisation als Bedingung forderten. Die Präferenz vieler Inder für einen männlichen Stammhalter sollte so konterkariert werden. In einigen Bundesstaaten (z.B. Gujarat und Maharashtra) hat sich das ,Tochterprämiensystem“ durchgesetzt, allerdings in vielen anderen nicht.

Die indische Regierung beschloss 2000 mit der „National Population Policy2000“(NPP), eine neue Bevölkerungspolitik zu formulieren. Es sollten rechtliche Grundlagen geschaffen werden, die eine große Fläche an Gesundheitsbelangen von Müttern und Kindern abdecken sollten. Die NPP 2000 möchte sich weg von 
Bevölkerungskontrolle und hin zu Zielen bewegen, die die Lebensqualität der Bürger verbessert, ihren Wohlstand fördert und ihnen die Chancen und Wahlmöglichkeiten gibt, die sie zu produktiven Mitgliedern der Gesellschaft machen. Sie sieht unkontrolliertes Bevölkerungswachstum als Hindernis für die Steigerung des Lebensstandards und dafür, dass Menschen ein gesundes, produktives Leben führen können. Die Inhalte dieses Programms sind zum einen die Einbeziehung der Männer in die Familienplanung (durch die Erhöhung der Verwendungsrate von Kondomen, die ziemlich niedrig ist) und die Reduzierung der Säuglingssterberate durch den Ausbau der Gesundheitsvorsorge in ländlichen Gebieten. Es sollen ca. 100 Mio. Menschen in städtischen Slums mit Dienstleistungen versorgt werden. Ein Ziel der NPP ist es auch, soziale Bedingungen zu bessern z.B. durch vermehrte Bereitstellung von Trinkwasser und die Stärkung der Stellung der Frau. Des weiteren soll auf längere Sicht eine Hebung des Bildungsniveaus erreicht werden. Als ein großer Fortschritt wird die direkte Einbindung oberster Minister und anderer Beamter in Schlüsselpositionen durch die Gründung einer Staatlichen Kommission für bevölkerungspolitische Rahmenpläne angesehen. Denn die Umsetzung der politischen Strategie findet in Indien ähnlich wie lange Zeit in China dezentral auf der Ebene der Bundesstaaten statt.

\subsubsection{Vergleich der Geburtenpolitiken Chinas und Indiens}

Nachfolgend sollen Geburtenpolitiken von China und Indien verglichen werden. Beide Staaten sahen sich Anfang der fünfziger Jahre aufgrund der gleichen Motive gezwungen, Maßnahmen zur Reduzierung des Bevölkerungswachstums einzuführen. China wie auch Indien hätten ohne die Einführung bevölkerungspolitischer Maßnahmen immensen wirtschaftlichen wie auch sozialen Problemen gegenüber gestanden. Vor allem das Ernährungsproblem spielte in beiden Staaten eine tragende Rolle.

Auch die eingeführten antinatalistischen Maßnahmen lassen Parallelen erkennen. So besteht eine Gemeinsamkeit darin, dass beide Staaten mit der Wahl ihrer geburtensenkenden Maßnahmen einen gewissen Wandel durchliefen. Man kann sagen, dass alle Arten von Geburtenpolitiken in China wie auch Indien Anwendung fanden, wenn auch zu unterschiedlichen Zeiten und unterschiedlicher Tragweite. China wie auch Indien versuchten anfangs ausschließlich mit appellativen Ansätzen, kombiniert mit der angebotsorientierten Bereitstellung von Verhütungsmitteln, ihre bevölkerungspolitischen Ziele zu erreichen. (China bei der Propagierung der Ein Kind Familie vor 1979 und Indien mit seinen „Small family happy family“- Kampagnen).

Beide Länder halten an Plansolls und finanziellen Steuerungsmechanismen fest. Insgesamt ist Indiens Bevölkerungspolitik (vom Zeitraum 1975-1977 abgesehen) liberaler gewesen als die chinesische. Allerdings machen die großen regionalen Unterschiede eine solche Aussage schwer. Es gibt chinesische Provinzen, in de- 
nen die Kontrolle stets lax war, und es gibt indische Provinzen, in denen die Durchsetzung von Plansolls strikt eingehalten wurde.

Interessant ist es nun, die Unterschiede im Zielerreichungsgrad zu betrachten. Momentan beträgt die Bevölkerungszahl Indiens ca. 1,03 Mrd. Menschen. Die Wachstumsrate beträgt 1,5 Prozent und die Fertilitätsrate liegt bei 3,01 Kindern pro Frau. Indien rangiert mit seiner absoluten Bevölkerungszunahme damit seit 1975 an erster Stelle weltweit und wird Schätzungen zufolge China 2050 mit seiner Bevölkerungszahl überholt haben. Nur 46,2 Prozent der Familien sind durch Familienplanungsprogramme erfasst.

China hat eine Bevölkerung von 1.3 Mrd. Menschen. Die jährliche Wachstumsrate beträgt 0,7 Prozent und die Fertilitätsrate liegt bei durchschnittlich 1.83 Kindern pro Frau. Nach chinesischen Angaben sind 95 Prozent der Bevölkerung Chinas in das Familienplanungsnetzwerk involviert. Und dies obwohl auch 50,5 Prozent der Gesamtbevölkerung Chinas in ländlichen Gebieten lebt und es auch da zu Umsetzungsschwierigkeiten der geburtenpolitischen Maßnahmen kommt.

Als Ergebnis zeigt sich, dass Effizienzerfolge mit ethisch umstrittenen Maßnahmen erkauft wurden. Kurz gefasst: „Je rigider die Maßnahmen, desto effizienter die Geburtenpolitik, aber auch desto ethisch problematischer!" Möglicherweise sind solche geburtenpolitische Maßnahmen, die noch ethisch vertretbar sind, unwirksam und jene, die wirksam sind, ethisch unvertretbar. Ob der chinesische Weg ethisch vertretbar ist, müssen die weiteren Abschnitte klären. Im nächsten Abschnitt wird eine Übersicht über den internationalen Konsens der letzten Weltbevölkerungskonferenzen (z.B. in Kairo 1994 und in Ndw York 1999) gegeben. Das Aktionsprogramm der International Conference on Population and Development (ICPD) von Kairo betont die Autonomie von Paaren und Einzelpersonen. ${ }^{438}$ Es scheint somit, im Vergleich zu China, genau am anderen Ende des Kontinuums zu stehen, welches von Unfreiwilligkeit bis zu Freiwilligkeit reicht.

\subsection{Der Konsens von Kairo}

\subsubsection{Reproduktive Gesundheit als neues Paradigma}

Das trotz heftiger Kontroversen schließlich einstimmig von 179 Staaten verabschiedete $^{439}$ Aktionsprogamm von Kairo führte 1994 das Paradigma der

\footnotetext{
${ }^{438} \mathrm{BiB}$ (1994), siehe z.B. 7.3 oder 7.12

${ }^{439}$ Vorbehalte zu einzelnen Teilen gaben zu Protokoll:

- von katholischer Seite (Vatikan, zahlreiche lateinamerikanische und wenige afrikanische Länder) wurde betont, dass das Leben mit der Befruchtung beginne, dass der Begriff ,reproduktive Gesundheit' aus ihrer Sicht Abtreibung nicht definitiv ausschließe und dass sie unter Familie keinesfalls andere Gemeinschaften als die eheliche Gemeinschaft zwischen Mann und Frau verstehen wollen.
} 
,reproduktiven Gesundheit' auf der politischen Bühne ein. Um die unterschiedlichen Ansätze, die seit 1974 die Debatte bestimmt hatten, ${ }^{440}$ zu versöhnen, wurde 1994 dieser Kunstbegriff geboren, ähnlich wie zwei Jahre zuvor in Rio der Begriff „,sustainable development“. Auch wenn die einzelstaatliche Souveränität in bevölkerungspolitischen Fragen betont wird, so soll nach Ansicht der internationalen Gemeinschaft Bevölkerungspolitik stets in einen breiteren Zusammenhang eingebunden werden. ,Reproduktive Gesundheit' (RH) ist in Anlehnung an die WTO-Definition von Gesundheit definiert als ,ein Zustand uneingeschränkten körperlichen, geistigen und sozialen Wohlbefindens - und nicht nur das Nichtvorhandensein von Krankheit und Gebrechen - bei allen Aspekten, die mit den Fortpflanzungsorganen und ihren Funktionen und Prozessen verbunden sind. Reproduktive Gesundheit bedeutet deshalb, dass Menschen ein befriedigendes und ungefährliches Sexualleben haben können und dass sie die Fähigkeit zur Fortpflanzung und die freie Entscheidung darüber haben, ob, wann und wie oft sie hiervon Gebrauch machen wollen. ${ }^{\text {(4411 }}$

Unter den Gesundheitsbedürfnissen der Frauen wurden v.a. der Schutz vor sexuell übertragbaren Krankheiten und vor Infektionen der Fortpflanzungsorgane, Vor- und Nachsorge bei der Geburt, die Gesundheitsversorgung von Neugeborenen zur Verminderung der Säuglingssterblichkeit, eine gynäkologische Basisversorgung für die Mütter und die Bekämpfung unsicherer Abtreibungspraktiken gefasst.

Damit wird ein Paradigmenwechsel vollzogen, da früher BevölkerungszielgröBen (,demographic targets') im Mittelpunkt standen. Das neue Paradigma macht das Individuum mit seinen Bedürfnissen zum Maßstab für Bevölkerungspolitik. Im Vergleich zu Familienplanungsprogrammen ist das Konzept der reproduktiven Gesundheit umfassender und folgt einem integrativen Ansatz. ${ }^{422}$ Unterscheidet man Bevölkerungsprogramme in angebotsseitige und nachfrageseitige, ${ }^{443}$ so verschob sich der Schwerpunkt mit der ICPD von der Angebotsseite (d.h. der Verfügbarkeit von Verhütungsmitteln) auf die Nachfrage- bzw. Motivationsseite.

Die Vorsitzende des Bundesinstituts für Bevölkerungsforschung, Charlotte Höhn, schreibt dazu in der Zeitschrift für Bevölkerungswissenschaft: „Aus bevölkerungswissenschaftlicher Sicht ist das Konzept der reproduktiven Gesundheit ein Beispiel, wie praktische Erfahrungen der Feldarbeiterinnen wohl gerade der NGO

\footnotetext{
- von islamischer Seite wurden die gleichen Einwände gemacht, zudem wurde die Verwendung des Begriffs ,individuals' (Einzelpersonen) kritisiert. Aus ihrer Sicht sollten ausschließlich (verheiratete) Paare die Addressaten des Kapitels über RH sein.

- von europäischer Seite äußerten die nordischen Staaten Enttäuschung darüber, dass die Konzepte der reproduktiven und sexuellen Gesundheit nicht weit genug gingen und dass das Recht auf Sexualität nicht anerkannt worden sei (Rosen 1994, 72 bzw. BiB 1994, 107-125)

${ }^{440}$ Siehe dazu den Abschnitt 5.5.2. Die Weltbevölkerungskonferenzen vor Kairo

${ }^{441} \mathrm{BiB}(1994), 7.2$

${ }^{442}$ Höhn (1995), 3

${ }^{443}$ Demeny (1993/94)
} 
und insbesondere der Frauengruppen in aller Welt die wissenschaftliche Gemeinschaft überflügelt, fast möchte man sagen überrumpelt haben." ${ }^{6444}$ Tatsächlich war der Einfluss der NRO-Vertreterinnen auf den Abschlusstext enorm. Während in Mexiko 1984 noch gar keine NRO teilnahmen, waren in Kairo die Vertreterinnen der Zivilgesellschaft mit rund 4.000 Personen sogar zahlreicher vertreten als die Regierungsvertreter (3.300 Personen). Dass der beschlossene Paradigmenwechsel nicht antizipiert wurde, zeigt auch der Weltbevölkerungsbericht von 1994 der UNFPA, der führenden multilateralen UN-Organisation auf diesem Gebiet. Er geht zunächst auf die konventionellen Familienplanungsprogramme ein, um dann - knapp - auf reproduktive Gesundheitsprogramme zu sprechen zu kommen. ${ }^{445}$ Die Bevölkerungswissenschaft sah sich in Kairo auch nicht in der Lage, die Frage der Politiker nach der Quantifizierung des neuen RH-Ansatzes zu beantworten. Die Bevölkerungswissenschaft hatte sich seit Ende der 1960er Jahre mit der Erfassung und Evaluation mit Familienplanungsprogrammen befasst, die in den Einzelstaaten in nationaler Souveränität entworfen und durchgeführt wurden. Dabei wurden neben der Wirksamkeit, also des Grades der Erreichung vorformulierter Ziele, auch stets die Kosten ermittelt. So war es in Kairo bezeichnend, dass im Finanzierungskapitel die zu erwartenden Kosten von Familienplanungsprogrammen als erste und als Hauptkomponente beziffert werden konnten, wohingegen die Kosten für die Verbesserung des Gesundheitszustands (sofern er sich auf die Fortpflanzung bezieht) und für HIV/AIDS-Bekämpfung als „,finanziell zusätzliche Kosten“ hinzugeschätzt wurden. ${ }^{446}$ „Damit entspricht der Finanzierungsansatz in seinem Aufbau nicht dem im Aktionsprogramm dargestellten Konzept, in welchem eben Familienplanung nur eine Unterkategorie des umfassenderen Gesundheitskonzeptes ist“", erläutert Höhn. ${ }^{447}$

\subsubsection{Stärkung von Frauen in allen Bereichen}

Ein Großteil des Programme of Action (PoA) stellt ,Empowerment' von Frauen in den Mittelpunkt, wobei der Begriff in der offiziellen Übersetzung mit ,Stärkung', ${ }^{448}$ in den wissenschaftlichen Auswertungen aber auch mit ,Entfaltung', ,Befähigung' und ,Ermächtigung' übersetzt wird. ${ }^{449}$ Das Konzept der Stärkung der Frauen in allen Lebensbereichen geht in geradezu revolutionärer Weise über frühere Konferenzergebnisse hinaus. Nicht nur die sexuelle Selbstbestimmung der Frauen wird eingefordert, sondern auch deren wirtschaftliche Unabhängigkeit und eine Übernahme von Haushaltspflichten durch die Männer. Wie deutlich tatsäch-

\footnotetext{
${ }^{444}$ Höhn (1995), 19

${ }^{445}$ A.a.O

${ }^{446}$ A.a.O., 20

${ }^{447}$ A.a.O.

${ }^{448}$ Vgl. Kapitel IV. in ICPD (1994) und BiB (1994)

${ }^{449}$ Rosen (1994), 77
} 
liche Gleichberechtigung - auch gegen die Praxis in manchen islamischen Ländern - eingefordert wurde, mag der folgende Absatz 4.6 des PoA verdeutlichen:

„Die Träger der staatlichen Verwaltung aller Ebenen sollten sicherstellen, dass Frauen in gleicher Weise wie Männer Eigentum und Landbesitz erwerben, unterhalten und veräußern, Kredite aufnehmen und in eigenem Namen Verträge abschließen und ihre gesetzlichen Erbrechte ausüben können." ${ }^{\text {“450. Besondere }}$ Aufmerksamkeit wird den Bildungschancen von Frauen gewidmet (Kapitel XI). Bildung wird einerseits als Ziel an sich gesehen, als „Teil des Wohlergehens“ und als Voraussetzung, „um sich in der heutigen komplexen Welt zurechtzufinden.“4451 Zum anderen ist sie aber auch Mittel zur Erreichung des Ziels der baldigen Stabilisierung der Weltbevölkerung. Es wird vermutet, dass „,der zunehmende Bildungsstand von Frauen und Mädchen zu einer weiterreichenden Stärkung der Frauen, zu einem höheren Heiratsalter und zur Verkleinerung der Familiengröße beiträgt. "“52 Spätestens bis 2015 sollen Mädchen bzw. Frauen überall den gleichen Zugang zu Bildungseinrichtungen bekommen wie Jungen bzw. Männer.

\subsubsection{Der Bezug auf zukünftige Generationen}

Die ,zukünftigen Generationen' werden in dem Aktionsprogramm zwölf Mal erwähnt, v.a. im Kapitel über die Wechselbeziehungen zwischen Bevölkerung, anhaltendem Wirtschaftswachstum und nachhaltiger Entwicklung (im Folgenden: Umweltkapitel), sowie in der Präambel. ${ }^{453}$ So heißt es in Absatz 1.2:

„Weltweit werden viele der wesentlichen natürlichen Hilfsquellen erschöpft, von denen das Überleben und Wohlergehen künftiger Generationen abhängen wird, und die Umweltverschlechterung nimmt zu, bedingt durch nicht nachhaltige Produktions- und Verbrauchsstrukturen, ein noch nie dagewesenes Bevölkerungswachstum, weitverbreitete und anhaltende Armut sowie soziale und wirtschaftliche Ungleichheit. Ökologische Probleme wie beispielsweise weltweite Klimaveränderungen, die weitgehend aus nicht nachhaltigen Produktions- und Verbrauchsstrukturen herrühren, stellen zusätzliche Gefährdungen für das Wohlergehen künftiger Generationen dar." ${ }^{454}$

Es wird in Erinnerung gerufen, dass die zwei Jahre zuvor verabschiedete Agenda 21 die Prüfung der Wechselbeziehungen zwischen Bevölkerung und Umwelt der Kairo-Konferenz überließ. ${ }^{455}$ Im Aktionsprogramm wird nun klar konstatiert, dass neben dem hohem Konsumniveau in den SEL auch das Bevölkerungswachstum in den Entwicklungsländern die globale Umwelt bedroht. ${ }^{456}$ Diese

\footnotetext{
${ }^{450} \mathrm{BiB}(1994), 4.6$

${ }^{451}$ A.a.O., 11.2

${ }^{452}$ A.a.O., 11.3

${ }^{453}$ Referenzen auf zukünftige Generationen finden sich in den Absätzen 1.2, 1.11, Grundsatz 3, Grundsatz $6,3.1,3.4,3.28,6.3,6.6,6.7,14.8$ u. 16.1.

${ }^{454} \mathrm{BiB}(1994), 1.2$

${ }^{455}$ A.a.O., 3.23

${ }^{456}$ A.a.O., 3.25
} 
Trends wiederum gefährdeten die Fähigkeiten künftiger Generationen, ihren eigenen Bedarf zu decken. ${ }^{457}$ Die drei Themen Bevölkerungswachstum, Umweltkrise und Generationengerechtigkeit werden also direkt miteinander in Verbindung gebracht. Durchgängig werden im Umweltkapitel ein zu schnelles Wachstum der Weltbevölkerung und eine zu groß werdende Weltbevölkerung als Bedrohungen für das Wohl kommender Generationen angesehen. Auch wird begrüßt, dass immer mehr Länder sich zu einer antinatalistischen Bevölkerungspolitik entschlossen haben ${ }^{458}$ Fragen der Bevölkerung und auch des Wirtschaftswachstums sollen im Kontext von ,Sustainable Development', das heißt hier: immer auch im ökologischen Kontext, diskutiert werden. ${ }^{459}$

Keineswegs wird Bevölkerungswachstum als Motor für Wirtschaftswachstum gesehen. Vielmehr heißt es im Aktionsprogramm: „Maßnahmen zur Verlangsamung des Bevölkerungswachstums, zur Linderung der Armut, zur Verwirklichung des wirtschaftlichen Fortschritts, zur Verbesserung des Umweltschutzes und zum Abbau nicht nachhaltiger Verbrauchs- und Produktionsmuster verstärken sich gegenseitig. ${ }^{*}{ }^{460}$

Und Absatz 1.11 (Präambel) fasst noch einmal das generelle Anliegen zusammen: "In den kommenden 5, 10 und 20 Jahren werden intensivierte Bemühungen bei einer ganzen Reihe von Bevölkerungs- und Entwicklungsmaßnahmen erforderlich sein, wobei der entscheidende Beitrag zu bedenken ist, den eine baldige Stabilisierung der Weltbevölkerung zur Verwirklichung einer nachhaltigen Entwicklung leisten würde. Das vorliegende Aktionsprogramm behandelt all diese wie auch andere Fragen in einem umfassenden und ganzheitlichen Rahmenkonzept, das die Lebensqualität der heutigen Weltbevölkerung und ihrer künftigen Generationen verbessern soll." ${ }^{\text {461 }}$

Auch wenn der ökologische Bezug selten isoliert, sondern stets im Rahmen von ,Sustainable Development ${ }^{462}$ ausgesprochen wird, so atmet der Text doch vielerorts die Ideen des ökologischen Nachhaltigkeitskonzeptes. Trotz der revisionistischen Bemühungen v.a. von Wirtschaftswissenschaftlern wie Julian Simon waren die Gefahren einer zu großen Erdbevölkerung 1994 noch im allgemeinen

\footnotetext{
${ }^{457}$ A.a.O., 3.28

${ }^{458}$ A.a.O., 1.8

${ }^{459}$ Siehe z.B. BiB (1994), 1.9: „Die bevölkerungs- und entwicklungsrelevanten Ziele und Maßnahmen des vorliegenden Aktionsprogramms werden in ihrer Gesamtheit auf die brisanten Herausforderungen und Wechselbeziehungen zwischen Bevölkerung und anhaltendem Wirtschaftswachstum im Rahmen einer nachhaltigen Entwicklung eingehen.“

${ }^{460}$ A.a.O., 3.14

${ }^{461}$ A.a.O., 1.11

${ }^{462}$ Im Laufe der 1990er Jahre setzte sich auf UN-Ebene die Definition durch, dass ,Sustainable Development' durch die drei Säulen wirtschaftliche Entwicklung, soziale Entwicklung und Umweltschutz definiert sei. Dies wurde 2002 in der Johannesburg Declaration on Sustainable Development 2002 festgeschrieben (Tremmel 2003a, 160).
} 
Bewusstsein. Der Text enthält - plakativ gesagt - eindeutig mehr Gedankengut von Ehrlich als von Simon.

\subsubsection{Die Ausführungen zu Bevölkerungspolitik und Familienplanung}

In Kairo war das neue Paradigma der ,reproduktiven Gesundheit' v.a. wegen der darin enthaltenen Abtreibungsproblematik umstritten. ${ }^{463}$ In dieser Studie ist es hingegen daraufhin zu untersuchen, welche Art von Bevölkerungspolitik es propagiert. Bevölkerungspolitik wird im Aktionsprogramm nicht abgelehnt - das wäre auch illusorisch angesichts des Bezugs auf nationale Souveränität und der weiten Verbreitung solcher Politiken - aber es wird klar gesagt, wie bevölkerungspolitische Maßnahmen aussehen sollen. Wie bereits gezeigt, ist Familienplanung im Aktionsprogramm in den breiteren Ansatz von RH integriert. Die alles überwölbende Leitidee ist „,das Grundrecht aller Paare und Individuen, frei und verantwortlich über die Anzahl, den Geburtenabstand und den Zeitpunkt der Geburt ihrer Kinder zu entscheiden und über die diesbezüglichen Informationen und Mittel zu verfügen. ${ }^{6464}$ Der zweite Teil dieser Formel bringt den Staat ins Spiel, da ihm aufgetragen wird, Abhilfe zu schaffen, wenn Paare keinen Zugang zu Verhütungsmitteln haben. Und zwar sollten Familienplanungsprogramme nicht nur den Zugang zu einem oder wenigen Kontrazeptiva gewährleisten, sondern darauf achten ,eine vollständige Auswahl an ungefährlichen und wirksamen Methoden zur Verfügung zu stellen“. ${ }^{465}$ Es wird konstatiert, dass „,das volle Angebot moderner Familienplanungsmethoden für mindestens 350 Millionen Paare in der Welt noch immer nicht verfügbar" sei. ${ }^{466}$

Im Aktionsprogramm der ICPD wird nicht nur jede Form von Zwang im Rahmen von Familienplanungsprogrammen abgelehnt, es wird den Regierungen auch empfohlen, von Plansolls Abstand zu nehmen: „Demografische Ziele sollten, auch wenn sie zu Recht Gegenstand staatlicher Entwicklungsstrategien sind, den

\footnotetext{
463 Hier standen sich die Frauengruppen, die USA, die skandinavischen Länder und einige Entwicklungsländer wie Indien, Bangladesch, Indonesien, Brasilien und Mexiko auf der einen Seite, sowie der Vatikan, einige katholische Länder und zahlreiche islamische Länder lange unversöhnlich gegenüber, bis eine Kompromissformel gefunden wurde. Danach kann jedes Land im Rahmen seiner Souveränität die juristische/ethische Lösung des Problems des Schwangerschaftsabbruchs wählen. Einig war man sich bei der Bekämpfung unsachgemäßer Methoden und bei dem Prinzip, Schwangerschaftsabbruch dürfe keine Methode der Familienplanung sein (Absatz 8.25). Die Sonderproblematik der Abtreibung hat mehrere Bevölkerungskonferenzen schwer belastet, und Änderungen in der Gesetzgebung haben oft die Geburtenraten stark beeinflusst, aber trotzdem ist sie im Rahmen der ethischen Bewertung von Geburtenpolitiken relativ uninteressant, weil alle Ethiker und Politiker sie als Methode der Bevölkerungspolitik ablehnen. Eine Wiedergabe der ethischen Debatte über den Interessenkonflikt zwischen Embryo und Mutter, also die Bewertung von Abtreibung als solcher, ist aus Gründen einer sinnvollen thematischen Begrenzung der Fragestellung hier nicht möglich.

${ }^{464} \mathrm{BiB}(1994)$, siehe z.B. 7.3 oder 7.12

${ }^{465}$ A.a.O., 7.12

${ }^{466}$ A.a.O., 7.13
} 
Trägern von Familienplanungsdiensten nicht in Form von Sollvorgaben oder Quoten für die Anwerbung von Betroffenen auferlegt werden. ${ }^{“ 467}$ In Bezug auf bestehende Programme wird gesagt: „Allen staatlichen Verwaltungsebenen wird eindringlich nahegelegt, Systeme zur Überprüfung und Bewertung von anwenderorientierten Diensten auszuführen, um einen Missbrauch seitens der Leiter und Träger von Familienplanungsdiensten aufzudecken, zu verhindern und zu überwachen und eine ständige Verbesserung der Qualität der Dienstleistungen sicherzustellen. Hierzu sollten die Regierungen für die Beachtung der Menschenrechte und der sittlichen und berufsethischen Normen bei der Erbringung von Familienplanungs- und damit zusammenhängenden reproduktiven Gesundheitsdiensten (...) Sorge tragen (...). . ${ }^{6468}$

Auch wenn (in anderen Kapiteln des PoA) mehrfach eine Stabilisierung der Weltbevölkerung als wünschenswert bezeichnet wird ${ }^{469}$ so enthält das Kapitel über Familienplanung (Abs. 7.12 - 7.26) keinerlei Empfehlungen über eine Begrenzung der Kinderzahl. Dies hätte als Einflussnahme in die freie Entscheidung der Individuuen bzw. der Paare ausgelegt werden können.

\subsubsection{Klares Bekenntnis zum Durchschnittsnutzenutilitarismus}

Im Hinblick auf die philosophische Debatte über Nutzensummenutilitarismus und Durchschnittsnutzenutilitarismus fällt auf, dass im Aktionsprogramm von Kairo eine nutzensummenutilitaristische Betrachtungsweise nirgendwo auftaucht. Vielmehr heißt es etwa in Abs. 3.16: „Das Ziel ist es, die Lebensqualität aller Menschen durch angemessene bevölkerungs- und entwicklungspolitische Grundsatzkonzeptionen und Programme zu erhöhen (...).“470 Und an anderer Stelle: „Bei der Ausübung dieses Rechts [auf freie Entscheidung über die Zahl ihrer Kinder, J.T.] sollten die Menschen die Bedürfnisse ihrer bereits lebenden und ihrer zukünftigen Kinder sowie ihre Verpflichtungen gegenüber der Gemeinschaft berücksichtigen. ${ }^{6471}$ Deutlich sprach auch die norwegische Ministerpräsidentin Brundtland in ihrer Eröffnungsrede diese Frage an: „People's needs will suffocate under their numbers. ${ }^{“{ }^{4} 72}$ Obwohl der NSU in der Philosophie immerhin noch als Minderheitenposition existiert, spielt er also im Aktionsprogramm von Kairo keine Rolle.

\subsubsection{Das Finanzierungs-Kapitel}

Bis zum Jahr 2015 soll für alle Menschen in den Entwicklungs- und Schwellenländern der Zugang zu einem breiten Angebot an Familienplanung und eine be-

\footnotetext{
${ }^{467}$ A.a.O., 7.12

${ }^{468}$ A.a.O., 7.17

${ }^{469}$ A.a.O., z.B. in 1.11 oder 6.3

${ }^{470}$ A.a.O., 3.16. Siehe auch 6.3 u. 7.3 .

${ }^{471}$ A.a.O., 7.3

${ }^{472}$ Brundtland (1994), 33
} 
gleitende Gesundheitsversorgung offen stehen. Den Einzelstaaten wird im PoA aufgetragen, ,den Umfang des nationalen ungedeckten Bedarfes an hochwertigen Familienplanungsdiensten zu veranschlagen“ und „Schritte zu unternehmen, um den Familienplanungsbedarf ihrer Bevölkerungen baldmöglichst zu decken“ spätestens bis 2015.473 Dabei ist zu bedenken, dass wegen des Bevölkerungswachstums ,die Zahl der Paare im fortpflanzungsfähigen Alter um ungefähr 18 Millionen pro Jahr ansteigen“4474 wird. „Um ihrem Bedarf zu entsprechen und die bestehenden großen Lücken in der Bereitstellung von Dienstleistungen zu schließen, wird sich das Angebot an Familienplanung und Kontrazeptiva in den nächsten Jahren sehr schnell vergrößern müssen." ${ }^{\text {"475 }}$ Die Kosten für die Umsetzung des Aktionsprogramms werden mit jährlich 17 Milliarden US-Dollar bis zum Jahr 2000, 18,5 Milliarden bis 2005 und auf 21,7 Milliarden bis 2015 veranschlagt. ${ }^{476}$ Davon sollten zwei Drittel die Entwicklungsländer selbst und ein Drittel die SEL übernehmen. ${ }^{477} \mathrm{Im}$ einzelnen entfallen auf die Komponente „Familienplanung“ 10,2 Mrd. \$ bis 2000, 11,5 Mrd. \$ bis 2005, 12,6 Mrd. \$ bis 2010 und 13,8 Mrd. \$ bis 2015. Für die Komponente „reproduktive Gesundheit“ (sofern nicht schon in der Komponente „Familienplanung“ miterfasst) sollen in den entsprechenden Zeiträumen 5 Mrd. \$, 5,4 Mrd. \$, 5,7 Mrd. \$ und 6,1 Mrd \$ ausgegeben werden. Gesondert ausgewiesen sind die Kosten für ein Programm zur Bekämpfung von sexuell übertragbaren Krankheiten (insb. HIV/AIDS) in Höhe von 1,3 Mrd. \$, 1,4 Mrd. \$, 1,5 Mrd. \$ und 1,5 Mrd \$ bis 2015. Schließlich werden als letzter Punkt die Maßnahmen für Forschung, Datenerhebung und Evaluation von Bevölkerungs- und Entwicklungsprogrammen mit 500 Mio \$, 200 Mio \$, 700 Mio \$ und 300 Mio $\$$ in den entsprechenden Zeiträumen veranschlagt. ${ }^{48}$ Das Aktionsprogramm hält fest, dass diese Mittel nicht ausreichen werden, um die angestrebte volle Gleichberechtigung von Frauen in allen Bereichen herzustellen und um die ehrgeizigen Ziele im Bildungsbereich und Bereich der Armutsbekämpfung zu verwirklichen. ${ }^{479}$ Dafür sollen die Geberländer zusätzliche Mittel aufbringen, die aber nicht quantifiziert werden.

Die ,zwei Drittel/ein Drittel'-Regelung des quantifizierten Finanzbedarfs ist vor dem Hintergrund zu sehen, dass 1994 noch 80 Prozent aller Finanzmittel für Familienplanungsmaßnahmen von den Entwicklungsländern selbst aufgebracht wurden, die diese Programme im Rahmen ihrer Entwicklungsstrategien in eigener Souveränität durchführten. ${ }^{480}$ Wegen des (schon allein durch das Bevölkerungs-

\footnotetext{
${ }^{473} \mathrm{BiB}(1994), 7.16$

${ }^{474}$ A.a.O., 7.13

${ }^{475}$ A.a.O.

${ }^{476}$ A.a.O., 13.15

${ }^{477}$ A.a.O., 13.16

${ }^{478}$ A.a.O., 13.15

${ }^{479}$ Die dafür benötigten Mittel wurden auf späteren Konferenzen wie dem Millenium Summit der UN quantifiziert.

${ }^{480}$ Höhn (1995), 20
} 
wachstum) bedingten steigenden absoluten Bedarfs an Kontrazeptiva wird nun ein größerer Beitrag der reicheren Länder eingefordert.

Im Rahmen der Verwirklichung des RH-Programms fordert das Aktionsprogramm von Kairo von den SEL weitere Maßnahmen: „Die internationale Gemeinschaft sollte auch Maßnahmen wie den Technologietransfer an Entwicklungsländer in Erwägung ziehen, um es ihnen zu ermöglichen, hochwertige Kontrazeptiva sowie andere Güter, die für reproduktive Gesundheitsdienste wesentlich sind, selbst herzustellen und zu verteilen, um die Selbstversorgungsfähigkeit dieser Länder zu stärken. Auf Ersuchen der betroffenen Länder sollte die WHO weiterhin Ratschläge zu Qualität, Sicherheit und Wirksamkeit der Familienplanungsmethoden erteilen. ${ }^{6481}$ Bezogen auf die Forschung wurde beschlossen: „Die Regierungen sollten mit Unterstützung der internationalen Gemeinschaft und Gebereinrichtungen, des privatwirtschaftlichen Bereichs, NRO und des Hochschulbereichs die biomedizinische, technologische, klinische, epidemiologische und sozialwissenschaftliche Grundlagen- und angewandte Forschung in verstärktem Maße unterstützen, um die Dienstleistungen im Bereich reproduktive Gesundheit auf eine festere Grundlage zu stellen, einschließlich der Verbesserung vorhandener bzw. Entwicklung neuer Methoden zur Fertilitätsregulierung, die auf die Erfordernisse der Anwender abgestimmt sowie annehmbar, leicht anwendbar, ungefährlich, frei von Lang- und Kurzzeitnebenwirkungen und von Auswirkungen bei der zweiten Generation sind, wirksam, erschwinglich und sowohl für unterschiedliche Altersgruppen und kulturelle Gruppen als auch für verschiedene Abschnitte des reproduktiven Zyklus geeignet sind. ${ }^{“ 482}$

\subsubsection{Die Weltbevölkerungskonferenzen vor Kairo}

\subsubsection{Die Debatte 1945-1974}

Wie ist das Ergebnis von Kairo im Hinblick auf frühere Weltbevölkerungskonferenzen einzuordnen? Nach dem Zweiten Weltkrieg schossen die Bevölkerungswachstumsraten vieler Länder in die Höhe. Die Zeit, in der die Menschheit um eine weitere Milliarde wuchs, schien sich immer mehr zu verkürzen. Unter diesen Umständen gab es einen internationalen Konsens, die Geburtenraten zu senken und so das Bevölkerungswachstum zu verlangsamen. Wissenschaftler betonten in ihren Studien, dass hohe Bevölkerungswachstumsraten in einem geringeren Wirtschaftswachstum, in Risiken für die Nahrungsmittelversorgung und in gesellschaftlicher und politischer Instabilität resultierten, später kam noch das Argument der Ressourcenknappheit hinzu. ${ }^{483}$ Bereits 1958 hatten Coale und Hoover ein mathematisches Modell aufgestellt, um nachzuweisen, dass Bevölkerungswachstum Industrialisierung und Wirtschaftswachstum gefährden kann. ${ }^{484}$ Sie schätzten,

\footnotetext{
${ }^{481} \mathrm{BiB}$ (1994), 7.25

${ }^{482}$ A.a.O., 12.12

${ }^{483}$ Coale/Hoover (1958); Notestein (1964); Ehrlich (1968); Piotrow (1973)

${ }^{484}$ Coale/Hoover (1958)
} 
dass Indien sein Pro-Kopf-Einkommen um 40 Prozent steigern würde, wenn es ihm gelänge, sein Geburtenniveau um 50 Prozent innerhalb einer Generation zu reduzieren. ${ }^{485}$ Einige asiatische Länder begannen zuerst damit, ihre hohen Geburtenraten aktiv zu senken. Die Japaner waren darin nach dem Krieg die ersten, bald gefolgt von Indien, das 1952 das erste eigentliche bevölkerungspolitische Programm verabschiedete. Ein paar Jahre später zogen auch Pakistan und Sri Lanka (damals noch Ceylon) sowie Ende der 60er Jahre mehrere andere asiatische Staaten nach. Obwohl sich diese Länder in dem Ziel einig waren, hohe Bevölkerungswachstumsraten zu verlangsamen, gab es Kontroversen darüber, wie dies am besten erreicht werden könne. ${ }^{486}$ Auf der einen Seite standen die vielen Entwicklungsländer und einige Sozialwissenschaftler, die in hohen Fertilitätsraten eine rationale und durchaus verständliche Reaktion auf die Unterentwicklung sahen: Subsistenzwirtschaft, Fehlen organisierter Sozialversicherungs- und Bildungssysteme, sowie relativ niedrige Kosten und hohe Gewinne bei großer Kinderzahl. ${ }^{487}$ Ihrer Meinung nach konnte nur durch Entwicklung und soziale Modernisierung eine Veränderung des Kosten-Nutzen-Verhältnisses von Kinderreichtum herbeigeführt und dadurch die Fertilität gesenkt werden. Belegt sahen sie dies durch Studien, die zeigten, dass die Fertilität sehr stark vom Bildungsniveau der Frauen, deren Wohnumfeld (städtisch oder ländlich) und deren Einkommen beeinflusst war. Ihr Motto war: „Entwicklung ist das beste Verhütungsmittel!" Auf der anderen Seite standen jene, die propagierten, dass v.a. Kontrazeptiva benötigt würden. Sie sahen Entwicklung und eine Stabilisierung der Geburtenraten als zwei getrennte Themen an. Das zweite Ziel ließe sich theoretisch (und kostengünstiger) auch ohne das erste erreichen. Die Verringerung der Geburtenraten würde automatisch für mehr Wohlstand sorgen.

\subsubsection{Bukarest 1974}

1974 - mitten im Kalten Krieg - hielten die Vereinten Nationen ihre erste Weltbevölkerungskonferenz in Bukarest ab. Die USA und viele ihrer Verbündeten strebten an, die Völkergemeinschaft davon zu überzeugen, dass ein schnelles Bevölkerungswachstum für Entwicklung, Frieden und Sicherheit hinderlich sei, und dass ein Aktionsplan aufgestellt werden müsse, der die Länder zu einer internationalen Kampagne für einen Rückgang der Fertilität insbesondere durch Familienplanungsprogramme verpflichtete. „Dem stand eine Koalition katholischer, sozialistischer und Drittweltländer entgegen, die in Frage stellte, ob ein schnelles Bevölkerungswachstum wirklich eine derartige Katastrophe darstellen würde. Andere wandten sich gegen die neo-kolonialistischen Untertöne in den Zielsetzungen der Industrienationen“, so fasst Sinding, der Direktor von IPPF, die Ausgangslage

\footnotetext{
${ }^{485}$ A.a.O., 13

${ }^{486}$ Sinding (2003), 1

${ }^{487}$ Sinding (2000)
} 
zusammen. ${ }^{488}$ Karl Marx hatte die Probleme, die Malthus dem Bevölkerungswachstum anlastete, stattdessen auf die ungerechte Verteilung von Macht und Wohlstand zurückgeführt, eine Position, die damals in den Entwicklungsländern viele Anhänger hatte.

Angesichts der Grundstimmung der 1970er Jahre konnte es ,auf dem Papier' nicht anders enden als in einem Sieg der entwicklungsorientierten und sozialistischen Weltanschauungen über Neomalthusianismus und Familienplanung. Das Ergebnis der Bukarester Weltbevölkerungskonferenz, der Weltbevölkerungsaktionsplan (WPPA), ähnelte in seinen Kernpunkten einem unkonditionierten Entwicklungshilfeprogramm. Gefordert wurden Verbesserungen des allgemeinen Lebensstandards sowie eine fairere Verteilung des Reichtums und der Chancengleichheit auf der Welt. Bevölkerungspolitisch regte es die Länder dazu an, den Zugang zu Familienplanungsdiensten ,für diejenigen, die diese wünschen“ zu erweitern. Bereits damals wurden also die Rahmenbedingungen dafür geschaffen, jedem und jeder als ein menschliches Grundrecht das Wissen und die Mittel zugänglich zu machen, um die Anzahl und den Altersabstand der eigenen Kinder selbst zu bestimmen. ${ }^{489}$ Die Formel, dass Paare und Einzelpersonen über Anzahl und Geburtenabstände ihrer Kinder frei, informiert und verantwortlich entscheiden, der Staat jedoch dafür sorgt, dass dieses Recht auch in der Praxis ausgeübt werden kann, war also im Grunde schon in Bukarest gefunden worden. Der WPPA enthielt keine quantitativen Zielgrößen für eine angestrebte durchschnittliche Kinderzahl ${ }^{490}$ Er betonte auch bereits die Souveränität einzelner Staaten in bevölkerungspolitischen Fragen, v.a. auf Wunsch zahlreicher afrikanischer Länder, die kurz davor die Unabhängigkeit errungen hatten. ${ }^{491}$

Sobald sich der propagandistische Schlachtenlärm verzogen hatte, begannen zur großen Überraschung der Experten Dutzende von Entwicklungsländern, Geburtenpolitik einen höheren Stellenwert einzuräumen. Indien, das in Bukarest eine erklärtermaßen sozialistische Haltung eingenommen hatte, führte nur zwei Jahre danach seine inzwischen verrufene Zwangssterilisationspolitik ein. Weitere drei Jahre später war es China, einer der Anführer der anti-amerikanischen Position in Bukarest, das seine berüchtigte Ein-Kind-Politik formulierte. ${ }^{492}$

\subsubsection{Mexiko 1984}

Die zweite Weltbevölkerungskonferenz fand 1984 in Mexiko City statt. Zu diesem Zeitpunkt hatten die meisten Länder der Welt bevölkerungspolitische Richt-

\footnotetext{
${ }^{488}$ Sinding (2003), 2. Siehe auch Macura (1987)

${ }^{489}$ In Abs. 29a heißt es: "All countries should respect and ensure the right of persons to determine, in a free, informed and responsible manner, the number and spacing of their children..."(zitiert nach UN Population Fund 2003, 16).

${ }^{490}$ Höhn (1995), 5

${ }^{491}$ UN Population Fund (2003), 16

${ }^{492}$ A.a.O.
} 
linien und Programme geschaffen. Die Fertilität ging zunehmend zurück - überall auf der Welt, außer in Afrika. ${ }^{493}$ Aber selbst in Afrika rückten die meisten Staaten von der fast durchgängig pronatalistischen Haltung ab, die sie in Bukarest eingenommen hatten. Bei der zweiten Weltbevölkerungskonferenz hatte sich auch die Datenlage verbessert: Dank dem World Fertility Survey war es 1984 bereits möglich, wirkliche Veränderungen in der Anwendung von Verhütungsmitteln und im Fortpflanzungsverhalten der Menschen zu erkennen.

Ironischerweise machten in Mexico City die Vereinigten Staaten eine Kehrtwendung. Nachdem sich fast die ganze Welt der Meinung angeschlossen hatte, die die USA in Bukarest so vehement vertreten hatten, räumte die US-Regierung unter Ronald Reagan nun 1984 einer Stabilisierung der Weltbevölkerung keine Priorität mehr ein und bezog eine scharfe Haltung gegen Abtreibungen. Außerdem vertrat sie die ideologische Position, dass die Marktkräfte auch in Bevölkerungsfragen die richtigen Lösungen produzieren würden und staatliche Familienplanungsprogramme daher überflüssig bis schädlich seien.

Die Abschlussdokumente von Mexiko, die Mexiko City Declaration und die Mexico City Empfehlungen zum WPPA, bestätigten im Grundsatz den WPPA, ergänzten ihn aber auch substantiell. Vor allem wurde die Rolle der Frau stärker betont, zudem wurden Umweltfragen stärker thematisiert.

\subsubsection{Zwischen 1984 und 1994}

Bis 1994 führten immer mehr Entwicklungsländer, auch in Afrika, Bevölkerungspolitiken ein. Während 1974 bei der ersten Weltbevölkerungskonferenz nur 27 Länder explizite Bevölkerungspolitiken durchführten und nur eine Handvoll mehr die Menschen in ihren Ländern bei Familienplanung assistierten, hatten 1994 immerhin 100 Länder explizite Bevölkerungspolitiken. ${ }^{494}$

Zwischen 1984 und 1994 setzte sich zudem endgültig die Einsicht durch, dass eine Stabilisierung der Weltbevölkerung v.a. indirekt angestrebt werden sollte und dass, wie Boutros Boutros-Ghali es ausdrückte, die Faktoren, die für rasches Bevölkerungswachstum verantwortlich sind, beachtet werden müssen. ${ }^{495}$ Unter Hinweis auf das indische Programm der Zwangssterilisation 1976-1978 und Chinas Bevölkerungspolitik forderten Frauen- und Bürgerrechtsgruppen, dass direkte bevölkerungspolitische Zielsetzungen vermieden werden sollten. ${ }^{496}$ Stattdessen sollten sich die Gesundheits-, Bildungs- und Entwicklungsprogramme vor allem auf Frauen konzentrieren. Integrierte Programme zur Einkommensschaffung und finanziellen Selbstständigkeit der Frauen sollten deren Lebenssituation verbessern. Dabei wurde auch thematisiert, dass die von Frauen gewünschte Kinderzahl wesentlich von gesellschaftspolitischen Voraussetzungen wie der Gleichberechti-

\footnotetext{
${ }^{493}$ A.a.O.

${ }^{494}$ Sadik (1994), 25

${ }^{495}$ Boutros-Ghali (1994), 22

${ }^{496}$ International Women's Health Coalition (1994)
} 
gung von Frauen in überwiegend patriarchalischen Gesellschaften abhängt. All dem wurde wie gezeigt in Kairo Rechnung getragen. Die Bevölkerungskonferenz in Kairo wurde gelegentlich deshalb auch als Vorläufer der Welt-Frauen-Konferenz in Peking 1995 bezeichnet. ${ }^{497}$

\subsubsection{Kairo +5}

Fünf Jahre nach der Verabschiedung des Aktionsprogramms traf sich in New York die internationale Gemeinschaft zur Konferenz Kairo +5 . Ihr Abschlussdokument liest sich wie eine flüssiger formulierte Kurzform des PoA. Bezüglich Bevölkerungspolitik wird noch mal pointiert: „In dem Aktionsprogramm werden zwar keine Zielvorgaben für Bevölkerungswachstum, -struktur und -verteilung gesetzt, doch wird die Auffassung deutlich, dass eine baldige Stabilisierung der Weltbevölkerung einen entscheidenden Beitrag zur Verwirklichung des übergeordneten Ziels einer nachhaltigen Entwicklung leisten würde. ““998 Betont wird „die Freiheit, Freiwilligkeit und Aufgeklärtheit der Entscheidungen“"von Paaren und Individuen über Zahl und Geburtenabstände der Kinder. Staatliche Bevölkerungspolitiken müssten „ohne jegliche Zwangsausübung“ auskommen. ${ }^{499}$ Für AIDSBekämpfung und Familienplanung seien ähnliche Maßnahmen nötig: „Die Regierungen sollten danach streben, sicherzustellen, dass alle Einrichtungen der gesundheitlichen Grundversorgung und der Familienplanung bis zum Jahr 2015 in der Lage sind, entweder direkt oder durch Überweisung ein möglichst breites Spektrum an sicheren und wirksamen Methoden der Familienplanung und der Empfängnisverhütung (...), sowie Barrieremethoden (beispielsweise Kondome für Männer und Frauen sowie Mikrobizide, sofern verfügbar) anzubieten, um Ansteckung zu verhindern." ${ }^{\text {"500 }}$

In einigen Bereichen wird eine Zwischenbilanz gezogen. „Die fünfjährliche Überprüfung der Fortschritte zeigt, dass die Umsetzung der Empfehlungen des Aktionsprogramms positive Ergebnisse gezeitigt hat. Viele Länder haben Schritte zur Einbeziehung von Bevölkerungsbelangen in ihre Entwicklungsstrategien unternommen. In den meisten Ländern ist die Sterblichkeit (...) weiter zurückgegangen. Die (...) festgelegte Definition der reproduktiven Gesundheit wird von einer steigenden Zahl von Ländern akzeptiert, wobei der Schwerpunkt mehr und mehr auf der Qualität der Betreuung liegt. Der zunehmende Einsatz von Familienplanungsmethoden deutet darauf hin, dass sich der Zugang zu Familienplanung verbessert hat und dass immer mehr Paare und Einzelpersonen die Zahl ihrer Kinder und die Geburtenabstände wählen können. ${ }^{501}$ Es gab jedoch auch Negatives zu bilanzieren: „Einige Länder und Regionen konnten jedoch nur begrenzte Fort-

\footnotetext{
${ }^{497}$ Sinding (2003), 3

${ }^{498}$ United Nations (1999), Abs. 7

${ }^{499}$ A.a.O., Abs. 52

${ }^{500}$ A.a.O., Abs. 53

${ }^{501}$ A.a.O., Abs. 9
} 
schritte verzeichnen und mussten teilweise sogar Rückschritte hinnehmen. Frauen und Mädchen sind nach wie vor Diskriminierung ausgesetzt. Die HIV/AIDS-Pandemie führte in vielen Ländern, insbesondere in Afrika südlich der Sahara, zu einem Anstieg der Mortalität. Infektionskrankheiten, parasitäre und durch Wasser übertragene Krankheiten wie Tuberkulose, Malaria und Bilharziose führen bei Erwachsenen und Kindern nach wie vor zu erhöhter Mortalität und Morbidität. (...) Millionen von Paaren und Einzelpersonen haben nach wie vor keinen Zugang zu Informationen und Dienstleistungen auf dem Gebiet der reproduktiven Gesundheit.“.502

\subsubsection{Die Rolle der USA}

In den 1960er und 1970er Jahren standen die USA an der Spitze der Bewegung, die ein $\mathrm{zu}$ schnelles Bevölkerungswachstum als Entwicklungshemmnis ansah. Und es waren die USA, die sich - unterstützt durch andere SEL - dafür einsetzten, dass die Weltbank und die Vereinten Nationen ,family planning' in ihre Entwicklungshilfeprogramme aufnehmen. 1994 regierten in den USA die Demokraten. Präsident Bill Clinton nahm in Fragen der reproduktiven und sexuellen Gesundheit eine progressive Haltung ein und ließ Pro-Life-Gruppen nicht über Gebühr Einfluss auf seine Politik nehmen. Die Abtreibungsfrage, eigentlich ein Randaspekt im Aktionsprogramm, wurde in Kairo v.a. durch den Vatikan thematisiert. Unterstützt wurde er dabei von islamistischen Regimen, die durch ihren Widerstand in dieser Frage den Kongress über Tage blockierten. Es war aber wohl die Aufbruchsstimmung nach dem Fall des Eisernen Vorhangs, die als historisch empfundene weltweite demografische Situation, sowie die erfolgreiche Vermittlung Europas und der USA während der Konferenz, die alle Teilnehmer letztlich zu der Auffassung brachten, dass diese Konferenz nicht scheitern dürfe.

Mit dem Wahlsieg von George Walker Bush in den USA im Herbst 2000 änderte sich die regierungsamtliche Haltung der USA zum Paradigma von ,Reproductive Health' wieder radikal. Passagen, die sexuelle Rechte von jungen Menschen betonten, widersprachen dem Weltbild der republikanisch geführten USAdministration. ${ }^{503}$ Tatsächlich ist das Aktionsprogramm hier erstaunlich progressiv. So wird die „begrenzte Möglichkeit vieler Frauen und Mädchen zur Einflussnahme auf ihr Sexual- und reproduktives Leben ${ }^{\text {"504 }}$ ebenso angeprangert wie die Tatsache, dass Frauen einen erheblich größeren Anteil an der Hausarbeit übernehmen als Männer. ${ }^{505}$ Das Recht von Jugendlichen auf selbstbestimmte sexuelle Aktivität wird zwar nicht anerkannt - dies war nicht durchsetzbar -, aber es wird auch nicht ausdrücklich bestritten.

\footnotetext{
${ }^{502}$ A.a.O., Abs. 10

${ }^{503}$ Vgl. auch den Vorbehalt des Vatikans (BiB 1994, 121).

${ }^{504} \mathrm{BiB}$ (1994), 7.3

${ }^{505}$ A.a.O., 181
} 
Das progressive Aktionsprogramm von Kairo ist somit ein „rotes Tuch“ für die Regierung von George W. Bush. Als seine erste Amtshandlung ließ Bush ein bereits unter der Präsidentschaft von Ronald Reagen bestehendes Gesetz wiederaufleben, nach dem nichtamerikanische NRO nicht mehr von der US-Entwicklungshilfeagentur USAID finanziert werden dürfen, ,if they in any way promote, provide, or refer patients for abortion. “506 Dieses Gesetz, in der RH-Community ,Global Gag Rule' (,Globale Knebelungs-Regel') genannt, führte dazu, dass UNFPA unter Reagan und nun unter Bush bisher 11 Jahre keine Förderung der USA erhielt und 500 Mio. \$ verlor. ${ }^{507}$ Die größte Nichtregierungsorganisation im Sektor von Reproduktiver Gesundheit und Familienplanung, IPPF, verlor im Jahr des Amtsantritts von George W. Bush zwanzig Prozent ihres Budgets (12 Mio. \$). Mehrere zehntausend Demonstrantinnen und Demonstranten protestierten am 25.4.2004 in einem Marsch für das Leben von Frauen in Washington DC für reproduktive und sexuelle Rechte und Selbstbestimmung von Frauen weltweit und gegen die ideologische Haltung der Bush-Regierung. ${ }^{508}$

Die „Allianz von Weißem Haus und Vatikan“"509 ist der Auffassung, dass Abstinenzappelle die beste Strategie gegen HIV/AIDS und ungewollte Schwangerschaften sind. Die religiös motivierte Rechte bekämpft daher vor allem das ,C' in dem sog. ABC-Ansatz der RH-Community, bei dem ,A' für ,abstinence“, ,B' für „Be faithful“" und ,C' für ,use Condoms“ steht. Angesichts des weitverbreiteten (und gesellschaftlich legitimierten) vorehelichen Geschlechtsverkehrs in Europa und der Tatsache, dass er in Schwarzafrika bei 29 Prozent und in Lateinamerika bei 24 Prozent aller 15-19jährigen vorkommt, ${ }^{510}$ kann man hier von einem Realitätsverlust der Republikaner in den USA sprechen. Diese bekämpfen das Aktionsprogramm von Kairo nichtsdestotrotz mit großer Energie. Bei dem Weltkindergipfel in New York im Mai 2002, der die Internationale Erklärung der Rechte des Kindes überarbeitete, bestand die US-Regierung darauf, ,reproductive health' durch ,basic health care' zu ersetzen. Und bei der 5. Asia-Pacific Population Conference in Bangkok im Dezember 2002 versuchte die US-Delegation wiederum, diesmal erfolglos, die Formulierungen des Aktionsprogramms von Kairo zu ändern. Dabei wurde den Regierungen asiatischer Ländern angedroht, dass Finanzhilfen der USA gekürzt werden, wenn sie sich gegen die US-Wünsche sperren. ${ }^{511}$ Im September 2003 kürzte die Bush-Regierung einer Gruppe brasilianischer Anti-AIDS-NGOs eine Finanzhilfe in Höhe von 8 Mio. \$, weil sie es ab-

\footnotetext{
${ }^{506}$ Sinding (2004), 44

${ }^{507}$ Schering AG (2004), 27

${ }^{508}$ Siehe o.V. (26.04.2004), 6 und www.ippf.org/womansday/index.htm. Rev. 2004-04-26

${ }^{509}$ Schering AG (2004), 20 u. 25 f.

${ }^{510}$ John Hopkins Bloomberg School of Public Health (2003), 27

${ }^{511}$ Schering AG (2004), 27
} 
lehnten, ihre Programme auf die Empfehlung von Abstinenz zu beschränken und die Benutzung von Kondomen propagierten. ${ }^{512}$

\subsubsection{Finanzielle Versprechen nicht gehalten}

Zum zehnjährigen Jubiläum des ICPD-Aktionsprogramms zieht die RH-Community (NGOs, Private-Public-Partnerships, öffentliche Stellen) eine ernüchternde Zwischenbilanz. Die SEL erfüllen ihre Verpflichtungen gerade zu 40 Prozent. Statt 5,7 Mrd. \$ zahlten sie nur 2,5 Mrd. \$. Die Entwicklungsländer gaben hingegen 7,1 Mrd. \$ für die Umsetzung des Aktionsprogramms aus. Insgesamt wird somit aber nur etwa halb so viel für die Implementierung des Aktionsprogramms ausgegeben, wie für den Zeitraum 2000-2005 pro Jahr vorgesehen war. ${ }^{513}$

Seit dem Ende des Zweiten Weltkriegs bzw. verstärkt seit den 1950er Jahren nimmt zwischenstaatliche Entwicklungshilfe (,Official Development Assistance' $=$ ODA) einen festen Platz im Gefüge der internationalen Beziehungen ein. Dabei wird 95 Prozent der ODA von den OECD-Staaten geleistet. Die nachfolgende Tabelle 9 zeigt, wie sich die Entwicklungshilfe für Gesundheit zwischen 1990 und 2001 entwickelte:

Tab. 9: Official Development Assistance für Gesundheit (Mio US \$)

\begin{tabular}{|l|r|r|r|r|r|r|}
\hline Land & $1990-92$ & $1993-95$ & $1996-98$ & $1999-2001$ & $\begin{array}{l}\text { Prozentsatz der } \\
\text { ODA fur Ge- } \\
\text { sundheit an der } \\
\text { gesamten ODA } \\
\text { 1999-2001 }\end{array}$ & $\begin{array}{l}\text { Protsatz } \\
\text { der geamamten } \\
\text { ODA am BIP } \\
\text { 2001 }\end{array}$ \\
\hline Australien & 14 & 43 & 83 & 124 & 3 & 0,25 \\
\hline Kanada & 31 & 57 & 36 & 69 & 2 & 0,22 \\
\hline Dänemark & 69 & 71 & 90 & 56 & 2 & 1,03 \\
\hline Frankreich & 71 & 65 & 100 & 59 & 2 & 0,32 \\
\hline Deutschland & 37 & 114 & 163 & 125 & 3 & 0,27 \\
\hline Italien & 94 & 31 & 26 & 38 & 1 & 0,15 \\
\hline Japan & 107 & 198 & 242 & 152 & 4 & 0,23 \\
\hline Niederlande & 61 & 97 & 140 & 145 & 4 & 0,82 \\
\hline Norwegen & 32 & 38 & 42 & 92 & 3 & 0,83 \\
\hline Spanien & 26 & 59 & 117 & 93 & 3 & 0,30 \\
\hline Schweden & 154 & 92 & 73 & 73 & 2 & 0,81 \\
\hline Schweiz & 31 & 19 & 30 & 34 & 1 & 0,34 \\
\hline UK & 134 & 98 & 233 & 500 & 14 & 0,32 \\
\hline USA & 383 & 800 & 733 & 1.108 & 30 & 0,11 \\
\hline Insgesamt & $\mathbf{1 . 2 8 6}$ & $\mathbf{1 . 8 4 1}$ & $\mathbf{2 . 2 0 1}$ & $\mathbf{2 . 8 1 7}$ & - & $\mathbf{0 , 2 2}$ \\
\hline
\end{tabular}

\section{Quelle: Sinding (2004), 40-43}

\footnotetext{
${ }^{512}$ Sinding (2004), 45

${ }^{513}$ Sinding (2004), 34
} 
Die Ausgaben für Gesundheit werden nach einer Systematik von Sinding unterteilt in die Ausgaben für Basisgesundheitsdienste, die dafür notwendigen Infrastrukturmaßnahmen, medizinische Dienstleistungen inkl. Forschung und Training, die Bekämpfung von Infektionskrankheiten, AIDS-Bekämpfung, Familienplanung, reproduktive Gesundheit und die Verwaltung der Programme. ${ }^{514}$

Während 1994 noch 563 Mio. \$ für Familienplanung von den Geberländern zur Verfügung gestellt wurden, waren es 2001 nur noch 356 Mio \$, dies entspricht einem Minus von 36,8 Prozent. Aufgestockt wurden hingegen im gleichen Zeitraum die Mittel für die AIDS-Bekämpfung von 193 Mio \$ auf 587 Mio \$ und auch für die Konzipierung und Verwaltung von bevölkerungspolitischen Programmen (von 14 auf 135 Mio \$). ${ }^{515}$ Durch die mangelhafte Finanzierung von Familienplanungsmitteln kann nach Angaben der UNFPA-Vorsitzenden Safiye Cagar nur der geringe Prozentsatz von 16 Prozent der benötigten Kondome in Afrika südlich der Sahara bereit gestellt werden. Insgesamt haben 105 Mio. Frauen zur Zeit keinen Zugang zu Kontrazeptiva, damit ist der ungedeckte Bedarf heute noch fast genauso hoch wie 1994 (120 Mio. Frauen). Diese Zahlen schließen nicht die beträchtliche und weiter zunehmende Zahl sexuell aktiver Unverheirateter ein. Um den Anteil von Menschen, die Kontrazeptiva verwenden, auch bei einer steigenden Weltbevölkerung zumindest konstant zu halten, müssen zwischen 2000 und 2025 rund 150 Millionen zusätzliche Nutzer versorgt werden. Angesichts von Millionen Menschen, denen ihr Wunsch nach Verhütungsmitteln schon heute nicht erfüllt werden kann, ist sehr fraglich, ob dies gelingt.

Seit der Verabschiedung der Millenium Development Goals (weitreichende und ambitionierte Vorhaben zur Bekämpfung von Armut, Hunger, Unbildung und Krankheit in den Entwicklungsländern) im Jahr 2000 hat sich die Aufmerksamkeit der Geberländer diesen zugewandt. Dabei ist die Erfüllung der Ziele von Kairo eine Voraussetzung dafür, dass die Millenium Development Goals erfüllt werden können. In den Worten des Generalsekretärs der Vereinten Nationen: ,the Millenium Development Goals (...) cannot be achieved if questions of population and reproductive health are not squarely addressed." 516

Zusammenfassend kann man sagen, dass derzeit der Konsens von Kairo nicht etwa umgesetzt, sondern von den USA aggressiv bekämpft wird, während die anderen reichen Länder das Problem des schnellen Bevölkerungswachstums verdrängen. Der Vorsitzende der IPPF fasst zusammen: „As a result we face a double challenge today: first, to persuade governments to stick to the commitments made in Kairo, and second, to counteract the conservative resistance that is being led by

\footnotetext{
${ }^{514}$ Sinding (2004), 42-43

${ }^{515}$ Sinding (2004), 43

${ }^{516}$ Rede von Kofi Annan bei der Fünften Asiatisch-Pazifischen Bevölkerungskonferenz in Bangkok, Thailand vom 11-17.12.2002, zitiert nach UN Population Fund (2003), 29
} 
the US government - which in the past was one of the strongest champions and architects of the Cairo consensus." ${ }^{517}$

Unter diesen Voraussetzungen muss man es als weise Entscheidung bezeichnen, dass UNFPA darauf verzichtet hat, 2004 eine neue politische Konferenz (Kairo + 10) einzuberufen. Massive Rückschritte in allen Bereichen wären zu erwarten gewesen.

Vergleicht man die Abschlussresolutionen der drei bevölkerungspolitischen Konferenzen, so ist das Aktionsprogramm von Kairo das progressivste. ${ }^{518}$ Es ist auch verglichen mit früheren Weltbevölkerungskonferenzen in Bezug auf die Wortwahl eine deutliche Veränderung zu beobachten. Während im WPPA und im Mexiko Plan of Action noch tunlichst die Wörter ,sexuell“ und „Sexualität“" vermieden wurden, nennt das PoA die Probleme beim Namen, inklusive ,weibliche Genitalverstümmelung“, „Kindstötung“, „Vergewaltigung“, „Inzest“, „Frauenhandel“" ,Kinderprostitution“ und ,--pornografie“. Diese Offenheit und Progressivität war seinerzeit sicherlich durch die politische Großwetterlage beeinflusst. ${ }^{519}$

\subsubsection{Kontrazeptiva heute und morgen}

Die beschreibende Übersicht über die bevölkerungspolitisch relevanten Vertragswerke auf internationaler Ebene (v.a. Kairo 1994 und New York 1999) und die heutige Meinung der wichtigsten Akteure dazu ist nun abgeschlossen. Eine weitere wichtige Information, die zur Bewertung von Geburten- und geburtenwirksamen Politiken weltweit nötig ist, bezieht sich auf die Anwendung von Kontrazeptiva. Weltweit nutzen 57 Prozent (620 Millionen) aller verheirateten Frauen im gebärfähigen Alter Verhütungsmittel. In den Entwicklungsländern sind es insgesamt 55 Prozent, aber die Skala reicht von 15 Prozent in Zentralafrika bis zu 68 Prozent in Lateinamerika und der Karibik. ${ }^{520}$

\footnotetext{
${ }^{517}$ Schering AG (2004), 12

${ }^{518}$ Natürlich erfüllen solche Resolutionen politischer UN-Konferenzen (im Gegensatz zu den technischen Konferenzen) niemals den Anspruch an Widerspruchsfreiheit. Auf UN-Konferenzen muss keine Gruppe ihre Überzeugungen und Ideale aufgeben. Wichtiger als Konsistenz und Kürze ist es, die Belange aller aufzunehmen. Dies führt dazu, dass z.B. unvereinbare Positionen friedlich in einem Abschlusstext nebeneinander stehen.

${ }^{519}$ Sexualität war lange Zeit eine Leerstelle im ganzen Diskurs über Bevölkerung, Familienplanung und Bevölkerungspolitik (Wichterich 1994, 34). Nur am Rande soll darauf hingewiesen werden, dass interessanterweise keine positive Korrelation zwischen der Sexualmoral und der Geburtenrate eines Landes zu bestehen scheint. Ganz im Gegenteil: Je stärker vorehelicher Geschlechtsverkehr gesellschaftlich legitimiert ist (und je häufiger er vermutlich dann auch stattfindet), desto weniger Geburten gibt es. Thailand hat eine lange Tradition der Prostitution. Neunzig Prozent der unverheirateten männlichen Thais (und leider immer mehr Ausländer) besuchen regelmässig Prostituierte. Dennoch ist die Geburtenrate niedriger als in den Nachbarländern. Die islamischen Länder haben eine sehr repressive Sexualmoral und die meisten Geburten. Vermittelt werden solche Zusammenhänge durch das Niveau von Aufklärung und Sexualerziehung eines Volkes. Je geringer seine Aufklärung ist, desto mehr ungewollte Schwangerschaften scheint es zu geben.

${ }^{520}$ John Hopkins Bloomberg School of Public Health (2003), 2-15
} 
Tab. 10: Methoden der Kontrazeption (in Prozent)

\begin{tabular}{|c|c|c|c|}
\hline Methode & SEL & WEL & Welt \\
\hline \multirow{9}{*}{$\begin{array}{l}\text { Weibliche Sterilisation } \\
\text { Männliche Sterilisation } \\
\text { Spirale } \\
\text { Pille } \\
\text { Kondom } \\
\text { Hormonspritzen } \\
\text { Vaginale Barrieremethoden } \\
\text { Rhythmus } \\
\text { Andere } \\
\end{array}$} & 10 & 33 & 26 \\
\hline & 5 & 12 & 10 \\
\hline & 8 & 24 & 19 \\
\hline & 20 & 12 & 15 \\
\hline & 19 & 6 & 10 \\
\hline & - & 2 & 1 \\
\hline & 3 & 1 & 2 \\
\hline & 13 & 5 & 7 \\
\hline & 22 & 6 & 10 \\
\hline
\end{tabular}

\section{Quelle: Fathalla (1994), 226}

In den Entwicklungsländern sind v.a. ländliche Gebiete unterversorgt. Verschiedene Studien zeigen, dass die Verwendungsraten von Kontrazeptiva um so höher sind, je näher und schneller erreichbar die nächste ,Versorgungsstelle' ist. In Deutschland ist sie gleichbedeutend mit dem Weg zu der nächstgelegenen Apotheke. In Thailand, wo es sehr viele Versorgungsmöglichkeiten in Tankstellen, Shops oder Polizeistationen gibt, ist der nächste Versorgungspunkt trotz geringerer Apothekendichte nur durchschnittlich drei km entfernt und kann in durchschnittlich 15 min erreicht werden. Die Prävalenz von Kontrazeptiva liegt bei 68 Prozent. In Uganda dagegen ist ein Versorgungspunkt durchschnittlich $19 \mathrm{~km}$ entfernt und kann erst in 60 min erreicht werden. Dort liegt die Prävalenz bei nur 5 Prozent. ${ }^{521}$

Dazu kommt, dass die Angebote häufig nicht auf die Verhältnisse zugeschnitten sind. Kontrazeptiva zu Marktpreisen sind für Dorfbewohner oft unerschwinglich, auf der anderen Seite werden die öffentlich subventionierten Kontrazeptiva zu häufig zu billig an die Mittelschicht in Entwicklungsländern abgegeben, die durchaus gewillt und in der Lage wäre, den Marktpreis dafür zu bezahlen.

\subsection{Arten von Geburtenpolitik}

\subsubsection{Vorbemerkung}

Es ist hilfreich für eine ethische Bewertung, wenn das, was es zu bewerten gilt, zunächst mal systematisiert wird. Allerdings gibt es, von der Unterscheidung zwischen pro- und antinatalistisch abgesehen, bisher keine einheitliche Systematisierung von Bevölkerungspolitiken in der Demografie. Erster Schritt dafür ist eine belastbare Definition von Bevölkerungspolitik.

${ }^{521}$ A.a.O., 35 


\subsection{2 ,Bevölkerung'}

Eine Bevölkerung ist eine Population von Menschen auf einem bestimmten Territorium (z.B. Baden-Württemberg, Deutschland, Europa, der Welt). Demgegenüber bezeichnen ,Volk' oder ,Nation' Großgruppen von Personen, die traditionell, kulturell, ethnisch oder politisch miteinander verbunden sind.

\subsection{3 ,Bevölkerungspolitik' und ,Geburtenpolitik'}

Im Handbuch Weltbevölkerung wird ,Bevölkerungspolitik' wie folgt definiert:

„Staatliche Maßnahmen zur Beeinflussung von Größe, Wachstum und räumlicher Verteilung einer Bevölkerung. Unter antinatalistischer Politik wird die Politik einer Regierung, Gesellschaft oder gesellschaftlichen Gruppe mit dem Ziel, über eine Begrenzung der Geburten das Bevölkerungswachstum zu verlangsamen, verstanden. “522

Diana Hummel definiert dagegen:

„Bevölkerungspolitik bezeichnet staatliche Interventionen, die demografische Veränderungen hinsichtlich der Zahl, Zusammensetzung, dem Altersaufbau, der Wachstumsrate oder Verteilung einer Bevölkerung bewirken sollen. Sie beinhaltet quantitative sowie qualitative (Hervorhebung von J.T.) Ziele. Analog zu den drei wesentlichen demografischen Komponenten Geburtenrate, Sterblichkeit und Migration lassen sich drei Teilbereiche unterscheiden: 1) Eine Politik, die auf die Beeinflussung der Fertilität einer Bevölkerung oder Bevölkerungsgruppe (Hervorhebung von J.T.) zielt.

2) Gesundheitspolitik mit dem Ziel der Reduzierung der Sterblichkeit oder Morbidität. Sie kann im krassen Fall aber auch auf die Anhebung der Mortalität abzielen.

3) Migrations- und Urbanisierungspolitik, die sowohl auf die Anzahl als auch Verteilung der Bevölkerung einwirkt, z.B. durch die Einwanderungsgesetzgebung oder Investitionen in die ländliche Infrastruktur, um die Migration in die Städte zu verlangsamen. Von Bevölkerungspolitik ist im Allgemeinen dann die Rede, wenn folgende Kriterien erfüllt sind: Eine Regierung formuliert offiziell bevölkerungspolitische Ziele. Gemeinsam mit einem systematischen Plan ihrer Implementierung werden Maßnahmen ergriffen, diese Ziele zu errei-

\footnotetext{
${ }^{522}$ Deutsche Stiftung Weltbevölkerung (1999), 90
} 
chen und auf Regierungsebene wird eine Institution zur Überwachung der Aktivitäten geschaffen. ${ }^{\text {(6523 }}$

Offensichtlich unterscheiden sich die Definitionen in der ethisch bereits entscheidenden Frage, ob der Begriff auch , qualitative' Maßnahmen umfasst, d.h. Maßnahmen, mit denen der Träger der Bevölkerungspolitik die innere Zusammensetzung einer Gesamtbevölkerung durch auf einen Teil der Bevölkerung gerichtete Maßnahmen ,verbessern' will. Die Definition von Hummel, die eine entschiedene Gegnerin jeder Art von Bevölkerungspolitik ist, subsumiert auch diese Bedeutung unter den Begriff. Ein hypothetisches Beispiel für eine ,qualitative' Geburtenpolitik wäre die Politik eines Landes, in von ihm besetzten Gebieten die Geburtenrate der lokalen Bevölkerung durch staatliche Maßnahmen unter die von neuankommenden Siedlern zu drücken. Dagegen wäre eine quantitative Geburtenpolitik, wenn die Regierung dieses Landes beschlösse, allen Menschen in ihrem Einzugsgebiet ein Kindergeld ab dem zweiten Kind zu bezahlen. ,Quantitative Bevölkerungspolitik' hat es also nur mit den Problemen des Zuviel oder Zuwenig von Menschen in einem Land bzw. Territorium zu tun, nicht aber damit, welche Menschen. ${ }^{524}$

Die internationale Politik versteht unter ,Bevölkerungspolitik' eine quantitative Politik, die alle von ihr betroffenen Menschen gleich bewertet. In dieser Bedeutung verwendet auch die UN den Begriff ,Bevölkerungspolitik'. Eine ,qualitative' (bzw: ,rassistische') Geburtenpolitik ist in der UN-Konvention von 1948 als ,Völkermord' geächtet. ${ }^{52}$ Artikel 2 zählt unter d) auch eine bestimmte Geburtenpolitik auf:

In dieser Konvention bedeutet Völkermord eine der folgenden Handlungen, die in der Absicht begangen wird, eine nationale, ethnische, rassische oder religiöse Gruppe als solche ganz oder teilweise zu zerstören:

a) Tötung von Mitgliedern der Gruppe;

b) Verursachung von schwerem körperlichem oder seelischem Schaden an Mitgliedern der Gruppe;

\footnotetext{
${ }^{523}$ Hummel (2000), 103

${ }^{524}$ Maßnahmen, die für einen hohen Bildungs- oder Qualifizierungsstand aller Menschen in einem Gebiet sorgen wollen, werden gemeinhin nicht als Bevölkerungspolitik bezeichnet, sondern anderen Politikfeldern wie der Bildung- oder Arbeitsmarktpolitik zugeordnet (Feucht 1999, 21). Dafür wird der Terminus ,qualitative Bevölkerungspolitik' also nicht benötigt.

${ }^{525}$ UNO (10.12.1948)
} 
c) vorsätzliche Auferlegung von Lebensbedingungen für die Gruppe, die geeignet sind, ihre körperliche Zerstörung ganz oder teilweise herbeizuführen;

d) Verhängung von Maßnahmen, die auf Geburtenverhinderung innerhalb der Gruppe gerichtet sind;

e) gewaltsame Überführung von Kindern der Gruppe in eine andere Gruppe.

Diese klare Ächtung spricht dafür, mit dem Begriff ,Bevölkerungspolitik' eine Politik zu bezeichnen, die rein quantitativ ist. Wenn eine rassistische oder diskriminierende Politik bezeichnet werden soll, so könnte dies durch ein Adjektiv vor dem Nomen kenntlich gemacht werden. Es gibt aber bei dieser Festlegung ein Problem: Dieser klare Ausschluss von Politiken, die Individuen aufgrund von Merkmalen wie Hautfarbe, Ethnie, Fähigkeiten, Intelligenz oder Religion diskriminieren, aus der Extension des Begriffs ,Bevölkerungspolitik' ist im Hinblick auf die Zuwanderungspolitik problematisch. Kaum ein Land betreibt eine diskriminierende Geburten- oder Gesundheitspolitik. Im Hinblick auf Zuwanderungspolitik betreiben dagegen fast alle Einwanderungsländer eine qualitative Politik, d.h. sie unterscheiden (diskriminieren) die Bewerber nach ihrem Alter, ihren Fähigkeiten, etc. Nur diejenigen dürfen einwandern, die die Bevölkerung des Einwanderungslandes nach Ansicht der Alteingesessenen, verbessern'. Wenn diese eine der drei Teilpolitiken von Bevölkerungspolitik ,qualitativ' ist, so folgt daraus, dass auch Bevölkerungspolitik ,qualitativ' ist. Die Rede von einer ,qualitativen Bevölkerungspolitik' aber würde den falschen Eindruck erwecken, dass von einer ,qualitativen Geburtenpolitik', also u. U. von Völkermord die Rede ist, da in wohl 90 Prozent der Fälle, in denen in Zeitungsartikeln oder in Äußerungen von Politikern von ,Bevölkerungspolitik' die Rede ist, darunter Geburtenpolitik verstanden wird. ${ }^{526}$ Weil die Migrationspolitik politisch oft umkämpft ist und die Sterberate überhaupt nicht direkt politisch beeinflusst werden kann, konzentrieren sich die bevölkerungspolitischen Maßnahmen eines Staates oft ausschließlich auf die Beeinflussung der Geburtenrate. ${ }^{527}$ So schreiben Dorbritz und Fleischacker über die Bevölkerungspolitik der DDR: „Die Zielstellungen auf dem Gebiet der Familienförderung waren zunächst allgemein im Sinne sozialpolitischer Orientierungen formuliert, stellten aber die politischen Grundlagen der pronatalistischen

\footnotetext{
${ }^{526}$ In der öffentlichen Debatte spielt der Begriff ,Geburtenpolitik' fast gar keine Rolle. Eine GeniosDatenbankanfrage zum Suchbegriff ,Bevölkerungspolitik' fand am 4.1.2004 in den Zeitschriften Handelsblatt, Tagesspiegel, Saarbrücker Zeitung, VDI Nachrichten und die ZEIT 209 Treffer in 173 Dokumenten. Dieselbe Anfrage zum Suchbegriff, Geburtenpolitik' fand zehn Treffer in neun Dokumenten.

${ }^{527}$ Görres-Gesellschaft (1985), 767
} 
Bevölkerungspolitik dar. Hiervon ausgehend definierte sich die DDR als ein Land, das eine aktive Bevölkerungspolitik, verstanden als Geburtenförderung (Hervorhebung J.T.) betreibt, das aber offiziell keine quantitativen Vorgaben über die Entwicklung der Bevölkerungszahl und -strukturen formulierte. “528

Wir haben also ein semantisches Dilemma: Nach Definitionsmöglichkeit 1 umfasst der Begriff „Bevölkerungspolitik“ quantitative Politiken (Geburten-, Gesundheits- und Migrationspolitik). Dies hat den Vorteil, dass es dem allgemeinen Sprachgebrauch entgegenkommt. Weil die Altersstruktur zumindest kurzfristig nicht und die Sterberate überhaupt nicht direkt politisch beeinflusst werden können, konzentrieren sich politische Maßnahmen auf die Beeinflussung der Geburtenrate. In 90 Prozent der Fälle setzt die Öffentlichkeit Bevölkerungs- und Geburtenpolitik gleich. Geburtenpolitik ist jedoch nur als quantitative Politik akzeptiert. Definitionsmöglichkeit 1 hat den Nachteil, dass sie nicht dem Kriterium „Innerer Wortsinn" entspricht.

Nach Definitionsmöglichkeit 2 umfasst der Begriff „Bevölkerungspolitik“ quantitative und qualitative Politiken (Geburten-, Gesundheits- und Migrationspolitik). Dies hat den Vorteil, dass es stringent ist: Selbst wenn nur einer der drei Teilbereiche i.d.R. als qualitative Politik betrieben wird, muss auch der Oberbegriff so bezeichnet werden. Der Nachteil von Definitionsmöglichkeit 2 ist, dass Bevölkerungspolitik von der Öffentlichkeit meist mit Geburtenpolitik assoziert wird. Qualitative Geburtenpolitik ist aber geächtet.

Nun gibt es durchaus Kriterien für die Güte einer Definition, z.B. die innere Struktur eines Ausdrucks, seine Etymologie und die Konvention, d.h. der Gebrauch durch die Mehrheit der Wissenschaftler. ${ }^{529}$ Nach diesen Kriterien wählt die Scientific Community dann aus. Allerdings ist sich eben die Demografie nicht einig, wie schon die beiden Eingangsbeispiele zeigten. ${ }^{530}$ Folgender Ausweg aus dem semantischen Dilemma erscheint meines Erachtens am sinnvollsten: Der Begriff „Bevölkerungspolitik“ ist gänzlich zu vermeiden, wenn die Frage der Quantität vs. Qualität ansteht. Statt dessen sollte Geburten-, Gesundheits- ${ }^{531}$ und Migrationspolitik verwendet werden und entsprechend ein Adjektiv hinzugefügt werden. Im folgenden Abschnitt geht es nur um quantitative Geburtenpolitik. ${ }^{532}$

\footnotetext{
${ }^{528}$ Dorbritz/Fleischhacker (1995), 163

${ }^{529}$ Siehe dazu ausführlich Tremmel (2004a), Tremmel (2003a).

${ }^{530}$ Wie Feucht $(1999,21)$ konstatiert, verwendet zwar eine große Mehrheit der Demografen den Begriff ,qualitative Bevölkerungspolitik' nicht, es gibt aber prominente Ausnahmen, z.B. Wingen (1975), 19.

${ }^{531}$ Eigentlich müsste richtigerweise von ,Politik zur Beeinflussung der Sterberate' gesprochen werden. Es besteht aber weltweit Einigkeit, dass politische Maßnahmen stets die Reduzierung der Sterblichkeit zum Ziel haben sollten. Aus früheren Epochen sind dagegen hinlänglich Beispiele für Bevölkerungspolitik durch Tötungen bekannt, etwa die Kindsmorde bei den Spartanern, das Töten von Alten bei den Inuit und anderen Naturvölkern oder die Euthanasie-Politik der Nationalsozialisten.

${ }^{532}$ Allerdings geht es insgesamt in dieser Arbeit nicht ausschließlich um Geburtenpolitik. Ein Land, das eine solche Geburtenpolitik implementiert, um ein höheres Naturkapital zu erreichen, hat aus
} 
Birg hat auf den interessanten Aspekt hingewiesen, dass es den Staaten nicht möglich ist, das Geburtenverhalten seiner Bürger nicht zu beeinflussen: „Wer diesen Standpunkt [dass sich Bevölkerungspolitik mit den Prinzipien einer Demokratie nicht vereinbaren lasse, J.T.] vertritt, übersieht, dass jede Art politischen Handelns (und Unterlassens) Auswirkungen auf die Zahl und Struktur der Bevölkerung hat. Sind diese Auswirkungen beabsichtigt, spricht man von Bevölkerungspolitik. Da die Wirkungen unabhängig davon eintreten, ob sie beabsichtigt sind oder nicht, können die bevölkerungspolitisch bedeutsamen demografischen Auswirkungen der Politik auch in einer Demokratie nicht vermieden, sondern nur anders genannt werden. ${ }^{\text {"5533 }}$

Sie sollten allerdings auch anders benannt werden. Wie in der Demografie üblich, wird in dieser Studie der Begriff, Geburtenspolitik' für beabsichtigte Maßnahmen reserviert.

Eine letzte zu klärende Frage ist, ob nur Staaten Geburtenpolitik machen können oder auch Stiftungen, Nichtregierungsorganisationen, Firmen oder mächtige Privatpersonen. Diese Definitionsfrage ist nicht auf Geburtenpolitik beschränkt, sondern betrifft auch Gesellschaftspolitik oder Frauenpolitik, letztlich jede Politik. Auch wenn einzelne Menschen bzw. die von ihnen gegründeten Organisationen durchaus einzelne Aufgaben des Staates übernehmen können, so sollte doch aus Klarheitsgründen der Begriff „Politik“ nicht auf ihr Tun angewandt werden.

\subsubsection{Familienpolitik}

Nicht nur die Unterscheidung zwischen ,Bevölkerungs-, und ,Geburtenpolitik' ist wichtig, sondern auch die Abgrenzung beider zu ,Familienpolitik'. Ein Gutachten des Bundesfamilienministeriums definiert: „Generell lassen sich unter Familienpolitik alle Maßnahmen verstehen, mit denen der Staat das Ziel verfolgt, das Wohlergehen von Familien positiv zu beeinflussen. In einer genaueren Definition umschreibt Familienpolitik direkte und indirekte finanzielle Transfers (z.B. Kinder- und Erziehungsgeld bzw. steuerliche Vergünstigungen) sowie Regelungen bezüglich Mutterschutz und Elternzeit und Einrichtungen der Kinderbetreuung. “5334

Diese Definition weist dem Staat nicht das Recht zu, Familiengründungen zu fördern, sondern nur bestehende Familien zu unterstützen. Ausgangspunkt ist die Gerechtigkeit zwischen Eltern und Kinderlosen. Familienpolitik ist also Teil der Sozialpolitik, Geburtenpolitik ist hingegen keine Sozialpolitik. Familienpolitik setzte historisch gesehen weit später als Geburtenpolitik bzw. die übrigen Arten der Bevölkerungspolitik ein. Weltweit sind schätzungsweise fast 5 Milliarden Menschen, 75 Prozent der Weltbevölkerung, von Geburtenpolitiken betroffen. Von Familienpolitik, um die es in dieser Studie nicht geht, sind hingegen weit

nachvollziehbaren Gründen kein Interesse daran, dass die Geburtenüberschüsse anderer Länder als Einwanderer bei ihm landen, vgl. Abschnitt 6.8.4. Gezielte Einwanderung.

${ }^{533} \operatorname{Birg}(2004 a), 12$

${ }^{534}$ Rürup/Gruescu (2003), 6 
weniger betroffen ${ }^{535}$ Entscheidend sind die Intentionen bei der Unterscheidung beider Politiken, selbst wenn die konkreten Maßnahmen durchaus identisch sein können. Sie müssen dies aber nicht: Selbst wenn erwiesen wäre, dass erhebliche finanzielle Transfers an bereits kinderreiche Familien die Geburtenrate nicht erhöhen, könnte es aus familienpolitischen Gründen geboten sein, diese Transfers durchzuführen.

\subsubsection{Klassifizierung von Geburtenpolitiken}

Generell stellt sich für die Demografie die Herausforderung der Klassifizierung und Bewertung von Geburtenpolitiken. Solche Politiken lassen sich zunächst nach dem Ziel in pro- oder antinatalistische einteilen. Betrachtet man wie hier nur die antinatalistischen, so gibt es zahlreiche weitere Klassifizierungskriterien. So kann man sie nach dem Durchdringungsgrad als vollständig oder teilweise etabliert einteilen, sie lassen sich nach der Motivation in angebots- oder nachfrageorientiert einteilen, nach dem Absender als staatlich oder nicht-staatlich klassifizieren. ${ }^{536} \mathrm{In}$ unserem Zusammenhang interessiert das Kriterium der ethischen Legitimierbarkeit. Der Übergang von der Sicherung der Wahlfreiheit zum Zwang, von ethischen zu unethischen Methoden ist fließend. Eingeteilt nach dem Grad ihrer ethischen Legitimierbarkeit, ist folgendes Kontinuum bei antinatalistischen Maßnahmen sinnvoll:

\section{1.) Der indirekte Ansatz}

Bei einem indirekten Ansatz verzichtet ein Staat komplett auf die direkte Beeinflussung der Kinderzahlen seiner Bürger. Stattdessen versucht die Regierung, andere Größen so zu beeinflussen, dass sich (wahrscheinlich) ein Rückgang der Geburtenrate ergibt. Dazu gehört die Verbesserung der Gesundheitsdienstleistungen, des Bildungsgrades und der Arbeitsmöglichkeiten, v.a. für Frauen. Es werden also Ziele propagiert, die auch von Pronatalisten eigentlich als wünschenswert angesehen werden. Daneben werden Individuen in die Lage versetzt, über Zahl und Geburtenabstand ihrer Kinder selbst zu entscheiden, indem man ihnen den Zugang zu Kontrazeptiva ermöglicht (wie bei allen aufgezählten Arten von Geburtenpolitik). Es wird alles getan, damit Menschen nicht nur auf dem Papier, sondern tatsächlich die freie Wahl haben. Wenn man hier überhaupt von ,Geburtenpolitik' sprechen kann, dann deshalb, weil die Regierung eine bestimmte demografische

\footnotetext{
${ }^{535}$ Die besten Ausführungen zur Abgrenzung von „Geburtenpolitik“ und „Familienpolitik“ liefert immer noch Schubnell (1990). Das im Auftrag des BMFSFJ erstellte Gutachten von Rürup und Gruescu hält die gute eigene Definition leider nicht stringent durch, sondern vermischt später Familienpolitik mit Geburtenpolitik. So schreiben die Autoren, dass zwar Irland und Italien in Bezug auf Transfers und Kinderbetreuung ähnliche Familienpolitiken verfolgen, die irische aber wegen der dortigen hohen Geburtenrate „erfolgreicher“ sei. Das Erreichen einer ausreichenden Kinderzahl wird ausdrücklich als Ziel einer „,nachhaltigen Familienpolitik“ genannt (Rürup/Gruescu 2003, 8 f.).

${ }^{536}$ Hauser (1991), 601-655; Görres-Gesellschaft (1985), 764-770; Hummel (2000), 103; Feucht (1999), 21
} 
Entwicklung anstrebt (z.B. Senkung der Geburtenrate) oder zumindest eine Meinung darüber hat, ob das eigene Geburtenniveau zu hoch, zu niedrig oder angemessen ist. Der Staat verfolgt diese quantitativen Ziele aber eben nur über Umwege.

\section{2.) Der appellative Ansatz}

Bei der nächsten Kategorie, die nun zweifellos eine Art von ,Geburtenpolitik' darstellt, propagiert der Staat aktiv die Kinderzahl pro Frau, die er für gemeinverträglich hält. Man sieht Plakate am Straßenrand mit glücklichen Zwei-KinderFamilien, darunter Slogans wie „Small family = happy family“. Es wird an Individuen appelliert, freiwillig ein bestimmtes Geburtenniveau nicht zu überschreiten. Die Bürger werden über die negativen Folgen für Entwicklung und Umwelt aufgeklärt, die rasches Bevölkerungswachstum hat. Dies geht einher mit der Deckung ihres Bedarfs an Verhütungsmitteln.

\section{3.) Der Ansatz finanzieller Vergünstigungen}

Der Staat bietet ,positive' finanzielle Anreize für das von ihm als gemeinwohlverträglich angesehene Reproduktionsverhalten. Dazu können Prämien für Sterilisationen oder für die Benutzung von langwirkenden Kontrazeptiva, für längere Pausen zwischen einzelnen Schwangerschaften, verbilligte Kredite für kleine Familien, sowie Auszeichnungen und öffentliche Belobigungen für, verantwortliche Elternschaft' gehören.

\section{4.) Der Ansatz der Begrenzung finanzieller Vergünstigungen}

Der Staat beschränkt bestehende finanzielle Vergünstigungen auf eine bestimmte Kinderzahl. Kindergeld, freier Schulbesuch, bezahlter Mutterschaftsurlaub, Erziehungsgeld und ähnliche staatliche Vergünstigungen gibt es bspw. nur für das erste Kind.

\section{5.) Der Ansatz finanzieller Sanktionen}

Der Staat setzt ,negative' finanzielle Anreize. Für überzählige Kinder ist z.B. eine Strafsteuer oder ein negatives Kindergeld zu zahlen. ${ }^{537}$

\section{6.) Der Rationierungs-Ansatz}

Bei der ,Rationierung' hingegen werden Überschreitungen der festgelegten Kinderzahl (zumindest in der Theorie) auch dann nicht geduldet, wenn jemand eine hohe Summe dafür zahlen würde. Wenn eine Familie die erlaubte Zahl von Kindern bereits erreicht hat, dann gilt eine neue Schwangerschaft als ,illegal' und die Frau wird gezwungen, abzutreiben, sofern sie das nicht von selbst tut. Auch

${ }^{537}$ 3.-5. sind finanzielle Steuerungsmechanismen. 
Zwangssterilisierungen könnten bei einem Rationierungsgesetz zur Anwendung kommen. Abb. 22 stellte diese Typen von Maßnahmen auf einem Kontinuum dar.

\section{Abb. 21: Kontinuum geburtenpolitischer Maßnahmen}

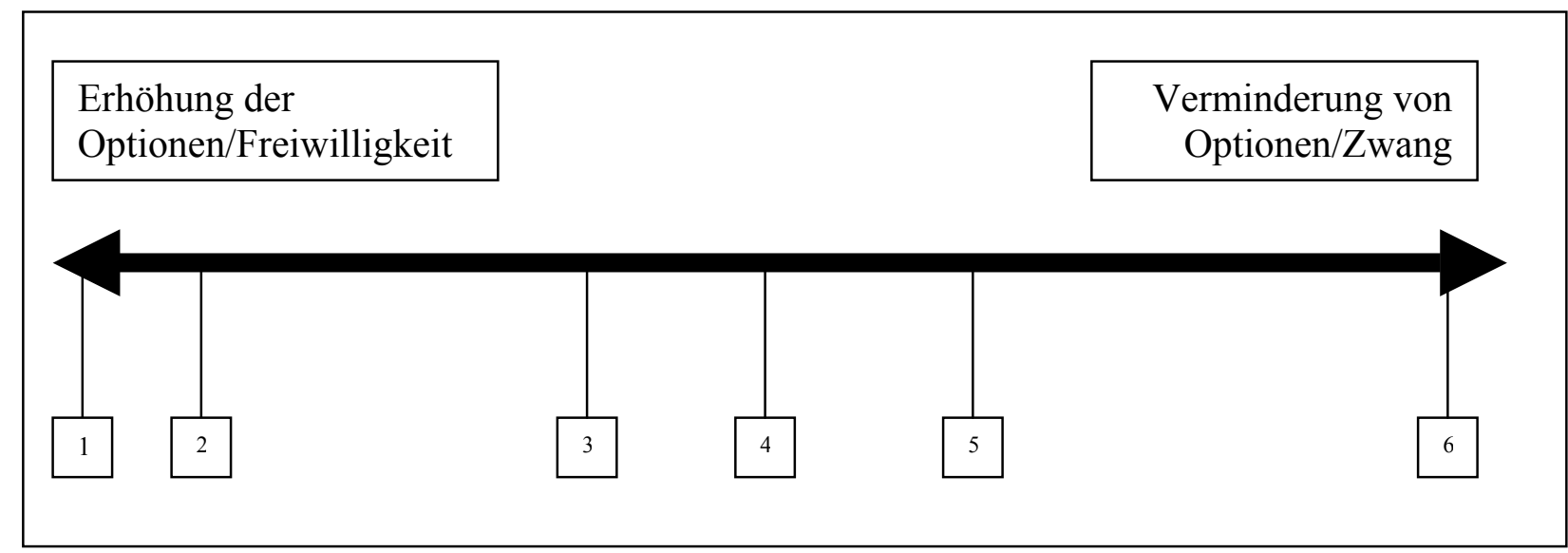

\section{Quelle: eigene Darstellung}

\subsection{Ethische Bewertung von Rationierung}

Am einfachsten erscheint die Bewertung von Typ 6. Es spricht sehr viel dafür, dass Rationierung unethisch ist. Es ist eine klare Verletzung des Menschenrechtskatalogs der Vereinten Nationen, der festlegt, dass niemand gefoltert oder einer grausamen, unmenschlichen oder degradierenden Behandlung oder Bestrafung unterzogen werden darf (13, Artikel 5). Eine Rationierung hat fast zwangsläufig schwerwiegende soziale Verwerfungen zur Folge, etwa massive Ängste vor einer ungenehmigten Schwangerschaft. Die Durchsetzung eines solchen Rationierungsgesetzes würde in der Praxis eine engmaschige Überwachung bedeuten und Denunziationen begünstigen. Zudem fällt de facto bei dieser Art von Geburtenpolitik die Last v.a. auf die Kinder, die bei verheimlichten Schwangerschaften geboren werden. Nehmen wir an, der Staat erlaube nur ein Kind pro Familie. Jemand, dessen gesamtes Glück bzw. Wohlbefinden von einem weiteren Kind abhängt, kann sich trotzdem diesen Wunsch nicht erfüllen. Sie kann nicht einmal mit jemandem tauschen, der gar kein Kind will, was unter utilitaristischen Gesichtspunkten offensichtlich eine schlechte Lösung ist.

Diese rigideste der hier aufgezählten Geburtenpolitiken wurde ansatzweise in Chinas zwischen 1979 und 1984 praktiziert. Es ist aber die Frage, ob ,Rationierung' ein angemessener Begriff für Chinas Ein-Kind-Politik in diesem Zeitraum ist. Es wurde zwar - wie bei einer Rationierungsstrategie - unmissverständlich eine bestimmte Norm (Ein-Kind-Ideal) propagiert, de facto ließ sich aber mit 
Geld viel regeln, so dass wir man in dieser Hinsicht eher von einem Ansatz finanzieller Sanktionen sprechen muss. ${ }^{538}$

Bei einer Rationierungsstrategie scheint es besonders oft zu Missbräuchen zu kommen, dies kann und sollte bei der ethischen Beurteilung mitberücksichtigt werden. $\mathrm{Zu}$ analytischen Zwecken sollte man die gesetzlich geregelte Geburtenpolitik gedanklich trennen von Exzessen und Missbräuchen. Korruption ist generell schlecht und Korruption im Zusammenhang mit Geburtenpolitik ist nicht schlechter als in anderen Zusammenhängen. Zudem sind Missbrauchsgefahren kein exklusives Merkmal von Rationierungsstrategien. Aber dort kamen in der Vergangenheit die größten Exzesse vor: Während die Notstandsgesetze in Kraft waren (1975-1977), wurden in Nordindien 6,5 Millionen Männer zwangssterilisiert. Aber auch noch 1994 wurden Frauen nach Angaben von Hummel aus dem benachbarten Nepal von indischen Beamten entführt und zur Zwangssterilisation in Massenlager verschleppt. ${ }^{539}$

In erster Linie muss sich die ethische Bewertung auf gesetzliche Regelungen beziehen. ${ }^{540}$ Eine solche Regelung ist z.B. das Anfang 2003 geplante indische Gesetz zur Geburtenkontrolle: Männer und Frauen, die mehr als zwei Kinder haben, sollen in Zukunft nicht mehr für politische Ämter kandidieren dürfen. Das Verbot soll Politiker zwingen, bei der Familienplanung mit gutem Beispiel voranzugehen. ${ }^{541}$ Durch dieses Gesetz wird ein Individuum, welches mehr als zwei Kinder hat, im Rahmen einer Rationierung von einem Beruf ausgeschlossen. Hierbei handelt es sich um einen Spezialfall der Rationierung. Während eine ausnahmslose Rationierung bei allen Bürgern großes Unglück erzeugt, da sie ihre gewünschte Lebensplanung unter keinen Umständen mehr realisieren können, steht hier ein Bürger vor der schweren Entscheidung, entweder bei der Berufswahl oder bei der gewünschten Kinderzahl Kompromisse zu machen. Solche Dilemmate zu erzeugen ist unethisch, selbst wenn es - was fast unmöglich erscheint - bei der Durchsetzung keine unangenehmen Nebenwirkungen wie illegale Kinder oder Bestechungsversuche gäbe.

\subsection{Ethische Bewertung des Konsenses von Kairo}

5.8.1 „Menschen dürfen nie als Objekte betrachtet werden“- Kritik am Ergebnis von Kairo

Nachfolgend soll die Form von Geburtenpolitik bewertet werden, die als indirekter Ansatz (Typ 1) bezeichnet wurde. Obwohl es Unterschiede in einzelnen For-

\footnotetext{
${ }^{538}$ Heute haben sich die Gewichte weiter verschoben. Nach den diversen Lockerungen kann heute jeder Chinese, wenn er finanzielle Einbußen in Kauf nimmt, mehr als ein Kind haben.

${ }^{539}$ Hummel (2000), 108

${ }^{540}$ Es sei denn, die Gesetze sind so ausgestaltet, dass Missbrauch unvermeidlich ist. Die Bestrafung von Kadern, die z.B. die chinesischen Gesetze verletzten, zeigen aber, dass diese Gesetze , nicht nur auf dem Papier stehen', sondern in ernsthafter Absicht erlassen wurden.

${ }^{541}$ o.V. 28.04.2003, 147
} 
mulierungen gibt, hat man sich in Kairo von diesem Ansatz leiten lassen. Daher kann die Bewertung des indirekten Ansatzes zusammen mit der Bewertung des Konsenses von Kairo behandelt werden. Hierbei wird zunächst auf die Bewertungen durch andere Gruppen oder Personen eingegangen, bevor eigene normative Überlegungen folgen.

Aus Sicht des Bundesinstituts für Bevölkerungsforschung entwirft das 98seitige, in 16 Kapitel gegliederte Dokument eine angemessene und erfolgversprechende Strategie für die nächsten 20 Jahre. „Das Aktionsprogramm ist freiheitlich, zeitgemäß und fortschrittlich, ohne futuristisch zu sein. Der holistische Gesamtansatz ist sehr problemadäquat, sowohl hinsichtlich des entwicklungspolitischen als auch des reproduktiven Gesundheitskonzepts. Diskriminierung und Zwang wird eindeutig abgelehnt", urteilt Charlotte Höhn. ${ }^{542}$

Das PoA hält wie gezeigt an Bevölkerungspolitik als zulässigem Handeln von Staaten gegenüber Paaren bzw. Individuen fest. Die Hauptstoßrichtung der Kritik am Aktionsprogramm von Kairo richtet sich gegen die Natur von Bevölkerungspolitik als solcher - und damit gegen alle Typen von 1 bis 6 . Bevölkerungspolitik wird ganz generell vorgeworfen, Menschen zum Zweck herabzuwürdigen. „Die Bevölkerungsprogramme und die Familienplanungsbürokratie mit ihrer hierarchischen Organisationsstruktur, die von staatlichen demografischen Vorgaben und quantitativen Zielsetzungen für die Fertilitätsreduzierung ausgehen, stehen in ihrer inhärenten Logik im Gegensatz zur Förderung der Selbstbestimmung von Frauen über ihre Fortpflanzungsfähigkeit", schreibt Hummel. ${ }^{543}$ Die Idee, die ,Menschenproduktion' wie jede andere Produktion vernunftsmäßig zu planen, sei technokratisch. Menschen dürften, so die Kritik, nie zum Material demografischer Planung werden. Die Kontinuität der Familienplanungsprogramme bestehe im Glauben an die politische Machbarkeit einer bestimmten Familiengröße. „Wo quantitative Zielvorgaben und Plansolls für die Senkung der Geburtenrate, die Art der Kontrazeptiva und die Zahl der zu gewinnenden Benutzerinnen festgelegt werden, sind die individuellen Bedürfnisse und die offiziellerseits postulierte Freiwilligkeit zwangsläufig sekundär und werden letztlich dem übergeordneten Ziel der Fertilitätssenkung untergeordnet. “" ${ }^{444}$

Die Publizistin Christa Wichterich ergänzt: „Das Verständnis von Familienplanung war stets ein reduziertes, nämlich die Vermeidung von Schwangerschaften. Und gesucht wurde immer nach einem einfachen Wenn-dann-Mechanismus. “" ${ }^{\text {"45 }}$

\footnotetext{
${ }^{542}$ Höhn (1995), 23

${ }^{543}$ Hummel (2000), 107

${ }^{544}$ A.a.O., 108. Ähnlich Aufhauser (2001). Zumeist werden diese Kritikpunkte von Frauen geäußert. Um dies deutlich zu machen, wird Kritiker und Kritikerinnen in diesem Abschnitt nicht alternierend verwendet.

${ }^{545}$ Wichterich (1994), 25
} 


\subsubsection{Fließende Übergänge zum Zwang}

Übergänge vom Drang zum Zwang sind nach Ansicht von Frauenrechtlerinnen fließend, Plansolls seien ein Indikator dafür. Sie programmierten nicht nur Menschenrechtsverletzungen vor, sondern öffneten auch der Korruption Tür und Tor. ${ }^{546}$

Die Sozialanthropologin Shalini Randeria greift Kerala heraus, um jede Art von staatlichen Zielvorgaben anzugreifen, weil sie nicht ohne negative Nebenwirkungen blieben. Der indische Bundesstaat Kerala gilt als Musterbeispiel für eine geglückte Familienplanungspolitik. Von 1961 bis 1971 hatte er die höchste Bevölkerungswachstumsrate in Indien (2,24 Prozent im Vergleich zu 1,95 Prozent in ganz Indien). Zwischen 1971 und 1991 sank diese auf 1,31 Prozent, dagegen war in Gesamt-Indien im selben Zeitraum nur ein Rückgang von 2,22 Prozent auf 2,11 Prozent zu verzeichnen. Randeria führt das, Wunder Kerala' vor allem darauf zurück, dass die indische Regierung und die internationalen Familienplanungsorganisationen die armen Bevölkerung durch Überredung und Bestechung zu Sterilisationen gedrängt hätten. Die Sterilisation mache 90 Prozent aller in Indien angewandten Methoden aus. Jeder oder jede Sterilisierte hätte als Belohnung ein ,Geschenk' in Form von Geld, Naturalien oder einem Radio erhalten. 90 Prozent aller vorgenommenen Sterilisationen seien an Frauen vorgenommen worden, obwohl die Operation bei Männern einfacher und weniger gefährlich ist. Oft hätten sich Frauen für die Sterilisation entschieden, wenn sie glauben, ihre Wunschzahl an Kindern erreicht zu haben, allerdings bereuten einige von ihnen ihre Entscheidungen später, z.B. wenn ein Kind später durch einen Unfall ums Leben gekommen sei ${ }^{547}$ Randeria kritisiert, dass in Indien reiche Frauen (wie in Europa) reversible Verhütungsmethoden anwendeten, während bei der armen Bevölkerung die Sterilisation am häufigsten angewandt werde. Die Vasektomien würden in „Massensterilisationslagern ${ }^{\text {" } 548}$ vorgenommen. Neben Sterilisationen würden LangzeitKontrazeptiva mit einer Wirkungsdauer zwischen zwei Monaten (wie die Net-EnSpritze) und fünf Jahren (wie Norplant ${ }^{549}$ ) verschrieben. Diese Mittel hätten oft starke Nebenwirkungen, insbesondere bei mangelernährten Frauen. Die Verhütungsmethode werde dabei neben den Sollzahlen von der Zentralregierung festgelegt.

Da die staatlichen Angestellten in Indien in jedem Distrikt eine bestimmte Zahl von Teilnehmern an Familienplanungsprogrammen vorweisen müssten, würden Statistiken massiv gefälscht. In anderen Fällen sollen sich Menschen mehrfach sterilisieren gelassen haben, um die Prämien zu erhalten oder Ärzte und Patientinnen sollen sich die Prämien geteilt haben.

\footnotetext{
${ }^{546}$ Randeria (1994)

${ }^{547}$ A.a.O., 245

${ }^{548}$ A.a.O., 246

${ }^{549}$ Norplant ist auch in Deutschland unter dem Handelsnamen Implanon erhältlich.
} 
Kritiker(innen) wenden sich aber auch gegen das in Kairo beschlossene Konzept des ,Ungedeckten Bedarfs' (,unmet need'). Die Bereitstellung von Entwicklungshilfemitteln für die Familienplanung gehe zu Lasten von Basisgesundheitsleistungen, Bildung und anderen Entwicklungshilfezielen. „Während im Rahmen von Strukturanpassungsprogrammen immer weiter gekürzt werde, steigen die Mittel für Familienplanungsprogramme“, kritisiert Hummel. ${ }^{550}$ In Indien sei 1992/1993 das Budget für Familienplanung mit 10,10 Mrd. Rupien fast doppelt so hoch gewesen wie die Ausgaben im Gesundheitsbereich. ${ }^{551}$ Verhütungsmittel, von deren Vertrieb zudem die Pharmaindustrie profitiere, würden zu einem kostengünstigen Substitut für Entwicklungspolitik,. Andere Kritiker(innen) verurteilen das Konzept das ,Ungedeckten Bedarfs' mit der Begründung, dass es nur auf die Bedürfnisse bestimmter (Alters)gruppen eingehe. So blieben bestimmte Gruppen wie Jugendliche oder unverheiratete Frauen und Männer ausgeschlossen. Außerdem berücksichtige es nur bestimmte Bedürfnisse. Der Bedarf vieler Frauen nach sicherer Abtreibung werde ignoriert. ${ }^{552}$ Des Weiteren wird eingewandt, dass keine eindeutige Korrelation zwischen dem Wunsch der Frauen, ihre Kinderzahl zu begrenzen, und der Anwendungsrate von Verhütungsmitteln bestehen muss. Denn wo Männer sich mehr Kinder wünschen als Frauen und diesen Wunsch auch durchsetzen können, da nutze auch die breitflächige Bereitstellung von Verhütungsmitteln nicht viel.

Angesichts der Forderung des Aktionsprogrammes, im Rahmen der allgemeinen Entwicklungshilfe auch die Mittel für RH-Maßnahmen aufzustocken, befürchten Kritiker(innen) eine Konditionalität. Diese Position „Entwicklungshilfe nur bei (antinatalistischer) Bevölkerungspolitik“" wird von Kritiker(innen) als ,rassistisch" bezeichnet. ${ }^{553}$

Diese Kritik kann grundsätzlich von Frauen und Männern geäußert werden. Es gibt eine radikalere feministische Kritik, die sich von der bereits dargestellten in einer Nuance unterscheidet. ${ }^{554}$ Diese Nuance ist, dass Frauen (statt: Paare) die Opfer der Bevölkerung'ingenieure' seien. „Die (feministische) Kritik dechiffriert die Bevölkerungspolitik als eine strukturell sexistische (Hervorhebung von J.T.) und rassistische Herrschaftsform und hat zu einer Delegitimierung hegemonialer Vorstellungen beigetragen“, erläutert Hummel. ${ }^{555}$

Manchmal wird die Kritik gegen Bevölkerungspolitik sogar ausgeweitet in die Ablehnung der Demografie als Wissenschaft. Im Sammelband von Wichterich wird kritisiert: „Damit überhaupt von einer ,Bevölkerungsfrage' gesprochen werden kann, muss von den einzelnen Menschen abstrahiert werden. Im Überbevöl-

\footnotetext{
${ }^{550}$ Hummel (2000), 110

${ }^{551}$ Randeria (1994), 251

${ }^{552}$ Hummel (2000), 115

${ }_{553}$ Randeria (1994), 240

${ }^{554}$ Hummel (2000), 19

${ }^{555}$ A.a.O
} 
kerungsdiskurs kommen weder Personen noch ihre Lebensbedingungen wirklich vor, sondern nur der ,Faktor Bevölkerung', eine variable Größe, an der und mit der die unterschiedlichsten ,Operationen' vorgenommen werden können. "“556. Hummel beklagt, „dass reale, lebendige Menschen im Begriff der Bevölkerung terminologisch ausgelöscht werden.“557 Sie würden zu „blutlosen Einheiten, die als eigenschaftslose Klassen manipuliert werden können. “558 In den demografischen Diskursen erscheine ,Bevölkerung' als eine von der Gesellschaft unabhängige ,Biomasse'. „Die Bevölkerungswissenschaft trennt so die planende Menschheit als Subjekt von der zu regulierenden Bevölkerung als - analog der ,Natur' zu beherrschendem Objekt. Diese ideologische Konstruktion legitimiert die Definition einer Gruppe von Menschen als überzählige, ökonomisch und ökologisch nicht integrierbare ,Überbevölkerung' und spricht ihnen damit die Existenzberechtigung ab. ${ }^{6559}$ Kritisiert wird, so fasst Hummel zusammen, dass die formale Demografie die Komplexität und Multidimensionalität des Phänomens Bevölkerungswachstum auf ihre einfachsten quantitativen Zusammenhänge reduziert, die sich formelhaft und statistisch darstellen lassen. Dadurch ergebe sich eine EntPolitisierung und quasi eine Verhüllung der gesellschaftlichen Beziehungen. ${ }^{560}$

Sehr in der Kritik steht auch die Sprache einiger Apologeten der Familienplanung bzw. Bevölkerungspolitik. Da werden Kriegsszenarien inszeniert, in denen ,menschliche Sprengsätze' ticken, ,Zeitbomben' gezündet werden, ,demografische Raketen' hochgehen, ,Millionengeschwader' anrücken und Bevölkerungen ,explodieren'. ${ }^{561}$

\subsubsection{Die Ökologie als Druckmittel}

Nach Ansicht der Kritiker(innen) haben sich die Motive der Familienplaner seit den 1950er Jahren nicht geändert. Die neue Richtung der Debatte seit dem Aufkommen der Umweltfrage wird mit taktischen Überlegungen des ,Bevölkerungsestablishments' begründet. Wichterich schreibt: „In der Ökologisierung der Debatte liegt jedoch ein wichtiger Argumentationswechsel. Wo die Menschen im Süden durch ihre Vermehrung angeblich ihre eigene »Unter«entwicklung verursachen, schaden sie nur sich selbst. Wo sie jedoch angeblich das ökologische Gleichgewicht stören, das zum Überleben der gesamten Menschheit notwendig ist, da schaden sie auch uns. ${ }^{{ }{ }^{662}}$ Die immer wechselnden Begründungen verdeckten die eigentlichen Interessen, nämlich eine Unterdrückung der Dritten Welt aus rassistischen und ökonomischen Motiven. ${ }^{563}$

\footnotetext{
${ }^{556}$ Heim/Schaz (1996), 199

${ }^{557}$ Hummel (2000), 100

${ }^{558}$ Duden (1993), 76

${ }^{559}$ Schultz (1994), 13

${ }^{560} \mathrm{Vgl}$. Hummel (2000), 101

${ }^{561}$ Wichterich (1994), 31

${ }_{562}^{56}$ A.a.O.

${ }^{563}$ A.a.O.
} 
Nur sehr vereinzelt wird auf eine ökologische begründete Sorge vor einer $\mathrm{Zu}$ nahme der Weltbevölkerung eingegangen. Teilweise drehen Kritiker(innen) den Spieß einfach um: „Mit dem Verweis auf die ökologische Gefährdung des Kinderreichtums wird insbesondere gegen arme Frauen, Slumbewohnerinnen, Kleinbäuerinnen und Tagelöhnerinnen ein Verantwortungsdiskurs geführt, in dem sie als die primär Verantwortlichen der Umweltdegradierung dargestellt werden. Frauen sind jedoch die Hauptbetroffenen von ökologischer Zerstörung. “"564 Heide Mertens schlägt unter der Überschrift Frauen und Ökologie: Opfer oder Schuldige? in die gleiche Kerbe: „Umweltzerstörung bedeutet aber für Frauen im Süden noch viel konkreter als bei uns - die Gefährdung der täglichen Existenz. Da, wo nichts mehr wächst, weil der Boden erodiert ist, die Wasserquellen versiegen und die Suche nach Brennholz und Futter für die Tiere immer weitere Wege erfordert, wird die Umweltzerstörung zur Überlebensfrage. Auch hier bedeutet das für Frauen vor allem mehr Arbeit. Wasser holen, Brennholz sammeln, Feldarbeit, die Notwendigkeit, zusätzliches Einkommen zu erwirtschaften (zum Beispiel durch den Verkauf von Brennholz), alles braucht mehr Zeit, Kraft und Energie.“6565

Aber auch im Norden gelte dieser Zusammenhang: „Überall scheint zu gelten, dass zunehmende Umweltzerstörung gerade den Frauen mehr Arbeit und Sorge bringt. (...) In den Industrieländern heißt das, Nahrungsmittel teurer und aufwendiger durch den Bio-Handel zu beschaffen, um Beeinträchtigungen durch Lebensmittelrückstände zu vermeiden. Die Kinder müssen mehr beaufsichtigt werden, damit ihnen durch Straßenverkehr und Umweltschadstoffe keine Unglücke geschehen. Für die Säuglingsnahrung muss Mineralwasser herangeschleppt werden, weil das Leitungswasser nitratbelastet ist. Der Versuch, umweltbewusst zu leben, bedeutet neue Aufgaben. Dazu gehören z.B. die Anlage eines Komposthaufens, aufwendiges Müllsortieren, Vermeiden von Fertiggerichten oder auch der Versuch, weitgehend auf Wegwerfwindeln und Auto zu verzichten. ${ }^{\text {" } 566}$

\subsubsection{Differenzierte Beurteilung der Kritik notwendig}

Damit wurde die Kritik an Typ 1-Ansätzen bzw. an ,Kairo' umfassend dargestellt. Sie ist zwar manchmal schrill und polemisch, ${ }^{567}$ aber dennoch nicht pauschal zu verwerfen. Entscheidend ist nun, en detail festzustellen, inwieweit die Kritik berechtigt ist.

Es ist mit Sicherheit extrem schwierig festzulegen, an welchem Punkt staatliche Appelle in Zwang übergehen. ${ }^{568}$ Allerdings desavouiert sich manche Kritik selbst,

\footnotetext{
${ }^{564}$ Hummel (2000), 76

${ }^{565}$ Mertens (1994), 187

${ }^{566}$ A.a.O., 184

567 So lautet etwa eine Überschrift von Diana Hummel: „Verhütungsmittel: Verwaltung der Gebärmütter“ (Hummel 2000, 11), bei Wichterich heisst es: „Der Griff nach dem Frauenkörper“ (1994, 10)

${ }^{568}$ Siehe dazu Abschnitt 5.9. Ethische Bewertung von finanziellen Steuerungsinstrumenten
} 
wenn sie diesen komplexen Sachverhalt aus politischen Gründen vereinfacht. Die plakativsten, krassesten Fälle, die von Kritiker(innen) aufgebracht wurden, sind naturgemäß in ethischer Hinsicht am einfachsten zu beurteilen. Ein Großteil der Einwände der Kritiker betrifft Missstände, die bereits von der internationalen Staatengemeinschaft in Kairo klar als unethisch addressiert wurden. Die Berichte über offensichtliche Menschenrechtsverletzungen, die sich vor allem in den 1960er und 1970er Jahren ereigneten, haben die Autoren des Aktionsprogramm veranlasst, jeden Zwang in Familienplanungsprogrammen mit klaren Worten zu verurteilen.

Die Kritiker(innen) negieren den Lernprozess, der von Bukarest bis Kairo stattgefunden hat, ja sie stellen mit Vergleichen zur Bevölkerungspolitik der Nationalsozialisten sogar die Arbeit der UN in eine Tradition mit der planmäßigen Menschenökonomie der Nazis. Der von allen Fachleuten hervorgehobene Paradigmenwechsel, der in Kairo gerade auf Druck von Frauengruppen beschlossen wurde, wird teilweise schlicht ignoriert. ${ }^{569}$ Dass man ausgerechnet der langjährigen UNFPA-Direktorin Nafis Sadik vorwarf, sie betreibe zusammen mit ihren vielen weiblichen Mitarbeitern eine gegen Frauen gerichtete Politik, erscheint weit hergeholt.

Ich möchte im Rahmen der umfassenden Bewertung der gegen Kairo vorgebrachten Kritikpunkte und meiner anschließenden eigenen Bewertung zunächst auf einige Kritikpunkte eingehen, die leicht zu widerlegen sind. Wie ist etwa die Kritik zu bewerten, dass Bevölkerungswissenschaft an sich unethisch sei? Mit demselben Argument müsste man die gesamten Sozialwissenschaften verurteilen, weil sie alle „reale, lebendige“ Menschen zu größeren Einheiten zusammenfassen (Klassen, Schichten, Geschlechter, Kohorten, Generationen oder eben einzelne Bevölkerungen). Diese Kritik läuft auf eine generelle Ablehnung quantitativer Sozialforschung hinaus, was offensichtlich wenig zielführend ist. Zumal erweckt das Aktionsprogramm von Kairo absolut nicht den Eindruck, als würde die Staatengemeinschaft ihre Augen vor den individuellen Bedürfnissen der Menschen verschließen. Der Generalsekretär der Vereinten Nationen, Boutros Boutros Ghali, hatte bei der Eröffnung der Konferenz explizit betont: „From the social standpoint, let us not forget that what we term ,the population' is not an indiscriminate mass. Each member of the population belongs to a culture, a society, a tradition. A population consists of multiple relationships, in which each community deserves our respect, and of which the family is the nucleus." ${ }^{570}$

Auch bezüglich der Angriffe auf die Sprache der Antinatalisten scheint mir eine früher berechtigte, gegenwärtig aber obsolete Kritik kultiviert zu werden. Tatsächlich sind die inkriminierten Formulierungen hochproblematisch und - wenn sie die Bevölkerungen des Südens auf diese Weise als Zerstörungspotenzial beschreiben - möglicherweise rassistisch. Sie stammen allerdings aus den 1970er

\footnotetext{
${ }^{569}$ Hummel (2000), 90-93

${ }^{570}$ Boutros-Ghali (1994), 23
} 
Jahren und waren selbst damals niemals Mainstream, sondern wurden vielmehr von allen seriösen Bevölkerungswissenschaftlern stets abgelehnt. Der Begriff der ,Bevölkerungsexplosion', wird dagegen durchaus von seriösen Demografen für das weltweite Bevölkerungswachstum in der zweiten Hälfte des 20. Jahrhunderts verwandt. ${ }^{571}$ Genauso spricht man von der ,Bevölkerungsimplosion' von Staaten. Der Begriff ,Bevölkerungsexplosion' soll also bildhaft ein Wachstum bezeichnen, bei dem die Wachstumsraten selbst zunehmen. In der Mathematik wird dies als hypergeometrisches Wachstum bezeichnet. Wissenschaftlich unbestritten ist die Tatsache, dass die Weltbevölkerung in den letzten Jahrhunderten zeitweise stärker als exponentiell wuchs und in der zweiten Hälfte des 20. Jahrhunderts unerwartet und historisch einmalig in eine eruptive Wachstumsphase eintrat. Ob der Begriff der ,Explosion' dafür eine glückliche Metapher zur Vermittlung des Sachverhalts war, ist Geschmackssache.

Auch die Behauptung, die Ökologie sei lediglich ein Druckmittel, braucht nicht ausführlich erörtert werden. Hier kann man aus wissenschaftlicher Sicht kurz und knapp einwenden, dass der mögliche Zusammenhang zwischen Umweltdegradierung und Frauenproblemen nicht geeignet ist, den von der PAT-Formel postulierten Zusammenhang zwischen $\mathrm{N}$ und $\mathrm{P}$ zu widerlegen.

Ob Frauen mehr als Männer von Umweltzerstörungen betroffen sind, wäre eine interessante, wenn auch wahrscheinlich schwierig zu beantwortende Frage, die nicht Teil meiner Fragestellung ist. Auch nicht näher eingegangen werden kann auf die kaum belegbaren Thesen von Ökofeministinnen, die unterstellen, dass Frauen ,von Natur aus' ökologisch wirtschaften, während das zerstörerische Potenzial der modernen Produktionsmethoden aus der für Männer charakteristischen Interaktion mit der Natur über Technologie und Werkzeuge erwachse. ${ }^{572}$

In dieser ersten Zurückweisung eines Teils der heftigen Kritik am Abschlussdokument von Kairo erscheint es sinnvoll, auf die vielleicht leicht zu übersehende Tatsache hinzuweisen, dass das Konzept der RH in gewisser Hinsicht als endgültiger Sieg über malthusianische Konzeptionen gewertet werden kann. Zumindest in seiner ersten, noch relativ zynischen Auflage betont Malthus die Notwendigkeit von ,Hemmnissen' ${ }^{573}$ Aus dieser Sicht muss bessere Gesundheitsvorsorge, wozu auch eine Verringerung der Säuglingssterblichkeit zählt, zu einer Beschleunigung der Bevölkerungswachstumsraten führen. Das Wachstum der Weltbevölkerung war ja in der zweiten Hälfte des 20. Jahrhunderts nicht deshalb so rasant gestiegen, weil die Menschen mehr Kinder als früher hatten, sondern vielmehr, weil aufgrund der Fortschritte bei Hygiene und Pharmazeutika nicht mehr so viele starben. In Kairo spielte die theoretisch denkbare zynische Schlussfolgerung, dass Krankheiten wie AIDS das Bevölkerungswachstum dämpften, keine Rolle. Viel-

571 Z.B. Hauser (1991), 246; Schmid (1994a), 11. Siehe auch die Definition dieses Begriffs im Brockhaus - Die Enzyklopädie in 24 Bänden. Internetausgabe. Rev. 2004-03-05

${ }^{572} \mathrm{Vgl}$. Mies (1988), 67f.

${ }^{573}$ Vgl. Abschnitt 2.4.1. Das Bevölkerungsgesetz von Thomas Robert Malthus. 
mehr besann man sich ganz auf das, was Al Gore in seiner Eröffnungsrede ansprach: „The most powerful contraceptive in the world is the confidence of parents that their children will survive. ${ }^{6574}$ Gesundheit ist ein Grundbedürfnis jedes Menschen. Und wenn dabei in verdaulichen Portionen noch etwas Familienplanungsbewusstsein mitgeliefert wird, so wird dieser Mix auch angenommen. Der Ansatz von Kairo berücksichtigte dies - scheinbar mit Erfolg. Die Bevölkerungswachstumsraten gingen seit 1994 in damals für nicht möglich gehaltenem Ausmaß zurück. Dies liegt zu einem bestimmten Grad sicherlich auch daran, dass frühere Ansätze, die die Menschen mit Kondomen und anderen Kontrazeptiva überschwemmten, „ohne sonst etwas für sie zu tun“, verständlicherweise Akzeptanzprobleme hatten.

Die grundsätzliche Kritik an der Legitimität jeder Bevölkerungspolitik ist in dem Sinne radikal, als dass sie selbst innerhalb der Frauenbewegung nicht mehrheitsfähig ist. ${ }^{575}$ Der gemeinsame Bezugspunkt aller feministischen Positionen ist die Betroffenheit von Frauen durch Bevölkerungspolitik und Familienplanung, wobei Analysen stets auch das Machtverhältnis zwischen den Geschlechtern mit einbeziehen. In der zentralen Frage, ob jede (also auch eine frauenfreundliche wie in Kairo beschlossene) Bevölkerungspolitik abzulehnen ist, herrscht Dissens. Bereits seit Mitte der siebziger Jahre konzentrierte sich die Bevölkerungspolitik auf eine Stärkung der Rolle der Frauen. Diese neue Ausrichtung geht nicht unwesentlich auf das Engagment von Feministinnen zurück, die in den wichtigen Institutionen wie der Rockefeller Foundation oder dem Population Council arbeiten. In der Entwicklungspolitik wurde schon vor 1994 der Women in Development-Ansatz paradigmatisch. ${ }^{576}$ Auf der Weltbevölkerungskonferenz in Kairo 1994 wurde - zumindest aus Sicht zahlreicher Aktivistinnen in der südlichen Hemisphäre Wirklichkeit, wofür die Frauenbewegung Jahrzehnte gekämpft hatte.

\subsubsection{Bewertung der Kritik an quantitativer Familienplanung}

Kritiker wenden sich wie gezeigt auch gegen das in Kairo beschlossene Konzept des ,Ungedeckten Bedarfs' (,unmet need'), welches Bestandteil jedes indirekten Ansatzes von Bevölkerungspolitik ist. Diese Kritik wird nachfolgend bewertet. Offensichtlich ist der Zugang zu Verhütungsmitteln eine notwendige Voraussetzung dafür, dass Paare selbstbestimmt über die Zahl ihrer Kinder entscheiden können. Insofern wird durchaus zu Recht der Deckung des ungedeckten Bedarfs von Verhütungsmitteln ein hoher Stellenwert eingeräumt. ${ }^{577}$ Die NGO und Pharmafirmen, die sich auf die Entwicklung und Verteilung von Kontrazeptiva spezialisiert haben, haben hier weiter ihr Betätigungsfeld. Die Strategie der Be-

\footnotetext{
${ }^{574}$ Gore (1994), 47

${ }^{575}$ Für die Diskussionen zwischen Feministinnen in Nord und Süd, siehe ausführlich Hummel (2000), 124-143

${ }^{576}$ Vgl. Buvinic (1994), 212; Heim/Schaz (1994), $144 \mathrm{f.}$

${ }^{577}$ ICPD (1994)
} 
darfsdeckung durch quantitative Verbreitung von Verhütungsmitteln wurde in eine qualitative Strategie eingebunden, ohne erstere jedoch aufzugeben. Dies erscheint völlig berechtigt und erfolgsversprechend, wie Abb. 23 zeigt.

\section{Abb. 22: Relation zwischen Verwendung von Kontrazeptiva und Ge- burtenrate}

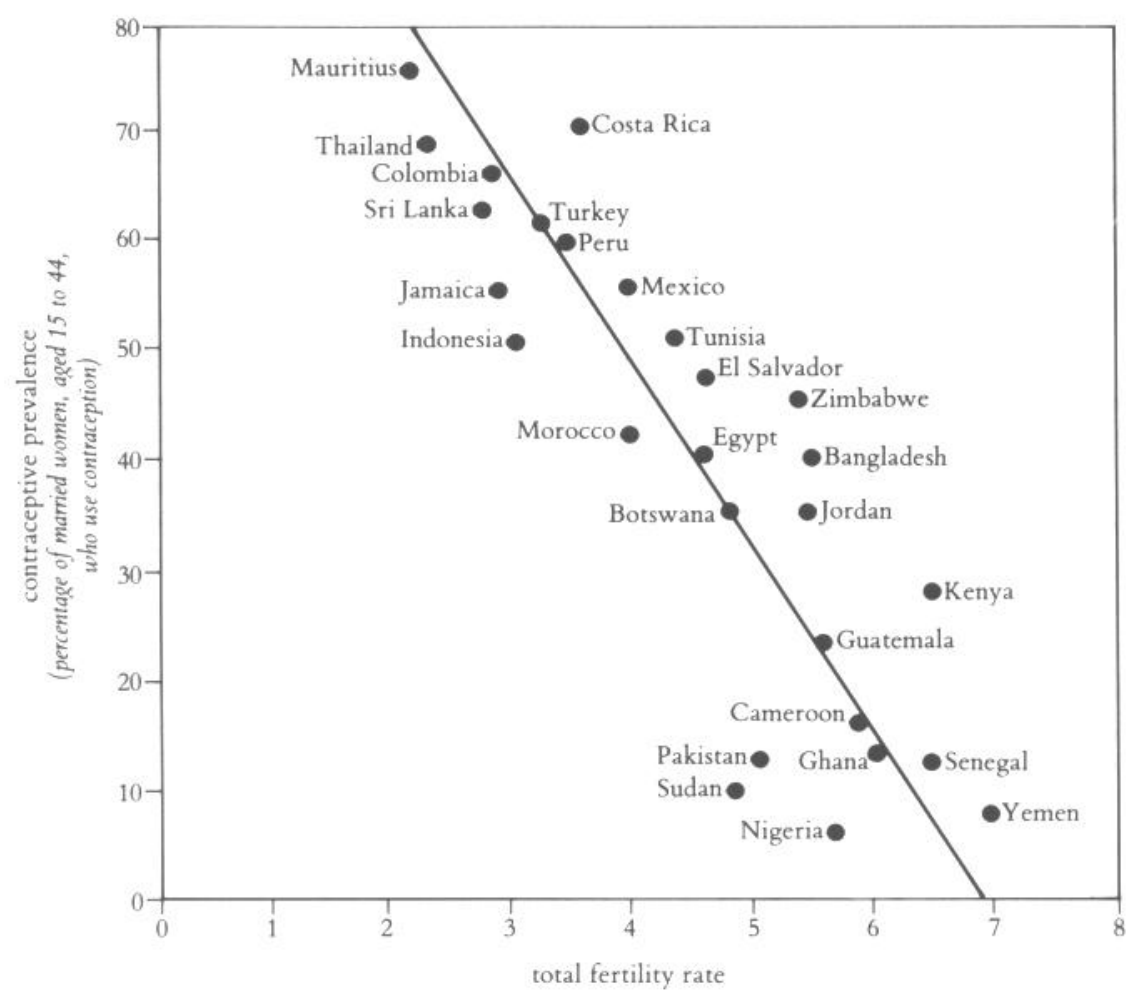

\section{Quelle; Cohen (1995), 74}

Wird der Rückgang der Geburtenrate in Relation zum Anstieg der Benutzerquote von Konrazeptiva in den vergangenen Jahrzehnten gesetzt, so ergibt sich als Faustregel, dass eine Steigerung der Anwendung moderner Kontrazeptiva um 15 Prozent mit einem Rückgang der Geburtenrate um ein Kind einhergeht. ${ }^{578}$ Andere Untersuchungen behaupten, dass 80 Prozent des Rückgangs der Geburtenrate hingegen auf den Anstieg der allgemeinen Lebensverhältnisse zurückzuführen sei, während der Einfluss von Familienplanungsmaßnahmen im engeren Sinn relativ unbedeutend sei. ${ }^{579}$ Höhn schreibt hingegen: „Diejenigen, die immer noch wissenschaftlich nachweisen wollen, dass Familienplanungsprogramme ausschlaggebend sind, seien daran erinnert, dass der säkulare Geburtenrückgang in Europa und Nordamerika ohne Familienplanungsprogramme und ohne moderne Verhü-

\footnotetext{
${ }^{578}$ Münz/Ulrich (1994), 15
}

${ }^{579}$ Schockenhoff (1996), 21 
tungsmittel erfolgte, vielfach gegen den erklärten Willen der Regierung. Die Motivation zur Beschränkung der Kinderzahl war in Nordamerika und Europa schon in der ersten Hälfte dieses Jahrhunderts [des 20. J.T.] offenbar so groß, dass sie die Menschen ihre Verhütungsmethoden finden ließ, auch ohne staatliche Förderung. “580 Höhn betont allerdings auch, dass dies nicht zu dem Schluss verleiten sollte, es sei nicht notwendig, den ungedeckten Bedarf an Verhütungsmitteln zu decken. Dies bleibe weiterhin nötig. ${ }^{581}$ Auch ohne Familienplanungsprogramme wäre die Fruchtbarkeit zurückgegangen - jedoch wesentlich langsamer. ${ }^{52}$ Ohne die weltweiten Anstrengungen zur Familienplanung wäre die Geburtenrate zwischen 1980 und 1985 statt auf 4,3 nur auf 5,4 Kinder pro Frau gesunken, was im Jahr 1990 einer höheren Gesamtbevölkerung von ungefähr 400 Mio. Menschen entsprochen hätte. ${ }^{58}$ Wenn alle Frauen, die sich gegen weitere Kinder aussprechen, auch wirklich keine bekommen müssten, würde die Anzahl der Geburten in Afrika um 27 Prozent reduziert, in Asien um 33 Prozent und in Lateinamerika um 35 Prozent. Außerdem könnte die Müttersterblichkeit um die Hälfte verringert werden. Wenn alle Paare in die Lage versetzt würden, ihre Familiengröße exakt zu planen, so könnte dies hinsichtlich der Bevölkerungsgröße der Entwicklungsländer im Jahre 2100 einen Unterschied von schätzungsweise 2,2 Milliarden Menschen bedeuten. Der Unterschied zwischen dem Einsatz wirkungsvoller Familienplanungsprogramme und gar keinen Programmen könnte weltweit 4,6 Mrd. Geburten ausmachen - das entspricht der gesamten Erdbevölkerung in den frühen achtziger Jahren. ${ }^{584}$

Es ist zynisch, wenn Feministinnen aus den reichen Ländern des Nordens ignorieren, dass öffentliche Stellen für die Verbreitung von Kontrazeptiva sorgen müssen, damit das Menschenrecht auf selbstbestimmte Sexualität nicht nur auf dem Papier steht. ${ }^{585}$ Familienplanungsprogramme sind weiterhin notwendig. Gleichzeitig stellt der allgemeine Zugang zu modernen Methoden der Empfängnisregelung nur eine notwendige, keine hinreichende Bedingung für eine ,Bevölkerungspolitik mit menschlichem Anlitz' (Klaus Leisinger) dar..$^{56}$

\footnotetext{
${ }^{580}$ Höhn (1995), 6

${ }^{581}$ A.a.O.

${ }^{582}$ Münz/Ulrich (1994), 10

${ }^{583}$ Schockenhoff (1996), 22

${ }^{584}$ Dabei wurden die oben genannten Zahlen hochgerechnet. Hierbei ist allerdings zu berücksichtigen, dass der Erfolg von ,Familienplanung' in der Vergangenheit teilweise eben auch auf unethische Mittel wie Rationierung und Zwangsmethoden zurückzuführen war.

${ }^{585}$ Im Rahmen eines Aufenthalts in einem abgelegenen Dorf in Jamaika konnte ich mir selbst ein Bild davon machen, wie dringend gewünscht die Versorgung mit Verhütungsmitteln ist. In vielen Dörfern kommt es zu frühem und häufigem Sexualverkehr in der Bevölkerung. Die jungen Männer, mit denen ich sprach, klagten über den Mangel an Verhütungsmitteln. Kondome seien für sie nur in der Stadt zu bekommen, also gingen sie ungewollt bei jedem ihrer Sexualkontakte das Risiko der Ansteckung mit Geschlechtskrankheiten und von ungewollten Vaterschaften ein.

${ }^{586}$ Leisinger (1994); ähnlich auch Schockenhoff (1996), 24
} 
Was ist in diesem Zusammenhang von dem Vorwurf zu halten, dass Frauen in der Dritten Welt bisher kaum zwischen verschiedenen Verhütungsmitteln wählen können? Christa Wichterich kritisiert: „Das Hochglanzbild einer »Cafeteria«, in der die Frauen das für sie geeignete Verhütungsmittel aus einem vielfältigen Angebot frei wählen können, ist lediglich die Supermarkt-Mentalität der Reproduktionstechnologie in den Staub der Dörfer des Südens phantasiert.“" ${ }^{687}$ Der Protagonist der Familienplanung in Thailand, Mechai Viavaidya, wird für den ihm zugeschriebenen Ausspruch „Familienplanung ist wie Sojasoße, man mischt sie unter alles“ von Wichterlich angegriffen: „Wo aber Pillen vom Friseur oder am Zigarettenbüdchen verkauft werden, ist vorprogrammiert, dass Beratung sowie medizinische Vor- und Nachsorge auf der Strecke bleiben. ${ }^{6588}$ Dagegen würden die Verhütungsmittel selbst immer mehr perfektioniert. Der Schwerpunkt liege heute neben einer Dominanz der Sterilisation in Entwicklungsländern in der Vergabe hochtechnologischer Langzeitkontrazeptiva, welche von den betroffenen Frauen schwer selbst kontrolliert werden könnten. ${ }^{589}$

Meiner Ansicht nach ist die Ausgabe von Verhütungsmitteln ohne Beratung tatsächlich kritisch zu bewerten, wobei aber zwischen Kondomen und anderen Verhütungsmitteln zu differenzieren ist. Kondome werden auch in Deutschland in öffentlichen Toiletten verkauft. Das in Thailand extrem erfolgreiche Konzept ,Capitalism condoms' (Kondome im Kapitalismus) von Mechai Viravaidya basiert auf der Idee, dass Kondome für jedermann extrem leicht zugänglich sein sollten. Im Rahmen dieses Programmes wurden Ladenverkäufer, Taxifahrer, Polizisten, Lehrer und Tankwarte gebeten, Kondome in ihren Bestand aufzunehmen und zu verkaufen. ${ }^{590}$ Daran ist nichts Unethisches. Ganz im Gegenteil erleichtern die kurzen Wege zum jeweils nächsten Versorgungspunkt Menschen die selbstbestimmte Entscheidung. Richtig ist aber, dass Verhütungsmittel, die in den SEL rezeptpflichtig sind, in den WEL nicht ohne Beratung abgegeben werden sollten. Sofern langanhaltende Kontrazeptiva (z.B. Drei-Monats-Spritze oder das Norplant-Implantat) ein ordentliches medizinisches Unbedenklichkeitsverfahren durchlaufen haben und mit Beratung abgegeben werden, spricht nichts gegen sie. Die Tatsache, dass eine Benutzerin die Wirkung eines gewählten Mittels nicht jederzeit rückgängig machen kann, muss kein Gegenargument sein. Es gibt viele Fälle, in denen eine junge Frau in Cebu oder Lagos den Wunsch einer Schwangerschaft mit Sicherheit für einen bestimmten Zeitraum ausschließen kann, z.B. wenn sie gerade ein Studium begonnen hat. Entscheidend ist, dass sie ihre Wahl informiert und selbstbestimmt trifft. Die Forderung, dass in Entwicklungsländern prinzipiell die gleichen Akzeptanzkriterien gelten müssen wie bei uns, ist aus

\footnotetext{
${ }^{587}$ Wichterich (1994), 18

${ }^{588}$ Wichterich (1994), 26

${ }^{589}$ Hummel (2000), 111

${ }^{590}$ Schering AG (2004), 23
} 
ethischer Sicht richtig. ${ }^{591}$ Dies ist aber kein Argument gegen langanhaltende Kontrazeptiva: Es stellt sich vielmehr die Frage, ob es nicht ethisch wäre, solche Methoden auch in SEL wie Deutschland populärer zu machen. Angesichts der Tatsache, wie häufig junge Frauen die Einnahme der ,Pille' vergessen und dann gegen ihren Willen schwanger werden, wäre dies ein Beitrag zu mehr Selbstbestimmung im Hinblick auf den Kinderwunsch.

Zusammenfassend lässt sich sagen, dass ein Teil der in der Literatur beschriebenen Kritik am Konsens der Staatengemeinschaft sich selbst zehn Jahre nach Kairo immer noch auf Punkte bezieht, die von der internationalen Gemeinschaft als ebenso ethisch problematisch bewertet wurden wie von den Kritiker(innen). Zugegebenermaßen setzen die Einzelstaaten, die 1994 auf ihrer nationale Souveränität bestanden, ${ }^{592}$ in ihren nationalen Bevölkerungsstrategien oft noch nicht alle in Kairo formulierten Kriterien um. Aber der Konsens von Kairo hat eine normative Wirkung auf viele Staaten ausgeübt und z.B. auch dazu beigetragen, dass die chinesische Führung ihre Bevölkerungspolitik umstrukturierte. ${ }^{593}$

Eine aus ethischer Sicht wichtige Frage ist aber, ob das, was die internationale Gemeinschaft im Rahmen von indirekten Ansätzen (Typ 1.) für ethisch legitim hält, in sich stimmig ist. Möglicherweise fiel die Kritik an Kairo auch deshalb so laut aus, weil die ethischen Maximen des Aktionsprogramms als contradictio in se gar nicht umgesetzt werden können. Dies wird im folgenden Abschnitt untersucht.

\subsubsection{Kann es eine selbstbestimmte, ,freie' Entscheidung geben?}

Schon in der in der UN-Konvention von 1948 findet sich das Verbot jeder qualitativen, bestimmte Bevölkerungsgruppen diskriminierenden Bevölkerungspolitik. Eine solche Politik wird als ,Völkermord' geächtet. ${ }^{594}$

Das Recht von Paaren bzw. Individuen, die Zahl ihrer Kinder selbst zu bestimmen, ist nicht explizit in der UN Universal Declaration of Human Rights verankert. Die Proklamation von Teheran, verabschiedet am 13.5.1968, formulierte aber schließlich den Grundsatz, dass Paare das Recht haben, frei und verantwortlich über die Anzahl und Geburtenabstände ihrer Kinder zu entscheiden. ${ }^{595}$ In Kairo findet sich der Grundsatz im Abschlussdokument wieder, ergänzt um die Pflicht des Staates, das Recht der Paare auf Familienplanung auch in der Praxis zu verwirklichen: „Alle Paare und Einzelpersonen ${ }^{596}$ haben das Grundrecht, frei und

\footnotetext{
${ }^{591}$ Schockenhoff (1996), 64

${ }^{592}$ Vgl. dazu die Eröffnungsrede der ICPD in Kairo vom Präsident des Gastgeberlandes, Hosni Mubarak. Der ägyptische Präsident schildert die ägyptischen Maßnahmen zur Familienplanung als sehr erfolgreich und betont, dass der Grundsatz der nationalen Souveränität in diesen Fragen für ihn nicht zur Disposition steht (Mubarak 1994).

${ }^{593}$ Meulenberg (2004), 33

${ }^{594}$ Siehe Abschnitt 2.2. Definition der Kernbegriffe.

${ }^{595}$ UN Population Division (2003a), 14

${ }^{596}$ Man beachte, dass hier nicht von ,Frauen' die Rede ist.
} 
eigenverantwortlich über die Anzahl und den Geburtenabstand ihrer Kinder zu entscheiden und die dafür nötigen Informationen, Aufklärung und Mittel zu haben.“ Dieser Rechtsanspruch geht davon aus, dass ein abstraktes Recht wenig wert ist, wenn man nicht auch die Mittel hat, es umzusetzen. Dieser gedankliche Schritt ist Begründungsbasis staatlicher Programme und bevölkerungspolitischer Institutionen für die Bereitstellung von Mitteln zur Familienplanung.

\section{Abb. 23: Kinderwunsch und Kinderzahl in Entwicklungsländern}

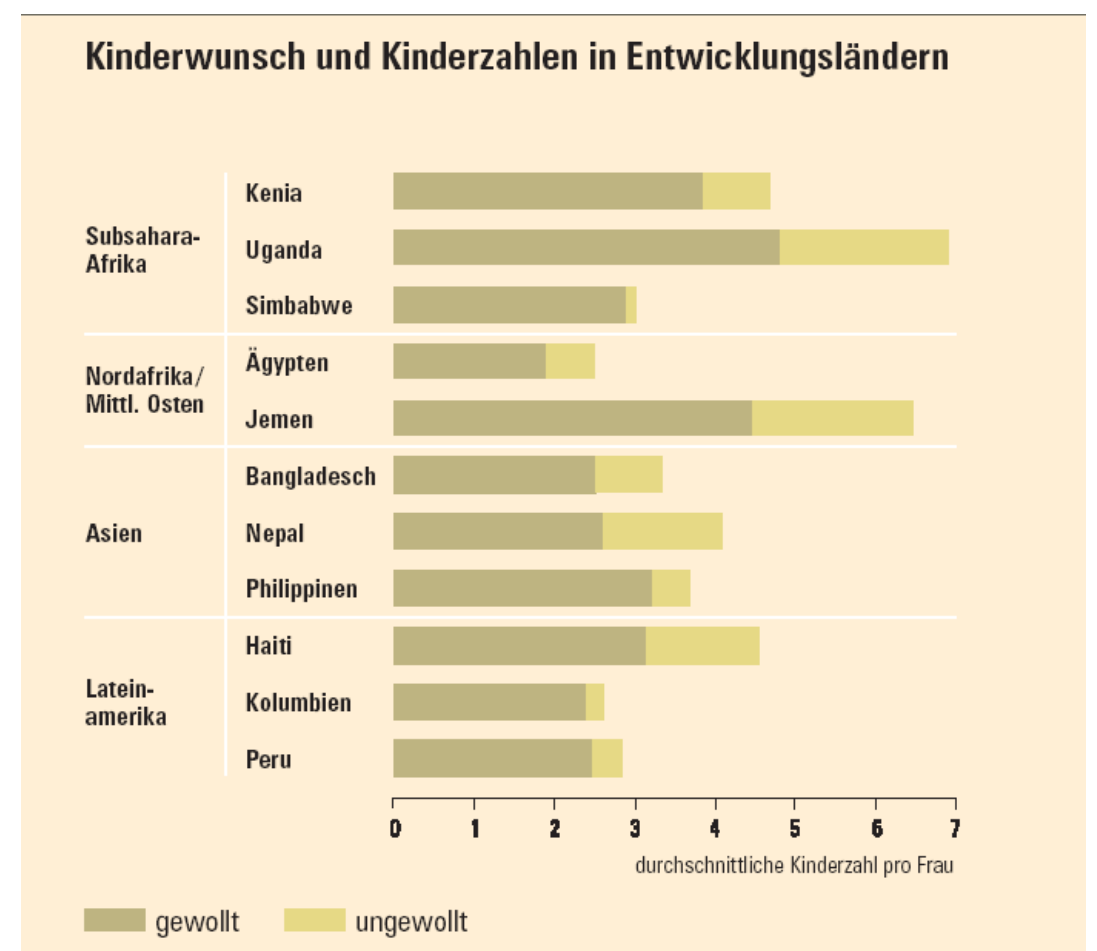

\section{Quelle: Deutsche Stiftung Weltbevölkerung,} http://www.dsw-online.de/grafiken.html

Die gewünschte Kinderzahl der Bürgerinnen von Entwicklungsländern wird im Rahmen von international vergleichbaren erhobenen Surveys erhoben, etwa dem World Fertility Service aus den späten 1970er und frühen 1980er Jahren und dem Demographic and Health Survey aus den späten 1980er Jahren. ${ }^{597}$ Auch im Rahmen des World Values Survey wird nach der als ideal angesehen Familiengröße gefragt. ${ }^{598}$ Diese Befragungen belegen, dass mittlerweile in fast allen Ländern der südlichen Hemisphäre die Mehrzahl der verheirateten Frauen die Zahl ihrer künftigen Geburten einschränken will. ${ }^{599}$ Ein weiterer Befund lautet, dass in fast allen Gebieten die gewünschte Kinderzahl unter der tatsächlichen liegt, auch wenn v.a.

\footnotetext{
${ }^{597}$ Siehe http://www.pop.psu.edu/data-archive/daman/dhs.htm

${ }^{598}$ Frage 108, siehe http://www.worldvaluessurvey.org/statistics/index.html

${ }^{599}$ Schockenhoff (1996), 18
} 
in Schwarzafrika, aber auch in den ölreichen Golfstaaten die gewünschte Kinderzahl noch sehr hoch ist. ${ }^{600}$

Hierbei ist zu klären, was in diesem Zusammenhang eigentlich ,frei' und was , eigenverantwortlich' heißen kann. Entgegen der scheinbaren Eindeutigkeit von Abb. 24 lässt sich auch die Auffassung vertreten, dass in jeder Gesellschaft Sexualität und reproduktive Entscheidungen durch ein komplexes Zusammen- und Gegeneinanderspielen von individuellen Hoffnungen und Wünschen auf der einen Seite und gesellschaftlichen Rahmenbedingungen, Normen und Ansprüchen auf der anderen Seite bestimmt sind. Wie man z.B. aus Wahlumfragen weiß, determinieren Verhaltensabsichten keinesfalls automatisch tatsächliches Verhalten. Es dürfte eine Rolle spielen, wer nach der gewünschten Kinderzahl fragt, wie alt die befragte Person zu diesem Zeitpunkt ist und unter welchen Rahmenbedingungen gefragt wird. Bezogen auf die Ergebnisse der oben genannten empirischen Studien wurde eingewandt, dass die gewünschte Kinderzahl - wie viele andere Präferenzen auch - über den Lebenszyklus nicht konstant ist. Eine Entscheidung über weitere Kinder wird von der Befragten erst nach der Geburt des jeweils vorherigen getroffen, da sich dadurch Lebenspläne und -bedingungen signifikant ändern. ${ }^{601}$

Der Kinderwunsch ist abhängig von einer Vielzahl wirtschaftlicher, kultureller, sozialer und letztlich auch biologischer Faktoren. ${ }^{602}$ Wichterich kritisiert: „Individuelle Familienplanung, wie strategisch und ausgetüftelt sie auch sein mag, ist letztendlich häufig auch in unseren Breitengraden eine krude Mischung aus Gewünschtem und Ungewolltem, von Plan und Zufall.“" ${ }^{“ 63}$ In den neuen Bundesländern hat ohne jede offizielle Bevölkerungspolitik nach der Wiedervereinigung der heftigste demografische Umbruch der deutschen Geschichte stattgefunden. Zwischen 1989 und 1993 sanken die jährlichen Geburtenzahlen auf 40 Prozent, von 200.000 auf ca. 80.000 Geburten. Dies zeigt, wie stark äußere Faktoren wie veränderte Rahmenbedingungen und Zukunftschancen bzw. - unsicherheiten die ,selbstbestimmten' Entscheidungen von Paaren beeinflussen.

\subsubsection{Kinderwunsch und Kinderzahl}

Der Kinderwunsch bewegt sich oft in Widersprüchen. Er konkurriert mit anderen Lebensentwürfen, er kann durch den überraschenden Arbeitsplatzverlust aufgeschoben und durch eine frühe Heirat mit dem oder der ,Richtigen' vorgezogen werden. Auch völlig unabhängig von staatlicher Bevölkerungspolitik steckt die normative Kraft der sozialen Realität die Handlungsspielräume ab. Kann ein Kinderwunsch als ,selbstbestimmt' bezeichnet werden, wenn er von dem Zwang zur eigenen sozialen Alterssicherung diktiert wird?

\footnotetext{
${ }^{600}$ Höhn (1995), 8

${ }^{601}$ Goldstein/Lutz/Testa (2003), 4

${ }^{602} \mathrm{Zu}$ beachten sind hier die steigenden Unfruchtbarkeitsraten weltweit, vgl. Heinrichs (2001).

${ }^{603}$ Wichterich (1994), 9
} 
Es ist erhellend, diesen Zusammenhang an den Aussagen von Pronatalisten in der nördlichen Hemisphäre zu spiegeln. Die Formel von der selbstbestimmten Entscheidung wird in Bezug auf Entwicklungsländer so interpretiert, dass der Staat aktiv werden soll, um diese Entscheidung durch Bereitstellung von Verhütungsmitteln in der Praxis zu verwirklichen. Umgekehrt soll z.B. in Deutschland der Staat geburtenförderliche Maßnahmen erlassen, um die Entscheidungsfreiheit der Bürger zu wahren. Der ehemalige Ministerialdirektor Max Wingen, einer der Befürworter staatlicher, geburteninduzierender Maßnahmen, schreibt: „Kann von wirklich freier und verantwortlicher Entscheidung dann gesprochen werden, wenn Eltern mit der Entscheidung für (mehrere) Kinder Gefahr laufen, sich und die (weiteren) Kinder in - wenn auch kulturspezifisch relative - Armut hineinzumanövrieren?" ${ }^{604}$ Wenn es ein durch UN-Beschlüsse und Grundgesetz garantiertes Menschenrecht gebe, frei verantwortlich und informiert über die Zahl der Kinder zu entscheiden, dann müsse der deutsche Staat nach Wingen dieses Recht auch einlösen. Die Entscheidungs- und Handlungsspielräume junger Paare müssten so erweitert werden, dass sie sich „unter zumutbaren Bedingungen“"605 für die Zahl der Kinder entscheiden können, die ihrem Lebensentwurf entspricht. „Unter zumutbaren Bedingungen" heißt bei Wingen letztlich ohne Verzicht auf andere Formen der Selbstverwirklichung und ohne bedeutsame Einkommensverluste. Man darf vermuten, dass die Antwort anders ausfiele, wenn man die Fragestellung variierte. Auf die Frage, wie viele Kinder sich eine Deutsche wünschen würde, wenn das Kindergeld 1.000 Euro pro Monat betrüge (oder 0 Euro), wenn es genug Betreuungsstätten gäbe (oder noch weniger) oder wenn es einfach nur mehr (oder weniger) Hoffnung auf einen wirtschaftlichen Aufschwung gäbe, wird man unterschiedliche Antworten bekommen. Das gleiche gilt auch für Menschen in China oder Brasilien.

\subsubsection{Fortpflanzung - eine Entscheidung der Frau, des Mannes oder des Paares?}

Die Formel von der selbstbestimmten Entscheidung ist noch in einer anderen Hinsicht möglicherweise ein Widerspruch in sich. Das Paradigma von der selbstbestimmten Entscheidung geht implizit davon aus, dass beide Ehepartner (bzw. in Ländern mit erlaubter Vielweiberei bzw. Vielmännerei alle Ehepartner) wie ein Individuum handeln. In der Realität variieren jedoch die Kinderwünsche von Männern und Frauen. Nur Frauen können Kinder austragen und gebären. Folgt daraus, dass nur Frauen entscheiden? Das Aktionsprogramm von Kairo scheint ausschließlich Frauen als seine Zielgruppe anzusehen, wie auch die meisten staatlich konzipierten Bevölkerungspolitiken davor und danach. „Der strategische Schlüssel zur Plan- und Steuerbarkeit des Bevölkerungs»körpers« ist ohne Zwei-

\footnotetext{
${ }^{604}$ Wingen (1.4.2004), 9

${ }^{605}$ A.a.O.
} 
fel der Frauenkörper" schreibt die Feministin Christa Wichterich. ${ }^{606}$ Der erste Anschein scheint ihr Recht zu geben. Lange Zeit flossen fast alle Mittel für die Entwicklung von Langzeitverhütungsmitteln in Kontrazeptiva für Frauen.

Wie aber unterscheidet sich der Kinderwunsch von Männern und Frauen eigentlich? Für einige afrikanische Länder wurde aufgezeigt, dass Männer sich häufig mehr Kinder wünschen und Frauen sogar an der Benutzung von Kontrazeptiva hindern. ${ }^{607}$

Tab. 11: Divergierende Kinderwünsche von Männern und Frauen

\begin{tabular}{|l|l|l|l|}
\hline \multirow{2}{*}{ Land } & \multicolumn{2}{l|}{ Durchschnittl. Zahl gewünschter Kinder } & Höherer Kinderwunsch \\
\cline { 2 - 4 } & Männer & Frauen & Männer \\
\hline Kamerun & 11,2 & 7,3 & 53 Prozent \\
\hline Niger & 12,6 & 8,5 & 48 Prozent \\
\hline Kenia & 7,6 & 5,3 & 43 Prozent \\
\hline Ghana & 4,8 & 4,8 & 0 \\
\hline
\end{tabular}

Quelle: Sachs $(1994,14)$

Gerade in patriarchalisch geprägten Gesellschaften findet ein Austausch der Geschlechter über Fragen der Verhütung oder auch nur der Sexualität allgemein nicht statt. Es ist für Frauen dort bislang schwer, ihre Bedürfnisse gegen die von Männern gewünschte Kinderzahl durchzusetzen. ${ }^{608}$

Hoffnungsvoll stimmt, dass auch und gerade in patriarchalischen Länder Frauen die Verhütungsfragen überlassen werden. In Lateinamerika entscheiden zwar in 46 Prozent aller Fälle die Paare gemeinsam über die Wahl der Kontrazeptiva, aber in 37 Prozent maßgeblich die Frauen und nur in 6 Prozent maßgeblich die Männer. ${ }^{609}$ Dadurch können Frauen die von ihnen präferierte Zahl von Kindern leichter durchsetzen.

Das Aktionsprogramm des ICPD konzentriert sich auf Frauen, also auf das Geschlecht, das meist weniger Kinder will. Durch die Erhöhung ihrer Kompetenz und ihres ,Verhütungswissens' behandelt man tatsächlich die Frauen als „Schlüssel“ einer wirksamen Familienplanung. ${ }^{610}$

Es zeigt sich also, dass Kinderwunschzahlen stets partnerabhängig und konditioniert sind. Dennoch kann man Messfehler natürlich einschränken. Besonders die Untersuchungen, die nicht nach Idealvorstellungen von Familiengröße und Kin-

\footnotetext{
${ }^{606}$ Wichterich (1994), 12

${ }^{607}$ Vgl. Lloyd (1996), 65-80; Sachs (1994) zitiert nach Hummel (2000), 116

${ }^{608}$ Cohen (1995), 71

${ }^{609}$ Corral (2004), 59. Der Restwert auf 100 Prozent entspricht ,keine Angabe'.

${ }^{610}$ In allen Ländern, in denen die Frauen den Männern nicht familiär untergeordnet sind, wird allerdings kaum der Mann alleine die Entscheidung über Zahl und Zeitpunkt der Kinder treffen. In den westlichen Ländern besprechen in der Regel die Partner untereinander, wann sie Kinder wollen. Die Entscheidung, wann Verhütungsmittel abgesetzt werden, um den Kinderwunsch in die Tat umzusetzen, ist eine gemeinschaftliche Entscheidung.
} 
derzahl, sondern gezielt danach fragen, ob die jeweils letzte Geburt zu ihrem Zeitpunkt erwünscht war, dokumentieren eine hohe Zahl unerwünschter Schwangerschaften. Als Gesamtziffer lässt sich aus den Befragungsergebnissen eine ungefähre Trendangabe errechnen, wonach jede dritte Geburt zu diesem Zeitpunkt ungelegen kam oder überhaupt unerwünscht war. ${ }^{611}$ Unter Einbeziehung der 30 bis 50 Millionen Abtreibungen pro Jahr fällt das Ergebnis noch weit ungünstiger aus. $\mathrm{Zu}$ dem brutalen Mittel einer Abtreibung als Mittel der Geburtenplanung greift nur, wer vorher keine wirksamen Formen der Empfängnisverhütung zur Verfügung hatte. Die bessere Versorgung mit wirksamen (d.h. auch lange wirksamen), sicheren Verhütungsmethoden ist offensichtlich ein Weg, um die Zahl der unerwünschten Schwangerschaften und die Zahl der Abtreibungen zu senken.

\subsubsection{Kann der Staat neutral bleiben?}

Die Weltgemeinschaft fordert den Verzicht von Zielvorgaben für die nachgeordneten Beamten, die Familienplanungsprogramme ausführen, obwohl der Staat insgesamt an demografischen Zielgrößen festhalten darf. Der Abschlusstext von Kairo +5 formuliert diesen nur scheinbar widersprüchlichen Punkt noch mal flüssig: „Bei dem Versuch, diese Richtwerte ${ }^{612}$ zu erreichen, sollen den Anbietern von Familienplanungsdiensten keine demografischen Ziele in Form von Zielwerten oder Quoten für die Anwerbung von Kunden vorgegeben werden, obgleich solche Ziele legitime Bestandteile der staatlichen Entwicklungsstrategien sind." ${ }^{\prime 13}$ Ein Land wie China kann demnach also weiterhin nationale Ziele im Hinblick auf Bevölkerungswachstum und-größe proklamieren, aber es darf, wenn es in Zukunft ethisch handeln will, nicht den Familienplanungsdiensten auf lokaler Ebene konkrete Zielgrößen vorgeben. Man stelle sich dieses Prinzip übertragen auf Deutschland vor. Die deutsche Regierung dürfte die Geburtenrate durch finanzielle Anreize fördern, aber sie dürfte nicht den staatlichen Krankenhäusern oder Pro Familia Vorgaben machen, dass in jedem Regierungsbezirk so viele Kinder geboren werden sollen. Diese direkte Übertragung - so unpassend sie auch scheint - zeigt, warum direkte Sollquoten in China oder anderen Ländern zu Fehlverhalten nachgeordneter Beamter führen können. Gleichzeitig erkennt die UN-Formulierung an, dass es unmöglich und nicht sinnvoll ist, von einem Staat $\mathrm{zu}$ verlangen, die Trends seiner Bevölkerungsentwicklung neutral zu sehen. Ein Staat darf demografische Ziele haben, aber er darf diese nur mit bestimmten Mitteln verfolgen.

\footnotetext{
${ }^{611}$ Münz/Ulrich (1994), 37

${ }^{612}$ Soweit eine Lücke zwischen der Verwendung von Empfängnisverhütungsmitteln und dem Anteil derjenigen besteht, die längere Zeiträume zwischen den Geburten ihrer Kinder wünschen oder die Anzahl ihrer Kinder begrenzen möchten, soll diese von den Ländern bis zum Jahr 2005 um mindestens 50 Prozent und bis zum Jahr 2010 um 75 Prozent verkleinert und bis zum Jahr 2050 ganz geschlossen werden.

${ }^{613}$ United Nations (1999), Abs. 58
} 
Man kann die Auffassung vertreten, das Recht des Staates auf demografische Zielgrößen bei gleichzeitiger Forderung selbstbestimmter Entscheidung von Individuen über Reproduktionsentscheidungen sei in gewisser Weise eine Quadratur des Kreises. Wenn sich aber ein Staat desinteressiert gegenüber Bevölkerungsprognosen zeigen müsste, so würde dies z.B. bedeuten, dass er auch dann nicht aktiv werden dürfte, wenn die Bevölkerung wie im Beispiel Deutschlands nach den Langzeitprognosen der UNO ohne Änderung des Gebärverhaltens bis 2300 von 82 auf 3 Millionen zurückgehen wird. ${ }^{614}$ Oder wenn sich die Bevölkerungsgröße wie im Falle von einigen Entwicklungsländern vervielfachen würde. Insofern hat es mehr Nachteile als Vorteile, dem Staat jegliches Nachdenken über eine ideal angesehen Bevölkerungsentwicklung und jede Formulierung von diesbezüglichen Zielen verbieten zu wollen.

Die Aufgabe eines Staates, das Wohl seiner Bürger zu mehren, ist seit langem anerkannt. Der Staat gewährleistet Sicherheit, betreibt Sozial-, Bildungs- und Umweltpolitik. Mit Migrationspolitik und Gesundheitspolitik (im Sinne einer Erhöhung der Lebenserwartung und einer Verbesserung der Gesundheit) betreiben Staaten auch bereits Bevölkerungspolitik, ohne dass dies moralisch umstritten ist. Zur Rolle von Staaten bezüglich Geburtenpolitik schreibt der Bevölkerungsethiker Callahan:

„It is only fairly recently, however, that governments have taken leading role in an antinatalist control of fertility (...). While many countries still do not have such policies, few international objections have been raised against the right of nations to develop them. So far, most government population policies have rested upon and been justified in terms of an extension of freedom of choice. Increasingly, though, it is being recognized that, since demographic trends can significantly affect national welfare, it is within the right of nations to adopt policies designed to reduce birthrates and slow population growth. (...) Is there any special reason to presume (...) that governmental intervention in the area of individual procreation (...) raises problems which, in kind, are significantly different from other kinds of interventions? (...) I see no special reason to think that the formation of interventionist, antinatalist, national population policies poses any unique theoretical difficulties. (...) In any case, the premise of my discussion will be that governments have as much right to intervene in procreation-related behavior as in other areas of behavior affecting the general welfare." ${ }^{\prime 15}$

\footnotetext{
${ }^{614}$ Vgl. dazu aber auch den Abschnitt 6.4. Projektionen sind keine Prognosen.

${ }^{615}$ Callahan (1976), $26 \mathrm{f}$.
} 
Wie Callahan im weiteren ausführt, hängt es aber eben von den konkreten Maßnahmen ab, ob staatliche Geburtenpolitik ethisch legitim ist oder nicht. Bisher haben wir nur festgestellt, dass Regierungen das Recht haben, demografische Zielgrößen als ,wünschenswert' zu bezeichnen. Noch ist nichts darüber gesagt, ob ein Staat einen systematischen Plan aufstellen darf, diese Ziele zu erreichen oder ob z.B. auf Regierungsebene eine Institution zur Überwachung der Aktivitäten geschaffen werden darf.

\subsubsection{Der Demokratie-Vorbehalt}

Mir erscheint es an dieser Stelle notwendig, bereits bei dem Recht, welches Regierungen legitimerweise haben, nämlich bei der Formulierung von Zielgrößen, hinzuzufügen, dass es sich dabei um demokratisch legitimierte Regierungen handeln muss. Es macht einen großen Unterschied, ob eine diktatorische oder eine demokratische Regierung eine Geburtenpolitik entwickelt und implementiert wird. Bei einer demokratischen Regierung werden die Ziele zwangsläufig bei jeder Wahl neu diskutiert und ggf. revidiert. Eine Minderheitenposition hat stets die Möglichkeit, zur Mehrheit zu werden. Angesichts der langen Zeiträume, in der jede Geburtenpolitik nur wirksam werden kann, könnte man einwenden, dass es dann de facto nicht zu einer kontinuierlichen Geburtenpolitik kommen würde. Mehr noch: In seinem berühmten Aufsatz The Tragedy of the Commons argumentiert Garrett Hardin, dass das Fortpflanzungsverhalten eines der Probleme sei, in denen das eigennützige Verhalten des Einzelnen zum Ruin des gesamten Systems führe. ${ }^{616}$

Dabei wird vorausgesetzt, dass die Kinderzahl, die sich eigennutzmaximierende Eltern zulegen würden, über dem Reproduktionsniveau liegt. Bayles schreibt dazu (bezogen auf die USA):

„The second premise is that under current conditions it is not in the interest of individual couples to limit the number of children they have to that compatible with the public good of a limited population size or rate of growth. The reasons why this premise is true vary between countries and cultures. (...) First, most people value having children. The reasons and causes for this evaluation are complex. There are historical factors in the sexual ethic which suggest that the purpose of marriage is to have children. Many couples want a child of each sex, and it may take three or four children for them to realize this goal. Children provide companionship, love, and a purpose to life. Further, psychologists have suggested that single children are not as well adjusted as those in larger

${ }^{616}$ Hardin (1968), 1243-1248 
families. (...) The second and perhaps more important factor making it in the interest of individual couples to have more children than are compatible with the public good is that the costs are partially externalized. People do not bear the full financial burden of their children. Single persons and couples without children pay taxes for public schools, sewage plants, police protection, garbage collection, highways, and other public services at the same or sometimes higher rates than couples with children." ${ }^{\prime 17}$

Hardin und Bayles setzen voraus, dass die Kinderzahl, die sich eigennutzmaximierende Eltern zulegen würden, über dem Reproduktionsniveau liegt. Kann daraus geschlossen werden, dass der ethische Demokratie-Vorbehalt falsch ist?

Hardin wurde empirisch widerlegt. Es aber auch ausreichend empirische Belege, dass sich Entwicklungsländer, die Demokratien sind, mit Mehrheitsentscheidung eine antinatalistische Geburtenpolitik auferlegen, selbst wenn jeder einzelne Bürger deutlich mehr als zwei Kinder haben will. Vernünftige Individuen sehen auch die Notwendigkeit von Besteuerung ein, obwohl niemand gerne Steuern zahlt. Indien, die größte Demokratie der Welt, hat eine antinatalistische Politik verabschiedet. Es spricht also vieles dafür, nur Demokratien das Recht zuzusprechen, Bevölkerungsziele zu formulieren und eine Geburtenpolitik zu implementieren. In Indien konnten Missbräuche - anders als in China - korrigiert werden, wie der Rücktritt der Regierung Indira Gandhi wegen Zwangssterilisationen zeigte. ${ }^{618}$

\footnotetext{
${ }^{617}$ Bayles (1976b), 47

${ }^{618}$ Es ist eine für die weitere Entwicklung der Welt wichtige Frage, ob ein demokratisches China eine antinatalistische Geburtenpolitik beibehalten würde. Diese Frage habe ich mit etwa 12 unterschiedlichen Chinesen, die in den USA, Großbritannien oder Deutschland studieren oder arbeiten, mehrfach diskutiert. Ohne aus ihren Aussagen irgendeine Repräsentativität ableiten zu wollen, so halte ich doch die Antworten der Exil-Chinesen, die ich bekam, im Rahmen von anekdotischer Evidenz für bemerkenswert. Das autoritäre bzw. diktatorische Regime in Bejing wurde in aller Schärfe verurteilt. Auf die Frage nach der Ein-Kind-Politik antworteten die Chinesen, dass diese zwar eigentlich richtig sei, aber mit zu vielen Menschenrechtsverletzungen einherginge. Konkret nachgefragt, ob China, wenn es demokratisch würde, eine antinatalistische Geburtenpolitik beibehalten würde, antworteten meine Gesprächspartner übereinstimmend mit Ja. Über die genaue Ausgestaltung dieser Politik gab es unterschiedliche Meinungen. Diese jungen Chinesen, die sicherlich zur Bildungselite ihres Landes gehören, vertraten aber einhellig die Auffassung, dass eine Senkung der Geburtenrate im Gemeinwohlinteresse sei. Ihrer Ansicht nach wäre es besser für China, wenn weniger Menschen darin lebten als heute. Konkret nachgefragt, welche Bevölkerungspolitik sie machen würde, wenn sie Premierministerin wäre, antwortete z.B. die 26jährige Jie Wang (Studentin von Comparative Education in Buffalo, USA), dass sie mehr auf Aufklärung setzen würde, damit Menschen von sich aus weniger Kinder wollten. Sie nannte es ein Problem, dass viele Bauern vermutlich so viele Kinder anstrebten, bis ein Junge darunter ist und hoffte, dass man dieses Verhaltensmuster durch freiwillige Kampagnen schwächen könne. Auf die Frage, ob sie eher auf Anreize oder auf Sanktionen setzen würde, antwortete sie: „Incentives probably don't work.“ Jie hatte im schulischen Biologieunterricht nur sehr unzureichende Informationen über Reproduktivität und Kontrazeptiva erhalten und hielt es für wichtig,
} 
Zusammenfassend lässt sich sagen, dass die bisherigen Überlegungen das Ergebnis brachten, dass Geburtenpolitik in Demokratien nicht generell ethisch unzulässig ist, sondern vielmehr die Wahl der Mittel das entscheidende Kriterium ist.

\subsubsection{Fazit}

In diesem Abschnitt erfolgte eine ausführliche Bewertung von Typ 1-Bevölkerungspolitiken. Da man sich in Kairo von diesem Ansatz leiten lassen, wurde die Bewertung des indirekten Ansatzes zusammen mit der Bewertung des Konsenses von Kairo behandelt. Hierbei wurde zunächst auf die Bewertungen durch andere Gruppen oder Personen eingegangen, bevor eigene normative Überlegungen erfolgten. Von den Argumenten gegen das PoA (und gegen den indirekten Ansatz) ist v.a. zwei relevant:

Erstens wurde geprüft, ob die Leitlinie von Typ 1-Politiken („Individuen bzw. Paare sollen frei und selbstbestimmt über die Zahl ihrer Kinder entscheiden") ein Widerspruch in sich ist. In Bezug auf die ,freie' Entscheidung von Individuen ist festzuhalten, dass diese stets partnerabhängig, konditioniert und sequentiell, also in diesem Sinne nicht wirklich ,frei' ist. Befragungen über die gewünschte Kinderzahl der Menschen auf der ganzen Welt sind mit Vorsicht zu genießen. Dennoch kann man Messfehler natürlich einschränken und auf diese Weise auf eine annähernde Wunschzahl von Kindern ermitteln. Besonders die Untersuchungen, die nicht nach Idealvorstellungen von Familiengröße und Kinderzahl, sondern gezielt danach fragen, ob die jeweils letzte Geburt zu ihrem Zeitpunkt erwünscht war, dokumentieren eine hohe Zahl unerwünschter Schwangerschaften in Entwicklungsländern.

Zweitens wurde die, u.a. von feministischer Seite erhobene Behauptung geprüft, dass es zwangsläufig zu einer Einschränkung der freien Entscheidung kommen müsse, wenn man Staaten weiter erlaubt würde, demografische Zielgrößen aufzustellen. Da der Verzicht eines Staates auf demografische Zielgrößen bzw. Stellungnahmen (z.B. in dem Sinne, dass die Geburtenrate des eigenen Landes zu hoch oder zu niedrig ist) dem Verzicht auf jegliche Art von Bevölkerungspolitik gleichkäme, ist diese Kritik gleichzusetzen mit der Meinung, dass Bevölkerungspolitik per se niemals ethisch legitim sein könne.

In Bezug auf die Frage, ob ein Staat sich demografische Zielgrößen setzen dürfe, ist das Ergebnis unserer Diskussion, dass diese in demokratischen Staaten legitim sind. Es gibt keine hinreichenden Argumente, von einem demokratischen Staat zu verlangen, dass er kein demografisches Bewusstsein, keine offizielle Regierungsmeinung zur Entwicklung seiner Bevölkerung haben dürfe. Wie im PoA festgelegt, sollten nachgeordneten Beamten aber keine Sollzahlen vorgegeben

die Sexualerziehung zu verbessern. Das Heiratsalter von 24 Jahren für Männer und 21 für Frauen (in ihrer Provinz) würde sie nicht senken. Jie sah in der schneller wachsenden Bevölkerung des regionalen Rivalen Indien eine Bedrohung. Dennoch hielt sie es absolut unerlässlich im Interesse Chinas, die antinatalistische Politik beizubehalten. Die Begründung: „China is overcrowded today“. 
werden, da die Gefahr des Mißbrauchs dann sehr hoch ist. Die Weltgemeinschaft hat für dieses Problem mit ihrer differenzierten Formulierung, die staatliche Zielvorstellungen erlaubt, aber Vorgaben an untergeordnete Familienplanungsstellen verbietet, einen ethisch akzeptablen Kompromiss gefunden.

Der indirekte Ansatz ist nicht in sich selbst widersprüchlich und er ist ethisch legitim.

\subsection{Ethische Bewertung von finanziellen Steuerungsinstrumen- ten}

5.9.1 Anreize und Sanktionen

In fast allen Staaten der Welt, unabhängig davon, ob sie eine Geburtenpolitik offiziell proklamieren oder nicht, gibt es finanzielle Anreize oder Sanktionen für Kinder. Werden damit geburtenpolitische Ziele verfolgt, dann fällt diese Politik in unserem Einteilungsschema unter die Typen 3, 4 oder 5.

Auch hier stellt sich wieder das Problem, dass es schwierig ist, einige wenige ethische Normen für die Vielzahl der tatsächlichen bevölkerungspolitischen Maßnahmen zu finden. Einige Beispiele aus der Praxis: In Bangladesh wurde die Vergabe von Nahrungsmittelhilfe von Sterilisationsnachweisen abhängig gemacht. ${ }^{619}$ In Laos machte ein Beamter dem Dorfältesten das Angebot, dass die Dorfgemeinschaft einen Brunnen spendiert bekommt, wenn alle Mitglieder des Dorfes für fünf Jahre auf ein drittes Kind verzichten. ${ }^{620}$ In vielen Entwicklungsländern sind Mikrokreditprogramme und, einkommensschaffende Projekte' (wie z.B. von der Grameen Bank) an Familienplanung gekoppelt. ${ }^{621}$ Im südindischen Bundesstaat Andhra Pradesh bekommen Familien verbilligte Kredite und subventioniertes Saatgut, wenn sie den Nachweis über eine Sterilisation vorlegen können ${ }^{622}$ In China werden wie beschrieben Strafzahlungen für jedes zusätzliche Kind fällig. In Vietnam ist es Familien mit mehr als drei Kindern untersagt, in ein Stadtzentrum oder in eine Industrial Zone zu ziehen und es können ihnen zusätzliche Steuern und mehr ,soziale Arbeiten' auferlegt werden. ${ }^{623}$ Ein-Kind-Familien können dagegen nach dem Ermessen lokaler Behörden gratis Bauland bekommen, von Steuern und sozialen Arbeitspflichten befreit und mit Kindergeld und anderen Prämien ausgezeichnet werden.

Dass finanzielle Sanktionen von Kritiker(innen) abgelehnt werden, ist wenig überraschend. Nach Ansicht vieler haben aber auch Anreizsysteme generell einen subtilen Zwangscharakter. De facto mache es für die ökonomische Situation der Paare keinen Unterschied, ob sie unter dem einen oder anderen System leben. Das

\footnotetext{
${ }^{619}$ Kasun (1988), 91

${ }^{620}$ So etwa Schockenhoff (1996), 51

${ }^{621}$,Kritiker' und ,Kritikerinnen' wird in diesem Abschnitt wieder nicht alternierend verwendet.

${ }^{622}$ Randeria (1995), 121

${ }^{623} \mathrm{Haub}(2003), 1$
} 
Prinzip von Freiwilligkeit und Selbstbestimmung sei in Frage gestellt, wenn aufgrund der ökonomischen Situation keine Alternativen vorhanden sind. ${ }^{624}$ „Das Gewaltverhältnis, das sich vorher in direktem Zwang zur Geburtenkontrolle äußerte, erscheint nun weniger sichtbar, ist deshalb aber nicht aufgehoben," kritisiert Schlebusch. ${ }^{625}$

Es ist in der Bevölkerungsethik umstritten, ob finanzielle Anreizsysteme anders zu bewerten sind als Sanktionssysteme. Bayles schrieb dazu: „Some philosophers contend that offering benefits and incentives is coercive while others contend that it is not." ${ }^{2626}$ Für einen homo oeconomicus ist beides gleich zu bewerten. Es sind aber zahlreiche Beispiele denkbar, bei denen es zumindest psychologisch sehr wohl einen Unterschied macht, ob es sich um einen finanziellen Bonus, die Beendigung eines finanziellen Bonus oder einen finanziellen Malus handelt. Nehmen wir an, das Gehalt für die eine Hälfte der Beschäftigten eines Betriebs wird um fünf Prozent erhöht, für die andere Hälfte um vier Prozent. Wenn nun dieses eine Prozent Unterschied sich dadurch ergibt, dass die weniger guten Mitarbeiter einen Malus auf die allgemeine Gehaltserhöhung von fünf Prozent erhalten, so wird der Ärger weit größer sein als wenn die bessere Hälfte der Belegschaft einen Bonus von einem Prozent auf eine allgemeine Gehaltserhöhung von vier Prozent erhalten. Per Definitionem sind Boni etwas, das als Zusatz auf das als ,normal' angesehene finanzielle Niveau bezahlt wird. Viele Menschen sind bereit darauf zu verzichten, wenn ihnen die an sie geknüpften Bedingungen nicht gefallen. Finanzielle Strafen aber erzeugen größeren Ärger. Der Ethiker Callahan schreibt: „In principle, incentive schemes are noncoercive; that is, people are not forced to take advantage of the incentive. Instead the point of an incentive is to give them a choice they did not previously have." ${ }^{\prime 27}$ Und Bayles ergänzt: "For example, if Jones is trying to decide whether to take a position with employer A or B and A increases the salary offered, A has not limited Jones' liberty to decide." ${ }^{228}$ Stellen wir uns jetzt vor, Jones habe bereits bei Arbeitgeber A unterschrieben und nun kündige der eine Gehaltskürzung um 10 Prozent an, falls Jones nicht eine Nachtschicht arbeite.

Wegen dieser psychologischen Wirkungen auf Menschen erscheinen mir finanzielle Sanktionen ethisch ein bisschen problematischer zu sein als Anreize. Eine Mittelstellung nehmen begrenzte Anreize ein. Ein Beispiel wäre, wenn ein Staat nur für die ersten zwei Kinder den Schulbesuch subventioniert.

$\mathrm{Ob}$ finanzielle Instrumente ethisch zulässig sind, ist nicht in erster Linie eine Frage von Boni oder Mali, sondern vielmehr, wie tiefgreifend sie in die finanzielle Stellung des Paares eingreifen. Je höher der positive oder negative finan-

\footnotetext{
${ }^{624}$ Hummel (2000), 109

${ }^{625}$ Schlebusch (1994), 162

${ }^{626}$ Bayles (1976), xiv

${ }^{627}$ Callahan (1976), 29

${ }^{628}$ Bayles (1976b), 42
} 
zielle Anreiz, desto eher ist es gerechtfertigt, von Zwang zu sprechen. Werden die Anreize prohibitiv hoch gesetzt, so besteht kein Unterschied mehr zwischen ihnen und einer Rationierungsstrategie. Eine geringe Höhe des finanziellen Steuerungsinstruments ist dagegen nicht als Verstoß gegen die Wahlfreiheit bzw. das Selbstbestimmungsrecht zu werten. Die Verteuerung von Rauchen oder von Autofahren durch Steuern ist ebenfalls ein Eingriff in die Wahlfreiheit der Individuen. All diese Steuerungsversuche lassen Individuen aber Freiheitsgrade, so dass die Schwelle zum ,Zwang' noch nicht erreicht ist.

\subsubsection{Die Vier-Fünftel-Regel}

Wer eine ganz bestimmte Zahl von Kindern will, der verliert bei moderaten finanziellen Anreizen oder Sanktionen auch dann nicht seine relative Einkommensposition in seiner Gesellschaft, wenn diese Kinderzahl von der staatlich propagierten Kinderzahl (weit) abweicht. Sind die finanziellen Anreize oder Sanktionen dagegen hoch, dann ändert sich die ökonomische Position eines Paares gravierend, wenn es eine andere Kinderzahl anstrebt als die vom Staat als ideal angesehene.

Welche exakte finanzielle Belastung noch ethisch neutral ist, lässt sich schwer festlegen. Irgendwo muss aber eine Grenze gezogen werden. Mir erscheint folgende Faustregel angemessen: Die Wahl einer vom Staat nicht gewünschten Kinderzahl darf nicht zu einer Einkommens-Verschlechterung von mehr als 20 Prozent gegenüber der Einkommenssituation führen, die jemand hätte, wenn er die vom Staat gewünschte und am stärksten geförderten Kinderzahl wählen würde. Diese Vier-Fünftel-Regel ist zugegebenermaßen nur eine grobe Orientierungsmarke. Man kann auch eine Drei-Viertel-Regel oder eine Fünf-Sechstel-Regel für angemessen halten. Wer jedoch zugibt, dass für die ethische Zulässigkeit von finanziellen geburtenpolitischen Maßnahmen irgendwo eine Grenze gezogen werden muss, sollte im Sinne konstruktiver Kritik eine andere vorschlagen, anstatt die Vier-Fünftel-Regel pauschal abzulehnen. Entscheidend ist, was man als verhältnismäßig ansieht. Nach dem Verhältnismäßigkeitsprinzip lässt die Vier-FünftelRegel meiner Ansicht nach dem Individuum gerade noch genug Freiheitsgrade, dass man noch nicht von Zwang sprechen muss.

Was bedeutet die Vier-Fünftel-Regel in der Praxis? Wenn also z.B. in Indien die demokratisch gewählte Regierung eine antinatalistische Bevölkerungspolitik realisieren will, die 2 Kinder zur Regel macht, so muss sie diese nach der VierFünftel-Regel so ausgestalten, dass ein Paar A, das sechs Kinder haben will und auch bekommt, finanziell nicht mehr als 20 Prozent schlechter gestellt wird als wenn es sich für nur zwei Kinder entschieden hätte. Wenn also z.B. ab dem vierten Kind eine monatliche Strafabgabe von 15 Prozent zu zahlen ist, ab dem dritten Kind das Schulgeld nicht mehr erlassen wird (wofür Paar A z.B. fünf Prozent des Einkommens aufwenden muss) und Zwei-Kinder-Familien einen verbilligten Kredit bekommen (wodurch für Paar A z.B. Opportunitätskosten von sechs Prozent seines Einkommens entstehen), so ist die Gesamtsumme maßgeblich. In 
diesem Beispiel addieren sich die Benachteiligungen von Paar A auf 26 Prozent. Paar A ist also in diesem Beispiel aufgrund der geburtenpolitischen Maßnahmen des Staates mehr als 20 Prozent finanziell schlechter gestellt und somit unangemessen stark benachteiligt.

Man könnte einwenden, dass Paar A mit fünf Kindern durch die Wahl seiner Kinderzahl ${ }^{629}$ dennoch ein höheres Lebenszeiteinkommen als bei zwei Kindern hat, da die Kinder für seine Altersversorgung aufkommen. Dies ist aber eine Frage der Rahmenbedingungen, und fließt in die Rechnung nicht mit ein. ${ }^{630}$

Nach der Vier-Fünftel-Regel ist z.B. die heutige Geburtenpolitik Chinas nicht ethisch legitimiert. In China werden in vielen Provinzen für ein ,überzähliges' Kind bei Städtern Lohnkürzungen von 20 Prozent der Löhne beider Eltern für eine Dauer von drei bis 14 Jahren erhoben. Dies allein wäre nach der Vier-Fünftel-Regel noch erlaubt, allerdings kommen weitere monetäre Benachteiligungen (im Vergleich zu Ein-Kind-Familien) hinzu, so dass der Vier-Fünftel-Wert überschritten wird.

Am einfachsten sind solche Berechnungen, wenn man zwei alternative Szenarien mit unterschiedlichen Kinderzahlen für dasselbe Paar vergleicht. Vergleicht man die Einkommenspositionen von zwei unterschiedlichen Paaren, so ist dies nur unter der Annahme möglich, dass diese unterschiedlichen Einkommenspositionen ausschließlich ihrer unterschiedlichen Entscheidung über die Zahl ihrer Kinder zuzuschreiben sind.

Paul Ehrlich hat 1968 für die USA vorgeschlagen, dass Eltern mit einem Einkommen von 25.000 \$ für die ersten zwei Kinder je 600 \$; für jedes weitere 1.200 $\$$ zusätzlich versteuern sollten. ${ }^{631}$ Bei einem angenommenen persönlichen Steuersatz von 15 Prozent bliebe einer Familie mit keinem Kind nach Abzug der Steuern ein verfügbares Einkommen $21.250 \$ ;{ }^{632}$ einer Familie mit vier Kindern bliebe noch $17.650 \$$ als verfügbares Einkommen. ${ }^{633}$ Für diese Familie wäre eine solche Regelung also mit der Vier-Fünftel-Regel vereinbar. ${ }^{634}$ Aber schon bei einem fünften Kind würde der Staat zu stark das Verhältnis zwischen beiden Familien verändern, da der kinderreichen Familie dann nur noch 16.450 \$ blieben.

Ehrlichs finanzieller Steuerungsmechanismus hat erstens den Nachteil, dass er für kinderreiche Familien keine Obergrenze für die relativ zu einer kinderlosen Familie erlittenen finanzielle Einbußen festlegt. Zweitens müssen Familien, die keine Steuern zahlen, auch keine finanziellen Sanktionen fürchten.

Besser ist ein finanzielles Steuerungssystem, welches die finanziellen Sanktionen direkt prozentual zum Einkommen bemisst. Nehmen wir an, ein Schwellen-

\footnotetext{
${ }^{629}$ Alle anderen das Lebenszeiteinkommen beeinflussenden Faktoren werden außen vor gelassen.

${ }^{630}$ Siehe Abschnitt 5.10. Ethische Bewertung ordnungspolitischer Ansätze.

${ }^{631}$ Ehrlich (1971), 108

${ }^{632} 25.000-(25.000 \cdot 0,15)$

${ }^{633} 25.000-(25.000 \cdot 0,15+2 \cdot 600+2 \cdot 1.200)$

${ }^{634} 21.250 \cdot 0,8=17.000$
} 
land in Asien mit einem Durchschnittsjahreseinkommen von $10.000 €$ führt im Rahmen einer antinatalistischen Geburtenpolitik folgende Abgabe ein, so würde sich die relative Einkommensposition nicht mehr als 20 Prozent ändern.

Tab. 12: Eine antinatalistische Bevölkerungspolitik im Rahmen der Vier-Fünftel-Regel - Malussystem (Staatsziel: max. 2 Kinder)

\begin{tabular}{|l|l|}
\hline Zahl der Kinder & Negatives Kindergeld \\
\hline 1. Kind & - \\
\hline 2. Kind & - \\
\hline 3. Kind & $5 \%$ des Jahreseinkommens \\
\hline 4. Kind & $10 \%$ des Jahreseinkommens \\
\hline 5. Kind & $15 \%$ des Jahreseinkommens \\
\hline 6. und weitere Kinder & $20 \%$ des Jahreseinkommens \\
\hline
\end{tabular}

\section{Quelle: Eigene Darstellung}

Wie schon erwähnt, können auch Boni einen Zwangscharakter annehmen, wenn sie keine Entscheidungsfreiheit mehr lassen. Tabelle 13 zeigt ein Bonussystem im Rahmen der Vier-Fünftel-Regel, welches als ethisch zulässig gelten kann. Würden aber die Prozentsätze verdoppelt, wäre es dies mit Sicherheit nicht mehr.

Tab. 13: Eine antinatalistische Bevölkerungspolitik im Rahmen der Vier-Fünftel-Regel - Bonussystem (Staatsziel: max. 2 Kinder)

\begin{tabular}{|l|l|}
\hline Zahl der Kinder & Positives Kindergeld \\
\hline 1. Kind & $20 \%$ des Jahreseinkommens \\
\hline 2. Kind & $\begin{array}{l}20 \% \text { des Jahreseinkommens } \\
\text { 3. Kind und weitere Kinder }\end{array}$ \\
$\begin{array}{l}\text { Wegfall der für das 1. und 2. } \\
\text { Kind gezahlten Boni }\end{array}$ \\
\hline
\end{tabular}

\section{Quelle: Eigene Darstellung}

\section{Gegenargumente gegen die Vier-Fünftel-Regel}

Welche Gegenargumente gibt es gegen die Vier-Fünftel-Regel?

1.) „Finanzielle Boni oder Mali sind sozial ungerecht, weil es reicheren Personen dann leichter fällt, ihre gewünschte Kinderzahl zu realisieren." Dieses Argument trifft am stärksten zu, wenn finanzielle Boni oder Mali als Einmalzahlungen ausgestaltet sind Dies ist z.B. bei der in Tab. 3 u. 4 beschriebenen Geburtenpolitik ausgeschlossen, bei denen die finanziellen Boni bzw. Mali als einkommensabhängige Zahlungen ausgestaltet sind. Man kann aber immer noch argumentieren, dass es jemand bei einem Jahreseinkommen von einer Million Euro leichter fällt, 200.000 Euro Strafsteuern zu zahlen als wenn jemand nur ein Jahreseinkommen 
von 20.000 Euro hat. Um diesem Einwand zu begegnen, sollte zunächst das Existenzminimum (des jeweiligen Landes) generell steuerfrei gestellt werden, so dass m.a.W. niemand durch die staatliche Geburtenpolitik in die Armut abrutscht. Das Problem, dass dann de facto Arme von der Geburtenpolitik ausgenommen wären, ließe sich in einem System, welches Boni für die Armen mit Mali für die restliche Bevölkerung kombiniert, vermeiden. Natürlich lässt sich die Vier-Fünftel-Regel auch abwandeln zu einer progressiven Besteuerung. Allerdings wird die übergeordnete Frage, ob ein progressives oder ein lineares Steuersystem gerechter ist, in verschiedenen Gesellschaften der Welt kontrovers diskutiert. Auf diese Diskussion kann hier aus Platzgründen nicht vertiefend eingegangen werden. Erlaubt sei aber der Hinweis, dass bisher niemand eine höhere Tabak- oder Mineralölsteuer für Reichere gefordert hat. Wie auch immer, wo die jeweilige Gesellschaft sich für ein progressives Steuersystem entschieden hat, so kann sie auch ein Malussystem progressiv auszugestalten. ${ }^{635}$

2.) „Die Vier-Fünftel-Regel ist in den am wenigsten entwickelten Gesellschaften nicht anwendbar, weil in solchen Ländern kein Geldsystem etabliert ist.“

Dieser Einwand ist richtig. Wenn in einem Land wie Laos der Dorfgemeinschaft ein Brunnen versprochen wird, wenn alle Mitglieder des Dorfes für fünf Jahre auf ein drittes Kind verzichten, so ist die ethische Legitimität nicht mit der VierFünftel-Regel zu bewerten. Diese Regel ist nur in monetarisierten Gesellschaftsverhältnissen anwendbar. Allerdings gibt es in anderen Ländern auch keine alternative Methode, die ethische Zulässigkeit des Angebots quantitativ zu bewerten.

3.) „Die Besser- oder Schlechterstellung eines Paares durch staatliche Geburtenpolitik lässt sich nicht berechnen." Gegen die Vier-Fünftel-Regel könnte eingewandt werden, dass es im konkreten Einzelfall gar nicht möglich sein wird, angesichts des Dickichts von finanziellen Sanktionen oder Anreizen zu beurteilen, ob zwei alternative Kinderwünsche aufgrund staatlicher Geburtenpolitik zu einer mehr als 20prozentigen Einkommensdifferenz führen würden. Dies scheint mir jedoch eher ein Argument für mehr Transparenz bei staatlichen Geburtenpolitiken als gegen die Vier-Fünftel-Regel zu sein.

4.) „Finanzielle Steuerungsmechanismen sind unnötig, weil es ethisch unproblematischere und ebenso wirksame Alternativen gibt". Natürlich sollte ein Staat stets die indirekten und appellativen Maßnahmen antinatalistischer Geburtenpolitik ausschöpfen, bevor er zu finanziellen Steuerungsmechanismen übergeht. Es gibt auch kein empirisches Beispiel für einen Staat, der einfach nur finanzielle Boni und Mali einsetzt. Bei allen Politiken ist z.B. auch die Bereitstellung von Kontrazeptiva, der Ausbau von Gesundheitssystemen und meist auch die Einrichtung von sozialen Sicherungssystemen, damit Kinder nicht mehr zum Zweck

\footnotetext{
${ }^{635}$ Bei einer Rationierung werden arme und reiche Paare mit dem Wunsch nach vielen Kindern zwar gleich, aber nur gleich schlecht gestellt. Ein Armer darf bei einer „Zwei-Kind-Politik“ selbst dann keine drei Kinder haben, wenn er dafür weitere Armut in Kauf nehmen würde. Es wird ihm ein geringer Trost sein, dass der Reiche sich auch mit zwei Kindern zufrieden geben muss.
} 
der Altersvorsorge geboren werden, eine durchgeführte Begleitmaßnahme. Gerade in den Ländern, in denen sich die Bevölkerung den Projektionen zu Folge bis 2050 verdreifachen wird, könnten solche Maßnahmen aber nicht ausreichen. Die Aussage, dass die unterschiedlichen Alternativen, die ein Staat im Hinblick auf Bevölkerungspolitik hat, gleichermaßen effizient sind, wurde bereits widerlegt. Es kann nicht bestritten werden, dass rigidere, strikter durchgesetzte Maßnahmen einen größeren Zielerreichungsgrad aufweisen. ${ }^{636}$

5.) „Zwang kann niemals ethisch legitimiert sein.“ Wer diesen Einwand erhebt, hat das Anliegen dieses Textes nicht verstanden, in dem es ja gerade darum ging, ab welchem Punkt finanzielle Steuerungsmechanismen als ethisch illegitimer Zwang bezeichnet werden sollten. Wer behauptet, jede Art von staatlichen Anreizen und Sanktionen mit dem Ziel, das Verhalten seiner Bürger zu beeinflussen, sei „Zwang“, der betreibt schlicht Missbrauch mit dem Begriff.

\subsection{Ethische Bewertung ordnungspolitischer Ansätze}

\subsubsection{Veränderung des Heiratsalters}

Eine weitere Möglichkeit für Regierungen sind Gesetze, die ordnungspolitisch die Rahmenbedingungen verändern. ${ }^{637}$ Es gibt eine Schnittmenge mit den indirekten Ansätzen. Allerdings ist bei den indirekten Ansätzen das Primärziel (wie Gesundheit, Gleichberechtigung oder Bildung) immer in sich begrüßenswert und die demografische Dividende eine gewünschte Nebenwirkung. Wenn dagegen z.B. das Heiratsalter im Jemen von 14 auf 15 hochgesetzt wird, so können damit direkt und ausschließlich geburtenpolitische Ziele verfolgt werden. Ordnungspolitische Ansätze sind daher nicht mit indirekten Ansätzen gleichzusetzen.

Eine ordnungspolitische Maßnahme wie die Heraufsetzung des Heiratsalters beschränkt grundsätzlich nicht das Recht von Individuen und Paaren, die Zahl ihrer Kinder zu bestimmen, dennoch senkt sie (im Erfolgsfall) die Geburtenraten. Ordnungspolitische Regelungen erscheinen prima facie ethisch unproblematisch, da sie niemanden diskriminieren. Von einem Eingriff in die Freiheitsrechte kann man nicht sprechen.

\subsubsection{Generationengerechtigkeit und umlagefinanzierte Rentensysteme in Entwicklungsländern}

Eine wichtige Veränderung der Rahmenbedingungen wäre z.B. die Einführung umlagefinanzierter Rentensysteme durch die Regierungen von Entwicklungsländern, um dort das Bevölkerungswachstum zu verringern. Kinder sind immer noch in vielen Ländern die einzige Altersvorsorge. Sie leisten in armen Ländern einen

\footnotetext{
${ }^{636}$ Natürlich sollte sich der Staat bei der Nennung der gewünschten Zahl von Kindern auch an die Vorstellungen seiner Bürger richten. So kann es nicht sein, das nur ein Kind vom Staat erlaubt wird und die Bürger lieber vier Kinder hätten. Hier muss der Staat realistische Zielgrößen aufstellen und durch finanzielle Maßnahmen zu erreichen versuchen.

${ }^{637}$ Diese Maßnahmen passen nicht richtig in das Freiwilligkeits-Zwang-Schema.
} 
unverzichtbaren Beitrag zum Familienunterhalt und zur ökonomischen Absicherung ihrer Eltern, gerade auch für die Risiken von Krankheit und Invalidität. ${ }^{638}$ Der Transfer von Hilfestellungen und Dienstleistungen, der in allen Gesellschaftsformen das Interaktionsgefüge zwischen den Generationen bestimmt, verläuft in vielen WEL fast ausschließlich innerhalb der Familien, und nicht - wie in den SEL über Märkte oder auf dem Weg der staatlichen Umverteilung. ${ }^{639}$ In den meisten Befragungen rangiert die Sorge um die eigene Absicherung im Alter in den Ländern Asiens, Afrikas und Lateinamerikas ganz oben auf der Skala der Gründe, die für eine hohe Kinderzahl angegeben werden. ${ }^{640}$ Bei einer vergleichenden interkulturellen Studie gaben 95 Prozent der Befragten in der Türkei, in Thailand, den Philippinen und auf Taiwan an, dass sie Kinder als ,Hilfen im Alter' betrachten, während in Deutschland oder den USA dieser Wert nur bei 10 Prozent lag. ${ }^{641}$

Umgekehrt zeigen Studien, dass die Einführung des Umlageverfahrens maßgeblich zur Absenkung der Geburtenrate beiträgt. ${ }^{642}$ Der Bremer Altersforscher Hartmut Dießenbacher erläutert diesen Zusammenhang: „Vor der Industrialisierung herrschte in den besitzenden Schichten folgendes Generationenverhältnis vor: (...) Du bekommst mein Eigentum, dafür ernährst Du mich im Alter. Das wird durch Altenteilverträge gut bezeugt. (...) Das hervorstechende Merkmal des neuen städtischen Industrieproletariats bestand darin, dass es im Alter nichts zu vererben hatte. Das einzige Eigentum des Lohnarbeiters ist seine Arbeitskraft. Diese ist im Alter verbraucht und unvererbar. (...) Für das erwerbstätige Ehepaar in der Mitte wurde die Ernährung der alten Eltern und der eigenen Kinder - besonders in Zeiten periodischer Massenarbeitslosigkeit - zur ökonomischen Last.“6643 Mit der Einführung von Altersversicherungssystemen wurde eine ökonomische Entkoppelung von Kinderzahl und Altersversorgung hergestellt. Das junge Lohnarbeiterpaar konnte nun damit rechnen, im Alter Rentenempfänger zu werden.

In vielen Schwellenländern, die das Stadium einer Agrargesellschaft hinter sich gelassen haben, wäre es möglich, den familiären Generationenvertrag durch ein umlagefinanziertes Rentensystemen zu ersetzen. Bei einem Umlageverfahren wird ein bestimmter Prozentsatz des Einkommens auf die Rentenzahlungen umgelegt. Im Grundsatz geht man dabei nach der einfachen Formel $\mathrm{A} * \mathrm{BH}=\mathrm{R} * \mathrm{RH}$ vor, wobei A die Zahl der Mitglieder der aktiven Generation (also der 1565jährigen), BH die Höhe von deren Zahlungen in das Rentensystem, R die Zahl der Mitglieder der Rentnergeneration und RH die Höhe der Renten ist. Um z.B. in Deutschland gemachte Fehler nicht zu wiederholen, sollte man von Anfang an darauf achten, dass nicht nur Arbeiter und Angestellte, sondern auch Selbstän-

\footnotetext{
${ }^{638}$ Schockenhoff (1996), 17

${ }^{639}$ A.a.O.

${ }^{640}$ A.a.O., 18

${ }^{641}$ Dießenbacher (25.5.1993), 12

${ }^{642}$ Birg (2001), 46

${ }^{643}$ Dießenbacher (25.5.1993), 12
} 
dige, Beamte und Mandatsträger ins umlagefinanzierte Rentensystem einbezogen sind und dass auch Miet- und Zinseinkünfte miteingerechnet werden.

Erhebt man zum Stichtag x einen zunächst moderaten Beitrag von z.B. 5 Prozent, so würde auf einen Schlag alten Menschen, die bisher nie eine staatliche Rente erhielten, eine solche Rente gezahlt werden können.

Man darf allerdings auch bei dieser Maßnahme keine eindeutigen Regressionen erwarten. In jedem Fall dauert der Lernprozess einige Zeit. Dennoch sind die Korrelationen so hoch, dass z.B. China Regionalversicherungen aufbaut, um Eltern die Zahlung einer Altersrente zu garantieren. ${ }^{644}$ Dießenbacher schreibt: „Die Regierungen bevölkerungsexplosiver Länder dürfen auf dieses Instrument zur Verringerung der Geburtenrate vertrauen." ${ }^{\text {(645 }}$

\subsection{Zwischenfazit}

Im Rahmen einer ethischen Bewertung müssen erst die Maßnahmen in sich auf ethische Legitimität bewertet werden, bevor sie instrumentell auf das Ziel der ökologischen Generationengerechtigkeit bezogen werden können. Die Mehrheit aller Staaten der Erde, 77 Prozent der Entwicklungsländer und 45 Prozent der SEL, verfolgt Bevölkerungspolitiken. Es ist nicht grundsätzlich angreifbar, wenn ein Staat im Rahmen seiner Aufgaben, das Wohl der Bürger zu mehren und Schaden von ihnen abzuwenden, eine Bevölkerungspolitik betreibt. In den letzten Abschnitten wurde eine Bewertung von antinatalistischen Geburtenpolitiken (Rationierungsmaßnahmen, indirekten Geburtenpolitiken, finanziellen Steuerungsinstrumenten und ordnungspolitischen Ansätzen) vorgenommen. ${ }^{646}$ Indirekte Geburtenpolitiken, wie sie vom Ansatz her das Aktionsprogramm von Kairo prägen, sind ethisch vertretbar. Auch eine Veränderung der Rahmenbedingungen übt keinen Zwang im engeren Sinne aus. Bei finanziellen Steuerungsinstrumenten hängt vor allem von der Höhe dieser Maßnahmen ab, ob man von Zwang sprechen muss. Je stärker die durch Geburtenpolitik gesetzten Anreize oder Sanktionen, desto problematischer sind sie in ethischer Hinsicht. Die Grenze legt die Vier-Fünftel-Maxime fest: Die Wahl einer vom Staat nicht gewünschten Kinder-

\footnotetext{
${ }^{644}$ A.a.O.

${ }^{645}$ A.a.O.

${ }^{646}$ Eine Kategorisierung pronatalistischer Politiken hätte als extremste Maßnahme das gesetzliche Verbot, Kontrazeptiva zu benützen, wie es in einigen frankophonen Staaten in Westafrika noch bis in die 1990er Jahre der Fall war (UN Population Fund 2003, 18). Zu den Ländern, in denen der Staat die Versorgung der Bevölkerung mit Kontrazeptiva immer noch in keiner Weise unterstützt, gehören Äquatorialguinea, Gabun, Libyen, Brunei Darussalam, Laos und Turkmenistan (UN Population Fund (2003, 22). Eine weit größere Zahl von Staaten verhindert oder erschwert Kontrazeptiva, die wirken, nachdem Ei- und Samenzelle bereits verschmolzen sind (Spirale, ,Pille danach'). Die Abtreibung, die wie beschrieben von der internationalen Gemeinschaft als Methode der Familienplanung stets geächtet wurde, ist in vielen Ländern, darunter auch SEL wie Irland oder Polen, verboten oder nur unter sehr restriktiven Bedingungen gestattet. Dies geschieht jedoch nicht aus pronatalistischen, sondern aus religiösen bzw. ethischen Erwägungen heraus.
} 
zahl darf nicht zu einer Einkommens-Verschlechterung von mehr als 20 Prozent gegenüber der Einkommenssituation führen, die jemand hätte, wenn er die vom Staat gewünschte und am stärksten geförderten Kinderzahl wählen würde.

Weitere Anforderungen an finanziell ausgerichtete staatliche Geburtenpolitiken sind, dass darauf geachtet werden muss, dass die ,überzählig' geborenen Kinder (also z.B. bei einer staatlichen Zwei-Kinder-Politik die Drittgeborenen jeder Familie) keine Nachteile haben. Solche Nachteile könnten in verkürztem Schulbesuch bestehen (z.B. wenn für zwei Kinder das Schulgeld vom Staat erlassen wird, für weitere Kinder aber nicht) oder in verschlechterten Gesundheitsdienstleistungen. Schon wegen des Gleichheitsgebotes wäre eine Geburtenpolitik, die in der Praxis zu solchen Ergebnissen führt, unethisch. Den Kindern ist die Tatsache, dass sie geboren werden, am allerwenigsten zur Last zu legen. ${ }^{647}$

Ethische Grundsätze können hier nicht jeden Einzelfall vorwegnehmen. In der Praxis hängt aber viel von den Detailregelungen ab, wenn man ein ethisches Urteil über eine bestimmte Bevölkerungspolitik abgeben will. ${ }^{648}$

\subsection{Mittel und Ziele}

\subsubsection{Die Rechte heutiger und künftiger Individuen}

Die Prüfung der Mittel ist abgeschlossen. Nun müssen sie auf das Ziel der ökologischen Generationengerechtigkeit bezogen werden. Die gefundenen Maximen, z.B. die Vier-Fünftel-Regel, berücksichtigen die Rechte heutiger Individuen. Jetzt müssen die Rechte heutiger Individuen gegen die Interessen zukünftiger Individuen abgewogen werden.

Wie im Aktionsprogramm der ICPD 1994 deutlich hervorgehoben, ist es in ökologischer Hinsicht vorteilhaft für kommende Generationen, wenn das Weltbevölkerungswachstum so schnell wie möglich gebremst und die Weltbevölkerung somit stabilisiert wird. Das weltweite Bevölkerungswachstum bedroht bestimmte Elemente der Natur, in erster Linie ihre Artenvielfalt. Wir haben Grund zu der Vermutung, dass zukünftige Generationen gerade der Biodiversität einen (noch) höheren Wert zumessen werden als wir heute.

Sind im Interesse kommender Generationen also alle Staaten verpflichtet, antinatalistische Geburtenpolitiken einzuführen? Zunächst einmal ist zu fragen, warum sich ein solches moralisches Gebot an die Staaten richten sollte. Der Adressat für ethische Handlungsmaximen ist zunächst das einzelne Individuum. Mit dem Menschenrecht auf freie Entscheidung über die Kinderzahl korrespondiert die Pflicht einer verantwortlichen Ausübung dieses Rechts. Jedes einzelne Paar könnte sich dafür entscheiden, auf gewollte Kinder zu verzichten. Einige Individuuen werden dies vermutlich auch heute schon tun. Es stellt sich aber die Frage, ob die ökologisch-demografischen Zusammenhänge ein so starkes Motiv darstel-

\footnotetext{
${ }^{647}$ Schockenhoff (1996), 49

${ }^{648}$ Callahan (1976), 34
} 
len können, dass ein Paar dafür ein freiwilliges Opfer erbringt. Zudem werden viele Menschen zumindest nicht exakt wissen, welche demografischen und ökologischen Folgewirkungen ihr persönliches Verhalten hat. ${ }^{649}$

Es gibt den interessanten Umstand, dass z.B. viele Raucher ein generelles Rauchverbot auf Inlandsflügen befürworten, aber dennoch nicht freiwillig auf das Rauchen verzichten, solange es noch nicht eingeführt ist. Ein ähnliches Beispiel betrifft die Einführung von Tempo 100 auf Autobahnen. Viele Fahrer halten diese Regelung zwar eigentlich für sinnvoll und richtig, gleichzeitig sehen sie aber nicht ein, warum sie - solange es eine solche Regelung noch nicht gibt - freiwillig höchstens $100 \mathrm{~km} / \mathrm{h}$ fahren und sich von anderen Autofahrern überholen lassen sollten ${ }^{650}$ Liegt hier ein Widerspruch im Wollen und Handeln vor? Meiner Ansicht nach ist sowohl die Position der Autofahrer wie die der Raucher legitim. Bei einer generellen Regelung akzeptieren alle aus Gründen des Gemeinwohls eine Einschränkung ihrer Freiheit. Dies ist ein kleineres Opfer als die freiwillige Einschränkung eines Lasters für nur einen Teil der Lasterhaften. ${ }^{651}$

In Gesellschaften, die ein hohes Bevölkerungswachstum sowie Ernährungs- und Umweltprobleme aufweisen und die gleichzeitig weniger individualisiert sind, werden manche Eltern durchaus bereit sein, im Rahmen einer verantwortlichen Elternschaft ganz ohne staatliche Appelle freiwillig auf (weitere) Kinder zu verzichten. In noch höherem Maße wird die Bevölkerung aber bereit sein, dem Staat zuzugestehen, eine (legitime) Bevölkerungspolitik zu etablieren.

\subsubsection{Ethische Dilemmata und ethisch doppelt gebotene Handlungs- strategien}

Offensichtlich geht es um eine Abwägung der Interessen heutiger Generationen, zukünftiger Generationen und des Staates. Es sind vier mögliche Fälle zu unterscheiden:

Fall 1: Staat wünscht Senkung der Geburtenrate, Individuen wünschen sich weniger Kinder als sie tatsächlich haben (Bsp: Afghanistan).

Fall 2: Staat wünscht Senkung der Geburtenrate, Individuen wünschen sich mehr Kinder als sie tatsächlich haben (Bsp: China).

\footnotetext{
${ }^{649}$ Vgl. Birg (1998), 247

${ }^{650}$ Knaus/Renn (1998), 137

${ }^{651}$ Entsprechend bekam auch die Umwelteinstellung „Wenn, dann müssen alle mitmachen!“ in der BMU-Studie zum Umweltbewusstsein höhere Zustimmungswerte als der Haltungstyp „Bei sich selber anfangen!“(BMU 2002, 30).
} 
Fall 3: Staat wünscht Erhöhung der Geburtenrate, Individuen wünschen sich weniger Kindern als sie tatsächlich haben (Bsp: Iran).

Fall 4: Staat wünscht Erhöhung der Geburtenrate, Individuen wünschen sich mehr Kindern als sie tatsächlich haben (Bsp: Deutschland).

In den Fällen 1 und 4 geht staatliche Politik auf die Bedürfnisse der Bürger ein und ist insofern ethisch unbedenklicher als in den Fällen 2 und 3. Dieses Ergebnis gilt aber nur für den intragenerationellen Kontext. Bewerten wir alle vier Fälle nun zusätzlich unter dem Kriterium der ökologischen Generationengerechtigkeit, so ist nur noch Fall 1 ethisch unproblematisch, den wir nachfolgend näher untersuchen.

Viele Frauen würden sich für weniger Kinder entscheiden, wenn Sie Zugang zu modernen Verhütungsmitteln hätten und wüssten, wie man diese anwendet. ${ }^{62}$ Die Frage des ,freien' Wunsches wurde bereits problematisiert, dadurch wird jedoch die grundsätzliche ethische Verpflichtung, dort zu helfen, wo Diskrepanz zwischen Kinderwunsch und Kinderzahl besteht, nicht in Frage gestellt. Überall dort, wo Individuen mangels anderer Optionen, mangels verfügbarer Verhütungsmittel oder mangels Sexualerziehung mehr Kinder haben müssen, als sie laut eigener Aussage wünschen, besteht also kein ethisches Dilemma, sondern eine Übereinstimmung von Individualinteressen und Interessen künftiger Generationen. In diesem Fall ist es ethisch doppelt geboten, daß sowohl die WEL als auch die SEL alle Anstrengungen unternehmen, den gewünschten Bedarf an Verhütungsmitteln im Rahmen qualitativ hochwertiger Gesundheitsdienstleistungen und Beratung zu decken.

Fall 2 wäre ein staatliches Handeln zu Gunsten der Interessen kommender Generationen, gegen die Interessen der Heutigen. Fall 3 scheint ein Verstoß gegen die Interessen heutiger und die zukünftiger Individuen zu sein. Fall 4 scheint eine Übereinstimmung von staatlichen Interessen und den individuellen Interessen der Heutigen, gegen die Interessen zukünftiger Generationen, zu sein.

Die gegenwärtige Situation in Deutschland und anderen reichen Ländern ist dadurch kennzeichnet, dass die Individuen sich mehr Kinder wünschen, als sie tatsächlich haben. Diesem Szenario müssen wir uns ihm im vierten Abschnitt dieser Arbeit zuwenden. Unser Zwischenergebnis, dass eine staatliche Förderung der Fertilität aus Sicht der ökologischen Generationengerechtigkeit negativ zu bewerten ist, dürfte gerade in Deutschland vielen Menschen unakzeptabel erscheinen. Ethische Prinzipien bestehen aber nicht im luftleeren Fall, sie müssen sich im konkreten Einzelfall einzelner Länderpolitiken bewähren. Es wäre unbefriedigend, die in der deutschen Debatte genannten Argumente nicht zu würdigen.

${ }^{652}$ Sinding (2003), 1 
Vorher aber wird das bisherige Ergebnis, welches sich aus der Verbindung der Mittel mit dem Ziel (ökologische Generationengerechtigkeit) ergab, zusammengefasst:

Die dritte (und wichtigste) Hypothese dieser Studie „Ist eine mit ökologischer Generationengerechtigkeit begründete Bevölkerungspolitik ethisch legitimierbar?" lässt sich in Bezug auf alle Fall 1-Länder eindeutig mit Ja beantworten. Der in der Einleitung dieser Studie dargestellte Interessenskonflikt zwischen heute und in Zukunft lebenden Menschen ist für viele Länder die falsche Prämisse. Es geht nicht darum, sich zwischen dem Kinderwunsch der heutigen und den Handlungsmöglichkeiten kommender Generationen entscheiden $\mathrm{zu}$ müssen. In der Praxis liegt statt eines ethischen Dilemmas in diesen Ländern das Gegenteil vor, nämlich eine ethisch doppelt gebotene Handlungsstrategie. 


\section{Demografischer Wandel und Bevölkerungspolitik in Deutschland}

\subsection{Vorbemerkungen}

In Deutschland gibt es keine antinatalistische Politik, dennoch bekommen die Deutschen weit weniger Kinder als zur Bestandserhaltung notwendig sind. Die Zahlen und Fakten des demografischen Wandels in Deutschland werden zu Beginn dieses Teils ausführlich dargestellt. Der vorausberechnete Bevölkerungsrückgang bis zum Jahr 2050 wird von den Eliten mit Sorge gesehen. In der gesellschaftlichen Debatte wird der demografische Wandel nicht nur als wohlstandsvernichtend und außenpolitisch fatal, sondern neuerdings auch als nicht generationengerecht bezeichnet. Hier wird also, genau umgekehrt zur ökologischen Debatte, eine sinkende Bevölkerung als Benachteiligung für die nächste Generation gesehen - nicht wegen ökologischer, sondern wegen wirtschaftlicher Zusammenhänge. Auch wenn ökonomische bzw. finanzielle Generationengerechtigkeit nicht im Mittelpunkt dieser Studie steht, so wäre es unbefriedigend, die Wechselwirkungen zwischen (dem ökologischen Aspekt von) Generationengerechtigkeit und Bevölkerungsentwicklung im weltweiten Maßstab darzustellen, ohne die gegenläufige Debatte zwischen (dem wirtschaftlichen Aspekt der) Generationengerechtigkeit und Bevölkerungsschrumpfung zu thematisieren.

\subsection{Die demografische Situation Deutschlands in Zahlen}

Die derzeitige Geburtenrate (Stand: 2003) liegt in Deutschland bei 1,34 Kindern pro Frau (1,26 in Ostdeutschland und 1,36 in Westdeutschland). ${ }^{653}$ Generell sind die Kinderzahlen in Großstädten niedriger als in ländlichen Gebieten. Langfristig ist ein deutlicher Rückgang der Zahl der Bevölkerung bei einem gleichzeitigen Altern zu erwarten. Bei ihrer 10. koordinierte Bevölkerungsvorausberechnung rechneten die Statistiker des Statistischen Bundesamtes neun Varianten durch. Wie Abb. 24 darstellt, ergab Variante 1, dass sich die Bevölkerungszahl von heute 82,531 Mio. ${ }^{654}$ bis zum Jahr 2050 auf 67,046 Millionen (81 Prozent des heutigen Niveaus) verringern könnte. ${ }^{655}$ Variante 1 ging von dem Szenario eines niedrigen Anstiegs der Lebenserwartung und von einem Wanderungssaldo von 100.000 Menschen aus. Variante 5 (mittlere Lebenserwartungszunahme, Wanderungssaldo von 200.000) führt dagegen im Jahr 2050 zu einem Bevölkerungsendbestand von 75,117 Mio. Menschen ${ }^{656}$ und Variante 9 (hohe Lebenserwartung, hoher Wande-

\footnotetext{
${ }^{653}$ Telefonische Auskunft des Statistischen Bundesamtes am 12.07.2005. Die zusammengefasste Geburtenziffer für 2004 wird bis September 2005 berechnet worden sein.

${ }^{654}$ Endbestand am 31.12.2003 nach telefonischer Auskunft des Statistischen Bundesamtes am 12.07.2005.

${ }^{655}$ Statistisches Bundesamt (2003), 38 (Tabelle 1)

${ }^{656}$ A.a.O., 42 (Tabelle 5)
} 
rungssaldo von 300.000) ergibt einen fast unveränderten Bevölkerungsendbestand im Jahre 2050 von 81,252 Millionen Menschen. ${ }^{67}$

\section{Abb. 24: Entwicklung der Bevölkerung in Deutschland bis 2050}

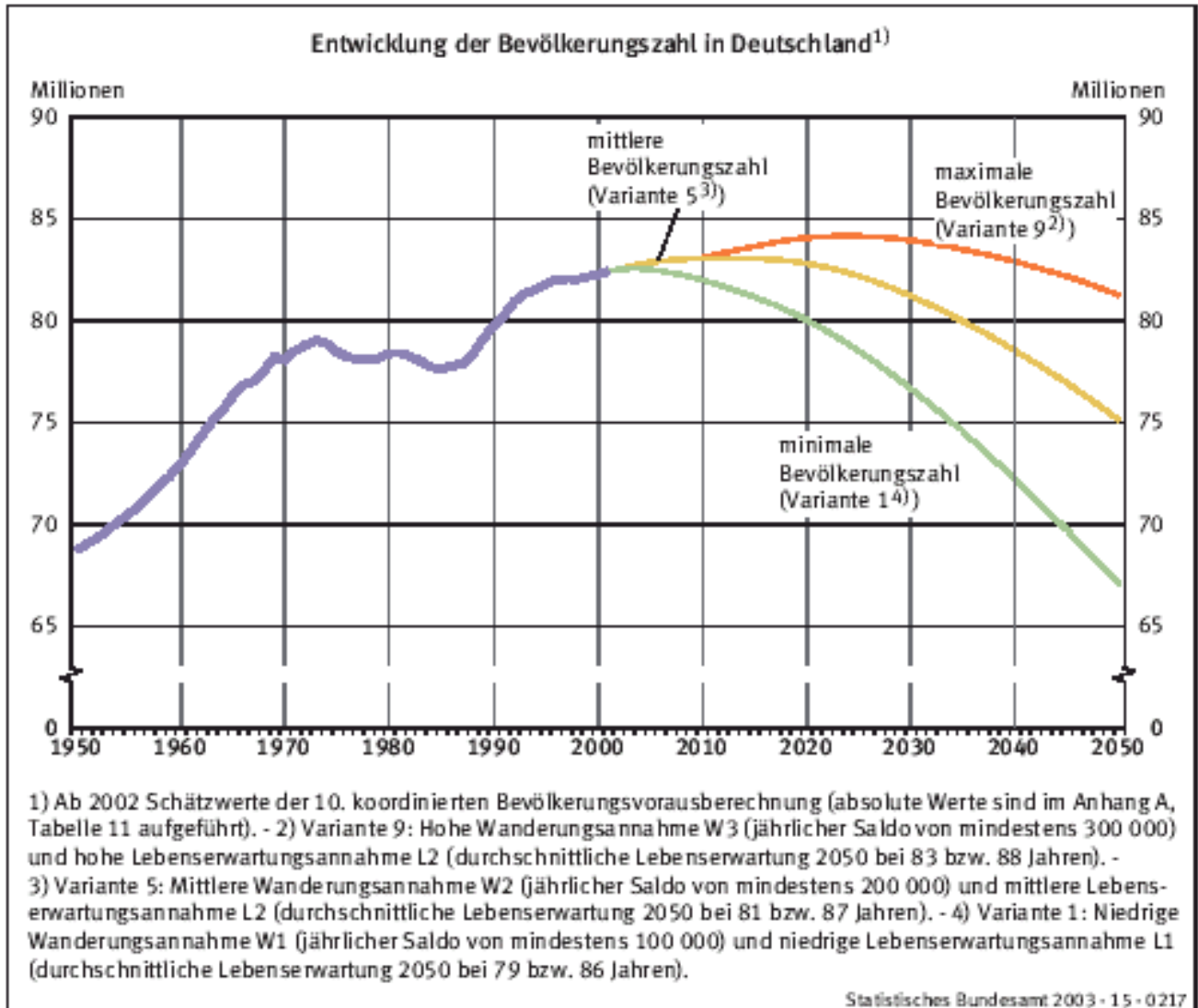

\section{Quelle: Statistisches Bundesamt (2003), 26}

Wie Abb. 24 zeigt, lebten noch nie so viele Menschen in Deutschland wie bisher und bis 2010 wird nach zwei der drei abgetragenen Varianten die Bevölkerung weiter steigen. Dies liegt allerdings allein an der Zuwanderung. Entsprechend der Annahme einer konstant bei 1,4 bleibenden Geburtenrate in allen 9 Varianten geht die Schere zwischen der Zahl der Sterbefälle und der Zahl der Geburten immer weiter auseinander. Soll die Elterngeneration gerade ersetzt werden (Nettoreproduktionsrate von 1), dürfte es keine freiwillige Kinderlosigkeit geben, und fast die Hälfte der Paare müssten sich für drei Kinder entscheiden.

Der erste demografische Wandel begann in Deutschland allerdings schon Mitte des 19. Jahrhunderts. ${ }^{658}$ Während Frauen 1860 durchschnittlich 5 Kinder hatten, sank der Wert bis 1920, als ein Zwischenminimum erreicht wurde, auf 1,9 Kinder pro Frau. Dieser erste demografische Übergang ist allerdings nicht mit dem zweiten demografischen Übergang zu vergleichen, der etwa 1965 einsetzte. Zwar

\footnotetext{
${ }^{657}$ A.a.O., 46 (Tabelle 9)

${ }^{658}$ Mackenroth (1953), 122-130
} 
wird das zur Bestandserhaltung nötige Niveau von 2,1 seit 1925 unterschritten, aber bis in die 1960er Jahre wurden etwa 90 Prozent der Kinder geboren, die zur Erhaltung des Bevölkerungsbestandes erforderlich waren. ${ }^{659}$ Dann kam es zum Geburtenrückgang der späten 1960iger Jahre (Pillenknick). Seitdem wird jede Elterngeneration nur zu 65 Prozent durch Nachkommen ersetzt (vgl. Abb. 25).

\section{Abb. 25: Lebendgeborene und Gestorbene in Deutschland bis zum Jahr 2050}

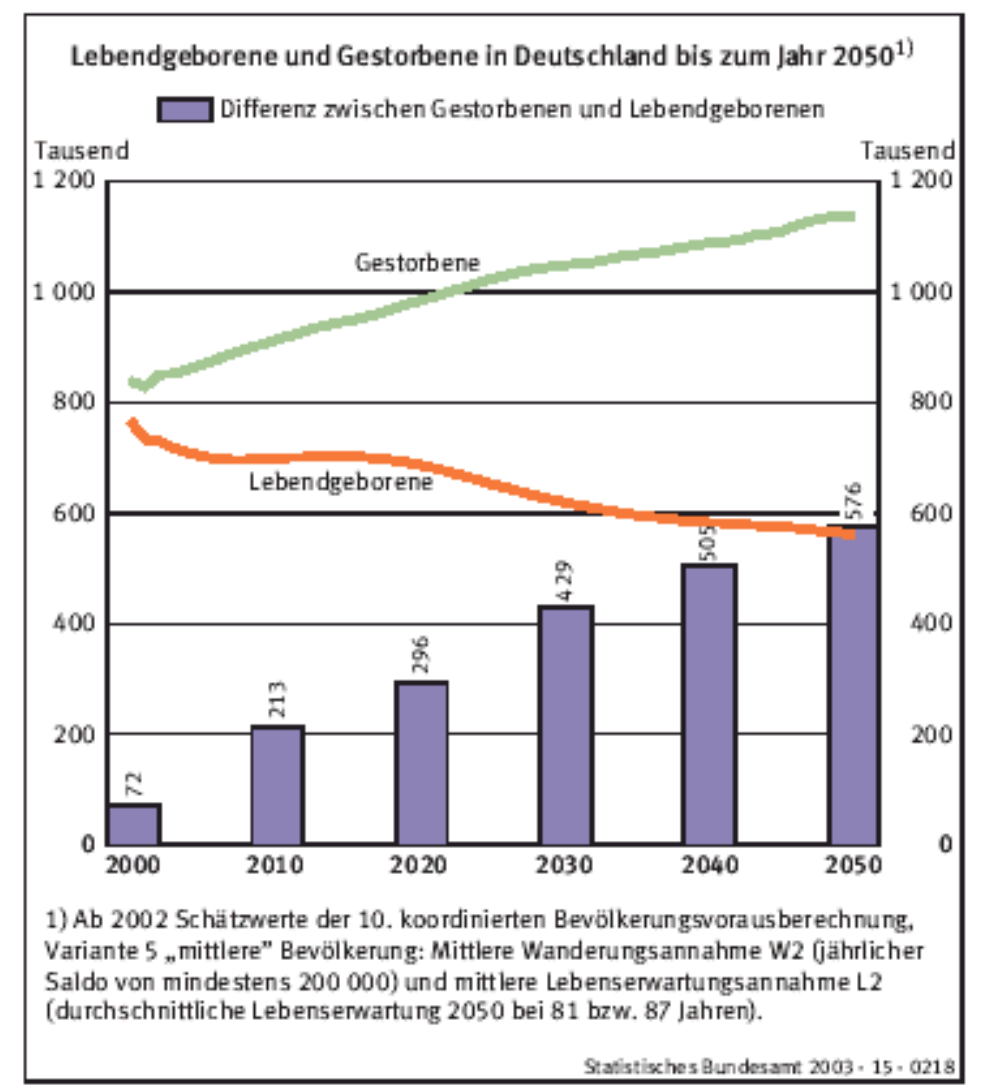

\section{Quelle: Statistisches Bundesamt (2003), 27}

Von den rund 82,5 Millionen Einwohnern Deutschlands leben 69 Millionen im früheren Bundesgebiet (einschließlich Berlin) und 13,5 Millionen in den neuen Bundesländern (ohne Berlin). Zwischen 1970 und 1990 betrug die Bevölkerungszahl stets nur zwischen 77,5 und 79,75 Mio. Menschen. Während die Bevölkerung auf Bundesebene also in den Jahren seit der Wiedervereinigung 1990 weiter gewachsen ist, haben regional in den 1990er Jahren bereits ein Drittel aller Kreise Einwohner verloren, darunter fast alle ostdeutschen. Seit 1990 hat die Bevölke-

${ }^{659}$ Ein Zwischenhoch gab es um 1960, als noch einmal in der Nachkriegszeit das Bestandhaltungsniveau erreicht wurde, vgl. Birg (2001), 50. 
rungszahl mit Ausnahme des Jahres 1998 ständig zugenommen ${ }^{660}$ Grund dafür waren die hohen Zahlen der Zuwanderer, v.a. vor der Änderung des Asylrechts (vgl. Abb. 26).

\section{Abb. 26: Saldo der Wanderungen über die Grenzen Deutschlands}

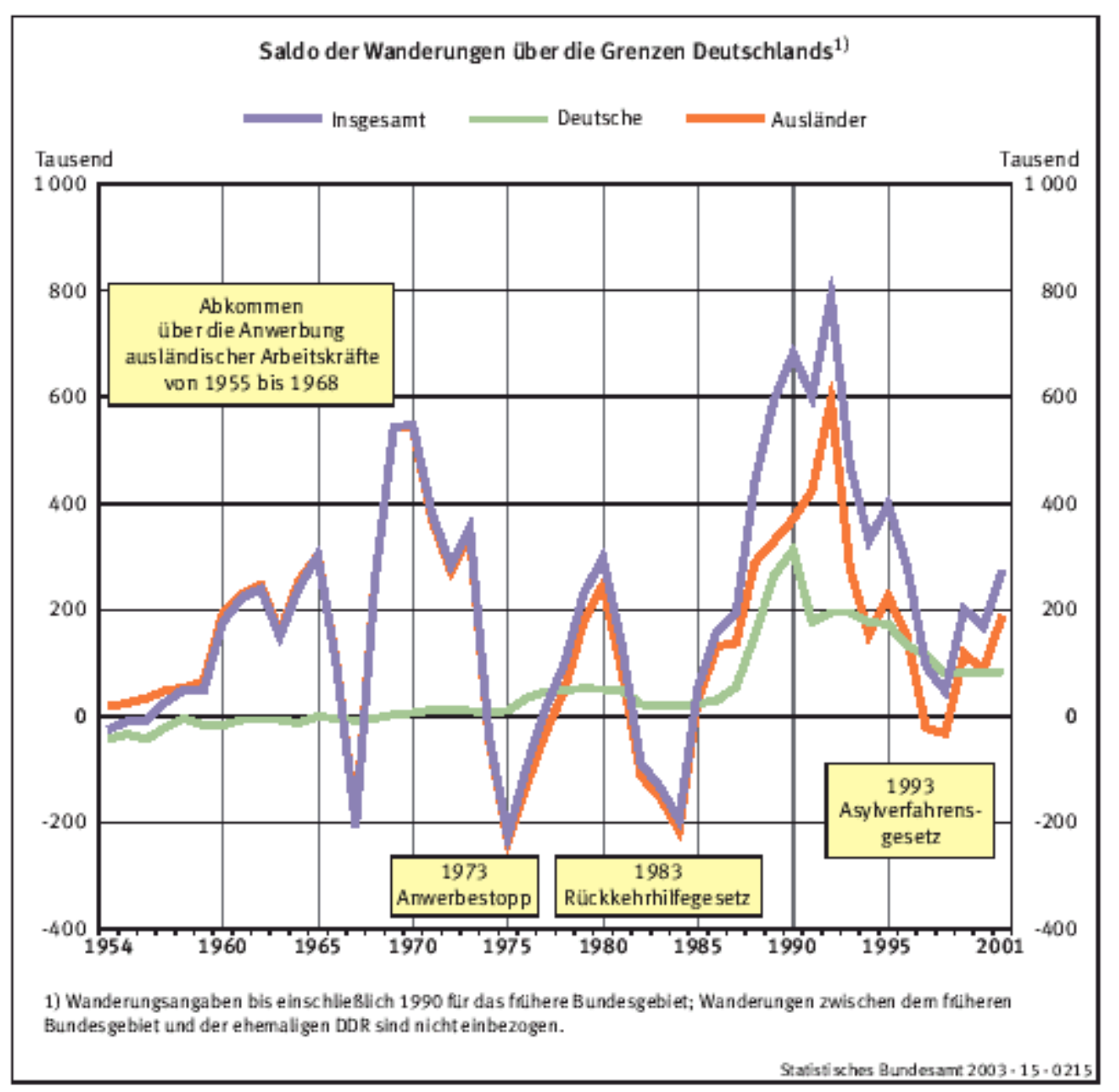

\section{Quelle: Statistisches Bundesamt (2003), 21}

Wegen der Veränderungen im Altersaufbau kommt es zu einer steigenden Zahl von Sterbefällen, obwohl die Lebenserwartung der Menschen zunimmt. Die Altersstruktur prägt generell die Entwicklung der nächsten Jahrzehnte. Die heute 35bis 40jährigen bilden die geburtenstärksten Jahrgänge und rücken allmählich ins Seniorenalter vor.

Schon lange ist die sogenannte Bevölkerungspyramide keine Pyramide mehr durch die Einschnitte der beiden Weltkriege nahm die Pyramide die Form einer

\footnotetext{
${ }^{660} 1998$ gab es einen Überschuss der Gestorbenen über die Lebendgeborenen, der die in diesem Jahr nur leicht positive Wanderungsbilanz überstiegen hat, so dass 1998 die Bevölkerung sank.
} 
,zerzausten Tanne' an. In 50 Jahren wird die Altersstruktur die Form einer sich ,verschlankenden Säule' oder eines ,ausgefransten Pilzes' annehmen.

\subsubsection{Lebenserwartung}

Seit 1855 hat sich die Lebenserwartung der Deutschen von 37 Jahren auf durchschnittlich 78 Jahre (Männer: 75,6 Jahren, Frauen: 81,3 Jahren im Durchschnitt der Jahre 2001-2003) ${ }^{661}$ mehr als verdoppelt. Noch vor hundert Jahren haben Eltern selten die Heirat ihres jüngsten Kindes erlebt. Heute erleben sie in der Regel sogar die Heirat ihrer Enkel mit. ${ }^{62}$

\section{Abb. 27: Fernere Lebenserwartung im Alter von 60 Jahren}

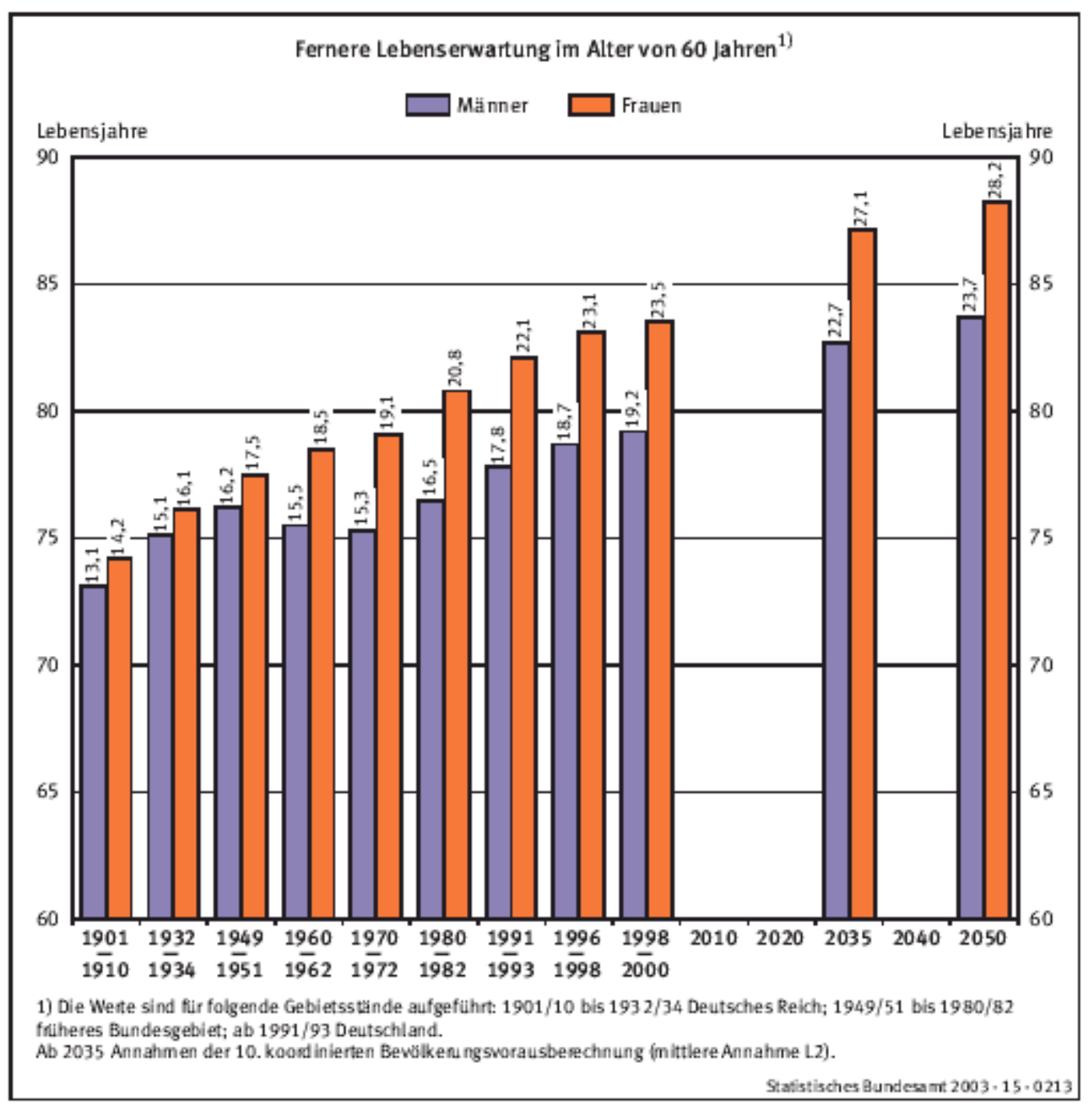

\section{Quelle: Statistisches Bundesamt (2003), 16}

\footnotetext{
${ }^{661}$ Telefonische Auskunft des Statistischen Bundesamtes. Generell berechnet Destatis diesen Wert nur im 3-Jahres-Durchschnitt. Wegen des heißen Sommers 2003 lag z.B. die Sterblichkeit ziemlich hoch. Solche verzerrenden Effekte will man ausschließen.

${ }^{662}$ BMJFFG (1986), 36
} 
Das Durchschnittalter der Bevölkerung hat sich seit 1950 von 35 auf 40 Jahre erhöht und wird bis Mitte dieses Jahrhunderts auf über 50 Jahre steigen. 60jährige Frauen haben dann noch knapp ein Drittel ihres Lebens (28,2 Jahre) vor sich.

\subsubsection{Altenquotienten}

Die wirtschaftlichen Folgen der Alterung der Bevölkerung werden u.a. mit dem Altenquotient gemessen, dessen Entwicklung in Abb. 29 dargestellt ist.

\section{Abb. 28: Altenquotienten nach verschiedenen Altersabgrenzungen}

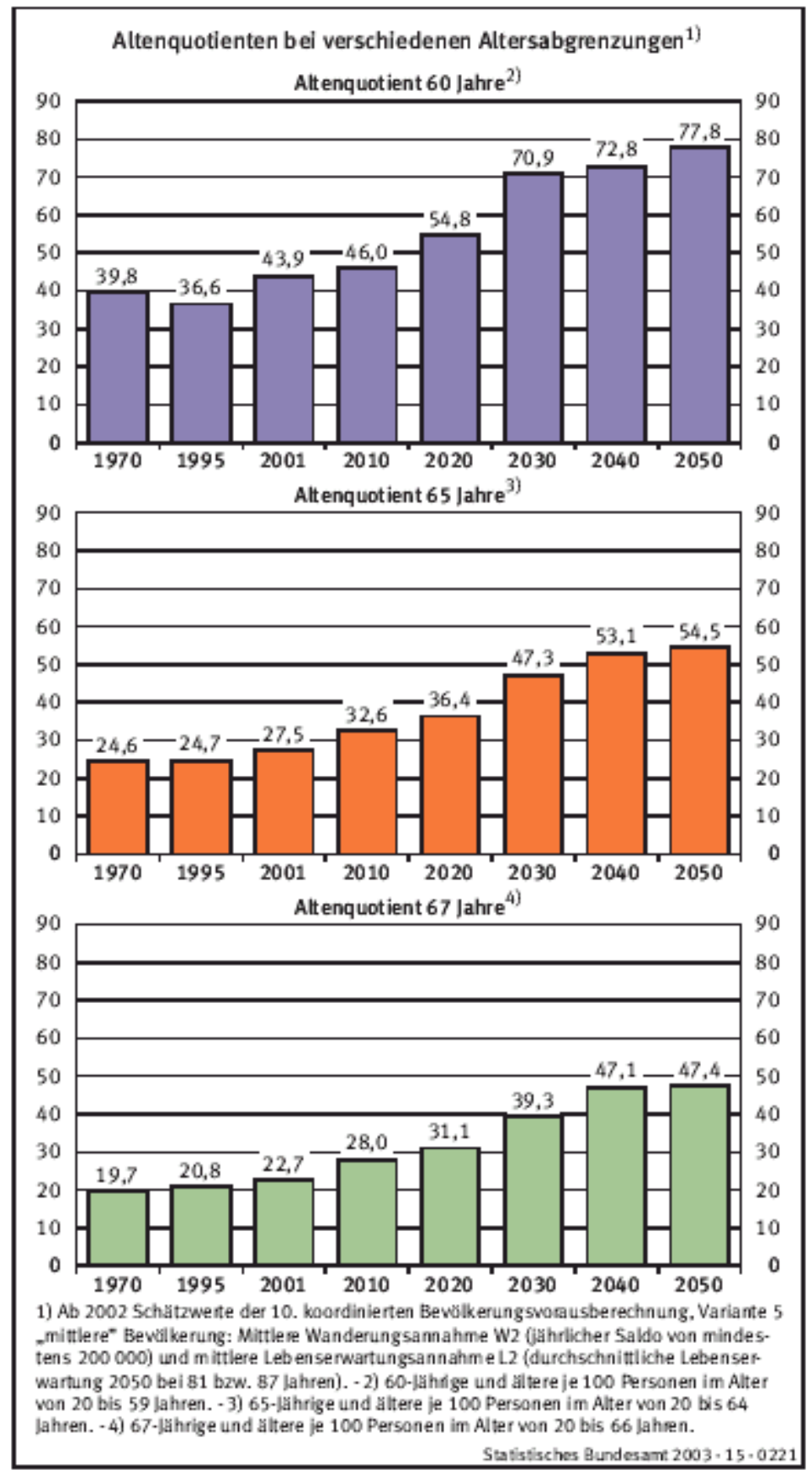

Quelle: Statistisches Bundesamt (2003), 32 
Je nachdem, wie er definiert wird, entwickelt er sich unterschiedlich ungünstig. Sollte sich das durchschnittliche Renteneintrittsalter nicht erhöhen, so ist das Verhältnis der Zahl der über 60jährigen zur Zahl der 20- bis 60jährigen relevant. Dieser Quotient, der 1910 noch bei bei 0,16 und 2001 bei 0,44 lag, wird nach der mittleren Variante (Variante 5) bis 2050 auf 0,78 ansteigen. Wenn dagegen - wie von der Politik geplant - das gesetzliche Renteneintrittsalter hochgesetzt und damit die durchschnittliche Lebensarbeitszeit verlängert wird, so steigt er nur auf 0,55 (bei einer Altersdefinition von 65 Jahren) bzw. 0,47 (bei 67 Jahren).

\subsubsection{Familien in Deutschland}

Der Anteil der Bevölkerung, in deren Haushalt es keine Kinder gibt, steigt seit Jahrzehnten. Im Jahr 1972 lebten im früheren Bundesgebiet 55 Prozent der Bevölkerung mit Kindern unter 18 Jahren zusammen (68,5 Prozent mit ledigen Kindern ohne Altersbegrenzung). Im Jahr 2000 ist dieser Wert auf 41,4 Prozent (bzw. 54 Prozent) gesunken.$^{663}$ Im Jahr 2000 gab es 1,77 Millionen Alleinerziehende mit Kindern unter 27 Jahren. Von den Alleinerziehenden waren 85,5 Prozent Frauen und 14,5 Prozent allein erziehende Männer. Der Anteil der Frauen, die zeitlebens kinderlos bleiben, steigt. Im Geburtsjahrgang 1940 sind nur 10,5 Prozent der Frauen zeitlebens kinderlos geblieben ${ }^{664}$ Schätzungen zufolge werden von den 1965 geborenen Frauen im Westen Deutschlands voraussichtlich 31 Prozent, im Osten 26 Prozent kinderlos bleiben. ${ }^{665}$ Von den nicht-kinderlosen Frauen ${ }^{666}$ entschied sich im Westen Deutschlands eine deutliche Mehrheit von 48,7 Prozent für 2 Kinder, gefolgt von 28,1 Prozent mit einem Kind und 23,2 Prozent mit 3 oder mehr Kindern (Osten: 60,2 Prozent mit 2 Kindern, 22,4 Prozent mit einem Kind und 16,4 Prozent mit 3 oder mehr Kindern). „Es ist in den jüngeren Geburtsjahrgängen eine Dominanz der Zwei-Kind-Familie bei hoher Kinderlosigkeit eingetreten, womit eine Polarisierung zwischen Familien und unverheirateten Paaren entstanden ist", resümiert das Bundesinistitut für Bevölkerungsforschung. ${ }^{667}$

\subsection{Zu viele Menschen, aber zu wenig Deutsche?}

\subsubsection{Die deutsche Debatte über den demografischen Wandel}

In weiten Kreisen der deutschen Bevölkerung, der politischen Klasse und der Wirtschaft wird der vorausberechnete demografische Wandel negativ bewertet. Befürchtet werden ein Verlust an Innovationsfähigkeit und Risikobereitschaft, eine Krise der Sozialversicherungssysteme, ein geringeres Wirtschaftswachstum, die rapide Verschärfung gesellschaftlicher Verteilungskonflikte und eine Verrin-

\footnotetext{
${ }^{663}$ BMFSFJ (2003), 33

${ }^{664} \mathrm{Bib}(2005), 35$

${ }^{665}$ A.a.O., Tabelle 14

${ }^{666}$ Zahlen liegen nur für den Jahrgang 1960 vor, nicht für den Jahrgang 1965.

${ }^{667} \mathrm{Bib}(2005), 35$
} 
gerung des Humanvermögens. So formuliert ein Bericht an die Hessische Staatskanzlei: „In Kürze wird sie [die Bevölkerung Deutschlands, J.T.], sich beschleunigend, schrumpfen. Der Verlust an Innovationsfähigkeit und der als Produktivkraft eigener Art geschätzten Risikobereitschaft wird immens. Während die sozialen Belastungen durch die Alterung und die notwendigen Integrationsanstrengungen wachsen, verliert die Wirtschaft an Dynamik. Die rapide Verschärfung gesellschaftlicher Verteilungskonflikte ist damit vorprogrammiert." ${ }^{\text {"668 }}$ Prognostiziert werden des weiteren ein "Legitimationsverlust des politischen Systems" ${ }^{\text {“669 }}$ wegen des immer geringer werdenden Gewichts der jungen Generation an der Wahlurne und eine „Beschädigung des Humanvermögens. “670 Diese negative Bewertung gibt die weit überwiegende Einstellung der ,Eliten' und Meinungsmacher in Deutschland wieder. In Leitartikeln führender Magazine wird vor der Schrumpfung gewarnt. So mahnt Ex-Bundespräsident Roman Herzog, dass die grassierende Kinderlosigkeit zu einem geistigen Verarmungsprozess führe. ${ }^{671}$ Die ZEIT veröffentlichte seit Anfang 2003 mehrere große Serien über die „,demografische Katastrophe““672 Eine Kostprobe daraus: „Deutschland verliert jährlich 200000 Einwohner, da mehr Menschen sterben als geboren werden. Es wächst ein demografisches Problem ungeheuren Ausmaßes heran, doch die Politiker ignorieren es. “673 GEO behauptet, dass das Ticken der „Zeitbombe Demografie“" noch von zu wenigen gehört würde und dass Bürgermeister beschönigend von „negativem Wachstum“ und „Stadtentwicklung rückwärts“" redeten. ${ }^{674}$

Die deutsche Bevölkerungswissenschaft war nach der NS-Herrschaft zurückhaltend geworden, demografische Zustände zu bewerten. ${ }^{675}$ Erst in den letzten Jahren ist diese Einstellung abgelöst worden durch eine pronatalistische Haltung, die heute an allen deutschen Lehrstühlen außer Rostock vertreten wird. Die meisten deutschen Bevölkerungswissenschaftler sehen Deutschland im „demografischen Dilemma“6 ${ }^{676}$ oder charakterisieren die Entwicklung mit Begriffen wie „demografischer Implosion“ und „Verzicht auf Nachwuchssicherung“.677 Herwig Birg warnt vor „drohenden kulturellen und sozialen Verwüstungen“6778 und meint resignierend: „Es wird in Deutschland im 21. Jahrhundert nie wieder so viele Kinder und junge Menschen geben wie heute. Die sich ständig wiederholenden, immer gleichen Schlagzeilen der kommenden Jahre und Jahrzehnte über abnehmende Schü-

\footnotetext{
${ }^{668}$ Hessische Staatskanzlei (2003), 22

${ }^{669}$ A.a.O., 22-30

${ }^{670}$ A.a.O., 22-30

${ }^{671}$ o.V. $(05.01 .2004), 38$

${ }^{672}$ Niejahr (02.01.2003), 9

${ }^{673}$ A.a.O.

${ }^{674}$ Gaede (2004), 3

${ }^{675}$ Mackensen (2003), 2

${ }^{676}$ Schmid (2004), 8

${ }^{677}$ Kaufmann (2004a)

${ }^{678}$ Birg (2001), 12
} 
ler- und Arbeitskräftezahlen werden unsere Nerven strapazieren und das Land mit einer tiefen Tristesse überziehen."679 In unseren europäischen Nachbarländern sparen besonders die Franzosen nicht mit drastischen Worten: „Im Vergleich zur demografischen Katastrophe ist der Zusammenbruch des Kommunismus unwichtig", schreibt der Ethnologe Claude Lévi-Strauss. ${ }^{600}$ Und Gérard-François Dumont nennt sein Buch gleich „Europa stirbt vor sich hin....“6681

Neuerdings wird der demografische Wandel nicht nur als wohlstandsvernichtend und außenpolitisch fatal, sondern auch als nicht generationengerecht bezeichnet. „Und schließlich ist es die nachhaltigste und folgenreichste Verletzung der Generationengerechtigkeit, wenn in einer Gesellschaft die nachwachsende Generation zu klein gehalten wird, um die ihr von den vorangehenden Generationen hinterlassenen Verpflichtungen zu schultern", schreibt der Bevölkerungssoziologe Franz-Xaver Kaufmann. ${ }^{682}$ Birg schlägt in die gleiche Kerbe: „Gegen die Beschäftigung mit Bevölkerungsproblemen kann man allerdings ins Feld führen, was die Menschen von heute eigentlich davon hätten, wenn sie ihre Energie in die Vermeidung der demografisch bedingten Zukunftsprobleme investierten, wo doch die Menschen z.B. des Jahres 2050 auch nichts für die jetzt Lebenden tun? Für das Nichtstun und für das Nicht-Wissen-Wollen gibt es viele Gründe, so dass ich als Autor die Beweislast spüre, darlegen zu müssen, warum ich dieses Buch überhaupt geschrieben habe. Der wichtigste Beweggrund ist die Aussicht, dass der demografische Niedergang Deutschlands (und Europas) rückblickend einmal als ein Vorzeichen für den Abschied unseres Landes aus seiner tausendjährigen Geschichte gedeutet werden könnte, ohne dass diese Gefahr den heutigen Zeitgenossen überhaupt bewusst war.“683

Und in der deutschen Zeitschrift für Bevölkerungswissenschaft plädiert Max Wingen für eine bevölkerungsbewusste Familienpolitik, da „die Verwirklichung von Generationengerechtigkeit (was meint, dass jede Generation der nächsten mindestens so viele Chancen und Handlungsspielräume hinterlassen sollte, wie sie selbst vorgefunden hat) eine Bevölkerungsentwicklung voraussetzt, die nicht durch jene drastischen Verwerfungen gekennzeichnet ist, wie sie seit Jahrzehnten absehbar und kaum noch korrigierbar sind." ${ }^{684}$

Aus diesen drei Äußerungen spricht eine erstaunliche Unwissenheit in Bezug auf das Konzept der Generationengerechtigkeit. Es sollte eine etwas intensivere Beschäftigung mit den voraussichtlichen Wünschen kommender Generationen erfolgen. Würde dies geschehen, dann wäre die ökologische Komponente des Konzeptes, Generationengerechtigkeit' nicht übersehen worden.

\footnotetext{
${ }^{679} \operatorname{Birg}(2004 \mathrm{a}), 13$

${ }^{680}$ Lévi-Strauss (1992), 55

${ }^{681}$ Dumont (1997)

${ }^{682}$ Kaufmann (2004a), 11. Vgl. auch Birg (2001), 160 u. 208

${ }^{683} \operatorname{Birg}(2001), 14$

${ }^{684}$ Wingen (01.04.2004); siehe ausführlicher auch Wingen (2002)
} 
Meistens werden in der deutschen Debatte ausschließlich mögliche negative Folgen der Schrumpfung benannt. ${ }^{655}$ Selten einmal kommt eine abweichende Meinung zu Wort, wie etwa Heik Afheldt in der ZEIT: „Aber was ist so schlimm, wenn die Zahl der Einwohner innerhalb eines Zeitraums von fünf Jahrzehnten um vielleicht zehn oder fünfzehn Millionen schrumpft - Deutschland, das so dicht besiedelte Land, ein wenig weniger dicht besiedelt sein wird? Mehr Luft zum Atmen, mehr Raum, um sich zu bewegen, mehr Straßen, um zu fahren, mehr Wohnfläche um zu leben? Vor 20 Jahren hat man die Wirkungen einer seinerzeit erwarteten schrumpfenden Einwohnerzahl überwiegend positiv beurteilt. (...) Was passiert denn auch, wenn aus einem Vierpersonenhaushalt von zwei Kindern eines auszieht? Ein Zimmer wird frei, das von den Eltern oder dem Bleibekind gerne zusätzlich genutzt wird. In Deutschland und den Nachbarländern ist die Wohnfläche pro Person in den vergangenen Jahren kräftig gestiegen, damit nahmen auch die Wohnkosten zu. Aber gleichzeitig sind die Pro-Kopf-Einkommen noch stärker gestiegen, obwohl (oder weil?) die Zahl derjenigen, die das Sozialprodukt erarbeiten, zurückgegangen ist. Die Reiseintensität der kleineren Zahl an Menschen nimmt zu ebenso wie die Zahl der Konzert- und Opernbesuche und andere Freizeitaktivitäten. Weil das Volumen an arbeitsfreier Zeit insgesamt auch bei schrumpfender Bevölkerung noch kräftig wächst, bleiben die Märkte für persönliche Dienstleistungen fast alle auf Wachstumskurs. ${ }^{\text {“686 }}$

\subsubsection{Der Richtungswechsel der deutschen Politik zwischen 1994 und 2004}

Der Nationale Bericht der Bundesregierung für die UN-Konferenz zu Bevölkerung und Entwicklung 1994 in Kairo, eines der wenigen offiziellen Dokumente zur deutschen Position bezüglich Bevölkerungspolitik, formuliert deutlich die Sorge, dass das Bestanderhaltungsniveau hierzulande unterschritten wird. Eine Bevölkerungspolitik in Deutschland wird indes abgelehnt: „Der Staat hat unterschiedliche Vorstellungen von Familienleben zu respektieren und die freie Entscheidung der Eltern über Zahl und Zeitpunkt der Geburt ihrer Kinder zu gewährleisten. “687 Hier nahm die deutsche Regierungspolitik inzwischen eine Kurskorrektur vor. So rief Bundesfamilienministerin Schmidt im Sommer 2003 in einer neuen Eindringlichkeit zur Steigerung der Geburtenrate in Deutschland auf. ${ }^{68}$ Frau Schmidt nahm für die Bundesregierung in Anspruch, sie werde künftig

\footnotetext{
${ }^{685}$ Unter den Büchern, auf die dies am deutlichsten zutrifft, sind: Deutsche Studiengemeinschaft (2003), Dumont (1997). Nur wenige Beiträge wägen Vorteile und Nachteile gegeneinander ab, z.B. Umweltakademie Baden-Württemberg (2004); Ehrenberg/Fuchs (1980), 248-252; Olechowski (1980); Silkenbeumer (1979)

${ }^{686}$ Afheldt (17.05.2001), 26. In eine ähnliche Richtung argumentiert Natorp (01.08.1988), 2

${ }^{687}$ Zitiert nach Wichterich (1994), 20

${ }^{688}$ o.V. (10.07.2003) Die Rede wurde von der Bundesfamilienministerin anlässlich der Präsentation einer gemeinsamen Kampagne mit der Bertelsmann-Stiftung gehalten. Die Kampagne will Beispiele familienfreundlicher Unternehmen untersuchen und bekannt machen, vgl. Schmidt/Mohn (2004).
} 
eine ,aktive Bevölkerungspolitik“ betreiben. ${ }^{689}$ Damit beendete sie eine fast sechzigjährige Abstinenz deutscher Regierungen auf diesem Gebiet, zumindest was die offizielle Regierungsmeinung angeht. Bei der nächsten UN-Befragung der Mitgliedsländer zu nationalen Bevölkerungspolitiken dürfte Deutschland voraussichtlich erstmals nicht ,No intervention' angeben. Frau Schmidt sagte warnend, Deutschland habe eine der niedrigsten Geburtenraten der Welt, es liege auf dem 180. Platz unter 191 Staaten der Erde. Weniger Kinder bedeuteten für ein reiches Land wie die Bundesrepublik auch weniger Wohlstand; ein Teil der aktuellen Wachstumsschwäche der deutschen Wirtschaft resultiere auch aus der geringen Kinderzahl, die eine konsumdämpfende Wirkung in vielen Branchen habe. Es gehe nicht darum, jenen Paaren „Kinder einzureden“, die kinderlos bleiben wollten, doch müßten jene stärker unterstützt werden, die einen Kinderwunsch hätten, ihn jedoch der Umstände wegen nicht verwirklichten. Frau Schmidt sagte, es seien „Mentalitätsveränderungen“ und „,neue Prioritäten“ notwendig; beides sei weder durch neue Gesetze noch durch politische Appelle zu erreichen. Sie setze vielmehr auf eine ,effektive und nachhaltige Freiwilligkeit“, um die Wirtschaft dazu zu bewegen, Arbeitsplätze frauen- und familienfreundlicher zu gestalten. Es gebe „eine Menge gesetzlicher Voraussetzungen“, die kinderfreundlich wirken sollten, sie nannte den Anspruch auf Teilzeitarbeit als Beispiel, doch änderten sich die Verhältnisse deswegen „keineswegs so, wie sie sich zu ändern hätten“. ${ }^{690}$

Vergleicht man diese Aussagen z.B. mit der deutschen Einleitung zu Paul Ehrlichs Buch Die Bevölkerungsbombe von 1971, so wird der Mentalitätswandel deutlich: „Zur Zeit wächst die Bevölkerung der Bundesrepublik jährlich (mit beträchtlichen Schwankungen) um etwa eine halbe Million. Erst im Jahr 2040 werden wir (bei unveränderter Wachstumsrate) die doppelte Anzahl von Einwohnern und damit eine Bevölkerungsdichte von fast 500 pro $\mathrm{km}^{2}$ haben. (...) Es ist kaum zu bezweifeln, dass wir eine solche Zahl von Menschen ernähren könnten, aber sind damit bereits alle Probleme gelöst? Wenn die Menschen dann nicht schlechter leben sollen als heute, muss alles verdoppelt werden: unser gesamtes Industriepotenzial, das Verkehrsnetz, die Wasserversorgung, das Bildungswesen, die ärztliche Betreuung und natürlich auch die Erholungsgebiete. Und hier mag es jedem deutlich werden, dass wir auf Probleme stoßen, die bereits heute bestehen und die trotz Wohlstand und Überernährung keineswegs als gelöst angesehen werden können.“' ${ }^{6911}$ Und weiter: „Die kleineren (aber immer noch beträchtlichen) Wachstumsraten in den hochentwickelten Ländern und die rein technischen Möglichkeiten, auch eine zunehmende Bevölkerung zu versorgen (die im Grunde ein Leben von der Substanz bedeuten), dürfen uns nicht davon abhalten, auch bei uns

\footnotetext{
${ }^{689}$ A.a.O.

${ }^{690}$ A.a.O.

${ }^{691}$ Ehrlich (1971), 9. Das Vorwort stammt von H. Glubrecht.
} 
eine entschiedene und effektive Bevölkerungspolitik als das dringendste Gebot der Stunde anzusehen. ${ }^{\text {“692 }}$

\subsubsection{Eine Welt im demografischen Gleichgewicht}

Die Debatte in Deutschland hat Auswirkungen auf die internationale Ebene. Seit den 1950er Jahren war es in weltweiten bevölkerungspolitischer Debatten stets um das Wie einer Stabilisierung der Weltbevölkerung gegangen, nicht um das $O b$. Offiziell wird diese Position auf UN-Ebene auch immer noch aufrecht erhalten. Inzwischen sind die Wachstumsraten allerdings so weit zurückgegangen, und zwar nicht nur in den Entwicklungsländern, sondern v.a. in Europa, dass die internationale Debatte der Agenda von Kairo kaum noch Beachtung schenkt. Die UN Population Division mutmaßt, dass europäische Länder wie Österreich, Dänemark, Frankreich, Italien oder die Schweiz die Unterstützung für Familienplanungsprogramme deshalb zurückgefahren haben, weil in ihren Ländern die Geburtenraten so niedrig sind ${ }^{693}$ Es ist tatsächlich zu vermuten, dass die deutsche Regierung unter dem Eindruck eines drohenden ,finis Germaniae' wenig Bereitschaft zeigt, ihre finanziellen Verpflichtungen aus dem Aktionsprogramm von Kairo zu erfüllen. Auch in der deutschen Bevölkerung werden sich beim derzeitigen Diskussionsklima wenig private Spender für geburtensenkende ,Reproductive Health'-Maßnahmen finden lassen.

Es ist zu befürchten, dass es bei Vertretern anderer Kontinente einen falschen Eindruck hinterläßt, wenn etwa die deutsche Parlamentariern Karin Kortmann, immerhin Mitglied des Parlamentarischen Beirats der Deutschen Stiftung Weltbevölkerung, bei einer hochrangigen Konferenz mit dem bezeichnenden Titel Ways out of the Crisis - Reproductive Health in Need of New Ideas ${ }^{694}$ ihren Vortrag mit den Worten beginnt: „Wir haben zu niedrige Geburtenraten.“6955 Gunta Lazdane von der WHO in Genf stellte bei der gleichen Konferenz die Frage, ob in Europa mit seinen niedrigen Geburtenraten ,Reproduktive Gesundheit' eine Rolle spiele ${ }^{996}$ Die meisten Teilnehmer waren sich einig, dass die Agenda von Kairo dringend mehr öffentliche Unterstützung braucht, wenn man sie retten wolle ${ }^{697}$ Es erscheinen in Deutschland nicht nur mehr Berichte über die ,demografische Katastrophe' (ZEIT) im eigenen Land, es erscheinen auch weniger Berichte über die Folgen des anhaltenden Bevölkerungswachstums auf weltweiter Ebene. 1994 schrieb Klaus Leisinger noch: „Ohne die Veränderung unserer Denk- und Verhaltensweisen, ohne biophile handlungsleitende Werte in Bezug auf unseren

\footnotetext{
${ }^{692}$ A.a.O., 10

${ }^{693}$ UN Poplation Division (2003), 7

${ }^{694}$ International Dialogue on Population and Sustainable Development „Ways out of the Crisis - Reproductive Health in Need of New Ideas." 14.10.2003. GTZ-Haus in Berlin.

${ }^{695}$ Schering AG (2004). Video des Vortrags von Karin Kortmann auf der Dokumentations-CD.

${ }^{696}$ Schering AG (2004), 23

${ }^{697}$ A.a.O., 23-26
} 
Energieverbrauchs-, Ressourcennutzungs- und Abfallstil, klingt eine von uns geführte bevölkerungspolitische Debatte hohl und heuchlerisch. ${ }^{“ 698}$ Heute ist das Problem, dass kaum noch über das rasante weltweite Bevölkerungswachstum und die Notwendigkeit antinatalistischer Maßnahmen gesprochen wird, weil die reichen Länder auf ihre eigenen Geburtenraten fixiert sind. Das Worldwatch Magazine resümiert: „Population arouses passions. No other topic covered recently in World Watch has driven so many people to sit down and dash off a strongly worded letter. At one time this passion was widespread; population seemed a more urgent issue in the 1970s. But it faded from public discourse and the media hardly mention it now." ${ }^{999}$ Die rückläufige Tendenz der jährlichen Zuwachsraten der Weltbevölkerung wird genutzt, um auf breiter Front Entwarnung zu geben. Im Hinblick auf das prognostizierte Wachstum der Weltbevölkerung um ein weiteres Drittel (und die ökologischen Folgen dieser Entwicklung) ist dies eine verfrühte und ungerechtfertigte Reaktion. ${ }^{700}$

Es ist bestürzend, dass der Implementationsplan von Johannesburg das Thema Bevölkerung so gut wie nicht mehr berücksichtigt, während es in der 1992 verabschiedeten Agenda 21 noch ausführlich erwähnt worden war. Ein Mitglied der deutschen Delegation bestätigte, dass es massiven Druck von Seiten der USA und des Vatikans gab, das Wort ,Bevölkerungswachstum' überhaupt zu erwähnen. ${ }^{701}$

Bestenfalls werden der Geburtenrückgang in den reichen Ländern im Norden der Hemisphäre und die notwendige Stabilisierung der gesamten Weltbevölkerung als zwei Themen dargestellt, die nichts miteinander zu tun haben und insofern als zwei komplett voneinander getrennte Probleme zu betrachten seien. Dieses neue Paradigma hat die weitverbreitete Ansicht abgelöst, die WEL würden zu schnell wachsen. Aber ist es durch die Fakten gedeckt? Europa ist Teil dieser Erde. Was in einem Teil geschieht, hat Auswirkungen auf das Gesamtsystem. Es kann nicht bestritten werden, dass die demografische Entwicklung in Europa, Japan und Nordamerika mitentscheidend dafür ist, wann die Weltbevölkerung stabilisiert wird. Das Ziel von Kairo war eine rasche Stabilisierung der Weltbevölkerung. Dieses Ziel wird zunehmend durch den Perspektivenwechsel in den SEL gefährdet. Wenn europäische Länder den Kampf gegen die Bevölkerungsschrumpfung zur neuen Priorität ausrufen, dann wird offensichtlich das Ziel einer möglichst frühen Stabilisierung der Weltbevölkerung aufgegeben.

\footnotetext{
${ }^{698}$ Leisinger (1994), 22

${ }^{699}$ Prugh/Ayres (2004), 13

700 Berechtigt ist allerdings eine differenzierte Strategie für die Sicherung der demografischen Zukunftsfähigkeit in den SEL, vgl. Abschnitt 6.8. Eine Strategie für den Übergang in Deutschland.

${ }^{701}$ Kröger/van Olst/Klingholz (2004), 50
} 


\subsubsection{Was sagt das Aktionsprogramm über die Geburtenentwicklung in den reichen Ländern?}

Obwohl schon 1994 in vielen SEL die Geburtenraten unter dem Reproduktionsniveau lagen, wird im Aktionsprogramm keineswegs eine Ankurbelung derselben empfohlen. Vielmehr gilt: „In allen Ländern braucht die Gesellschaft mehr Aufklärung über die Auswirkungen der Wechselbeziehungen zwischen Bevölkerung und Umwelt, damit Verhaltensänderungen herbeigeführt sowie das Konsumverhalten beeinflusst werden können und eine nachhaltige Bewirtschaftung natürlicher Hilfsquellen gefördert wird. ${ }^{702}$ Die hier angesprochenen Verhaltensänderungen können wohl nur so interpretiert werden, dass auch in den SEL bei Bevölkerungspolitiken die Grenzen ökologischer Nachhaltigkeit im Blick behalten werden sollten. Zwar heißt es kryptisch: „Länder, die ihren demografischen Übergang abgeschlossen haben, sollten die notwendigen Schritte unternehmen, um ihre demografischen Trends im Rahmen ihrer sozialen und wirtschaftlichen Entwicklung zu optimieren. ${ }^{\text {"703 }}$ Einer pronatalistischen Bevölkerungspolitik wird aber nirgendwo das Wort geredet. Vielmehr wird immer wieder die Notwendigkeit betont, aus Verantwortung für zukünftige Generationen den demografischen Übergang in den Entwicklungsländern ,as soon as possible ${ }^{6704}$ abzuschließen und dadurch die Weltbevölkerung bald $\mathrm{zu}$ stabilisieren. ${ }^{705}$ Auch wenn (v.a. auf Betreiben konservativer Länder) ein ganzes Kapitel zur Förderung der Familie aufgenommen wurde (Kapitel V), so wird damit im Aktionsprogramm keine Erhöhung der Geburtenraten impliziert. Auch wenn alles in diplomatischer Sprache formuliert ist, so gilt: Der Süden darf sich aufgefordert fühlen, weiter seine Geburtenraten zu senken, der Norden soll seine Konsum- und Produktionsmuster ändern und seinen Überkonsum ${ }^{706}$ beenden. ${ }^{707}$ Ein demografischer Beitrag des Nordens zur Stabilisierung der Weltbevölkerung wird zwar nicht verlangt, aber es ist bezeichnend für die Stimmung der damaligen Zeit, dass die Premierministerin von Norwegen, Gro Harlem Brundtland, die mit 1,3 Kindern pro Frau außerordentlich niedrige Geburtenrate Italiens in ihrer Eröffnungsrede in folgender Weise erwähnte: „For example Buddhist Thailand, Moslem Indonesia and Catholic Italy demonstrate that relatively sharp reductions in fertility can be achieved in an amazingly short time. ${ }^{\text {“708 }}$ Wenige Minuten zuvor hatte sie die antinatalistische Grundhaltung vieler Delegierter noch mal deutlich gemacht: „In many countries where population growth is higher than economic growth, the problems are exacerbated each year.

\footnotetext{
${ }^{702} \mathrm{BiB}(1994), 11.16$

${ }^{703}$ A.a.O., 6.4

${ }^{704}$ A.a.O., 6.3

${ }^{705}$ A.a.O.

706 Das Wort ,Überkonsum' fällt zwar nicht im Aktionsprogramm, aber in den Einleitungsreden formulieren die Redner ihre Kritik am Konsumverhalten des Nordens deutlich (vgl. Brundtland 1994).

${ }^{707} \mathrm{BiB}(1994)$, z.B. Grundsatz 6, 3.25 oder 3.28

${ }^{708}$ Brundtland (1994), 35
} 
The costs of future social needs will be soaring. The punity in inaction will be severe, a nightmare for ministers of finance and a legacy which future generations do not deserve. But the benefits of policy change are so great that we cannot affort not to make them. We must measure the benefits of successful population policies in savings (...). Egyptian calculations show that every pound invested in family planning saves 30 pounds in future expenditures on food subsidies, education, water, sewage, housing and health." 709

Alle Länder werden im Aktionsprogramm zudem aufgefordert, „demografische Faktoren bei Umweltverträglichkeitsprüfungen " einzubeziehen. ${ }^{710}$

Im Rahmen des RH-Programms sollen allerdings auch finanzielle Mittel für die Verhütung bzw. Behandlung von Unfruchtbarkeit aufgebracht werden, da diese ebenfalls zu den möglichen Krankeiten der Fortpflanzungsorgane gezählt wird. ${ }^{711}$ Die Tatsache, dass dadurch die Weltbevölkerung weniger schnell stabilisiert werden kann, wird nicht thematisiert bzw. den Gesundheitszielen untergeordnet.

\subsubsection{Sind die reichen Länder überbevölkert?}

Die Angehörigen der heutigen auf der Erde lebenden Generation nehmen mindestens so viele Ressourcen in Anspruch, wie alle Menschen zusammen in der bisherigen Geschichte für sich in Anspruch genommen haben. ${ }^{712}$ Biologen versuchen den Grad der menschlichen Beeinflussung der Ökosysteme durch die Inanspruchnahme der sogenannten Nettoprimärproduktion (NPP) zu erfassen. Die NPP ist definiert als der Betrag an Sonnenenergie, der innerhalb eines Jahres durch Photosynthese in biochemische Energie umgewandelt wird und den die Pflanzen nicht für ihre eigenen Lebensprozesse benötigen. Sie stellt den Teil der Biomasse dar, der für den Menschen und für alle anderen Lebewesen zur Verfügung steht und die grundlegende Nahrungsquelle allen Lebens ist. Schon die heutige Inanspruchnahme von über 40 Prozent durch den Mensch ist ein deutliches Zeichen, dass er einen ungebührlich hohen Anteil an der NPP für seine eigenen Zwecke vereinnahmt. ${ }^{713}$ In den nächsten Jahren droht jedoch eine Verdoppelung. ${ }^{714}$

Dabei ist die Bilanz menschlicher Umweltbelastungen schon heute eine extreme Hypothek für künftige Generationen. Wir sind verantwortlich für die Emission von 60.000.000 Tonnen $\mathrm{CO}_{2}$ in die Atmosphäre, für den Verlust von 55.000 Hektar Tropenwald und 20.000 Hektar Ackerland, für die Vernichtung von mindestens 73 Arten und für die Entfischung der Meere mit 220.000 Tonnen pro Tag. ${ }^{715}$

\footnotetext{
${ }^{709}$ A.a.O., 34

${ }^{710} \mathrm{BiB}$ (1994), 3.29

${ }^{711}$ A.a.O., 13.14

${ }^{712}$ Knaus/Renn (1998), 21

${ }^{713}$ Vgl. Vitousek/Ehrlich/Matson (1986). Zitiert nach Knaus/Renn (1998), 39

${ }^{714}$ Dieren (1995), 67

${ }^{715}$ Kreibich (2003), 229. Die Zahl der ausgerotteten Tier- und Pflanzenarten basierend auf Wilsons Schätzung, vgl. Abschnitt 3.2. Verlust der Biodiversität.
} 
Diese Umweltsünden werden nicht zu gleichen Anteilen von SEL und Entwicklungsländern begangen. Vielmehr sind die reichen Länder trotz ihres kleineren Anteils an der Weltbevölkerung die Hauptverursacher der globalen ökologischen Krise. ${ }^{716}$ Ein Maß für den jährlichen Konsum von natürlichen Ressourcen ist der ökologische Fussabdruck (ecological footprint). Der Footprint einer Bevölkerung umfasst das gesamte biologisch produktive Land oder die Meeresflächen, die benötigt werden, um alle pflanzlischen Nahrungsmittel, Fleisch, Meeresfrüchte, Holz und Textilien herzustellen, die Energieversorgung zu gewährleisten und die dazu nötige Infrastruktur zu errichten. ${ }^{717}$ Zur Berechnung des Footprints werden Fragen gestellt wie:

- Welche Weidefläche brauchen die Rinder, deren Fleisch wir essen?

- Wie gross müssen die Felder sein, um sämtliche von uns konsumierten Agrarprodukte anzubauen - von Getreide über Gemüse bis zu Baumwolle oder Kaffee? Berücksichtigt wird auch die Fläche, welche der Anbau von Futtermittel für Nutztiere benötigt.

- Welche Meeresfläche brauchen die Fische, die wir verspeisen?

\section{Abb. 29: Ökologischer Fußabdruck nach Regionen}

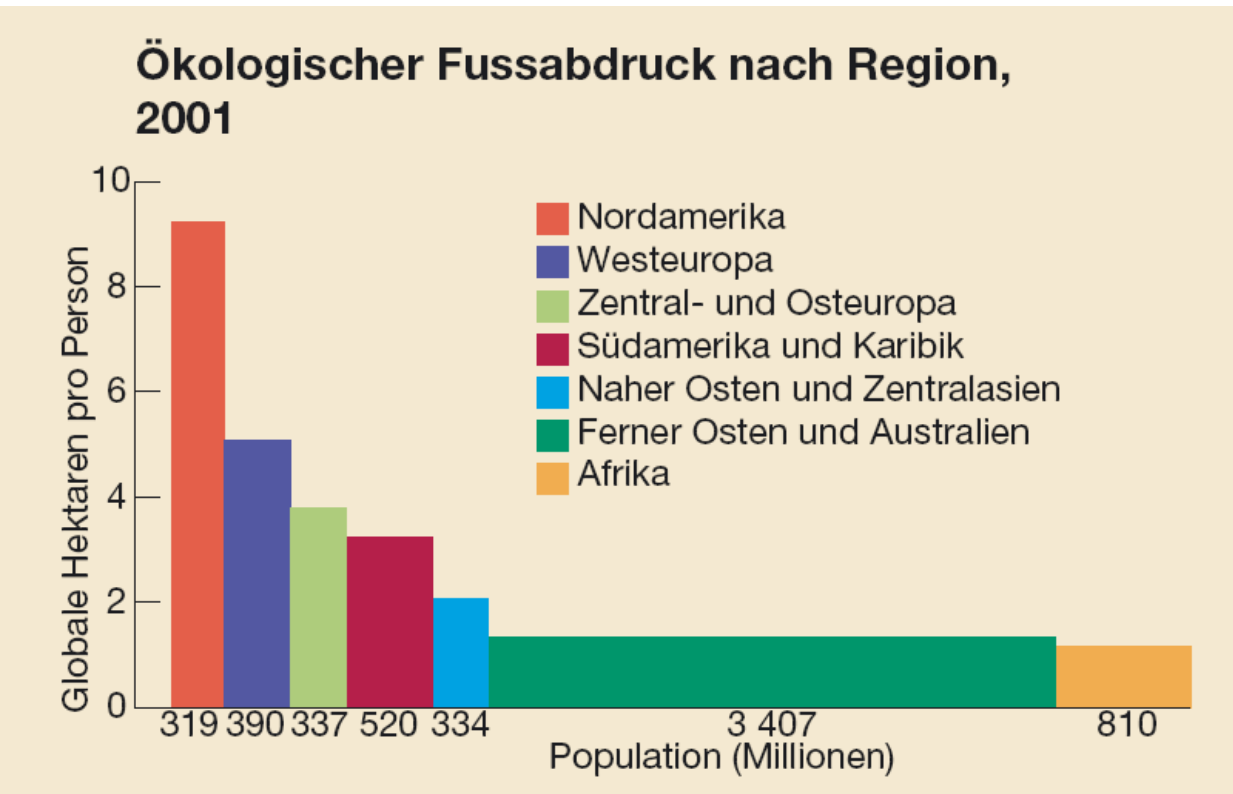

Quelle: World Wide Fund for Nature (2004b): Living Planet Report 2004. Deutsche Zusammenfassung. http://www.wwf.de/service/publikationen/.

\footnotetext{
${ }^{716}$ Leisinger (1994), 22

${ }^{717}$ World Wide Fund for Nature (2004b), 1
} 
- Wie viel Fläche benötigen wir für Infrastruktur wie Gebäude oder Verkehrswege?

- Welche Waldfläche ist nötig, um das $\mathrm{CO} 2 \mathrm{zu}$ binden, das wir durch die Verbrennung von fossilen Energieträgern freisetzen? ${ }^{718}$

Die Methodologie der Berechnung ist kompliziert und umstritten. Aber selbst bei einer alternativen Berechnungsweise würde der Footprint die Unterschiede im Konsum natürlicher Ressourcen aufzeigen, die zwischen Ländern bestehen. Ein durchschnittlicher Afrikaner oder Asiate beanspruchte 2001 einen Footprint von weniger als 1,3 Hektaren, bei einem Westeuropäer belief er sich hingegen auf 5,1 Hektaren, und ein Nordamerikaner verbrauchte durchschnittlich gar 9,2 Hektaren (vgl. Abb. 29). Deutschland liegt im weltweiten Ländervergleich auf Platz 21, hat also einen relativ hohen Footprint. ${ }^{719}$

Auf die inhärenten Probleme von Berechnungen zur Ermittlung der maximalen Zahl von Menschen, die die Erde tragen kann, wurde bereits eingegangen. Dennoch ist es illustrativ, in bedingten Aussagen den unterschiedlichen Ressourcenverbrauch in verschiedenen Teilen der Welt zu verdeutlichen. „Die Erde hat eine Tragekapazität von 700 Mio. Menschen mit dem Ressourcenverbrauch der Industrieländer und $9 \mathrm{Mrd}$. Menschen mit dem gegenwärtigen Ressourcenverbrauch der Entwicklungsländer", schreiben Kopfmüller und Mitautoren. ${ }^{720}$ Wenn die SEL die gleichen Wachstumsraten der Bevölkerung aufweisen würden wie die Entwicklungsländer, wären die ökologischen Probleme um ein Vielfaches größer als sie ohnehin sind. ${ }^{721}$

\subsubsection{Stabilisierung ist nicht genug}

Die Schrumpfung der SEL kann nach Ansicht vieler Wissenschaftler nur der Auftakt zu einer Schrumpfung der gesamten Weltbevölkerung sein. Vor dem Hintergrund der heutigen Überbeanspruchung des Naturkapitals hat sich ein Teil der Ökologiebewegung von Anfang an nicht nur für eine Stabilisierung der Weltbevölkerung, sondern für eine maßvolle Schrumpfung stark gemacht. ${ }^{722}$ Der Anthropologe Ken Smail formuliert: „Looking past the near-time concerns that have plagued population policy on a political level, it is increasingly apparent that the long-term sustainability of civilisation will require not just a leveling-off of human numbers as projected over the coming half-century, but a colossal reduction in both population and consumption. (...) Prudent and increasingly reliable scien-

\footnotetext{
${ }^{718}$ A.a.O., 2

${ }^{719}$ A.a.O., 1

${ }^{720}$ Kopfmüller u.a. (2001), 53

${ }^{721}$ In den die USA prangerte Ehrlichs Buch Population Bomb bereits 1968 die negativen Folgen des Bevölkerungswachstums in seinem Land an. Als Reaktion darauf kam es dort zur Gründung der Zero Population Growth-Initiative. Siehe: www.populationconnection.org

${ }^{722}$ Vgl. z.B. Harrison (1994)
} 
tific estimates suggest that the Earth's long-term sustainable human carrying capacity, at what might be defined as an 'adequate' to 'moderately comfortable' developed-world standard of living, may not be much greater than 2 to 3 billion. It may be considerably less, particularly if the normative lifestyle (level of consumption) aspired to is anywhere close to that of the United States. “723

In den nächsten Jahrhunderten wird sich der exponentielle Wachstumstrend der Weltbevölkerung laut den Prognosen der UN Population Division nicht fortsetzen. Mit 9,2 Milliarden wird das Wachstum der Weltbevölkerung 2075 seinen Höhepunkt erreichen und dann bis 2300 auf neun Milliarden leicht abnehmen. ${ }^{724}$

Im Idealfall wird sich die Menschheit danach in einem Gleichgewicht einpendeln, welches ökologisch nachhaltig ist und dann auf Jahrhunderte Bestand hat. Dieses wird unter dem heutigen Niveau liegen. Wenn dies aber die langfristige Entwicklung ist, die die Menschheit nehmen wird oder zumindest nehmen sollte, ist unter diesen Umständen die prognostizierte Schrumpfung in Deutschland und anderen reichen Ländern nicht neu zu bewerten? Abb. 30 zeigt, dass 2050 nicht nur die deutsche, sondern auch die Weltbevölkerung ihre Form von einer Pyramide zu einer schmaler werdenden Säule weiterentwickelt haben werden. Sind die reichen Länder Vorreiter eines demografischen Wandels, der als wünschenswert und nachahmenswert für die ganze Welt gelten kann? Sollten also z.B. wir Deutsche die Schrumpfung, statt sie emotional abzulehnen, nicht vielmehr positiv bewerten und die dabei gemachten Erfahrungen anderen Nationen zur Verfügung stellen? Die Nachteile, die in der Debatte gegen Schrumpfung und Alterung geltend gemacht werden, lassen sich in interne und externe Faktoren einteilen. Mit internen Faktoren sind die Folgen der Schrumpfung im eigenen Land gemeint. Als externe werden die Faktoren bezeichnet, die die Rolle Deutschlands im Gefüge der internationalen Beziehungen betreffen. Bevor sie dargestellt werden, soll aber zunächst darauf eingegangen werden, ob die Bevölkerungsvorausberechnung des Statistischen Bundesamtes überhaupt zuverlässig ist.

\footnotetext{
${ }^{723}$ Smail (2004), 58

${ }^{724} \mathrm{http} / / /$ www.un.org/esa/population/publications/longrange2/longrange2.htm
} 


\section{Abb. 30: Entwicklung der deutschen Bevölkerung und Weltbevöl- kerungsentwicklung}

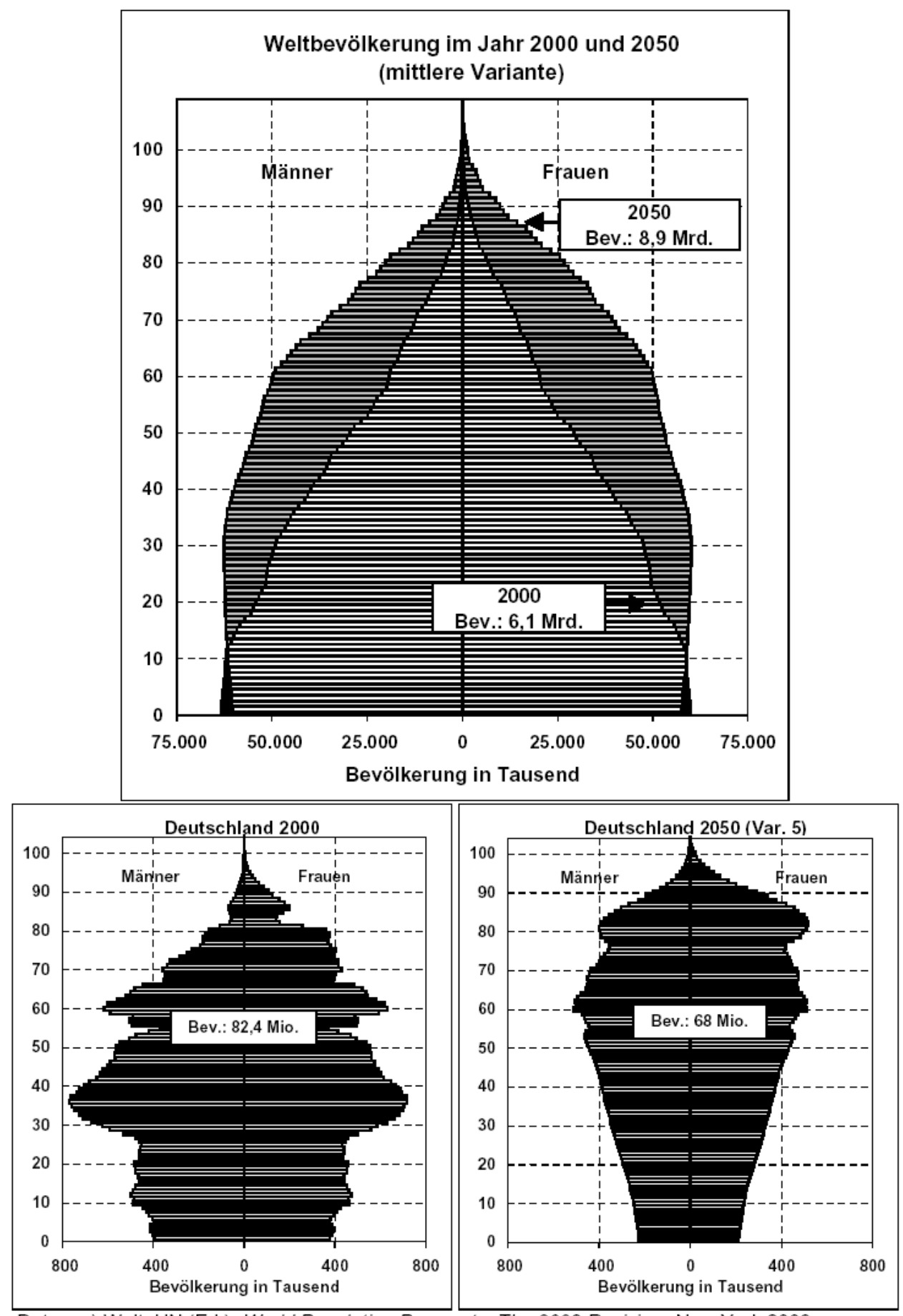

Daten: a) Welt: UN (Ed.), World Population Prospects: The 2002 Revision. New York 2003.

b) Deutschland: Birg H., Die demographische Zeitenwende. München, 2003.

\section{Quelle: Birg (2004a), 13}




\subsection{Projektionen sind keine Prognosen}

$\mathrm{Zu}$ Beginn dieses sechsten Abschnitts wurde die vom Statistischen Bundesamt vorausberechnete demografische Entwicklung ausführlich dargestellt. Wie sicher können wir sein, dass diese Vorhersagen eintreffen? Nachfolgend wird zunächst der Unterschied zwischen Projektionen und Prognosen erläutert, dann werden die Annahmen der 10. koordinierte Vorausberechnung des Statistischen Bundesamtes problematisiert.

Der Statistik-Professor Gerd Bosbach bezweifelt die Richtigkeit der Prognosen über die Schrumpfung der Gesellschaft: „So wie es für 2050 vorausberechnet wurde, muss es nicht kommen! Jede Prognose gilt nur bis zu unvorhergesehenen Brüchen in der Entwicklung. Und davon wird es in den kommenden 50 Jahren viele geben. (...) Was hätte man 1950 bei einer Prognose für 2000 alles übersehen? Antibabypille, Trend zur Kleinfamilie, Zuwanderung aus südlichen Ländern und nicht zuletzt der Zusammenbruch des Ostblocks mit der Zuwanderung vieler Aussiedler." ${ }^{\text {"7 } 25}$

In seinen Modellrechnungen geht das Statistische Bundesamt davon aus, dass die Geburtenrate bis 2050 bei 1,4 Kindern pro Frau bleibt. Für die alten Bundesländer wird also angenommen, dass sie auf dem heutigen Niveau bleibt. In den neuen Bundesländern, wo sie im Moment noch darunter liegt, erwartet Destatis, dass dieser Quotient bis 2011 auf 1,4 ansteigt und dann bis 2050 ebenfalls konstant bleibt.

Wie Abb. 31 zeigt, verlief die Geburtenentwicklung in beiden Teilen Deutschlands recht unterschiedlich. Im früheren Bundesgebiet erreichte die Geburtenrate Mitte der 1980er Jahre ihr Tief bei 1,3 Kindern pro Frau, bevor sie wieder auf 1,45 anstieg. In der ehemaligen DDR sank die Geburtenrate ab Mitte der 1960er Jahre bis auf 1,54 Kinder $1975 \mathrm{ab}$, bevor sie wegen der umfangreichen staatlichen Fördermaßnahmen bis 1980 auf erstaunliche 1,94 Kinder pro Frau anstieg. Vor dem Hintergrund solcher Schwankungen erscheint die Annahme einer konstanten Geburtenrate von 1,4 bis 2050 gewagt. Wenn diese entscheidende Annahme durch die Annahme ersetzt würde, dass bis 2015 wieder eine Geburtenrate von 1,7 erreicht würde, dann würde die Bevölkerungsgröße bei über 82 Mio. stabilisiert und die gesamte Situation würde sich entspannen. ${ }^{726}$

\footnotetext{
725 Bosbach (07.03.2004). Bosbachs Thesenpapier „Demografische Entwicklung - kein Anlass zur Dramatik“ ist u.a. im Internet verfügbar unter www.memo.uni-bremen.de/docs/m0404.pdf

${ }^{726}$ Dieses Szenario (welches relativ hohe Einwanderungsraten voraussetzt), ist zur zur Zielmarke der „bevölkerungsorientierten nachhaltigen Familienpolitik“ von Ministerin Renate Schmidt geworden, vgl. BMFSFJ (2005). Man könnte denken, dass erst bei einer Geburtenrate von 2,1 die Bevölkerungszahl stabilisiert wird. Diese Ansicht verkennt aber, dass sich auch die Variablen Mortalität und Migration verändern. Nach einer vom BMFSFJ in Auftrag gegebenen Studie von Bomsdorf reicht die Geburtenziffer von 1,7 Kindern pro Frau aus, um den Bevölkerungsumfang zu stabilisieren und die Altersstruktur zu verbessern (BMFSFJ 2005).
} 


\section{Abb. 31: Zusammengefasste Geburtenziffern in Deutschland}

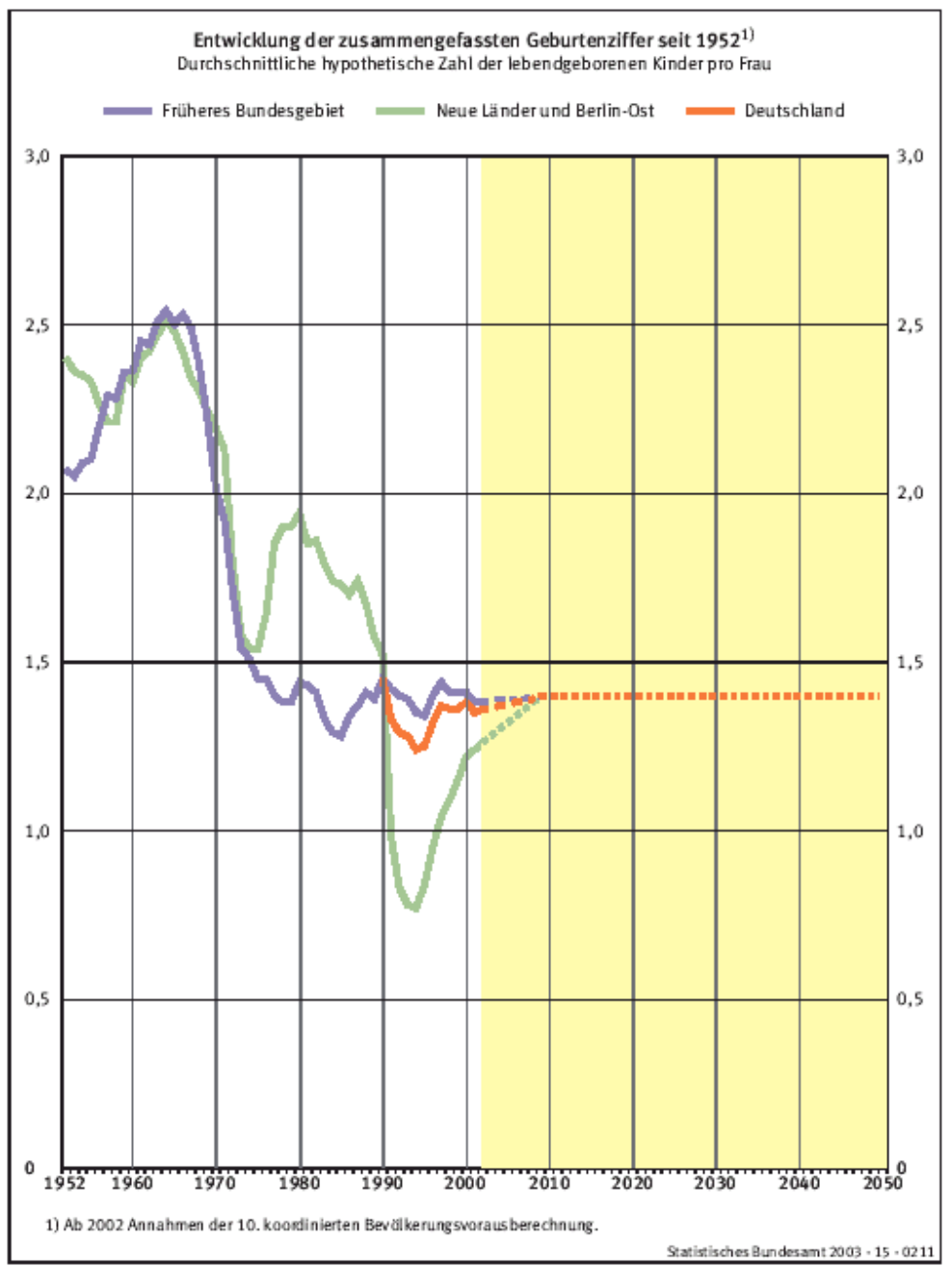

\section{Statistisches Bundesamt (2003), 11}

Schon einmal, bei der ersten Bevölkerungsvorausberechnung mit Basisjahr 1951, orientierte sich das Statistische Bundesamt zu lange Zeit an der damaligen Ausgangssituation. Die Geburtenrate wurde lange zu niedrig angesetzt, mit der Folge, dass der Mitte der 1950er Jahre einsetzende Geburtenanstieg die Berechnungen über den Haufen warf. Die erste Bevölkerungsvorausberechnung von 1951 unterschätzte das Wachstum der Bevölkerung um rund 15 Prozent (vgl. Abb. 32).

Bevölkerungswissenschaftler unterscheiden zwischen ,Projektionen' und ,Prognosen'. Prognosen zielen darauf ab, die tatsächlichen Bevölkerungszahlen zu einem bestimmten Zeitpunkt in der Zukunft zu ermitteln. Im Gegensatz dazu sind Projektionen bedingte Wenn-Dann-Aussagen, die - von Rechenfehlern abgesehen - nicht falsch sein können. 


\section{Abb. 32: Vorausberechnete und tatsächliche Bevölkerungsent- wicklung}

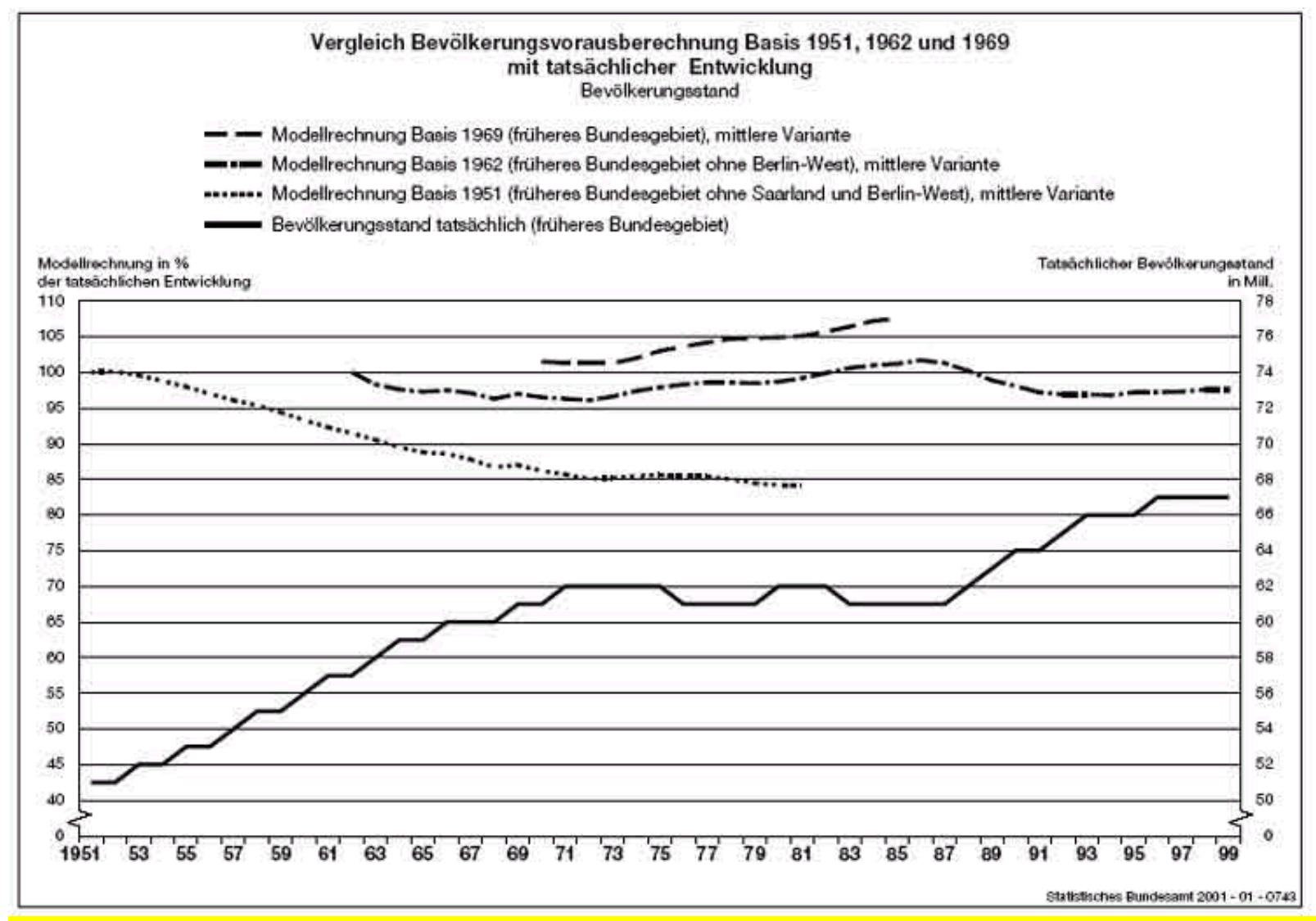

\section{Quelle: Bretz (2001), Schaubild 1}

Eine der wichtigsten Entscheidungen bei der 10. koordinierten Vorausberechnung war es wie gesagt, die heutige Geburtenrate von rund 1,4 zu extrapolieren, ohne hier unterschiedliche Varianten zu berechnen. Wie fragwürdig solche Extrapolationen heutiger Geburtenraten in die Zukunft sind, zeigt sich anschaulich, wenn man die Berechnungen nicht im Jahr 2050 enden lässt, sondern bis ins Jahr 2300 weiterführt. Würden die Fertilitätsraten auf heutigem Niveau bleiben, so ergeben sich nach den Langzeitprognosen der UNO die in Tabelle 14 dargestellen Zahlen für verschiedene Länder.

Tab. 14: Langzeitprojektionen der UNO

\begin{tabular}{|l|l|l|}
\hline Land & Bevölkerung 2000 & Bevölkerung 2300 \\
\hline Niger & 10,7 Mio. & 23,6 Billionen \\
\hline Uganda & 23,5 Mio. & 18,6 Billionen \\
\hline Dem. Rep. Kongo & 48,6 Mio. & 16,3 Billionen \\
\hline Jemen & 18,7 Mio. & 8,5 Billionen \\
\hline
\end{tabular}




\begin{tabular}{|l|l|l|}
\hline Angola & 12,4 Mio. & 8,5 Billionen \\
\hline Äthiopien & 65,6 Mio. & 7,6 Billionen \\
\hline Nigeria & 114,7 Mio. & 7,4 Billionen \\
\hline Afghanistan & 21,4 Mio. & 4,2 Billionen \\
\hline Pakistan & 132,6 Mio. & 3,7 Billionen \\
\hline$\ldots$ & $\ldots$ & $\ldots$ \\
\hline Lettland & 2,4 Mio. & 9.000 \\
\hline Estland & 1,4 Mio. & 10.000 \\
\hline Bulgarien & 8,1 Mio. & 19.000 \\
\hline Litauen & 3,5 Mio. & 98.000 \\
\hline Tschechien & 10,3 Mio. & 62.000 \\
\hline Griechenland & 10,9 Mio & 229.000 \\
\hline Ungarn & 10 Mio & 271.000 \\
\hline Rumänien & 22,5 Mio. & 387.000 \\
\hline Spanien & 40,7 Mio. & 617.000 \\
\hline Italien & 57,5 Mio. & 660.000 \\
\hline$\ldots .$. & $\ldots$ & $\ldots$ \\
\hline Deutschland & 82,2 Mio. & 3,057 Mio. \\
\hline
\end{tabular}

\section{Quelle: UN Population Division (2003b)}

So würden dann in Uganda 18,6 Billionen ${ }^{727}$ Menschen leben und und in Lettland nur noch 9.000, also weniger als in einem mittelgroßen Dorf. Tabelle 19 verdeutlicht die drastischen Entwicklungen von exponentiellem positivem oder negativem Wachstum. Der gesunde Menschenverstand sagt uns, dass diese Zahlen nicht eintreffen werden. Die UNO hat deshalb in einem mittleren Szenario berücksichtigt, dass sich die Fertilitätsraten ändern können: „Innerhalb des Zeitraums 1950 und 2175 wird die zusammengefasste Geburtenrate rund hundert Jahre lang unter das Fertilitätsniveau sinken, dann wird sie wieder auf das Bestanderhaltungsniveau steigen und dort bis 2300 bleiben." ${ }^{\prime 28}$

Legt man dieses Szenario zu Grunde, so sagt die UN in ihrer mittleren Projektion für Deutschland folgende Bevölkerungsentwicklung voraus:

\begin{tabular}{|l|l|l|l|l|}
\hline 2000 & 2050 & 2100 & 2200 & 2300 \\
\hline 82,282 Mio. & 79,145 Mio. & 73,069 Mio. & 79,397 Mio. & 85,334 Mio. \\
\hline
\end{tabular}

\footnotetext{
${ }^{727}$ Es handelt sich tatsächlich um „Billionen“. Es liegt kein Übersetzungsfehler vor, der dadurch verursacht worden wäre, dass das deutsche Wort „Milliarde“ mit englisch „billion“ übersetzt wird. Die Weltbevölkerung würde auf 244 Milliarden bis 2150 und sogar 134 Billionen (engl: trillion) in 2300 explodieren (UN Population Division 2003b, 3).

${ }^{728}$ UN Population Division (2003b), 14. Obwohl nicht explizit angegeben, dürfte die UN Population Division somit für Deutschland mit einem Anstieg der Geburtenrate auf 2,1 bis zum Jahr 2070 ausgegangen sein, da Deutschland seit 1970 - und somit 100 Jahre - das Bestanderhaltungsniveau unterschreitet.
} 
Die höchste Bevölkerungszahl innerhalb der nächsten 300 Jahre würde Deutschland also im Jahr 2300 erreichen, und dann wäre Deutschland noch dichter besiedelt als heute. Diese Bevölkerungsprojektion passt gar nicht in das Bild, dass Birg und andere von der ,demografischen Katastrophe' zeichnen. Und ist - bezogen auf das Jahr 2050 - der Unterschied zwischen der mittleren Projektion des Statistischen Bundesamtes (rund 75 Mio) und der UNO (rund 79 Mio) nicht frappierend?

Auch die Beamten des Statistischen Bundesamtes sagen ausdrücklich, dass sie Trendwenden nicht vorhersagen können und dass ihre berechneten Ergebnisse, also z.B. der Bevölkerungsstand Deutschlands im Jahr 2050, nur dann eintreffen, wenn sich die Annahmen im nachhinein als richtig herausstellen. ${ }^{729}$ Problematisch ist aber, dass die Öffentlichkeit die Zahlen des Statistischen Bundesamt als die , voraussichtlich eintreffende Zukunft' interpretiert. Die Medien und die Bevölkerung interpretieren eine Projektion als eine Prognose. Damit soll nicht einem generellen Verzicht auf Projektionen und Prognosen das Wort geredet werden. Würden die Sozialwissenschaften ganz auf Prognosen (für Bevölkerungs-, aber z.B. auch Arbeitsmarkt-, oder Rentenentwicklungen) verzichten, so käme dies ihrer Abdankung als gesellschaftlich nützliche Wissenschaften nahe. Gerade die Demografie ist in der glücklichen Lage, wegen der relativ geringen Variabilität demografischer Daten - so sind alle Rentner des Jahres 2050 heute schon geboren - den Blick in die Zukunft wagen zu können. ${ }^{730}$ Es sollte aber stärker über die Annahmen und weniger über die Ergebnisse diskutiert werden.

Nachdem der begrenzte Nutzen von Extrapolationen über längere Zeiträume gezeigt wurde, stellt sich die Frage, was konkret für und gegen die Annahme spricht, dass die deutsche Fertilitätsrate so niedrig bleibt, wie sie derzeit ist. Die westdeutsche Geburtenrate liegt schon seit 1972, also seit über 30 Jahren deutlich unter dem Ersatzniveau. Statistisch gesehen hat sich die Fertilitätsrate Deutschlands allerdings seit dem Minusrekord des Jahres 1994 von 1,24 auf 1,37 Kinder pro Frau erhöht. Dies wurde maßgeblich dadurch verursacht, dass sich die Fruchtbarkeit der Frauen im Osten nach dem Rekordtief nach der Wende $(0,75$ Kinder pro Frau) stetig erhöht und sich somit der Fertilität im Westen angenähert hat. Derzeit wird viel über die Notwendigkeit diskutiert, die Fertilität weiter zu steigern. Die Bundesregierung hat ihre jahrzehntelange Abstinenz aufgegeben und will nun eine aktive Geburtenpolitik betreiben. Dabei bezeichnet die Familienministerin Renate Schmidt die gesetzte Zielmarke von 1,7 Kindern pro Frau als „ein realistisches Ziel“".731 Das zeige auch der Blick auf die unterschiedlichen Fertilitätsraten in den Ländern der Europäischen Union. ${ }^{732}$

\footnotetext{
${ }^{729}$ Statistisches Bundesamt (2003), 9

${ }^{730} \mathrm{Schmid} /$ Heigl/Mai (2000), 35

${ }^{731}$ BMFSFJ (2005), 3

${ }^{732}$ A.a.O.
} 
Die vom Statistischen Bundesamt gewählte Annahme setzt voraus, dass alle Versuche der Eliten in Deutschland, die Geburtenzahlen wieder zu erhöhen, scheitern. Wenn man aber vernünftigerweise annimmt, dass diese Anstrengungen zumindest einen gewissen Effekt haben, dann bedeutet es gleichzeitig, dass die Bevölkerung sich anders entwickeln wird, als in der 10. koordinierten Vorausberechnung modelliert.

Ist die Bevölkerungsvorausschätzung der UN oder die des Statistischen Bundesamtes realistischer? Bei dieser Frage sollte berücksichtigt werden, dass nicht zuletzt von uns abhängt, welche Zukunft eintrifft. Die Zukunft ist nicht determiniert. Die Wenn-Dann-Rechnungen der Bevölkerungsstatistiker haben ja nicht zuletzt den Sinn, heutige Handlungsmuster zu verändern, so dass ihre Projektionen nicht eintreffen. Wie ausführlich im Kapitel Eine Strategie für den Übergang in Deutschland dargelegt werden wird, können und sollten wir die Geburtenrate in Deutschland bis 2020 auf 2,1 erhöhen. ${ }^{733}$ Im Sinne dieser Prognose als einer ,Konspiration mit der Zukunft' ${ }^{734}$ wird die Bevölkerungszahl Deutschlands bis 2050 auf 73,5 Mio. Menschen abnehmen und sich bis 2100 bei rund 70 Mio. einpendeln. ${ }^{735}$ Von diesem Szenario wird im Folgenden ausgegangen.

\subsection{Interne Folgen der Schrumpfung}

\subsubsection{Bevölkerung und Wirtschaftswachstum}

Wenn die Schrumpfung in Deutschland vehement abgelehnt wird, dann i.d.R. nicht mit ökologischen, sondern mit nicht-ökologischen Argumenten. Diese lassen sich wie gesagt in interne wie externe einteilen. Zu den internen gehört, dass Bevölkerungswachstum mit Wirtschaftswachstum korreliert sei und eine schrumpfende Bevölkerung den Preis niedrigeren Wachstums (oder gar einer Stagnation oder Rezession) zu zahlen habe.

Es wurde bereits im Rahmen der Bevölkerungsdebatte (Malthus, Ehrlich, Simon) darauf hingewiesen, dass es im klassischen volkswirtschaftlichen Denken keine ,zu geringe' Bevölkerung geben könne, da Bevölkerung neben Boden und Kapital einer der Produktionsfaktoren ist. Wir sahen auch, dass von Antinatalisten hervorgehoben wird, dass kleinere Bevölkerungswachstumsraten mit höherem Wirtschaftswachstum einhergehen. Wer hat recht? Es ist leicht einzusehen, dass nicht nur die absolute Bevölkerungsgröße, sondern auch ihre Struktur Auswirkungen auf den Produktionsfaktor ,Arbeit' haben muss. Eine kleinere Bevölkerung kann einen höheren Anteil von Menschen im erwerbsfähigen Alter haben als eine größere Bevölkerung. In diesem Zusammenhang liefert die schon im $\mathrm{Zu}$ -

\footnotetext{
${ }^{733}$ Allerdings gehört zur demografischen Zukunftsfähigkeit Deutschlands auch dazu, die Chancen der Schrumpfung zu nutzen.

${ }^{734}$ Godet (1997), zitiert nach Schmid/Heigl/Mai (2000), 27

${ }^{735}$ Birg (2001), 114. Siehe ausführlich zu den Bedingungen, unter denen dieses Szenario eintreffen kann, den Abschnitt Aktive pronatalistische Geburtenpolitik.
} 
sammenhang mit Kriegsgefahren dargestellte Youth Bulge-Theorie auch ökonomische Erklärungsansätze. ${ }^{736}$ Wir müssen uns vergegenwärtigen, dass in Gesellschaften mit konstant hohen Geburtenraten kein ,Youth Bulge', kein überproportionaler Anteil an Menschen zwischen 15 und 30 Jahren, entstehen kann. Denn es wachsen immer mehr Unter15jährige nach und die stellen einen weit größeren Anteil. Einen ,Youth Bulge' gibt es vielmehr in jenen Ländern, bei denen die ganz jungen Jahrgänge weniger werden, d.h. wenn auf eine Phase eines hohen Bevölkerungswachstums eine Phase gebremsten Bevölkerungswachstums folgt. Diese Entwicklung führt dazu, dass man sich weniger um die Versorgung der Kinder kümmern muss. Bei bei den Eltern ist mehr Geld vorhanden, Kaufkraft und Nachfrage steigen. Auch der Staat kann mehr in wirtschaftliche Entwicklung investieren. Da es meistens arbeitsintensive Prozesse sind, die in diesen Ländern zu wirtschaftlicher Entwicklung führen, werden viele Arbeitskräfte gebraucht. China ist dank seiner Ein-Kind-Politik genau in dieser Phase. Die Chinesen sind seit einem Jahrzehnt Motor der Weltwirtschaft und übertrafen mit ihrem Wachstum von 9,1 Prozent auch 2003 andere südostasiatische Staaten. ${ }^{737}$ Sie wachsen zur Zeit aus dieser Phase, in der viele leistungsfähige Arbeitskräfte benötigt werden, heraus und werden bald eine zweite Phase der wirtschaftlichen Entwicklung erreichen, die nicht mehr so arbeitsintensiv ist. In dieser zweiten Phase ist es aus volkswirtschaftlicher Sicht nahezu optimal, dass dann die geburtenschwachen Jahrgänge auf den Arbeitsmarkt kommen. ${ }^{738}$ Reiner Klingholz resümiert: „Man kann also eine demografische Dividende einfahren, aber dafür gibt es nur ein enges Zeitfenster von 10-15 Jahren. Und die Chinesen wie auch die anderen asiatischen Länder haben das geschafft. Ob etwa der Jemen da jemals hinkommen wird, ist sehr fraglich. “739

Eine Korrelation zwischen hohem Bevölkerungswachstum und hohem Wirtschaftswachstum gibt es nicht. Die ökonomische Youth-Bulge Theorie erklärt aber nur die wirtschaftlichen Auswirkungen bei einem Übergang von hohen zu niedrigen Wachstumsraten der Bevölkerung, nicht aber bei Minuswachstum, wie wir es in Deutschland zu erwarten haben. Für Minuswachstum wären neue Modelle nötig, die von den Wirtschaftswissenschaften noch kaum entwickelt wurden.

\subsubsection{Wachstum oder Wachstum pro Kopf?}

Die OECD hat berechnet, dass in der Europäischen Union die durchschnittliche jährliche Wachstumsrate des Bruttoinlandsprodukts von heute etwa 2,3 Prozent auf durchschnittlich rund 0,5 Prozent im Zeitraum der Jahre 2025 bis 2050 sinken

\footnotetext{
${ }^{736}$ Klingholz/Tremmel (2004)

${ }^{737}$ Japan: 2,7 \%, Thailand 6,4 \%, Südkorea 3,1 \%, Taiwan 3,2 \%, Hongkong 3,3\%, Singapur 1,1\%, Malaysia 5,2 \%, Philippinen 4,5\%, Indonesien 4,1\%.

${ }^{738}$ Klingholz/Tremmel (2004)

${ }^{739}$ A.a.O.
} 
wird. ${ }^{740}$ Hauptursachen sind der Rückgang der Erwerbsfähigen und die Verlangsamung des technischen Fortschritts. ${ }^{741}$ Auch die durchschnittlichen jährlichen Wachstumsraten des Pro-Kopf-Einkommens werden der Studie zufolge sinken aber viel weniger stark. Sie sollen von 1,9 Prozent zwischen den Jahren 2000 und 2010 auf 1,1 Prozent in der Dekade zwischen 2040 und 2050 sinken. ${ }^{72}$

Es ist eine wichtige Frage, ob das BIP oder das BIP/Kopf der Indikator ist, an dem wir uns orientieren müssen. Wenn man z.B. von Deutschland in die Schweiz umzieht, so zieht man in ein Land mit einem viel niedrigeren BIP. Aber selbstverständlich ist deshalb in der Schweiz nicht der Lebensstandard geringer, ganz im Gegenteil. Birg diskutiert beide Maßstäbe und erwähnt dabei, dass für das ProKopf-Einkommen Bevölkerungsschrumpfung sogar besser sein kann als Bevölkerungswachstum. ${ }^{743}$ Birg wendet sich schließlich dennoch gegen den Maßstab des Pro-Kopf-Einkommens, weil dann „mit einer permanenten Bevölkerungsschrumpfung " zu rechnen sei. ${ }^{744}$ Mit anderen Worten: Die Bevölkerungsschrumpfung würde uns zwar reicher machen, aber dieser zunehmende Wohlstand (als unabhängige Variable) würde dann die Geburtenrate (als abhängige Variable) weiter zum Sinken bringen. Hintergrund ist das in Birgs Büchern mehrfach dargestellte „demo-ökonomische Paradoxon“, welches eine Kausalität zwischen steigendem Pro-Kopf-Einkommen und sinkender Geburtenrate behauptet. ${ }^{75}$ Nach Birg sei die Pro-Kopf-Geburtenzahl in jenen Ländern besonders niedrig, in denen das ProKopf-Einkommen ein überdurchschnittlich hohes Niveau erreicht habe. Diese interessante und durch zahlreiche Beispiele fernöstlicher, südamerikanischer und afrikanischer Staaten gestützte Theorie wird aber empirisch schon durch das Beispiel der Golfstaaten in Frage gestellt. Obwohl diese Staaten zu den reichsten Ländern der Welt gehören, haben sie hohe bis sehr hohe Geburtenraten (SaudiArabien 4,06; Quatar 3,03; Vereinigte Arabische Emirate 2,53). Zweitens lässt sich das demo-ökonomische Paradoxon im europäischen Vergleich nicht nachweisen, wie Abbildung 33 zeigt

Drittens zeigt sich im historischen Verlauf vieler Staaten, z.B. auch der Bundesrepublik Deutschland und der ehe. DDR, keine signifikante Korrelation zwischen BIP und Geburtenrate. In beiden Staaten nahm das BIP in den letzten fünf Jahrzehnten nahezu stetig zu, während die Geburtenrate großen Schwankungen unterlag.

\footnotetext{
${ }^{740}$ OECD (1998), 47, Tabelle 3

${ }^{741}$ Es wird davon ausgegangen, dass von der Politik keine speziellen Maßnahmen aufgrund der Alterung ergriffen werden („,Business-as-usual“-Szenario).

${ }^{742}$ OECD (1998), 49, Tabelle 5

${ }^{743} \operatorname{Birg}(2001), 164$

${ }^{744}$ A.a.O.

${ }^{745}$ Birg (2001), 24
} 


\section{Abb. 33: Demo-ökonomisches Paradoxon}

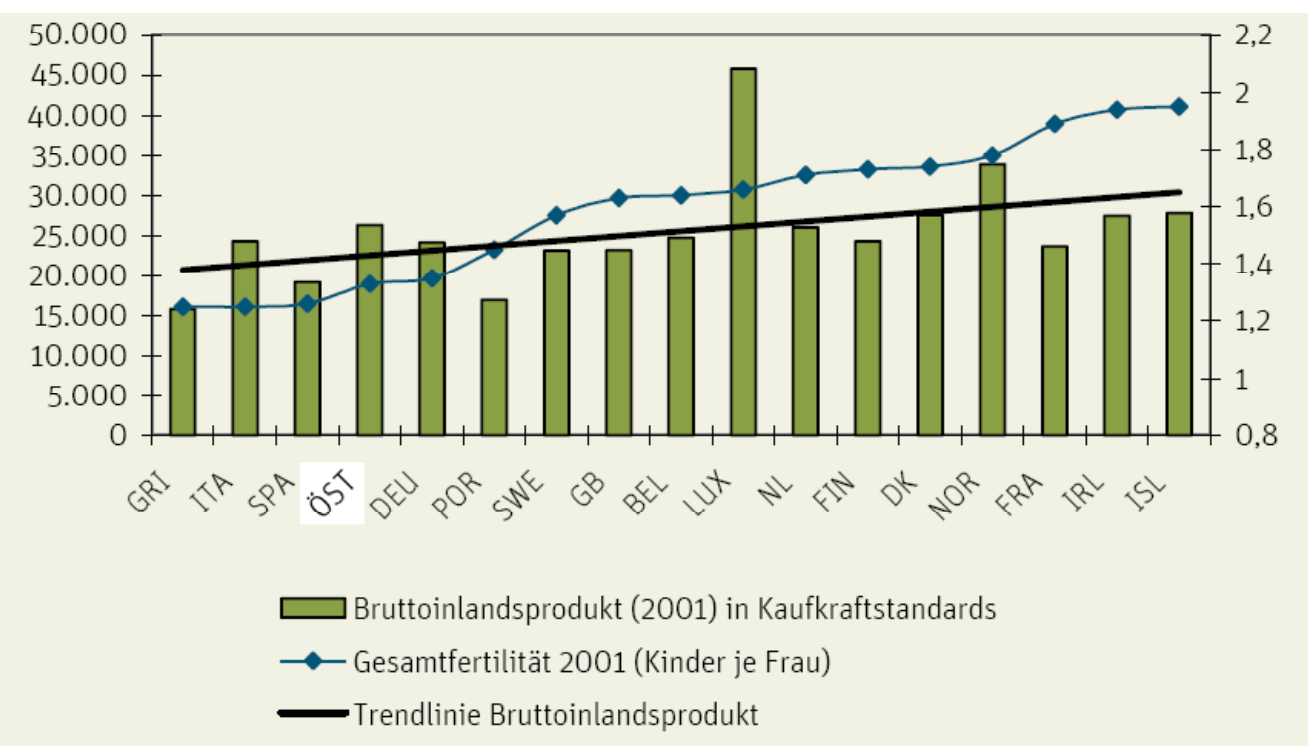

\section{Korrelationskoeffizient BIP/Gesamtfertilität $=0,43$ \\ Quelle: Kröhnert/van Olst/Klingholz (2005), 4. Zahlen von Eurostat (2002)}

Die Hypothese des demo-ökonomischen Paradoxons ist also durch viele Gegenbeispiele stark in Frage gestellt. Wie Birg sogar selbst sagt, steigern die Folgen der Schrumpfung, z.B. die Zunahme der Wohnfläche pro Person, auch wieder den Kinderwunsch. ${ }^{746}$ In keinem Fall ist die demo-ökonomische Hypothese ein Argument gegen die Verwendung des Indikators BIP/Kopf.

Es ist unverständlich, dass in den Abendnachrichten und den Tickern der Nachrichtenagenturen bisher stets die Veränderung des BIP bzw. BSP im Mittelpunkt steht. Stefanie Wahl vom Institut für Wirtschaft in Gesellschaft in Bonn schreibt in einem Sammelband über die Folgen der Bevölkerungsschrumpfung: „Namhaften Untersuchungen zufolge ist es nicht ausgeschlossen, dass in Zukunft das jährliche Wachstum der deutschen Wirtschaft demografiebedingt langfristig 1 Prozent nicht überschreitet. ${ }^{6747}$ Der Mannheimer Volkswirtschaftler Börsch-Supan sieht eine Bedrohung „unseres Wirtschaftswachstums“ durch den Rückgang der Erwerbstätigen. ${ }^{748}$ Es ist zu hoffen, dass solche und ähnliche Aussagen in Zukunft das BIP/Kopf als Maßstab nehmen werden - womit sie gleichzeitig ihre Bedrohlichkeit verlieren würden. Schon weil die Politiker ein systemisches Interesse an Erfolgsnachrichten haben, ist auch durchaus damit zu rechnen, dass in einer schrumpfenden Gesellschaft langfristig das BIP/Kopf (oder das verfügbare

\footnotetext{
${ }^{746}$ Vgl. Birg (2001), 137. In diesem Fall muss also der steigende Wohlstand (in diesem Fall gemessen durch Wohnfläche pro Person) positiv korreliert sein mit steigender Geburtenzahl.

${ }^{747}$ Wahl (2004), 19

${ }^{748}$ Börsch-Supan (2004b), 38
} 
Volkseinkommen/Kopf) der Maßstab wird, an dem sich unsere Gesellschaft orientiert.

In einer schrumpfenden Gesellschaft kann das BIP/Kopf weiter zunehmen, auch wenn das BIP sinkt. Dies war z.B. im Jahr 2001 in Ostdeutschland der Fall.

\section{Tab. 15: Wirtschaftswachstum pro Kopf in Ostdeutschland}

\begin{tabular}{|l|r|r|r|}
\hline \multicolumn{3}{|l|}{ Neue Länder ohne Berlin (Angaben in \%) } & \\
\hline Jahr & $\begin{array}{r}\text { Wirtschafts- } \\
\text { wachstum }\end{array}$ & $\begin{array}{r}\text { Bevölkerungs- } \\
\text { wachstum }\end{array}$ & $\begin{array}{r}\text { Wirtschafts- } \\
\text { wachstum } \\
\text { pro Kopf }\end{array}$ \\
\hline 1994 & 11,4 & $-0,48$ & 11,94 \\
\hline 1995 & 4,48 & $-0,39$ & 4,89 \\
\hline 1996 & 3,22 & $-0,30$ & 3,53 \\
\hline 1997 & 1,64 & $-0,34$ & 1,99 \\
\hline 1998 & 0,44 & $-0,48$ & 0,92 \\
\hline 1999 & 2,59 & $-0,48$ & 3,08 \\
\hline 2000 & 1,38 & $-0,70$ & 2,09 \\
\hline 2001 & $-0,17$ & $-0,87$ & 0,71 \\
\hline 2002 & 0,12 & $-0,82$ & 0,95 \\
\hline
\end{tabular}

\section{Quelle: Statistisches Bundesamt, https://www-} genesis.destatis.de/genesis/online/Online (Tabelle 81000-0001)

Ähnlich irreführend wie das Lamento über ein sinkendes (absolutes) BIP ist die Argumentation, dass in Deutschland mit der Schrumpfung eine Entwertung des Humankapitals drohe. ${ }^{79} \mathrm{Zu}$ diesem Schluss kann nur kommen, wer Humankapital nicht pro Kopf, sondern absolut ausrechnet. Nach dieser Berechnungsweise wären 100 Millionen schlecht ausgebildete Menschen mit einem jeweiligen Humankapitalwert von z.B. 10 eine bessere Alternative als 50 Millionen gut ausgebildete mit einem Wert von 18.

In beiden Fällen, beim BIP wie beim Begriff ,Humankapital', gelten die gleichen Argumente wie in der Debatte über eine nutzensummenutilitaristische Sichtweise versus einen personenorientierten Utilitarismus.

\subsubsection{Auslastung der Infrastruktur- und Versorgungssysteme}

Wäre Infrastruktur proportional abbaubar, so würden wegen der Schrumpfung zurückgehende Steuereinnahmen für Kommunen c.p. nicht problematisch sein, weil eben öffentliche Güter und Dienstleistungen bei einer geringeren Bevölkerung nicht teurer für jeden Einzelnen würden. Diese Proportionalität ist bei manchen Gütern und Dienstleistungen uneingeschränkt, bei anderen teilweise und bei anderen nicht gegeben. Wasser- und Abwassersysteme sind Beispiele für Versorgungssysteme, die für eine bestimmte Mindestzahl an Nutzern ausgelegt sind. Würden diese Leitungsnetzen zu wenig ausgelastet, so träten Funktionsstörungen

\footnotetext{
${ }^{749}$ Hessische Staatskanzlei (2003), 38
} 
auf. ${ }^{750}$ Eine stärkere Proportionalität herrscht bei Schulen und Kindergärten. Bei einer Schulklasse, deren minimale Größe auf 16 festgelegt ist, ist ein Rückgang von 30 auf 16 durchaus eine Erleichterung für Lehrer und (die verbleibenden) Kinder, erst ein weiterer Rückgang führt zur Auflösung der Klasse. ${ }^{751}$ Vollständige Proportionalität gibt es beispielsweise bei der Taktung von Stadtbussen. Ein anderes Beispiel wären städtische Broschüren (Postwurfsendungen), die entsprechend in geringerer Auflage produziert werden können. Auch hier fällt die Antwort nach den Effekten der Schrumpfung also differenziert aus.

\subsubsection{Entwicklung der Nachfrage}

„Steckt die Wirtschaft in der Altersfalle?“ - fragt ein Buchtitel. ${ }^{72}$ Um dies zu beantworten, soll zunächst analytisch zwischen den Wirkungen der Schrumpfung und der Alterung unterschieden werden, selbst wenn diese in Kombination auftreten. ${ }^{753}$ Der Vorteil der getrennten Betrachtung zeigt sich, wenn wir beispielsweise die Auswirkungen auf die Nachfrage betrachten. In einer schrumpfenden Gesellschaft sinkt, alles andere unverändert, die Nachfrage. In einer alternden Gesellschaft muss dies nicht so sein. Eine ältere Bevölkerung fragt andere Güter nach. Insbesondere dürfte die Nachfrage nach Grsundheits- und Pflegeleistungen steigen, aber auch nach Urlaubsreisen, Kultur, Qualifizierungs- und Weiterbildungsleistungen für Ältere. Häufig wird in diesem Zusammenhang auf die hohe Kaufkraft der Älteren hingewiesen. ${ }^{754}$ Das Gesamtvermögen der Über65jährigen wird auf mehr als 2,2 Billionen Euro geschätzt. Ein Rentner-Paar hat mit durchschnittlich $19.000 €$ im Jahr sogar etwas mehr zur Verfügung als eine Familie mit Kindern. ${ }^{755}$

Unterschiedliche Annahmen gibt es über die Entwicklung der Konsum- und Sparquoten, über Abhängigkeit des Konsums von der Zahl der Haushalte (statt der Haushaltsgröße) und über die weitere Entwicklung der Einkommenssituation verschiedener Kohorten. ${ }^{756}$ Insgesamt sind die Entwicklungen vielschichtig und zum Teil gegenläufig. Es spricht aber einiges dafür, dass sich die Wirtschaft an

\footnotetext{
${ }^{750}$ Troge (2004), 34

${ }^{751}$ Dies gilt zumindest für einzügige Dorfschulen. Bei anderen Schulen kann es allerdings schon zur Zusammenlegung von Klassen und somit zu Personalabbau.

${ }^{752}$ Tichy/Tichy (2001)

${ }^{753}$ Die gesellschaftlichen Folgen der demografischen Alterung werden bereits sehr breit diskutiert und erforscht (vgl. Baltes/Mittelstrass 1992; Klose 1993; Schmid/Heigl/Mai 2000; Deutscher Bundestag 2002; Schimany 2003; Leipert 2003), die der Schrumpfung erheblich weniger. Beide sind natürlich nicht unverknüpft. Wenn die Stabilisierung der Weltbevölkerung das Ziel ist, dann ist eine Alterung der Gesellschaft, zumindest in vielen Ländern, eine notwendige Bedingung dafür.

${ }^{754}$ Klose (1993)

755 Banze/Porwollik (2003), 27. Siehe zur Einkommenssituation von Rentnerhaushalten auch VDR (2003), 483.

${ }^{756}$ Für genauere Analysen alterspezifischer Konsumausgaben siehe Börsch-Supan (2004a), 9 f.; Deutscher Bundestag (2002), 165-167
} 
die veränderten Nachfragestrukturen anpassen kann, ohne dass es zu größeren Erschütterungen kommen wird. ${ }^{757}$

\subsubsection{Wohnungsmarkt}

Im Hinblick auf den Wohnungsmarkt führt eine Schrumpfung c.p. zunächst zu einer geringeren Nachfrage nach Wohnungen bei gleichbleibendem Angebot, also zu Leerständen oder zumindest zu sinkenden Mieten und Immobilienwerten. Diesem Trend steht aber ein gegenläufiger Trend als Folge der Alterung entgegen.

\section{Abb. 34: Vorausschätzung der Ein- und Mehrpersonenhaushalte bis 2050}

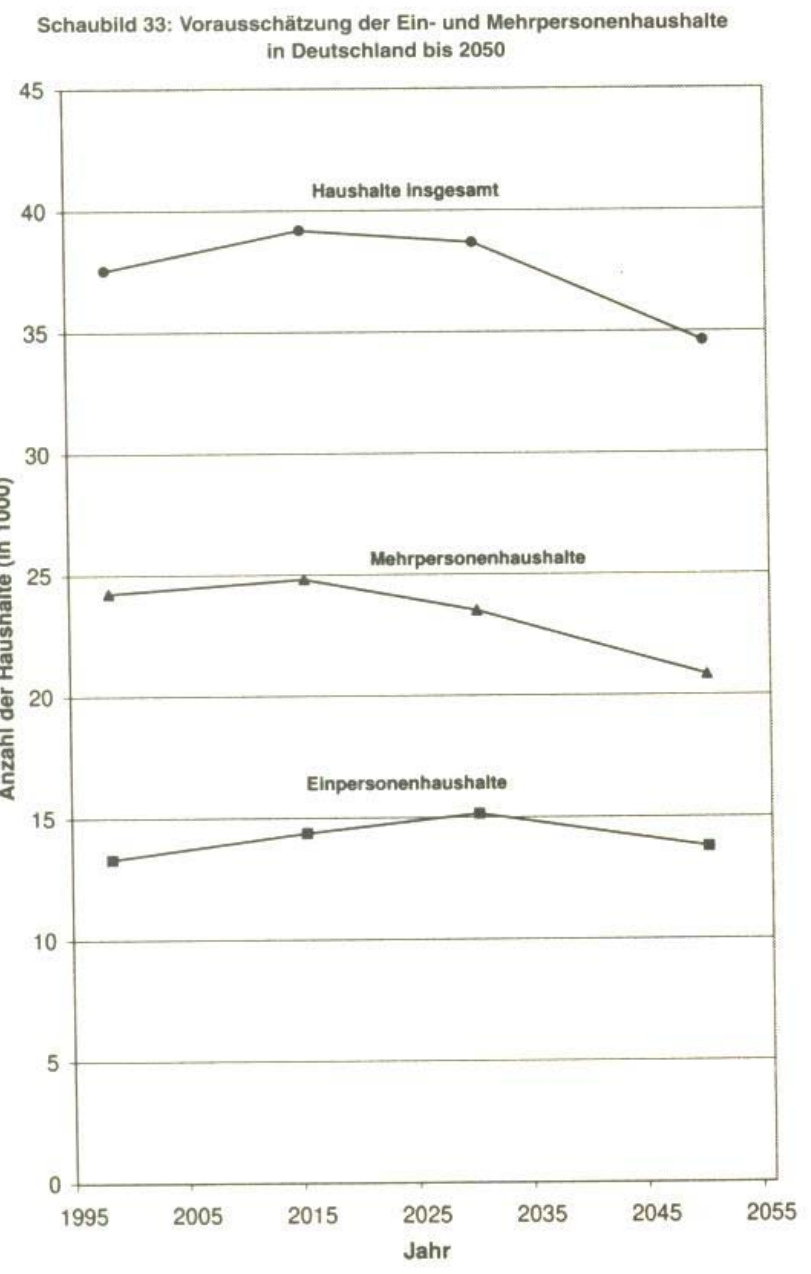

\section{Quelle: Birg (2001), 146}

Hinterbliebene wollen möglichst lange in ihren alten, relativ großen Wohnungen bleiben, so dass c.p. die durchschnittliche Haushaltsgröße steigt. Die Zahl der Einpersonenhaushalte wird bis 2030 von heute 13,3 Mio. auf 15,2 Mio. im Jahr

${ }^{757}$ Vgl. Abschnitt 6.8.2.9. Demografische Daten bei jeder Planung berücksichtigen. 
2030 zunehmen. Insgesamt wird die Zahl der Haushalte aber ab ca. 2015 zu sinken beginnen. ${ }^{758}$ Dies stellt Abbildung 34 dar. Auch hier ist eine genaue Prognose aber schwierig. Der Bedarf an Wohnraum ändert sich nicht nur in Abhängigkeit vom Alter und Familienstand, sondern das Eheschließungsverhalten und die Geburtenrate werden z.T. auch von der Verfügbarkeit von Wohnraum beeinflusst. ${ }^{759}$

\subsubsection{Sozialversicherungssysteme}

Eine der zweifellos problematischen Begleiterscheinungen des demografischen Wandels ist die Belastung der Sozialversicherungssysteme durch die Alterung der Gesellschaft. Kurz gesagt: Der demografische Wandel führt zu ungleichen Belastungen für verschiedene Generationen. Dies birgt den Keim zu einem Generationenkonflikt. Denn die 20-60jährigen erbringen die meisten Leistungen, ihre Nettobilanz gegenüber dem Staat ist positiv, wohingegen andere Altersgruppen mehr staatliche Leistungen beanspruchen als sie an den Staat durch Steuern etc. zahlen. Für die Probleme der Sozialversicherungssysteme gibt es allerdings umfassende und durchgerechnete Reformvorschläge. Für die Rentenversicherung hat die sog. Rürup-Kommission im Herbst 2003 ein Lösungsmodell vorgelegt, dessen zentrale Bestandteile ein ,Nachhaltigkeitsfaktor' sowie eine schrittweise Erhöhung des gesetzlichen Renteneintrittsalters auf 67 Jahre sind. Dieses Modell würde im Falle der prognostizierten Alterung der Gesellschaft zu einer Aufteilung der Belastung zwischen Jung und Alt führen würde. ${ }^{760}$ Der ,Nachhaltigkeitsfaktor' wurde - anders als die Erhöhung des Renteneintrittsalters - von der Bundesregierung übernommen und am 17.3.2004 in Gesetzesform gegossen. ${ }^{761}$ Diese Reform braucht hier nicht nochmals ausführlich dargestellt werden, vielmehr soll ein unterbelichteter Aspekt hervorgehoben werden. Da bei der verabschiedeten Reform die Modellrechnungen des Statistischen Bundesamtes zu Grunde gelegt wurden, wird in gewisser Weise die Alterung und Schrumpfung als gegeben hingenommen. Alle Lösungen, die Expertenkommissionen in den letzten Jahren für die Reform der Sozialversicherungssysteme vorgeschlagen haben, waren ,Anpassungsstrategien', keine ,Umkehrstrategien'. Eine solche Anpassung der Sozialversicherungssysteme an den demografischen Wandel wird von denjenigen, die in einer Stabilisierung der deutschen Bevölkerung auf heutigem Niveau den Königsweg sehen, denn auch kritisch gesehen. So schreibt Susanne Mayer in einem Artikel

\footnotetext{
${ }^{758} \operatorname{Birg}(2001), 144$

${ }^{759} \operatorname{Birg}(2001), 137$

${ }^{760}$ Bundesministerium für Gesundheit und Soziale Sicherung (2003), 103

${ }^{761}$ Vor dem ,Nachhaltigkeitsfaktor' wurden bereits der sog. Demografische Faktor und der ,Abschlagsfaktor' (Riester 2000) entwickelt. Nicht alle Vorschläge konnten sich politisch durchsetzen. Teilweise wurden sie nach Wahlsiegen der Opposition wieder zurückgenommen. Ein sinnvoller Reformvorschlag ist nicht zuletzt auch das Modell einer Teilungslösung (vgl. Tremmel 2003d; Zechmeister/Tremmel 2001). Sie berücksichtigt, dass Prognosen irren können und dass sich deshalb nicht genau vorhersagen lässt, wie sich die Rahmenbedingungen im Detail entwickeln (Schmähl 2000, 52 u. 69).
} 
namens Her mit den Kindern! Plädoyer für eine moderne Bevölkerungspolitik, die den Namen verdient über die Rentenreformpolitik der letzten Jahre: „Kinder kamen nur vor als ,demografischer Faktor', in der Rentenanpassung. Als ließe sich irgendetwas anpassen, wenn in wenigen Jahrzehnten 16 Millionen weniger Menschen erwerbstätig sind - keine Arbeitslose, sondern Leute, die es nicht gibt - und gleichzeitig 10 Millionen mehr Ältere zu versorgen sind. “762

Eine Anpassungstrategie für den demografischen Wandel zu entwickeln, ist also in Bezug auf die Sozialversicherungssysteme nicht nur möglich, es ist bereits geschehen und diese Strategie ist auch bereits teilweise zum Gesetz geworden. ${ }^{763}$ Trotz dieses relativen Erfolgs muss darauf hingewiesen werden, dass die demografisch bedingte ungleiche Belastung der Generationen ein Faktor ist, der es fast unmöglich macht, die Maxime der Generationengerechtigkeit im isolierten Teilsystem Rente einzuhalten. ${ }^{764}$ Renten-Generationengerechtigkeit ist dadurch definiert, dass die Rendite der jungen (temporalen) Generation nach Möglichkeit besser, zumindest aber nicht schlechter sein darf als für ihre Vorgänger-Generation. ${ }^{765}$ Doch dieses Ziel ist trotz diverser Reformen noch nicht erreicht worden: Noch immer ist die Rendite der jüngeren Jahrgänge schlechter als die der älteren. ${ }^{766}$

Häufig wird als Ausweg der Umbau unseres maßgeblich auf dem Umlageverfahren basierenden Rentensystems in Richtung Kapitaldeckung vorgeschlagen ${ }^{767}$ Dem steht aber die sog. Age-Wave-These gegenüber, nach der kapitalgedeckte Rentensysteme in ähnlicher Weise anfällig gegenüber Alterung sind, da der Verkaufsdruck auf die Aktienmärkte massiv zunimmt, wenn die geburtenstarken Jahrgänge in Rente gehen und ihre Depots auflösen bzw. verringern. ${ }^{768}$ Ein Absinken der Rendite ergibt sich bei beiden Alterssicherungssystemen. Nichtsdestotrotz ist eine Stärkung der kapitalgedeckten Säule im Rahmen des bestehenden DreiSäulen-Systems (gesetzliche, betriebliche und private Alterssicherung) sinnvoll, da kapitalgedeckte Renten wegen der zumindest teilweise vorhandenen Anlagemöglichkeiten im Ausland etwas weniger von der deutschen demografischen Entwicklung abhängig sind als die umlagefinanzierte Rente. Eine solche Stärkung der kapitalgedeckten Säule hat die Bundesregierung deshalb bereits im Zuge der Rentenreform 2001 durchgeführt (,Riester-Rente').

\footnotetext{
${ }^{762}$ Mayer (2004), 18

${ }^{763}$ Allerdings fehlt wie gesagt noch die Anhebung des Renteneintrittsalters. Diese in der Bevölkerung höchst unpopuläre Maßnahme wurde von der Regierung Schröder aus wahltaktischen Gründen nicht angepackt.

${ }^{764}$ Vgl. zur Debatte über Generationengerechtigkeit in isolierten Bezügen die Ausführungen über Ökologische Generationengerechtigkeit.

${ }^{765}$ Tremmel (2003d), 421-436; Tremmel (1997), $209 \mathrm{ff}$.

${ }^{766}$ Ohsmann/Stoltz (2004), 62

767 Das Washingtoner Center for Strategic and International Studies (CSIS) berechnet einen ,Aging Vulnerability Index'. Ihm liegt die Annahme zu Grunde, dass Länder mit einer starken umlagefinanzierten Säule besonders verwundbar gegenüber der Altersproblematik sind und deshalb einen Standortnachteil gegenüber Ländern wie den USA, Australien oder Großbritannien haben.

${ }^{768}$ Hypovereinsbank (26.04.2001)
} 
Die wichtigste noch ausstehende Reform ist eine drastische Verlängerung der Lebensarbeitszeit. Durch die Fortschritte in der Medizin ist heute ein Siebzigjähriger noch so fit wie früher ein Sechzigjähriger. Nach Erkenntnissen der Gerontologie hat das vierte Lebensalter die Merkmale des früheren dritten angenommen: „Die Älteren halten sich für jünger, sehen im Durchschnitt jünger aus, sind hinsichtlich ihrer Gesundheit, ihrer Selbständigkeit und Kompetenz jünger und vitaler als frühere Generationen." ${ }^{\text {"769 }}$ Bei einer Lebenserwartung von rund 78 Jahren ist die Lebensarbeitszeit mit durchschnittlich 38 Jahren deutlich zu kurz. ${ }^{770}$ Nur ein Drittel der 60-64jährigen Männer arbeitet heute noch. Anfang der 1970er Jahre, als die Menschen etwa vier Jahre jünger starben und die körperlichen Arbeitsbedingungen weit schwerer als waren als heute, waren es noch mehr als drei Viertel. ${ }^{771}$ Wie gezeigt ist die Entwicklung des Altenquotienten weit weniger dramatisch, wenn man die Altersabgrenzung 67 wählt, von einer Altersabgrenzung 70 ganz zu schweigen. Bei der Altersabgrenzung 67 läge er bei 0,47 im Jahr 2050 statt bei 0,44 bei der Altersabgrenzung 60 heute, also kaum höher (vgl. Abb. 28). Besser als eine starre Erhöhung des gesetzlichen Renteneintrittsalters ist es aber, durch eine Mindesteinzahldauer von z.B. 40 Jahren das tatsächliche Renteneintrittsalter zu erhöhen und gleichzeitig den Renteneintritt zu flexibilisieren.

\subsubsection{Arbeitsmarkt und Beschäftigung}

Auf der Positivseite der Bilanz der Schrumpfung wird häufig die demografische Entlastung des Arbeitsmarktes genannt. Dies floss z.B. als Annahme in die Berechnungen der Rürup-Kommission zur Nachhaltigkeit in den sozialen Sicherungssystemen ein. ${ }^{772}$ Zentrale Ursache hierfür ist der Rückgang der Bevölkerung im erwerbsfähigen Alter. Die Zahl der Erwerbspersonen geht nach Angaben von Schimany bis 2040 auf rund 32 Mio. (und damit um etwa 9 Mio. Menschen) zurück. ${ }^{773}$ Der Direktor des Max-Planck-Instituts für demografische Forschung, James Vaupel, hält es für sicher, dass Deutschland unabhängig von einem möglichen erneuten Anstieg der Geburtenziffer bis spätestens 2030 mit einem Arbeitskräftemangel konfrontiert sein wird. ${ }^{774}$ Wie er vertreten die meisten Fachleute die Meinung: „Arbeit wird knapp“. ${ }^{775}$

Jürgen Borchert wendet sich mit folgenden Argumenten gegen die These von den sinkenden Arbeitslosenquoten: „Für die herrschende Auffassung, dass die Arbeitslosigkeit aus Gründen der Bevölkerungsentwicklung und der Verknappung des Arbeitsangebots nach 2010 von selbst verschwinden wird, spricht bei einer

\footnotetext{
${ }^{769}$ Schroeter (2000), 91

${ }^{770}$ Opaschowski (2004), 79

${ }^{771}$ Börsch-Supan (2004b), 38

772 Bundesministerium für Gesundheit und Soziale Sicherung (2003)

${ }^{773}$ Schimany (2003), 449

${ }^{774}$ Vaupel (08.04.2004), 41

${ }^{775}$ So z.B. plakativ Börsch-Supan (2004a)
} 
Beibehaltung des gegenwärtigen Systems jedoch wenig. Denn es ist zum einen nicht zu übersehen, dass steigende Sozialversicherungsbeiträge auch den Rationalisierungsdruck erhöhen. Andererseits ist wegen der Alterung und Schrumpfung der Bevölkerung nicht ersichtlich, woher die Produktivitätszuwächse kommen sollen, die nicht ohne ausreichenden und qualifizierten Nachwuchs zu erreichen sind. Nach allem, was aus der Vergangenheit zu lernen ist, muss somit mit einer weiteren Zunahme der Massenarbeitslosigkeit in den mittleren und unteren Lohnsegmenten sowie dem Ausweichen in Schattenwirtschaft und neue Formen der Selbstständigkeit gerechnet werden, womit sich die Problemspirale dynamisch weiter dreht." ${ }^{6776}$

$\mathrm{Ob}$ die heutige Sockelarbeitslosigkeit durch den Bevölkerungsrückgang quasi „in Luft aufgelöst wird“, ist tatsächlich zweifelhaft. In modernen Ökonomien, die ständigem Innovations- und Rationalisierungsdruck sowie stetigem Strukturwandel unterworfen sind, können die Angebots- und Nachfrageseite des Arbeitsmarktes so gut wie nie zur Deckung kommen. ${ }^{777}$ Auch wenn bei einem verkrusteten, unflexiblen Arbeitsmarkt die Demografie nicht alle Probleme lösen kann, so ist sie doch Teil der Lösung für die heutige Massenarbeitslosigkeit. Dies wird von der Enquete-Kommission Demographischer Wandel in ihrem sorgsam formulierten Resümee berücksichtigt: „Im demografischen Wandel wird sich für den deutschen (aber auch für den europäischen) Arbeitsmarkt längerfristig eine deutliche Entlastung von der Angebotsseite her ergeben. Von dieser Seite besteht die Chance, die Arbeitslosigkeit zu überwinden, um sich der Vollbeschäftigung anzunähern. Dem sollten die Arbeitsnachfrage reduzierende Belastungen möglichst wenig entgegenwirken." ${ }^{\text {"7 }} 78$

Auch wenn derzeit über die Frage, ob die demografische Entwicklung zu sinkender Arbeitslosigkeit führt, ein Glaubenskrieg ausgetragen zu werden scheint, so überwiegen aus meiner Sicht deutlich die Indizien, dass es einen solchen Rückgang geben wird, sobald der demografisch bedingte Rückgang des Erwerbspersonenpotenzials eingesetzt hat. Dies gilt selbst dann, wenn die nachrückenden Generationen höhere Erwerbsquoten aufweisen aufgrund der steigenden Erwerbstätigkeit von Frauen und älteren Menschen. Ein solcher Rückgang der Arbeitslosigkeit aber würde die negativen Auswirkungen der Alterung auf die Sozialversicherungssysteme selbst mildern, worauf die Gewerkschaften zu Recht hinweisen: „Mehr Beschäftigung entschärft Demografie: Von denen, die aktiv sein könnten, sind lediglich 69 Prozent erwerbstätig. (...) Es kann langfristig erreicht werden, dass 90 Prozent der 15-65jährigen arbeiten. Die ,tickende Zeitbombe' Bevölkerungsentwicklung wird so deutlich entschärft.“'779

\footnotetext{
${ }^{776}$ Hessische Staatskanzlei (2003), 39

${ }^{777} \mathrm{Schmid} /$ Heigl/Mai (2000), 119

${ }^{778}$ Deutscher Bundestag (2002), 176

${ }^{779}$ Verdi (Oktober 2003), 10
} 


\subsubsection{Die Bedeutung des Jugendquotienten}

Von den Gegnern jeglicher Schrumpfung Deutschlands werden selten die wirtschaftlichen Folgen eines steigenden Jugendquotienten thematisiert. Volkswirtschaftlich gesehen kosten nicht nur die Alten, sondern auch Kinder und Jugendliche Geld. Unter20jährige erhalten - über Kindergärten, Schulen, Gesundheitsleistungen und nicht zuletzt wegen des kostenfreien Erststudiums - mehr Geld aus den staatlichen Systemen als sie über Steuern und Sozialabgaben in diese einzahlen. Der Koblenzer Statistiker Bosbach: „Bei seriösen Betrachtungen darf nicht nur der Altenquotient, sondern muss auch der Jugendquotient dargestellt werden. Die Summe beider, der Gesamtquotient, ist eine aussagekräftige Größe über die von den Erwerbsfähigen zu versorgenden Menschen“". ${ }^{780}$ Nach der mittleren Prognose des Statistischen Bundesamtes wird der Jugendquotient zwischen 2001 und 2050 von 38 auf 34 fallen, der Altenquotient von 44 auf 78 steigen und somit der Gesamtquotient von 82 auf 112 zunehmen, ${ }^{781}$ also um 37 Prozent steigen. Bosbach folgert daraus, da sich allein dadurch die Problematik bereits halbiere, da die ,Demografie-Pessimisten' immer nur den um 77 Prozent steigenden Altenquotienten nennen würden. ${ }^{782}$

Der Ökonom Meinhard Miegel hat berechnet, dass derzeit jedes Jahr 75 Milliarden Euro eingespart werden, weil der Jugendquotient zurückgeht. ${ }^{783}$ Es dauert 25 bis 30 Jahre, bis sich eine Steigerung der Geburtenrate in Deutschland ökonomisch auszahlen würde. Erst wenn die Kinder aufgezogen und ausgebildet sind, sind sie irgendwann so produktiv, dass sie mehr zur Volkswirtschaft beitragen, als sie kosten - dann steigern sie das BIP/Kopf. Der Zyklus der finanziellen Beziehungen zwischen dem Staat und seinen Bürgern ist nun mal dreigeteilt. Erst kostet das Individuum im statistischen Durchschnitt Geld für Versorgung, Erziehung und Ausbildung, dann arbeitet es produktiv, zahlt Steuern und Beiträge, und schließlich ist es alt und muss versorgt werden. Der Ertrag der zweiten Phase muss im Schnitt der Volkswirtschaft für alle drei reichen. Auch wenn es zu einem steigenden Jugendquotienten langfristig keine Alternative gibt, ${ }^{784}$ so hat doch eine Gesellschaft, die gleichzeitig einen hohen Alten- und einen hohen Jugendquotienten hat, von allen denkbaren Konstellationen die schwerste.

\subsubsection{Innovationsfähigkeit}

Das Durchschnittsalter der Beschäftigten in Deutschland wird von 39 Jahren (Stand: 2000) bis 2050 auf 44 Jahre steigen. ${ }^{785}$ Wenn ältere Arbeitnehmer grund-

\footnotetext{
${ }^{780}$ Bosbach (2003), 6

${ }^{781}$ Statistisches Bundesamt (2003), 42

${ }^{782}$ Bosbach (2003), 6

${ }^{783}$ Mayer (04.03.2004), 18

${ }^{784}$ Siehe Abschnitt 6.8.3. Aktive pronatalistische Geburtenpolitik.

${ }^{785}$ Börsch-Supan (2004a), 6
} 
sätzlich eine geringere physische Leistungsfähigkeit und Defizite im Umgang mit neuen Technologien (Stichwort: Computer, Internet) aufweisen, so wird c.p. mit dem größeren Anteil älterer Menschen in den Belegschaften die Arbeitsproduktivität sinken. ${ }^{786}$ Die Lücke der Arbeitsproduktivität ${ }^{787}$ durch die Alterung wird von manchen Autoren mit etwa 10 Prozent angegeben. ${ }^{78}$ Unter den Komponenten, die für eine nachlassende Leistungsfähigkeit Älterer verantwortlich gemacht werden, wird v.a. die Innovationsfähigkeit diskutiert.

Um die These von der nachlassenden Innovationskraft zu prüfen, ist es fruchtbar, in einem Gedankenexperiment zu überlegen, wie sich Innovationsfähigkeit und Fortschritt verändern würden, wenn die durchschnittliche Lebenserwartung der Menschen bzw. die Generationenfolge eine ganz andere wäre als die tatsächliche. ${ }^{789}$ Mannheim schreibt dazu: „Durch eine Verlängerung der Lebensdauer der Individuen würde das Tempo des Fortschritts gehemmt; durch eine Verkürzung der Lebensdauer etwa auf die Hälfte oder ein Viertel des gegenwärtiges Maßes würde das Tempo in entsprechender Weise beschleunigt werden, und zwar deshalb, weil im ersteren Falle die hemmende, konservierende Rolle der alten Leute infolge ihrer längeren Lebensdauer länger, im letzteren Falle durch ihr schnelleres Verschwinden kürzer in Wirkung treten würde. ${ }^{\text {" } 790}$ Man stelle sich eine Gesellschaft vor, in der eine Generation ewig leben und keine weitere Generationenfolge stattfinden würde. In einer solchen Gesellschaft würden Prozesse der Kultur- und Wissensschöpfung nicht mehr von neuen Jahrgängen geleistet werden können. Die Orientierungen und Denkmuster, die die einzige Generation im Zuge ihrer Sozialisation annahm, bliebe auf alle Zeiten erhalten. Die Bereitschaft, etwas grundsätzlich Neues auszuprobieren, ist in der Jugend am größten. Mannheim schreibt dazu: „Dass die Jugend weitgehend ohne Erfahrung ist, bedeutet für diese eine Minderung des Ballastes, eine Erleichterung des Weiterlebens. (...) Alt ist man primär dadurch, dass man in einem spezifischen, selbsterworbenen, präformierenden Erfahrungszusammenhang lebt, wodurch jede neue mögliche Erfahrung ihre Gestalt und ihren Ort bis zu einem gewissen Grade im vorhinein zugeteilt erhält, wogegen im neuen Leben die formierenden Kräfte sich erst bilden und die Grundintentionen die prägende Gewalt neuer Situationen noch in sich zu verarbeiten vermögen. Ein ewig lebendes Geschlecht müsste selbst vergessen lernen können, um das Fehlen neuer Generationen zu kompensieren. ${ }^{\text {" } 791}$

\footnotetext{
${ }^{786}$ Prognos (2002), 35. Nach Prognos steigen die Kosten für Unternehmen auch wegen der Vergütungsstrukturen, die i.d.R. auf dem Senioritätsprinzip basieren oder an dieses angelehnt sind.

787 Die Arbeitsproduktivität ist definiert durch das mengenmäßige oder wertmäßige Produktionsergebnis, geteilt durch den Arbeitseinsatz.

${ }^{788}$ Börsch-Supan (2004a), 6. Niejahr (28.08.2003) gibt 15 Prozent an.

${ }^{789}$ Dieses Gedankenexperiment habe ich bisher gefunden bei Jonas (1980), 32; Williams (1978), 133162 und Mannheim (1928). Auch Hume und Comte haben es nach Angaben von Mannheim $(1928 ; 158)$ durchgespielt.

${ }^{790}$ Mannheim (1928), 158

${ }^{791}$ A.a.O., 179
} 
Anders gesagt: Alt ist man, wenn man keine neuen Erkenntnisse mehr annehmen kann, die den bisherigen Überzeugungen und Weltanschauungen radikal widersprechen. Ein so definiertes ,geistiges Altern' trifft sicher auf fast alle Menschen zu. ${ }^{792}$ Es sind nun mal die ,jungen Männer, die die Staaten erschüttern. “"793

Durch die Generationenfolge wird stets das künstliche Kapital, v.a. die gesellschaftlichen Arrangements und Kulturinhalte, akkumuliert, aber auch teilweise verworfen. Für die nachwachsenden Jahrgänge hat historisch Gewachsenes nicht mehr dieselbe Relevanz wie für frühere Generationen. Das Absterben früherer Generationen führt dazu, dass es zu ,gesellschaftlichem Vergessen' kommt. Wegen der begrenzten Kapazität des menschlichen Gehirns kann jedes Individuum nur einen bestimmten Prozentsatz seines gesamten Wissens sowohl weitergeben wie auch aufnehmen. Die ältere Generation wird - über formelle und informelle Bildungsprozesse ${ }^{794}$ - das weiterzugeben versuchen, was ihr am wichtigsten ist. Der Rest wird ersetzt durch das, was die nachwachsende Generation neu schafft. Schimany weist darauf hin, dass für diesen spannungsgeladenen Prozess von Erneuern und Bewahren aber auch ein gewisse Mindest-Lebensdauer notwendig ist. Wie wir sahen, war die durchschnittliche Lebenserwartung für die meiste Zeit der Menschheitsgeschichte nur 20-25 Jahre. Erst die steigende Lebenserwartung in den letzten beiden Jahrhunderten begünstigte die Tradierung von Erfahrungen und Informationen, da die von Eltern- und Kindergeneration gemeinsam durchlebte Zeit anstieg. ${ }^{795}$

Die Vertreter der These von der nachlassenden Innovationskraft Deutschlands können ins Feld führen, dass die 25-44jährigen mit Abstand die aktivsten Unternehmensgründer sind. ${ }^{796}$ „Natürlich steckt auch in den Alten Potenzial, aber Weisheit hilft wenig, wo Innovation nötig wäre“, schreibt der Berliner Bevölkerungswissenschaftler Ralf Ulrich. ${ }^{797}$ Die These von der nachlassenden Innovationskraft berücksichtigt aber nicht, dass sich die Alten von morgen deutlich im Ausbildungsstand von den Alten von gestern unterscheiden. Rund ein Drittel der 65- bis unter 70jährigen des Jahres 2040 werden die Hochschulreife haben. Jeder sechste wird ein Hochschulstudium abgeschlossen haben. Dagegen weist unter der gleichaltrigen Altersgruppe heute nur jeder zehnte ein Abitur und

\footnotetext{
${ }^{792}$ Eine der seltenen Ausnahme von dieser Regel scheint Wittgenstein zu sein, dessen Spätwerk, die Philosophischen Untersuchungen, den Thesen seines ersten Hauptwerks, des Tracatus, radikal widersprach.

${ }_{793}$ Ein Ausspruch von Cicero, der dabei leider die Frauen vergaß.

${ }^{794}$ Die meisten Kulturinhalte werden nicht bewusst übertragen. Sie sickern vielmehr von Generation zu Generation durch, ohne dass Eltern und Kinder dies merken. „Was bewusst gelehrt, anerzogen wird, gehört zu jenem Bestand, der im Laufe der Geschichte irgendwann und irgendwo problematisch und reflexiv geworden ist.“ (Mannheim 1928, 182).

${ }^{795}$ Schimany (2003), 105

${ }^{796}$ Klingholz (2004), 92

${ }^{797}$ Zitiert nach Klingholz, a.a.O.
} 
nur jeder 17. einen Universitätsabschluss auf. ${ }^{798}$ Nicht nur die Lebenszeit dehnt sich aus, auch die aktiv-gesunde Zeit nimmt im Durchschnitt zu. „Die heute 70jährigen sind also mindestens genauso fit, geistig und körperlich, wie die 65jährigen der vorangegangenen Generation“, schreibt der Altersforscher Paul Baltes. ${ }^{799}$

Das entscheidende Argument dürfte sein, dass sich der Verlust an Innovationsfähigkeit durch lebenslanges Lernen zumindest bremsen lässt. Dies lässt sich plakativ so zusammenfassen: „Wenn es mehr Ältere und weniger Jüngere gibt, so müssen wir die Älteren länger jung halten.“ Für rohstoffarme Länder bilden seine Bewohner die wichtigste Ressource. Sie muss folglich so effizient wie möglich eingesetzt werden. Dazu hat z.B. die Enquete-Kommission Demographischer Wandel ausführliche Vorschläge unterbreitet. ${ }^{800}$ Zur Steigerung der geistigen Flexibilität sollte man - um nur einen Vorschlag herauszugreifen - über ein fest etabliertes Studium für 45-50jährige, einen Senior-Masters, nachdenken, damit Menschen, die dann ja erst in Mitte ihrer Erwerbsphase stehen, sich weiterqualifizieren und ggf. noch mal neu orientieren können.

Der Mitherausgeber der FAZ, Frank Schirrmacher, beklagt, dass die Unterstellung, Ältere seien weniger innovativ, eine selbsterfüllende Prophezeiung sei ${ }^{801}$ Bei einer Langzeitstudie in Ohio wurde über 20 Jahre hinweg das Altern einer ganzen Stadt untersucht. Dabei konnte nach Schirrmachers Angaben gezeigt werden, dass „diejenigen, die das Alter für eine erfüllte Phase ihres Lebens ansahen und über ältere Menschen positiv dachten, im Schnitt siebeneinhalb Jahre länger lebten als diejenigen, die vom Alter nichts erwarteten." ${ }^{\text {"802 }}$

Durchaus positiv ist, dass der übertriebene Jugendkult in den Personalabteilungen der Unternehmen zurückgehen dürfte. In den vergangenen Jahren haben Unternehmen in Deutschland ihre Belegschaften drastisch verjüngt. Fast 60 Prozent aller Betriebe beschäftigen derzeit keine Mitarbeiter über 50. ${ }^{803}$ Der Anteil der Über50jährigen an allen Arbeitslosen liegt bei 30 Prozent, bei staatlichen Weiterbildungsmaßnahmen beträgt er nur 7,5 Prozent, d.h. das meiste Geld für Umschulung und Weiterqualifizierung wird in junge Arbeitslose investiert. Im Moment sind 56 Prozent aller Langzeitarbeitslosen zwischen 50 und 65 Jahren alt. In Zukunft werden engagierte Fünfundfünfzigjährige weniger Probleme haben, einen Arbeitsplatz zu finden. Die ZEIT titelt: „Die Bellheim-Republik. Vergreisung? Verarmung? Die Alten werden die Retter sein ${ }^{\text {“804 }}$ Der Titel spielt an auf die Geschichte des Peter Bellheim, die in dem Film Der große Bellheim mit Mario

\footnotetext{
${ }^{798}$ Cornelius (2004), 57

${ }^{799}$ Baltes (2005), 44

${ }^{800}$ Deutscher Bundestag (2002), 177-215

${ }^{801}$ Schirrmacher (2004). Ähnlich Gronemeyer (2004) und Opaschowski (2004).

${ }^{802}$ Schirrmacher (2004), 29

${ }^{803}$ Opaschowski (2004), 160

${ }^{804}$ Niejahr (28.08.2003)
} 
Adorf in der Hauptrolle verfilmt wurde. Es ist die Geschichte eines Pensionärs, den die Hiobsbotschaften über die wirtschaftliche Lage seiner Kaufhauskette dazu ermuntern, der Langeweile seines Pensionärsdaseins zu entfliehen, um mit alten Weggefährten sein altes Imperium zu retten. Solche Geschichten gibt es nicht nur im Film: Der 64jährige Jean-Rene Fourtou wurde zur Rettung des Vivendi-Imperiums gerufen, der 71jährige Robert A. Lutz bei General Motors. Und der 79jährige Alan Greenspan leitet als US-amerikanischer Notenbankchef die internationalen Finanzströme. Die FAZ kommt zu der Aussage: „Überall grassiert das Bellheim-Syndrom. ${ }^{“{ }^{\circ 05}}$ Aber auch hier sollten gesellschaftliche Maßnahmen den demografisch bedingten Trend unterstützen. $\mathrm{Zu}$ den Gestaltungselementen, welche die Nachfrage nach älteren Arbeitnehmern steigern würden, gehört die Abschaffung des Senioritätsprinzips und anderer Regelungen (z.B. beim Kündigungsschutz), welche ältere Arbeitnehmer unabhängig von ihrer Produktivität und Innovationskraft derzeit teurer machen als jüngere.

Bisher haben wir diskutiert, wie die Innovationskraft von der Alterung beeinflusst wird. Eine andere Frage ist, wie sich die Innovationskraft zur Bevölkerungsgröße verhält. Hier ist wieder zwischen einer Pro-Kopf-Perspektive und einer absoluten Perspektive zu unterscheiden. Ist Deutschland innovativer als Österreich, Israel oder Holland? Zu Beginn des 19. Jahrhunderts lebte in Deutschland ungefähr ein Viertel der heutigen Bevölkerungszahl. Und dennoch brachte diese Gesellschaft Beethoven, Mozart, Goethe, Schiller, Hegel oder Herder hervor. Ein mit 70 Millionen Menschen bevölkertes Deutschland kann innovativer sein als Deutschland heute. Entscheidend für die Innovationskraft ist nicht die Bevölkerungsgröße, sondern Ausbildungs- und Wissensstand. Der menschliche Geist ist zwar potenziell die größte und unerschöpflichste aller Ressourcen, aber eben nur, wenn er trainiert und ausgebildet wird. Das Bildungsniveau ist teilweise auch abhängig von der Wachstumsrate einer Bevölkerung. Die stark wachsenden muslimischen Gesellschaften sind nicht die am besten ausgebildetsten und nicht die innovativsten, da der Staat mit dem Bau von Bildungseinrichtungen kaum nachkommt. Eine nur schrumpfende, aber nicht alternde Gesellschaft, die die Bildungs-Infrastruktur ihrer Vorgänger-Generation erbt und erhält, hätte beste Chancen, ihr Bildungsniveau dramatisch zu erhöhen. Eine schrumpfende und alternde Gesellschaft sieht sich zwei gegenläufigen Trends ausgesetzt. Auch hier sind die Effekte von Schrumpfung und Alterung also analytisch zu trennen.

In der hier gebotenen Kürze kann dieser Abschnitt wie folgt zusammengefasst werden: Die nachlassende Innovationskraft stellt zwar ein potenzielles Problem dar. Die problematischen Wirkungen könnten jedoch durch gesellschaftliches Gegensteuern (Schlagwort:'Lebenslanges Lernen') deutlich vermindert werden.

${ }^{805}$ Heydebreck (04.08.2002) 


\subsubsection{Die Macht der Generationen an der Wahlurne}

Ein weiteres Problem ist die politische Repräsentanz der jungen Generation: Im Jahr 2050 werden mit 27,58 Millionen voraussichtlich mehr als doppelt so viele Übersechzigjährige leben wie Unterzwanzigjährige (12,09 Millionen). Der Anteil der Übersechzigjährigen wird von 20 auf 36,7 Prozent steigen und der Anteil der Unterzwanzigjährigen von 22 auf 16,1 Prozent sinken. ${ }^{806}$ Die junge Generation gerät mehr und mehr in eine strukturelle Minderheitenposition. Es sind zahlreiche Befürchtungen laut geworden, dass die ältere Generation aufgrund ihrer numerischen Überlegenheit die Politik und das gesellschaftliche Leben dominieren wird. ${ }^{807}$ Einschneidende Rentenreformen könnten dann z.B. politisch erheblich schwerer durchzusetzen sein als heute.

Aus demokratietheoretischen Gründen (Gleichwertigkeit jeder Stimme) verbieten sich Vorschläge, alten Menschen das Wahlrecht zu entziehen ${ }^{808}$ oder ihre Stimmen niedriger zu gewichten. ${ }^{809}$ Diskussionswürdig erscheinen aber Vorschläge, durch eine Wahlrechtsreform auch Unterachtzehnjährigen eine Stimme zu geben, entweder durch ein Wahlrecht ohne fixe Altersgrenze ${ }^{810}$ oder durch ein Elternwahlrecht. ${ }^{811}$ Würde das Wahlrecht auf die Unterachtzehnjährigen erweitert, so kämen zu den 67 Mio. heutigen Wählerstimmen 15 Mio. hinzu. ${ }^{812}$ Eine solche Veränderung der Stimmenverteilung bliebe nicht ohne Auswirkung auf die politischen Entscheidungen der gewählten Volksvertreter. Gleichzeitig würde auf diese Weise das fundamentale Defizit des Demokratieprinzips, nämlich der Ausschluss eines beträchtlichen Teils des Staatsvolkes von der politischen Partizipation, beseitigt.

\subsubsection{Zwischenfazit}

Wie lautet ein Fazit unserer Diskussion der ,internen Faktoren'? Für manche internen Probleme, z.B. das Übergewicht der älteren Generation an der Wahlurne, oder die nachlassende Innovationskraft gibt es bereits Lösungsansätze, die die Probleme zumindest entschärfen könnten. Andere Probleme erweisen sich als Scheinprobleme wie etwa das abnehmende (absolute) Humankapital. Alle Argumente zusammenfassend deutet aber vieles darauf hin, dass der prognostizierte demografische Wandel in wirtschaftlicher Hinsicht eher negative Auswirkungen

\footnotetext{
${ }^{806}$ Alle Zahlen haben den Stand 2003.

${ }^{807}$ Tremmel (1996), 59; Gronemeyer (1989); Schüller (1995); Schüller (1997); Podszun (2000); Klöckner (2003); Gronemeyer (2004)

${ }^{808}$ Schüller (1995), 180. Nach Schüllers Vorschlag wäre dies nur zulässig, wenn deren geistige Kraft nachweislich nachlässt.

${ }^{809}$ Klöckner (2003), 80

${ }^{810}$ SRzG (2004), Weimann (2002)

${ }^{811}$ Siehe z.B. den Gruppenantrag „Mehr Demokratie wagen durch ein Wahlrecht von Geburt an“ von 47 Bundestagsabgeordneten, eingebracht am 11.09.2003; Bundestagsdrucksache 15/1544

${ }^{812}$ Bei dieser Rechnung wurde nicht zwischen Deutschen und Ausländern unterschieden, da letztere auf kommunaler Ebene wählen dürfen.
} 
auf kommende Generationen haben wird. Generationengerechtigkeit im isolierten Teilsystem der Rentenversicherung wird dadurch nahezu unerreichbar, wenn man sie so definiert, dass die nächste Generation eine gleichhohe oder höhere Rendite haben sollte wie die heutige.

\subsection{Externe Faktoren}

\subsubsection{Machtpolitik oder Kooperation als Maxime von Außenpolitik?}

Externe Faktoren sind auf die relative Stellung eines Landes im Kontext der internationalen Beziehungen bezogen. In langen Epochen der Menschheitsgeschichte galt die Bevölkerungszahl als Quelle politischer, wirtschaftlicher und militärischer Macht. ${ }^{813}$ Historikern gilt etwa die geringere Geburtenrate Roms im Vergleich zu seinen Nachbarvölkern als einer der Hauptgründe für den Untergang des Weltreiches. ${ }^{814}$ Frankreich war während der Napoleonischen Kriege auch deshalb zunächst so erfolgreich, weil das Land damals von allen europäischen Mächten die meisten Einwohner hatte und nach Einführung der allgemeinen Wehrpflicht damit die meisten Soldaten aufbieten konnte. ${ }^{815}$ Mit der Weiterentwicklung der Waffentechnik zu Massenvernichtungswaffen wie z.B. den Nuklearwaffen, sank die Bedeutung einer großen Bevölkerung für militärischen Erfolg gegen Null. China ist dank seiner weit größeren Zahl an Nuklearsprengkörper heute seinem Nachbarn Indien militärisch überlegen, obwohl dieser schneller wächst und bald über eine größere Bevölkerung verfügen wird. Dieses Beispiel zeigt, dass eine hohe Bevölkerungswachstumsrate sogar militärisch von Nachteil sein kann, weil das Gesundheits- und Bildungssystem Ressourcen bindet, die nicht (bzw. nur um den Preis eines niedrigen HDI des eigenen Landes) in Aufrüstung investiert werden können.

Aber die Bevölkerungsgröße ist immer noch z.B. verknüpft mit Stimmenanteilen in internationalen Organisationen oder dem EU-Parlament. Wenn die heutigen Geburtenraten konstant blieben, dann würde Frankreich langfristig Deutschland überrunden und in der EU das bevölkerungsstärkste Land werden - falls bis dahin die Türkei kein Mitglied ist. Erstmals hat das 58-Millionen-Land Frankreich 2002 mit 779000 Geburten absolut mehr Kinder hinzubekommen als Deutschland mit seinen 82 Millionen Einwohnern. ${ }^{816}$ Gäbe es heute schon eine Weltdemokratie, in der wichtige Entscheidungen nach dem Prinzip ,Ein Mensch - eine Stimme' gefällt würden, so entfielen auf China und Indien zusammen mehr als ein Drittel aller Stimmen.

Mit der zunehmenden Bedeutung von weltweiten Verschmutzungsrechten könnte es in Zukunft auch dazu kommen, dass große Bevölkerungen von ihren

\footnotetext{
${ }^{813}$ Foucault (1989)

${ }^{814}$ Demandt (1984), 354

${ }^{815}$ Münz (2003), 6

${ }^{816}$ Hessische Staatskanzlei (2003), 32
} 
Regierungen als Faktor angesehen werden, der bei der weltweiten Ressourcenverteilung Vorteile bringt.

In israelischen Regierungskreisen ist man - trotz der militärischen Überlegenheit - über wenige Dinge mehr besorgt als über die unterschiedlichen Geburtenraten von israelischen Frauen (2,9 Kinder) und Palästinenserinnen (6 Kinder) ${ }^{817}$ Die Entscheidung, einen Sperrzaun zwischen Israel und dem Westjordanland zu bauen, hat sehr viel mit der Bevölkerungsprognose zu tun, dass es unter diesen Umständen in 50 Jahren mehr Palästinenser als Israelis geben wird.

Der Soziologe Karl Otto Hondrich bringt ein anderes Beispiel: „Seit einem halben Jahrhundert ist diese Macht [der politische Islamismus, J.T.] auf dem Vormarsch. Ihre äußeren Merkzeichen - die antikolonialen Freiheitskämpfe, die Gründung islamischer Staaten von Marokko bis Indonesien, die weltwirtschaftliche Bedeutung des Öls - verdecken allerdings die tieferen Gründe des Machtzuwachses: die merkwürdige Verschränkung von religiöser Statik und demografischer Dynamik. Sie bringen die Weltverhältnisse zum Tanzen - nach Takten, für die der Westen kein Ohr hat. Während er die großen Töne ökonomischer, politischer und militärischer Machtmusik anschlägt, vollzogen sich die Machtverschiebungen zugunsten des Islam über lange Zeiten fast lautlos und unbemerkt: durch Geburtenüberschüsse und Wanderungen von islamische in christliche Länder, durch die Ausbreitung von islamischen Gemeinden, durch Inanspruchnahme von Gast- und Minderheitenrechten zugunsten der Muslime. “818

Der Berater der US-Regierung, Robert Kagan, beschwört ebenfalls die Demografie: „Heute liegt das Durchschnittsalter der Amerikaner bei 35,5 Jahren, das der Europäer bei 37,7 Jahren. Im Jahr 2050 wird das Durchschnittsalter der Amerikaner bei 36,2 liegen, das der Europäer dagegen bei 52,7. Dies bedeutet unter anderem, dass die finanzielle Belastung durch die Altersversorgung in Europa viel stärker wachsen wird als in den Vereinigten Staaten. Und dies wiederum bedeutet, dass den Europäern in den kommenden Jahren und Jahrzehnten weniger Geld für die Verteidigung zur Verfügung stehen wird. “819

Was ist zu diesen auf den ersten Blick sehr überzeugenden Argumenten zu sagen? Die These vom deutschen Machtverlust in der EU folgt einer Denkschule, die die internationalen Beziehungen dadurch charakterisiert sieht, dass alle Staaten stets versuchen, ihre eigenen Interessen auf Kosten anderer Staaten durchzusetzen. ${ }^{820}$ Statt Win/Win-Situationen anzustreben, wird von Win/Loose-Szenarien ausgegangen, bei denen sich ein Staat auf Kosten von anderen behaupten muss. Auch wenn sicherlich die längste Zeit der europäischen Geschichte dieses Para-

\footnotetext{
${ }^{817}$ Niejahr (02.01.2003), 9

${ }^{818}$ Hondrich (2002), $187 \mathrm{f}$.

${ }^{819}$ Kagan (2003), $104 \mathrm{f}$.

${ }^{820}$ In der Politologie wird diese Position oft als ,Realismus' und die (wichtigste) Gegenposition als ,Konstruktivismus' bezeichnet. Der Begriff ,Realismus' ist aber völlig verfehlt, da ja gerade in Frage steht, wie ,richtig' bzw. ,realistisch' diese Denkschule die Wirklichkeit beschreibt.
} 
digma realistisch war, so ist es seit dem Ende des 2. Weltkriegs nicht mehr handlungsleitend für die Außenpolitik europäischer Staaten. Die ganze Entstehungsgeschichte der EU ist ein empirischer Gegenbeweis gegen eine solche Charakterisierung der internationalen Beziehungen. Nach der nur von ihren Anhängern als ,realistisch' bezeichneten Denkschule ist weder die Entstehung der EU noch die friedliche Abschaffung des Apartheid-Systems in Südafrika durch den damaligen weißen Präsidenten de Klerk erklärbar. Hätte dieses Modell maßgeblichen Erklärungswert, dann hätten die Nuklearwaffen Frankreichs und Großbritanniens diesen Ländern zu einer beherrschenden Stellung in Europa verholfen - v.a. auf Kosten Deutschlands. Tatsächlich haben zahlreiche empirische Studien ergeben, dass sich Regierungen und ihre Mitglieder meist nicht so verhalten, wie es ihren egoistischen Interessen entsprechen würde. ${ }^{821}$ Sie orientieren sich in ihrem Verhalten an ,geteilen Werten, kollektiven Weltdeutungen und wechselseitigen Verhal-

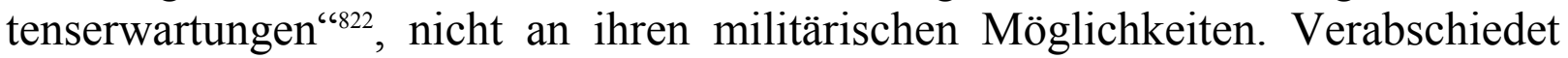
man sich vom machtpolitischen Denkschema, so verlieren die ,externen Argumente' gegen Bevölkerungsschrumpfung an Bedeutung. Innerhalb der EU schrumpfen andere Länder noch stärker als Deutschland. In Großbritannien fordern Ökologen, die den 'Ökologischen Fußabdruck' ihres Landes berechnet haben: „The UK population needs to be gradually reduced from 59 million to a maximum 30 million, which could be done without coercion on family size. ${ }^{\text {“6223 }}$ Und in den USA hat die ähnliche Forderungen vertretende Initiative Zero Population Growth (heute: Population Connection) rund 55.000 Mitglieder.

Im Sinne eines auf Kooperation angelegten Umgangs der reichen Länder mit der Schrumpfung könnten Best-Practice-Beispiele ausgetauscht und ein regelmäBiger Erfahrungsaustausch initiiert werden.

Die Debatte zwischen eigennutzmaximierender und kooperativ ausgerichteter Denkschule in der Außenpolitik kann und muss hier nicht weiter vertieft werden. Wie so häufig wird es vermutlich von den Umständen abhängen, ob die eine These etwas mehr Geltungskraft beanspruchen kann als die andere. Wenn die These vom Machtzuwachs durch Bevölkerungswachstum uneingeschränkt stimmen würde, so bliebe nichts anderes übrig als ein ,Rattenrennen', bei dem jeder das Falsche tun muss, obwohl er weiß, dass es die schlechtere Alternative für ihn ist (begrenzt wiederholtes Gefangenendilemma). Für Deutschland wäre das eine schlechte Option. Zum Glück lebt Deutschland inzwischen in Frieden mit ihren Nachbarn und hat keine territoralen Streitigkeiten bzw. Gebietsansprüche - damit befindet es sich in einer fundamental anderen Situation als z.B. Israel.

\footnotetext{
${ }^{821}$ Für einen Überblick siehe Hasenclever (2000).

${ }^{822}$ Hasenclever (2000), 14

${ }^{823}$ www.optimumpopulation.org
} 


\subsubsection{Bevölkerungsdichte in Nord und Süd}

Einer radikalen Verzerrung unterliegt die Sichtweise, dass die SEL insgesamt weniger stark bevölkert seien als die Entwicklungsregionen. Selbst wenn die Wachstumsraten im Moment in den Entwicklungsländern höher sind, so ist doch außer Asien kein anderer Kontinent der Welt gegenwärtig so dicht besiedelt wie Europa. Europas Entwicklungspfad führte dazu, dass heute die europäische Bevölkerungsdichte immer noch rund dreimal so hoch ist wie in Afrika oder Lateinamerika und fast fünf mal so hoch wie in Nordamerika (ohne Mexiko). Diese Zahlen relativieren die weitverbreitete Ansicht, andere Kontinente wie z.B. Afrika oder Südamerika seien überbevölkert, unser eigener aber nicht.

Eine gängige Meinung in den Ländern auf der Nordhalbkugel ist, dass das „demografische Gleichgewicht in der Welt mit zunehmendem Tempo zerstört" ${ }^{\star 824}$ werde. Die Frage ist, womit sich dieses Gleichgewicht, also der status quo, rechtfertigen lässt.

Generell begann der demografische Übergang in den europäischen Ländern und den USA ein bis zwei Jahrhunderte früher als im Rest der Welt, wo er sich im 20. Jahrhundert z.T. in einem Viertel der Zeit vollzog. ${ }^{825}$ Während der ersten Phase der Transition - dem Rückgang der Sterberaten bei unverändert hohen Geburtenraten - wuchs Europa stärker als jede andere Region auf der Welt (vgl. Abb. 3538).

\section{Abb. 35-38: Besiedelungsdichte der Welt 1700, 1800, 1900, 1998}

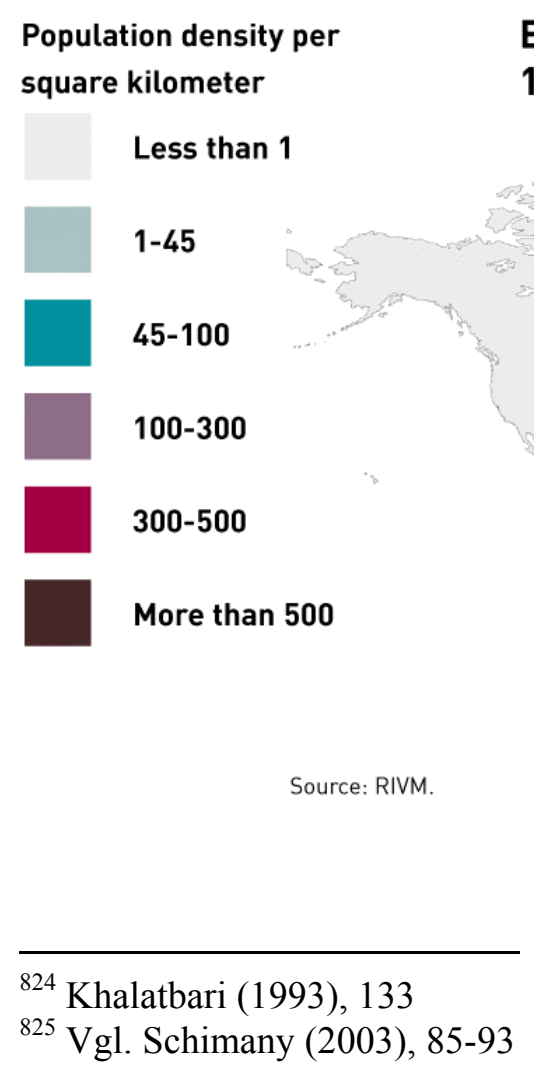

\section{EXPANSION OF THE HUMAN POPULATION 1700}

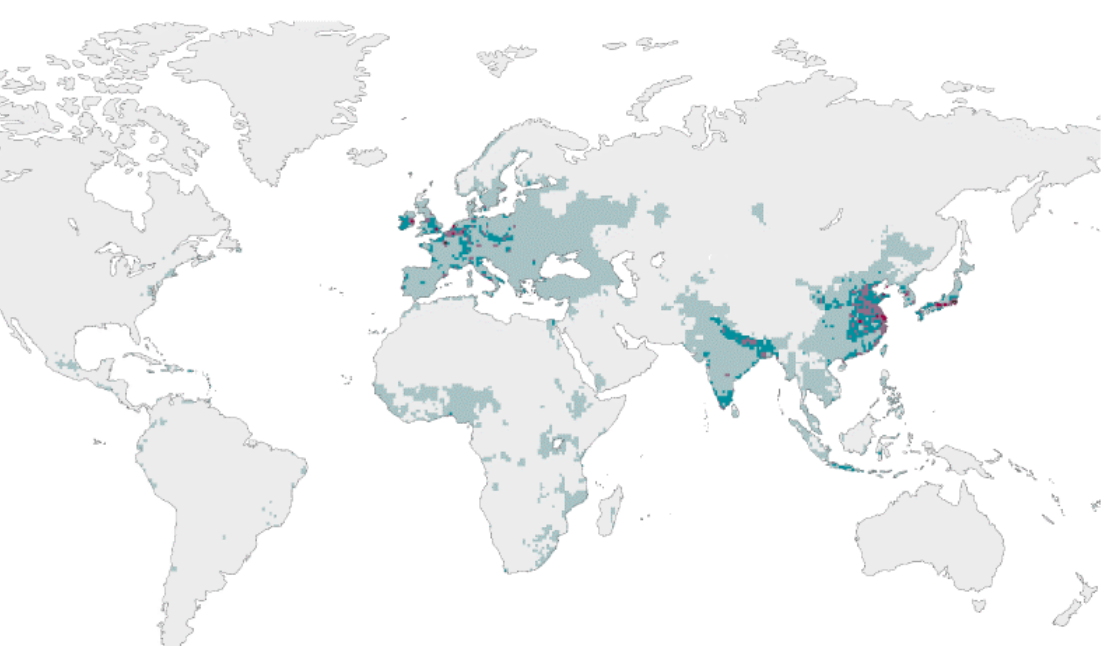

${ }^{825}$ Vgl. Schimany (2003), 85-93 
Der Wirtschaftsnobelpreiträger indischer Abstammung Amartya Sen weist darauf hin, dass sich die Anteile der europäischen und der nichteuropäischen Völker seit der Mitte des achtzehnten Jahrhunderts dramatisch zu Gunsten Europas verschoben haben. ${ }^{826}$ Zur Mitte des 18. Jahrhunderts hatte Europa etwa 50 Prozent mehr Einwohner als Afrika.

Gegen Ende des 18. Jahrhunderts waren es schon doppelt so viele und in der zweiten Hälfte des 19. Jahrhunderts erreichte Europa das Dreifache der Bevölkerungszahl Afrikas. Im 20. Jahrhundert holte Afrika den Rückstand in nur drei Jahrzehnten auf. Bei den gegenwärtigen Wachstumsraten würden Asien und Afrika nach Sens Angaben zusammen erst um das Jahr 2050 wieder jenen Anteil an der Weltbevölkerung erreichen, den sie im achtzehnten Jahrhundert gehabt haben. Die Entwicklungsländer machen also derzeit einen demografischen Schub durch, wie Europa und die Vereinigten Staaten ihn in den vergangenen zweihundert Jahren hinter sich gebracht haben.

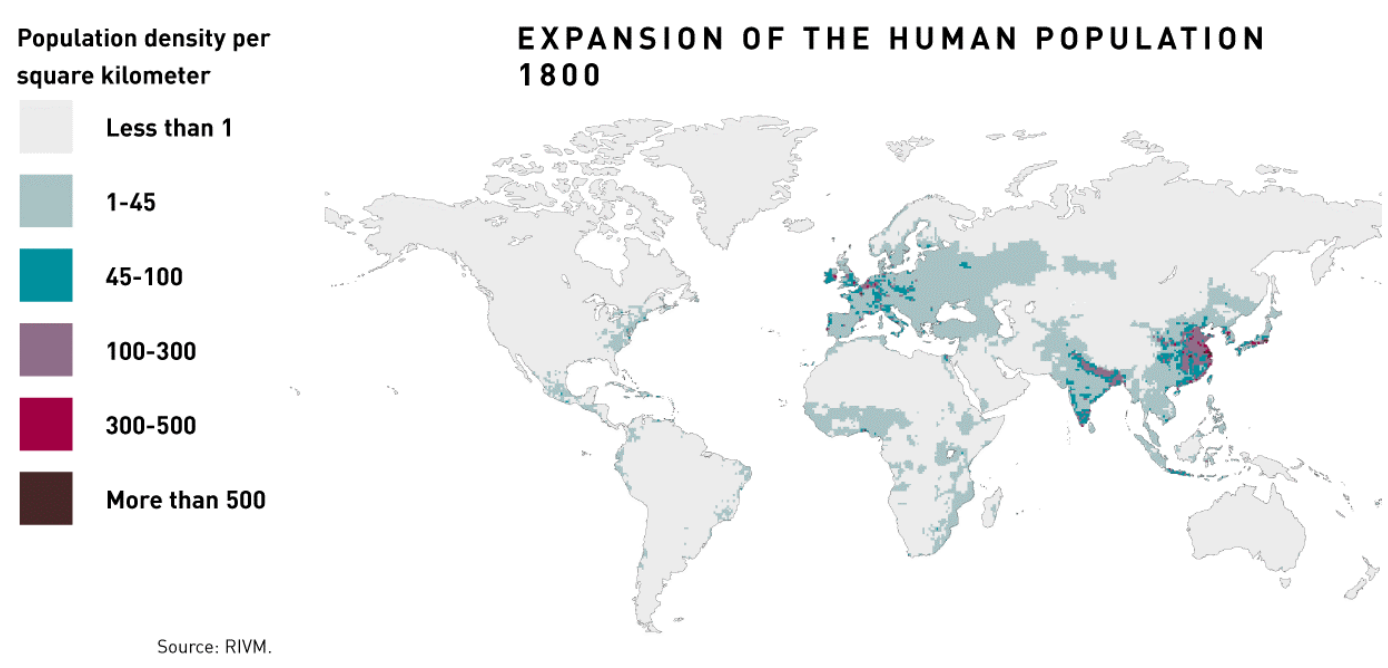

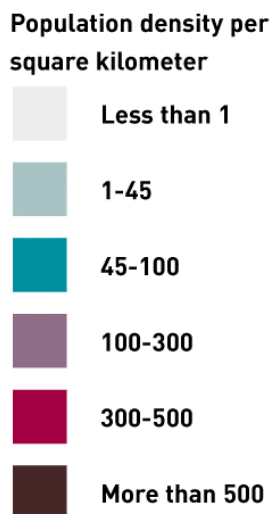

EXPANSION OF THE HUMAN POPULATION 1900

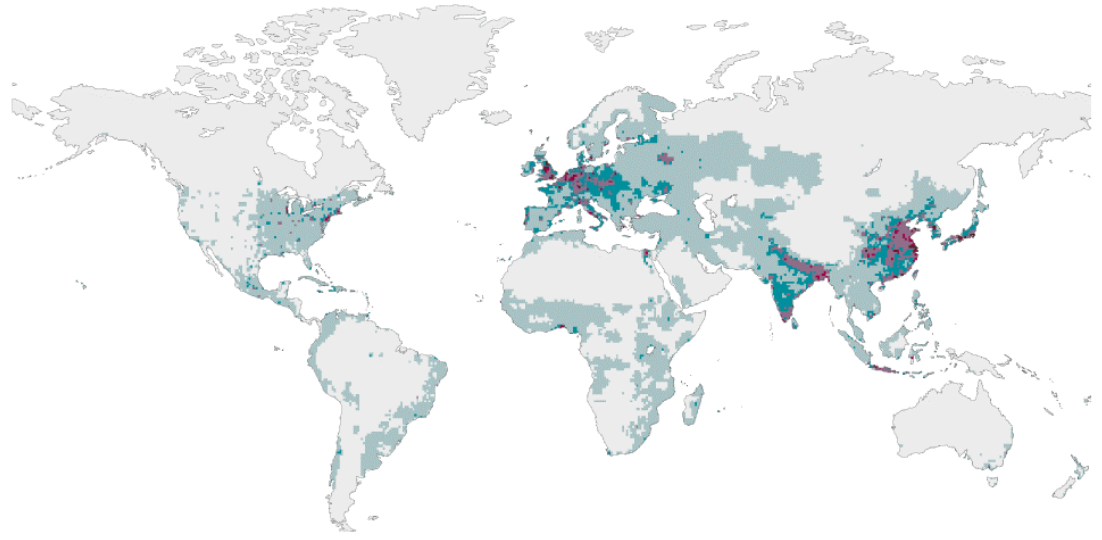




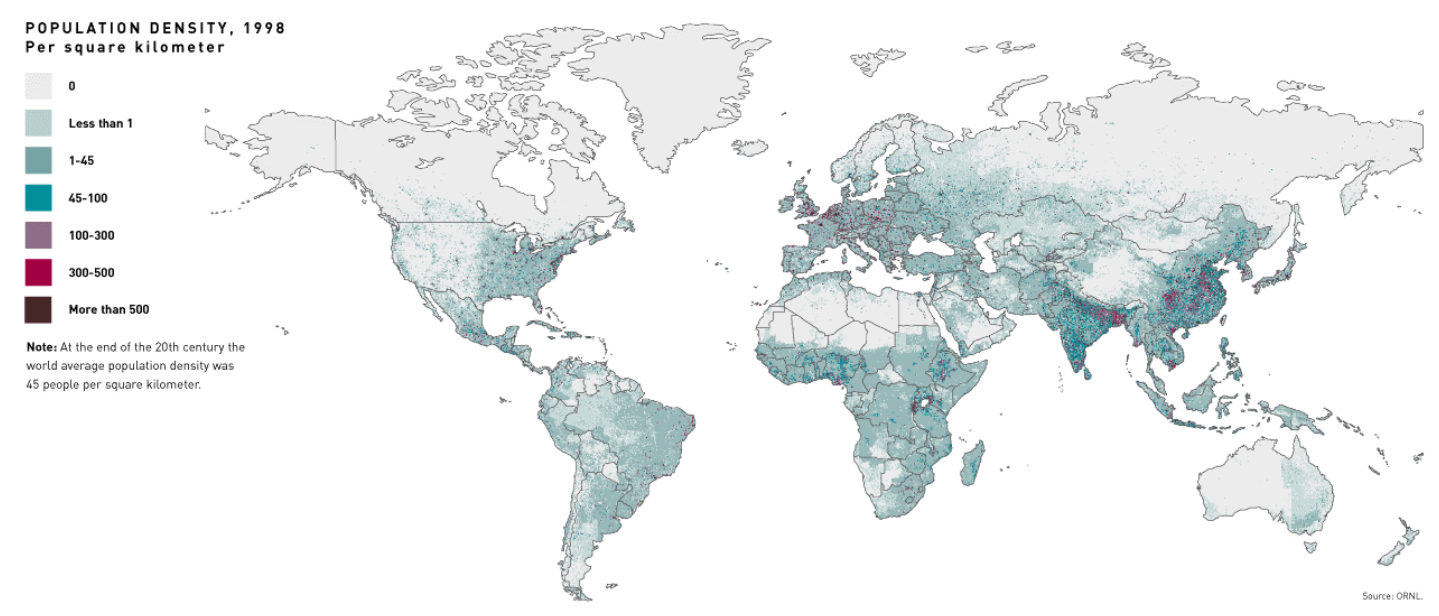

Quelle: American Association for the Advancement of Sciences / Harrison, Paul / Pearce, Fred (2001) (Hg.): AAAS Atlas of Population and Environment

Wie ist dieses Argument zu beurteilen? Auch wenn das Wachstum der Weltbevölkerung von 1700 bis ca. 1880 v.a. in den heutigen SEL stattfand, ${ }^{827}$ so ist doch zu bedenken, dass sie damals nur rund um 700 Mio. Menschen wuchs. Die außereuropäischen Zuwächse finden hingegen derzeit mit einem weit höheren absoluten Zuwachs statt. ${ }^{828}$ Hinzu kommt, dass die Sequenz der einzelnen Entwicklungsstufen in den vormodernen Gesellschaften Afrikas, Asiens und Lateinamerikas nicht dem Verlauf folgt, der den insgesamt günstigen Anpassungsprozeß in Europa kennzeichnete. ${ }^{829}$ Hier bauten die Modernisierungsschübe aufeinander auf, so dass die kommerzielle Revolution des frühen ebenso wie die Agrarrevolution des späten 18. Jahrhunderts die erforderliche Kapital- und Ernährungsbasis für die industrielle Revolution des 19. Jahrhunderts bereitstellten konnte. Dagegen traf die plötzlich einsetzende Bevölkerungszunahme die Entwicklungsländer im 20. Jahrhundert gesellschaftlich, politisch und ökonomisch unvorbereitet. Zudem öffneten mehrere Auswanderungswellen dem europäischen Bevölkerungsüberschuss zusätzliche Ventile. Von 1816 bis 1920 wuchs beispielsweise die Bevölkerung Deutschlands von 23,6 auf 64,9 Millionen, also um das Zweieinhalbfache. Im selben Zeitraum verlor Europa 10 Prozent des Nettobevölkerungszuwachses durch Emigration, allein nach Amerika zog es mehrere Millionen Deutsche. ${ }^{830}$ Die europäische Bevölkerungsexplosion ging einher mit dem europäischen Kolonia-

\footnotetext{
${ }^{827}$ Vgl. Schockenhoff (1996), 5

${ }^{828} \mathrm{Zu}$ den Unterschieden des gegenwärtigen Bevölkerungswachstums in Afrika, Asien und Lateinamerika zum europäischen Wachstum im 18. und 19. Jahrhundert, siehe z.B. Schmid (1976), 277-296.

${ }^{829}$ Schockenhoff (1996), $14 \mathrm{f}$.

${ }^{830}$ Dießenbacher (25.5.1993), 12
} 
lismus. 1815 waren 55 Prozent der Erdoberfläche von Europa erobert, 1914 waren es 84,4 Prozent. ${ }^{831}$

Daraus kann man sicher ableiten, dass Schuldzuweisungen aus Europa in Richtung auf die heute wachsenden Kontinente unberechtigt sind, solange Europa die Prinzipien ökologischer Nachhaltigkeit verletzt. Aber aus Sens Fingerzeig auf die frühere europäische ,Bevölkerungsexplosion' lässt sich nicht die Schlussfolgerung ableiten, dass das rasche Bevölkerungswachstum in einigen Ländern der südlichen Hemisphäre dadurch im Sinne der ökologischen Generationengerechtigkeit ethisch legitimiert sei. Im 19. Jahrhundert war die Weltbevölkerungsdichte der Welt viel geringer und die Artenvielfalt viel höher als heute. Alles in allem sind die Rahmenbedingungen einfach nicht vergleichbar, zumal der Anteil Europas an der Weltbevölkerung bereits stark zurückgegangen ist, von 22 Prozent (1900) auf sieben Prozent (2004).

\subsubsection{Zwischenfazit}

Bewertet man abschließend die externen Faktoren, die als Gegenargumente gegen eine moderate Schrumpfung ist Feld geführt werden, so ist ein differenziertes Urteil notwendig. In einer Welt, in der Deutschland als einziges Land schrumpfen würde, wäre dies von großem Nachteil für die Deutschen. Tatsächlich schrumpft Deutschland nach den meisten Projektionen aber kaum stärker als der europäische Durchschnitt. Europa wiederum scheint der Vorreiter für die demografische Entwicklung der ganzen Welt zu sein. Außenpolitisch könnte Europa auf internationalen Bevölkerungskonferenzen ein gutes Beispiel sein, wenn es der Schrumpfung auch positive Aspekte abgewönne. Dies würde für die Aufrechterhaltung des Ziels der möglichst baldigen Stabilisierung der Weltbevölkerung von großer Bedeutung sein. Es gilt also den Blick auf die Chancen der Schrumpfung zu richten, v.a. die ökologischen Potenziale dieses Prozesses. Dies allerdings muss eingebunden sein in eine Gesamtstrategie für die demografische Zukunftsfähigkeit Deutschlands, wie sie in den nächsten Kapiteln dargestellt wird.

\section{7 „Hilfe wir schrumpfen!“ - Rationalität und biologisches Erbe}

Eine rationale Debatte über die Schrumpfung tut Not. Aber ist sie überhaupt möglich? Viele Menschen reagieren auf die prognostizierte Schrumpfung Deutschlands mit instinktiver Abwehr. ${ }^{832}$ Dies ist evolutionsbiologisch nur zu verständlich, denn jede Spezies ist, um ihr eigenes Überleben zu sichern, zunächst mal auf Vermehrung gepolt. „Hilfe, wir schrumpfen!“ - von allen Hinterlassenschaften der Evolution in unserem Stammhirn ist die Polung auf Wachstum der eigenen Art, Nation oder Sippe am mächtigsten. Seit Jahrtausenden haben kinder-

${ }^{831}$ A.a.O.

${ }^{832}$ Hager/Schenkel (2003), 4 
reiche Menschen ihre Erbanlagen in größerem Umfang weitergegeben als andere. Unser Erbgut ist von ihren Genen bestimmt. Keine Art hat jemals ihre Fortpflanzung beschränkt, ohne durch äußere Umstände dazu gezwungen gewesen zu sein. Bei allen Tier- und Pflanzenarten beschränkt das komplizierte Geflecht aus ökologischen Abhängigkeiten die Fortpflanzungsraten. Feldmauspopulationen können im Jahr um das 24fache, Mehlkäferpopulationen sogar um das Zehnmilliardenfache wachsen ${ }^{833}$ Dass die Welt trotzdem nicht von Mehlkäfern bevölkert ist, liegt daran, dass die Umstände (klimatische Bedingungen, Nahrungsmittelknappheit, etc.) dem Wachstum Einhalt gebieten. Der Mensch hat zwar eine geringere biologische Fortpflanzungsrate, aber er hat es verstanden, sich über diese Schranken hinweg zu setzen, so dass sie seine Fortpflanzung zumindest im Moment nicht mehr hemmen. Dies ist neu für die menschliche Spezies. In seiner gesamten Geschichte war der Mensch damit beschäftigt, für sich selbst, seine Familie, seinen Clan oder seine Nation das Überleben zu sichern. Fortpflanzung ist ein wesentlicher Teil dieser Überlebenssicherung. Als Ergebnis wurde einer hohen Kinderzahl ein intrinsischer Wert zugeschrieben und in zahlreichen Religionen, frühen Gesetzestexten und Gesellschaftsnormen verankert. Über Jahrtausende galt es als moralisch richtig und gut, viele Kinder zu haben. ${ }^{834}$ Die entscheidende Frage ist, ob der Mensch schnell genug in der Lage ist, sein ,bewährtestes' Prinzip aufzugeben. ${ }^{835}$

In der Natur gibt es kein ewiges Wachstum, sondern einen steten Wechsel von Wachsen und Schrumpfen. Damit die Blüten im Frühling sprießen können, müssen sie im Herbst vergehen. Ein Acker, der immerzu gepflügt wird, trägt keine Frucht. ${ }^{836}$ In ihrem Buch Schrumpfungen konstatieren Frithjof Hager und Werner Schenkel: „Um so erstaunlicher ist es, dass das mit dem Wachstum verbundene Schrumpfen viel weniger untersucht ist. Es gibt keine Theorie der Schrumpfung, obwohl Schrumpfung ein Synonym für praktische Ökologie sein könnte. “6837 Auch wenn dieser Vergleich sich nicht auf die Weltbevölkerung übertragen lässt - hier wäre ein Oszillieren kaum wünschenswert - so zeigt er doch, dass Schrumpfen nicht ausschließlich negativ bewertet werden kann.

\subsection{Eine Strategie für den Übergang in Deutschland}

\subsubsection{Vorbemerkungen}

Um den demografischen Übergang bis zur Erlangung eines neuen, niedrigen Bevölkerungsgleichgewichts in Deutschland zu bewältigen, brauchen wir eine dreiteilige Strategie, bestehend erstens aus der tatsächlichen Realisierung der positiven Potenziale der Schrumpfung, zweitens einer pronatalistischen Wiederankur-

\footnotetext{
${ }^{833}$ Harrison (1994), 25

${ }^{834}$ Callahan (1976), 18

${ }^{835}$ Vgl. Kratochwil (1996), 115-119

${ }^{836}$ Hager/Schenkel (2003), 5

${ }^{837}$ A.a.O.
} 
belung der Geburtenraten aufgrund der Problematik des Bevölkerungsmomentums und drittens einer gezielten Einwanderungsteuerung.

\subsubsection{Die Chancen der Schrumpfung nutzen}

\subsubsection{Bevölkerungsentwicklung und Naturschutz in Deutschland}

Eher selten gehen die Gegner der Bevölkerungsschrumpfung in Deutschland auf ihre ökologischen Wirkungen ein. Dabei schreibt z.B. die Umweltakademie Baden-Württemberg: „Natürlich bietet die zu erwartende Schrumpfung der Bevölkerung auch Chancen für Natur und Umwelt. Die Entlastungseffekte in der Fläche bedeuten allein durch den zurückgehenden Flächenverbrauch zunächst positive Auswirkungen für Ökologie und Landschaft. Für die Umwelt scheint prinzipiell eine Entlastung in Sicht. Die baulichen Wucherungen der Städte gehen zurück. “6838 Diese Argumente gilt es näher zu betrachten.

\subsubsection{Fortdauernde Bodenversiegelung und Artensterben}

In Deutschland nimmt die Siedlungs- und Verkehrsfläche (SuV) täglich um 93

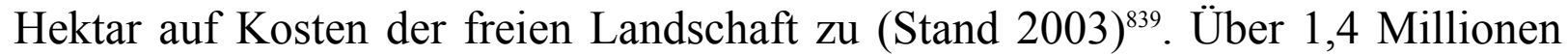
Hektar Bodenfläche, mehr als in jedem anderen europäischen Land, wurden seit 1970 durch Straßen, Parkplätze, Industrieanlagen und Wohnblocks verbaut. Zwar ist das BSP in den 1990er Jahren stärker gewachsen als die SuV, dennoch kann von einer Entkoppelung des Flächenverbrauchs von der wirtschaftlichen Entwicklung noch nicht gesprochen werden ${ }^{840}$. Während bei den anderen Umwelteinsatzfaktoren wie Wasser, Energie oder Rohstoffen, z.T. aufgrund des Einsatzes von umweltfreundlicheren Technologien und Prozessen, ein deutlich geringerer Input pro Einheit wirtschaftlicher Leistung erreicht werden konnte, gelang dies bei der Flächenproduktivität nicht. Die ,Urbanisierung' der noch ländlich geprägten Gebiete schreitet weiter voran. Problematisch ist zum einen die Inanspruchnahme von Grünland, zum anderen die unterlassene Renaturierung von bebautem, aber inzwischen brachliegendem Land. Von den verbauten 93 Hektar pro Tag wird knapp die Hälfte für Wohnzwecke neu in Anspruch genommen. Circa 30 Hektar davon liegen auf der grünen Wiese - der Rest auf ehemaligen Gewerbe-, Bahn- oder militärischen Flächen. ${ }^{841}$ In der ,Strategie für eine Nachhaltige Entwicklung' 2002 wurde der aktuelle ,Flächenfraß' als vorrangiges Umweltproblem identifiziert und gefordert, diesen bis 2020 auf 30 Hektar/Tag zu reduzieren. ${ }^{842}$

\footnotetext{
${ }^{838}$ Umweltakadamie Baden-Württemberg (2004), 102

${ }^{839}$ Zwischen 1997 und 2000 waren es durchschnittlich 129 ha pro Tag (89 ha/Tag in den alten und 40 ha/Tag in den neuen Ländern). Der Rückgang in den letzten Jahren ist im Wesentlichen auf den konjunkturell bedingten Rückgang der Bauinvestitionen zurückzuführen, vgl. Bundesregierung Deutschland (2004), 44-46.

${ }^{840}$ Siedentop/Kausch (2004), 37

${ }^{841}$ Troge (2004), 29

${ }^{842}$ Bundesregierung Deutschland (2002), 99
} 
Das Problem der Flächeninanspruchnahme muss in Zusammenhang mit den Zerschneidungseffekten durch Verkehrstrassen gesehen werden. ${ }^{843}$ Durch ein immer dichteres Straßennetz werden Tierarten von ihren Futterquellen abgeschnitten und gehen zugrunde. Wenn man die betonierten Feldwege dazurechnet, so erreicht das bundesdeutsche Straßennetz eine Dichte von $4 \mathrm{~km} / \mathrm{km}^{2}$. Unser Straßennetz ist inzwischen über 1 Mio. Kilometer lang. Aber nicht nur die Straßen, sondern auch Schienen, Leitungs- und Gasrohrtrassen und Kanäle zerschneiden die zusammenhängenden Biotope in immer kleinere Inseln. Das „Haus im Grünen" wird durch staatliche Subventionen wie der Eigenheimzulage und der Entfernungspauschale weiterhin attraktiv gemacht. Die Zersiedelung der Landschaft durch Zweithäuser auf dem Land wird dadurch gefördert.

Nach Angaben des Bundesamt für Naturschutz ist Flächenverbrauch in Zusammenhang mit Zersiedelung die Hauptursache für den Rückgang von zahlreichen Arten. ${ }^{84}$ Der effektivste Artenschutz besteht auch nach Ansicht von des Greifswalder Biologen Gorke im Schutz von Lebensräumen. ${ }^{845}$ Das Bundesamt für Naturschutz hat eine ,Rote Liste Biotope' erstellen lassen, die die Lebensräume katalogisiert. Die heimische Artenwelt verteilt sich auf etwa 500 Ökosystemtypen. Allerdings ist die überwiegende Zahl der interessanteren Ökosystemtypen auf nur 3 Prozent der Fläche der BRD konzentriert. Hier finden sich z.B. Hochmoore, Fluss-Auen, Wattenflächen und die Gebirgsregionen. Die restlichen 97 Prozent beherbergen nur wenige Biotoptypen. Dies sind die land- und forstwirtschaftlich genutzten Bereiche und die Siedlungen. Über zwei Drittel (69 Prozent) aller Biotoptypen sind gefährdet. ${ }^{846}$ „Der mit über 15 Prozent relativ hohe Anteil von völliger Vernichtung bedrohter Typen verdeutlicht, dass bei Fortwirken der Gefährdungsursachen mit dem vollständigen Verlust einer großen Zahl von Biotoptypen gerechnet werden muss", schreibt das Bundesamt für Naturschutz. ${ }^{847}$ Die Zersiedelung der Landschaft führt auf lokaler Ebene auch zu Veränderungen des Klimas, z.B. durch Überwärmung, Kaltluftstaus oder die Reduzierung der Luftfeuchtigkeit. Dies hat besonders auf empfindliche Biotope einschneidende Wirkungen und kann zum Verdrängen sensibler Tier- und Pflanzenarten führen ${ }^{848}$ Zudem kommt es zu Beeinträchtigungen des lokalen Wasserhaushalts. So verstärkt sich beispielsweise die Hochwassergefährdung durch Überbauung von Wasserrückhalteflächen und Besiedelung der Überschwemmungsgebiete. ${ }^{849}$

\footnotetext{
${ }^{843}$ Haaren/Nadin (2003), 347

${ }^{844}$ Bundesamt für Naturschutz (2002), 80

${ }^{845}$ Gorke (1999), $278 \mathrm{f}$.

${ }^{846}$ Bundesamt für Naturschutz (22.10.2002)

${ }^{847}$ A.a.O.

${ }^{848}$ Troge (2004), 30

${ }^{849}$ A.a.O.
} 
Wie im globalen Maßstab, so wirkt sich die Habitatszerstörung natürlich auch in Deutschland negativ auf die Populationsgrößen und letztlich auf die Artenvielfalt aus. In der Bundesrepublik sind schätzungsweise 45.000 Tierarten heimisch, von denen 16.000 hinsichtlich ihrer Gefährdung im Rahmen der Roten Liste bewertet wurden ${ }^{850}$ Inzwischen sind drei Prozent der bewerteten Arten ausgestorben oder verschollen, 40 Prozent mehr oder weniger stark gefährdet, bei drei Prozent ist eine Vorwarnung ausgesprochen worden und bei weiteren drei Prozent sind die Daten unzureichend. ${ }^{851}$ Nur die Hälfte der in Deutschland lebenden Arten (51 Prozent) gelten als ungefährdet. Die Zahl der gefährdeten oder schon ausgestorbenen Tierarten hat sich in Deutschland zwischen 1900 und 2002 rapide erhöht - nicht nur bei den Säugetierarten (51 Prozent gefährdet oder ausgestorben), sondern auch bei Brutvögeln (44 Prozent), Kriechtieren (79 Prozent) und Lurchen (67 Prozent). ${ }^{852}$

Solange die Bevölkerungsgröße weiter steigt und Deutschland immer dichter besiedelt wird, besteht wenig Hoffnung, daß die Zerschneidung der Landschaft zumindest teilweise rückgängig gemacht wird, und wieder größere Flächen als Naturschutzgebiete ausgewiesen werden können.

Bezogen auf alle Formen von Schutzgebieten hatte die Enquete-Kommission „Schutz des Menschen und der Umwelt“ bereits 1998 angeregt, 15 Prozent der Fläche Deutschlands dem Naturschutz zu widmen. Dies entspricht dem europäischen Durchschnittswert, der in Deutschland bis heute nicht erreicht wird. So weist Nordrhein-Westfalen nur 5,4 und Niedersachen nur 6,5 Prozent seiner Landesfläche für den Naturschutz aus, während die anderen Bundesländer immerhin neun bis zwölf Prozent unter Naturschutz stellten bzw. dies angekündigt haben. Der Anteil von Nationalparks, die zu mindestens 50 Prozent frei von regulativen Eingriffen des Menschen bleiben müssen, liegt an der terrestrischen Landesfläche in Deutschland sogar bei nur 0,55 Prozent. ${ }^{853}$

Wie schwer es oft fällt, heimischen Tieren und Pflanzen der Raum zu überlassen, den brauchen, zeigen die Auseinandersetzungen um das Naturschutzgebiet Bayrischer Wald. Dort wurde ein Gebiet von 10.000 Hektar wieder der Natur überlassen. Aufgebrachte Bürger leisteten lange Zeit erbitterten Widerstand gegen das Verbot, das Gebiet wirtschaftlich zu nutzen.

\footnotetext{
${ }^{850}$ Bundesamt für Naturschutz (1998), 19

${ }^{851}$ A.a.O.

${ }^{852}$ A.a.O., 20 f. Bei den Pflanzen stehen rund 40 Prozent auf der Roten Liste, von denen knapp 4 Prozent ausgestorben oder verschollen sind (Bundesamt für Naturschutz 2000, 17).

${ }^{853}$ Bundesamt für Naturschutz (2004). Telefonische Erläuterungen von Dr. Volker Scherfhose, Fachgebietsleiter Großschutzgebiete am 2.3.2005. Inklusive der drei Wattenmeer-Nationalparks beträgt der Anteil an der Fläche Deutschlands rund 2,5 Prozent. Da die Nord- und Ostsee aber kein Lebensraum für den Menschen ist, spielt hier die Verdrängungsproblematik eine untergeordnete Rolle. Nach Ansicht von Dr. Scherfhose begünstigt die vorausberechnete Schrumpfung die Anstrengungen der Naturschutzverbände, die Fläche von 0,55 Prozent deutlich zu steigern und insbesondere mehr zusammenhängende Nationalparks einzurichten.
} 
Es sind - mit Ausnahme der Niederlande - v.a. die dichtbesiedelsten Länder Europas, in denen das Artensterben am weitesten vorangeschritten ist (vgl. Abb. 39).

\section{Abb. 39: Rote Listen im europäischen Vergleich (Säugetiere)}

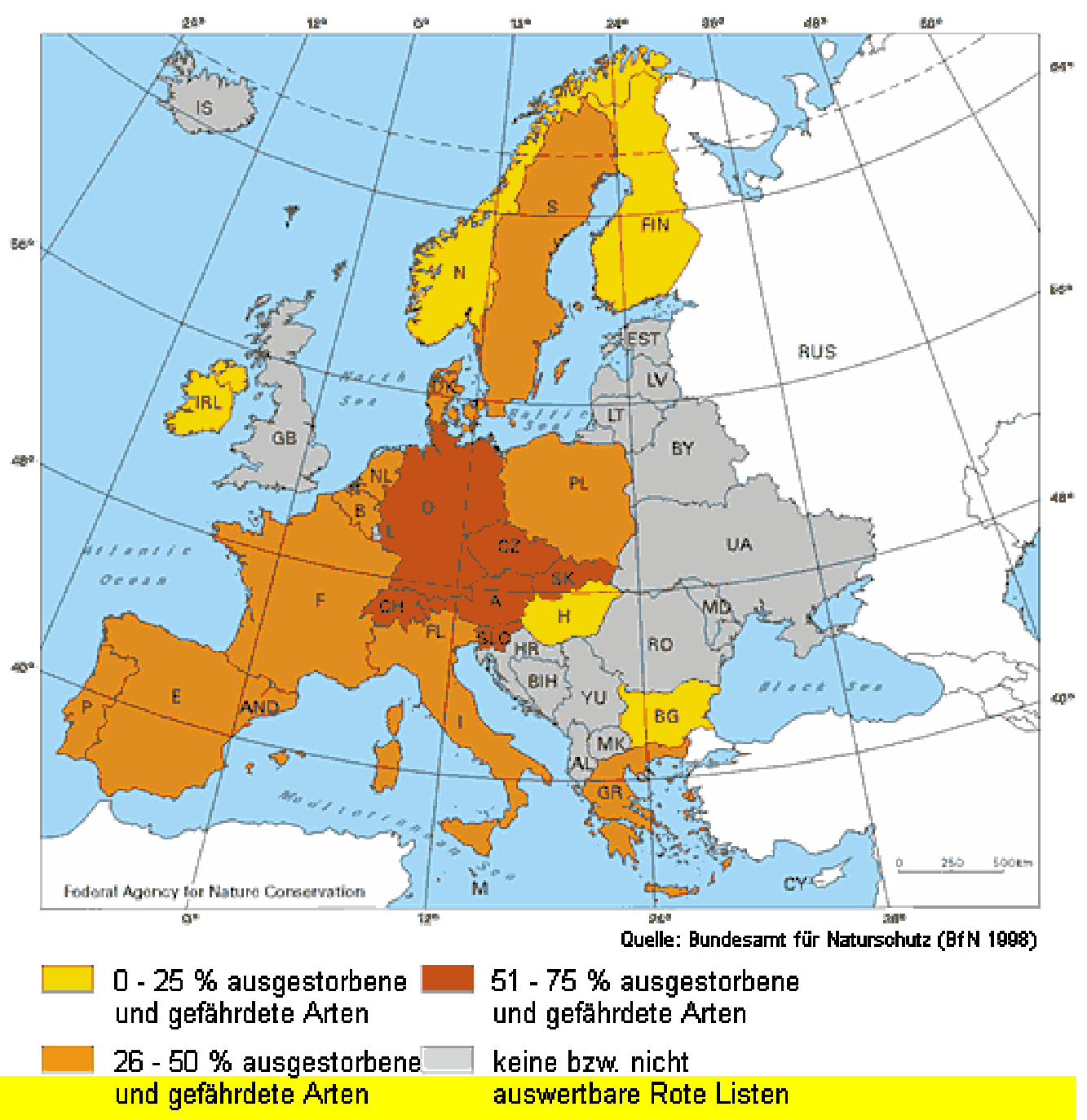

\section{Quelle: www.bfn.de/03/030101_abb34.htm}

Hier bietet sich durch die demografische Entwicklung erstmals seit Jahrhunderten die Chance zur Trendwende.

\subsubsection{Bewusstes Schrumpfen}

In vielen Regionen Deutschlands (und Europas) ist der Schrumpfungsprozess nicht mehr aufzuhalten, deshalb sollten wirksame Strategien für einen Umgang damit entwickelt werden. Wir brauchen städtebauliche Ansätze, die unsere Städte auf den Rückgang der Bevölkerung einstellen und sogar Chancen dieser Entwicklung zu identifizieren versuchen. Ostdeutschland, das seit der Wende von 16 
Mio. auf 14,5 Mio. Einwohner ${ }^{854}$ schrumpfte und damit knapp 10 Prozent seiner ursprünglichen Bevölkerung verlor, hat im Zeitraffer durchgemacht, was in den nächsten 30 Jahren in Gesamtdeutschland passieren wird. Schrumpfungsprozesse sind aber kein typisch deutsches Problem, sondern weltweit zu beobachten - in Glasgow in den englischen Midlands ebenso wie in Detroit im Rustbelt in den nordöstlichen Staaten der USA. In Osteuropa und Russland gehören , Geisterviertel' in vielen Städten schon zum Stadtbild ${ }^{855}$ Diese Beispiele zeigen somit aber auch, wie dringend eine Strategie für die Schrumpfung benötigt wird. Denn die Potentiale werden nur realisiert, wenn weitere Rahmenbedingungen verändert werden: „Denn solange Gewerbesteuer und Einkommensteuernachteile für die kommunalen Finanzen eine entscheidende Rolle spielen, solange sich öffentliche Einrichtungen nur rentieren, wenn eine entsprechende Anzahl von Nutzern vorhanden ist und solange das Planungsrecht hierzu unverändert bleibt, werden weiterhin aus Gründen der Attraktivität Wohn- und Gewerbegebiete ausgewiesen“, schreibt die Umweltakademie Baden-Württemberg. ${ }^{856}$

\subsubsection{Herausforderungen für den Städtebau}

Angesichts der demografischen Entwicklungen in vielen SEL steht der Städtebau vor einer epochalen Aufgabe. „Die Möglichkeiten reichen vom Wohnungsrückbau bis zur vollständigen Entsiedelung und Renaturierung einiger Regionen. Aller Erfahrung nach ist es sinnvoll, den Rückbau von den Stadträndern nach innen zu organisieren und kein Geld in die Sanierung von Außenbezirken zu stecken. Um dabei Fehlplanungen zu verhindern und weil der demografische Wandel nicht vor Ländergrenzen halt macht, sollten die betroffenen Kommunen und Kreise länderübergreifend zusammenarbeiten“, schreibt das Berlin-Institut in seiner Studie zur demografischen Zukunftsfähigkeit Deutschlands. ${ }^{857}$ Der Abriss überflüssiger Wohnhäuser, die Transformation von Landschaftsbrachen und Infrastrukturen sowie die Aufwertung funktionsfähiger Stadtteile stellen eine organisatorische, planerische und künstlerische Herausforderung dar. „Es sind noch viele Überlegungen notwendig, welche Maßnahmen aus städtebaulicher Sicht für Infrastruk-

\footnotetext{
${ }^{854}$ Die Zahl 16,02 Mio für 1990 ist inklusive Ost-Berlin gerechnet. Seit 2000 werden die Zahlen für Ostdeutschland ohne Berlin erhoben, dies waren 13,5 Mio. Schätzt man die Menschen hinzu, die im ehe. Ostteils leben, so kommt man auf rund 14,5 Mio.

${ }^{855}$ Auf der Website www.ortzukunft.de sind die Vorschläge vorgestellt, die 22 internationale Architekten, Künstler und Landschaftsgestalter für ,rückwärts wachsende Städte' unter dem Motto ort.zukunft: weniger ist mehr erarbeitet haben.

Unter www.bauhaus-dessau.de sind die Ergebnisse einer Internationale Bauausstellung in SachsenAnhalt, die die Praxis des Stadtrück- und umbaus durch modellhafte Projekte profilieren will, dargestellt.

In dem Projekt Schrumpfende Städte (www.shrinkingcities.com) untersuchen Architekten, Wissenschaftler und Künstler die jüngere Entwicklung von Detroit, Ivanovo, Manchester/Liverpool und Halle/Leipzig.

${ }^{856}$ Umweltakademie Baden-Württemberg (2004), 8

${ }^{857}$ Kröhnert/van Olst/Klingholz (2004), 92
} 
tur- und Instandhaltungskosten, Energieeinspar- und $\mathrm{CO}_{2}$-Emissionsminderungsziele langfristig am sinnvollsten sind, um Siedlungen mit schrumpfender Bevölkerung weiter zu entwickeln“, schreibt Troge. ${ }^{858}$ Einige Bestandteile einer solchen Strategie sind aber bereits identifizierbar.

\subsubsection{Eine attraktive Vision für Planer und Architekten entwickeln}

Es ist eine attraktive Vision, durch weitsichtige städtebauliche Planung im Zuge der nächsten 60 Jahren die schlimmsten architektonischen Sünden der Vergangenheit zu beseitigen. In den nächsten 30 Jahren können wir unsere Städte schöner machen! Es ist zentral, für die unterschiedlichen Strategien des Umbaus neue und andere Marketingkonzepte zu entwickeln, die Schrumpfung nicht mehr als zu beseitigendes Übel, sondern als kreativ zu gestaltende Tatsache aufzeigen. Sie müssen die Potenziale aufzeigen und sie bei den vielfältigen Akteuren, die am Umbauprozess beteiligt sind, publik machen. Begriffe wie ,Shrink positiv', ,Weniger ist mehr', ,Lean City' und ,Stadtverschlankung' schaffen gesellschaftliche Akzeptanz für die Schrumpfung.

Der Künstler Wolfgang Kil gibt in der Internetausgabe seines Buches Luxus der Leere zu bedenken: „Planer und Architekten wurden bisher dazu erzogen, ihr Glück im Entstehen von etwas Neuem zu finden. Nun sollen sie vorschlagen, was nicht geschehen, oder gar: was ersatzlos verschwinden soll. Dabei stehen sie sich mit ihrem traditionellen Berufsbild vielfach selbst im Weg. “659

Abschied von der Doktrin des Wachstums zu nehmen ist heute - gut 30 Jahre nach dem Bestseller des Club of Rome immer noch eine ungewohnte Idee für Politiker, Planer und Architekten. Die jetzt geforderten Strategien zielen nicht mehr auf ,dichter' und ,höher', sondern jetzt geht es um ,kleiner', ,langsamer', vielleicht ,eleganter'. Wo früher um Zuwächse (an Bauvolumen, Wohnfläche, Ausstattung usw.) gerungen wurde, geht es heute um Freisetzungspotenziale. ${ }^{860}$

Sinnvoll wäre es, auf Bundesebene einen hochdotierten Preis für die Nutzung der Chancen der demografischen Schrumpfung auszuschreiben. Preiswürdige Konzepte wären dezentrale Versorgungsanlagen (z.B. Planzenkläranlagen) und mobile, temporäre und arbeitsteilige Angebotsformen (z.B. städtisches Ruftaxi statt regulärer Buslinie) oder eine Nutzung leerstehender Hallen als Show-Rooms (z.B. für Oldtimer-Messen ${ }^{861}$ oder für bisher im Freien stehende Gebrauchtwagen). Denn auch das Schrumpfen braucht ein Ziel. Häußermann und Siebel beschreiben das Dilemma: „Einerseits bietet das Schrumpfen sicherlich Chancen für ein von Zeitzwängen und Raumknappheit entlastetes Leben. Zeit und Platz zu haben ist wohl der größte Luxus, den eine Gesellschaft, die den Hunger abgeschafft hat, bieten kann. Andererseits wissen wir immer weniger, wie ein humanes, von Zeit-

\footnotetext{
${ }^{858}$ Troge (2004), 33

${ }^{859}$ Kil (2004). www.luxus-der-leere.de

${ }^{860}$ A.a.O.

${ }^{861}$ o.V. (23.08.04), 87
} 
zwängen und Raumnot entlastetes Leben in den großen Städten aussehen soll. Und solange wir das nicht wissen, werden auch Alternativen zur gegenwärtig dominierenden Stadtpolitik schwer durchzusetzen sein, selbst wenn diese dominierende Politik in den schrumpfenden Städten unrealistisch und mit schweren negativen sozialen, stadtstrukturellen und ökologischen Folgen belastet ist.“" ${ }^{\text {‘862 }}$

\subsubsection{Gezielterer Abriss}

Anstatt abzureißen, was sich von selbst entleert bzw. entleert hat, müssten bestimmte Liegenschaften identifiziert werden, die zuerst entleert werden sollen, um sie dann abzureißen. ${ }^{863} \mathrm{Im}$ Osten hat man z.T. zuerst die schlechtesten Plattenbauten saniert, weil die als erstes zusammen zu fallen drohten. Zwei Jahre später wurden sie dann abgerissen, weil alle Leute weggezogen waren. ${ }^{864}$ In Brandenburg wurden bis Ende 2003 in 24 Städten rund 9.000 Wohnungen abgerissen. ${ }^{865}$ Der große Abriss steht noch bevor: Im Herbst 2003 meldete das Land Brandenburg 165.000 Wohnungen über Bedarf. ${ }^{866}$ Allein im sächsischen Leipzig stehen mit 58.000 so viele Wohnungen leer, wie die Stadt Ulm insgesamt besitzt. Insgesamt sind in Ostdeutschland eineinhalb Millionen Wohnungen überzählig. ${ }^{867}$ Darin könnten nominell so viele Menschen Unterkunft finden, wie in Rostock, Magdeburg, Halle, Leipzig, Dresden, Chemnitz und Erfurt insgesamt leben. „Und da lauert auch die ungeheuerlichste Provokation: Könnten diese Städte dann nicht von der Landkarte verschwinden?" fragt Kil. ${ }^{868}$

In den meisten ostdeutschen Kommunen sind die Einwohnerzahlen seit der deutschen Vereinigung gesunken, z.B. in Cottbus minus 13,8 Prozent, in Magdeburg minus 16 Prozent, in Greifswald minus 17 Prozent und in Schwerin, Rostock und Zwickau etwa jeweils minus 20 Prozent (vgl. Abb. 41). Görlitz verlor sogar fast ein Viertel seiner Einwohner.

Dabei ist allerdings zu berücksichtigen, dass ein Großteil der Schrumpfungen in diesem Zeitraum auf Wanderungsbewegungen innerhalb Deutschlands, von Ost nach West, zurückzuführen ist.

\footnotetext{
${ }^{862}$ Häußermann/Siebel (2003), 85

${ }^{863}$ Beispielsweise existiert in Oberursel im Taunus ein sehr hässliches städtisches Altersheim in schöner Wohnlage. Bürgermeister und Stadtverordnete haben gegen Widerstände die Entscheidung getroffen, dieses zu entmieten, um es abzureißen. Die Fläche soll entweder einem angrenzenden Naturschutzgebiet zugeschlagen werden oder erneut als Bauland für Luxuswohnungen ausgewiesen werden.

${ }^{864}$ Kröhnert/van Olst/Klingholz (2004), 92

${ }^{865} \mathrm{Kröhnert/van} \mathrm{Olst/Klingholz} \mathrm{(2004),} 42$

${ }^{866}$ Kil (2004)

${ }^{867}$ Kil gibt diese Zahl an. Niejahr $(02.01 .2003,9)$ spricht von 1,3 Millionen leerstehenden Wohnungen.

${ }^{868}$ Kil (2004)
} 


\section{Abb. 40: Veränderung der Einwohnerzahlen ausgewählter ostdeut- scher Städte 1988 bis 1999}

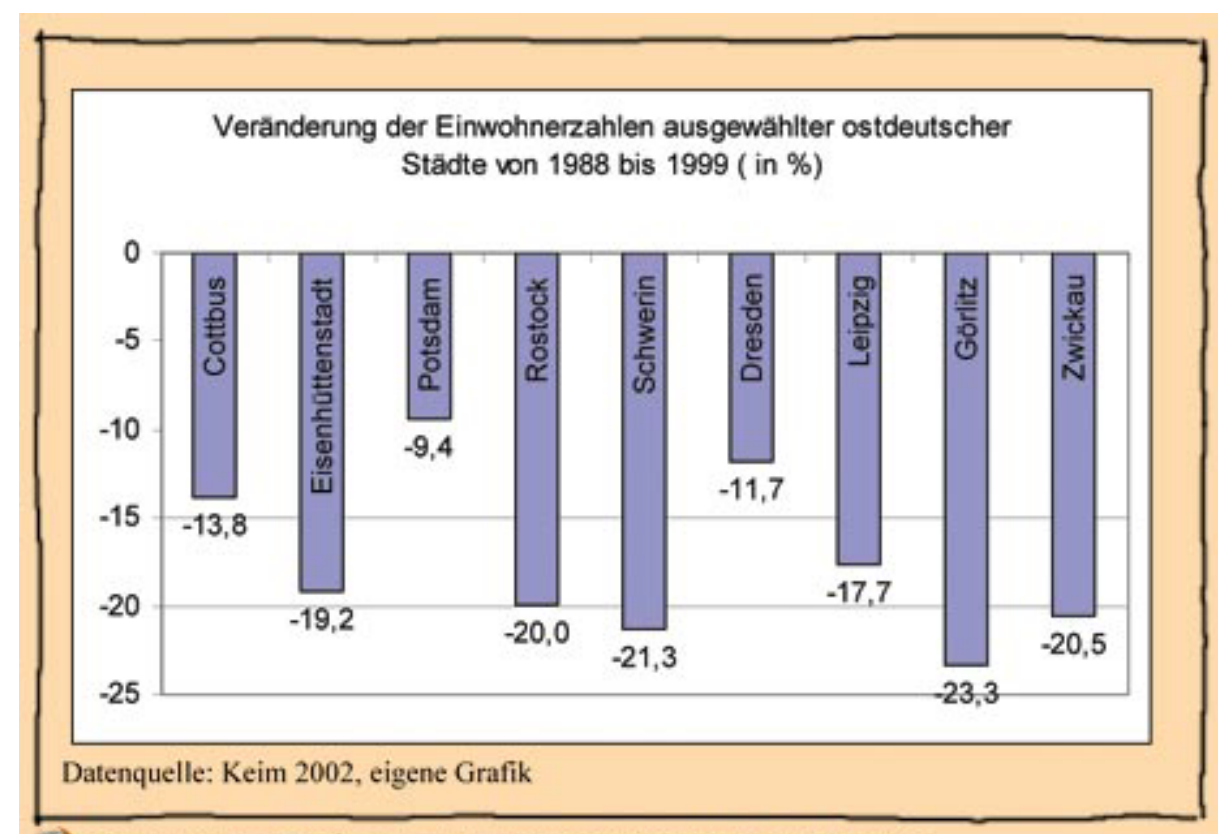

BOKUN-INSTITT FîR WETTEEVÖLERUNG WND GLOBALE ENTWICKLWNG

Quelle: Berlin-Institut für Weltbevölkerung und globale Entwicklung, http://www.berlin-institut.org/pages/fs/fs_grafiken.html

Anderthalb Millionen nicht mehr benötigte Wohnungen zwingen zur gründlichen Planung von Abrissen. So sollte z.B. zur Auflage gemacht werden, dass einzelne Häuser nicht aus geschlossenen Zeilen herausgebrochen werden. Aus energetischer Sicht ist dies nicht vorteilhaft, weil sich dadurch die relative Fläche der Außenwände vergrößert. Bei Leerstand innerhalb der Gebäudekomplexe wiederum fließt Wärme von bewohnten in unbewohnte Räume, was ebenfalls zu energetischen Verlusten führt. ${ }^{869}$

\subsubsection{Wohnen auf der, Grünen Wiese' unattraktiver, in den Städten attraktiver machen}

Bereits in den letzten Jahren war zu beobachten, dass die Deutschen von entlegenen ländlichen Gebieten in Ballungsräume ziehen. Dort lassen sie sich jedoch auch im Westen - im Umland nieder, nicht in den Innenstädten. Wie Abbildung 41 zeigt, liegen die Kreise mit den höchsten Zuwanderungsraten ausnahmelos in den Speckgürteln der Städte, wohingegen Berlin, München oder Frankfurt an Einwohnern verlieren.

${ }^{869}$ Troge (2004), 33 


\section{Abb. 41: Deutschland zieht um}

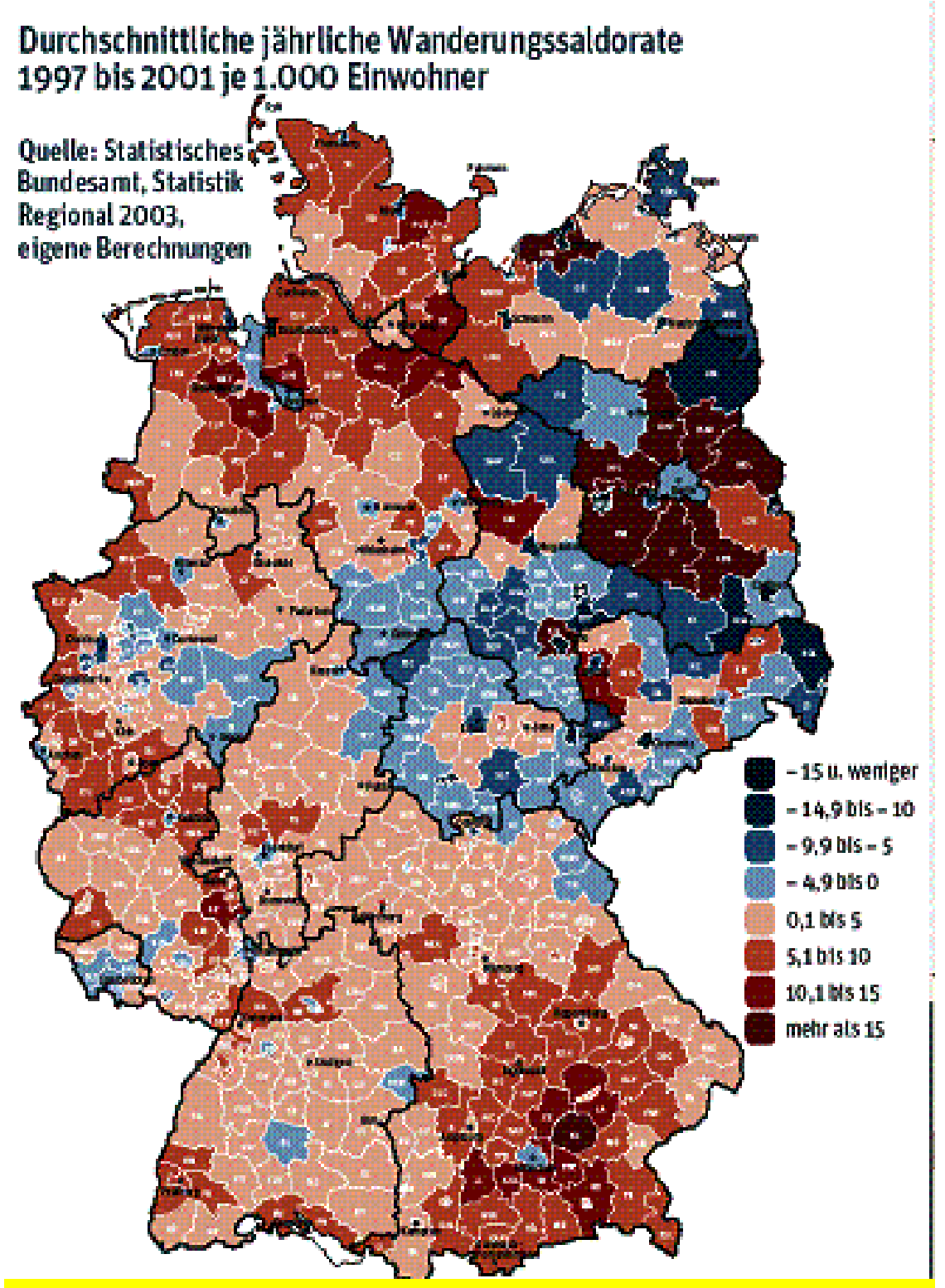

\section{Quelle: Kröhnert/van Olst/Klingholz (2004), 13}

Dies schafft ökologische Probleme durch das tägliche Pendeln und die Zersiedelung des Umlandes. „Pendlerpauschale und Eigenheimzulage, in Wirklichkeit versteckte Subventionen der Bauwirtschaft, beschleunigen den Zerfall der Städte. Erfolgreiche Ansätze der Re-Urbanisierung, wie etwa in Leipzig, sollten unterstützt und vervielfältigt werden," schreiben die Autoren der Studie Deutschland $2020{ }^{870}$ Unter dem Stichwort ,perforierte Stadt' entwickelten Stadtplaner in Leipzig das Leitbild einer Stadt, die auf weniger Dichte setzt. Dabei werden die

\footnotetext{
${ }^{870}$ Kröhnert/van Olst/Klingholz (2004), 92
} 
günstigen Wohnungspreise durchaus als Marketingargument für die Stadt genutzt. $220 \mathrm{~m}^{2}$ Wohnfläche für 600 Euro Warmmiete überzeugen auch kühle Rechner.

Tab. 16: Wohnfläche pro Person in Leipzig 1849-2001

\begin{tabular}{|l|l|l|l|l|l|l|l|l|}
\hline & 1849 & 1879 & 1891 & 1913 & 1939 & 1960 & 1985 & 2001 \\
\hline $\begin{array}{l}\text { Wohnfläche } \\
\text { pro Person } \\
\text { (in qm }^{2} \text { ) }\end{array}$ & 40 & 25 & 27 & 26 & 42 & 54 & 68 & 131 \\
\hline
\end{tabular}

\section{Quelle: Doehler-Behzadi/Lütke-Daldrup (2004), 34 f.}

Solch komfortablen Wohnverhältnisse, nicht zu sprechen von Grünflächen und Parks, verursachen paradoxerweise sogar wieder Zuzüge. ${ }^{871}$

Wie in vielen anderen Städten auch hat es Leipzig mit gegenläufigen Trends zu tun: wachsen und schrumpfen zugleich. Der Buchtitel Plusminus Leipzig bringt das auf den Punkt. ${ }^{872}$ In Leipzig hat man eingesehen, dass Schrumpfungsprozesse ebenso geplant werden müssen wie Wachstumsprozesse. Der wichtigste Grundsatz ist, dass von Leerstand und sozialer Ghettoisierung bedrohte Quartiere umso erfolgreicher als Wohnstandorte gehalten werden können, je enger Stadtplaner und Wohnungseigentümer ihre Vorhaben miteinander abstimmen. ${ }^{873}$ In Gelsenkirchen will man Hauseigentümer motivieren, ihren Bestand $\mathrm{zu}$ modernisieren, Wohnungen zusammenzulegen und Höfe zu entkernen. ${ }^{874}$ Wo früher heruntergekommene Areale standen, entstehen Parks und Abenteuer-Kindergärten.

\subsubsection{Demografische Daten bei jeder Planung berücksichtigen}

Die Strategien des Schrumpfens müssen für die sozialen Schichten und Nutzergruppen differenziert ausgearbeitet werden: für alte Menschen, für Nutzer, die weiter billige Wohnungen nachfragen und für mobile Bevölkerungschichten, die in bestimmten Lebensphasen solche Bauten als Zwischennutzung für andere Wohnformen annehmen. Auf die Schwierigkeiten bei Bevölkerungsprognosen wurde bereits hingewiesen - aber auch auf die Notwendigkeit, die Annahmen mehr als bisher zu diskutieren und somit die Forschung in diesem Bereich eher zu verstärken als zu reduzieren. Der Rahmentrend einer Schrumpfung und Alterung Deutschlands steht für die große Mehrzahl der Bevölkerungsstatistiker nicht in Frage. Die notwendigen Konsequenzen werden von Politikern und Planern häufig jedoch nicht gezogen.

Während z.B. Verkehrsexperten mehrheitlich von einem Rückgang der Verkehrsleistungen ausgehen, plant das Bundesverkehrsministerium für die Spanne

\footnotetext{
${ }^{871}$ Kil (2004)

${ }^{872}$ Doehler-Behzadi/Lütke-Daldrup (2004)

${ }^{873}$ Klingholz (2004), 128

${ }^{874}$ Klingholz (2004), 126-130
} 
von 1997 bis 2015 mit einem 16-prozentigen Wachstum des Verkehrsaufkommens. ${ }^{875}$ Angesichts der Tatsache, dass schon seit 1999 auf Deutschlands Straßen weniger gefahren wird, ${ }^{876}$ ist dies eine Verschwendung von finanziellen Ressourcen. Der Rückgang der Kilometerleistung wird sich noch beschleunigen, wenn ab 2015 die geburtenstarken Jahrgänge das fahrfreudigste Alter verlassen. ${ }^{877}$ Die wachstumsorientierte Perspektive der Bundesverkehrswegeplanung bedarf dringend der Korrektur. ${ }^{878}$ Das gleiche gilt auch für die Fördertatbestände des Gemeindefinanzierungsgesetzes.

Anders als das Bundesverkehrsministerium richtet z.B. die (leicht schrumpfende) Gemeinde Ludwigsburg ihre Bedarfsplanung bei Kindergartenplätzen, Schulen, Friedhofsflächen, Einrichtungen für Ältere und Sportstätten an demografischen Daten für den Zeitraum von 20 Jahren aus.

\section{Abb. 42: Bevölkerungsentwicklung und kommunale Infrastruktur- bereiche}

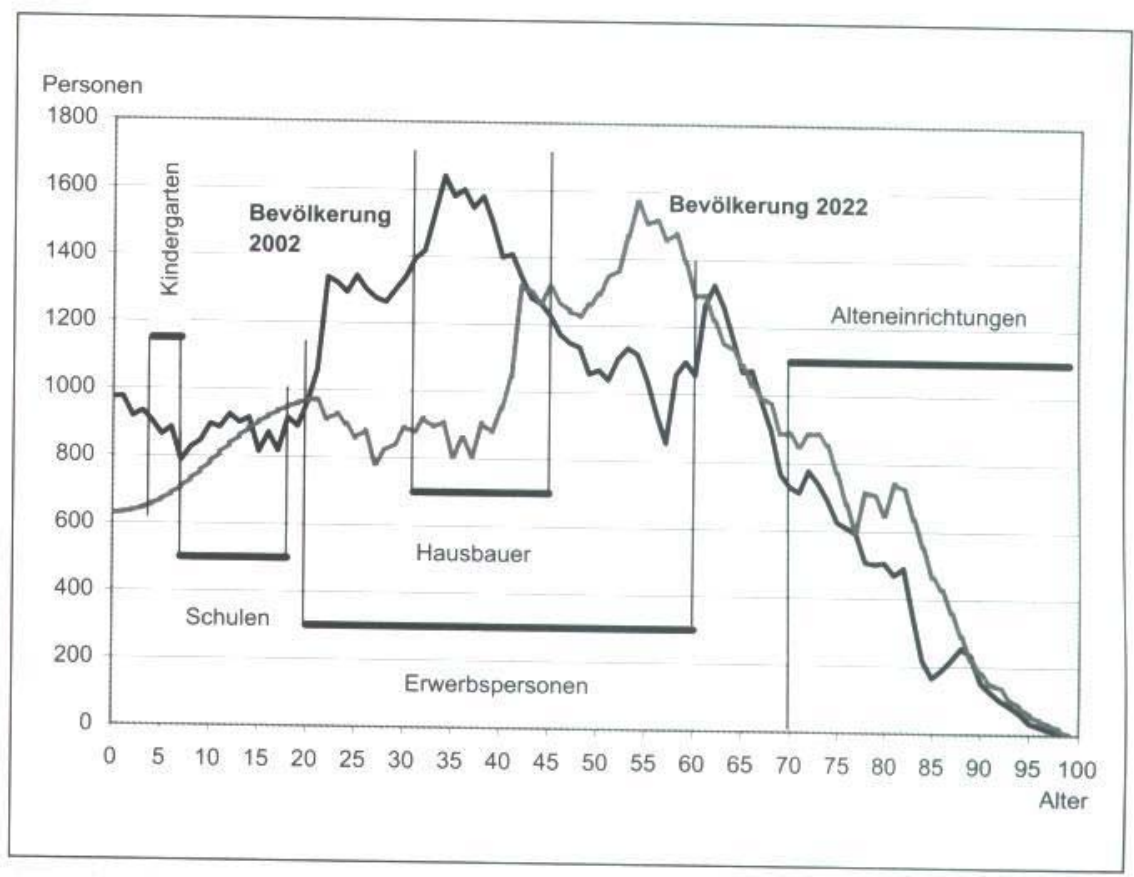

\section{Quelle: Köhl (2004), 85}

Ludwigsburg geht anhand dieser Daten z.B. von einer Bedarfssteigerung an Friedhofsfläche in Höhe von 47 Prozent, einer Abnahme der Kindergartenbevölkerung von über 40 Prozent und einer Reduzierung der Bevölkerung im Führer-

\footnotetext{
${ }^{875}$ Bundesverkehrsministerium (2003), 60

${ }^{876}$ Bundesverkehrsministerium (2002/2003), zitiert nach Kröhnert/van Olst/Klingholz (2004), 92

${ }^{877}$ Chlond/Manz/Zumkeller (2002)

${ }^{878}$ Gans (2004)
} 
scheinalter um etwa 13 Prozent, jeweils bis 2050, aus. ${ }^{879}$ Gerade solche Prognosen auf regionaler Ebene sind geeignet, den Flächenbedarf bestimmter Altersgruppen zu verdeutlichen. Wie Abb. 42 zeigt, nimmt bis 2050 der Zahl der Hausbauer stark ab. Nur ein bestimmtes Segment der Erwerbspersonen bildet die Gruppe der ,Hausbauer', nämlich jene, welche bereits lange genug im Beruf stehen, um sich den Bau eines Hauses leisten zu können und die gleichzeitig durch ihre Familiengründungen auch den Wunsch danach verspüren. Ein alleinstehender Single fühlt sich häufig in einer komfortablen Stadtwohnung wohler als in einem großen Haus auf dem Land, selbst wenn er sich letzteres leisten könnte.

Ludwigsburg ist die Ausnahme: Ein Symposium der Umweltakademie BadenWürttemberg zur Schrumpfung kommt zu dem Ergebnis, dass „es auf kommunaler Ebene zur Feststellung der Lage konkreter Daten über den Bevölkerungswandel bedarf. Viele Kommunen verfügen hier noch nicht über spezifische Daten und spielen weiterhin planerisches Russisch Roulette. ${ }^{6880}$

\subsubsection{Keine öffentliche Förderung mehr für die Erschließung neuer Bauflächen in stark schrumpfenden Regionen}

Die herrschende Meinung ist in schrumpfenden Regionen immer noch, dass mit einer konsequenten Wachstumspolitik (insbesondere High-Tech-Förderung) Schrumpfungsbereiche kompensiert werden könnten. ${ }^{881}$ Wo es kein Wachstum mehr gibt, wird (oft schuldenfinanziertes) ,Scheinwachstum' inszeniert. ${ }^{882}$ Dass gerade in Ostdeutschland der Flächenverbrauch in den 1990er Jahren noch stärker gestiegen ist als im Westen, ${ }^{883}$ deutet auf völlig unrealistische Wachstumserwartungen und eine überzogene Angebotsplanung der Kommunen hin. Denn gerade dort renaturiert Deutschland am schnellsten (vgl. Abb. 43). Anstatt ausgewählte Zentren, die noch realistische Erfolgschancen haben, zu stärken, versickern die Fördermittel im Raum. Statt, geordneten Rückzugs' aus manchen Ortschaften animiert das herrschende Wachstumsparadigma manche Bürgermeister $\mathrm{zu}$ „kommunalpolitischen Verzweiflungstaten“" ${ }^{884}$ etwa die schuldenfinanzierte Erstellung schlüsselfertiger Industrieparks, in die niemals Industrie einziehen wird. Viele heute geplante und realisierte Bau- und Gewerbegebiete werden in naher Zukunft leer stehen. Die Aufrechterhaltung einer überdimensionierten Infrastruktur lässt die Gebührenbelastung der Haushalte spürbar steigen. „Immer weniger Einwohner werden für immer stärker überdimensionierte Netze bezahlen müssen. Eine quantitative Reduktion des Flächenverbrauchs ist daher

\footnotetext{
${ }^{879}$ Köhl (2004), 85

${ }^{880}$ Umweltakademie (2004), 103

${ }^{881}$ Solche Aussagen werden von den Stadt- und Regionalsoziologen Hartmut Häußermann und Walter Siebel als ,Propaganda` bezeichnet (Häußermann/Siebel 2003, 83).

${ }^{882}$ Hannemann (2003), 99

${ }^{883}$ Siedentop/Kausch (2004), 41

${ }^{884}$ Jessen (2004)
} 
auch aus ökonomischen Gründen dringend geboten", folgern die Raumplaner Siedentop und Kausch ${ }^{885}$ Die Städte, die sich mit der unvermeidlichen Schrumpfung arrangieren, sind die Städte der Zukunft. Anstatt intelligent mit der Schrumpfung umzugehen, erfolgen öffentliche Investitionen immer noch, als würde Deutschland in den nächsten Jahren massiv wachsen.

\section{Abb. 43: Deutschland renaturiert}

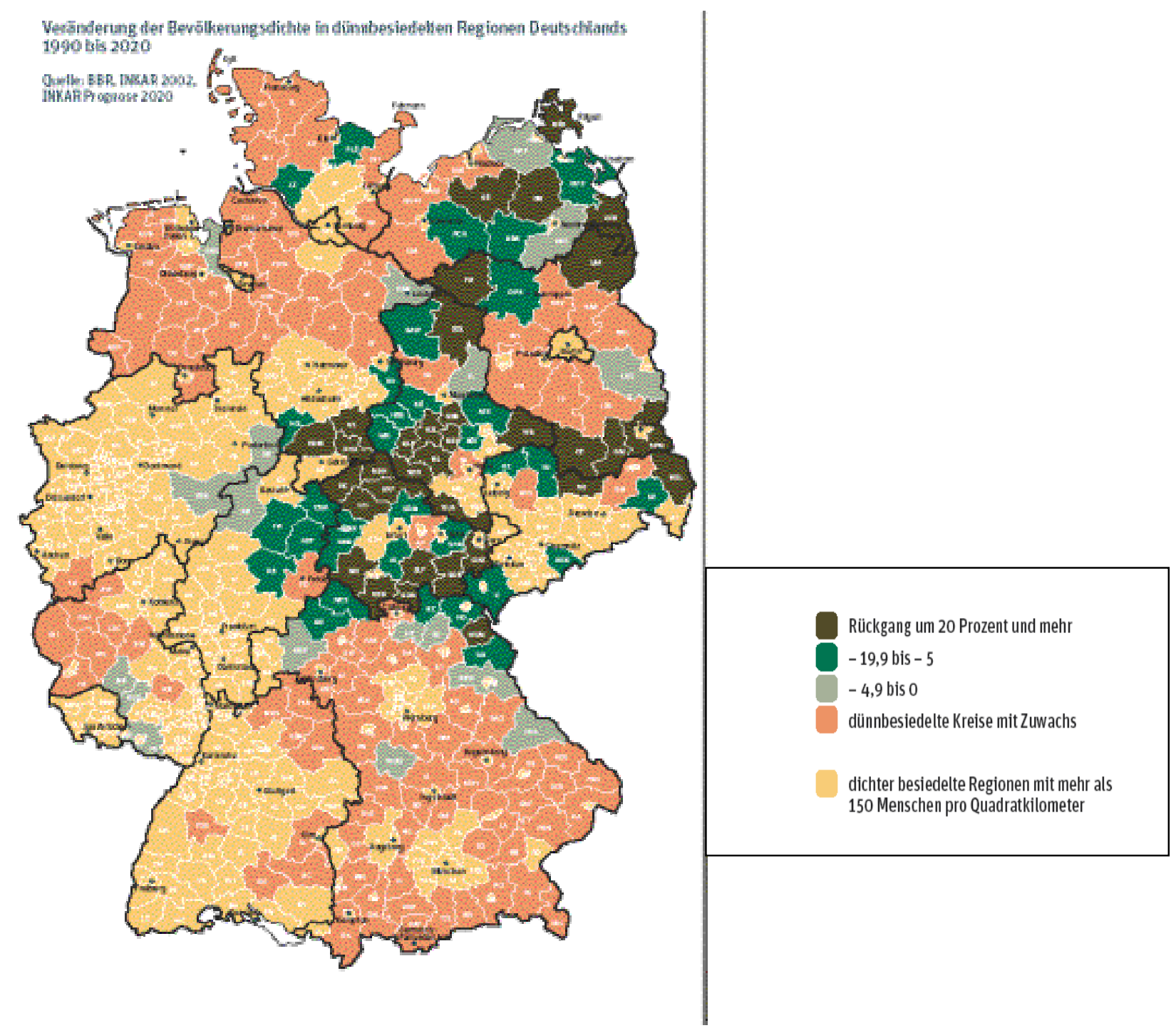

\section{Quelle: Quelle: Kröhnert/van Olst/Klingholz (2004), 21}

Deutschland und Europa könnten in Zukunft wieder sehr viel mehr intakte Ökosysteme haben als heute. Im nationalen Maßstab sollten v.a. für Ostdeutschland gezielt Pläne entwickelt werden, um zusammenhängende Naturschutzgebiete einzurichten. Sieben der insgesamt 15 deutschen Nationalparks befinden sich bereits

\footnotetext{
${ }^{885}$ A.a.O., 47
} 
in den neuen Bundesländern, da Ostdeutschland weite Gebiete mit wenig gestörtem Naturraum besitzt. In Regionen, in denen Menschen abwandern und die Landwirtschaft extensiviert wird, erobert die Natur Terrain zurück. In der Vergangenheit hat man jedoch eher einzelne Dörfer ausgedünnt, anstatt die Aufgabe bzw. Zusammenlegung von Dörfern aktiv zu fördern. Nur durch letzteres könnten aber zusammenhängende Naturschutzgebiete geschaffen werden, die es ermöglichen, dass auch scheue Großtiere wie der europäische Bison, Luchs und Wolf wieder nach Deutschland zurückkehren.

Nun hat Deutschland wegen seiner territorialen Offenheit deutlich weniger endemische Arten als Inseln oder Regenwaldgebiete. ${ }^{886}$ „Würde das Saarland ausgelöscht, würde keine Art aussterben. Mit den Hawaii-Inseln würden dagegen 850 endemische Blütenpflanzen, 956 Mollusken, 5188 Insekten und 60 Vogelarten für immer von diesem Planeten verschwinden“", schreibt der Biogeograf Müller. ${ }^{887}$ Dies ist richtig, entbindet uns aber nicht von unserer eigenen ethischen Verpflichtung zum Artenschutz. Zwar können ausgestorbene Arten nicht wiedergewonnen werden, aber für verdrängte Arten bietet ein Bevölkerungsrückgang hierzulande große Potenziale. ${ }^{888}$ Wie die folgende Fallstudie zeigt, müsste schon vieles falsch gemacht werden, wenn dadurch die ,Rote Liste' nicht dünner würde.

\subsubsection{Fallstudie: Die Rückkehr der Wölfe in die Lausitz}

Unter der Überschrift „Wenn ländliche Regionen sich entvölkern, gewinnt die Natur" berichtet das Berliner Institut für Weltbevölkerung und globale Entwicklung über die Rückkehr der Wölfe in die Lausitz. ${ }^{889}$ Zwei Wolfsrudel leben inzwischen dauerhaft in der Oberlausitz, nahe der Grenze zu Polen. Vierzehn Welpen kamen allein im Frühjahr 2004 zur Welt. Nach Ansicht von Steffen Flath, Umweltminister des Freistaates Sachsen, ist dieser einzige frei lebende Wolfsbestand Deutschlands ein Grund zur Freude und „ein Symbol für eine einzigartige Naturlandschaft" ${ }^{890}$ Die Rückkehr der Wölfe zeigt, dass die Zunahme der Artenvielfalt in Deutschland nicht mit der Alterung, sondern nur mit der Schrumpfung korreliert ist. Um es überspitzt zu sagen: Die Natur kehrt zurück, wo keine bzw. wenig Menschen sind - egal, ob junge oder alte.

\footnotetext{
${ }^{886}$ Ethisch folgt daraus möglicherweise tatsächlich eine größere Verpflichtung zum Artenschutz von artenreichen WEL. Wer eine bestimmte Ressource nicht besitzt, kann auch nicht ethisch verpflichtet sein, sie zu erhalten. Im Falle des Artenschutzes liegen die Dinge aber komplizierter. Die Artenvielfalt in den WEL hat auch für Menschen in den SEL einen Wert, was sich u.a. an ihrer Zahlungsbereitschaft ablesen lässt („Was wäre es Ihnen wert, die letzten Tiger vor dem Aussterben zu retten?“). Da die Erhaltung dieser Arten aber de facto nur für die Menschen jener Länder, in denen diese Arten heimisch sind, ökonomische Einbußen bedeutet, besteht die ethische Verpflichtung der SEL, einen finanziellen Ausgleich zu leisten. Im Falle des Tigers, der z.B. in Indien vorkommt, würde also ein finanzieller Ausgleich fällig.

${ }^{887}$ Müller (2003), 198

${ }^{888}$ Umweltakademie Baden-Württemberg (2004), 102

${ }^{889}$ Berlin-Institut (9.10.2003)

${ }^{890}$ A.a.O.
} 
Wie das Berlin-Institut für Weltbevölkerung in seinem Newsletter schildert, war Canis lupus ursprünglich in ganz Europa verbreitet. Im 18. und 19. Jahrhundert wurde der Wolf jedoch in weiten Teilen Mitteleuropas ausgerottet. Jäger stellten ihm nach, und die Wälder, die ihm - und seinen Beutetieren - Lebensraum boten, verschwanden. Nur in sehr abgelegenen Gegenden konnte der scheue Räuber sich behaupten.

Einzelne umherstreifende Wölfe wurden jedoch immer wieder auch in besiedelten Gebieten beobachtet. Die Oberlausitzer Population gründet sich wahrscheinlich auf ,Einwanderer', die vor vier Jahren aus Polen kamen. In der vergleichsweise unberührten Wildnis des Truppenübungsplatzes Oberlausitz fanden sie offenbar ideale Bedingungen, um sesshaft zu werden. Der Truppenübungsplatz ist aus Sicherheitsgründen nicht öffentlich zugänglich. Das Berlin-Institut führt die Ansiedlung der Wölfe direkt auf den Bevölkerungsrückgang zurück: „Der Landkreis Niederschlesische Oberlausitz ist mit 79 Einwohnern je Quadratkilometer eher dünn besiedelt. Zwischen 1990 und 1999 hat die Einwohnerzahl um 6,9 Prozent abgenommen, vor allem, weil der Braunkohlebergbau und die Energieerzeugung nach der Wende wegbrachen.

In der nördlich des Truppenübungsplatzes gelegenen Stadt Weißwasser, wo es zu DDR-Zeiten noch eine Glasindustrie gab, sackte die Einwohnerzahl von 37.388 im Jahre 1987 binnen vierzehn Jahren auf 25.000. Die Westwanderungsrate betrug dort nach Angaben des Leipziger Leibnitz-Institutes für Länderkunde im Jahre 2001 4,3 Prozent, das heißt, von jeweils 1.000 Einwohnern versuchten 43 ihr Glück im alten Bundesgebiet. Das ist der höchste Landflucht-Wert der neuen Bundesländer."“891

Nach Ansicht des Berlin-Instituts für Weltbevölkerung und globale Entwicklung bieten sich für die Wölfe gute Überlebensbedingungen auch in anderen Regionen. Denn der Schwund durch Geburtenrückgang und Abwanderung beschränkt sich nicht auf die Randlage in Sachsen. Im Herzen Deutschlands, im Thüringer Wald, ist nach den Prognosen des Bundesamtes für Bauwesen und Raumordnung zwischen 2000 und 2020 ein Rückgang um bis zu 18,6 Prozent zu erwarten - mehr noch als in der Oberlausitz (14,6 Prozent) oder im benachbarten Landkreis Löbau-Zittau (16,8 Prozent).

Nicht nur im Osten, auch im Hunsrück oder im Pfälzerwald weisen die Bevölkerungzahlen eine abnehmende Tendenz auf. Wo Industrie und Gewerbe fehlen, wo bäuerliche Betriebe stillgelegt werden, weil Landwirtschaft sich nicht mehr lohnt, gewinnt die Natur Oberhand. Damit sind gute Voraussetzungen für die Wiederansiedlung von Tieren geschaffen, die lange keine Chance hatten. Im Pfälzerwald leben bereits seit einiger Zeit wieder Luchse. Sie kamen aus Frankreich, wo durch Landflucht ganze Regionen praktisch verwaist sind.

${ }^{891}$ A.a.O. 


\subsubsection{Die Menschen begleiten}

Wichtig ist aber auch, die negativen Folgen in der Übergangszeit zu thematisieren und das Los der verbleibenden Einwohner schrumpfender Dörfer zu erleichtern. In den Regionen, die von der Schrumpfung betroffen sind, leiden die Menschen in der Regel darunter. Die ökologischen Chancen sind zwar Förstern und Naturschützern bewusst, aber nicht der Mehrheit. Es sind v.a. die Jungen und Qualifizierten, die weggehen. Der Abbau der verbleibenden Infrastruktur wird von den weniger Mobilen und den Älteren, die dableiben, als Verlust empfunden.

Wenn in ihrem Ort die Hälfte der Leute wegzieht, das Postamt, die Sparkasse und das Schwimmbad geschlossen werden und Bus, Bahn und der Brötchendienst nicht mehr kommen, dann geht mit der Infrastruktur auch Lebensqualität verloren. Dies ist ein schrittweiser Prozess. In einer dicht besiedelten Stadt wird es durch den demografischen Wandel in aller Regel zunächst ein Mehr an Lebensqualität geben: Endlich findet man wieder einen Parkplatz in der eigenen Strasse, endlich kann der Wunsch vom Einfamilienhaus in der Stadt verwirklicht werden, weil die Baulücke nebenan nicht sofort durch ein neues Reihenhaus geschlossen wird. Erst wenn der demografische Wandel ein weitergehendes Stadium erreicht, ${ }^{892}$ kann sich das Gefühl von Vereinsamung und Zurückgelassenwerden einstellen. Für die weit überwiegende Zahl von Städten ist es das Ziel, den Rückbau auf einem niedrigeren Niveau zu stabilisieren. ${ }^{893}$ Für einige Dörfer, deren Einwohnerzahl weit unter die kritische Masse sinkt, ist es dagegen wie beschrieben sinnvoller, sie ganz aufzugeben anstatt ihre (dann) überdimensionierte Infrastruktur weiter zu erhalten. Wolfgang Kil weist darauf hin, dass man die Verlierer des Wandels, also die Immobilen (Alte, Arme, Arbeitslose, Ausländer bzw. Aussiedler) die in den ,verlorenen Dörfern' am längsten ausharren werden, nicht alleine lassen darf: „Schrumpfungsprozesse planen heißt also, in erster Linie die Lebensbedingungen und Interessenlagen von Verlierern wahrzunehmen und $\mathrm{zu}$ verteidigen. (...) $\mathrm{Zu}$ denen, die ,als letzte das Licht ausmachen', sollten unbedingt Architekten und Planer gehören. Für die unvermeidlichen und schmerzhaften Verluste bedarf es Vorkehrungen, Veranstaltungen, Planungsverfahren und Realisierungsschritte, die - über alle technischen und organisatorischen Notwendigkeiten hinaus - den Betroffenen signalisieren, dass ihre Lebenszeit in und mit diesen Gehäusen nicht umsonst und folgenlos war. Wagen wir ruhig den Vergleich: Das Wegnehmen einer Stadt bedarf des Rates des Experten genauso, wie es ihr Aufbau einstmals brauchte. Das ist wie mit den Ärzten - deren Beistand ist beim Beginn eines Menschenlebens genauso vonnöten wie an dessen Ende. [...] Die Auseinandersetzung mit ,vergehenden' Städten ist also ein Vorgang von allerhöchster Verbindlichkeit. Sie setzt die Bereitschaft voraus, nicht nur Einstiegsdiagnosen oder flotte Masterpläne abzuliefern, sondern ganz direkt und persönlich Teilnehmer eines langen

${ }^{892}$ Wie im Abschnitt 6.8.3. Aktive pronatalistische Geburtenpolitik beschrieben, kann durch gesellschaftliche Strategien versucht werden, dass ein weitergehendes Stadium gar nicht erreicht wird.

${ }^{893}$ Häußermann/Siebel (2003), 81 
und mühseligen Prozesses zu werden. Die Aufgabe heißt nicht Beglücken, sondern Begleiten. “ ${ }^{\text {8994 }}$

Zusammenfassend kann man sagen, dass noch erheblicher Forschungsbedarf besteht, um die Potenziale optimal nutzen zu können. Zu den wichtigsten Aufgaben zählt aber die Durchsetzung eines Paradigmenwechsels hinsichtlich der Schrumpfung. In Gesamtdeutschland lebten 1950 rund 68 Mio. Menschen, nach der niedrigen Projektion des Statistischen Bundesamtes werden es 2050 wieder so viele sein. Ist dies wirklich eine so erschreckende Vision?

\subsubsection{Umweltzerstörung durch Kinderlosigkeit?}

Ein interessantes, wenn auch falsches Argument ist, dass Kinderlosigkeit indirekt für Umweltzerstörungen verantwortlich sei. So schreibt Jürgen Borchert: „Schwieriger ist allerdings die Tatsache zu erkennen, dass Kinderlosigkeit in Deutschland auch ökologisch verheerend wirkt, weil sie - in Energiemaßstäben gemessen - paradoxerweise wie ein massiver Beitrag zur Überbevölkerung wirkt. Die infolge der Kinderersparnis entstehenden Einkommensüberhänge wandern nämlich nahezu zwangsläufig in den ökologisch besonders schädlichen Luxuskonsum: Fernreisen, exotische Ernährungsgewohnheiten, übermäßiger Wohnraumverbrauch mit hohen Energiekosten, etc. ${ }^{6895}$ Bochert geht davon aus, dass die Umweltbilanz einer Durchschnittsfamilie mit ein bis zwei Kindern im Durchschnitt günstiger ist als die eines Singles. Dies erscheint höchst unwahrscheinlich. Durch die Familiengründung steigt die benötigte Wohnraumgröße und die Städte verlieren Einwohner an das Umland. Die Ausdehnung der Siedlungsflächen erzeugt Verkehr, dieser forciert die weitere Versiegelung und Überbauung. ${ }^{896} \mathrm{Da}-$ durch leiden Flora und Fauna. Viele Kinderlose frönen zudem nicht, wie Borchert unterstellt, einem luxusriösem Lebensstil.

Die Beziehungen zwischen Familiengröße und Umweltbelastung werden im Grunde schon durch die PAT-Formel erfasst. Die beschriebenen Schwierigkeiten, die Beziehung zwischen Bevölkerungszunahme und den verschiedenen Umweltproblemen quantitativ-exakt auszudrücken, darf nicht dazu führen, dass diese Beziehung gänzlich bestritten wird. In einer Modellrechnung kam Nancy Birdsall zu dem Ergebnis, dass es weniger als die Hälfte kosten würde, $\mathrm{CO}_{2}$-Emissionen indirekt durch Verhinderung von (ungewollten) Geburten zu reduzieren, anstatt sie im nachhinein durch Ökosteuern zu reduzieren. ${ }^{897}$

\footnotetext{
${ }^{894}$ Kil (2004)

${ }^{895}$ Hessische Staatskanzlei (2003), 129 f.; Ähnlich Birg (2004b), 23

${ }^{896}$ Troge (2004), 30

${ }^{897}$ Birdsall (1994), zitiert nach UN Population Division (2001b), 37
} 


\subsubsection{3 „Demografische Nachhaltigkeit“?}

Die öffentliche Fixierung auf die Risiken des Bevölkerungsrückgangs hat zugenommen. Vor diesem Hintergrund versuchen pronatalistisch eingestellte Personen und Institutionen, den Neologismus „demografische Nachhaltigkeit“" im öffentlichen Bewusstsein zu etablieren. So schreibt Herwig Birg: „Viele Menschen bewegen die Sorgen über eine ökologisch nachhaltige Entwicklung unseres Landes und unseres Planeten. Dabei kommt es ihnen meist nicht in den Sinn, dass zur ökologischen Nachhaltigkeit eigentlich auch eine demografische Nachhaltigkeit (Herv. J.T.) gehört. Dass auch der Mensch eine natürliche Spezies ist, deren abnehmende Zahl in Deutschland und in anderen Industrieländern nicht weniger alarmierend ist als die der zurückgehenden Populationen einiger Tier- und Pflanzenarten, scheint niemanden wirklich zu kümmern. Die Bevölkerungsabnahme wird sogar oft begrüßt, weil sie eine positive Dividende für die Umwelt abzuwerfen scheint. Dabei wird übersehen, dass mit den geringfügigen positiven Wirkungen auf die natürliche Umwelt gleichzeitig negative Auswirkungen auf die soziale und gesellschaftliche Mitwelt verbunden sind.“6898

Inhaltlich verbinden die Propagandisten des Begriffes „demografische Nachhaltigkeit" damit die Vorstellung, dass - wie bei der ökologischen oder finanziellen Nachhaltigkeit - ein Fließgleichgewicht einzuhalten sei. So wie die Menge der den Gewässern entnommenen Fischbestände nicht die Reproduktionsrate der erneuerbaren Ressource „Fisch“ übersteigen oder die Höhe der Staatsausgaben langfristig nicht die Höhe der Staatseinnahmen übersteigen sollte, so sollte eine Bevölkerung gleich bleiben oder wachsen, auf keinen Fall aber schrumpfen. Diese Definition verkennt nicht nur die heutige Definition von Nachhaltigkeit, die auch starke intragenerationelle Aspekte hat ${ }^{899}$ sondern sie ist auch völlig sachfremd. Der in Ökologie und Finanzwissenschaft mit erklärendem Adjektiv (ökologische Nachhaltigkeit, finanzielle Nachhaltigkeit) verwendete Begriff der Nachhaltigkeit lässt sich nicht auf demografische Zusammenhänge übertragen. Wie in dieser Studie gezeigt, können nachrückende Generationen durchaus Vorteile haben, wenn die Bevölkerungsgröße sinkt. Die sehr komplexen Zusammenhänge zwischen Generationengerechtigkeit und Bevölkerungsgröße sollen nun mit dem Begriff ,demografische Nachhaltigkeit" auf eine einfache Norm reduziert werden: „Wir alle haben die Pflicht dafür zu sorgen, dass die Bevölkerung nicht abnimmt!“. Wenn dies sachlich nicht gerechtfertigt ist, warum unternehmen interessierte Kreise dennoch den Versuch, einen derartigen Begriff durchzusetzen? Begriffe wie dieser begründen Sichtweisen auf reale Verhältnisse und wer den Definitionskampf gewinnt, der setzt seine Interessen auch in der realen Welt durch. „Wer die Sprache beherrscht, beherrscht die Menschen“, so Schelsky. ${ }^{900}$ Und einem chinesischen Sprichwort zufolge antwortete Kung Fu Tse auf die Frage, was

\footnotetext{
${ }^{898}$ Birg (2001), 12

${ }^{899}$ Vgl. den Abschnitt „Begriffsdefinitionen“.

${ }^{900}$ Zitiert nach Heringer (1982), 19
} 
er als erstes nach einer Regierungsübernahme anpacken wolle: „Sicherlich die Richtigstellung der Begriffe“.901 Tatsächlich gab es Versuche der Sprachmanipulation bei Schlüsselwörtern in fast allen Diktaturen. In der NaziZeit wurde sogar per Erlass festgelegt, dass das Wort ,tapfer' nur auf deutsche Soldaten anzuwenden sei. ${ }^{902}$ Auch der amtliche Sprachgebrauch von , antifaschistisch' in der DDR ist bezeichnend.

Nun gibt es in einer Demokratie viele Vorkehrungen dagegen, dass sprachliche Sinngebung als Mittel der Herrschaft so eingesetzt wird wie in Orwells 1984. Aber es ist auch nicht zu bestreiten, dass in unserem Medienzeitalter politische Entscheidungen sprachlich hübsch verpackt werden müssen, um eine Chance auf Durchsetzung zu haben. Wenn eine Partei ihre Wählerstimmen maximieren will, so kommt sie zwangsläufig in die Versuchung, die Kernbegriffe der Politik in ihrem Sinne zu instrumentalisieren, das heißt: umzudefinieren. Da dies alle Parteien gleichermaßen versuchen, besteht auch nicht die Gefahr, dass dies vollständig gelingt, zumindest nicht in Ländern mit Meinungsfreiheit. Pawlowski spricht in diesem Zusammenhang von ,persuasiven Definitionen'. Bei der persuasiven Definition wird versucht, eine emotionale Reaktion auszulösen. Durch geschicktes Definieren werden die mit bestimmten sprachlichen Ausdrücken verbundenen emotionalen Assoziationen ausgenützt und auf andere Ausdrücke übertragen. ${ }^{903}$

Damit dies nicht beim Begriff ,demografische Nachhaltigkeit“ gelingt, sollten seriöse Bevölkerungssoziologen und -ethiker frühzeitig einen Riegel vorschieben. Denn dieser Begriff ist wirklich dem Denken rechtsradikaler Kreise geschuldet, die damit ihre Ängste vor dem Aussterben der Deutschen in die Mitte der Gesellschaft tragen wollen. ${ }^{904}$

\subsubsection{Zusammenfassung}

Gilt also der Zusammenhang zwischen Bevölkerungswachstum und Artenverlust auch in umgekehrter Richtung? Was ist von der These zu halten, dass mit einem Bevölkerungsrückgang die Artenvielfalt zunimmt (These 1). Überspitzt gefragt: Kann durch die Bevölkerungsschrumpfung in Deutschland der Living Planet Index wieder verbessert werden? So wie in Bezug auf Gebiete in anderen Teilen der Welt die Kausalität zwischen zunehmender Bevölkerungsdichte und Artenverlust gilt, so gilt diese Beziehung natürlich auch für Deutschland. Dagegen gibt es weit weniger empirische Hinweise, dass eine abnehmende Bevölkerungsdichte eine Zunahme an Arten bewirken würde. Zunächst einmal erschafft die Natur neue Arten nur in Zeitskalen, die im Bereich von Hundertausenden bzw. Millionen von Jahren liegen. Wenn also im Zeitraum von wenigen Jahrzehnten die Bevölkerungsdichte in einem Gebiet sinkt, so führt dies nicht zu einem Feuerwerk von

\footnotetext{
${ }^{901}$ Heringer (1982), 24

${ }^{902}$ Heringer (1982), 30

${ }^{903}$ Pawlowski (1980), 244

${ }^{904}$ Vgl. für andere Beispiele eines solchen Denkens: Butterwegge (2003)
} 
ganz neuen Lebensformen. These 1 kann also nicht bestätigt werden. Anders sieht es bei den ausgewanderten Arten aus. Es spricht viel dafür, dass Arten, die es zwar nicht mehr in Deutschland, aber in seinen weniger dicht besiedelten Nachbarländern gibt, bei einer abnehmenden Bevölkerungsdichte in Deutschland wieder heimisch werden würde (These 2). Als Beispiel wurde schon die aus Polen eingewanderte Wolfspopulation genannt. Ebenso ist es wahrscheinlich, dass die Bestände heimischer Arten zunehmen, wenn die Bevölkerungsdichte sinkt (These 3). Also können schrumpfende Länder in den gemäßigten, artenärmeren Regionen neben dem Erhalt ihrer eigenen Arten viel für den Populationsschutz tun. Viele Arten, die heute nur noch ex situ (d.h. nicht mehr in der freien Wildbahn, sondern in Zoos) überleben, könnten wir leicht rückbürgern, wenn die Landbevölkerung dem zustimmen würde. ${ }^{905}$ Das gilt z.B. für den in Deutschland im Freiland ausgerotteten Wisent, den Braunbär, den Luchs oder den Wolf. Voraussetzung ist, dass große zusammenhängende Nationalparks ausgewiesen werden. Die bisher ausgewiesenen Nationalparks sind zu klein, um die Ansiedelung solcher ausgewanderter Arten zu ermöglichen. Der Luchs bespielsweise benötigt in freier Wildbahn ein Revier von mindestens 100 Quadratkilometern, die relativ kleinen Flächen, die in Deutschland als Nationalparks ausgewiesen sind, reichen ihm nicht zum Leben. Erfolge bei der Reduzierung der menschlichen Flächeninanspruchnahme vergrößern also die Chancen für die Erhaltung der Artenvielfalt für zukünftige Generationen. Der Nachhaltigkeitsbeirat Baden-Württembergs empfiehlt, dass der Flächenverbrauch in diesem Bundesland ,in einem überschaubaren Zeitraum, z.B. bis zum Jahr 2020, auf ein Viertel des heutigen Niveaus zurückgefahren werden soll, also auf durchschnittlich drei Hektar pro Tag." ${ }^{\text {“906 Und }}$ bezüglich der Erreichbarkeit dieses Ziels fährt er fort: „Ein solches Ziel ist insbesondere angesichts der erwarteten Bevölkerungsentwicklung in Baden-Württemberg durchaus zu erreichen. ${ }^{\text {“907 }}$

Allerdings ist bei These 2 und 3 der Zusammenhang nicht monokausal. Eine quantitative Prognose - etwa dass bei einer Senkung der Bevölkerungsdichte um 10 Prozent die Bestände der Tierarten generell um 10 Prozent steigen - wäre unseriös.

\subsubsection{Aktive pronatalistische Geburtenpolitik}

\subsubsection{Berücksichtigung des Trägheitseffektes}

Wie Demografen hervorheben, ist aufgrund des Trägheitseffekts (,population momentum') eine gewisse Schrumpfung praktisch unumkehrbar, nur ein weiteres Absinken nach 2050 ließe sich noch vermeiden, wenn frühzeitig Maßnahmen

\footnotetext{
${ }^{905}$ Müller (2003), 205

${ }^{906}$ NBBW (2004), 4

${ }^{907}$ A.a.O.
} 
unternommen werden. ${ }^{908}$ Nach Berechnungen von Herwig Birg würde es selbst dann, wenn die Geburtenrate schrittweise bis 2030 auf das bestanderhaltende Niveau ansteigen würde, bis 2080 dauern, bis die Schrumpfung zum Stillstand käme und die Geburtenbilanz wieder ausgeglichen wäre (vgl. Abb. 25). Auch wenn außerdem 150 Tausend jüngere Menschen pro Jahr einwanderten, würde sich an der langen Zeitspanne bis zum Ende der Schrumpfung nicht viel ändern, die Geburtenbilanz bliebe auch dann bis 2068 negativ. Und selbst wenn pro Jahr 300 Tausend jüngere Menschen netto einwanderten, wäre die Geburtenbilanz bis 2060 defizitär. ${ }^{909}$ Eckart Bomsdorf kommt zwar zu dem Schluss, dass eine Geburtenrate von 1,7 (zu erreichen bis 2015) ausreicht, aber er setzt bei seinen Berechnungen eine relativ hohe Zuwanderung voraus. ${ }^{910}$ In jedem Fall schärfen solche Berechnungen das Bewusstsein, dass das Bevölkerungsmomentum auch in Richtung Schrumpfung mit einem schwerfälligen Tanker vergleichbar ist, der beizeiten gestoppt werden muss. Vier Familien mit jeweils einem Kind haben eben mehr Nachkommen als eine Familie mit 3 Kindern. Wer eine Bevölkerung von z.B. 70 Millionen als Zielgröße nimmt, der darf nicht erst dann, wenn dieser Wert erreicht ist, pronatalistische Maßnahmen einleiten. Dann ist es sozusagen schon 30 Jahre nach 12 Uhr. Kaufmann betont die zunehmende Wucht der demografischen Implosion: „Bei den seit drei Jahrzehnten andauernden, die Reproduktion nur noch zu zwei Dritteln gewährleistenden Fertilitätsverhältnissen in der Bundesrepublik haben 1.000 Frauen nur noch 667 Töchter, 444 Enkelinnen und 296 Urenkelinnen. “911 Wie Abb. 44 zeigt, geht die Basis für die eigenständige Reproduktionsfähigkeit Deutschlands, also die Zahl der Frauen im Alter von 1549 Jahren, ohne eine Kurskorrektur immer stärker zurück.

Der zweite Teil der Strategie für die demografische Zukunftsfähigkeit Deutschlands umfasst Maßnahmen, um die Geburtenrate wieder auf das Bestandhaltungsniveau von 2,1 Kindern pro Frau steigern. Dieses Plädoyer für eine aktive Geburtenpolitik steht nicht im Widerspruch zu dem ersten Teil der Strategie für die demografische Zukunftsfähigkeit Deutschlands, also zur Nutzung der Chancen der Schrumpfung. Aus der negativen Bewertung von - aus ökologischer Sicht relativer oder absoluter Überbevölkerung folgt ja nicht der Wunsch nach Selbstauslöschung, sondern ein Plädoyer für eine Stabilisierung der Bevölkerung auf ökologisch nachhaltigem Niveau. Wenn die derzeitige Fertilitätsrate von 1,4 anhielte, wird nach den Langzeitprojektionen der Population Division Deutschland im Jahr 2300 nur noch rund 3 Mio. Einwohner haben, so viele wie heute Berlin. Dies kann niemand wollen.

\footnotetext{
${ }^{908}$ Kaufmann (2004a), 9

${ }^{909}$ Birg (2004a), 13

${ }^{910}$ BMFSFJ (2005)

${ }^{911}$ Kaufmann (2004a), 9
} 


\section{Abb. 44: Anzahl der Fraven im Alter von 15-49 Jahren}

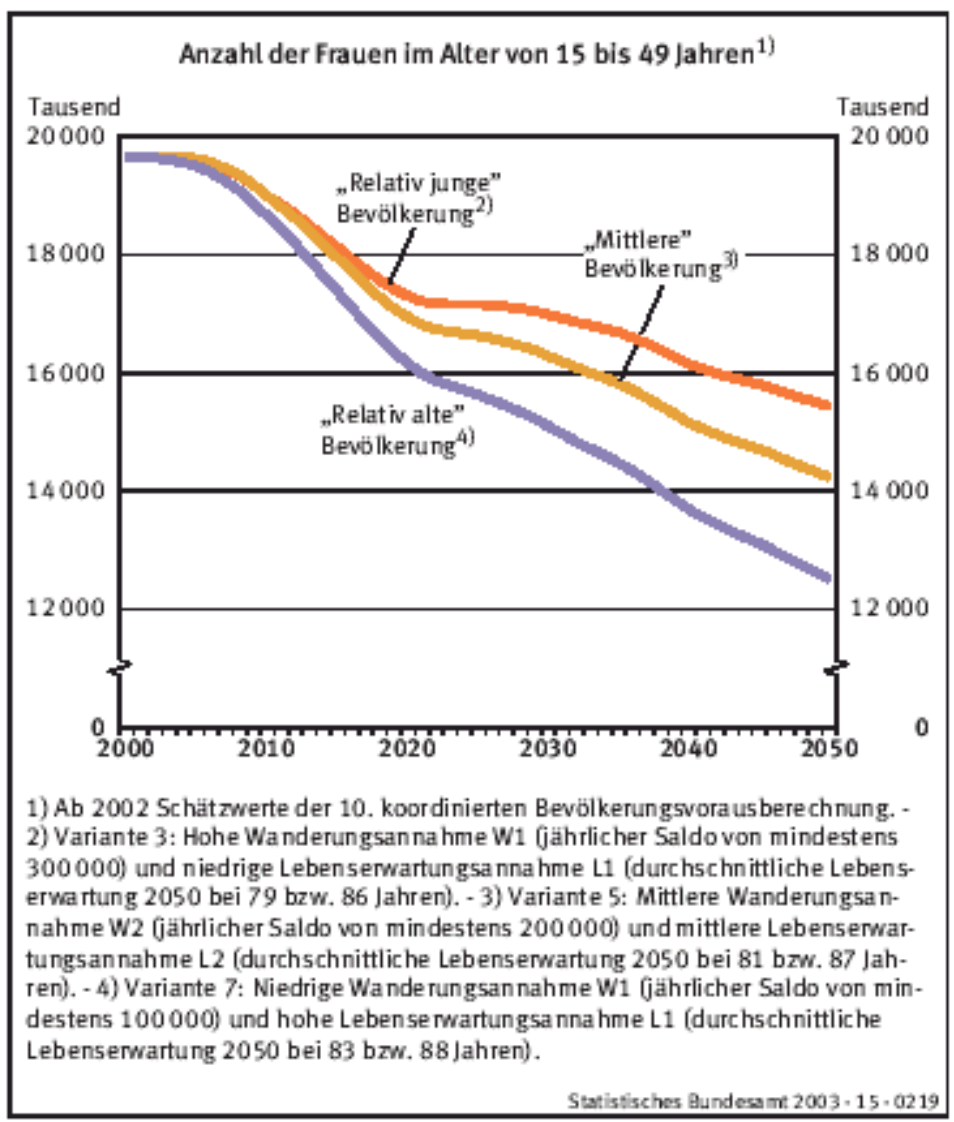

\section{Quelle: Statistisches Bundesamt (2003), 27}

Ein erstrebenswertes Szenario ist wie gesagt, dass die Bevölkerungszahl Deutschlands bis 2050 auf rund 73 Mio. Menschen abnimmt und bis 2100 bei rund 70 Mio. ein neues Gleichgewicht erreicht. Unter welchen Bedingungen könnte dieses Szenario eintreffen? Dafür müsste sich die Geburtenrate spätestens bis zum Jahr 2010 auf 1,7 Kinder pro Frau, bis 2015 auf 1,85 Kinder pro Frau und bis 2020 auf 2,1 Kinder pro Frau erhöhen. Geburtenpolitik muss also nicht abrupt einsetzen, vielmehr kann man über den Zeitraum von 15 Jahren Erfahrungen sammeln und die Maßnahmen, die sich als wirksam herausstellen, sukzessive verstärken. Für dieses Szenario des weiteren ist eine - relativ geringe - jährliche Nettoeinwanderung von etwa 60.000 Personen notwendig. Es wird von einem zehn Prozent stärkeren Anstieg der Lebenserwartung als bei den Simulationsrechnungen des Bielefelder Institut für Bevölkerungsforschung und Sozialpolitik ausgegangen..$^{12}$

912 Das Bielefelder Institut für Bevölkerungsforschung und Sozialpolitik (IBS) rechnet zahlreiche Projektionen für die zukünftige Bevölkerungsentwicklung Deutschlands durch. Die Annahmen und Ergebnisse werden im Internet unter http://www.ibs.uni-bielefeld.de/personal/simrechnung veröffentlicht. Das oben dargestellte Szenario entspricht einer Mischung aus den Varianten A24 und B24. In bei- 


\subsubsection{Gründe für den Geburtenrückgang}

Gerade am Beispiel Deutschland zeigt sich jedoch, dass viele Demografen grundsätzlich daran zweifeln, ob der Staat überhaupt messbar das Geburtenverhalten seiner Bevölkerung im Sinne einer pronatalistischen Politik beeinflussen kann. ${ }^{913}$ Als Beispiel wird etwa der Honecker-Buckel in der ehe. DDR angeführt. Durch staatliche Anreize versuchte die damalige Regierung Honecker, die Fertilitätsraten zu heben. Allerdings, so zeigte sich, zogen die DDR-Bürger nur ohnehin geplante Geburten vor, die Fertilitätsrate sank schon bald wieder auf das frühere Niveau ab..$^{914}$

Um zu beurteilen, ob eine pronatalistische Geburtenpolitik Aussicht auf Erfolg hätte, ist es ratsam, sich die Gründe für den Geburtenrückgang seit den 1960er Jahren in Deutschland zu vergegenwärtigen. ${ }^{915}$

- die besseren Möglichkeiten der Familienplanung. Seit den 1960er Jahren steht Frauen in Deutschland eine große Auswahl von billigen und unkompliziert anzuwendenden Kontrazeptiva zur Verfügung (,Pille', Spirale, Kondome, Diaphragma, Sterilisationen, etc.). Einen ungedeckten Bedarf an Verhütungsmitteln gibt es kaum noch, selbst wenn immer wieder die Diskussion über Kondomautomaten an Schulen oder die ,Pille danach' ohne Rezept hochkocht. Die leichte Verfügbarkeit von zuverlässigen Verhütungsmitteln ist eine entscheidende Ursache für den Geburtenschwund, der auch zeitlich ziemlich genau mit der Einführung der Anti-Baby-Pille zusammenfällt („Pillenknick“). Viele der vorher geborenen Kinder waren eben zumindest zum Zeitpunkt der Zeugung keine Wunschkinder. In den USA (mit einer Geburtenrate von 2,0) sind auch heute noch nahezu die Hälfte aller Schwangerschaften unerwünscht oder kommen zumindest zeitlich ungelegen..$^{916}$

- der Verlust des ,,instrumentellen “ Faktors von Kindern. Durch den Wertewandel seit der 68er-Revolte ist es für Deutsche unwichtiger geworden, einen Namensträger bzw. ,Stammhalter' zu haben. Früher hingegen (und in anderen Kulturen heute noch) spielte dies für Eltern eine große Rolle. Häufig sollte unter den Kindern zumindest ein Sohn sein, um die Familiendynastie oder zumindest den Familienstammbaum weiter $\mathrm{zu}$ führen. ${ }^{917}$ Ein Unternehmer, der Kinder als

\footnotetext{
den Varianten erreicht die Geburtenrate schneller als im oben dargestellten Szenario das Ersatzniveau, dafür nimmt die Lebenserwartung etwas langsamer zu.

${ }^{913}$ Höhn/Schubnell (1996), 3; Schwarz (1987), 409; Schwarz (1992)

${ }^{914}$ Birg (2001), 80. Ob ein beobachtetes Ansteigen der Geburtenzahlen auf einer Verkürzung der Geburtenabstände oder auf einer echten Zunahme der endgültigen Familiengröße beruht, lässt sich mit Hilfe der sog. Kohortenanalyse zeigen.

915 Siehe dazu z.B. Schimany (2003), 157-228; Grünheid (2003), 9-15; Miegel (2002), 22 f.; Opaschowski (2004) 48; Lehr (2003), 43-45

${ }^{916}$ Cincotta/Engelmann (2001), 64

${ }^{917}$ Der Wunsch nach dem Sohn ist bekanntlich der Vater vieler Töchter.
} 
Nachfolger sucht, kann heute nicht mehr sicher sein, dass diese ganz andere Lebenswege einschlagen. Und den Familiennamen kann in Deutschland inzwischen auch die Tochter weitergeben.

Durch die Einführung staatlicher Rentensysteme, zunächst durch Bismarck 22.6.1889, dann als umlagefinanzierten Generationenvertrag durch Adenauer 1957, verloren Kinder ihre instrumentelle Bedeutung als persönliche Alterssicherung. Während früher auch bei uns die Arbeitskraft des Kindes ganz konkret zur Wohlstandmehrung der Eltern beitrug, sind Kinder heute zu Kostenfaktoren geworden. „Die Ergebnisse der Kindererziehung werden bei uns vergesellschaftet, indem die Kinder zu Steuer- und zumeist auch zu Sozialabgabenzahlern werden. Die Kosten für die Erziehung müssen aber privat getragen werden. Früher erwuchs aus der Kinderlosigkeit eine Bedrohung für die eigene Existenz. Heute entsteht daraus ein deutlicher materieller Vorteil, den immer mehr Menschen für sich reklamieren“", schreibt die Politikerin von der Leyen. ${ }^{918}$

- die Verlängerung der Adoleszenz. Kinder bedeuten Sich-Festlegen, Verbindlichkeit, Erwachsenwerden. „Und wer will heute schon erwachsen sein - wo doch allerorten das Ideal des Jungseins oder zumindest der Jugendlichkeit propagiert wird? Das Erwachsenen-Alter ist für viele keine erstrebenswerte Entwicklungsphase mehr", schreibt die ZEIT. ${ }^{19}{ }^{19}$ Diese Verlängerung der geistigen Adoleszenzphase fällt zusammen mit den verlängerten Ausbildungszeiten, die sich manchmal bis in das vierte Lebensjahrzehnt hineinziehen. Wer studiert, ist oft lange finanziell von den Eltern abhängig. Tiefgreifender ist der Trend zum Individualismus und zur Selbstverwirklichung. Kinder haben immer stärker ,expressiven Charakter', d.h. sie werden dann eingeplant, wenn sie ein Bedürfnis der Individuen erfüllen und somit zu ihrem selbstgewählten Lebensentwurf beitragen. ${ }^{920}$

„Manche Singles vergnügen sich auf der Dauersuche nach dem richtigen Partner mit möglichst vielen falschen Partnern", schreibt der SPIEGEL. ${ }^{921}$ Aber bei dieser endlosen Suche bleibt die biologische Uhr nicht stehen und irgendwann müssen weibliche Singles dann feststellen, dass es nun für Kinder zu spät ist. Frauen fragen sich zudem, wofür eigentlich so enorme volkswirtschaftliche Ressourcen in ihre Berufsausbildung investiert wurden, wenn sie nach dem Ende des Studiums nicht zumindest einige Jahre Vollzeit arbeiten. Die Schwangerschaftswahrscheinlichkeit beträgt aber bei 25jährigen pro Zyklus 23 Prozent, bei 40jährigen aber nur 5 Prozent.

Männer scheinen gelegentlich ihren biologischen Vorteil auszuspielen, auch im höheren Alter noch Kinder bekommen zu können. Viele scheinen eine ernsthafte,

\footnotetext{
${ }^{918}$ Leyen (2003), 15. Siehe vertiefend auch den Abschnitt 6.8.3.3. Die Debatte über die, Rente nach Kinderzahl'.

${ }^{919}$ Billerbeck (2003), 32

${ }^{920}$ Schimany (2003), 226

${ }^{921}$ o.V. (5.1.2004), 41
} 
verbindliche Beziehung, eine Heirat und die Kinderplanung daher bis in die späten 30er Jahre oder noch länger hinaus zu schieben. ${ }^{922}$

Der Hauptgrund, der Frauen daran hindert, Kinder zu bekommen, sind nicht etwa die hohen Kosten eines Kindes oder die mangelnden Betreuungsmöglichkeiten, sondern schlicht das Fehlen des ,richtigen“ Partners:

\section{Tabelle 17: Gründe von Frauen in Deutschland gegen ein (weiteres) Kind, nach Altersgruppen (Anteile der "sehr wichtig“ und "wichtig“ Antworten in \%)}

\begin{tabular}{|l|l|l|l|l|}
\hline Rang & Grund & $20-29$ & $30-39$ & $40-49$ \\
\hline 1. & Ich lebe allein und habe keinen festen Partner & 83,1 & 74,4 & 56,3 \\
\hline 2. & $\begin{array}{l}\text { Ich habe schon so viele Kinder wie ich möchte } \\
\text { bzw. ich möchte keine Kinder }\end{array}$ & 41,7 & 76,2 & 80,1 \\
\hline 3. & $\begin{array}{l}\text { Ich mache mir zu viele Sorgen darüber, wel- } \\
\text { che Zukunft meine Kinder erwartet }\end{array}$ & 59,4 & 55,2 & 53,9 \\
\hline 4. & $\begin{array}{l}\text { Ich möchte meinen jetzigen Lebensstandard } \\
\text { beibehalten }\end{array}$ & 61,8 & 52,4 & 47,2 \\
\hline 5. & $\begin{array}{l}\text { Ein (weiteres) Kind würde zu hohe Kosten } \\
\text { verursachen }\end{array}$ & 47,8 & 41,4 & 42,6 \\
\hline 6. & Ich bin/mein Partner ist zu alt & 11,8 & 20,8 & 58,6 \\
\hline 7. & Mein Partner ist dagegen & 31,6 & 25,5 & 36,7 \\
\hline 8. & $\begin{array}{l}\text { Ich könnte mein Leben nicht mehr so genie- } \\
\text { Ben wie bisher }\end{array}$ & 39,9 & 27,8 & 29,6 \\
\hline 9. & $\begin{array}{l}\text { Ich könnte es nicht mit meiner Berufstätigkeit } \\
\text { vereinbaren }\end{array}$ & 41,6 & 35,7 & 36,1 \\
\hline 10. & Ich müsste Freizeitinteressen aufgeben & 44,5 & 22,0 & 23,2 \\
\hline 11. & Mein Gesundheitszustand erlaubt es nicht & 25,9 & 20,5 & 29,0 \\
\hline 12. & $\begin{array}{l}\text { Meine Partnerschaft funktioniert nicht so, wie } \\
\text { ich es mir vorstelle }\end{array}$ & 26,6 & 17,1 & 20,8 \\
\hline
\end{tabular}

\section{Quelle: Bib (2005), 37}

Der wichtigste, aber auch der siebtwichtigste und der zwölftwichtigste Grund beziehen sich auf die Partnersituation. ${ }^{923}$ Es ist nicht verwunderlich, dass für 83,1 Prozent der Unter30jährigen das Fehlen eines Partners ein sehr wichtiger bzw.

\footnotetext{
${ }^{922}$ Es spricht relativ viel dafür, dass die Werthaltung von Männern maßgeblich mitverantwortlich ist für die geringe Kinderzahl. Das Männerverhalten und ihre Erwartungen sind relativ schlecht wissenschaftlich untersucht, vgl. Hank/Tölke (2005); Klingholz (2004), 102.

${ }^{923}$ Dieses Ergebnis wird auch von anderen Studien bestätigt. So nannten in einer forsa-Umfrage vom Okt. 2004 die Befragten als Hauptgrund für den Verzicht auf (weitere) Kinder: „Weil ich keinen geeigneten Partner habe." Vgl. Pohl (2004).
} 
wichtiger Grund für den Verzicht auf (weitere) Kinder darstellt. Aber auch bei den Frauen in der Altersgruppe von 30 bis 39 Jahren spielt dieser Grund eine wichtige Rolle (74,4 Prozent). Durch die langen Ausbildungszeiten haben insbesondere Akademikerinnen ohnehin nur ein kleines biologisches Fenster. Dieses muß zeitlich zusammenfallen mit dem Fenster, in dem ein williger Partner zur Verfügung steht. Dies ist oft nicht gegeben, so dass viele sehr gut ausgebildete Frauen kinderlos bleiben. 57,5 Prozent der Männer und 37,8 Prozent der Frauen zwischen 30 und 34 waren im Jahr 2002 kinderlos. Bei den Männern zwischen 35 und 39 lag die Quote der Kinderlosen bei 33,6 Prozent, bei den Frauen in der gleichen Altersgruppe bei 17,4 Prozent. ${ }^{924}$ Dieses Ergebnis kann nicht verwundern, wenn man sich die unterschiedlichen Kinderwünsche von Männern und Frauen in Deutschland ansieht: Frauen wollen im Durchschnitt 1,74 und Männer 1,57 Kinder haben. West-Ost-Unterschiede sind im Durchschnitt kaum noch anzutreffen. Der Kinderwunsch der Frauen ist im Osten mit 1,78 Kindern (Westen: 1,73) etwas höher und bei den Männern mit 1,46 Kindern (Westen: 1,59) etwas niedriger. ${ }^{925}$ Es sind also deutlich mehr Männer als Frauen gewollt kinderlos - so dass schon von einem ,Zeugungsstreik' gesprochen wird. ${ }^{926}$ Dieser ist nicht auf ökonomische Kalküle zurückzuführen. In qualitativen Interviews wird deutlich, dass viele Menschen das Gefühl haben, nicht einmal ihr eigenes Leben hinreichend zu meistern. ${ }^{927}$ Unter diesen Umständen erscheint es ihnen absurd, auch noch Verantwortung für ein Kind zu übernehmen. Andere geben zu Protokoll, dass ihnen ihr Wunsch nach Freiheit und Unabhängigkeit unvereinbar mit einem Kind erscheint. Partnerschaft gehört zwar zum eigenen Glück dazu, aber oft nur im Sinne von Selbstbestätigung und sexueller Erfüllung. Kinder kommen darin nicht vor. Der Familiensoziologe Thomas Klein schreibt: „Am Beginn einer Partnerschaft denkt kaum jemand direkt an Kinder. Kündigt sich das Ende einer Beziehung an, hüten sich die meisten Leute, ein Kind in die Welt zu setzen. ${ }^{“{ }^{c} 28}$ So summieren sich die Zeiten, in denen der Kinderwunsch keine Rolle spielt. Paare, die seit acht Jahren zusammen sind, haben dagegen auch heute mit derselben Wahrscheinlichkeit Nachwuchs, wie ihn die eigenen Eltern oder Großeltern hatten. Angesichts dieser Befunde hält Klein die in Deutschland praktizierte Familien- und Sozialpolitik für verfehlt. Finanzielle Anreize des Staates werden nicht abgerufen, weil sie junge Leute ohne eine stabile Partnerschaft kaum interessieren. Ein Teil des Geldes wäre in Zuschüssen für Paarberatungsstellen besser investiert. ${ }^{929}$

\footnotetext{
${ }^{924}$ Schmitt (2005), 23. Tabelle 1.

${ }^{925} \mathrm{BiB}(2005), 36$

${ }^{926}$ Dinklage (2005)

${ }^{927}$ A.a.O.

${ }^{928}$ Klingholz (2004), 102

${ }^{929}$ A.a.O.
} 
Die ehemalige Familienministerin Ursula Lehr betont, dass das tradierte Rollenverständnis, welches Frauen den Bereich ,Kinder' zuweist, bröckelt und dass Frauen heute selbstbewusster sind als früher: „Während in der ersten Hälfte unseres Jahrhunderts die Frau solange im Elternhaus lebte, bis geheiratet wurde (und sie so zur Anpassung an die Lebensgewohnheiten anderer gezwungen war), nach der Heirat sehr schnell Kinder kamen, die wiederum eine Anpassung verlangten, geht sie heute mit 18 oder 20 Jahren aus dem Haus und lebt selbständig, allein. Ein solches mehrjähriges Alleinwohnen führt zu einer verstärkten Ausbildung der Individualität; es bilden sich Eigenheiten und Gewohnheiten, eine Zeit in der oft ein ganz individueller eigener Lebensstil kreiert wird, der dann schon eine Anpassung an einen Partner, erst recht aber an Kinder, sehr erschwert. Dies ist eine Ursache dafür, dass so viele heute das Single-Dasein vorziehen und dass, wenn sie einmal heiraten, dann oft das gewünschte zweite Kind nicht mehr kommt." ${ }^{\text {"c930 }}$ Man kann allerdings auch argumentieren, dass Männer verstehen müssen, dass Deutschland erst wieder höhere Geburtenraten erleben wird, wenn sie fünfzig Prozent der Mühen der Kinderaufzucht schultern: „Ohne einen neuen Geschlech-

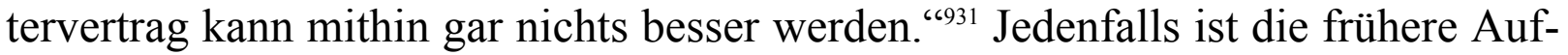
gabenverteilung zwischen Eltern verlorengegangen, eine neue ist noch nicht gefunden. Die Frauen sind nicht mehr mehrheitlich bereit, 100 Prozent der Erziehungsarbeit alleine zu machen, die Männer sind mehrheitlich (noch) nicht bereit, 50 Prozent zu übernehmen. Nur vier Prozent der Männer beantragen die sog. Elternzeit.

- die schlechte Vereinbarkeit von Beruf und Familie. Ein weiterer Grund für die niedrige Geburtenrate in Deutschland ist die schlechte Vereinbarkeit von Beruf und Kinderbetreuung. Während die Betreuungsquote für Kinder im Alter unter drei Jahren in Dänemark bei 64 Prozent und in Schweden bei einem Drittel liegt, beträgt sie in Westdeutschland 3,4 Prozent (vgl. Abb. 46). ${ }^{932}$ Nach einem Beschluss der rot-grünen Bundesregierung sollen bis 2010 für 15 Prozent der Unterdreijährigen Betreuungsangebote zur Verfügung stehen. ${ }^{933}$ Die Bundesregierung hat zudem 2002 beschlossen, Länder und Kommunen beim Ausbau der Ganztagsbetreuung mit einem 4-Milliarden-Programm im schulischen Bereich und mit 1,5 Milliarden Euro jährlich im elementaren Bereich zu unterstützen. Allerdings ist eine Förderung nur möglich bei Investitionen, die laufenden Kosten tragen aus

\footnotetext{
${ }^{930}$ Schriftliche Fassung des Vortrags von Ursula Lehr in Wipperführt am 16.11.2003 bei einer Tagung der Aktion Gemeinsinn e.V.

${ }^{931}$ Gaschke (2004), 3

${ }^{932}$ Zum Teil widersprüchliche Angaben ergeben sich aus Rürup/Gruesecu (2003), 32; Kröhnert/van Olst/Klingholz (2005), 6; Schmidt (2003), 3. So geben Rürup/Gruesecu z.B. für die Niederlande 6 Prozent an, Kröhnert/van Olst/Klingholz aber 40 Prozent. In Ostdeutschland beträgt die Betreuungsquote der Unter3jährigen 34,8 Prozent (Rürup/Gruesecu 2003, 32).

933 o.V. (15.07.2004), 4. Damit würde die Zahl der Plätze von derzeit rund 60.000 auf 230.000 steigen.
} 
verfassungsrechtlichen Gründen die finanzielle Not leidenden Länder und Kommunen.

Fraglich ist generell, ob (west)deutsche Frauen dieses Angebot überhaupt nutzen wollen. Die Autorin Barbara Vinken behauptet in einer kulturhistorischen Untersuchung des Selbstbildes westdeutscher Mütter, dass diese möglicherweise weniger als andere Europäerinnen bereit sind, die Kinderbetreuung aus der Hand zu geben. ${ }^{934}$

Bessere Betreuungsmaßnahmen können das Geburtenniveau moderat, aber nicht drastisch steigern. Auch in Ländern, in denen die Betreuungsquote höher liegt als in Deutschland, liegen die Geburtenraten unter dem Bestandhaltungsniveau.

${ }^{934}$ Vinken (2001) 
Abb. 45 u. 46: Geburtenraten und Betreuungsquoten in der EU
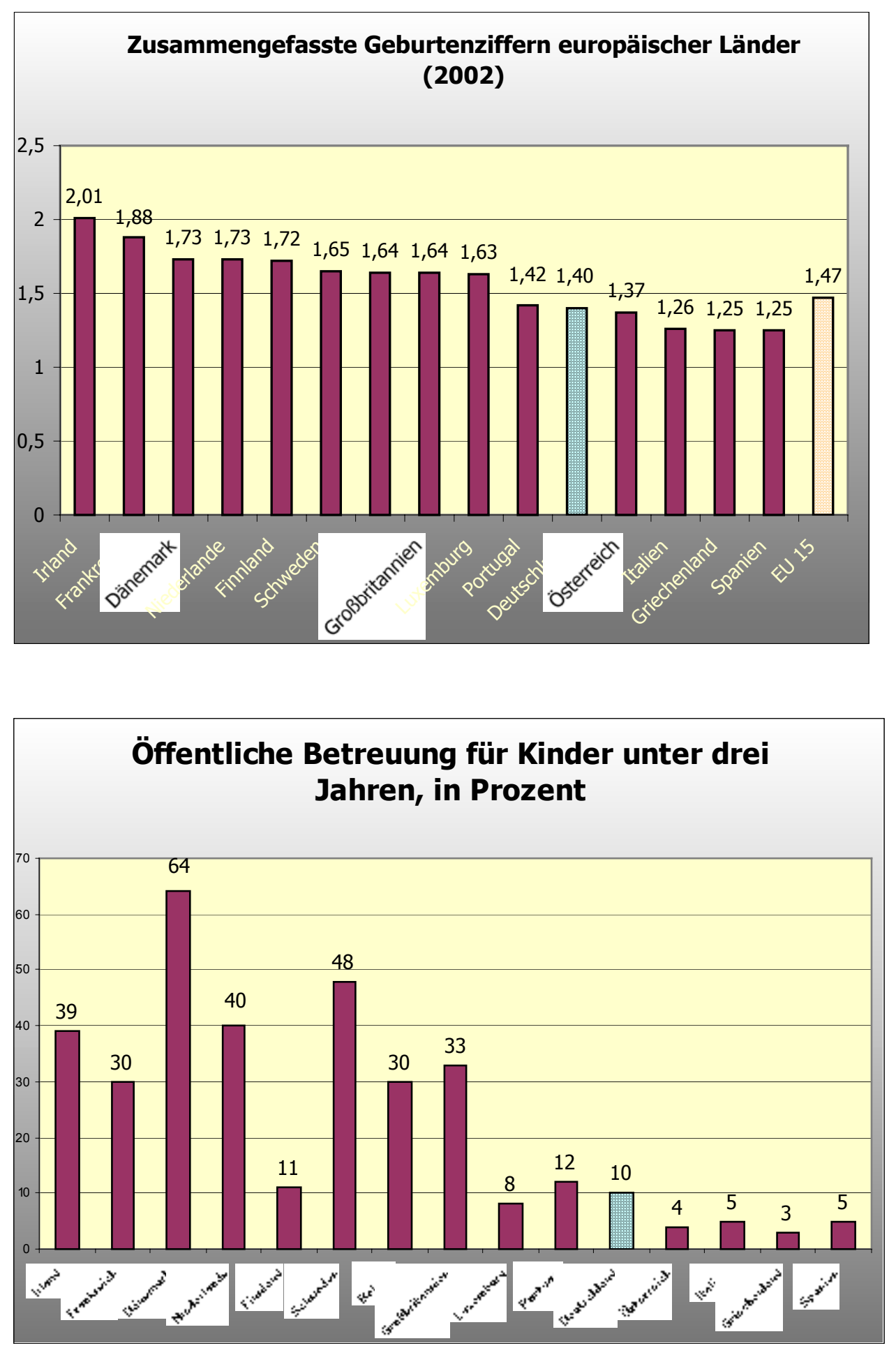

Quelle: eigene Darstellung, Daten von Eurostat 2002, Deutschland West: 3,4\%. Deutschland Ost: $34,8 \%$

Betrachtet man die fünf geburtenstärksten Länder im Hinblick auf ihre Betreuungssituation, so wird schnell deutlich, dass es nur einen relativ schwachen, kei- 
nesfalls monokausalen Zusammenhang gibt. Auffällig ist aber, dass die Länder mit niedrigen Geburtenraten in der Regel auch niedrige Betreuungsquoten aufweisen. In den neuen Bundesländern, in denen eine flächendeckende Versorgung mit institutioneller Kindesbetreuung besteht, war die Geburtenrate nach der Wiedervereinigung auf ein weltweit einmaliges Tief von 0,75 abgesackt und hat trotz Nachholens der ausgesetzten Geburten - seitdem die westdeutsche Geburtenrate zwar wieder eingeholt, aber nicht überflügelt. Auch die große Studie über die demografische Zukunftsfähigkeit Deutschlands, die Anfang 2004 vom BerlinInstitut für Weltbevölkerung und globale Entwicklung durchgeführt wurde, fand kaum Korrelationen zwischen der Versorgung mit Betreuungseinrichtungen und der Geburtenrate. Vereinzelt gilt sogar: Dort wo die Dichte der Kindertagesstätten hoch ist, sind die Kinderzahlen niedrig und dort wo es wenige Kindertagesstätten gibt, weil die meisten Frauen Hausfrauen sind, gibt es viele Kinder. ${ }^{935}$ Der kinderreichste Kreis in Deutschland ist der Kreis Cloppenburg im Münsterland mit einer Gesamtfertilitätsrate von 1,92 Kindern je Frau. ${ }^{936}$ Auch in den anliegenden Kreisen in Westniedersachsen ist die Geburtenrate ungewöhnlich hoch. Dies sind ländliche, katholisch geprägte Kreise, wo ein traditionelles Rollenverständnis vorherrscht und Frauen v.a. Hausfrauen und Kindererzieherinnen sind. Nur 34 Prozent der Frauen zwischen 15 und 65 sind dort berufstätig (Bundesdurchschnitt 65,3 Prozent). Das Angebot an Kindergartenplätzen liegt rund 15 Prozentpunkte unter dem bundesdeutschen Durchschnitt von 60,7 (je 100 Kinder). Mit anderen Worten: Cloppenburg wurde von der Modernisierung und vom Wertewandel der letzten 40 Jahre noch relativ wenig erfasst. Aus dem ,Fall Cloppenburg' eine Strategie für die Zukunft abzuleiten, wäre jedoch fatal. Es ist offensichtlich, dass dies kein Modell für deutsche Großstädte sein kann, schon weil in einer alternden Gesellschaft Frauen verstärkt als Erwerbstätige gebraucht werden. Das Modell der Zukunft sehen Rürup und Gruescu deshalb in einer Mischung aus verbesserter Infrastruktur für Kinder und mehr Zeitpolitik. ${ }^{937}$

Dass eine familienfreundliche Politik in einem begrenzten Rahmen Wirkung auf die Geburtenrate hat, zeigen Beispiele wie Frankreich oder Estland. In Frankreich ist es von 1993 bis 2000 gelungen, die Anzahl der Kinder pro Frau von 1,65 auf $1,88 \mathrm{zu}$ erhöhen. ${ }^{938}$ Dort besteht ab dem 3. Lebensjahr ein Rechtsanspruch auf Aufnahme in eine Vorschule (école maternelle). Diese besuchen ein Drittel der Zweijährigen und über 90 Prozent der Dreijährigen. ${ }^{939}$ Dort arbeiten 72,3 Prozent aller Frauen mit zwei Kindern und immerhin 51 Prozent aller Mütter mit drei Kindern. Ein gewisser Mentalitätswechsel wird aber ebenso unverzichtbar sein, wenn Deutschland sich an Frankreich orientiert. Der ,sortie de clinique' etwa

\footnotetext{
${ }^{935}$ Klingholz (2004), 92

${ }^{936} \mathrm{Kröhnert/van} \mathrm{Olst/Klingholz} \mathrm{(2004),} 33$

${ }^{937}$ Rürup/Gruescu (2003), 63

${ }^{938}$ Bosbach (07.03.2004)

${ }^{939}$ Veil (2002)
} 
würde in Deutschland immer noch Müttern den Vorwurf einbringen, ihre Kinder zu vernachlässigen. In Frankreich versteht man darunter, dass Säuglingspflegerinnen nach dem Verlassen der Klinik für einige Zeit ins Haus kommen, um sich von $8 \mathrm{Uhr}$ abends bis $8 \mathrm{Uhr}$ morgens um das Neugeborene zu kümmern. Nächtliches Aufstehen inklusive Stillen entfällt für die Französinnen, die sich diesen Service leisten können. ${ }^{940}$ Eine ,Vereinbarung' von Beruf und Kindern ist etwas anderes als die ,Vereinbarkeit' - der aktive Wunsch, Kinder wegen des Berufs auch mal in fremde Hände zu geben, muss zur Vereinbarkeit dazukommen. Bisher liegen deutsche Mütter mit einer Auszeit von fünf Jahren nach der Geburt international an der Spitze. Dies zu ändern, könnte der Schlüssel zum Erfolg einer pronatalistischen Bevölkerungspolitik sein - nicht die noch weitere Erhöhung des Kindergeldes. Es verwundert nicht, dass gerade Spanierinnen, Italienerinnen und Griechinnen noch geringere Geburtenraten haben als die deutschen - in diesen Ländern dominiert ein Mutterbild, welches Frauen Schuldgefühle vermittelt, sobald sie ihre Kinder in Betreuung geben. Für „Rabenmütter“ gibt es kein französisches Wort. Immerhin scheint es einen langsamen Bewusstseinswandel zu geben. In der Population Policy Acceptance Studie 2003 hatte im Vergleich zur Vorgängerstudie 1992 eine deutliche Orientierung in Richtung Vereinbarkeit stattgefunden. 89,4 Prozent der befragten Frauen und Männer zwischen 20 und 39 Jahren wünschen sich bessere Möglichkeiten zur Tagesbetreuung von Kindern ab drei Jahren bis zum Schulalter; 82,7 Prozent wünschen sich bessere Tagesbetreuungsmöglichkeiten von Kindern unter drei Jahren. ${ }^{941}$ Allerdings zeigte die Studie von McKinsey, dem Stern, dem ZDF und der AOL durchgeführte Studie Perspektive Deutschland,${ }^{942}$ dass immer noch drei von zehn Frauen glauben, dass die Entwicklung des Kindes durch die Berufstätigkeit der Mutter leidet. Bei den Männern glaubt das mehr als die Hälfte..$^{943}$

Die Familienministerin Renate Schmidt will die Tagesbetreuung der unter Dreijährigen ausbauen und bis 2010 rund 230.000 neue Krippenplätze schaffen. So sinnvoll diese Maßnahme ist, so sehr ist vor überzogenen Hoffnungen im Hinblick auf geburtenpolitische Ziele zu warnen. Dies wird ohne große Effekte bleiben, wenn Kinder nicht wieder hip und cool bei jungen Leuten werden.

- zu geringe oder verfehlt wirkende direkte finanzielle Anreize (Kindergeld). Ein Gutachten von Rürup und Gruescu führte 2003 zu einem Paradigmenwechsel in der deutschen Familien- und Geburtenpolitik. Das Gutachten wies darauf hin, dass Frauen in Deutschland häufiger als in anderen Ländern gar kein Kind bekommen. Haben Frauen in Deutschland aber ein Kind bekommen, so sinken die Hemmnisse für ein zweites. Ziel der Geburtenpolitik - vom Gutachten verschämt

\footnotetext{
${ }^{940}$ o.V. (05.01.2004), 46

${ }^{941} \mathrm{BiB}(2005), 41$

${ }^{942}$ www.perspektive-deutschland.de

${ }^{943}$ Kluge (2004), 45
} 
Familienpolitik genannt - müsse es also sein, dafür zu sorgen, dass mehr Frauen in Deutschland überhaupt Mütter würden. ${ }^{944}$ Zweitens würden Kinder immer direkte Kosten (für Windeln, Spielsachen, Ausbildung etc.) und indirekte Kosten verursachen. Diese indirekten Kosten oder Opportunitätskosten ergäben sich aus dem Einkommensverlust, der laut Rürup und Gruescu generell bei dem Elternteil anfalle, dass wegen Geburt und Erziehung kein Einkommen auf dem Arbeitsmarkt erzielen könne (in den meisten Fällen die Mutter) ${ }^{945}$ Die Opportunitätskosten sind um so höher, je mehr die Eltern bzw. die Mutter vor der Geburt verdient hat. Dies erkläre auch, dass in Westdeutschland 44,3 Prozent der 35-39jährigen Akademikerinnen kinderlos seien. Aus diesen Zusammenhängen leiten die Autoren die Forderung nach einem einkommensabhängigen Elterngeld ab, welche sich dann auch Familienministerin Renate Schmidt zu eigen machte und die inzwischen im Wahlmanifest der SPD, welches im Sommer 2005 verabschiedet wurde, steht.

Dieser interessante Vorschlag zur Umsteuerung bei den direkten finanziellen Anreizen kann hier nicht ausführlich diskutiert werden. Es sollten aber keine überzogenen Hoffnungen darin gesetzt werden. Neuere Untersuchungen zeigen, dass die ökonomische Theorie der Fertilität, d.h. die Theorie, dass rationale Individuen in einer ökonomischen Kosten-Nutzen-Rechnung über die Zahl der Kinder entscheiden, relativ geringen Erklärungswert besitzt. ${ }^{946}$ „Der Haushalt bzw. das Individuum stellt die erwarteten Kosten des erwünschten Kindes den mit diesem verbundenen erwarteten Nutzen gegenüber und ,verrechnet" beide Positionen. (...) Übersteigt der Nutzen eines Kindes für ein Individuum oder Paar die Kosten, wird der Kinderwunsch realisiert. (...) Auch wenn der Nutzen eines Kindes für die Eltern schwer zu messen ist, ist die Anzahl der realisierten Kinderwünsche (...) umso größer, je niedriger die Kosten von Kindern sind“, schreiben Rürup und Gruescu. ${ }^{947}$ Das ist nicht falsch, trifft aber nicht den Kern des Problems. Ebenso könnte man sagen: Menschen schaffen sich ein Segelboot an, wenn der Nutzen die Kosten übersteigt. Die meisten Menschen werden sich auch dann kein Segelboot anschaffen, wenn der Staat die Kosten für Segelboote drastisch senkt. Mit rund $150 \mathrm{Mrd}$. Euro im Jahr unterstützt der deutsche Staat Familien und Kinder, ohne dass dies $\mathrm{zu}$ einer messbaren Erhöhung der Geburtenrate geführt hat. ${ }^{948}$ Deutschland liegt bei direkten finanziellen Leistungen („Kindergeld“) bereits auf dem 2. Platz in Europa, ohne dass dies die Geburtenraten kausal zu beeinflussen scheint (vgl. Abb. 48). Der Spitzenreiter - Luxemburg - zahlt für jedes zweite Kind 400 Euro und für jedes dritte Kind sogar 727 Euro Kindergeld, ohne dass die Geburtenrate nennenswert über der deutschen liegt.

\footnotetext{
${ }^{944}$ Rürup/Gruescu (2003), 13 und passim.

${ }^{945}$ Rürup/Gruescu (2003), 20

${ }^{946}$ Klingholz (2004), 101

${ }^{947}$ Rürup/Gruescu (2003), 19

${ }^{948}$ Rürup/Gruescu (2003), 36
} 


\section{Abb. 48: Kindergeld in Europa}

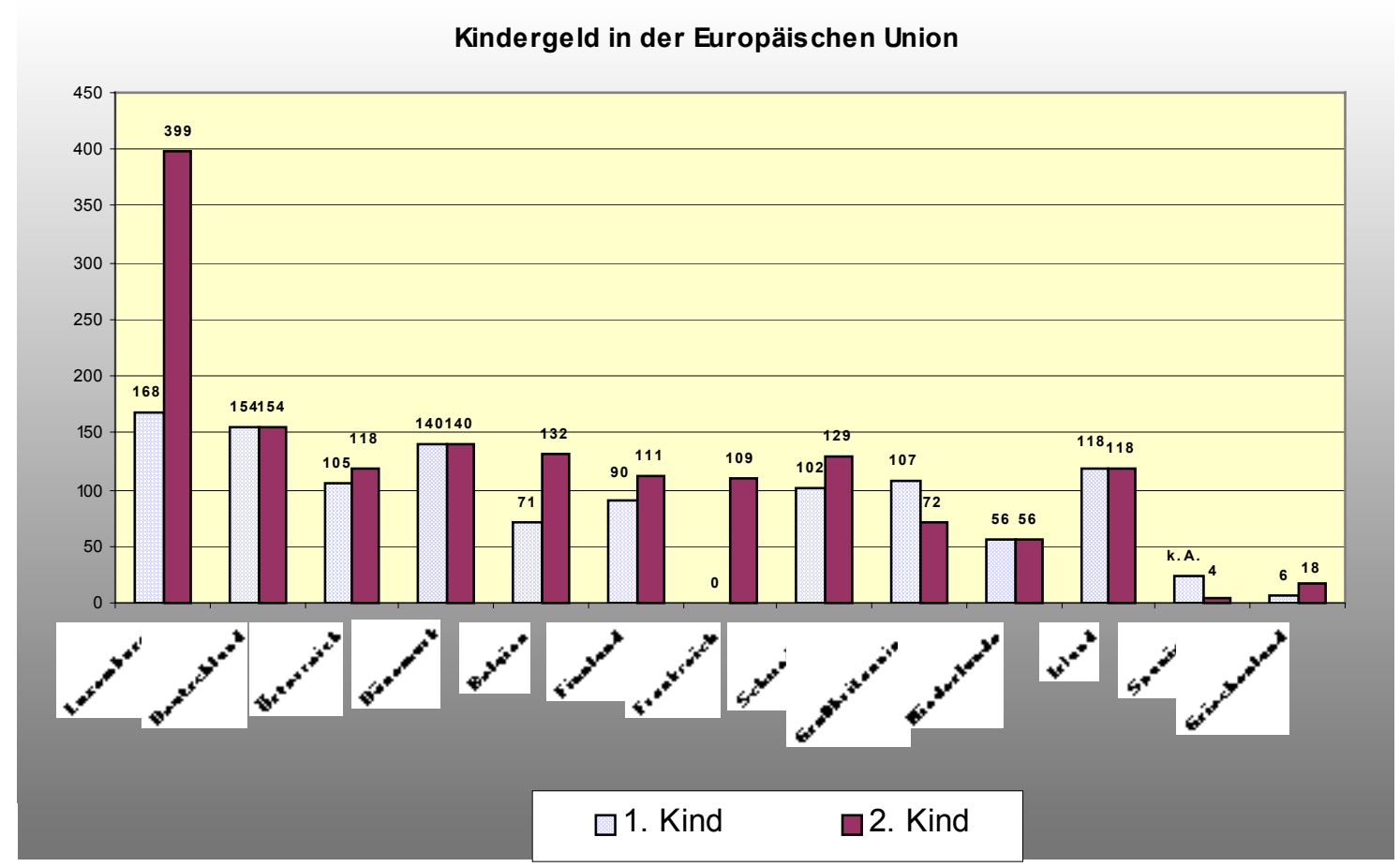

\section{Quelle: eigene Darstellung, Daten von Eurostat 2002949}

Klingholz resümiert: „Der Entschluss bei jungen Menschen für oder gegen Kinder fällt auf einer anderen Ebene als nur mit dem Blick auf das Finanzielle. Das ist überall auf der Welt so. Die meisten Kinder haben Leute in Ländern, die sehr viel ärmer sind als wir und wo die Versorgungssicherheit als Andere als gewährleistet ist." ${ }^{\text {"950 }}$

Gewichtet man über eine Conjoint-Analyse einzelne Wünsche der Bürger an den Staat gegeneinander, so zeigt sich, dass die Vorstellungen der Bürger deutlich Betreuung gegenüber finanziellen Anreizen präferieren. „Unter den heutigen Rahmenbedingungen können sich nur etwa ein Drittel der Frauen im Alter von 20 bis 39 Jahren ohne eigene Kinder und ohne expliziten Kinderwunsch vorstellen, ein Kind zu bekommen. Würde man das Kindergeld von 150 Euro auf 300 Euro

\footnotetext{
${ }^{949}$ Dabei ist zu berücksichtigen, dass die Regelungen weit komplexer sind, als in der Tabelle abgebildet. Die Zahlungen unterscheiden sich zunächst nach der Dauer. In Deutschland wird das Kindergeld in der Regel (es gibt zahlreiche Ausnahmen!) bis zum 18. Lebensjahr gezahlt, bei Berufsausbildung oder Studium bis zum 27. Lebensjahr. In anderen Ländern gelten wieder andere Grenzwerte. In jedem europäischen Land gibt es andere Abstufungen und Zusatzleistungen, z.B. bei Behinderung des Kindes, bei Arbeitslosigkeit des Kindes, bei Alleinerziehung durch Mutter oder Vater. In einigen Ländern variiert das Kindergeld nach dem Alter des Kindes. In einigen Ländern ist das Kindergeld einkommensabhängig ausgestaltet, so dass kein fester Wert angebbar ist. Italien und Portugal wurden deshalb gar nicht in die Tabelle aufgenommen. Für einen umfassenden Überblick siehe Rürup/Gruescu (2003), $26 \mathrm{f}$.

${ }^{950}$ Klingholz/Tremmel (2004)
} 
verdoppeln, die sonstigen Rahmenbedingungen jedoch gleich lassen, würde dieser Wert gerade einmal um drei Prozentpunkte steigen. Mit anderen Worten: Selbst eine signifikante Erhöhung der direkten Subventionierung von Familien mit Kindern hätte kaum messbare Effekte auf die Kinderquote. Würde der Staat jedoch die Kinderbetreuung verbessern, so würde sich der Anteil der Frauen, die sich für ein Kind entscheiden, um 24 Prozentpunkte erhöhen - selbst bei ansonsten unveränderten Rahmenbedingungen“, schreibt der McKinsey-Vorsitzende Jürgen Kluge. ${ }^{951}$

Der Staat kann nicht alles. Nur etwa die Hälfte der Bürger zeigt sich in ihrem Kinderwunsch durch geburten- bzw. familienpolitische Maßnahmen wie finanzielle Anreize überhaupt beeindruckt. ${ }^{952}$ Bei den anderen verpufft jede Art staatlicher Einflussversuche. Gesellschaftliche Vorbilder, Meinungsmacher und Trendsetter sind ebenso wichtig wie der Akteur Staat, wenn unsere Gesellschaft die Geburtenrate gemeinsam steigern will.

Als Fazit lässt sich sagen, dass zuvorderst ein gesellschaftlicher Wertewandel, flankiert durch ein besseres Betreuungsangebot und mehr Teilzeitstellen geeignet sein dürften, die Geburtenrate um bis zu 0,5 bis 0,8 Kinder pro Frau zu steigern und somit knapp wieder das Reproduktionsniveau zu erreichen. Die Geburtenrate in Westdeutschland liegt seit 30 Jahren bei etwa 1,4. Diese große Konstanz ist besorgniserregend, allerdings liegt sie in anderen Industrieländern um 0,4-0,7 höher, so dass offensichtlich zumindest bis zu diesem Niveau eine Steigerung möglich ist, ohne dass Errungenschaften der Moderne aufgegeben werden müssen. Um eine deutlich darüber hinausgehende Geburtenrate zu realisieren, müsste man im Rahmen einer pronatalistischen Politik zu Steuerungsinstrumenten greifen, die heute erst ansatzweise diskutiert werden. Viele Maßnahmen, die in anderen, unmoderneren Teilen der Welt üblich sind, verbieten sich von selbst. Wenn etwa Ehen von den Eltern arrangiert würden wie in vielen Entwicklungsländern, hätte das einen deutlich geburtensteigernden Effekt. Auch die Einschränkung der Verfügbarkeit von Verhütungsmitteln und ein Verbot der Abtreibung wird wohl kaum eine Mehrheit hierzulande finden. ${ }^{953}$ Wie wir bereits bei der Besprechung von antinatalistischen Maßnahmen in WEL sahen, spielt das Rentensystem eine große Rolle. Daher soll auf die Debatte über Kinderkomponenten im deutschen System etwas ausführlicher eingegangen werden. ${ }^{954}$

\footnotetext{
${ }^{951}$ Kluge (2005), 46

${ }^{952} \mathrm{Bib}(2005), 42$

${ }^{953}$ Als Ceausescu in Rumänien Ende der 1960er Jahre im Zuge pronatalistischer Maßnahmen die Abtreibung verbot, stieg die Geburtenrate von 1,8 auf 4,0 an. Allerdings sank sie schon kurz darauf wieder, da illegale Abtreibungen zur Normalität wurden (Kröger/van Olst/Klingholz 2004, 58).

954 Bei den Ursachen für die niedrigen Geburtenraten in Deutschland spielt die angeblich gestiegene Unfruchtbarkeit wohl nur eine geringe Rolle, da durch den Fortschritt bei den Befruchtungstechniken (ICSI etc.) heute auch Paaren zu Kindern verholfen werden kann, die früher kinderlos geblieben wären.
} 


\subsubsection{Die Debatte über eine ,Rente nach Kinderzahl'}

Einer der weitreichendsten Reformvorschläge, der in Deutschland ernsthaft unter geburtenpolitischen Vorzeichen diskutiert wird, betrifft die gesetzliche Rentenversicherung. Der Umlageversicherung wird seit der Einführung 1957 vorgeworfen, sie führe zu einer Absenkung der Geburtenraten. ${ }^{955}$ Nachdem diese Kritik jahrzehntelang kaum Resonanz in der Politik fand, hat mit der CSU kürzlich immerhin eine der großen Volksparteien die Forderung nach einem grundlegenden Umbau des Rentensystems auf einem Parteitag (am 17.11.2003) verabschiedet. Auch mehrere Urteile des Bundesverfassungsgerichts (v.a. das ,Mütterurteil' vom 7.7.1992 und das Urteil zur Pflegeversicherung vom 3.4.2001) haben die Befürworter eines grundlegenden Umbaus der Sozialversicherungssysteme ermutigt. ${ }^{956}$

Zwei Reformmodelle sind in der deutschen Debatte zu unterscheiden: die Kinderversicherung (Drei-Generationen-Vertrag) und das Kapitaldeckungsverfahren für Kinderlose.

\subsection{Kinderversicherung}

Die Kinderversicherung wurde bereits von den geistigen Vätern des Umlageverfahrens von 1957, also von Winfried Schreiber, Oswald von Nell-Breuning und Gerhard Mackenroth, erdacht, aber von Adenauer mit der Begründung „Kinder haben die Leute immer" nicht umgesetzt. ${ }^{957}$ Grundgedanke ist, dass es neben der Transferleistung der Erwerbstätigen für die Rentnergeneration auch eine Transferleistung für die Kindergeneration gibt. Nach dem Schreiber-Plan für eine ,Kindheits- und Jugendrente ${ }^{958}$ soll jeder Erwerbstätige ab dem 35. Lebensjahr einen prozentualen Anteil seines Lohneinkommens an Familien mit Kindern in der Erziehungsphase zahlen. Die Beitragshöhe richtet sich nach der Zahl der Kinder des Beitragszahlers; Verheiratete mit sechs Kindern und mehr wären von der Beitragspflicht befreit.

Dem gegenwärtigen (Zwei)-Generationenvertrag wird vorgeworfen, dass ihm diese nachwuchssicherende Komponente fehle und es dadurch zu Ungerechtigkeiten zwischen Eltern und Kinderlosen kommen würde. Die später erwachsenen

\footnotetext{
955 Schreiber (1955); Mackenroth (1957); Nell-Breuning/Fetsch (1981); Kaufmann/Leisering (1985); Burkhardt (1985); Borchert/Nell-Breuning (1986); Almsick (1988); Oeter (1989); Borchert (1993); Adam (1996); Werding (1998); Sinn (1997); Sinn (1998)

956 Die Sozialministerin Ulla Schmidt (SPD) will das Urteil des Bundesverfassungsgerichts zur Pflegeversicherung dergestalt umsetzen, dass Erwachsene, die älter als 23 Jahre und jünger als 65 Jahre sind und keine Kinder haben, für die Pflegeversicherung einen um 0,25 Prozentpunkte höheren Beitragssatz zahlen sollen (also 1,95 statt 1,7 Prozentpunkte), vgl. o.V. (06.07.04), 1. Die CDU möchte stattdessen eine Besserstellung der Eltern mit 5 Euro im Monat für jedes Kind im Alter bis 18 Jahren, also ein Bonus- statt ein Malusmodell.

${ }^{957}$ So beschreibt es zumindest Borchert (1993), 61.

${ }^{958}$ Schreiber (1955), $97 \mathrm{f}$.
} 
Kinder müssten, so die Argumentation, nicht nur ihre eigenen alten Eltern finanzieren, sondern auch noch die kinderlosen Alten. ${ }^{959}$

\subsection{Kapitaldeckungsverfahren für Kinderlose}

Eine Alternative zur Kinderversicherung wird u.a. vom Münchner Ökonom HansWerner Sinn in einem Kapitaldeckungsverfahren für den kinderlosen Teil der Bevölkerung gesehen. ${ }^{960}$ Die Grundprinzipien sind folgende: Für Erwerbstätige mit Kindern gilt wie bisher das Umlageverfahren. Kinderlose sind von diesem Generationenvertrag ausgeschlossen, sie sparen im wesentlichen im Kapitaldeckungsverfahren für sich selbst. Aus der umlagefinanzierten Rentenversicherung werden nur Sockelbeträge für bedürftige Alte ohne Kinder aufgebracht.

Sinn erläutert: „Jede Generation wird in einem solchen System nur dann eine auskömmliche Rente haben, wenn sie eine von zwei Alternativen gewählt hat: Entweder muss sie Humankapital gebildet haben, indem sie Kinder in die Welt gesetzt und großgezogen hat. Oder sie muss gespart und somit direkt und indirekt Realkapital gebildet haben, um vom Verzehr dieses Kapitals zu leben. Eine Generation, die weder Human- noch Realkapital bildet, muss im Alter hungern. ${ }^{\text {"6961 }}$ Die 2001 eingeführten Anreize zur vermehrten Kapitalbildung (,Riester-Rente') hält Sinn allerdings für problematisch, denn sie verringerten nicht die „Fehlanreize für die Familienplanung“. ${ }^{962}$ Sinn führt weiter aus: „Die Staffelung von Umlagerente und Riester-Rente nach der Kinderzahl ist gerecht, weil sie dem Verursacherprinzip und dem Leistungsfähigkeitsprinzip folgt. (...) Man mag gegen den Vorschlag einwenden, mit der Zahlung des Rentenbeitrags erbrächten junge, kinderlose Bürger bereits eine Leistung für die eigene Rente, und insofern sei es ungerecht, sie auf dem Wege des Riester-Sparens zu einer zweiten Leistung zu zwingen. Dieses Argument verkennt, dass es historisch immer zu den normalen Pflichten einer jeden Generation gehörte, zwei Leistungen zu erbringen: In der leistungsfähigen Lebensphase muss man seine Eltern und seine Kinder ernähren. Die erste dieser beiden Leistungen wird heute in Form der Rentenbeiträge erbracht, die ja in vollem Umfang an die heutigen Rentner fließen. Doch die zweite Leistung wird von vielen Menschen nicht mehr erbracht, weil sie sich gegen Kinder entscheiden.

\footnotetext{
${ }^{959}$ So z.B. Almsick (1988). Dieser Argumentation ist das Bundesverfassungsgericht bei dem von Jürgen Borchert erstrittenen ,Mütterurteil' vom 7.7.1992 und in dem Urteil zur Pflegeversicherung vom 3.4.2001 teilweise gefolgt. In der Urteilsbegründung des letztgenannten Urteils heißt es: „Die gleiche Belastung mit Versicherungsbeiträgen führt zu einem erkennbaren Ungleichgewicht zwischen dem Gesamtbetrag, den Kindererziehende in die Versicherung einbringen und dem Geldbetrag von Kinderlosen. Hierin liegt eine Benachteiligung von erziehenden Versicherten, die im Beitragsrecht auszugleichen ist.“

${ }^{960} \operatorname{Sinn}(2003)$ 76-78 u. 83-87; Sinn (1997), 10

${ }^{961} \operatorname{Sinn}(14.01 .2003)$

${ }^{962}$ A.a.O.
} 
So gesehen ist es sehr wohl gerecht, nun auch diesen Menschen eine zweite Leistung in Form des Riester-Sparens abzuverlangen. “963

Die im Zuge der Riester'schen Rentenreform vom 26.1.2001 vorgesehenen Rentenniveaukürzungen und das kompensierende Sparen sollten nach Sinns Ansicht auf Kinderlose konzentriert werden. Menschen, die schon mehrere Kinder großziehen, an der Riester-Rente zu beteiligen sei gleichbedeutend mit einer dreifachen Last: „Als Beitragszahler ernähren sie die jetzt Alten, als Eltern finanzieren sie über die Kosten der Kindererziehung die Renten aller zukünftigen Rentenbezieher, und als Riester-Sparer müssten sie zusätzlich ihre eigenen Renten finanzieren." "964 Stattdessen soll Kinderlosen die Rente um die Hälfte gekürzt werden, allerdings ohne die bisher aufgebauten Anwartschaften anzutasten. ${ }^{965}$

Sinn setzt voraus, dass es die Pflicht jeder Generation sei, dafür zu sorgen, dass ihre Nachfolger-Generation ebenso groß ist wie sie selbst: „Die Staffelung der Umlagerente nach Kinderzahl ist nicht nur gerecht, sie wird darüber hinaus zu einer wünschenswerten Änderung der Familienplanung führen. (...) Manch ein bislang noch unschlüssiges junges Paar wird sich unter diesen Umständen doch für Kinder entscheiden. Alle Erfahrung zeigt, dass gerade auch die Familienplanung sehr stark auf ökonomische Anreize reagiert. (...) Die sog. Social Security Hypothesis, nach der die Ausgestaltung des Rentensystems den Kinderwunsch mitbeeinflusst, ist von der einschlägigen Fachliteratur auch für Deutschland empirisch bestätigt worden. “ 966

\subsection{Kritik der, Rente nach Kinderzahl'}

Zunächst ist festzustellen, dass bei Sinn familienpolitische und geburtenpolitische Argumente vermischt werden. Familienpolitisch ist die Argumentation, dass ein solches System Gerechtigkeit zwischen Eltern und Kinderlosen herstellen und die Eltern für ihren Konsumverzicht entschädigen würde. Geburtenpolitisch ist die Argumentation, dass die umlagefinanzierte Rentenversicherung deshalb zu reformieren sei, weil sie eine der wichtigsten Ursachen für den Geburtenrückgang sei. ${ }^{967}$

Selbst wenn man eine geburtenfördernde Politik grundsätzlich für sinnvoll hält, kann man der Ansicht sein, dass es gewichtige Gegenargumente gegen das spezielle Instrument ,Rente nach Kinderzahl' gibt? Wie soll mit Eltern umgegangen werden, deren Kinder sterben, so lange sie noch klein sind? Soll ein 60jähriger, der spätes Vaterglück erreicht, dann doch noch in die gesetzliche Rentenversicherung einbezogen werden, nachdem er sein ganzes Leben im Kapitaldeckungsver-

\footnotetext{
${ }^{963}$ A.a.O.

${ }^{964}$ A.a.O.

${ }^{965}$ A.a.O.

${ }^{966}$ A.a.O.; siehe auch Sinn (1997)

${ }^{967}$ A.a.O.
} 
fahren für Kinderlose war? Wem gehört der Rentenanspruch, wenn Eltern sich scheiden lassen? Inwieweit leisten Kinder überhaupt einen Beitrag zur Finanzierung der gesetzlichen Rente, wenn sie arbeitslos sind, oder aber als Beamte oder Selbständige nicht in die Rentenversicherung einzahlen? Wer bekommt im Fall von Adoption den Anspruch? Der notwendige administrative Aufwand für ein solches hybrides System ist „grotesk““.968

Als Versuch, den immensen Aufwand des Sinn-Modells zu vermeiden und dennoch Geburten über das Rentensystem zu fördern, kann der CSU-Vorschlag gelten. Der zwischen November 2003 und März 2004 mehrfach modifizierte Vorschlag sieht keine ,Rente nach Kinderzahl' nach dem Sinn-Modell vor, sondern eine Entlastung der Eltern beim Rentenbeitrag von 50 Euro je Kind. ${ }^{969} \mathrm{Um} \mathrm{zu}$ vermeiden, dass Arbeitgeber mit zwei Beitragssätzen rechnen müssen, sollen die Rentenversicherungsträger fiktive $\mathrm{Zu}-$ und Abschläge auf das Gehalt berechnen. So soll das Gehalt eines Elternteils ab der Geburt seines Kindes fiktiv um 500 Euro im Monat gemindert werden. Bei einem Rentenbeitragssatz von 20 Prozent ergibt sich daraus eine Entlastung von 50 Euro je Kind. Um Aufkommensneutralität und eine Umverteilung zwischen Kinderlosen und Eltern zu erhalten, soll das Einkommen eines Kinderlosen fiktiv um 14 Prozent erhöht werden, woraus sich eine Mehrbelastung von 70 Euro im Monat ergäbe. Kinderlose mit einem Einkommen über der Bemessungsgrenze von 5.150 Euro (Stand: 31.03.2004) müssten dann statt 502 Euro 572 Euro Rentenbeitrag im Monat zahlen, unabhängig von ihrem Alter. Der Arbeitgeberbeitrag zur Rentenversicherung soll unverändert bleiben. ${ }^{970}$

Die CSU vertritt also das Sinn-Modell in einer stark abgeschwächten Variante. $\mathrm{Ob}$ eine dergestalt verwaltungstechnisch machbare ,Beitragsstaffelung nach Kinderzahl' das Gebärverhalten spürbar steigern würde, ist keinesfalls „empirisch bewiesen“, wie Sinn schreibt. Vielmehr heben viele Demografen die Wirkungslosigkeit moderater finanzieller Anreize hervor, da Paare vor allem aus tieferliegenden sozio-psychologischen Überlegungen heraus über die Zahl ihrer Kinder entscheiden. ${ }^{971}$ Der Demograf Schwarz schreibt: „Mit Erziehungsgeld, Erziehungsurlaub und Anrechnung der Erziehungszeiten in der Altersversorgung wurde 1986 ein bedeutendes neues Instrument der Familienpolitik geschaffen und bis heute weiterentwickelt. Es liegt daher nahe, gerade an diesem Beispiel zu untersuchen, ob es auch Auswirkungen auf die Geburtenentwicklung hatte. Diese erweisen sich als vorübergehende Effekte bei jedem Stadium der Weiterentwicklung als wahr-

\footnotetext{
${ }^{968}$ Diese Formulierung wählt Herfeld (2001), 88.

969 o.V. (09.03.2004), 11. Inzwischen steht der Vorschlag auch im Wahlprogramm von CDU/CSU für die vorgezogene Bundestagswahl 2005.

${ }^{970}$ CDU, SPD, FDP und die Rentenversicherungsträger lehnen das Modell ab. Die CDU will stattdessen die Anrechnungszeiten für Kinder, die nach 1992 geboren sind, von drei auf fünf Jahre erhöhen.

${ }^{971}$ Schwarz (1992)
} 
scheinlich, aber immer als minimal." ${ }^{\text {"72 }}$ Ein weiteres Gegenargument gegen Sinns Modell sind die langen Umstellungszeiten. Sinn spricht selbst von der Notwendigkeit der Sicherung der Anwartschaften aus dem heutigen System. Dies heißt im Klartext, dass die ,Rente nach Kinderzahl' erst nach einer jahrzehntelangen Übergangsfrist etabliert werden könnte, da ein Kinderloser, der heute schon eine Anwartschaft in Höhe von z.B. 1.000 Euro im Monat erworben hat, für sein gesamtes restliches Leben eine Rente ohne Abschläge wegen Kinderlosigkeit beziehen darf - dies muss schon aus Gründen des Vertrauensschutzes so sein und ist verfassungsrechtlich gesichert. Hier zeigt sich eine Besonderheit des Umlageverfahrens, nämlich dass der Einstieg sehr kurzfristig, der Ausstieg aber nur über Jahrzehnte möglich ist. Zusammenfassend kann man sagen, dass der CSU-Vorschlag - anders als das Sinn-Modell - eine verwaltungstechnisch machbare, aber dennoch ordnungspolitisch wenig sinnvolle und im übrigen so gut wie wirkungslose Reform der Rentenversicherung wäre.

\subsection{Kritik der, Kinderversicherung'}

Bei der Kinderversicherung wird die Umverteilung zu Gunsten von Familien, die heute über den Familienlastenausgleich maßgeblich aus Steuern aufgebracht wird, durch einen neuen Ast des Sozialversicherungssystems geregelt (ähnlich der Pflegeversicherung). Angesichts der Probleme, die die einseitige Lohnabhängigkeit der Sozialversicherungssysteme verursacht, ist der Vorschlag sehr problematisch. Beiträge werden nur auf Lohneinkommen der Arbeitnehmer, Steuern hingegen auch auf Kapital- und Zinseinkünfte erhoben. Generell sollte nicht vergessen werden, dass das Rentensystem im Moment intragenerationell ungerecht ist, da u.a. Beamte, Selbständige und Mandatsträger nicht einbezogen sind. ${ }^{973}$ Steuern müssen dagegen von allen, auch von den Rentnern bezahlt werden - daher ist bei allen gesamtgesellschaftlichen Aufgaben Steuerfinanzierung geboten. Hierfür gibt es Vorschläge von der Erhöhung des Kindergeldes (z.B. auch um 50 Euro im Monat) bis zur Erhöhung des Kinderfreibetrags. All diese Vorschläge haben zudem den Vorteil, die Situation von Familien dann zu verbessern, wenn die Kinder klein sind und der Finanzbedarf am höchsten ist.

Zusammenfassend lässt sich sagen, dass die beiden Vorschläge ,Kinderversicherung' und ,Rente nach Kinderzahl' zwar gutgemeint, aber zu unpraktikabel und ordnungspolitisch fehlsteuernd sind. Um die Geburtenrate in Deutschland zu erhöhen, sind wie gesagt ein gesellschaftlicher Wertewandel, stärkere finanzielle Anreize und ein besseres Betreuungsangebot geeignete Maßnahmen. Die in diesem Maßnahmenbündel enthaltenen ,finanziellen Anreize' müssen aber wohlüberlegt sein. Wie gezeigt, eignen sich Eingriffe ins Rentenrecht nicht.

\footnotetext{
${ }^{972}$ A.a.O., 197

${ }^{973}$ Tremmel (1997), $233 \mathrm{f}$.
} 
Einen Katalog wirksamer, sinnvoller und ethisch vertretbarer pronatalistischer Maßnahmen im Detail (genaue Bezifferung, Zeitplan) zu entwickeln, ist hier nicht Teil der Aufgabenstellung. Die gesamte deutsche Bevölkerungswissenschaft sollte ihre Forschungsanstrengungen zu dieser Fragestellung verstärken. Auf einen die Leistungsseite betreffenden Aspekt soll aber kurz hingewiesen werden. Es erscheint vor dem Hintergrund der Ausführungen zu antinatalistischen Bevölkerungspolitiken sinnvoll und klug, auch bei pronatalistischen Politiken das Elterngeld einkommensabhängig zu gestalten. Bisher erhalten Eltern (bis zu einem Nettoeinkommen des arbeitenden Elternteils von bis zu 30.000 Euro jährlich) ein einheitliches Erziehungsgeld von 300 Euro. Besser wäre es, jungen Eltern ca. 60 Prozent ihres letzten Nettoverdienstes zu zahlen. Dadurch könnten verstärkt Eltern aus der gut ausgebildeten Mittelschicht ermuntert werden, mehr Kinder zu bekommen.

\subsubsection{Kinderwunsch und Kinderzahl}

Die theoretisch möglichen und in der Vergangenheit z.T. auch praktizierten Missbräuche und Auswüchse antinatalistischer Politik sind bereits thematisiert worden. Es darf aber nicht vergessen werden, dass eine pronatalistische Politik genauso stark in den Freiheitsbereich des Einzelnen eingreift. Die nationalsozialistische Geburtenpolitik steht uns hier noch als Menetekel vor Augen. Ein kürzlich gesendeter BBC-Film über pronatalistische Politiken in Europa prophezeit für 2024 Demonstrationen junger Menschen, die sich gegen den gesellschaftlichen Druck, mehr und früher Kinder zu bekommen, wehren. ${ }^{974}$ In jedem Fall besteht auch hier die Gefahr, dass in der Praxis staatliche Maßnahmen angewandt würden, die das im Aktionsprogramm von Kairo festgeschriebene Recht auf Selbstbestimmung verletzten.

Es ist für die ethische Bewertung pronatalistischer Politiken wichtig, ob wir in Deutschland eine Konstellation haben, in der sowohl der Staat wie auch die Individuen sich eine Erhöhung der Geburtenrate wünschen, oder ob eine Konstellation vorliegt, in der nur der Staat die Erhöhung der Geburtenraten wünscht. Das Ziel einer Geburtenpolitik kann darin bestehen, die Hindernisse aus dem Weg zu räumen, die der Verwirklichung schon vorhandener Kinderwünsche entgegenstehen. ${ }^{975}$ Oder Geburtenpolitik kann darauf gerichtet sein, einen Kinderwunsch überhaupt erst entstehen lassen.

Gerade wegen der schon thematisierten Komplexität der Frage nach der gewünschten Kinderzahl ist es überraschend, dass wirklich umfassende repräsentative Befragungen lange Zeit Mangelware waren. Klingholz schätzt die gewünschten Kinderzahlen in Deutschland auf 1,8 bis 2,1 Kinder, also knapp unter dem Reproduktionsniveau. ${ }^{976}$ Birg nimmt an, dass sich die Deutschen 2,1 Kinder

\footnotetext{
${ }^{974} \mathrm{BBC}(2004)$

${ }^{975}$ Birg (2001), 64

${ }^{976}$ Klingholz/Tremmel (2004)
} 
wünschen.$^{977}$ Einen deutlichen Erkenntnisgewinn erbrachte die Untersuchung The emergence of sub-replacement family size ideals in Europe von Joshua Goldstein, Wolfgang Lutz und Maria Testa. ${ }^{978}$ Basierend auf Daten der repräsentativen Eurobarometer-Umfrage 2001 weisen sie nach, dass deutsche Frauen sich im Median mit nur 1,7 Kinder eine Kinderzahl wünschen, die deutlich unter dem Bestandhaltungsniveau liegt. ${ }^{979}$ Methodisch wurden in der Eurobarometer-Studie mögliche Missverständnisse beim Verständnis der Fragestellungen minimiert. Erstens wurden die Erhebungen in allen Ländern vom gleichen Interviewer-Team durchgeführt, zweitens wurden zwei Fragen gestellt:

(1) Generally speaking, what do you think is the ideal number of children for a family?

(2) And for your personally, what would be the ideal number of children you would like to have or have liked to have had?980

Während in früheren Untersuchungen die Frage nach der gewünschten Kinderzahl oft mehrdeutig formuliert war, erlaubte es diese Vorgehensweise, explizit die persönlichen Ideale (Frage 2) zu erfragen.

Die vom Bundesinstitut für Bevölkerungsforschung 2005 veröffentlichten Ergebnisse der Population Policy Acceptance Study betätigen diese niedrigen Kinderwünsche: „Die Zahlen zeigen aber auch und das haben wir in diesem Ausmaß nicht erwartet, dass der Kinderwunsch in Deutschland inzwischen niedriger ist als bei früheren Untersuchungen. Nach den Berechnungen auf der Grundlage der neuen Daten der PPAS werden im Durchschnitt nur noch 1,7 Kinder gewünscht. (...) Das ist eine neue Situation, mit der Deutschland zu einem Ausnahmeland in Europa geworden ist. Es ist eindeutig festzustellen: Die Familien in Deutschland haben so viele bzw. wenige Kinder, wie sie sich wünschen. Und die häufig besprochene Spanne zwischen tatsächlicher Kinderzahl und Kinderwunsch, auf der viele familienpolitische Hoffnungen ruhten, deren geburtenförderndes Potential aber schon immer angezweifelt wurde, gibt es nicht mehr." ${ }^{\text {"981 }}$

Nehmen wir an, es handele sich bei Deutschland tatsächlich um eine Situation, in der der Staat mit 2,1 eine höhere Geburtenrate wünscht als die Individuen $(1,7)$, letztere aber deutlich über der tatsächlichen von 1,4 liegt. Dann ist die Steigerung von 1,4 auf 1,7 im Rahmen einer gegenwartsethischen Betrachtung ethisch nicht nur in jedem Fall unproblematisch, sondern sogar geboten. Berücksichtigt man nun zusätzlich die intergenerationelle Perspektive, so lässt sich zwar nicht bestreiten, dass dadurch die Schrumpfung abgebremst und dadurch die Entlastungseffekte für die Umwelt geringer würden, aber die Schrumpfung ginge ja

977 Diskussion im Anschluss an die Referate von Herwig Birg u. Paul Kirchhof, in: Hessische Staatskanzlei (2003), 204

${ }^{978}$ Goldstein/Lutz/Testa (2004)

${ }^{979}$ A.a.O., 11

${ }^{980}$ A.a.O., 6

${ }^{981} \mathrm{BiB}$ (2005), 10 
dennoch weiter. Da es aber auch im Sinne der ökologischen Generationengerechtigkeit nicht das Ziel sein kann, Deutschland unverhältnismäßig stark zu entvölkern, erscheinen pronatalistische Maßnahmen mit dem Ziel, eine Geburtenrate von 1,7 zu erreichen, bei Einhaltung der Vier-Fünftel-Regel in jedem Fall ethisch gerechtfertigt.

Wäre auch eine Politik zur Erhöhung des Kinderwunsches der Deutschen von 1,7 auf 2,1 ethisch gerechtfertigt? Man müsste zu dem Zeitpunkt, zu dem die Bevölkerung auf ein niedrigeres Niveau wie z.B. 70 Mio gesunken ist, prüfen, ob die Prinzipien ökologischer Nachhaltigkeit nun eingehalten werden. Ist dies so, so spricht aus Sicht der ökologischen Generationengerechtigkeit nichts gegen pronatalistische Maßnahmen, welche auf eine Geburtenrate von 2,1 zielen. Ist dies nicht so, dann muss erneut abgewogen werden, ob sich die ökologischen Vorteile einer weiteren Schrumpfung vor dem Hintergrund der Nachteile in anderen Bereichen vertreten lassen.

\subsubsection{Fazit}

Als Fazit lässt sich ziehen, dass es kein Widerspruch ist, Deutschland auf die Schrumpfung einzustellen und ihre Chancen zu nutzen, und gleichzeitig Maßnahmen zu ergreifen, um mittelfristig die Geburtenraten wieder deutlich zu steigern. Aus der negativen Bewertung von - aus ökologischer Sicht - relativer oder absoluter Überbevölkerung folgt ja nicht der Wunsch nach Selbstauslöschung, sondern ein Plädoyer für eine Stabilisierung der Bevölkerung auf ökologisch nachhaltigem Niveau.

Die Mehrzahl der Deutschen hat nicht aus Gründen ökologischer Rücksichtnahme so wenige Kinder. ${ }^{982}$ Die Gründe für die geringe Geburtenrate sind u.a. die besseren Möglichkeiten der Familienplanung, der Verlust des ,instrumentellen' Faktors von Kindern, die schlechte Vereinbarkeit von Beruf und Familie sowie die Verlängerung der Adoleszenz. Die verlängerten Ausbildungszeiten spielen ebenso eine Rolle wie der Umstand, dass Kinder heute ,nicht mehr einfach so kommen', sondern zu einem selbstgewählten Lebensentwurf beitragen. Da Beziehungspartner immer mehr zu ,Lebensabschnittspartnern' werden, summieren sich die Zeiten, in denen der Kinderwunsch keine Rolle spielt. Paare, die seit acht Jahren zusammen sind, haben dagegen auch heute mit derselben Wahrscheinlichkeit Nachwuchs, wie ihn die eigenen Eltern oder Großeltern hatten. Die Theorie, dass rationale Individuen in einer ökonomischen Kosten-Nutzen-Rechnung über die Zahl der Kinder entscheidet, besitzt relativ geringen Erklärungswert. Deshalb ist von verstärkten direkten finanziellen Anreizen, auch wenn diese weiterhin erhalten werden sollten, nur eine geringe Steigerung der Geburtenrate zu erwarten. Deutschland liegt beim ,Kindergeld' bereits auf Platz 2 in Europa. Um die Geburtenrate in Deutschland zu erhöhen, ist ein Mix aus verschiedenenn Maßnah-

${ }^{982}$ Dass dies trotzdem für einige ein Motiv sein könnten, vermutet Natorp (01.08.1998). 
men notwendig, darunter auch ein gesellschaftlicher Wertewandel und ein besseres Betreuungsangebot.

\subsubsection{Gezielte Einwanderung}

\subsubsection{Nachholende Integration notwendig}

Seit 1972 sterben mehr Menschen in Deutschland, als geboren werden. Nur durch Einwanderung wurde ein Schrumpfen in den letzten 30 Jahren verhindert. Einwanderung wird von den Gegnern jeder Schrumpfung auch für die nächsten Jahrzehnte als Mittel gesehen, den demografischen Wandel aufzuhalten. Deutschland müsse aus demografischen Gründen zum Einwanderungsland werden und seine Grenzen weiter öffnen, so wird gefordert.

Dass Einwanderung in keiner Weise die ersten beiden Teile der dreifachen Strategie ersetzen kann, wird klar, wenn man sich ein Rechenexempel der UN ansieht. Wollte Deutschland durch die Einwanderung verhindern, dass das Durchschnittsalter der Bevölkerung steigt, so müssten 188 Mio. Menschen bis 2050 mehr ein- als auswandern. ${ }^{983}$ Bei diesem Experiment kämen pro Jahr 3,4 Millionen Zuwanderer ins Land. Dadurch würde die Bevölkerungszahl von 82 Mio. auf 299 Mio. zunehmen. ${ }^{984}$ Ganz Deutschland würde verstädtert, die Bodenversiegelungsdichte würde sich extrem beschleunigen. Diese enormen Zahlen ergeben sich aus der Tatsache, dass auch Einwanderer altern.

Eine rationale Einwanderungspolitik muss auch die Frage stellen, aus welchen Ländern die Einwanderer kommen sollen. Wie schon gesagt liegt in den meisten europäischen Nachbarländern die Gebärfreudigkeit der Frauen auf einem ähnlichen niedrigen Niveau wie bei uns. Nun gibt es natürlich bei rund 3 Milliarden zusätzlichen Erdenbürgern in den nächsten 50 Jahren theoretisch keinen Mangel an Zuwanderern - doch sie kommen aus Ländern, die beträchtliche kulturelle Unterschiede zu unserem Kulturkreis aufweisen. Würde man schlicht die am schnellsten wachsenden Regionen in geografischer Nähe zu Europa berücksichtigen, so würde der Anteil islamischer Einwanderer einen Großteil davon ausmachen.

Heik Afheldt weist darauf hin, dass anders als bei den klassischen Einwanderungsbeispielen nicht große, dünn besiedelte Landstriche zu füllen wären, ${ }^{985}$ sondern dass es diesmal darum ginge, allmählich entstehende Leerstände aufzufüllen. ${ }^{986}$ Dies erhöht erheblich das Konfliktpotenzial zwischen Alteingesessenen, die auf Anpassung an ihre Kultur bestehen und Neuankömmlingen, die ihre eigene Kultur auch auf neuem Territorium etablieren wollen.

\footnotetext{
${ }^{983}$ UN Population Division (2000)

${ }^{984}$ A.a.O.

${ }^{985}$ Freilich auf Kosten der Indianer, Zulus oder Aborigines.

${ }^{986}$ Afheldt (17.05.2001), 26
} 
Es steht außer Frage, dass ein buntes Miteinander der Kulturen per se ein positiver Wert ist. Festivals wie der Berliner Karneval der Kulturen oder das vielfältige Angebot ausländischer Restaurants bereichern Deutschland ohne jeden Zweifel. Im Rahmen einer Strategie für die demografische Zukunftsfähigkeit Deutschlands ist jedoch das wichtigste Beurteilungskriterium, ob aus Arbeitsmarktaspekten eine Einwanderungsstrategie sinnvoll ist. „Zuwanderer tragen seit Ende der 1980er Jahre nicht mehr zur Sanierung der deutschen Sozialkassen bei, sondern belasten sie“, schreibt Klingholz in einem Artikel über die demografische Zukunftsfähigkeit Deutschlands. ${ }^{987}$ Denn der Erwerbstätigenanteil unter den knapp 8 Millionen in Deutschland lebenden Ausländern ist erheblich gesunken. Die Arbeitslosigkeit ist unter Ausländern doppelt so hoch wie unter Deutschen. ${ }^{988}$ Sozialhilfe beziehen sie gemessen an ihrem Bevölkerungsanteil dreimal so häufig. ${ }^{989}$ Deutlich zeigen sich die Mißerfolge der bisherigen Integrationspolitik - trotz eingeschränkter Vergleichbarkeit von deutschen und nicht-deutschen Tatverdächtigen ${ }^{990}$ - auch bei den Kriminalitätsraten. In den alten Ländern mit Gesamt-Berlin beträgt der Anteil nicht-deutscher Tatverdächtiger bei ,illegaler Einfuhr von Kokain“ 59,7 Prozent, bei „Mord und Totschlag“ 31,3 Prozent, bei „Vergewaltigung und sexueller Nötigung“ 30,6 Prozent, und bei „Raub und räuberischer Erpressung“ 29,8 Prozent. ${ }^{991}$ Dabei sind die EU-Inländer relativ unauffällig. Das Gros der nicht-deutschen Tatverdächtigen (2001: 87,6 Prozent) kommt von außerhalb der EU. Da die Fertilitätsraten in der EU ähnlich rückläufig sind, müssten auch Einwanderer, die eine Schrumpfung der deutschen Bevölkerung ausgleichen sollen, von außerhalb der EU kommen und wären somit ,integrationsferner“.

Die lange vermutete Hypothese, dass seit 1960 eingewanderte Migranten und ihre Nachkommen mit der Zeit ebenso integriert sein werden wie die polnischen und slowenischen Zuwanderer ins Ruhrgebiet Ende des 19. Jahrhunderts, hat sich nicht bewahrheitet. Junge Migranten der dritten Generation sprechen häufig schlechter Deutsch als jene der zweiten. ${ }^{992}$ Sie sind in stärkerem Maße arbeitslos als ihre Eltern. Die Angehörigen der dritten Generation sind häufig nicht bereit, anspruchslos und hart zu arbeiten wie ihre Eltern, die als ,Gastarbeiter' ins Land kamen. „Aufgewachsen in Deutschland, stellen sie aber Ansprüche wie Einheimische, werden zwangsläufig enttäuscht und sind noch schlechter zu integrieren“,

\footnotetext{
${ }^{987}$ Klingholz (2004), 93

${ }_{988}$ A.a.O.

${ }^{989}$ Am 31.12.2003 bekamen 2,209 Mio. Deutsche (rund 2,94 Prozent der deutschen Wohnbevölkerung) und 619.000 Ausländer (rund 8,5 Prozent der ausländischen Wohnbevölkerung) Sozialhilfe. Siehe Statistisches Bundesamt. Tabelle 22121-0001. https://www-genesis.destatis.de/genesis/online/Online. Rev. 2004-06-29

${ }_{990}$ Der/die durchschnittliche nicht-deutsche Tatverdächtige ist männlicher, jünger und lebt eher in Städten als sein/ihr deutsches Pendant. Zahlen, in denen diese kriminalitätsbegünstigenden statistischen Merkmale herausgerechnet wurden, veröffentlicht das Bundeskriminalamt nicht.

${ }^{991}$ Bundeskriminalamt (2003), 28-30

${ }^{992}$ Klingholz (2004), 94
} 
resümiert Klingholz. ${ }^{993}$ Statt den Aufstiegsmustern des neuen Heimatlandes zu folgen, setzten viele noch immer eher auf eine Einkommens- statt auf eine Bildungskarriere, so eine These der Leipziger Soziologin Heike Diefenbach. ${ }^{994} \mathrm{Im}$ Streben nach schnellem finanziellen Erfolg verglichen sie sich vor allem mit Angehörigen der eigenen Migranten-Gruppe - und dies um so stärker, wenn sie in Stadtvierteln und Schulen unter sich blieben. Angesichts dieser Bestandsaufnahme sind die Aussagen der Demografen, dass der Ausländeranteil in der wichtigen Altersgruppe der 20-40jährigen in den Städten zukünftig den Anteil der Deutschen überflügeln wird, besorgniserregend. Herwig Birg schrieb 1998: „Unabhängig von den möglichen bzw. notwendigen Änderungen des Staatsangehörigkeitsrechts lässt sich sagen: Die Deutschen i.S. der heutigen Definition der Staatsangehörigkeit werden ihre Bevölkerungsmehrheit im nächsten Jahrhundert an die Zugewanderten und ihre Nachkommen verlieren. Zuerst wird dies in den großen Städten der Fall sein, wo heute bereits in einigen Städten ein Viertel bis ein Drittel Ausländer sind, später flächendeckend auch im Landesdurchschnitt. In Berlin (West) wird z.B. der Anteil der Ausländer bei den Unter20jährigen von heute 25 Prozent schon bis 2015 auf 52 Prozent zunehmen. ${ }^{\text {" } 995}$

In den letzten Jahrzehnten ging wegen einer verfehlten Einwanderungs- und Integrationspolitik die Schere zwischen der zugewanderten Bevölkerung und dem Rest immer weiter auf. Klingholz zieht daraus die Schlussfolgerung: „Wir haben nicht nur mehr Ausländer, sondern wir haben immer mehr schlecht integrierte Ausländer." ${ }^{\text {"9996 }}$

Es ist aus demografischen Gründen sinnvoll und notwendig, in Zukunft um bestimmte Einwanderer zu werben. Problematisch ist, dass im Moment gleichzeitig die Integrationskosten für die versäumte Integration der letzten Jahrzehnte anfallen. Wegen der Langfristigkeit demografischer Trends wird die Integration der hohen Zahl von Ausländern, die Deutschland in den 1990er Jahren aufgenommen hat, noch einige Zeit in Anspruch nehmen. Abbildung 48 zeigt die Dimension der Zuwanderung in Staaten der Europäischen Union zwischen 1990 und 1996.

\footnotetext{
993 A.a.O.

${ }^{994}$ A.a.O.

${ }^{995} \operatorname{Birg}(1998), 245$

${ }^{996}$ Klingholz (2004), 94
} 


\section{Abb. 48: Zuwanderungen in Staaten der Europäischen Union und der Schweiz, 1990 bis 1996 (kumuliert)}

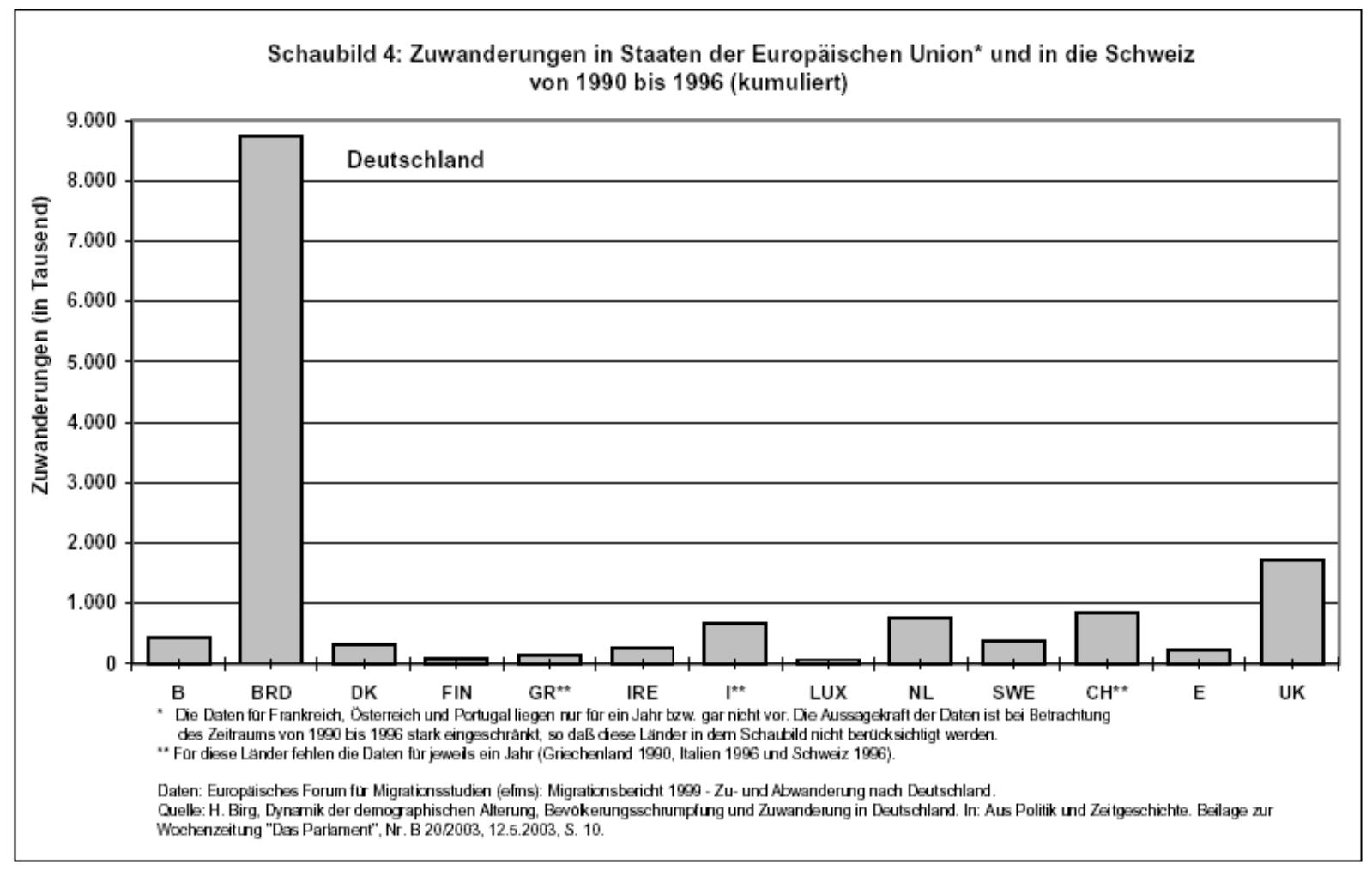

\section{Quelle: Birg (2004), 13}

Zwischen 1996 und 2002 ging dann die Nettozuwanderung auf ca. 175.000 jährlich zurück. ${ }^{997}$ In Bezug auf zukünftige Zuwanderung sollten die Fehler vermieden werden, die in der Vergangenheit gemacht wurden. In Deutschland erfolgte in den letzten Jahrzehnten die (unkontrollierte) Einwanderung maßgeblich über das Asylrecht. Daraus sollte die Konsequenz gezogen werden, die humanitäre Einwanderung über das Asylrecht auf ein Maß zu begrenzen, das vom Zielland festgelegt wird. Zwar gehören die enormen Einwandererzahlen der frühen 1990er Jahre Vergangenheit an, nachdem die sog. Drittstaaten-Regelung eingeführt wurde. Aber die Änderung von Artikel 16a war ein Kompromiss, der den Individualklageweg für Asylbewerber bestehen ließ.998 Dieser Rechtsanspruch existiert jedoch in keinem klassischen Einwanderungsland, da diese Länder selbst über die Zahl der Einwanderer bestimmen wollen, die sie aus rein humanitären Gründen aufnehmen. Der Individualklageweg sollte daher im Rahmen einer europäischen Harmonisierung abgeschafft werden. Herwig Birg schreibt dazu: „Wir brauchen ein Einwanderungsgesetz, durch das die Quoten für Einwanderer aus humanitären

\footnotetext{
997 Barth (2004), 27

${ }^{998}$ Von den Gerichten werden nur 3-4 Prozent der Asylbewerber anerkannt.
} 
Gründen, insbesondere für Flüchtlinge und Asylbewerber, und die Quoten für die aus Arbeitsmarktgründen benötigten Zuwanderer, jährlich aufs neue getrennt voneinander festgelegt werden, so dass sich die Gesamtquote aus der Summe der Einzelquoten ergibt. ${ }^{\text {“999 }}$ Im Sommer 2004 ist nach fünf Jahren Verhandlungen schließlich ein Einwanderungsgesetz verabschiedet worden, welchem wir uns nun zuwenden.

\subsubsection{Deutschlands erstes Einwanderungsgesetz}

Die künftige Akzeptanz von Migranten, die Offenheit der Gesellschaft ihnen gegenüber und die Attraktivität Deutschlands als Einwanderungsland hängen entscheidend davon $\mathrm{ab}$, ob die unkontrollierte Einwanderung eingedämmt wird und diejenigen in die Gesellschaft eingegliedert werden, die bereits im Land sind. Es ist zu erwarten, dass das im Sommer 2004 verabschiedete Einwanderungsgesetz die nachholende Integration beschleunigt und zugleich eine bessere Steuerung der weiteren Zuwanderung zulässt. ${ }^{1000}$ Der Kompromiss erleichtert den Zuzug von Hochqualifizierten, Selbständigen und qualifizierten Engpaß-Arbeitern. ${ }^{1001}$ Das neue Einwanderungsgesetz, mit dem Deutschland eine jahrzehntelange chaotische Praxis beseitigt, enthält eine sofortige und unbefristete Niederlassungserlaubnis für Hochqualifizierte wie Wissenschaftler und Spezialisten mit einem Mindestgehalt von 84.000 Euro im Jahr, wenn sie einen Arbeitsplatz nachweisen können. Eine zunächst auf drei Jahre befristete Aufenthaltserlaubnis erhalten Selbständige, sofern an ihrem Zuzug ein wirtschaftliches Interesse besteht. Hiervon wird ausgegangen, wenn die Bewerber nicht älter als 45 Jahre sind und mindestens eine Million Euro investieren. Nach drei Jahren erwerben sie den Anspruch auf einen Dauer-Aufenthalt in Deutschland. Das sog. Punktesystem wurde zum Bedauern der Wirtschaft aus dem Gesetzentwurf gestrichen. Es hätte im Rahmen eines jährlich festzulegenden Kontingentes einen flexiblen Zuzug von Fachkräften auch ohne Nachweis eines konkreten Arbeitsplatzes ermöglicht.

Angesichts der bisherigen Integrationsbilanz wird der nachholenden Integration von bereits in Deutschland lebenden Ausländern eine hohe Bedeutung zugemessen. Als eine der wichtigsten Maßnahmen finanziert der Bund Sprachkurse für im Land lebende Zugewanderte ohne deutsche Sprachkenntnisse. Dieses Einwanderungsgesetz ist der Beginn einer am eigenen Bedarf orientierten Einwanderungspolitik und insofern zu begrüßen. Negativ ist, dass das Asylgesetz nicht ebenfalls geändert wurde.

Zusammenfassend kann man sagen, dass Einwanderung nach den bisherigen Erfahrungen nicht als Königsweg gesehen werden sollte, um den demografischen Wandel in Deutschland zu bewältigen. Eine massive Ausweitung der Zuwande-

\footnotetext{
${ }^{999} \operatorname{Birg}(2001), 18$

${ }^{1000}$ Ein bestimmtes Maß an Zuwanderung geschieht über den Familiennachzug automatisch, da wir bereits eine hohe Zuwanderungsbevölkerung haben.

${ }^{1001}$ o.V. (28.5.2004)
} 
rung würde nicht nur die ökologischen Chancen der Schrumpfung verbauen, sondern auch erhebliche Widerstände in der Bevölkerung hervorrufen. Vorrangige Aufgabe ist zunächst die nachholende Integration, bevor man sich neue Integrationslasten auflädt. Hand in Hand mit Erfolgen bei der nachholenden Integration sollte Deutschland in den nächsten Jahrzehnten mehr und mehr seine Grenzen für neue Einwanderer öffnen, wobei die Kontingente für die aus humanitären Gründen und die aus Arbeitsmarktgründen aufgenommenen Zuwanderer beide vom Zielland festgelegt werden. Für letztere Gruppe bietet das neue Einwanderungsgesetz eine gute Grundlage, auf der künftig aufgebaut werden kann.

Als dritten Baustein einer Strategie für seine demografische Zukunftsfähigkeit braucht Deutschland hochqualifizierte Einwanderer. In bereits vorgestellten Szenario wandern etwa 60.000 Menschen jährlich netto nach Deutschland ein. Dadurch könnte die Differenz geschlossen werden, die nach einer Steigerung der Geburtenrate noch zum Bestandhaltungsniveau bestehen bleiben könnte. Der tatsächliche Bedarf richtet sich langfristig u.a. nach der tatsächlichen Entwicklung der Geburtenrate - steigt sie schneller an, ist er niedriger und umgekehrt.

\subsection{Demografischer Wandel in Deutschland - Fazit}

Allen Prognosen nach wird Europa in den nächsten Jahrzehnten der einzige Kontinent sein, in dem es jedes Jahr ,mehr Platz' geben wird. Dieser Wandel wird sehr unterschiedlich bewertet. Die einen schwärmen von Vollbeschäftigung und malen eine Zukunft aus, in der Kinder nicht mehr in überfüllten Klassen lernen müssen, in der Parkplatznot und chronisch verstopfte Straßen der Vergangenheit angehören und das Schlangestehen ein Ende hat. ${ }^{1002}$ Andere sprechen dagegen davon, dass wir unsere Infrastruktur nicht aufrecht erhalten können und verarmen werden.

In ökologischer Hinsicht aber sind die Entlastungseffekte für die Natur nicht zu bestreiten. Auch wenn die ökologische Belastung stärker mit der Haushaltszahl als mit der Einwohnerzahl korreliert sein sollte und deshalb der Zusammenhang nicht einfach dem Dreisatz folgt, so bietet der demografische Wandel große Potenziale, v.a. für die Probleme der Artenvielfalt und der Bodenversiegelung. Die PAT-Formel gilt grundsätzlich nicht nur wachsende, sondern auch für schrumpfende Gesellschaften. ${ }^{1003}$

Welches wirtschaftliche Szenario bei der Schrumpfung das wahrscheinlichste ist, ist schwieriger vorauszusagen. Hier besteht noch großer Forschungsbedarf. Die Wirtschaftswissenschaft hat Schrumpfungsprozesse bisher weitgehend vernachlässigt. An zahlreichen volkswirtschaftlichen Fakultäten kann man ,Wachstumstheorie' studieren - ,Schrumpfungstheorie' wird bisher nirgendwo angebo-

\footnotetext{
${ }^{1002}$ Vgl. Vortrag von Pero Mícíc auf den Rheingauer Impulsen am 31.10.2003, www.FutureManagementGroup.com. Rev. 2004-02-14

${ }^{1003}$ Tremmel (2001), 16
} 
ten. Trotz vieler Fragezeichen, z.B. über die zukünftige Entwicklung der Arbeitslosenquote und der Innovationskraft der Belegschaft, deutet aber vieles darauf hin, dass der prognostizierte demografische Wandel in wirtschaftlicher Hinsicht eher negative Auswirkungen auf kommende Generationen haben wird.

Die reichen Länder stehen damit heute schon vor einer Grundsatzentscheidung: Entweder sie streichen die ökologische Dividende schrumpfender Bevölkerungen ein und machen sich bewusst, dass dies Probleme, u.a. für das Rentensystem, mit sich bringen wird. Oder sie tun durch eine Förderung der Immigration und eine Ankurbelung der Geburtenraten alles, um die Krise des Rentenversicherungssystems abzuwehren, und schlagen die ökologische Dividende aus. Dies ist eine fundamentale Wahl, die wir treffen müssen. Hier wurde eine dreiteilige Strategie vorgeschlagen, die die Chancen nutzen und die Probleme zumindest abmildern könnte.

Grundsätzlich wird jede Bewertung des demografischen Wandels in Deutschland von Zwiespältigkeit geprägt sein. Dies sei noch mal am Beispiel der zukünftigen Entwicklung der Innovationskraft hervorgehoben: Deutschland wird je nach Sichtweise dadurch in 50 Jahren eine mutlose und erschlaffte oder aber eine beruhigtere und gefestigtere Nation sein. Optimisten können ins Feld führen, dass ein deutscher Staat ohne, youth bulge', ohne Testosteron-Überschüsse ein Segen sei. Europas friedliche Einigung wird möglicherweise durch diesen Trend unterstützt. Pessimisten können einwenden, dass Deutschland dann im internationalen Wettbewerb zurückfallen könnte und generell ,langweiliger' werde. Niemand mehr wird Deutschland für Revolutionen oder große gesellschaftliche Neuentwürfe zu begeistern vermögen können. Vernunft und Weisheit werden stärker die Politik bestimmen als Idealismus und Veränderungsbereitschaft. Die Bevölkerung wird weniger unternehmerisch und weniger einfallsreich sein, aber auch reifer, selbstsicherer, weniger leicht zu provozieren.

Die Hoffnung beider sollte sein, dass hundert Jahre später dann weltweit ein solch alterungsbedingter Wertewandel einsetzen wird. Dies schließlich ist eine Aussicht, die man durchaus positiv werten kann. 


\section{Resümee}

Ziel der Studie war, Antworten auf die Fragen zu geben, welche die Erklärung der Mehrzahl der lebenden Nobelpreisträger von 1993 (Warnung der Wissenschaftler an die Menschheit) aufgeworfen hatte. Dazu galt es die folgende Hypothese zu prüfen:

Bevölkerungspolitik ist ethisch vertretbar, wenn sie durchgeführt wird, um ökologische Generationengerechtigkeit zu erreichen.

Diese Hypothese ist nur sinnvoll, wenn sich zwei vorgelagerte Hypothesen bestätigen, nämlich

Es besteht eine Kausalbeziehung zwischen Bevölkerung und Naturbelastung.

Und:

Die heute lebende Generation hat eine Verantwortung für zukünftige Generationen, u.a. in ökologischer Hinsicht. Intertemporale Generationengerechtigkeit ist ein sinnvolles Konzept.

Die erste vorgelagerte Hypothese ist empirischer Natur. Die Untersuchung zeigte, dass ein enger Zusammenhang zwischen dem Verlust an Biodiversität und Bevölkerungswachstum besteht. Allerdings lässt sich das Artensterben nicht monokausal auf Bevölkerungszunahme zurückführen. Daraus darf man aber auch nicht den falschen Umkehrschluss ziehen, dass die Bevölkerung für das globale Artensterben nur ein Faktor unter vielen sei. Selbst wenn Menschen theoretisch einfach ,zusammenrücken können', so ist dies in der Praxis keine Option. Daher bietet eine stabile bzw. rückläufige Weltbevölkerung große Chancen für den Erhalt von Artenreichtum und biologischer Vielfalt auf unserem Planeten. Für andere Umweltprobleme mag das Bevölkerungswachstum im Konzert der Kausalfaktoren eine weniger große Rolle spielen, aber selbst dann führt es oft zu größerer Umweltbelastung. Die erste Hypothese dieser Studie kann also als bestätigt angesehen werden.

Auch die zweite vorgelagerte Hypothese ließ sich bestätigen. Von den Grundsätzen intragenerationeller Gerechtigkeit ist vor allem die Formel, dass Gleiches gleich, Ungleiches seiner Eigenart entsprechend verschieden zu behandeln sei, etabliert. Generationen setzen sich aus Individuen zusammen, die alle die gleiche Menschenwürde besitzen. Prima facie sind ,Generationen' gleichwertig zueinander. Allerdings ist letztlich nicht die bloße Erhaltung des Status Quo das Ziel - sonst befänden wir uns alle noch auf dem Niveau der 
Neandertaler - sondern Fortschritt in der Lebensqualität ist erstrebenswert. Mit Hilfe dieser Überlegungen, wurde ,Generationengerechtigkeit' wie folgt definiert:

Generationengerechtigkeit ist erreicht, wenn die Angehörigen der heutigen Generation A den Angehörigen der nächsten Generation B die Möglichkeit geben, sich ihre Bedürfnisse mindestens im gleichen Ausmaß wie A zu erfüllen.

Da antinatalistische Bevölkerungspolitiken häufig mit dem Wohlergehen und den Interessen kommender Generationen begründet werden, wird dann untersucht, ob und wenn ja, wie man die Bedürfnisse und Interessen zukünftiger Generationen ermitteln kann. Die Grundbedürfnisse aller Menschen - heutiger und künftiger sind ähnlich. Angesichts der in allen Teilen der Welt steigenden Lebenserwartung bei gleichzeitigem Bevölkerungswachstum ergeben sich wenig Anhaltspunkte, dass die Menschheit derzeit durch eine Zerstörung der lebenserhaltenden ökologischen Systeme die Basis für die Erfüllung der Grundbedürfnisse zukünftiger Generationen untergräbt. Die Lebenserwartung dürfte auch in Zukunft weiter ansteigen. Allerdings ist das ungebremste Artensterben eine klare Verletzung unserer Pflichten gegenüber kommenden Generationen. Dieses einzuschränken wird wiederum nicht ohne eine möglichst rasche Stabilisierung der Weltbevölkerung möglich sein.

Die Antwort auf die dritte und wichtigste Hypothese der Studie (Legitimität von Bevölkerungspolitik) lautet: Es kommt darauf an. Zunächst wurde geprüft, ob überhaupt irgendeine Art von Bevölkerungspolitik ethisch vertretbar sein kann. Staaten haben das Wohl ihrer Bürger zu fördern. Dazu kann auch gehören, dass demokratische Staaten demografische Zielgrößen haben bzw. Stellungnahmen (z.B. in dem Sinne, dass die Geburtenrate des eigenen Landes zu hoch oder zu niedrig ist) abgeben. Bevölkerungspolitische Ziele sind also nicht generell unethisch. Das Spektrum möglicher geburtenpolitischer Maßnahmen reicht von einer Vergrößerung der Optionen bis hin zum Zwang. Um diese Einteilung greifbarer zu machen, wurde die wohl umstrittenste und zugleich am besten untersuchte Bevölkerungspolitik der Welt - die chinesische - im Detail dargestellt. Dieses Beispiel zeigte bereits, dass in der Praxis keine Idealtypen, sondern Mischformen vorherrschen.

Ein Staat darf in jedem Fall durch indirekte Maßnahmen (Verbesserung der Gesundheitsvorsorge, der Bildungschancen von Frauen und der verbesserten Aufklärung) seine demografischen Ziele anstreben. Es ist ihm erlaubt, dabei die quantitative Deckung des ,ungedeckten Bedarfs' an Kontrazeptiva in einen integrierten Ansatz einzubinden. Diese Erkenntnis bestimmt seit der Weltbevölkerungskonferenz in Kairo 1994 die bevölkerungspolitische Debatte.

Im Rahmen direkter Maßnahmen sind finanzielle Steuerungsmechanismen dann legitim, wenn sie der Vier-Fünftel-Regel folgen. Das heißt: Der Staat muss sicherstellen, dass durch seine Geburtenpolitik keiner seiner Bürger, der eine vom 
Staat als ,unerwünscht' angesehene Kinderzahl wählt, dadurch mehr als ein Fünftel seines Einkommens verliert im Vergleich zu einer Wahl der vom Staat als ,ideal' angesehenen und entsprechend ökonomisch geförderten Kinderzahl. Rationierungspolitik ist generell als unethisch einzustufen.

Bezüglich der Kernfrage der Studie sind vier mögliche ethisch relevante Konstellationen zu unterscheiden:

Fall 1: Staat wünscht Senkung der Geburtenrate, Individuen wünschen sich weniger Kinder als sie tatsächlich haben.

Fall 2: Staat wünscht Senkung der Geburtenrate, Individuen wünschen sich mehr Kinder als sie tatsächlich haben.

Fall 3: Staat wünscht Erhöhung der Geburtenrate, Individuen wünschen sich weniger Kindern als sie tatsächlich haben.

Fall 4: Staat wünscht Erhöhung der Geburtenrate, Individuen wünschen sich mehr Kindern als sie tatsächlich haben.

Davon ist nur Fall 1 ethisch unproblematisch. Fall 1 ist die Situation in vielen schnell wachsenden Entwicklungsländern, in denen Paare bzw. Individuen sich weniger Kinder wünschen als sie haben müssen und der Staat versucht, Kontrazeptiva bereit zu stellen. Hier liegt eine doppelt gebotene ethische Handlungsstrategie vor: Das Handeln im Interesse heutiger Menschen ist identisch mit dem Handeln im Interesse zukünftiger Generationen. Bei Fall 2 sind die Interessen kommender Generationen, v.a. auf Erhalt der biologischen Vielfalt, abzuwägen gegen die Interessen heutiger Menschen. Bei Fall 3 schadet eine Regierung angesichts des heutigen Bevölkerungsdrucks auf die Umwelt scheinbar heutigen Zeitgenossen ebenso wie zukünftigen Generationen. Auf den ersten Blick scheint sich ein solches Verhalten kaum rechtfertigen zu lassen. Bei Fall 4 handelt der Staat im Einklang mit den Interessen seiner Bürger. Auch hier sind - wie bei Fall 2 aber die Interessen heutiger Menschen gegen die künftiger Generationen abzuwägen.

Nach der Betrachtung der Situation in Deutschland im letzten Hauptteil der Studie können auch die Fälle 3 und 4 unter dem Kriterium der ökologischen Generationengerechtigkeit bewertet werden. Hierbei gerät das Urteil über die pronatalistisch eingestellten Regierungen in Fall 4-Szenarios ins Wanken. In den alternden reichen Ländern sprechen sich immer mehr Regierungen für eine pronatalistische Politik aus. Dies ist im Zusammenhang mit ökologischer Generationengerechtigkeit ein komplexes und ambivalentes Thema, dessen Bewertung zwiespältig ausfällt. Nun hat Deutschland wegen seiner territorialen Offenheit deutlich weniger endemische Arten als Inseln oder Regenwaldgebiete. Dennoch können Länder in den gemäßigten, artenärmeren Regionen neben dem Erhalt ihrer eigenen Arten auch viel für den Populationsschutz tun, wenn sie schrumpfen. 
Viele Arten, die heute nur noch ex situ (d.h. nicht mehr in der freien Wildbahn, sondern in Zoos) überleben, könnten wir leicht rückbürgern, wenn die Landbevölkerung dem zustimmen würde. Das gilt z.B. für den in Deutschland im Freiland ausgerotteten Wisent, den Braunbär, den Luchs oder den Wolf. Voraussetzung ist, dass große zusammenhängende Nationalparks ausgewiesen werden. Die bisher ausgewiesenen Nationalparks sind zu klein, um die Ansiedelung solcher ausgewanderter Arten zu ermöglichen. Erfolge bei der Reduzierung der menschlichen Flächeninanspruchnahme vergrößern also die Chancen für die Erhaltung der Artenvielfalt für zukünftige Generationen.

So wie in Bezug auf Gebiete in anderen Teilen der Welt die Kausalität zwischen zunehmender Bevölkerungsdichte und Artenverlust gilt, so gilt diese Beziehung natürlich auch für Deutschland. Gilt der Zusammenhang zwischen Bevölkerungswachstum und Artenverlust auch in umgekehrter Richtung? Was ist von der These zu halten, dass mit einem Bevölkerungsrückgang die Artenvielfalt zunimmt (These 1). Es gibt kaum empirische Hinweise, dass eine abnehmende Bevölkerungsdichte eine Zunahme an neuen endemischen Arten bewirken würde. Zunächst einmal erschafft die Natur neue Arten nur in Zeitskalen, die im Bereich von Hundertausenden bzw. Millionen von Jahren liegen. Wenn also im Zeitraum von wenigen Jahrzehnten die Bevölkerungsdichte in einem Gebiet sinkt, so führt dies nicht zu einem Feuerwerk von ganz neuen Lebensformen. These 1 kann also nicht bestätigt werden. Anders sieht es bei den ausgewanderten Arten aus. Es spricht viel dafür, dass Arten, die es zwar nicht mehr in Deutschland, aber in seinen weniger dicht besiedelten Nachbarländern gibt, bei einer abnehmenden Bevölkerungsdichte in Deutschland wieder heimisch werden würde (These 2). Ebenso ist es wahrscheinlich, dass die Bestände heimischer Arten zunehmen, wenn die Bevölkerungsdichte sinkt (These 3). Allerdings ist bei These 2 und 3 der Zusammenhang nicht monokausal. Eine quantitative Prognose - etwa dass bei einer Senkung der Bevölkerungsdichte um 10 Prozent die Bestände der Tierarten im gleichen Gebiet im Durchschnitt um 10 Prozent steigen - wäre unseriös.

Trotz dieser Differenzierungen gilt: Eine Schrumpfung der Bevölkerung Deutschlands bietet große Chancen, die Flächenversiegelung zu verlangsamen, zusammenhängende Schutzgebiete auszuweisen und die Roten Listen bedrohter heimischer Tier- und Pflanzenarten zu verkürzen. Zudem würde der ökologische Fußabdruck Deutschlands in der Welt voraussichtlich kleiner. Dies alles ist im Sinne der ökologischen Generationengerechtigkeit positiv zu bewerten. Andererseits kann es nicht darum gehen, die Zahl der Menschen unbegrenzt schrumpfen $\mathrm{zu}$ lassen. Aus dem Plädoyer für eine Stabilisierung der Bevölkerung auf ökologisch nachhaltigem Niveau folgt die Notwendigkeit, entsprechende Stabilisierungsmaßnahmen auch tatsächlich einzuleiten. Es besteht zumindest theoretisch die Gefahr des Überschießens nach unten, wenn jede Generation ihre Vorgängergeneration nur zu zwei Dritteln ersetzt. Insofern kann eine ethische Bewertung nicht pauschal für die Fälle 3 und 4 abgegeben werden. In beiden 
Fällen kann das pronatalistische staatliche Handeln ausnahmsweise gerechtfertigt sein, wenn ohne staatliche Interventionen die Bevölkerung sehr stark abnähme. In jedem Fall ist Fall 4 aber unproblematischer als Fall 3, da der Staat bei Fall 4 im Einklang mit den Wünschen der heutigen Bürger handelt, wenn er die Geburtenrate zu erhöhen versucht. Die Grenze zwischen einem Fall 3-Szenario und einem Fall 4-Szenario liegt in Deutschland nach den Daten einer repräsentativen Eurostat-Umfrage bei einer Geburtenrate von 1,7 Kindern pro Frau. Dementsprechend ist die Steigerung der Geburtenrate von 1,4 auf 1,7 im Rahmen einer intratemporalen Betrachtung ethisch unproblematisch. Berücksichtigt man nun zusätzlich die intergenerationelle Perspektive, so lässt sich zwar nicht bestreiten, dass dadurch die Schrumpfung abgebremst und dadurch die Entlastungseffekte für die Umwelt geringer würden, aber die Schrumpfung ginge ja dennoch weiter. Da es auch im Sinne der ökologischen Generationengerechtigkeit nicht das Ziel sein kann, Deutschland unverhältnismäßig stark zu entvölkern, erscheinen pronatalistische Maßnahmen mit dem Ziel, eine Geburtenrate von 1,7 zu erreichen, bei Einhaltung der Vier-Fünftel-Regel in jedem Fall ethisch gerechtfertigt. Fraglich ist, ob eine Politik zur Erzielung einer Geburtenrate von 2,1 ethisch gerechtfertigt wäre. Dazu wäre zu dem Zeitpunkt, zu dem die Bevölkerung auf ein niedrigeres Niveau wie z.B. 70 Mio. gesunken ist, zu prüfen, ob die Prinzipien ökologischer Nachhaltigkeit nun eingehalten werden. Ist dies so, so spricht aus Sicht der ökologischen Generationengerechtigkeit nichts gegen pronatalistische Maßnahmen, welche auf eine Geburtenrate von 2,1 zielen. Ist dies nicht so, dann muss erneut abgewogen werden, ob sich die ökologischen Vorteile einer weiteren Schrumpfung vor dem Hintergrund der Nachteile in anderen Bereichen vertreten lassen.

In jedem Fall sollte aber im mit 83 Mio. bevölkerten Deutschland eine tatsächliche Schrumpfung eingeleitet und nicht etwa durch eine überzogene Einwanderungspolitik oder eine zu massive Geburtenpolitik von Anfang an verhindert werden. Diese Studie zeigt, dass ein Rückgang der Bevölkerung nicht per se generationenungerecht ist. Dies folgt bereits theoretisch aus den Überlegungen zu DNU und NSU und lässt sich auch praktisch durch viele Beispiele einer wachsenden Lebensqualität bei gleichzeitiger Verbesserung der ökologischen Rahmendaten zeigen. Deshalb ist der Begriff „demografische Nachhaltigkeit“ auch irreführend. In dieser Studie wird eine dreiteilige Strategie vorgeschlagen, um den demografischen Übergang bis zur Erlangung eines neuen, niedrigen Bevölkerungsgleichgewichts in Deutschland zu bewältigen. Der erste Teil lässt sich mit ,Die Chancen der Schrumpfung nutzen' zusammenfassen. Damit sind Ansätze gemeint, die den Rückgang der Bevölkerung nicht umzukehren versuchen, sondern unsere Gesellschaft darauf einstellen und sogar Chancen dieser Entwicklung zu identifizieren versuchen. Aus ökologischer Sicht sind diese Chancen bereits beschrieben worden. Im größeren Maßstab können v.a. in Ostdeutschland gezielt Pläne entwickelt werden, welche Dörfer aufgegeben werden sollten, um zusammenhängende 
Naturschutzgebiete einzurichten. Aber auch im Westen könnten durch weitsichtige städtebauliche Planung im Zuge der nächsten 50 Jahre die schlimmsten architektonischen Sünden der Vergangenheit beseitigt werden. Anstatt abzureißen, was sich von selbst entleert hat, müssten in jeder Gemeinde diejenigen Liegenschaften identifiziert werden, die zuerst entleert werden sollen, um sie dann abzureißen.

Eine wichtige Herausforderung ist die Dämpfung negativer Auswirkungen des demografischen Wandels auf die Wirtschaft. Hier ist allerdings eher die Alterung das Problem, die generell gedanklich häufig nicht hinreichend von der Schrumpfung getrennt wird. Viele Probleme, z.B. der Verlust an Innovationsfähigkeit, lassen sich nicht gänzlich vermeiden, aber sie lassen sich zumindest dämpfen.

Der zweite Teil der Strategie umfasst Maßnahmen, um die Geburtenrate wieder auf Bestandhaltungsniveau zu steigern. Dieses Plädoyer für eine aktive Bevölkerungspolitik steht nicht im Widerspruch zu den bisherigen Politikempfehlungen. Wenn die derzeitige Fertilitätsrate von 1,4 anhielte, wird nach den Langzeitprojektionen der UN Population Division Deutschland im Jahr 2300 nur noch rund 3 Mio. Einwohner haben, so viele wie heute Berlin. Dies ist keine attraktive Vorstellung. Das heißt für Deutschland, dass die Faktoren, die dafür verantwortlich sind, dass sich Paare gegen Kinder entscheiden, abgebaut werden müssen. Dazu sind u.a. ein gesellschaftlicher Wertewandel, stärkere finanzielle Anreize und ein besseres Betreuungsangebot notwendig.

Der dritte Teil der Strategie beschäftigt sich mit Einwanderung. Angesichts der verfehlten Integrationspolitik der letzten Jahrzehnte wird verstärkte Migration als Königsweg zur Bewältigung des demografischen Wandels abgelehnt, auch wenn Einwanderung in bestimmtem Maße notwendig ist.

Auch wenn die Geburtenraten im weltweiten Maßstab zurückgegangen sind, ist die Stabilisierung der Weltbevölkerung frühestens in der zweiten Hälfte des 21. Jahrhunderts zu erwarten. Die zunächst in den reichen Ländern und den Schwellenländern einsetzende Schrumpfung, die sich nach und nach auch auf die Entwicklungsländer ausweitet, eröffnet Potenziale für die Erreichung der ökologischen Nachhaltigkeit im globalen Maßstab. Die Weltbevölkerung könnte nach UN-Projektionen ab 2075 (bei 9,2 Mrd.) stagnieren und dann relativ konstant bleiben bzw. bis 2300 leicht abnehmen. Wenn die reichen Länder tatsächlich in das Stadium der Schrumpfung einträten, so könnten sie auf internationalen Bevölkerungskonferenzen ihre Vorleistungen betonen und damit allen Verfechtern von Neokolonialismus- und Rassismusthesen den Wind aus den Segeln nehmen. „Keines der auf dem Erdgipfel 1992 diskutierten (...) Probleme wäre wirklich schwerwiegend, wenn auf der Welt nur 500 Millionen Menschen mit Nahrung, Kleidung und einem Zuhause versorgt werden müssen“, schreibt Ernst-Ulrich von Weizsäcker. ${ }^{1004}$

${ }^{1004}$ Weizsäcker (1995), 291 
Es besteht weiterer Forschungsbedarf. Umweltwissenschaftler sollten stärker die positiven Effekte des Schrumpfens der Bevölkerungen der Nordhalbkugel untersuchen - die direkten Effekte für die Ökologie in diesen Ländern selbst, sowie die indirekten durch die Reduzierung ihres ökologischen Fußabdrucks und auf der internationalen Bühne, wenn globale demografische Zielgrößen verhandelt werden. In den Umweltgutachten des SRU und des WBGU fehlt bisher eine detaillierte Untersuchung dieser Beziehungen.

Wer heute aus ökologischen Gründen für einen moderaten Rückgang der deutschen Bevölkerung eintritt, kommt sich vor wie ein Rufer in der Wüste. Mit einem gewichtigen Unterschied: Auch wenn seine Argumente fast überall auf Unverständnis stoßen, so kann er sich doch freuen: Selbst diejenigen, die bestreiten, dass die Schrumpfung auch positive Effekte hat, verhalten sich in der Regel in ihrem Privatleben genau so, wie er es sich wünscht. 


\section{Literaturverzeichnis}

AAAS/American Association for the Advancement of Sciences (2001) (Hg.): AAAS Atlas of Population and Environment. Bearbeitet von Harrison, Paul / Pearce, Fred. New York

Acker-Widmaier, Gerald (1999): Intertemporale Gerechtigkeit und nachhaltiges Wirtschaften. Zur normativen Begründung eines Leitbildes. Marburg

Adam, Konrad (1996): Für Kinder haften die Eltern. Die Familie als Opfer der Wohlstandsgesellschaft, Weinheim

Adorno, Theodor (1976): Einleitung. In: Adorno, Theodor: Der Positivismusstreit in der deutschen Soziologie. 5. Auflage (1. Auflage 1969). Darmstadt/Neuwied

Afheldt, Heik (17.05.2001): 65 Millionen reichen aus. In: Die Zeit. 21/2001 (17.05.2001). S. 26

Ahrens, John (1983): Preparing for the future. An essay on the Rights of Future Generations. Bowling Green/Ohio

Albert, Hans (1980): Traktat über kritische Vernunft. 4. Auflage. Tübingen

Alfred-Herrhausen-Gesellschaft (2000) (Hg.): Generationengerechtigkeit. Leitbild für das 21. Jahrhundert. Berlin

Allenby, Braden R. (1999): Industrial Ecology. Policy Framework und Implementation. New Jersey

Almsick, Josef von (1988): Die gesetzliche Rentenversicherung als Instrument der Familien- bzw. Bevölkerungspolitik? In: Die Sozialversicherung. 6/1988. S. 144-151

Appleman, Philip (1976) (Hg.): The Norton Critical Edition of Thomas Robert Malthus 'An Essay on the Principles of Population (1798)'. New York

Arrhenius, Gustaf (2000): Mutual Advantage Contractarianism and Future Generations. In: Theoria 65. S. 25-35

Auerbach, Bruce E. (1995): Unto the Thousandth Generation. Conceptualizing Intergenerational Justice. New York/Frankfurt am Main

Aufhauser, Elisabeth (2001): Diskursfeld Bevölkerungspolitik. Zwischen „Menschenökonomie“ und „Menschenrechten“. In: Journal für Entwicklungspolitik. 1/2001 (17. Jg.). S. 7-28

Bähr, Jürgen (1997): Bevölkerungsgeographie. Verteilung und Dynamik der Bevölkerung in globaler, nationaler und regionaler Sicht. Stuttgart

Baltes, Paul B. (2005): Oma muss ran. In: Die Zeit. 19.05.2005 (60. Jg.). S. 44-45

Baltes, Paul B. / Mittelstraß, Jürgen (1992): Zukunft des Alterns und gesellschaftliche Entwicklung. Berlin 
Bandarage, Asoka (1997): Women, Population and Global Crisis. A Political-Economic Analysis. New Jersey

Bandman, Bertram (1982): Do Future Generations have the Right to Breathe Clean Air?" In: Political Theory 10. S. 95-102

Banze, Sonja / Porwollik, Ulrich (2003): Wie Götter in Deutschland. In: Welt am Sonntag. 27.04.2003. S. 27

Barry, Brian (1989): Theory of Justice. Berkeley

Barry, Brian (1978): Circumstances of Justice and Future Generations. In: Barry, Brian / Sikora, Richard (1978) (Hg.): Obligations to Future Generations. Philadelphia. S. 204-248

Barry, Brian (1977): Justice between Generations. In: Hacker, P. M. / Raz, J. (Hg.): Law, Morality and Society. Essays in Honour of H. L. A. Hart. Oxford. S. 268-284

Barry, Brian / Sikora, Richard (1978) (Hg.): Obligations to Future Generations. Philadelphia

Barth, Hans J. (2004): Die "Allianz für die Familie" im Kontext volkswirtschaftlicher und demographischer Trends. In: Schmidt, Renate / Mohn, Liz (2004): Familie bringt Gewinn. Innovation durch Balance von Familie und Arbeitswelt. Gütersloh S. 26-31

Bayles, Michael D. (1980): Morality and Population Policy. Alabama

Bayles, Michael D. (1976a): Introduction. In: Bayles, Michael D. (Hg.): Ethics and Population. Cambridge. S. xi-xxviii

Bayles, Michael D. (1976b): Limits to the Right to Procreate. In: Bayles, Michael D. (Hg.): Ethics and Population. Cambridge. S. 41-55

BBC (24.03.2004): IF...the generations fall out. Film. Zu beziehen über die BBC oder überinfo@srzg.de

Becker, Rolf (1997) (Hg.): Generationen und sozialer Wandel: Generationsdynamik, Generationsbeziehungen und Differenzierung von Generationen. Opladen

Beckerman, Wilfred (2004): Intergenerational Justice. In: Generationengerechtigkeit! Nr.2/2004 (4. Jg.). S. 1-5

Berlin-Institut (9.10.2003): „Wölfe statt Menschen.“ In: Newsletter DEMOS. 2/2003. $(9.10 .2003)$

BiB/Bundesinstitut für Bevölkerungsforschung (2005) (Hg): Einstellungen zu demographischen Trends und zu bevölkerungsrelevanten Politiken. Ergebnisse der Population Policy Acceptance Study in Deutschland. Bearbeitet von Dorbritz, Jürgen / Lengerer, Andrea / Ruckdeschel, Kerstin. Sonderheft der Schriftenreihe des BiB. Wiesbaden

BiB/Bundesinstitut für Bevölkerungsforschung (2004) (Hg): Bevölkerung: FaktenTrends-Ursachen-Erwartungen. Die wichtigsten Fragen. Wiesbaden 
BiB/Bundesinstitut für Bevölkerungsforschung (1994) (Hg): Internationale Konferenz 1994 über Bevölkerung und Entwicklung (ICPD 1994). Materialien zur Bevölkerungwissenschaft. Sonderheft 26. Wiesbaden

Billerbeck, Liane von (2003): Wie man in Deutschland erwachsen wird. In: Die Zeit. 46/2003 (6.11.2003). S. 32

Birdsall, Nancy (1994): Another look at population and global warming. In: Population, Environment and Development. Proceedings of the UN Expert Group on Population, Environment and Development, UN Headquarters, 20-24. Januar 1992. New York. S. 39-54

Birg, Herwig (2004a): Schrumpfen oder wachsen? Bevölkerungspolitik in der Demokratie. In: Generationengerechtigkeit! 1/2004. S. 12-13

Birg, Herwig (2004b): Ablehnende Stellungnahme zur These: „Die Schrumpfung der Bevölkerungen Deutschlands und anderer Industrieländer ist zu begrüßen, da langfristig die gesamte Weltbevölkerung auf einem niedrigeren Niveau als heute stabilisiert werden muß?" In: Generationengerechtigkeit! 1/2004. S. 23

Birg, Herwig (2001): Die demographische Zeitenwende: der Bevölkerungsrückgang in Deutschland und Europa. München

Birg, Herwig (1998): Demografisches Wissen und politische Verantwortung. In: Zeitschrift für Bevölkerungswissenschaft. 3/1998. (23. Jg.). S. 221-251

Birg, Herwig (1996): Die Weltbevölkerung. Dynamik und Gefahren. München

Birg, Herwig (1994): Perspektiven des globalen Bevölkerungswachstums - Ursachen, Folgen, Handlungskonsequenzen. In: Nuscheler, Franz / Fürlinger, Ernst (Hg.):

Weniger Menschen durch weniger Armut? Bevölkerungswachstum - globale Krise und ethische Herausforderung. Salzburg. S. 11-46

Birg, Herwig / Filip, Detlef / Flöthmann, Ernst-Jürgen (1990): Paritätsspezifische Kohortenanalyse des generativen Verhaltens in der Bundesrepublik Deutschland nach dem 2. Weltkrieg. Bielefeld

Birnbacher, Dieter (2003): Verantwortung für zukünftige Generationen - Reichweite und Grenzen. In: Stiftung für die Rechte zukünftiger Generationen (Hg.): Handbuch Generationengerechtigkeit. München. S. 84-104

Birnbacher, Dieter (1988): Verantwortung für zukünftige Generationen. Stuttgart

Birnbacher, Dieter (1986): Prolegomena zu einer Ethik der Quantitäten. In: Ratio. 1/1986 (Band 28). S. 30-45

Birnbacher, Dieter (1980) (Hg.): Ökologie und Ethik. Stuttgart

Birnbacher, Dieter / Brudermüller, Gerd (2001) (Hg.): Zukunftsverantwortung und Generationensolidarität. Würzburg

Birnbacher, Dieter / Hoerster, Norbert (2000) (Hg.): Texte zur Ethik. 11. Auflage (1. Auflage 1976). München 
Birnbacher, Dieter / Schicha, Christian (1996): Vorsorge statt Nachhaltigkeit - Ethische Grundlagen der Zukunftsverantwortung. In: Hans Kastenholz / Karl-Heinz Erdmann / Manfred Wolff (Hg.): Nachhaltige Entwicklung: Zukunftschance für Mensch und Umwelt. Berlin. S. 141-156

Bischöfe in Deutschland (2004) (Hg.): Die Bibel: Einheitsübersetzung. Stuttgart

Blume, Georg (2003): Großeltern im Schrebergarten. Wie Tradition und Sozialismus in China das Altwerden erleichtern. In: Die Zeit. 3/2003 (09.01.2003). S. 7

Borchert, Jürgen (1993): Renten vor dem Absturz. Frankfurt am Main

Borchert, Jürgen/ Nell-Breuning, Oswald von (1986): Die Alterssicherung hängt in der Luft. In: Zeitschrift für Sozialreform. 4/1986 (32. Jg.). S. 205-214

Börsch-Supan, Axel (2004a): Aus der Not eine Tugend - Zukunftsperspektiven einer alternden Gesellschaft. Discussion Paper 54-2004. Mannheimer Forschungsinstitut Ökonomie und Demographischer Wandel

Börsch-Supan, Axel (2004b): Aus der Not eine Tugend. In: Frankfurter Allgemeine Zeitung. Nr. 188/2004 (14.8.2004). S. 38

Börstinghaus, Volker (2002): Generationsbilanzen - Theorie, Empirie und Konsequenzen für die EU. Frankfurt am Main

Bosbach, Gerd (07.03.2004): „Die Politik will von den Problemen ablenken, die sie nicht lösen kann.“ Interview über die Vergreisung Deutschlands. In: Süddeutsche Zeitung online.

http://www.sueddeutsche.de/deutschland/artikel/829/26803/print.html. Rev. 200403-08

Bosbach, Gerd (2003): Thesenpapier ,Demografische Entwicklung - kein Anlass zur Dramatik“. www.memo.uni-bremen.de/docs/m0404.pdf. Rev. 2004-03-04. Inhaltsgleich in den Gewerkschaftlichen Monatsheften Nr. 2/2004 und in der Frankfurter Rundschau vom 23.02.04

Boserup, Ester (1981): Population and Technology. Oxford

Boserup, Ester (1965): The Conditions of Agricultural Growth. London

Boutros-Ghali, Boutros (1994): Key Note Address. In: BiB/Bundesinstitut für Bevölkerungsforschung (1994) (Hg): Internationale Konferenz 1994 über Bevölkerung und Entwicklung (ICPD 1994). Materialien zur Bevölkerungwissenschaft, Sonderheft 26. Wiesbaden. S. 18-24

Bouthoul, Gaston (1972), : Kindermord aus Staatsräson. Der Krieg als bevölkerungspolitischer Ausgleich. Stuttgart

Brand, Karl-Werner (2004): Strohhalme bieten keinen Halt. Kommentar 1 zu Jörg Tremmels Beitrag. In: GAIA. 1/2004. S. 35-37

Bretz, Manfred (2001): Zur Treffsicherheit von Bevölkerungsvorausberechnungen. In: Wirtschaft und Statistik 11/2001. S. 906-921 
Brockhaus - Die Enzyklopädie in 24 Bänden. 20., neu bearbeitete Auflage. Leipzig

Bronger, Dirk (1996): Indien - Größte Demokratie der Welt zwischen Kastenwesen und Armut. Gotha

Brost, Marc / Uchatius, Wolfgang: Wie geht's uns denn heute? Alle schauen auf das Bruttoinlandsprodukt. Doch Wohlstand hängt von viel mehr ab als von dieser Zahl. In: Die Zeit. 2/2004 (31.12.2003). S. 19

Brouns, Ellen (2003): Ist Wildnis planbar? Werte- und Interessenkonflikte in der raumbezogenen Umweltplanung anhand ausgewählter Wildnisprojekte. München

Brumlik, Micha (1995): Gerechtigkeit zwischen den Generationen. Berlin

Brumlik, Micha (1992): Über die Ansprüche Ungeborener und Unmündiger. Wie advokatorisch ist die Diskursethik? In: Advokatorische Ethik. Bielefeld. S.108-142

Brumlik, Micha / Brunkhorst, Hauke (1993) (Hg.): Gemeinschaft und Gerechtigkeit. Frankfurt am Main

Brundtland, Gro Harlem (1994): Key Note Address. In: BiB/Bundesinstitut für Bevölkerungsforschung (1994) (Hg): Internationale Konferenz 1994 über Bevölkerung und Entwicklung (ICPD 1994). Materialien zur Bevölkerungwissenschaft, Sonderheft 26. Wiesbaden. S. 33-38

Bude, Heinz (2001): Generation Berlin. Berlin

Bude, Heinz (2000): Qualitative Generationsforschung. In: Flick, Uwe / von Kardorff, Ernst / Steinke, Ines (Hg.): Qualitative Forschung. Ein Handbuch. Hamburg. S. 187-194

Bühler, Franz (1949): Verfassungsrevision und Generationenproblem. Freiburg (Schweiz)

BUND/Bund für Umwelt und Naturschutz Deutschland (1988) (Hg.): Umweltbilanz Die ökologische Lage der Bundesrepublik. Hamburg

Bundesamt für Naturschutz (2004): Daten zur Natur. Bonn (im Druck)

Bundesamt für Naturschutz (2003) (Hg.): Naturschutzbegründungen. (Bearbeitet von S. Körner, A. Nagel und U. Eisel). Bonn

Bundesamt für Naturschutz (2002): Daten zur Natur. Bonn

Bundesamt für Naturschutz (22.10.2002): Rote Liste gefährdeter Biotoptypen. www.bfn.de. Rev. 2004-06-20

Bundesamt für Naturschutz (2000) (Hg.): Bundesweite Rote Listen. Bilanzen, Konsequenzen, Perspektiven. (Bearbeitet von M. Binot / H. Gruttke / G. Ludwig, Gerhard / U. Riecken). Bonn

Bundesamt für Naturschutz (1998) (Hg): Rote Liste gefährdeter Tiere Deutschlands. (bearbeitet von M. Binot, R. Bless, P. Boye, H. Gruttke und P. Pretscher). Bonn 
Bundeskriminalamt (2003) (Hg.): Polizeiliche Kriminalstatistik Bundesrepublik Deutschland. Berichtsjahr 2002. Wiesbaden. S. 28-30.

http://www.bmi.bund.de/dokument/Bestellservice/ix_92274.htm. Rev. 2004-05-05.

Bundesministerium für Gesundheit und Soziale Sicherung (2003) (Hg.): Nachhaltigkeit in der Finanzierung der Sozialen Sicherungsysteme (Bericht der Kommission/Vors. Bert Rürup). Berlin

BMFSFJ/Bundesministerium für Familie, Senioren, Frauen und Jugend (2005): Perspektiven für eine nachhaltige Familienpolitik. Ergebnisse des Gutachtens von Prof. Dr. Eckart Bormsdorf. Berlin

BMFSFJ/Bundesministerium für Familie, Senioren, Frauen und Jugend (2003): Die Familie im Spiegel der amtlichen Statistik. Berlin

BMJFFG/Bundesministerium für Jugend, Familie, Frauen und Gesundheit (1986): Vierter Familienbericht. Die Situation älterer Menschen in der Familie (Drucksache 10/6145). Bonn

BMU/Bundesministerium für Umwelt, Naturschutz und Reaktorsicherheit (2002) (Hg.): Umweltbewusstsein in Deutschland 2002. Ergebnisse einer repräsentativen Bevölkerungsumfrage. Berlin. http://www.empirischepaedagogik.de/ub2002neu/indexub2002.htm. Rev. 2004-06-25

Bülow, Marco (2004): Generation Zukunft. Berlin

Bundesregierung Deutschland (2004): Fortschrittsbericht 2004 zur Strategie für eine Nachhaltige Entwicklung. Berlin

Bundesregierung Deutschland (2002): Unsere Strategie für eine nachhaltige Entwicklung. Berlin

Bundesverkehrsministerium (2003): Bundesverkehrswegeplan. Berlin

Bundesverkehrsministerium (2002/2003): Verkehr in Zahlen. Berlin

Burkhardt, Wilfried (1985): Drei-Generationen-Solidarität in der gesetzlichen Rentenversicherung als zwingende Notwendigkeit. Berlin

Butterwegge, Christoph (2002): Stirbt „das deutsche Volk“ aus? In: Butterwegge, Christoph / Cremer, Janine / Häusler, Alexander u.a.: Themen der Rechten - Themen der Mitte. Zuwanderung, demografischer Wandel und Nationalbewusstsein. Opladen 2002

Bündnis 90/Die Grünen (2002): Die Zukunft ist grün - Grundsatzprogramm von Bündnis 90/Die Grünen. Berlin

Butterwegge, Christoph / Klundt, Michael (2001) (Hg.): Kinderarmut und Generationengerechtigkeit. Familien- und Sozialpolitik im demografischen Wandel. Opladen

Buvinic, Mayra (1994): Population Policy and Family Planning Programmes: Contribution from a Focus on Women. In: Graham-Smith, Francis (Hg.): Population - the complex reality. Cambridge. S. 211-228 
Callahan, Daniel (1980): What Obligations do we have to Future Generations? In: Partridge, Ernest (Hg.): Responsibilities to Future Generations. Environmental Ethics. Buffalo N.Y. S. 73-85

Callahan, Daniel (1976): Ethics and Population Limitation. In: Bayles, Michael D. (1976) (Hg.): Ethics and Population. Cambridge. S. 19-40

Callicott, Baird (2003): Eigenwert der Natur. Gedanken über Ethik und Pragmatik. In: Natur und Kultur. Nr. 2/2003. S. 68-89

Center for International Earth Science Information Network. www.ciesin.org. Rev. 2003-08-24

Chamie, Joseph (2004): Die Neue Internationale Bevölkerungsordnung. In: Hessische Staatskanzlei (2004) (Hg.): Hilfe wir schrumpfen! Die Gesellschaft im demographischen Wandel - Prognosen und Handlungsoptionen. Dokumentation der Rheingauer Impulse 2003. Wiesbaden. S. 11-18

Chesnais, Jean-Claude (1992): Demografic transition. Oxford

Chlond, Bastian / Manz, Wilko / Zumkeller, Dirk (2002): Stagnation der Verkehrsnachfrage - Sättigung oder Episode. In: Internationales Verkehrswesen. Nr. 9/2002. Jg. 54. S. 396-403

Christlich Soziale Union (17.11.2003): Die Rente der Zukunft - familienfreundlich, generationengerecht und solidarisch. Konzept der CSU zur Reform der gesetzlichen Rentenversicherung. http://www.csu.de/home/UploadedFiles/Dokumente/031117_Rentenkonzept.pdf. Rev. 2003-12-10

Cincotta, Richard / Engelmann, Robert (2001): Mensch, Natur! (Hrsg. von der Deutschen Stiftung Weltbevölkerung). Stuttgart

Coale, Ansley / Hoover, Edgar (1958): Population Growth and Economic Development in Low-Income Countries. Princeton

Cohen, Joel E. (1995): How many people can the earth support? New York

Commoner, Barry (1971): Wachstumswahn und Umweltkrise. München

Comte, Auguste (1822/1914): Plan der wissenschaftlichen Arbeiten die für eine Reform der Gesellschaft notwendig sind. Leipzig (Originalauflage 1822)

Condorcet, Marie-Jean-Antoine-Nicolas de Caritat, Marquis de (1795/1976): Esquisse d'un Tableau Historique des Progrès de L'Esprit Humain. (Dt. Ausgabe: Entwurf einer historischen Darstellung der Fortschritte des menschlichen Geistes, hrsg. von Alff, Wilhelm / Schweppenhäuser, Hermann. Frankfurt am Main 1976)

Cornelius, Ivar (2004): Eine demografische Zeitenwende? Die Entwicklung der Bevölkerung in Baden-Württemberg und ihre Auswirkungen. In: Umweltakademie Baden-Württemberg (2004) (Hg.): Bevölkerungsrückgang. Konsequenzen für Flächennutzung und Umwelt. Stuttgart. S. 41-57 
Corral, José L. (2004) : CELSAM : Family planning for young people in Latin America. In: Schering AG (Hg.): International Dialogue on Population and Sustainable Development ,Ways out of the Crisis - Reproductive Health in Need of New Ideas”. 14. October 2004. GTZ-House. Conference Proceedings. Berlin. S. 59-66

Dahms, Hans-Joachim (1994): Positivismusstreit. Die Auseinandersetzung der Frankfurter Schule mit dem logischen Positivismus, dem amerikanischen Pragmatismus und dem kritischen Rationalismus. Frankfurt am Main

Daly, Herman (1991): Elements of Environmental Macroeconomics. In: Constanza, Robert (Hg.): Ecological Economics: the Science and Management of Sustainability. New York. S. 32-46

Daniels, Norman (1988): Am I my Parents`keeper? An Essay on Justice Between the Young and the Old. Oxford

Delattre, Edwin (1972): Rights, Responsibilites, and Future Persons. In: Ethics 82. S. $254-258$

Demandt, Alexander (1984): Der Fall Roms. München

Demeny, Paul (1993/94): Politische Maßnahmen zur Reduzierung eines hohen Geburtenniveaus: Eine Sache der Nachfrage. In: Zeitschrift für Bevölkerungswissenschaft. Heft 3/1993-94 (19. Jg.). S. 365-375

Derr, Thomas S. (1980): The Obligation to the Future. In: Partridge, Ernest (Hg.): Responsibilities to Future Generations. Environmental Ethics. Buffalo N.Y. S. 3745

De-Shalit, Avner (1992): Environmental Policies and Justice Between Generations. On the Need for a Comprehensive Theory of Justice Between Generations. European Journal of Political Research 21. S. 307-316

Dobson, Andrew (1996): Conservation and Biodiversity. New York: Scientific American Library

Doehler-Behzadi, Marta / Lütke-Daldrup, Engelbert (2004) (Hg.): Plusminus Leipzig 2030. Stadt in Transformation. Leipzig

Deutsche Bundesbank (2001): Zur langfristigen Tragfähigkeit der öffentlichen Haushalte - eine Analyse anhand der Generationenbilanzierung. In: Monatsbericht Dezember 2001. S. 29-44

Deutscher Bundestag (2002): Abschlussbericht der Enquete-Kommission „Demographischer Wandel“. Herausforderungen unserer älter werdenden Gesellschaft an den Einzelnen und die Politik. Berlin

Deutscher Bundestag (1998): Abschlussbericht der Enquete-Kommission „Schutz des Menschen und der Umwelt“" des 13. Deutschen Bundestags: Konzept Nachhaltigkeit: Vom Leitbild zur Umsetzung. Bonn 
Deutscher Bundestag (1994): Zwischenbericht der Enquete-Kommission „Schutz des Menschen und der Umwelt“. Die Industriegesellschaft gestalten. Perspektiven für einen nachhaltigen Umgang mit Stoff- und Materialströmen. Bonn

Deutsche Shell Holding GmbH (2002) (Hg.): Jugend 2002. Zwischen pragmatischem Idealismus und robustem Materialismus. 14. Shell Jugendstudie. Konzeption und Koordination von Klaus Hurrelmann, Mathias Albert und Infratest Sozialforschung. Frankfurt am Main

Deutsche Stiftung Weltbevölkerung (1999) (Hg.): Handbuch Weltbevölkerung. Begriffe, Fakten, Konzepte. Erweiterte Deutsche Erstausgabe. Stuttgart. (Basierend auf der vierten internationalen Ausgabe des Population Handbook, hrsg. vom Population Reference Bureau)

Deutsche Studiengemeinschaft (2003) (Hg.): Bevölkerungspolitik. Leonberg

Diekmann, Andreas / Jaeger, Carlo C. (1996): Aufgaben und Perspektiven der Umweltsoziologie. In: Diekmann, Andreas / Jaeger, Carlo C. (Hg.): Sonderheft Umweltsoziologie der KZfSS. Sonderheft 36. Opladen. S. 11-27

Dieren, Wouter van (1995): Mit der Natur rechnen. Berlin

Dießenbacher, Hartmut (1998): Die Kriege der Zukunft. Die Bevölkerungsexplosion gefährdet den Frieden. München/Wien

Dießenbacher, Hartmut (25.5.1993): Alterssicherung ist der Schlüssel zur Eindämmung des Bevölkerungswachstums. In: Frankfurter Allgemeine Zeitung. 25.05.2004. S. 12

Dinklage, Meike (2005): Der Zeugungsstreik. Warum die Kinderfrage Männersache ist. München

Dobson, Andrew (2000): Drei Konzepte ökologischer Nachhaltigkeit. In: Natur und Kultur. 1-2/2000. S. 62-85

Dorbritz, Jürgen / Fleischhacker, Jochen (1995): Der Übergang von der Bevölkerungszur Familienpolitik in den neuen Bundesländern - ein Beitrag zum familienpolitischen Diskurs in Deutschland. In: Zeitschrift für Bevölkerungswissenschaft, Jg. 20. Heft 2/1995. S. 159-185

Duden, Barbara (1993): „Bevölkerung“. In: Sachs, Wolfgang (Hg.): Wie im Westen, so auf Erden. Ein polemisches Handbuch zur Entwicklungspolitik. Reinbek. S. 71-88

Dumont, Gérard-François (1997): Europa stirbt vor sich hin...: Bevölkerungspolitik vor dem Bankrott. Wege aus der Krise. Aachen

Dunlap, Riley E. / Lutzenhiser, Loren A. / Rosa, Eugene A. (1994): Understanding Environmental Problems: A Sociological Perspective. In: Bürgenmeier, Beat (Hg.): Economy, Environment and Technology. A Socio-Economic Approach. Armonk/New York. Sharpe

Durkheim, Emile (1965): Regeln der soziologischen Methode. Neuwied/Berlin. (Franz. Originalausgabe 1895) 
Dworkin, Ronald (1981a): What is Equality. Part 1: Equality of Welfare. In: Philosophy \& Public Affairs. 10/1981. S. 185-246

Dworkin, Ronald (1981b): What is Equality. Part 2: Equality of Resources. In: Philosophy \& Public Affairs. 4/1981. S. 283-345

Easterlin, Richard A. (1980): Birth and fortune: The impact of numbers on personal welfare. New York

Ederer, Günter / Ederer, Peer (1995): Das Erbe der Egoisten. Wie unsere Generation die Zukunft Deutschlands verspielt. München

Ehmke, Horst (1953): Grenzen der Verfassungsänderung. Berlin

Ehrenberg, Herbert / Fuchs, Anke (1980): Sozialstaat und Freiheit. Frankfurt am Main

Ehrlich, Paul R. (1971): Die Bevölkerungsbombe. München. (Engl. Original: The Population Bomb. New York 1968)

Ehrlich, Paul R. / Ehrlich, Anne H. / Daily, Gretchen C. (1995): The Stork and the Plow. The Equity Answer to the Human Dilemma. New York

Ehrlich, Paul R. / Holdren, John P. (1971): The impact of population growth. In: Science 171. S. $1212-1217$

Ehrlich, Paul R. / Ehrlich, Anne H. (1970): Population, Resources, Environment: Issues in Human Ecology. San Francisco

English, Jane (1977): Justice between Generations. In: Philosophical Studies. Heft 31. S. 91-104

Epstein, Richard A. (1992): Justice across the generations. In: Laslett, Peter / Fishkin, James S. (Hg): Justice between age groups and generations. London. S. 84-106

Erdmann, Karl-Heinz / Küchler-Krischun, Jonna / Schell, Christiane (2000): Darstellung des Naturschutzes in der Öffentlichkeit. Bonn

Eurostat (2002): Material Use in the European Union 1998-2000, European Commission. Eurostat Working Papers and Studies 23-1-2003

Fathalla, Mahmoud F. (1994): Fertility Control Technology: A Women-Centered Approach to Research. In: Sen, Gita/ Germain, Adrienne / Chen, Lincoln C. (Hg.): Population Policies Reconsidered. Health, Empowerment, and Rights. Boston. S. 223-234

FAO (2003): The State of Food Insecurity in the World 2003. New York. http:/www.fao.org/sof/sofi/index_en.htm. Rev. 2004-05-10

FAO (2001): State of the World's Forests 2001. Rom

FAO (1983): Potential population supporting capacities of lands in the developing world. Technical report of project INT/75/P13 "Land resources for populations in the future. FPA/INT/513. Rom. (Bearbeitet von Higgins, G.M. / Kassam, A.H. / Naiken, L. / Fischer, G. / Shah, M.M) 
FDP (1997): Wiesbadener Grundsätze. Beschluss des F.D.P.-Bundesparteitages in Wiesbaden vom 24. Mai 1997. http://www.liberale.de/fdpbv/grundsatz/wiesbaden/ Rev. 2001-08-08

Fehige, Christoph / Wessels, Ulla (1998) (Hg.): Preferences. Berlin

Feucht, Ralf (1999): Beeinflussung demographischer Tatbestände durch den Staat. Eine Analyse zur bevölkerungspolitischen Kennzeichnung ausgewählter Politikbereiche in Deutschland. Baden-Baden

Fischer-Kowalski, Marina (2003): Gesellschaftlicher Metabolismus, Territorium und Nachhaltigkeit. In: GAIA. 1/2003. Editorial

Fischer-Kowalski, Marina / Amann, C. (2001): Beyond IPAT und Kuznets Curves Globalisation as a Vital Factor in Analysing the Environment Impact on SocioEconomic Metabolism. In: Population and Environment. Heft 23. S. 7-47

Fogt, Helmut (1982): Politische Generationen. Opladen

Foucault, Michel (1989): Der Wille zum Wissen. Sexualität und Wahrheit. Frankfurt am Main

Fremlin, J.H. (1964): How many people can the world support? In: New Scientist. Heft 415. (29.10.1964). S. 285-287

Fux, Beat (1994): Der familienpolitische Diskurs. Berlin

Gaede, Peter-Matthias (05.05.2004): Editorial. In: GEO. 05.05.2004. S. 3

Gans, Paul (2005): Regionale Bevölkerungsentwicklung Indiens. Online-Lexikon des Berlin-Instituts. http://www.berlininstitut.org/pdfs/Gans_Bevölkerungsentwicklung_Indien.pdf. Rev. 2005-01-16

Gans, Paul (2004): Tendenzen der räumlich-demografischen Entwicklung. Vortrag auf der Tagung der Akademie für Raumforschung und Landesplanung und des Bundesamtes für Bauwesen und Raumordnung „Demografischer Wandel im Raum. Was tun wir?"“ am 17./18.06.2004 in Magdeburg

Gaschke, Susanne (2004): Das kinderlose Land. In: Die Zeit. 15.01.2004 (59. Jg.). S. 3

Geist, Helmut (1993): Wie tragfähig ist das Tragfähigkeitstheorem? In: Massarat, Mohssen / Wenzel, Hans-Joachim / Sommer, Birgit / Széll, György (Hg.): Die Dritte Welt und wir. Bilanz und Perspektiven für Wissenschaft und Praxis. Freiburg. S.191-211

Gethmann, Carl F. / Kloepfer, Michael / Nutzinger, Hans G. (1993) (Hg.): Langzeitverantwortung im Umweltstaat. Bonn

Gibbs, Wayt (2001): On The Termination of Species. In: Scientific American. Heft 285 (Nov.2001). S. 40-49

Gille, Martina / Krüger, Winfried (2000): Unzufriedene Demokraten : politische Orientierungen der 16- bis 29jährigen im vereinigten Deutschland (DJI-Jugendsurvey). Opladen 
Glawak, C.: Unzerschnittene, verkehrsarme Räume in Deutschland 1999. In: Natur und Landschaft 76 (11). S. 481-484

Godet, Michel (1997): La Prospective Stratégique. In: Futuribles. Nr. 219/April 1997. S. 45-57

Godwin, William (1793): An Enquiry Concerning Political Justice. Hrsg. von Marshall, Peter. Princeton 1984

Goldstein, Joshua / Lutz, Wolfgang / Testa, Maria Rita (2003): The emergence of SubReplacement Family Size Ideals in Europe. Vienna Institute of Demography. Wien (European Demographic Research Papers Nr. 2). http://www.oeaw.ac.at/vid/publications/EDRP_No2.pdf. Rev. 2004-08-10

Gore, Al (1994): Key Note Address. In: BiB/Bundesinstitut für Bevölkerungsforschung (1994) (Hg): Internationale Konferenz 1994 über Bevölkerung und Entwicklung (ICPD 1994). Materialien zur Bevölkerungwissenschaft. Sonderheft 26. Wiesbaden. S. $45-50$

Gorke, Martin (2000): Was spricht für eine holistische Umweltethik? In: Natur und Kultur. Nr. 2/2002. S. 87-105

Gorke, Martin (1999): Artensterben. Von der ökologischen Theorie zum Eigenwert der Natur. Stuttgart

Görres-Gesellschaft (1985) (Hg.): Staatslexikon: Stichwort „Bevölkerungspolitik“. In: Band 1. Recht, Wirtschaft, Gesellschaft. Freiburg. S. 764-770

Gosseries, Axel (2002): Intergenerational Justice. In: LaFollette, Hugh (Hg.): The Oxford Handbook of Practical Ethics. Oxford. S. 459-484

Government of India (2005): Department of Family Welfare. http://www.mohfw.nic.in/dofw\%20website/Health\%20\&\%20Poulation\%20indicat ors/hpi\%20frame.htm. Rev. 2004-01-18

Gronemeyer, Reimer (2004): Kampf der Generationen. Düsseldorf

Gronemeyer, Reimer (1989): Die Entfernung vom Wolfsrudel. Düsseldorf

Grünheld, Evelyn (2003): Junge Frauen in Deutschland - Hohe Ausbildung contra Kinder? In: BIB-Mitteilungen. 1/2003 (15.3.2003). 24 Jg. S. 9-15

Grütz, Jens (1999): Generational Accounting - Buchhaltung für die Generationen: Einige Anmerkungen zu Vorgehen und Aussagekraft. In: Soziale Sicherheit 4/99

Guggenberger, Bernd (27.01.1990): Die neue Mehrheit der Lebenden. In: Frankfurter Allgemeine Zeitung. 23/1990. (27.01.1990), Tiefdruckbeilage

Haaren, Christina von / Nadin, Vincent (2003): Die Flächeninanspruchnahme in Deutschland im Vergleich zu der Situation in England. In: Raumforschung und Raumordnung. 5/2003 (61. Jg.). S. 345-356

Häberle, Peter (1998): Ein Verfassungsrecht für künftige Generationen - Die ,andere“ Form des Gesellschaftsvertrages: der Generationenvertrag. In: Ruland, Franz 
(Hg.): Verfassung, Theorie und Praxis des Sozialstaats: Festschrift für Hans F. Zacher zum 70. Geburtstag. S. 215-233

Habermas, Jürgen (1976): Zur Rekonstruktion des Historischen Materialismus. Frankfurt am Main

Hager, Frithjof / Schenkel, Werner (2003) (Hg.): Schrumpfungen. Wachsen durch Wandel. Ideen aus den Natur- und Kulturwissenschaften. München

Hammer, Thomas (Hg.): Großschutzgebiete - Instrumente nachhaltiger Entwicklung. München

Hannemann, Christine (2003): Zukunftschance Schrumpfung. Stadtentwicklung in Ostdeutschland - eine Chance. In: Hager, Frithjof / Schenkel, Werner (Hg.): Schrumpfungen. Wachsen durch Wandel. Ideen aus den Natur- und Kulturwissenschaften. München. S. 99-105

Hanns-Martin-Schleyer-Stiftung (1984) (Hg.): Die Lebensalter in einer neuen Kultur? Zum Verhältnis von Jugend, Erwerbsleben und Alter. Köln

Hardin, Garrett (1968): The Tragedy of the Commons. In: Science. Vol. 162 (13.12.1968). S. 1243-1248

Harrison, Paul (1994): Die dritte Revolution. Antworten auf Bevölkerungsexplosion und Umweltzerstörung. Heidelberg/Berlin/Oxford. (Engl. Originalausgabe: The Third Revolution. Population, Environment and a Sustainable World. New York 1992)

Hasenclever, Andreas (2000): Die Macht der Moral in der internationalen Politik. Militärische Interventionen westlicher Staaten in Somalia, Ruanda und Bosnien-Herzegowina. Frankfurt am Main

Haub, Carl (2005): Bevölkerungspolitik in Indien. Online-Lexikon des Berlin-Instituts. http://www.berlin-institut.org/pdfs/Haub_Bevpol_Indien.pdf. Rev. 2005-01-16

Haub, Carl (2003): Bevölkerungspolitik in Vietnam. Online-Lexikon des Berlin-Instituts. http://www.berlin-institut.org/pdfs/Haub_Vietnam.pdf. Rev. 2004-03-20

Hauff, Volker (1987) (Hg.): Unsere gemeinsame Zukunft. Der Bericht der Weltkommission für Umwelt und Entwicklung. Greven

Hauser, Jürg A. (1991): Bevölkerungs- und Umweltprobleme in der Dritten Welt, Band 2. Bern/Stuttgart

Häußermann, Hartmut / Siebel, Walter (2003): Neue Entwicklungstypen von Großstädten. In: Hager, Frithjof / Schenkel, Werner (Hg.): Schrumpfungen. Wachsen durch Wandel. Ideen aus den Natur- und Kulturwissenschaften. München. S. 75-85

Hayward, T. (1994), 142: The meaning of political ecology. In: Radical Philosophy. Nr. 66

Heidegger, Martin (1927): Sein und Zeit. 14. Auflage. Tübingen. 1977

Heilig, Gerhard K. (1996): How many people can be fed on earth? In: Lutz, Wolfgang (Hg.): The Future Population of the Earth. What can we assume today? S. 196-249 
Heim, Susanne / Schaz, Ulrike (1996): Berechnung und Beschwörung: Überbevölkerung - Kritik einer Debatte. Berlin/Göttingen

Heim, Susanne / Schaz, Ulrike (1994): „Das Revolutionärste, was die Vereinigten Staaten je gemacht haben“. Vom Aufstieg des Überbevölkerungsdogmas. In:

Wichterich, Christa (Hg.): Menschen nach Maß. Bevölkerungspolitik in Nord und Süd. Göttingen. S. 129-150

Heinrichs, Jürgen (2001) (Hg.): Globalisierung der Unfruchtbarkeit. München

Heinsohn, Gunnar (2003): Söhne und Weltmacht. Terror im Aufstieg und Fall der Nationen. Zürich

Herfeld, Anna-Maria (2001): Reformansätze zur Alterssicherung in Deutschland und intergenerationelle Gerechtigkeit. Frankfurt am Main.

Heringer, Hans (1982): Sprachkritik - die Fortsetzung der Politik mit besseren Mitteln. In: Heringer, Hans (Hg.): Holzfeuer im hölzernen Ofen. Aufsätze zur politischen Sprachkritik. Tübingen 1982

Hesselberger, Dieter (2000): Das Grundgesetz. 11. Auflage. Bonn

Hessische Staatskanzlei (2004) (Hg.): Hilfe wir schrumpfen! Die Gesellschaft im demographischen Wandel - Prognosen und Handlungsoptionen. Dokumentation der Rheingauer Impulse 2003. Wiesbaden

Hessische Staatskanzlei (2003) (Hg.): Die Familienpolitik muss neue Wege gehen! Der „Wiesbadener Entwurf" zur Familienpolitik. Wiesbaden

Hewitt, Paul S. (23.3.2004): Die Geopolitik des globalen Alterungsprozesses. In: Frankfurter Allgemeine Zeitung. 70/2004. (23.3.2004). S. 39

Heydebreck, Amelie von (04.08.2002): Die Entmachtung der Jugend. Die Alten schlagen zurück: Im deutschen Kunst- und Kulturbetrieb haben junge Menschen immer weniger zu melden. In: FAZ Sonntagszeitung. 4.8.2002

Heywood, V.H. / Stuart, S.N. (1992): Species Extinction in Tropical Forests. In:

Whitmore, T.C. / Sayer, J.A. (Hg.): Tropical Deforestation and Species Extinction. New York. S. 91-118

Hillmert, Steffen / Mayer, Karl Ulrich (2004): Geboren zwischen 1964 und 1971. Neuere Untersuchungen zu Ausbildungs- und Berufschancen in Westdeutschland. Wiesbaden

Hofmann-Riediger, Monika (1992): Metaethik. In: Pieper, Annemarie (Hg.): Geschichte der neueren Ethik. Band 2: Gegenwart. Tübingen/Basel. S. 55-76

Höhn, Charlotte (1995): Der Weg nach und von Kairo. Bevölkerungswissenschaftliche Betrachtungen zu den Ergebnissen der Internationalen Konferenz über Bevölkerung und Entwicklung (ICPD) 1994. In: Zeitschrift für Bevölkerungswissenschaft. 1/1995 (20. Jg.). S. 3-26 
Höhn, Charlotte / Schubnell, Hermann (1986): Bevölkerungspolitische Maßnahmen und ihre Wirksamkeit in ausgewählten europäischen Industrieländern. Teil 1. In: Zeitschrift für Bevölkerungswissenschaft. 1/1986. S. 3-51

Holdren, John P. / Ehrlich, Paul R. (1974): Human population and the global environment. In: American Scientist 62. S. 282-292

Holstein, Lars (2003): Nachhaltigkeit und neoklassische Ökonomik: der homo oeconomicus und die Begründung intergenerationeller Gerechtigkeit. Marburg

Hondrich, Karl Otto (2002): Wieder Krieg. Frankfurt am Main

Hondrich, Karl Otto / Koch-Arzberger, Claudia (1992): Solidarität in der modernen Gesellschaft. Frankfurt am Main

Honneth, Axel (1992): Kampf um Anerkennung. Frankfurt am Main

Höpflinger, François (1997): Bevölkerungssoziologie. Weinheim/München

Horn, Christoph / Scarano, Niko (2002): Einführung. In: Horn, Christoph / Scarano, Niko (2002) (Hg.): Philosophie der Gerechtigkeit. Frankfurt am Main. S. 9-16

Hösle, Vittorio (1999): Zur Philosophie der Geschichte der Sozialwissenschaften. In: Hösle, Vittorio: Die Philosophie und die Wissenschaften. München. S. 125-165

Hösle, Vittorio (1991): Philosophie der ökologischen Krise. München

Hummel, Diana (2000): Der Bevölkerungsdiskurs. Demografisches Wissen und politische Macht. Opladen

Hypovereinsbank (26.04.2001): Age Wave - Zur Demographieanfälligkeit von Aktienmärkten. In: Policy Brief 4/2001

ICPD/International Conference on Population and Development (1994): Report of the ICPD in Cairo, 5.-13.9.1994. www.unfpa.org. Rev. 2004-03-12

International Women's health Coalition (1994): Women's Declaration on Population Politics. http://www.iwhc.org/wd.html. Rev. 2002-04-06

Irrgang, Bernhard (2002): Natur als Ressource, Konsumgesellschaft und Langzeitverantwortung: zur Philosophie nachhaltiger Entwicklung. Dresden

IUCN (1990) (Hg.): Conserving the Worlds Biological Diversity. Gland. (Bearbeitet von McNeely, Jeffrey / Miller, Kenton / Reid, Walter / Mittermeier, Russell / Werner, Timothy)

Jessen, Johann (2004): Demografischer Wandel in Großstadtregionen. Vortrag auf der Tagung der Akademie für Raumforschung und Landesplanung und des Bundesamtes für Bauwesen und Raumordnung „Demografischer Wandel im Raum. Was tun wir?" am 17./18.06.2004 in Magdeburg

John Hopkins Bloomberg School of Public Health (2003): Population Reports. Vol. XXXI. Nr. 2 (Frühling 2003) 
Jonas, Hans (1980): Technology and Responsibility: The Ethics of an Endangered Future. In: Partridge, Ernest (1980) (Hg.): Responsibilities to Future Generations. Environmental Ethics. Buffalo N.Y. S. 23-36

Jonas, Hans (1979): Das Prinzip Verantwortung. Versuch einer Ethik für die technologische Zivilisation. Frankfurt am Main

Jüdes, Ulrich: Sprachverwirrung. Auf der Suche nach einer Theorie des Sustainable Development. In: Politische Ökologie. Heft 52. April/Mai 1997 (Jg.15). S. 1-12

Kagan, Robert (2003): Macht und Ohnmacht. Amerika und Europa in der neuen Weltordnung. Berlin

Kant, Immanuel (1785): Idee zu einer allgemeinen Geschichte in weltbürgerlicher Absicht. Hrsg von Weischedel, Wilhelm. Frankfurt am Main. 1968

Karkal, Malini (1996): Patriarchal Demography. Tracing India's History. In: Political Environments 4/1996 (Summer/Fall). S. 29-32

Kasun, Jaqueline (1988): The War against Population. The Economics and Ideology of Population Control. San Francisco

Kaufmann, Franz-Xaver (2004a): Ohne Nachwuchs keine Zukunft: Nachwuchssicherung als politisches Ziel. In: Generationengerechtigkeit! 1/2004. S. 9-11

Kaufmann, Franz-Xaver (2004b): Ablehnende Stellungnahme zur These: „Die Schrumpfung der Bevölkerungen Deutschlands und anderer Industrieländer ist zu begrüßen, da langfristig die gesamte Weltbevölkerung auf einem niedrigeren Niveau als heute stabilisiert werden muß? "In: Generationengerechtigkeit! 1/2004. S. 23

Kaufmann, Franz-Xaver (1990): Zukunft der Familie. München

Kaufmann, Franz-Xaver/Leisering, Lutz (1985) (Hg.): Studien zum Drei-Generationenvertrag. Institut für Bevölkerungsforschung und Sozialpolitik (IBS), Universität Bielefeld. Materialien Nr.15. Bielefeld

Ketelhodt, Friederike von (1993): Verantwortung für Natur und Nachkommen. Pfaffenweiler

Kettner, Mathias (1992): Diskursethik und Verantwortung für zukünftige Generationen. In: Friedrich Jahresheft 1992. S. 124-126

Khalatbari, Parviz / Otto, Johannes (1999) (Hg.): 200 Jahre Malthus. Schriftenreihe des Bundesinstituts für Bevölkerungswissenschaft. Heft 96. Wiesbaden

Khalatbari, Parviz (1993): Die Bevölkerungsexplosion - Ein Risiko für die bestehende Zivilisation! In: Studenteninitiative Wirtschaft \& Umwelt: Earth Summit '92. Perspektiven für eine neue Weltordnung. Münster. S. 125-152

Kil, Wolfgang (2004): Luxus der Leere. Wuppertal

Kleger, Heinz (1986): Gerechtigkeit zwischen Generationen. In: Müller-Schmied, Peter (Hg.): Begründung der Menschenrechte. Stuttgart. S. 145-191 
Klingholz, Reiner (2004): Deutschland 2020. In: GEO. 05.05.2004. S. 89-140

Klingholz, Reiner / Tremmel, Jörg (2004): Ein Gespräch zwischen Reiner Klingholz und Jörg Tremmel über Generationengerechtigkeit und Bevölkerungspolitik in Nord und Süd. In: Generationengerechtigkeit. Nr. 3/2004 (4. Jg.). Im Erscheinen

Klose, Hans-Ulrich (1994) (Hg.): Altern der Gesellschaft. Antworten auf den demographischen Wandel. Köln

Klose, Hans-Ulrich (1993): Altern hat Zukunft: Bevölkerungentwicklung und dynamische Wirtschaft. Opladen

Klöckner, Bernd (2003): Die gierige Generation. Wie die Alten auf Kosten der Jungen abkassieren. Frankfurt am Main

Kluge, Jürgen (2004): Deutschland spricht. Erwartungen an eine familienfreundliche Politik. Ergebnisse der Online-Umfrage „Perspektive Deutschland 2003“. In: Schmidt, Renate / Mohn, Liz (2004): Familie bringt Gewinn. Innovation durch Balance von Familie und Arbeitswelt. Gütersloh. S. 41-47

Kohli, Martin / Szydlik, Marc (2000) (Hg.): Generationen in Familie und Gesellschaft. Opladen

Köhl, Werner (2004): Sinkende Bevölkerungszahlen und dann? Herausforderungen für die Kommunen und das Flächenmanagement am Beispiel der Stadt Ludwigsburg. In: Umweltakademie Baden-Württemberg (2004) (Hg.): Bevölkerungsrückgang. Konsequenzen für Flächennutzung und Umwelt. Stuttgart. S. 83-99

Kopfmüller, Jürgen / Brandl, Volker / Jörissen, Juliane / Paetau, Michael / Banse, Gerhard / Coenen, Reinhard / Grunwald, Armin (2001): Nachhaltige Entwicklung integrativ betrachtet. Konstitutive Elemente, Regeln, Indikatoren. Berlin

Knaus, Anja / Renn, Ortwin (1998): Den Gipfel vor Augen. Unterwegs in eine nachhaltige Zukunft. Marburg

Kratochwil, Anselm (1996): Die Umweltkrise aus ökologischer Sicht. Historische Entwicklung und akuelle Bilanz. In: Herrenalber Protokolle. Schriftenreihe der Evangelischen Akademie Baden. Karlsruhe. S. 7-152

Kreibich, Rolf (2003): Generationengerechtigkeit im Zeitalter globaler Umweltkrisen. In: Stiftung für die Rechte zukünftiger Generationen (Hg.): Handbuch Generationengerechtigkeit. München. S. 221-242

Kreibich, Rolf / Weizsäcker, Ernst Ulrich von / Renn, Ortwin / Radermacher, FranzJosef / Borchert, Jürgen / Thunert, Martin (2003): Vorwort. In: Stiftung für die Rechte zukünftiger Generationen (Hg.): Handbuch Generationengerechtigkeit. München. S. 9-16

Kröhnert, Steffen / van Olst, Nienke / Klingholz, Reiner (2005): Emanzipation oder Kindergeld? Wie sich die unterschiedlichen Kinderzahlen in den Ländern Europas erklären. Studie des Berlin-Instituts für Weltbevölkerung und Entwicklung. http://www.berlin-institut.org/pdfs/emanzipation_oder_kindergeld.pdf. Rev. 200504-08 
Kröger, Inga / van Olst, Nienke / Klingholz, Reiner (2004): Das Ende der Aufklärung. Der internationale Widerstand gegen das Recht auf Familienplanung. Hrsg. vom Berlin-Institut für Weltbevölkerung und globale Entwicklung. Berlin

Kröhnert, Steffen / van Olst, Nienke / Klingholz, Reiner (2004): Deutschland 2020. Die demografische Zukunft der Nation. Hrsg. vom Berlin-Institut für Weltbevölkerung und globale Entwicklung. Berlin

Kröhnert, Steffen (2004): Jugend und Kriegsgefahr. Welchen Einfluss haben demografische Veränderungen auf die Entstehung von Konflikten? Eine Untersuchung des Berlin-Instituts für Weltbevölkerung und globale Entwicklung. http://www.berlininstitut.org/pages/fs/fs_studie_jugendundkrieg.html. Rev. 2004-07-01

Kronberger Kreis (1994) (Hg.): Mehr Langfristdenken in Gesellschaft und Politik. Bad Homburg

Lambrecht, Petra / Mertens, Heide (1989): Small family happy family. Münster

Laslett, Peter (1971): The Conversation between Generations. In: Laslett, Peter (Hg.): The Proper Study. Royal Institute of Philosophy Lectures. Bd. 4. London

Laslett, Peter / Fishkin, James S. (1992) (Hg): Justice between age groups and generations. London

Leggewie, Claus (1998): Nachwort. In: Stiftung für die Rechte zukünftiger Generationen (Hg.): Die 68er. Warum wir Jungen sie nicht mehr brauchen. Freiburg. S. 171179

Leggewie, Claus (1995): Die 89er - Portrait einer Generation. Hamburg

Lehr, Ursula (2003): Herausforderung alternde Gesellschaft. In: Die Politische Meinung. Monatsschrift zu Fragen der Zeit. September 2003 (48. Jg.). S. 40-47

Leipert, Christian (2003) (Hg.): Demographie und Wohlstand. Neuer Stellenwert für Familie in Wirtschaft und Gesellschaft. Opladen

Leisering, Lutz (1990): Sozialstaat und demographischer Wandel. Frankfurt am Main

Leisinger, Klaus M. (1994): Hoffnung als Prinzip. Bevölkerungspolitik mit menschlichem Anlitz. Hannover

Leist, Anton (1991): Intergenerationelle Gerechtigkeit. In: Bayertz, Kurt: Praktische Philosophie. Hamburg. S. 322-360

Lélés, Susanna (1991): Sustainable Development. A critical review. In: World Development. Vol. 19/6 (1991). S. 607-621

Leopold, Aldo (1992): Am Anfang war die Erde: Plädoyer zur Umweltethik. Darmstadt

Lévi-Strauss, Claude (1992): Interview mit Jürgen Altwegg. Frankfurter Allgemeine Zeitung. Magazin. Heft 622 (3.1.1992). S. 55

Leyen, Ursula von der (2003): Ist die derzeitige Familienpolitik ein Unrecht an kommenden Generationen? In: Generationengerechtigkeit! 3/2003 (3. Jg.). S. 15 
Li, Yongping/Peng, Xizhe (2000): Age and Sex Structures. In: Peng, Xizhe / Guo, Zhigang (Hg.): The Changing Population of China. Oxford. S. 64-76

Lloyd, Cynthia B. (1996): Family and Gender Issues for Population Policy. In: United Nations (Hg.): Population and Women. Proceedings of the United Nations Expert Group Meeting on Population and Women in Gabarone, Botswana, 22-26 June 1992. New York, S. 65-80

Locke, John (1690/1980): Second Treatise of Government. Hrsg. von MacPherson. Indianapolis

Lomborg, Björn (2002): Apocalypse no! Wie sich die menschlichen Lebensgrundlagen wirklich entwickeln. Lüneburg

Lorz, Oliver (2003): Intergenerative Umverteilung in der repräsentativen Demokratie. Berlin/Heidelberg/New York

Lude, Armin (2001): Naturerfahrung und Naturschutzbewusstsein. Innsbruck/Wien/München

Lüscher, Kurt / Liegle, Ludwig (2003): Generationenbeziehungen in Familie und Gesellschaft. Konstanz

Lüscher, Kurt / Schultheis, Franz (1995) (Hg.): Generationenbeziehungen in ,ppostmodernen" Gesellschaften. Konstanz

Maass, Harald (16.6.2004): 1000 Yuan für ein Einzelkind. In: Frankfurter Rundschau. $137 / 2004$ (16.6.2004). S. 16

MacArthur, R./Wilson, E. (1967): The Theory of Island Biogeography. Princeton

Mackenroth, Gerhard (1957): Die Reform der deutschen Sozialpolitik durch einen deutschen Sozialplan. In: Böttcher, Erik (Hg.): Sozialpolitik und Sozialreform, Tübingen. S. 43-73

Mackenroth, Gerhard (1972): Grundzüge einer historisch-soziologischen Bevölkerungstheorie. In: Köllmann, Wolfgang / Marschalck, Peter (Hg.): Bevölkerungsgeschichte. Köln. S. 29-33

Mackenroth, Gerhard (1953): Bevölkerungslehre. Theorie, Soziologie und Statistik der Bevölkerung. Berlin

Mackensen, Rainer (2003): Stichwort ,Bevölkerungsforschung'. Online-Lexikon des Berlin-Instituts. http://www.berlin-institut.org/pdfs/Mackensen_Forschung.pdf. Rev. 2004-03-20

Mackensen, Rainer (1998): Bevölkerungsfragen auf Abwegen der Wissenschaften. Zur Geschichte der Bevölkerungswissenschaft in Deutschland im 20. Jahrhundert. Opladen

Mackensen, Rainer (1997): Bevölkerungswissenschaft: Bringing Man back in. In: Zeitschrift für Bevölkerungswissenschaft. Heft 2-3/1997 (Jg.22). S. 195-215 
Mackensen, Rainer (1989): Geschichtliche Prägung und prognostisches Potential der Bevölkerungstheorie. In: Mackensen, Rainer / Thill-Thouet, Lydia / Stark, Ulrich (Hg.): Bevölkerungsentwicklung und Bevölkerungstheorie in Geschichte und Gegenwart. Frankfurt am Main/New York. S. 10-25

Macura, Milos (1987): The Significance of the UN International Population Conferences. Population Bulletin. Nummern 19/20. 1986

Malthus, Thomas R. (1803): An Essay on the Principle of Population; or View of its Past and Present Effects an Human Happiness; with an Inquiry into our Prospects Respecting the Future Removal or Mitigation of the Evils which is Occasions. A new Edition, very much enlarged. London. Hrsg. von M. Fogatry (1958). London/New York

Malthus, Thomas R. (1798): An Essay on the Principle of Population as it Affects the Future Improvement of Society, with Remarks on the Speculation of Mr. Godwin, M. Condorcet, and other Writers. London. Übersetzt und hrsg. von Christian M. Barth (1977). München

Mannheim, Karl (1928): Das Problem der Generationen. In: Kölner Vierteljahreshefte für Soziologie. Heft 7/1928. S. 157-185. Fortsetzung des Artikels in Heft 8/1928. S. $309-330$

Martin, Paul S. (1984): Prehistoric Overkill: The Global Model. In: Martin, Paul S. / Klein, Richard G.: Quaternary Extinctions: A Prehistoric Revolution. Tucson. S. $367-403$

Mayer, Susanne (2004): Her mit den Kindern! Plädoyer für eine moderne Bevölkerungspolitik, die den Namen verdient. In: Die Zeit. 04.03.2004. S. 18

Maxeiner, Dirk / Miersch, Michael (2002): Lexikon der Öko-Irrtümer. Fakten statt Umweltmythen. Taschenbuchsonderausgabe (Originalausgabe 1998). München

Maxeiner, Dirk / Miersch, Michael (1999): Öko-Optimismus. Überarbeitete Lizenzausgabe (Originalausgabe 1998). München

Max-Neef, Manfred (1995): Economic Growth and the quality of life: A threshold hypothesis. Ecological Economics 15. S. 115-118

McElroy, Marjorie/Yang, Dennis Tao (2000): Carrots and Sticks: Fertility Effects of China's Population Policies. In: American Economic Review. Heft 90. 2/2000

Meadows, Dennis L. (2004): "Wir haben 30 Jahre verloren.“ In: Die Zeit. Nr. 2/2004 (31.12.2003). S. 20

Meadows, Dennis L. / Meadows, Donella H. / Randers, Jørgen (1992): Die neuen Grenzen des Wachstums. Stuttgart

Meadows, Dennis L. / Meadows, Donella H. / Zahn, Erich / Milling, Peter (1972): Die Grenzen des Wachstums. Stuttgart 
Merli, M. Giovanni/Raftery, Adrian E. (2000): Are Births Underreported in Rural China? Manipulation of Statistical Records in Response to China's Population Policies. In: Demography. Heft 37. 1/2000. S. 109-126

Mertens, Heide (1994): Frauen, Natur und Fruchtbarkeit - Die Bevölkerungsdebatte und die ökologische Tragfähigkeit der Erde. In: Wichterich, Christa: Menschen nach Maß. Bevölkerungspolitik in Nord und Süd. S. 181-200

Mesarovic, Mihajlo / Pestel, Eduard (1977): Menschheit am Wendepunkt. Reinbek bei Hamburg

Meulenberg, Claudia (2004): DefinitelyProbably One. A Generation Comes of Age Under China's One Child Policy. In: Worldwatch Magazine. Sept./Okt. 2004. S. 31-33

Miegel, Meinhard (2003): Die deformierte Gesellschaft. Berlin/München

Mies, Maria (1988): Patriachat und Kapital - Frauen in der internationalen Arbeitsteilung. Zürich

Mohl, Hans (1993): Die Altersexplosion. Droht uns ein Krieg der Generationen? Stuttgart

Mohr, Hans (1987): Natur und Moral. Ethik in der Biologie. Darmstadt

Mubarak, Hosni (1994): Opening Address. In: BiB/Bundesinstitut für Bevölkerungsforschung (1994) (Hg): Internationale Konferenz 1994 über Bevölkerung und Entwicklung (ICPD 1994). Materialien zur Bevölkerungwissenschaft, Sonderheft 26. Wiesbaden. S. 9-17

Müller, Paul (2003): MacDonaldisierung, Schrumpfung, Aussterben. In: Hager, Frithjof / Schenkel, Werner (2003) (Hg.): Schrumpfungen. Wachsen durch Wandel. Ideen aus den Natur- und Kulturwissenschaften. München. S. 195-219

Münz, Rainer (2003): Mehr Kinder, mehr Macht. In: Die Zeit. 3/2003 (09.01.2003). S. 6

Münz, Rainer / Ulrich, Ralf (1994): Bevölkerungswachstum und Familienplanung in Entwicklungsländern. In: Demographie aktuell. Nr. 4/1994

Myers, Norman / Mittermeier, Russell A. / Mittermeier, Christina G. / Fonseca, Gustavo / Kent, Jennifer (2001): Biodiversity hotspots for conservation priorities. In: Nature 403 (24.2.2000). S. 853-858

NABU (2004): Innenentwicklung versus Grün in der Stadt? www.nabu.de. Rev. 2004$06-20$

NABU (23.01.2004): Pressemitteilung vom 23.01.2004: Jahrhundertwerk Natura 2000 jetzt vollenden. www.nabu.de. Rev. 2004-06-20

Naess, Arne (1997): Die tiefenökologische Bewegung: Einige philosophische Aspekte. In: Krebs, Angelika (1997) (Hg.): Naturethik. Frankfurt am Main. S. 182-220

Narveson, Jan (1978): Future People and Us. In: Barry, Brian / Sikora, Richard (1978)

(Hg.): Obligations to Future Generations. Philadelphia. S. 38-60 
Narveson, Jan (1976): Moral Problems of Population. In: Bayles, Michael D. (1976) (Hg.): Ethics and Population. Cambridge. S. 59-80

Natorp, Klaus (01.08.1988): Weniger kann auch mehr sein. In: Frankfurter Allgemeine Zeitung. 01.08.1988. S. 2

NBBW/Nachhaltigkeitsbeirat der Landesregierung Baden-Württemberg (2004): Neue Wege zu einem nachhaltigen Flächenmanagement in Baden-Württemberg. Sondergutachten. Februar 2004. Stuttgart

Nell-Breuning, Oswald von / Fetsch, Cornelius, G. (1981) (Hg.): Drei Generationen in Solidarität - Rückbesinnung auf den echten Schreiber-Plan. Köln

Niejahr, Elisabeth (28.08.2003): Die Bellheim Republik. In: Die Zeit. 36/2003 (28.08.2003). S. 1

Niejahr, Elisabeth (02.01.2003): Land ohne Leute. In: Die Zeit. 2/2003 (02.01.2003). S. 9-11

Notestein, Frank (1964): Population Growth and Economic Development. Lecture presented at the Ceylon Association for the Advancement of Science. Wiederabgedruckt in Population and Development Review. Vol. 9. No. 2 (June 1983)

Nullmeier, Frank (2004a): Die politische Karriere des Begriffs „Generationengerechtigkeit" und seine wissenschaftliche Bedeutung. In: Generationengerechtigkeit! Nr. 4/2004 (4. Jg.). Im Erscheinen

Nullmeier, Frank (2004b): Generationengerechtigkeit - aus politikwissenschaftlicher Sicht. In: VDR/Verband Deutscher Rentenversicherungsträger (2004): Generationengerechtigkeit - Inhalt, Bedeutung und Konsequenzen für die Alterssicherung. Frankfurt am Main. S. 65-73

Nutzinger, Hans G. / Held, Martin (2001) (Hg.): Nachhaltiges Naturkapital: Ökonomik und zukunftsfähige Entwicklung. Frankfurt am Main/New York

OECD (1998): The macroeconomic implications of ageing in a global context. Economic Department Working Paper no. 193. Paris

Oeter, Ferdinand (1989): Der unsoziale Sozialstaat. München

Ohsmann, Sabine / Stoltz, Ulrich (2004): Entwicklung der Rendite in der gesetzlichen Rentenversicherung. In: Die Angestelltenversicherung. Heft 2/2004 (Jg. 51). S. 5662

Olechowski, Richard (1980) (Hg.): Geburtenrückgang, besorgniserregend oder begrüssenswert? Bericht über das Interdisziplinäre Symposium der Österreichischen Gesellschaft für Familie und Kind am 6.10.1979. Wien/Freiburg/Basel

Olson, Robert L. (1994): Alternative Images of a Sustainable Future. In: Futures. Heft 26. S. $156-169$

Opaschowski, Horst (2004): Der Generationenpakt. Das soziale Netz der Zukunft. Darmstadt 
Opaschowski, Horst (2000): Bindung auf Dauer ist nicht mehr im Trend. In: GeneralAnzeiger vom 4.1.2000

Ott, Konrad (2004): Theoriebildung statt Definitionswirrwarr. Kommentar 2 zu Jörg Tremmels Beitrag. In: GAIA. 1/2004. S. 38-39

o.V. (23.08.2004): Oldies in Glasboxen. In: Der Spiegel. 35/2004 (23.08.2004). S. 87

o.V. (15.07.2004): Bundesregierung beschließt Ausbau der Tagesbetreuung. In: Frankfurter Allgemeine Zeitung. 162/2004 (15.07.2004). S. 4

o.V. (06.07.2004): Zuschlag für Kinderlose stößt auf Widerstand. In: Süddeutsche Zeitung. 153/2004 (06.07.2004). S. 1

o.V. (29.05.2004): Intime Schwester. In: Der Spiegel. 23/2004 (29.05.2004). S. 108

o.V. (28.05.2004): Zuzug von Hochqualifizierten und Selbständigen wird erleichtert. In: Frankfurter Allgemeine Zeitung. 123/2004 (28.05.2004). S. 15

o.V. (26.04.2004): Abtreibung als Wahlkampfthema. In: Frankfurter Allgemeine Zeitung. 97/2004 (26.04.2004). S. 6

o.V. (09.03.2004): CSU will Eltern bei der Rente künftig besser stellen. In: Frankfurter Allgemeine Zeitung. 58/2004 (09.03.2004). S. 11

o.V. (05.01.2004): Land ohne Lachen. In: Der Spiegel. 1/2004 (05.01.2004). S. 38-53

o.V. (27.10.2003): Baby-Boom bei Dämmerlicht. In: Der Spiegel. 44/2003

(27.10.2003). S. 87

o.V. (10.07.2003): „Aktive Bevölkerungspolitik“ - Familienministerin Schmidt besorgt über niedrige Geburtenrate. In: Frankfurter Allgemeine Zeitung. 57/2003 (10.7.2003). S. 5

o.V. (28.04.2003): Politikverbot für Kinderreiche. In: Der Spiegel. 18/2003 (28.04.2003). S. 147

o.V. (08.03.2002): Kinder, Krippe, Karriere. In Europa gibt es viele Geburten, wo der Staat für Betreuung sorgt. In: Tagesspiegel (08.03.2002). S. 6

o.V. (13.10.1999): Annan begrüßt sechsmilliardsten Erdenbürger. UN wählen bosnisches Baby aus Sarajevo symbolisch aus. In: Tagesspiegel (13.10.1999). S. 9

Parfit, Derek (1987): Reasons and Persons. Oxford. 3. überarb. Auflage (1. Auflage 1984)

Parfit, Derek (1981): Future Generations: Further Problems. In: Philosophy and Public Affairs. 7/1981. S. 113-172

Partridge, Ernest (1990): On the Rights of Future Generations. In: Donald Scherer: Upstream-Downstream. Issues in Environmental Ethics. Philadelphia. S. 40-66

Partridge, Ernest (1980) (Hg.): Responsibilities to Future Generations. Environmental Ethics. Buffalo N.Y.

Pawlowski, Tadeusz (1980): Begriffsbildung und Definition, Berlin/New York 
Pearce, David / Turner, Kerry R. (1990): Economics of Natural Resources and the Environment. London

Penck, Albrecht (1925): Das Hauptproblem der physischen Anthropogeographie. In: Zeitschrift für Geopolitik. 2/1925. S. 330-348

Petechsky, Rosalind Pollak (1995): The Body as Property: A Feminist Re-Vision. In: Ginsburg, Faye D. / Rapp, Rayna (Hg.): Conceving the New World Order. The Global Politics of Reproduction. Berkeley/Los Angeles/London. S. 387-406

Pfeiffer, Ulrich / Domitra, Michael (2003) (Hg.): Generationengerechtigkeit, Wachstum, Demografie: Positionspapier zum Kongress des Managerkreises vom 25.11.2003. Schriften der Friedrich-Ebert-Stiftung. Bonn

Pfordten, Dietmar von der (1996): Ökologische Ethik. Zur Rechtfertigung menschlichen Verhaltens gegenüber der Natur. Reinbek

Pimm, Stuart L. / Raven, Peter: Extinction by numbers. In: Nature. Vol. 403 (24.2.2000). S. 843-845

Pimm, Stuart L. / Russell, G. J. / Gittleman, John L. / Brooks, Thomas M. (1995): The Future of Biodiversity. In: Science 269 (1995). S. 347-350

Piotrow, Phyllis T. (1973): World Population Crisis: The United States Response. New York

Podszun, Rupprecht (2000): Die verkalkte Republik oder das Märchen vom Jugendkult. Köln

Pohl, Britta (19.11.2004): Mehr Kinder. Mehr Leben. Forsa-Befragung der Zeitschrift ELTERN in Zusammenarbeit mit Gruner+Jahr. Unterlagen bei der Pressekonferenz am 19.11.2004

Posner, Roland (1990): Das Drei-Kammer-System: Ein Weg zur demokratischen Organisation von kollektivem Wissen und Gewissen über Jahrtausende. In: Posner, Roland (Hg.): Warnungen an eine ferne Zukunft. Atommüll als Kommunikationsproblem. München. S. 259-305

Prognos (2002): Prognos Deutschland Report 2002-2020. Basel

Prugh, Thomas / Ayres, Ed (2004): Population and its Discontents. Editors Introduction. In: Worldwatch Magazine. Sept./Okt. 2004. S. 13

Pulte, Peter (1972): Die Bevölkerungslehre. München/Wien

Qu, Geping/Li, Jinchang (1994): Population and the Environment in China. Boulder/London

Raffelhüschen, Bernd / Walliser, Jan (1997): Was hinterlassen wir zukünftigen Generationen? Ergebnisse der Generationenbilanzierun. In: Knappe, Eckhard / Winkler, Albrecht (Hg.): Sozialstaat im Umbruch: Herausforderung an die deutsche Sozialpolitik. Frankfurt am Main. S. 65-89 
Raffelhüschen, Bernd / Feist, K. (2000): Möglichkeiten und Grenzen der Generationenbilanzierung. In: Wirtschaftsdienst. Heft 80. S. 440-447

Randeria, Shalani (1995): Die sozio-ökonomische Einbettung reproduktiver Rechte: Frauen und Bevölkerungspolitik in Indien. In: Feministische Studien. Mai/1995 (13. Jg.). S. 119-132

Randeria, Shalini (1994): Das Wunder Kerala. Eine Erfolgsgeschichte indischer Bevölkerungspolitik? In: Wichterich, Christa (Hg.): Menschen nach Maß. Bevölkerungspolitik in Nord und Süd. Göttingen. S. 239-262

Rat von Sachverständigen für Umweltfragen (2002): Umweltgutachten 2002. Langfassung. Bundestagsdrucksache 14/8792. Berlin

Raven, Peter H. (1994): Defining Biodiversity. In: Nature Conservancy. Heft 44. (Jan./Feb. 1994). S. 11-15

Rawls, John (1979): Eine Theorie der Gerechtigkeit. Frankfurt am Main (Originalausgabe: A Theory of Justice. Cambridge 1971)

Renn, Ortwin (1996a): Rolle und Stellenwert der Soziologie in der Umweltforschung. In: Diekmann, Andreas / Jaeger, Carlo C. (Hg.): Sonderheft Umweltsoziologie der KZfSS. Sonderheft 36. Opladen. S. 11-27

Renn, Ortwin (1996b): Kann man die technische Zukunft vorhersagen? Zum Stellenwert der Technikfolgenabschätzung für eine verantwortbare Zukunftsvorsorge. In: Pinkau, Klaus / Stahlberg, Christina (Hg.): Technologiepolitik in demokratischen Gesellschaften. Stuttgart. S. 23-51

Ricardo, David (1994): Über die Grundsätze der politischen Ökonomie und der Besteuerung. Marburg. (Originaltitel: Ricardo, David (1817): On the Principles of Political Economy and Taxation. London)

Riester, Walter (2000): Rentenreform 2000: Sicherheit für die Älteren - Bezahlbarkeit für die Jüngeren. In: ifo Institut für Wirtschaftsforschung e.V. (Hg.): ifo Schnelldienst 28-29/2000. 53. Jg. (17.10.2000). München, S. 5-8

Rolston, Holmes III (1974): Is there an ecological ethics? In: Ethics. Heft 85. S. 93-109

Rosen, Klaus-Henning (1994): Bericht über die Ergebnisse der ICPD. In:

$\mathrm{BiB} /$ Bundesinstitut für Bevölkerungsforschung (1994) (Hg): Internationale Konferenz 1994 über Bevölkerung und Entwicklung (ICPD 1994). Materialien zur Bevölkerungwissenschaft. Sonderheft 26. Wiesbaden. S. 67-84

Rosenmayr, Leopold (1990): Generation als gestaltete Geschichte. In: Rosenmayr, Leopold (Hg.): Die Kräfte des Alterns. Wien. S. 148-160

Ross, David (1930): Ein Katalog von Prima-facie-Pflichten. Aus: Ross, David: The Right and the Good. Oxford. Abgedruckt in: Birnbacher, Dieter / Hoerster, Norbert (2000) (Hg.): Texte zur Ethik. 11. Auflage (1. Auflage 1976). München. S. 253269 
Rürup, Bert / Gruescu, Sandra (2003): Nachhaltige Familienpolitik im Interesse einer aktiven Bevölkerungsentwicklung. Gutachten im Auftrag des BMFSFJ. Berlin

Sachs, Aaron (1994): Erst kommt der Sex und dann? Männer und Familienplanung in einer überbevölkerten Welt. In: World-Watch. Nr. 2/1994 (April/Mai). (3. Jg.) S. $10-16$

Sadik, Nafis (1994): Introductory Speech. In: BiB/Bundesinstitut für Bevölkerungsforschung (1994) (Hg): Internationale Konferenz 1994 über Bevölkerung und Entwicklung (ICPD 1994). Materialien zur Bevölkerungwissenschaft. Sonderheft 26. Wiesbaden. S. 25-33

Saladin, Peter /Zenger, Christoph (1988): Rechte künftiger Generationen. Basel/Frankfurt am Main

Scharping, Thomas (2003): Bevölkerungspolitik in China. Online-Lexikon des BerlinInstituts. http://www.berlin-institut.org/pdfs/Scharping_China.pdf. Rev. 2004-0320

Schimany, Peter (2003): Die Alterung der Gesellschaft. Ursachen und Folgen des demographischen Umbruchs. Frankfurt am Main/New York

Schirrmacher, Frank (2004): Das Methusalem-Komplott. München

Schlebusch, Cornelia (1994): Bevölkerungspolitik als Entwicklungsstrategie. Historisches und Aktuelles zu einem fragwürdigen Argument. Frankfurt am Main

Schlieben-Lange, Brigitte (Hg.) (2000): Generationen. Stuttgart

Schmähl, Winfried (2000): Alterssicherung in Deutschland an der Jahrtausendwende Konzeptionen, Maßnahmen und Wirkungen. In: VDR (Hrsg.): Deutsche Rentenversicherung, Heft 1-2 (Januar/Februar) 2000. Frankfurt am Main

Schmid, Josef (2004): Generationengerechtigkeit - ein globales Problem? In: Generationengerechtigkeit! 1/2004. S. 7-8

Schmid, Josef (2003): Stichwort ,Bevölkerungssoziologie'. Online-Lexikon des BerlinInstituts. http://www.berlininstitut.org/pdfs/Schmi_\%20Bevoelkerungssoziologie.pdf. Rev. 2004-03-20

Schmid, Josef (1994a): Die wachsende Weltbevölkerung. Ursachen, Folgen, Bewältigung. In: Aus Politik und Zeitgeschichte. B 35-36 (02.09.1994). S. 11-20

Schmid, Josef (1994b): Bevölkerung, Umwelt, Entwicklung. Forschungsrichtungen und aktuelle Argumentation. In: Schmid, Josef (Hg.): Bevölkerung - Umwelt - Entwicklung. Eine humanökologische Perspektive. Opladen. S. 17-42

Schmid, Josef (1984): Bevölkerung und soziale Entwicklung. Der demografische Übergang als soziologische und politische Konzeption. Boppard am Rhein

Schmid, Josef (1976): Einführung in die Bevölkerungssoziologie. Reinbek

Schmid, Josef /Heigl, Andreas /Mai, Ralf (2000): Sozialprognose. Die Belastung der nachwachsenden Generation. München 
Schmidt, Renate / Mohn, Liz (2004): Familie bringt Gewinn. Innovation durch Balance von Familie und Arbeitswelt. Gütersloh

Schmidt, Renate (2003): Zukunft der Familie und sozialer Zusammenhalt. In: Generationengerechtigkeit! 3/2003 (3. Jg.). S. 1-4

Schmitt, Christian (2005): Kinderlosigkeit bei Männern. Geschlechtsspezifische Determinanten ausbleibender Elternschaft. In: Tölke, Angelika/Hank, Karsten (Hg.): Männer - das vernachlässigte Geschlecht in der Familienforschung. Sonderheft 4 der Zeitschrift für Familienforschung. Wiesbaden

Schockenhoff, Eberhard (1996): Bevölkerungspolitik und Familienplanung in der Dritten Welt. In: Berichte aus den Sitzungen der Joachim-Jungius-Gesellschaft der Wissenschaften. 3/1996 (Jg. 14). S. 3-69

Schreiber, Winfried (1955): Existenzsicherung in der industriellen Gesellschaft. Köln

Schreiber, Matthias / Margraf, Christine (2003): Eine Chance für die Vielfalt. In: BUNDmagazin. 1/2003. S. 22-23

Schroeter, Klaus R. (2000): Altersstrukturwandel als „ungeplanter Prozess“. In: Backes, Gertrud M. (2000): Soziologie und Alter(n) : neue Konzepte für Forschung und Theorieentwicklung. Opladen. S. 79-108 Schubnell, Hermann (1990):

Bevölkerungs- und Familienpolitik - Definitionen, Bezug zur Gesamtpolitik und Probleme der Wirkungsanalyse. In: Birg, Herwig/Mackensen, Rainer (Hg.):

Demographische Wirkungen politischen Handelns. Frankfurt am Main. S. 33-53

Schüller, Heidi (1997): Wir Zukunftsdiebe. Wie wir die Chancen unserer Kinder verspielen. Berlin

Schüller, Heidi (1995): Die Alterslüge. Für einen neuen Generationenvertrag. Berlin

Schultz, Susanne (1994): Feministische Bevölkerungspolitik? Zur internationalen Debatte um Selbstbestimmung. In: Eichhorn, Cornelia / Grimm, Sabine (Hg.): Gender Killer. Texte zu Feminismus und Politik. Berlin/Amsterdam. S. 11-23

Schultz, T. Paul/Yi, Zeng (1995): Fertility of Rural China: Effects of Local Family Planning and Health Programs. In: Journal of Populations Economics 8, Heft 4. S. 329-350

Schwarz, Karl (1992): Bevölkerungspolitische Wirkungen familienpolitischer Maßnahmen. In: Zeitschrift für Bevölkerungswissenschaft 2/1992. S. 197-208

Schwarz, Karl (1987): Demographische Wirkungen der Familienpolitik in Bund und Ländern nach dem Zweiten Weltkrieg. In: Zeitschrift für Bevölkerungswissenschaft 4/87. S. 409-450

Schering AG (2004): International Dialogue on Population and Sustainable Development „Ways out of the Crisis - Reproductive Health in Need of New Ideas”. 14. October 2004. GTZ-House. Conference Proceedings. Berlin

Sen, Amartya (1994): Die Menschenbombe. Ein globales Problem - Verblendung und Wirklichkeit. In: Lettre International. 27/1994 (IV. Quartal). S. 6-13 
Siedentop, Stefan / Kausch, Steffen (2004): Die räumliche Struktur des Flächenverbrauchs in Deutschland. In: Raumforschung und Raumordnung. Heft 1/2004 (62. Jg.). S. 36-49

Silkenbeumer, Rainer (1979) (Hg.): Geburtenrückgang, Risiko oder Chance. Hannover Simon, Julian L. (1998): The Ultimate Resource 2. Princeton

Simon, Julian L. (1992): There is no environmental, population, or resource crisis. In: Tyler-Miller, G.: Living in the Environment. Principles, Connections, Solutions. Belmont

Simon, Julian L. (1981): The Ultimate Resource. Princeton

Simon, Julian L. (1977): The Economics of Population Growth. Princeton

Sinding, Steven W. (2004): Family Planning and Reproductive Health: Where is the international community headed? In: Schering AG: International Dialogue on Population and Sustainable Development „Ways out of the Crisis - Reproductive Health in Need of New Ideas". 14. October 2004. GTZ-House. Conference Proceedings. Berlin. S. 31-47

Sinding, Steven W. (2003): Internationale Bevölkerungskonferenzen. Online-Lexikon des Berlin-Instituts. http://www.berlin-institut.org/pdfs/Sinding_Konferenzen.pdf. Rev. 2004-03-20

Sinding, Steven W. (2000): The great population debates: how relevant are they for the $21^{\text {st }}$ century? American Journal of Public Health. Vol. 90. Nr. 12 (Dezember). S. $1841-1847$

Sing, Elmar (2003): Generationengerechtigkeit und Unternehmen. In: Generationengerechtigkeit! Nr. 2/2003. S. 5-7

Singer, Peter (1976): A Utilitarian Population Principle. In: Bayles, Michael D. (1976) (Hg.): Ethics and Population. Cambridge. S. 81-99

Sinn, Hans-Werner (2003): Das demographische Defizit. Die Fakten, die Folgen, die Ursachen und die Politikimplikationen. In: Leipert, Christian (Hg.): Demographie und Wohlstand. Neuer Stellenwert für Familie in Wirtschaft und Gesellschaft. Opladen. S. 57-88

Sinn, Hans-Werner (14.01.2003): Rente nach Kinderzahl. In: Frankfurter Allgemeine Zeitung (14.01.2003)

Sinn, Hans-Werner (1998): The Pay-as-you-go Pension System as a Fertility Insurance and Enforcement Device. CES Working Paper Series No. 154. München

Sinn, Hans-Werner (1997): The Value of Children and Immigrants in a Pay-as-you-go Pension system: A Proposal for a Partial Transition to a Funded System. CES Working Paper Series No. 141. München

Smail, Kenneth (2004): Global Population Reduction. Confronting the Inevitable. In: Worldwatch Magazine. Sept./Okt. 2004. S. 58-59 
Spaemann, Robert (1990): Glück und Wohlwollen. Versuch über Ethik. Stuttgart SPIEGEL SPEZIAL (1999): Generationen im Konflikt. Jung gegen Alt. 2/1999

SRU/Rat von Sachverständigen für Umweltfragen (2002): Umweltgutachten 2002. Langfassung. Bundestagsdrucksache 14/8792. Berlin

Statistisches Bundesamt (2003): Bevölkerung Deutschlands bis 2050. 10. koordinierte Bevölkerungsvorausberechnung. Presseexemplar. Wiesbaden

Steinmann, Gunter (1988): Bevölkerungswachstum, Ressourcen und wirtschaftliche Entwicklung. In: Steinmann, Gunter / Zimmermann, Klaus F. / Heilig, Gerhard (Hg.): Probleme und Chancen demographischer Entwicklung in der dritten Welt. Berlin. S. 19-35

Stiftung für die Rechte zukünftiger Generationen (2004): Positionspapier des AK Kinderrechte, http://www.srzg.de/ndeutsch/5publik/2ppap/1ppap.htm. Rev. 2004-07-02

Stiftung für die Rechte zukünftiger Generationen (2003) (Hg.): Handbuch Generationengerechtigkeit. 2. überarbeitete Auflage. München

Stiftung für die Rechte zukünftiger Generationen (1998) (Hg.): Die 68er. Warum wir Jungen sie nicht mehr brauchen. Freiburg

Stiftung für Kommunikationsforschung (1988) (Hg.): Der brüchige Generationenvertrag. Köln

Stoyke, Michael (2004): Bevölkerungspolitik in China. In: Generationengerechtigkeit! Nr.1/2004. S. 16-18

Süßmilch, Johann Peter (1741/1765): Die göttliche Ordnung in den Veränderungen des menschlichen Geschlechts aus der Geburt, dem Tode und der Fortpflanzung desselben erwiesen. 2 Bände. Berlin

Terborgh, J.: Requiem for Nature. Washington D.C.

Tichy, Roland / Tichy, Andrea (2001): Die Pyramide steht Kopf? Die Wirtschaft in der Altersfalle. München

Tölke, Angelika / Hank, Karsten (2005) (Hg.): Männer - das vernachlässigte Geschlecht in der Familienforschung. Sonderheft 4 der Zeitschrift für Familienforschung. Wiesbaden

Tremmel, Jörg (2004a): ,Nachhaltigkeit' - definiert nach einem kriteriengebundenen Verfahren. In: GAIA. Nr. 1/2004. S. 26-34

Tremmel, Jörg (2004b): Generationengerechtigkeit - eine Ethik der Zukunft. In: Natur und Kultur. Transdisziplinäre Zeitschrift für ökologische Nachhaltigkeit. 1/2004 (5/1). S. 45-64

Tremmel, Jörg (2004c): Is a Theory of Intergenerational Justice Possible? A Response to Beckerman. In: Generationengerechtigkeit! Nr.2/2004 (4. Jg.). S. 6-9

Tremmel, Jörg (2004d): Institutionelle Verankerung der Rechte nachrückender Generationen. In: Zeitschrift für Rechtspolitik. 37. Jg. (15.3.2004). S. 44-46 
Tremmel, Jörg (2003a): Nachhaltigkeit als politische und analytische Kategorie. Der deutsche Diskurs um nachhaltige Entwicklung im Spiegel der Interessen der Akteure. München

Tremmel, Jörg (2003b): Generationengerechtigkeit - Versuch einer Definition. In: Stiftung für die Rechte zukünftiger Generationen (Hg.): Handbuch Generationengerechtigkeit. 2. überarbeitete Auflage. München. S. 27-80

Tremmel, Jörg (2003c): Positivrechtliche Verankerung der Rechte nachrückender Generationen. In: Stiftung für die Rechte zukünftiger Generationen (Hg.): Handbuch Generationengerechtigkeit. 2. überarbeitete Auflage. München. S. 349-382

Tremmel, Jörg (2003d): Generationengerechtigkeit und Rentenbesteuerung. In: Rose, Manfred (Hg.): Integriertes Steuer- und Sozialsystem. Heidelberg. S. 421-436

Tremmel, Jörg (2003e): Generationengerechtigkeit aus ökonomischer Sicht. Abdiskontierung - die ökonomische Sicht der Zukunftsbewertung in der Diskussion. In: Generationengerechtigkeit! 2/2003 (3. Jg.). S. 19-21

Tremmel, Jörg (2001): Ist Deutschland überbevölkert? PAT - Die Weltformel der Bevölkerungswissenschaft. In: Generationengerechtigkeit. 2/2001. S. 16

Tremmel, Jörg (1998a): Der Generationsbetrug. In: Universitas. Heft 628. Oktober 1998 (53.Jg.). S. 915-919

Tremmel, Jörg (1998b): 68er und 89er - Zeit für einen Generationswechsel. In: Stiftung für die Rechte zukünftiger Generationen (Hg.): Die 68er. Warum wir Jungen sie nicht mehr brauchen. Freiburg. S. 213-288

Tremmel, Jörg (1997): Wie die gesetzliche Rentenversicherung nach dem Prinzip der Generationengerechtigkeit reformiert werden kann. In: Gesellschaft für die Rechte zukünftiger Generationen (Hg.): Ihr habt dieses Land nur von uns geborgt. Hamburg. S. 149-240

Tremmel, Jörg (1996): Der Generationsbetrug. Frankfurt am Main

Tremmel, Jörg / Viehöver, Martin (2000): Ökologische Steuerreform und Gerechtigkeit zwischen den Generationen. In: GAIA. 1/2000. S. 62-63

Tremmel, Jörg / Laukemann, Marc / Lux, Christina (1999): Die Verankerung von Generationengerechtigkeit im Grundgesetz - Vorschlag für einen erneuerten Art. 20a GG. In: Zeitschrift für Rechtspolitik. 10/1999 (32.Jg.). S. 432-438

Troge, Andreas (2004): Kommunen, Bevölkerungsentwicklung und Umwelt: Risiken erkennen - Chancen wahrnehmen. In: Umweltakademie Baden-Württemberg (2004) (Hg.): Bevölkerungsrückgang. Konsequenzen für Flächennutzung und Umwelt. Stuttgart. S. 29-40

UBA/Umweltbundesamt (1997): Nachhaltiges Deutschland - Wege zu einer dauerhaft umweltgerechten Entwicklung. Berlin

Udall, Stewart L. (1980): Foreword. In: Partridge, Ernest (1980) (Hg.): Responsibilities to Future Generations. Environmental Ethics. Buffalo N.Y. S. xi-xii 
Ulrich, Bernd (2003): Kanzlers trübe Truppe. In: DIE ZEIT. 20.11.2003 (58. Jg.). S. 1

Umweltakademie Baden-Württemberg (2004) (Hg.): Bevölkerungsrückgang. Konsequenzen für Flächennutzung und Umwelt. Stuttgart

UN Environment Programme (2000): Global Environmental Outlook. New York

UNFPA (2001): Weltbevölkerungsbericht 2001. Bevölkerung und Umwelt. (Übersetzt und hrsg. von der Deutschen Stiftung Weltbevölkerung. Stuttgart: Balance Verlag)

UN Population Division (2005): World Population Prospects. The 2004 Revision. Highlights. New York

UN Population Division (2003a): Fertility, Contraception and Population Policies. New York

UN Population Division (2003b): World Population in 2300. New York. http://www.un.org/esa/population/publications/longrange2/longrange2.htm. Rev. 2004-02-23. Rev. 2003-12-20

UN Population Division (2003c): World Population Prospects. The 2002 Revision. Executive Summery. New York

UN Population Division (2001a): National Population Policies 2001. New York

UN Population Division (2001b): World Population Monitoring. Population, Environment and Devlopment. New York

UN Population Division (2000): Replacement Migration. New York

UNO (10.12.1948): Resolution 217 A(III): Die Allgemeine Erklärung der Menschenrechte. http://www.unhchr.ch/udhr/lang/ger.htm. Rev. 2005-03-08

United Nations (1999): Schlüsselmaßnahmen zur weiteren Durchführung des Aktionsprogramms der Internationalen Konferenz über Bevölkerung und Entwicklung. Resolution der Generalversammlung. 21. Sondertagung. A/RES/S-21/2. New York

UNFPA: Die Welt im Gleichgewicht. Weltbevölkerungsbericht 1992. Bonn

Unnerstall, Herwig (1999): Rechte zukünftiger Generationen. Würzburg

Vaupel, James W. (08.04.2004): Deutschlands größte Herausforderung. In: Frankfurter Allgemeine Zeitung. 84/2004 (08.04.2004). S. 41

Vaupel, James W. / Oeppen, Jim (2002): Broken Limits to Life Expectancy. In: Science. 10.5.2002 (296 Jg.). S. 1029-1035

Veil, Mechthild (2002): Ganztagsschule mit Tradition: Frankreich. In: Aus Politik und Zeitgeschichte. Beilage zur Wochenzeitung „Das Parlament“. B 41/2002. S. 29-37

VDR/Verband Deutscher Rentenversicherungsträger (2004): Generationengerechtigkeit - Inhalt, Bedeutung und Konsequenzen für die Alterssicherung. Jahrestagung 2003 des Forschungsnetzwerks Alterssicherung (FNA) am 4. u. 5. Dezember 2003 in Erfurt. DRV-Schriften (Bd. 51). Frankfurt am Main 
VDR/Verband Deutscher Rentenversicherungsträger (2003): Stellungnahme zum „Wiesbadener Entwurf“. In: Hessische Staatskanzlei (Hg.): Die Familienpolitik muss neue Wege gehen! Der „Wiesbadener Entwurf“ zur Familienpolitik. Wiesbaden. S. 481-499

Verdi/Vereinte Dienstleistungsgewerkschaft (Oktober 2003): Mythos Demografie. Berlin

Viehöver, Martin (2002): Der Beitrag einer reformierten Grundsteuer zur nationalen Nachhaltigkeitsstrategie. Diplomarbeit. www.viehoever.de/grundsteuer. Rev. 2004-03-08

Vinken, Barbara (2001): Die deutsche Mutter. Der lange Schatten eines Mythos. München

Vitousek, P./Ehrlich, A. /Matson, P (1986): Human appropriation of the products of photosynthesis. In: Bio Science. Heft 34. S. 368-373

Vlastos, Gregory (1984): Justice and Equality. In: Waldron, Jeremy (Hg.): Theories of Rights. Oxford Readings in Philosophy. Oxford

Vorholt, Hubert (1984): Die Erklärung des generativen Verhaltens durch Richard A. Easterlin. IBS-Materialien Nr. 19. Bielefeld

Wagner, Angelika (2004): Der "ökologische Fußabdruck“ der Menschheit. In: Generationengerechtigkeit! Nr.1/2004 (4. Jg.). S. 14-15

Wahl, Stefanie (2004): Folgen der Bevölkerungsentwicklung für Wirtschaft und Gesellschaft. In: Umweltakademie Baden-Württemberg (2004) (Hg.): Bevölkerungsrückgang. Konsequenzen für Flächennutzung und Umwelt. Stuttgart. S. 13-28

Walzer, Michael (1992): Sphären der Gerechtigkeit. Frankfurt am Main/New York

WBGU/Wissenschaftlicher Beirat der Bundesregierung für Globale Umweltveränderungen (1999): Welt im Wandel. Umwelt und Ethik. Sondergutachten. Marburg

WBGU/Wissenschaftlicher Beirat der Bundesregierung für Globale Umweltveränderungen (1996): Welt im Wandel. Herausforderung an die deutsche Wissenschaft. Jahresgutachten 1996. Berlin

Weber, Max (1904): Die "Objektivität" sozialwissenschaftlicher und sozialpolitischer Erkenntnis. In: Weber, Max: Gesammelte Aufsätze zur Wissenschaftslehre (hrsg. von Winckelmann, Johannes). 7. Auflage. Tübingen. 1988. S. 146-214

Weber, Max (1913): Äußerungen zur Werturteilsdiskussion im Ausschuß des Vereins für Sozialpolitik. Als Manuskript gedruckt 1913. In: Nau, Heino (1996) (Hg.): Der Werturteilsstreit: die Äußerungen zur Werturteilsdiskussion im Ausschuss des Vereins für Sozialpolitik (1913). S. 147-186

Weber, Max (1917): Der Sinn der „Wertfreiheit" der soziologischen und ökonomischen Wissenschaften. In: Weber, Max: Gesammelte Aufsätze zur Wissenschaftslehre (hrsg. von Winckelmann, Johannes). 7. Auflage. Tübingen. 1988. S. 489-540 
Weber, Max (1980): Wirtschaft und Gesellschaft. Grundriß der verstehenden Soziologie. 5. Auflage. Tübingen

Weimann, Mike (2002): Wahlrecht für Kinder. Eine Streitschrift. Weinheim

Werding, Martin (1998): Zur Rekonstruktion des Generationenvertrages. Ökonomische Zusammenhänge zwischen Kindererziehung, sozialer Alterssicherung und Familienlastenausgleich. Tübingen

Weiss, Edith Brown (1989): In Fairness to Future Generations. Tokyo/New York

Weizsäcker, Ernst Ulrich von (1995): Faktor Vier. Doppelter Wohlstand - halbierter Naturverbrauch. München

White, Tyrene (2000): Domination, Resistance and Accommodation in China's OneChild Campaign. In: Perry, Elizabeth J. / Selden, Mark (Hg.): Chinese Society: Change, Conflict, and Resistance. London/New York. S. 102-119

Wichterich, Christa (1994): Menschen nach Maß - Bevölkerung nach Plan. In: Wichterich, Christa (Hg.): Menschen nach Maß. Bevölkerungspolitik in Nord und Süd. Göttingen. S. 9-37

Wiersbinski, Norbert / Erdmann, Karl-Heinz / Lange, Hellmuth (1998): Zur gesellschaftlichen Akzeptanz von Naturschutzmaßnahmen. Bonn

Wilson, Edward (2004): Die Zukunft des Lebens. München: Goldmann Vollständige Taschenbuchausgabe (Original: The Future of Life. 2002)

Wilson, Edward O. (1992): The Diversity of Life. Boston

Wilson, Edward O. (1984): Biophilia. Oxford

Williams, Mary B. (1978): Discounting vs. Maximum Sustainable Yield. In: Barry, Brian / Sikora, Richard (Hg.): Obligations to Future Generations. Philadelphia. S. 169-185

Wingen, Max (2004): Die Geburtenkrise ist überwindbar: Wider die Anreize zum Verzicht am Nachkommenschaft. Grafschaft

Wingen, Max (01.04.2004): Bevölkerungsbewusste Familienpolitik. In: Frankfurter Allgemeine Zeitung. 78/2004 (01.04.2004). S. 9

Wingen, Max (2002): Ein erneutes Plädoyer für eine bevölkerungsbewusste Familienpolitik. In: Zeitschrift für Bevölkerungswissenschaft. 1/2002 (27. Jg.). S. 69-85

Wingen, Max (1975): Grundfragen der Bevölkerungspolitik. Stuttgart

Wissenschaftlicher Beirat beim Bundesministerium der Finanzen (2001): Nachhaltigkeit in der Finanzpolitik: Konzepte für eine langfristige Orientierung öffentlicher Haushalte, dem Bundesfinanzministerium am 7. Dezember 2001 übergebenes Gutachten. http://www.bundesfinanzministerium.de/Anlage9127/Gutachten-zurNachhaltigkeit-in-der-Finanzpolitik.pdf. Rev. 2004-08-03

Wolf, Clark (1997): Person-Affecting Utilitarianism and Population Policy; or, Sissy Jupe's Theory of Social Choice. In: Fotion, N. / Heller, J.C.: Contingent Future 
People: On the Ethics of Deciding Who will live, or not, in the Future. Dordrecht. S. 99-122

Wolf, Clark (1996): Social Choice and Normative Population Theory: A Person-Affecting Solution to Parfit's Mere-Addition Paradox. In: Philosophical Studies 81 (2-3). S. 263-282

Wolf, Clark (1995): Property Rights, Lockean Provisos, and the Interests of Future Generations. In: Ethics. Heft 105 (4). S. 791-818

Wong, John (2001): China's Sharply Declining Fertility: Implications for Its Population Policy. In: Issues and Studies 37, Heft 3. US Census Bureau: www.census.gov/cgibin/ipc/idbsprd. Rev. 2003-12-09

World Commission on Environment and Development (1987): Our Common Future. Oxford/New York/Toronto

Worldwatch Institute (2002): Vital Signs. The Trends That Are Shaping Our Future. New York/London

Worldwatch Institute (2000): Wie viel ist zu viel? 19 Dimensionen zur Bevölkerungsentwicklung. Hrsg. von der Deutschen Stiftung Weltbevölkerung. Stuttgart. (Engl.Originalausgabe: Beyond Malthus. New York 1999)

Worldwatch Institute (1993): Full House: Reassessing the Earth's Population Carrying Capacity. New York

World Wide Fund for Nature/WWF (2004a): Living Planet Report 2004. http://www.panda.org/news_facts/publications/index.cfm. Rev. 2005-01-12

World Wide Fund for Nature/WWF (2004b): Living Planet Report 2004. Deutsche Zusammenfassung. http://www.wwf.de/service/publikationen/. Rev. 2005-01-12

World Resources Institute/UNEP/UNDP/World Bank (2000): World Resources 2000/2001. Washington D.C.

Xie, Zhenming (2000): Population Policy and the Family-Planning Programme. In: Peng, Xizhe / Guo, Zhigang (Hg.): The Changing Population of China. Oxford

Zahrnt, Angelika (1993): Zeitvergessenheit und Zeitbesessenheit in der Ökonomie - und ihre ökologischen Folgen. In: Held, Martin / Geissler, Karlheinz (Hg.): Ökologie der Zeit. Vom Finden der rechten Zeitmaße. Stuttgart. S. 111-120

Zechmeister, Michael / Tremmel, Jörg (2001): Die Teilungslösung. In: Soziale Sicherung. 2/2001. S. 23-30

Zhang, Junsen (1990): Socioeconomic Determinants of Fertility in China. In: Journal of Populations Economics 3, Heft 2. S. 105-123 